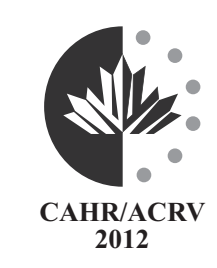

CAHR 2012

A Turning Point in the Fight Against HIV: Meeting New Challenges $21^{\text {st }}$ Annual Canadian Conference on HIV/AIDS Research

ACRV 2012

Un point tournant dans la lutte contre le $\mathrm{VIH}$ : de nouveaux défis à relever $21^{\mathrm{e}}$ Congrès canadien annuel de recherche sur le VIH/sida

$$
\begin{gathered}
\text { April 19-22 / Du } 19 \text { au } 22 \text { avril } 2012 \\
\text { Montreal, Quebec }
\end{gathered}
$$

\title{
ABSTRACTS / RÉSUMÉS
}

CAHR Executive Committee / Comité directeur de I'ACRV

Message from the CAHR President / Message du président de l'ACRV

Message from the Co-Chairs of CAHR 2012 / Message des coprésidents

du congrès de l'ACRV 2012

Oral Presentations / Exposés oraux

Poster Presentations / Présentations des affiches

CAHR 2012 - Publish Only / ACRV 2012 - Publient seulement

Author index / Index des auteurs

\section{Departments}

Calendar of Events 


\section{$21^{\mathrm{TH}}$ ANNUAL CANADIAN CONFERENCE ON HIV/AIDS RESEARCH $21^{\mathrm{e}}$ CONGRÈS CANADIEN ANNUEL DE RECHERCHE SUR LE VIH/sida CAHR COMMITTEES / COMITÉS DE L'ACRV}

\section{CAHR EXECUTIVE COMMITTEE / COMITÉ DIRECTEUR DE L'ACRV}

President / Président: Dr. Jonathan Angel

President Elect / Président désigné: Dr. Robert Hogg

Past President / Ancien président: Dr. William Cameron

Treasurer / Trésorière: Dr. Curtis Cooper

Executive Director / Directeur général: Andrew Matejcic

\section{CAHR COUNCIL MEMBERS / MEMBRES DU CONSEIL DE L'ACRV}

Track A / Volet A : Basic Sciences / Sciences fondamentales: Dr. Matthias Götte

Track B / Volet B : Clinical Sciences / Sciences cliniques: Dr. Jean-Pierre Routy

Track C / Volet C: Epidemiology and Public Health Sciences / Épidémiologie et sciences de la santé publique: Dr. Margaret Millson

Track D / Volet D : Social Sciences / Sciences sociales: Dr. Saara Greene

Secretary / Secrétaire: Dr. Carole Strike

Community Representative / Représentant communautaire: Darien Taylor

\section{CONFERENCE ORGANIZING COMMITTEE / COMITÉ ORGANISATEUR}

Conference Co-Chairs / Coprésidents du congrès: Dr. Pierre Côté and Dr. Jean-Pierre Routy

\section{Track Chairs / Coprésidents des volets}

Track A / Volet A : Basic Sciences / Sciences fondamentales

Dr. Bluma Brenner

Dr. Éric Cohen

Track B / Volet B : Clinical Sciences / Sciences cliniques

Dr. François Coutlée

Dr. Richard Lalonde

Track C / Volet C : Epidemiology and Public Health Sciences / Épidémiologie et sciences de la santé publique

Dr. Julie Bruneau

Dr. Nitika Pant Pai

Track D / Volet D : Social Sciences / Sciences sociales

Dr. Gilbert Émond

Christine Vézina

Community Representative / Représentant communautaire

Bruno Lemay

Sponsorship Coordinator / Coordonnateur des commandites : Dr. Brian Conway

Conference Advisory Members / Membres du comité consultatif du congrès :

Dr. Jonathan Angel, Andrew Matejcic, Robert O’Neill

\section{CONFERENCE COORDINATOR / ORGANISATEUR PROFESSIONNEL DU CONGRÈS}

Sea to Sky Meeting Management Inc.

206, 201 Bewicke Avenue, North Vancouver, British Columbia V7M 3M7, Canada

Telephone / Téléphone : 604-984-6455; Fax / Télécopieur : 604-984-6434

www.seatoskymeetings.com; info@cahr-conference-acrv.ca

President / Présidente : Sarah Lowis, CMP, CMM

Project Director / Directeur de projet : Ian Holliday

Registration Manager / Coordonnatrice des inscription : Glenda Freeman 


\section{Track A / Volet A : Basic Sciences / Sciences fondamentales}

Jonathon Angel

Nicole Bernard

Bluma Brenner

James Brooks

Alan Cochrane
Eric Cohen

Keith Fowke

Anne Gatignal

Matthias Götte

Michael Grant
Charu Kaushiv

Larry Kleiman

Chen Liang

Andrea Mouland

Mario Ostrawski

\section{Track B / Volet B : Clinical Sciences / Sciences cliniques}

$\begin{array}{ll}\text { Jean-Guy Baril } & \text { John Gill } \\ \text { Brian Conway } & \text { Marina Klein } \\ \text { Pierre Côté } & \text { Bernard Lessard } \\ \text { Francois Coutlée } & \text { Mona Loutfy } \\ \text { Alexandra de Pokomandy } & \text { Marie Munoz } \\ \text { Claude Fortin } & \text { Martin Potter }\end{array}$

Anita Rachlis

Michel Roger

Danielle Rouleau

Jean-Pierre Routy

Stephen Shafran

Mariek Smieja

\section{Track C / Volet C : Epidemiology and Public Health Sciences Epidémiologie et sciences de la santé publique}

$\begin{array}{ll}\text { Alix Adrien } & \text { Bob Hogg } \\ \text { Chris Archibald } & \text { Mira Johri } \\ \text { Suzanne Brissette } & \text { Gilles Lambert } \\ \text { Vera Caine } & \text { Peggy Millson } \\ \text { Joe Cox } & \text { Seyed Moghadas }\end{array}$

Carole Morissette

Stephen Moses

Stephanie Nixon

Mario Ostrowski

Robert Remis
Art Poon

Michel Tremblay
Sylvie Trottier

Benoit Trottier

Sharon Walmsley

\section{Track D / Volet D : Social Sciences/ Sciences sociales}

Barry Adam

David Brennan

Roy Cain

Liviana Calzavara

Stéphanie Claivaz-Loranger

José Côté
Colleen Ann Dell

Richard Elliott

Marilou Gagnon

Jacqueline Gahagan

Fransisco Ibanez-Carrasco

Thomas Kerr
Alana Klein

René Lavoie

Maria Nengeh Mensah

Jennifer Poudrier

Dominic Prévost-Beaulieu

Janice Ristock
Élise Roy

Ameeta Singh

Mark Tyndall

David Thompson

Catherine Worthington 
$\mathrm{T}$ he Canadian Association for HIV Research (CAHR) is the professional organization for HIV/AIDS research in Canada and by Canadians. CAHR is composed of researchers in all disciplines of HIV/AIDS science, for the purpose of improving prevention and treatment with an ultimate goal of eradication and cure. The annual CAHR conference is the premier gathering in Canada for those working in the field of HIV, as well as policy makers, persons living with HIV, and other individuals committed to ending the pandemic. It is a chance to assess where we are, evaluate recent scientific developments and lessons learned, and together chart a course forward.

Of course, as we think about the major advances that have been made, we must remind ourselves that HIV has not gone away. It is estimated that 34 million people are living with AIDS, of which 2.3 million are children most of whom are in subSaharan Africa. Here in Canada, HIV remains a pressing issue with an estimated 2,300 to 4,300 new infections each year, with certain populations over-represented in the epidemic.

We can be proud that Canadian researchers remain at the forefront of HIV research, focusing on all aspects of the disease, from exploring the basic foundation of the disease, understanding the intersection of aging and complications of HIV/AIDS, improving care and treatment, to developing interventions to reduce the risks and rates of complications. Once again, this year's program will present new scientific knowledge and offer many opportunities for structured dialogue on the major issues facing the global response to HIV. A variety of sessions - from abstract-driven presentations to symposia, bridging, and plenary sessions - will meet the needs of all participants. Other related activities, including ancillary meetings and the New Investigator Workshop, will contribute to an exceptional opportunity for professional development and networking.

CAHR 2012 will be a tremendous opportunity for researchers and community members from coast to coast to share the latest scientific advances in the field, learn from one another's expertise, and develop new ways to treat and prevent HIV. I hope you enjoy the conference, find it to be a worthwhile learning experience, and thank you in advance for your contributions, participation, and support.
I'Association canadienne de recherche sur le VIH L(ACRV) est un organisme professionnel se consacrant à la recherche sur le VIH/sida au Canada. L'ACRV est composée de chercheurs multidisciplinaires en VIH/sida dont l'objectif est d'améliorer les moyens de prévention et les traitements pour éventuellement éradiquer l'épidémie. Le congrès de l'ACRV est le principal lieu de rassemblement des intervenants dans le domaine du VIH, des décideurs, des personnes vivant avec le VIH et d'autres personnes s'engageant à mettre fin à l'épidémie. Il offre une occasion d'évaluer la situation actuelle, les récents progrès scientifiques et les nouvelles leçons apprises, et d'établir un plan d'action.

Évidemment, quand nous pensons aux percées majeures réalisées, nous devons nous rappeler que le VIH n'est pas pour autant disparu. En effet, le nombre de séropositifs dans le monde est estimé à 34 millions, dont 2,3 millions d'enfants, la majorité vivant en Afrique subsaharienne. Au Canada, le VIH reste une question urgente : de 2300 à 4300 nouvelles infections sont diagnostiquées chaque année et certains groupes de population sont sous-représentés.

Nous devons être fiers du fait que les chercheurs canadiens demeurent au premier plan de la recherche sur le VIH. Ceux-ci se penchent sur tous les aspects de la maladie, de l'étude des bases de la maladie et du lien entre le vieillissement et les complications du VIH/ sida, en passant par l'amélioration des soins et des traitements et la mise en place d'interventions visant à réduire les risques et les taux de complications. Cette année encore, la présentation des nouvelles connaissances scientifiques est prévue au programme, et de nombreuses occasions de dialogue structuré seront offertes concernant les principaux obstacles liés à la lutte mondiale contre le VIH. Une variété de séances - présentations d'abrégés, colloques, séances intermédiaires et séances plénières - répondra aux besoins des différents participants. Des activités connexes, dont des réunions auxiliaires et l'atelier destiné aux nouveaux chercheurs dans le domaine du VIH, offriront en outre une excellente occasion de perfectionnement professionnel et de réseautage.

Le congrès de l'ARCV 2012 constitue une occasion privilégiée pour les chercheurs d'un bout à l'autre du pays d'échanger sur les progrès scientifiques les plus récents en matière de $\mathrm{VIH}$, d'apprendre de leurs homologues et d'établir de nouvelles stratégies de traitement et de prévention du VIH. Espérant que le congrès sera pour vous une expérience d'apprentissage utile et agréable, je vous remercie d'avance de votre contribution, de votre participation et de votre soutien.

\author{
Dr Jonathan Angel \\ President / Président \\ Canadian Association for HIV Research (CAHR) / Association canadienne de recherche sur le VIH (ACRV)
}




\section{MESSAGE FROM THE CO-CHAIRS OF CAHR 2012/ MESSAGE DES COPRÉSIDENTS DU CONGRÈS DE L'ACRV 2012}

W elcome to the 21st Annual Canadian Conference on HIV/AIDS Research (CAHR 2012). The theme of CAHR 2012, "A Turning Point in the Fight Against HIV: Meeting New Challenges", was chosen by the Organizing Committee to recognize a defining moment in the epidemic. For the first time, we have started to curb the HIV epidemic, representing the turning point in the global response to controlling the infection. In the absence of a vaccine, the proven effectiveness of "treatment as prevention" highlights the need and our responsibility to make antiretroviral medication available worldwide. The cure of a single HIV infected patient by stem cell transplantation paves the road for an alternative strategy making HIV eradication research a priority.

Management of people infected with HIV continues to be an important challenge despite this success. Longterm drug toxicity, persistence of immune inflammation, premature aging, and co-infection with hepatitis remain unmet health issues. Vulnerable populations in Canada such as injection drug users, women, and Aboriginals are still facing unanswered challenges to improve their quality of life and access to early treatment. We need to overcome the emotional, social, and financial barriers to lessen the epidemic in these hard-to-reach populations. In addition, criminalization of HIV transmission or exposure poses a new challenge as antiretroviral therapy has been proven to reduce transmission. Canada's current legislation needs to be critically reviewed as it may discourage people from being tested for HIV, thereby

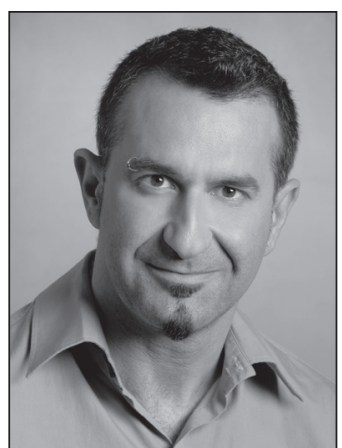

Dr Pierre Côté

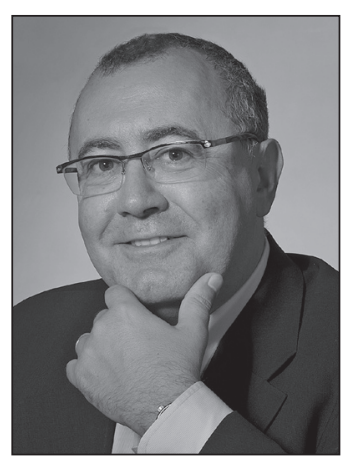

Dr Jean-Pierre Routy

Bienvenue au $21^{\mathrm{e}}$ Congrès canadien annuel de recherche sur le VIH/sida (congrès de l'ACRV 2012). Le thème du congrès, Un point tournant dans la lutte contre le $\mathrm{VIH}$ : de nouveaux défis à relever, a été choisi par le comité organisateur pour souligner l'étape déterminante franchie dans la lutte contre l'épidémie. Pour la première fois, nous avons commencé à juguler l'épidémie, ce qui constitue un point tournant dans les efforts déployés dans le monde entier pour en venir à bout. En l'absence d'un vaccin, l'efficacité démontrée du « traitement comme moyen de prévention » fait ressortir la nécessité d'offrir des médicaments antirétroviraux à l'échelle mondiale ainsi que notre responsabilité à cette fin. La guérison d'un patient infecté par le VIH grâce à une greffe de cellules souches ouvre la voie à une stratégie novatrice faisant de la recherche visant à éradiquer le VIH une priorité.

La prise en charge des personnes infectées par le VIH demeure un défi important malgré cette réussite. La toxicité des médicaments à long terme, la persistance de l'inflammation du système immunitaire, le vieillissement prématuré et la co-infection VIH/VHC sont autant de problèmes de santé non encore résolus. Au Canada, les groupes vulnérables, notamment les utilisateurs de drogues injectables, les femmes et les Autochtones, se heurtent toujours à des difficultés en ce qui a trait à l'amélioration de leur qualité de vie et à l'accès à un traitement dès le début de la maladie. Nous devons surmonter les obstacles émotionnels, sociaux et financiers pour atténuer l'épidémie dans ces groupes difficiles à jeopardizing early treatment.

CAHR 2012 provides a venue for conference participants to meet and share the outcomes of new research, honour new investigators, acknowledge the achievements of major contributors to the field, and elect a Council to carry on the work of the Association. We will learn and get involved together to build a stronger community to resolve what we still consider, even today, as impossible.

In Montreal, you will experience a bilingual city whose passion, joie de vivre and rich cultural heritage are legendary. Montrealers love to greet visitors and show off their city's charms, so expect a very warm welcome. It's like a taste of Europe right in North America. Enjoy CAHR 2012 'à la Montréal'.

joindre. En outre, la criminalisation de la transmission du VIH ou de l'exposition au VIH constitue un nouveau défi, la thérapie antirétrovirale ayant démontré son efficacité à réduire la transmission. La législation canadienne actuelle doit être entièrement revue, car elle pourrait décourager les gens à se soumettre à des tests de dépistage du $\mathrm{VIH}$, ce qui pourrait nuire à une intervention précoce.

Le congrès de l'ACRV 2012 est un lieu de rassemblement où les participants peuvent échanger et partager les résultats des nouvelles études, rendre hommage aux nouveaux chercheurs, souligner les réalisations des principaux collaborateurs dans le milieu et élire les membre du conseil d'administration de l'ACRV. Ensemble, nous enrichirons nos connaissances et solidifierons nos liens pour trouver une solution à l'épidémie, même si encore aujourd'hui, ce dénouement semble impossible.

Lors de votre séjour à Montréal, vous ferez l'expérience d'une ville bilingue animée d'une passion, d'une joie de vivre et d'un héritage culturel légendaires. Très accueillants, les Montréalais sont fiers de montrer au monde entier les charmes de leur ville unique, ville à saveur européenne au cœur de l'Amérique du Nord. Bon congrès « à la Montréal »!

Dr Pierre Côté, Dr Jean-Pierre Routy

CAHR 2012 Conference Co-Chairs / Coprésidents du congrès de l'ACRV 2012 
Track A: Basic Sciences: HIV Structure, Function and Cell Biology

Volet A : Sciences fondamentales : Structure, fonction et biologie cellulaire du VIH

\section{O001}

\section{THE HIV ENVELOPE GLYCOPROTEIN: A TENSEGRITY ARCHITECTURE}

Finzi, Andrés ${ }^{1}$; Pacheco, Beatriz²; Gu, Chris²; Wang, Liping²; Sodroski, Joseph²; Mao, Youdong²

\section{${ }^{1}$ Montreal, QC; ${ }^{2}$ Boston, MA, USA}

The entry of primate immunodeficiency viruses into target cells requires structural conservation in the envelope glycoprotein (Env) trimer. Meanwhile, structural plasticity and variability in the Env trimer across different strains of the viruses are needed to evade the immune system. How the two counteracting factors coexist in a single structure remains mysterious. The atomic structure of the HIV-1 Env trimer recently solved by cryoelectron microscopy suggests a hypothetical answer to this question, ie,, that the Env trimer has a tensional integrity (tensegrity) architecture. Here we substantiate the tensegrity hypothesis through the use of Env mutants and structural homology modelling. A series of single changes in conserved residues located at the tensional junctions and predicted to maintain the native tensegrity in the structure results in dramatic breakdown of the HIV-1 envelope glycoprotein trimer. This novel structural mechanism may be a useful guide for pharmacologic or prophylactic intervention in human immunodeficiency virus (HIV) infections.

AF is supported by amfAR Mathilde Krim Fellow Phase II Grant \#10809250-RKVA, a Bill and Melinda Grand Challenges Exploration Grant and a CRCHUM starting package.

\section{O002 \\ CONTRIBUTION OF HOST NUCLEOPORIN 62 IN HIV-1 INTEGRASE CHROMATIN ASSOCIATION AND VIRAL DNA INTEGRATION}

Ao, Zhujun; Jayappa, Kallesh; Wang, Binchen; Zheng, Yingfeng; Wang, Xiaoxia; Peng, Jinyu; Yao, Xiaojian

Winnipeg, MB

BACKGROUND: HIV-1 integration is promoted by viral integrase (IN) and its cellular cofactors. The lens epithelium derived growth factor (LEDGF/p75), an IN interacting cellular co-factor, has been shown to play an important role in HIV-1 chromatin targeting and integration. However, whether other cellular cofactors is also involved in above viral replication steps is still elusive. Nucleoporins (Nups), the major components of nuclear pore complex, not only facilitate the movement of receptors between the nucleus and the cytoplasm, but also interact with transcriptionally active genes in nucleoplasm and regulate gene expression. Here we analyze the functional role of Nup62 in HIV infection and its involvement in chromatin targeting and integration.

RESULTS: By using a cell based co-immunoprecipitation method, we show that the nucleoporins 62 (Nup62) can specifically interact with HIV-1 IN in 293T cell and HIV-1 infected CD4 $4^{+} \mathrm{T}$ cells and the IN binding region of Nup62 was mapped to its C-terminal coil-coil domain. We further demonstrated that Nup62 binds to chromatin and also interacts with HIV-1 IN in both soluble nuclear extract and chromatin-bound fractions by subcellular fractionation method. Interestingly, knockdown of Nup62 by shRNA significantly reduced the association of IN with host chromatin and impaired HIV-1 replication in $\mathrm{CD}^{+} \mathrm{T}$ cells and macrophages. Using quantitative real time PCR analysis, we demonstrated that HIV-1 infection in Nup62-knockdown cells is remarkably blocked at integration step. Furthermore, the expression of the C-terminal domain of Nup62 alone in CD4+ T cells significantly inhibited HIV-1 infection. CONCLUSION: Our results indicate that the cellular Nup62 is specifically recruited by HIV-1 IN and contributes to an efficient viral DNA integration. This study provides new insights into the early stage of HIV replication and suggests possible new target for HIV prevention and treatment.

\section{O003}

\section{TIAR PLAYS A CENTRAL ROLE IN REGULATING HIV-1 RNA} PROCESSING

\section{Cochrane, Alan}

Toronto, ON

Replication of HIV-1 is critically dependent upon the regulated processing of its RNA. Provirus transcription generates a single $9 \mathrm{~kb}$ RNA that is processed into over $40 \mathrm{mRNAs}$ by suboptimal splicing. Imbalance of viral RNA splicing results in a loss of new virions assembly and arrests the replication of this virus. Given that HIV-1 RNA splicing is largely regulated and performed by host cellular factors, we investigated various host proteins for their role in regulating HIV-1 RNA processing, with the goal of identifying new targets for therapeutic intervention. Depletion of TIA-1, TIAR and ELAV used shRNAs and the impact of the depletion on HIV-1 protein expression and viral RNA splicing was evaluated. These factors are normally implicated in regulating RNA stability and translation but more recent evidence has also demonstrated that they can regulate RNA splicing. Of the three factors, depletion of TIAR was found to have the most significant effect, resulting in almost complete loss of expression of the HIV-1 structural protein Gag and Env with only limited effect on the viral regulatory proteins Tat and Rev. Subsequent analysis of HIV-1 RNA determined that depletion of TIAR resulted in a dramatic reduction in incompletely spliced viral RNAs levels and increased accumulation of fully spliced viral RNA, consistent with an oversplicing phenotype. Analysis of splice site use did not detect any changes upon TIAR depletion. In contrast, depletion of the highly homologous TIA-1 resulted in increased viral protein levels that correlated with increases in all HIV-1 RNAs. Depletion of ELAV had a more limited effect, resulting a selective reduction in HIV-1 Env synthesis without affecting the abundance of the corresponding RNA, suggesting that this factor is acting at the level of translation. In conclusion, this study has identified TIAR as a key regulator of HIV-1 RNA processing. Further understanding of its mechanism of action will provide important insights into the regulation of viral RNA splicing that will prove useful in the design of therapeutic interventions to control this infection.

\section{4 \\ EVALUATING THE ROLE OF HIV-1 GAG IN THE BLOCKAGE OF STRESS GRANULES}

Valiente-Echeverria, Fernando A; Melnychuk, Luca; Vyboh, Kishanda; Ajamian, Lara; Mouland, Andrew

Montreal, QC

HIV-1 is member of the lentivirus subfamily of the Retroviridae and the etiologic agent of AIDS. Following HIV-1 infection, the host cell responds by mounting a robust, anti-viral immune response in order to create an inhospitable environment for viral replication. During a stress response, cells initiate to both the shut-off of mRNA translation (protein synthesis) and the assembly of stress granules (SGs). We have previously shown that HIV-1 expression impairs the assembly of SGs in T cells favoring the assembly of Staufen1-containing HIV-1-dependent ribonucleoproteins (RNPs), known as SHRNPs. In this work, we attempted to understand how HIV-1 blocks SG assembly. We have mapped the viral determinants that mediate the HIV-1-induced blockade to SG assembly. Here we demonstrate that Gag, specifically Capsid domain (CA), controls SG assembly and interferes with the cellular stress response pathway. Moreover, immunoprecipitation followed by proteomic analyses identified elongation factor 2 (eEF2), a component of the translational machinery, as a Gag-interacting protein. Depletion of eEF2 by siRNA in HIV-1-infected cells resulted in the assembly of SGs in HIV-1-expressing cells, whereas the expression of siRNA-resistant eEF2 impaired SG assembly. Finally, in live cell imaging assays we observed that Gag not only blocks the assembly of SGs, but also mediates the disassembly of SG. Understanding how HIV-1 interacts with the host to achieve these anti-viral countermeasures will contribute to our understanding of HIV-1 pathogenesis. This work is supported by a grant from the Canadian Institutes of Health Research (MOP-38111) and F.V.-E. is a recipient of a Postdoctoral Fellowship from Becas Chile. 


\section{5}

\section{UPF1 SHUTTLING MEDIATES HIV-1 VRNA EXPORT BY} BYPASSING THE REQUIREMENT OF UPF2

\section{Ajamian, Lara; Vyboh, Kishanda; Abel, Karen; Mouland, Andrew J} Montreal, QC

The HIV-1 ribonucleoprotein complex includes Gag, the genomic RNA (vRNA) and several cellular proteins including upframeshift protein 1 (UPF1), a key factor in nonsense-mediated mRNA decay (NMD). UPF1, 2 and 3 function together in this RNA quality control mechanism to eliminate mRNAs that harbor pre-termination codons (PTC) to prevent the synthesis of deleterious proteins. In earlier work, we demonstrated that UPF1 controlled vRNA stability and translation and these effects were independent of its functions in NMD. Here we reveal that UPF1 promotes HIV-1 nucleocytoplasmic vRNA export and this is dependent on the nucleocytoplasmic shuttling function of UPF1 mediated by its nuclear export and localization sequences (NES, NLS). In addition, UPF1 relieved the nuclear retention of vRNA in the absence of the viral protein Rev. UPF1 co-precipitated with Rev, CRM1 and DDX3 as well as the nucleoporin $\mathrm{p} 62$ supporting a dominant role for UPF1 in HIV-1 vRNA trafficking during the late expression phase of HIV-1. The major binding partner of UPF1 for the classical NMD pathway is UPF2. We had previously shown that UPF2 was excluded from the Staufen-HIV-1 RNP complex. We therefore decided to verify if this interaction was important in HIV-1. We identified UPF2 as being a negative regulator of HIV-1 vRNA export. UPF1 in complex with UPF2 (and UPF3aL) results in the nuclear sequestration of the HIV-1 vRNA. Our results describe a novel unique regulatory circuit between the UPF proteins essential for HIV-1 vRNA export such that the exclusion of UPF2 from the HIV-1 RNP complex is necessary for UPF1 to efficiently mediate the export the HIV-1 vRNA. In conclusion, we have shown that UPF1's shuttling function is essential for HIV-1 vRNA nucleocytoplasmic export and this is mediated by excluding an interaction between UPF1 and UPF2, an association essential for NMD.

\section{O006}

\section{DETECTION OF THE ANTISENSE PROTEIN (ASP) OF HIV-1} AND ITS POTENTIAL IMPLICATION IN AUTOPHAGY

Torresilla, Cynthia ${ }^{1}$; Larocque, Emilie ${ }^{1}$; Landry, Sébastien ${ }^{2}$;

Masson, Jean-Yves ${ }^{3}$; Mesnard, Jean-Michel ${ }^{4}$; Barbeau, Benoit ${ }^{1}$

${ }^{1}$ Montréal, QC; ${ }^{2}$ San Diego, CA, USA; ${ }^{3}$ Québec, QC; ${ }^{4}$ Montpellier, France

BACKGROUND: HIV-1 produces its proteins from a single transcript. The possible existence of a protein expressed from an antisense transcript has however been suggested. We have previously demonstrated the existence of such an antisense and polyadenylated transcript. ASP (AntiSense Protein) is the resulting protein from this transcript and is predicted to be highly hydrophobic. Due to the difficulty in demonstrating its existence, the aim of our study was to detect the protein in mammalian cell lines and to determine a potential function.

METHODS: Both the codon-optimized version of the ASP sequence (ASP*) and the non-optimized version (ASP) were tested. COS-7, 293T, Jurkat and U937 cell lines were transfected with expression vectors for both Myc-tagged ASP versions and analysed by confocal microscopy, FACS and Western blot.

RESULTS: We first demonstrated that ASP was detected by FACS analyses in all transfected cell lines with a more pronounced signal in ASP*-expressing cells. In COS-7 and 293T, ASP expression was detected by Western blot analyses and confocal microscopy, most importantly with ASP*. ASP localized in the cytoplasm of cells in a punctuated manner, which was reminiscent of autophagosomes. A significant increase in ASP-positive cells was noted in transfected COS-7 and 293T cells when autophagy was inhibited at its early steps. In addition, the punctuated localization of ASP was also lost in these conditions, suggesting that these structures could be autophagosomes. The induction of autophagy was confirmed with the overexpression of LC3-II in presence of ASP and the co-localization of ASP with LC3 positive punctua.

CONCLUSION: This study provides the first evidence that the antisense transcript of HIV-1 can lead to the synthesis of ASP in mammalian cells. The induction of autophagy following ASP expression might explain the inherent difficulty in detecting this viral protein and further justify its presumed low abundance in infected cells.
Track B: Clinical Sciences: Anti-HIV Treatment Modalities Volet B : Sciences cliniques : Modalités de traitement du VIH

\section{7}

EFFECT OF RANDOMIZED COMBINATION ANTIRETROVIRAL THERAPY (CART) ON VIRAL SUPPRESSION OFF THERAPY IN PATIENTS WITH ACUTE/ EARLY HIV INFECTION (CTN 214 STUDY)

Margolick, Joe ${ }^{2}$; Apuzzo, Linda ${ }^{2}$; Tossonian, Harout $^{1}$; Singer, Joel ${ }^{1}$; Fraser, Chris ${ }^{3}$; Loutfy, Mona ${ }^{4}$; Rachlis, Anita ${ }^{4}$; Kasper, Ken ${ }^{6}$;

El-Helou, Philippe ${ }^{5}$; Conway, Brian ${ }^{1}$

${ }^{1}$ Vancouver, BC; ${ }^{2}$ Baltimore, MD, USA; ${ }^{3}$ Victoria, BC; ${ }^{4}$ Toronto; ${ }^{5}$ Hamilton, ON; ${ }^{6}$ Winnipeg, $\mathrm{MB}$

BACKGROUND: Early treatment with cART may allow more effective immune-mediated suppression of viral replication, a possibility that has been supported in recent randomized trials in acute infection. Little is known about the natural history of acute/early HIV infection in this group or the response to cART if initiated at this stage of disease.

METHODS: We studied viral suppression in patients diagnosed within the first year of HIV infection who were randomized to immediate treatment with cART for 12 months or deferred treatment. We compared the number of patients in the deferred treatment arm who had not initiated cART at 24 months after study entry to that in the immediate treatment arm 24 months after cessation of cART, and the distributions of viral load in each group at this time point.

RESULTS: 113 patients were enrolled (57 in immediate and 56 in deferred treatment arm). The arms did not differ significantly in proportion of those infected within 2 months of study entry. 11 (19.2\%) patients in the immediate treatment arm had not reinitiated cART at 24 months off treatment, while only $4(7.1 \%)$ patients in the deferred treatment arm had not initiated cART by 24 months on study $(\mathrm{p}=0.09)$. Viral load set point at 24 months in those who had not (re)initiated cART was $<10,000$ copies $/ \mathrm{ml}$ in $10(17.5 \%)$ patients in the immediate treatment arm and $3(5.4 \%)$ in the deferred treatment arm $(p=0.07)$.

CONCLUSIONS: The group randomized to immediate cART had more people who remained off therapy, and more with low viral loads $(<10,000$ copies $/ \mathrm{ml})$ at 24 months without treatment than did the group randomized to deferred therapy. This suggests a benefit of immediate cART to the immune response that controls HIV replication in patients treated within the first year of HIV infection.

\section{O008}

FRACTIONAL EXCRETION OF PHOSPHATE (FEPO ${ }_{4}$ ), A MARKER OF RENAL TOXICITY: BIOLOGICAL VARIATION, DECISION LIMIT AND REFERENCE CHANGE VALUE

Belanger, Marie-Claire; Petitclerc, Claude; Hamel, Melanie;

\section{Baril, Jean-Guy}

Montreal, QC

BACKGROUND: Tenofovir (TDF) has been associated with renal tubular toxicity. $\mathrm{FePO}_{4}>20 \%$ has been recommended as screening tool for tubular toxicity. $\mathrm{FePO}_{4}$ decision limits were not established in HIVTDF or other antiretroviral drugs $(\mathrm{Tx})$ treated patients. $\mathrm{FePO}_{4}$ reference change value (RCV) for monitoring renal toxicity is not available.

METHODS: Treated and untreated HIV and non HIV patients were collected for serum $(\mathrm{S})$ and urine $(\mathrm{U})$ to validate $\mathrm{FePO}_{4}$. Within-subject variation was determined using Taylor approximations for $\mathrm{U}$ phosphate/S phosphate and $\mathrm{S}$ creatinine/U creatinine using known within-subject variation for each component and calculation of the variance of product of two ratios; $\mathrm{FePO} 4$ analytical variation was computed using quality controls. Urinary neutrophil gelatinase-associated lipocalin (NGAL), a biomarker of impaired renal function has been measured in a subset of HIV-TDF (41) RESULTS: $\mathrm{FePO}_{4}$ was analysed from non-HIV (79), non treated HIV (85), HIV-TDF (413) and HIV-Tx (228). Within-subject variation $\mathrm{FePO}_{4}$ : 26.2\%, Between-subject variation: $53.9 \%$, RCV: $75,7 \%$. S phosphate distribution shifted to lower values for HIV-TDF $(-0.05 \mathrm{mmol} / \mathrm{L}, \mathrm{P}<0.05)$ compared to non-HIV and non-treated HIV. $\mathrm{FePO}_{4}$ distribution shifted to higher values $(+2,73 \%, \mathrm{P}<0.01)$ for HIV-TDF compared to non-HIV Using a cut-off NGAL: $10 \mathrm{ng} / \mathrm{mL}$ and $\mathrm{FePO}_{4}: 20 \%$, HIV-TDF $(\mathrm{n}=41)$ 
yielded the following: Among $26 \mathrm{FePO}_{4}<20 \%, 17$ had NGAL $<10$ and $9 \mathrm{NGAL}>10$. Among 15 patients with $\mathrm{FePO}_{4}>20 \%, 10$ had NGAL $<10$ and 5 had NGAL $>10$.

CONCLUSION: $\mathrm{FePO}_{4}$ shift distribution to higher values could be partly explained by tubular damage as indicated by NGAL $>10 \mathrm{ng} / \mathrm{ml}$ across the entire range of $\mathrm{FePO}_{4}(4-35 \%)$. $\mathrm{FePO}_{4}>20 \%$ with negative NGAL may suggest a higher cut-off value for TDF. In view of NGAL positivity in the mid range of $\mathrm{FePO}_{4}$, an increase of $\mathrm{FePO}_{4}>75 \%$ (RCV) from previous values might be indicative of tubular damage independently of the cut-off value.

\section{O009}

RENAL MONITORING FOR PATIENTS ON TENOFOVIR: AN ANALYSIS OF INTERNATIONAL RECOMMENDATIONS Baril, Jean-Guy $^{1}$; Foisy, Michelle ${ }^{2}$; Harris, Marianne ${ }^{3}$;

Tan, Darrell ${ }^{4}$; Cooper, Ryan²; El Helou, Phillipe ${ }^{6}$; Hamel, Melanie'; Smith, Graham ${ }^{4}$; Stewart, Kris ${ }^{5}$; Werb, Ronald ${ }^{3}$

${ }^{1}$ Montreal, QC; ${ }^{2}$ Edmonton, AB; ${ }^{3}$ Vancouver, BC; ${ }^{4}$ Toronto, ON; ${ }^{5}$ Sasktoon, SK; ${ }^{6}$ Hamilton, ON

INTRODUCTION: Tenofovir (TDF) is a widely used, effective component of many cART regimens, with a good tolerability profile. However, some patients experience clinically significant defects in renal function, including proximal tubular dysfunction. The frequency and significance of subclinical proximal tubular defects are not well established. The Canadian HIV Renal Monitoring Group, comprising 8 HIV specialists and 2 nephrologists, has convened to review the evidence of TDF-related nephrotoxicity and to promote a consistent, evidence-based approach to renal monitoring in patients on TDF. The group undertook an initial survey of existing guidelines to identify areas of overlap or disagreement among them.

METHODS: Five major recent HIV treatment guidelines were identified by Medline searches, and their renal monitoring recommendations were compared.

RESULTS: Table 1 summarizes the recommendations regarding renal monitoring in $\mathrm{HIV}^{+}$patients. Specific guidance regarding TDF is shown where available. Recommendations on monitoring of glomerular function were generally consistent, although the frequency and timing differed, as did the preferred equation for estimating glomerular filtration rate. Regarding proximal tubular dysfunction, recommendations regarding serum phosphate monitoring differed substantially among the various documents; there was no agreement on the necessity for any other specific monitoring, nor on the preferred approach to, or frequency of, testing.

CONCLUSIONS: International guidelines offer relatively consistent recommendations on monitoring glomerular function in HIV patients but equivocal guidance on monitoring proximal tubular dysfunction. Canadian patients and caregivers would benefit from clear, practical guidance on renal monitoring, particularly for $\mathrm{HIV}^{+}$patients who are on, or are being considered for, treatment with TDF.

\begin{tabular}{|c|c|c|c|c|c|}
\hline & \multicolumn{2}{|c|}{ Infectious Disease European AIDS } & \multicolumn{2}{|c|}{ DHHS AntiretroviralBritish HIV } & \multirow[b]{2}{*}{$\begin{array}{l}\text { Tenofovir- } \\
\text { Associated } \\
\text { Kidney Toxicity } \\
\text { in HIV-Infected } \\
\text { Patients: A } \\
\text { Review of the } \\
\text { Evidence (Hall, } \\
\text { 2011) }\end{array}$} \\
\hline & $\begin{array}{l}\text { Society of } \\
\text { America (IDSA) } \\
\text { Guidelines } \\
(2009)\end{array}$ & $\begin{array}{l}\text { Clinical Society } \\
\text { (EACS) } \\
\text { Guidelines } \\
\text { (Version 6: } \\
\text { October, 2011) }\end{array}$ & $\begin{array}{l}\text { Guidelines for } \\
\text { Adults and } \\
\text { Adolescents, } \\
\text { October 14, } 2011\end{array}$ & $\begin{array}{l}\text { Association } \\
\text { Guidelines for } \\
\text { the Routine } \\
\text { Investigation } \\
\text { and Monitoring } \\
\text { (2012) }\end{array}$ & \\
\hline $\begin{array}{l}\text { Country of } \\
\text { origin }\end{array}$ & USA & EU & USA & UK & UK \\
\hline $\begin{array}{l}\text { Regular renal } \\
\text { monitoring } \\
\text { recommen- } \\
\text { dations (all } \\
\text { patients) }\end{array}$ & $\begin{array}{l}\text { Urinalysis; } \\
\text { eGFR } \\
\text { (Cockcroft- } \\
\text { Gault } \\
\text { equation) }\end{array}$ & $\begin{array}{l}\text { Urinalysis; } \\
\text { eGFR } \\
\text { (aMDRD } \\
\text { equation); } \\
\text { urine protein } \\
\text { / creatinine } \\
\text { ratio if pro- } \\
\text { teinuria on } \\
\text { urinalysis or } \\
\text { if eGFR } \\
<60 \mathrm{ml} / \mathrm{min}\end{array}$ & $\begin{array}{l}\text { Urinalysis; } \\
\text { eGFR } \\
\text { (MDRD } \\
\text { equation) } \\
\text { (M) }\end{array}$ & $\begin{array}{l}\text { Urinalysis; } \\
\text { eGFR }\end{array}$ & $\begin{array}{l}\text { Not } \\
\text { applicable }\end{array}$ \\
\hline
\end{tabular}

Additional More frequent More frequent More frequentMore frequent Fractional

\begin{tabular}{|c|c|c|c|c|c|}
\hline $\begin{array}{l}\text { monitoring } \\
\text { recommen- } \\
\text { dations for } \\
\text { TDF patients }\end{array}$ & $\begin{array}{l}\text { urinalysis } \\
\text { and eGFR }\end{array}$ & $\begin{array}{l}\text { eGFR; urine } \\
\text { phosphate; } \\
\text { assess for } \\
\text { proximal } \\
\text { tubular dys- } \\
\text { function and } \\
\text { renal } \\
\text { Fanconi } \\
\text { syndrome }\end{array}$ & $\begin{array}{l}\text { urinalysis if } \\
\text { patient is at } \\
\text { high risk }\end{array}$ & $\begin{array}{l}\text { urinalysis } \\
\text { and eGFR; } \\
\text { serum phos- } \\
\text { phate; } \\
\text { assess for } \\
\text { new-onset } \\
\text { or worsening } \\
\text { proteinuria } \\
\text { and/or gly- } \\
\text { cosuria; } \\
\text { fasting } \\
\text { serum and } \\
\text { urine phos- } \\
\text { phate if } \\
\text { tubular injury } \\
\text { is suspected }\end{array}$ & $\begin{array}{l}\text { excretion of } \\
\text { phosphate; } \\
\text { urine glu- } \\
\text { cose; urine } \\
\text { retinol bind- } \\
\text { ing protein }\end{array}$ \\
\hline
\end{tabular}

\section{O010}

A PILOT, PROSPECTIVE, OPEN-LABEL SIMPLIFICATION STUDY TO EVALUATE THE SAFETY, EFFICACY AND PHARMACOKINETICS OF ONCE DAILY LOPINAVIRRITONAVIR MONOTHERAPY IN HIV-HCV CO-INFECTED PATIENTS: THE MONOCO STUDY

Cooper, Curtis ${ }^{1}$; La Porte, Charles ${ }^{1}$; Tossonian, Harout ${ }^{2}$;

Sampalis, John ${ }^{3}$; Ackad, Nabil ${ }^{3}$; Conway, Brian ${ }^{2}$

1Ottawa, ON; ${ }^{2}$ Vancouver, BC; ${ }^{3}$ Montreal, QC

BACKGROUND: Safe, effective, easy to dose antiretroviral therapy that minimizes hepatic complication risk is essential in optimizing HIV$\mathrm{HCV}$ treatment. Nucleoside-sparing boosted protease inhibitor monotherapy may achieve this goal.

METHODS: A prospective, open-label pilot simplification study of QD lopinavir/ritonavir (LPV/r) monotherapy in HIV-HCV co-infected patients was conducted in Canada. Patients on highly active antiretroviral therapy (HAART) with undetectable HIV RNA for $>6$ months were eligible. The primary outcome was maintenance of HIV-RNA $<50 \mathrm{c} / \mathrm{mL}$ through week 48. HIV-RNA, immune measures, metabolic markers and pharmacokinetics (PK) were assessed.

RESULTS: Twenty participants received QD LPV/r monotherapy. Mean baseline age was 46.9 years, CD4 467 cells/ $/ \mathrm{L}$, and duration of virologic suppression pre-switch was 146 weeks. By per protocol analysis, $85.7 \%$ and $71.4 \%$ maintained virologic suppression at weeks 24 and 48 . Virologic breakthrough (HIV RNA $>50 \mathrm{c} / \mathrm{mL}$ on 2 consecutive measures) occurred in 7 patients [mean standard error (SE) time to breakthrough $=38.3$ (4.8) weeks]. Resuppression occurred with improved adherence in 2 participants and improved adherence plus addition of nucleosides in two others. LPV Cmin was $<1 \mathrm{mg} / \mathrm{L}$ in 8 patients, associated with virologic breakthrough in 2 cases but no development of resistance. No clinically significant changes in CD4, lipids or glucose were noted. Three participants developed transient $\geq 5$-fold liver enzyme elevations. None of 9 severe adverse events (SAEs) were LPV/r or liver-related. Six discontinued participation for withdrawal of consent $(n=1)$, poor adherence $(n=3)$ or drug intolerance $(n=2)$.

CONCLUSIONS: QD LPV/r monotherapy in HIV-HCV co-infected individuals offers a safe and effective approach to the management of the HIV infection, with a predictable pharmacokinetic profile. Virologic breakthrough is uncommon and resistance was not observed. This approach, in concert with careful adherence and virologic monitoring, may be considered to optimize the long-term management of co-infected individuals. 


\section{O011}

ISOLATED HIV-1 RNA SEMEN SHEDDING: THE EFFECT OF AN INTENSIFIED HIGHLY ACTIVE ANTIRETROVIRAL THERAPY REGIMEN

Osborne, Brendan J1; Sheth, Prameet $\mathbf{M}^{1}$; Yi, Tae Joon ${ }^{1}$;

Kovacs, Colin ${ }^{1}$; Benko, Erika ; la Porte, Charles ${ }^{2}$;

Brumme, Zabrina ${ }^{3}$; Le, Anh ${ }^{3}$; Baraki, Bemuluyigza ${ }^{3}$;

Brockman, Mark ${ }^{3}$; Mazzulli, Tony' ${ }^{1}$; Kaul, Rupert ${ }^{1}$

${ }^{1}$ Toronto; ${ }^{2}$ Ottawa, ON; ${ }^{3}$ Vancouver, BC

BACKGROUND: Effective antiretroviral therapy (ART) reduces HIV viral load (VL) in blood and semen to undetectable levels and dramatically reduces HIV transmission. However, isolated semen HIV-1 shedding (IHS) may persist despite and undetectable blood VL and a lack of clinical STIs. Understanding IHS and it's potential impact on HIV transmission is a public health priority.

METHODS: Paired blood and semen samples were collected from HIVpositive men prior to initiating ART and at weeks 2, 4, 8, 12, 16, 20 and 24. Standard 3-drug treatment (sART) was intensified by the addition of maraviroc and raltegravir (iART) in some men, in an open-label fashion.

RESULTS: Compared to standard ART, intensified ART was associated with a more rapid reduction in the semen VL ( $p=0.043)$. Over 6 months, IHS was detected at $\geq 1$ visit in $2 / 13(15 \%)$ participants in the iART group and in $12 / 25(48 \%)$ on sART. A semen $\mathrm{VL}>3.8 \log _{10}$ was defined as high-level IHS, due to the association with enhanced infectivity in vitro. Overall, iART was associated with reductions in low-level IHS $(p=0.048)$, but not in high-level IHS. Specifically, one participant $(1 / 13$; $7 \%)$ demonstrated prolonged high-level IHS, despite rapid and sustained suppressed viremia. Semen virus isolated from IHS individuals was R5trophic, wild-type, and drug sensitive. Plasma drug levels in both blood and semen of intensification drugs remained well above the minimum therapeutic concentration required for HIV suppression and often at concentrations in semen several fold higher than blood. Variations in plasma drug concentrations were not associated with frequency or detection of IHS.

CONCLUSIONS: This observational study provides evidence that an intensified ART regimen reduces, but cannot wholly prevent, the occurrence of isolated semen HIV during the first 6 months of therapy. Given the possible public health implications of IHS, the biological basis of this phenomenon merits further investigation.

\section{O012}

\section{HIV EXPERTS ON EARLY INITIATION OF ART FOR PREVENTION: UNCERTAINTY AND WEIGHING THE RISKS AND BENEFITS}

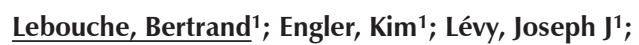

Gilmore, Norbert ${ }^{1}$; Spire, Bruno ${ }^{2,3}$; Rozenbaum, Willy ${ }^{4}$;

Routy, Jean-Pierre ${ }^{1}$

${ }^{1}$ Montreal, QC; ${ }^{2}$ Pantin; ${ }^{3}$ Marseille; ${ }^{4}$ Paris, France

OBJECTIVES: The finding that successful ART can almost eliminate the risk of heterosexual HIV transmission is a major scientific breakthrough. This potential has generated novel approaches to prevention including "Test and Treat" (T\&T). Our qualitative study, drawing on the perspectives of French HIV experts, aims to better understand if, and how, a T\&T approach might be applied in France and to learn more about issues of prevention with early ART. Findings were expected to be insightful for Canada (e.g., similar epidemics, treatment contexts).

METHOD: In 2011, 19 HIV French experts participated in a semi-structured interview on implementing T\&T in France. Expertise was typically defined on the basis of contribution to the 2010 French ART guidelines. Participants' HIV expertise included clinical care, epidemiology, virology, and community activism. Interviews lasted an hour, on average, and explored opinions on T\&T, who and how to test and treat, and what public health discourses and evaluations should accompany it. Analyses of interview content on who and how to treat are presented here. Thematic analysis of the transcribed interviews was supported by Atlas.ti 5.2.

RESULTS: The decision to treat earlier than current guidelines recommend (>500 CD4) was associated with uncertainty that involved weighing the risks and benefits in terms of patient health, the risk of transmission, patient choice, population health and cost. Key considerations in the choice of preventive treatment varied but included adherence, toxicity and/or side effects, efficacy, regimen simplicity, resistance, diffusion (of ART in genitals) and customization.

DISCUSSION: Despite the widely accepted efficacy of ART-based prevention, experts had uncertainties about early or preventive treatment of HIV-infection. Opinions also diverged over what might be considered optimal treatment strategies. Our analyses emphasize the need for greater dialogue to advance T\&T in France. Results are discussed in relation to a similar study we conducted among Quebec HIV experts.

Track C: Epidemiology and Public Health Sciences: Testing Strategies and Surveillance in Canada: Where are we? Volet C : Épidémiologie et sciences de la santé publique : Stratégies d'essai et surveillance au Canada : où en sommes-nous?

\section{O013}

FACTORS THAT PREDICT VOLUNTARY HIV TESTING IN THE GENERAL CANADIAN POPULATION: RESULTS OF THE SRC-CANFAR 2011 NATIONAL SURVEY

Worthington, Catherine ${ }^{1}$; Calzavara, Liviana ${ }^{2}$; Allman, Dan²; Tyndall, Mark ${ }^{3}$; Adrien, Alix ${ }^{4}$; White, Samantha ${ }^{2}$; Kelly, Christopher ${ }^{2}$

${ }^{1}$ Victoria, BC; ${ }^{2}$ Toronto; ${ }^{3}$ Ottawa, ON; ${ }^{4}$ Montreal, QC

BACKGROUND: HIV testing remains a central strategy for HIV prevention and can link those who test positive to HIV treatment. To inform HIV testing provision, we examined HIV testing and predictors of HIV testing in the general Canadian population.

METHODS: In May 2011, a bilingual survey of people 16 years of age or older was conducted in all provinces and territories in Canada. The survey used a dual mode methodology comprising a recruited online panel supplemented by random digit dial telephone interviewing. The margin of error for the total sample was \pm 2.1 percentage points $(95 \% \mathrm{CI})$. Bivariate analyses ( $\chi^{2}$, wald test) were conducted to compare those who tested voluntarily compared with those who had not tested/not tested voluntarily.

RESULTS: Weighted to the population, the sample was 2,139. Overall, $53 \%$ reported at least one lifetime HIV test. When ancillary reasons for testing (including blood donation (20\%), insurance (10\%) immigration (2\%) and research (1\%)) were excluded, $29 \%$ reported testing at least once voluntarily. Of these, approximately half (49\%) had tested more than once. Predictors of voluntary HIV testing included age $(p<0.001)$, education $(\mathrm{p}<0.01)$, household income $(\mathrm{p}<0.05)$, single marital status $(p<0.001)$, casual sexual partner (past year) $(p<0.001)$, member of a sexual minority $(p<0.001)$, sought HIV information (past year) $(p<0.001)$, higher HIV knowledge $(\mathrm{p}<.001)$, and knowing someone who died of AIDS $(p<0.01)$. While region was associated with HIV testing $(p<0.001$, with those living in the Atlantic region less likely to test for HIV), availability of anonymous test reporting in province/territory was not significantly related to testing $(\mathrm{p}=0.35)$.

CONCLUSION: Factors related to personal HIV risk behaviour, familiarity with HIV, and region of residence predict voluntary HIV testing among the general Canadian population, but availability of anonymous HIV test reporting in province/territory does not predict voluntary HIV testing. The percentage of the population that has tested for HIV is little changed from 2003

\section{O014}

WILL HIV SELF TESTING BE ACCEPTED BY LOW TO MEDIUM RISK EDUCATED POPULATIONS? A PILOT CROSS SECTIONAL STUDY IN STUDENTS OF MCGILL UNIVERSITY, MONTREAL

Pant Pai, Nitika; Bhargava, Madhavi; Sharma, Jigyasa;

Balram, Bhairavi; Bois, Cindy; Joseph, Lawrence;

Tellier, Pierre-Paul

Montreal, QC

BACKGROUND: HIV self-testing offers an alternative proactive screening solution to know one's sero-status in private. To bring more 
people to test globally, there is a huge momentum towards exploring this alternative although worldwide data on its performance and preference for linkages in educated populations are currently unavailable.

METHODS: In an ongoing cross sectional study, 100 confirmed seronegative students were recruited between September to December 2011 at the McGill University Student Health Center, Montreal. Concordance between oral HIV self-tests conducted by students and tests performed by health-care professionals (hcps) at point-of-care was computed. Secondary outcomes on preferences for counseling and treatment linkages, costs and attitudes towards self-testing were also evaluated.

RESULTS: The median age of student participants was 22 years (18 to 45 years), $37 \%$ were male, $80 \%$ undergraduates, with $52 \%$ out-ofprovince students. About $99 \%$ of participants were single; and $49 \%$ had multiple partners, with $78 \%$ self-reporting a history of casual sex in the past 6 months.

Concordance between self-tests conducted by students and tests by hcps was high at $100 \%$ with median time to self-test at 21 minutes (20.4 to 25.2 minutes). Self-tests were reported to be convenient (98\%), time-efficient (96\%), and pain free (84\%). About $81 \%$ preferred self-tests and further $71 \%$ expressed the desire to buy them over-the-counter. About $65 \%$ students preferred confirmatory testing with hospitals being the preferred site. Preferred post-test counseling options were community clinics $78(41 \%)$, phones $53(28 \%)$, pharmacies $31(16 \%)$ and online counseling 29 (15\%).

CONCLUSION: Results from this first global study in an educated population suggests that the provision of a) personalized counseling, expedited confirmatory testing and treatment linkages; and b) inexpensive and accurate oral HIV tests will increase the uptake of HIV self tests in them. A high concordance and preference for self-testing calls for studying linkages in controlled trials.

\section{O015}

\section{A PEER HIV TESTING AND LINKAGE TO CARE PILOT AMONG IDUS IN VANCOUVER DURING A TREATMENT AS PREVENTION (TASP) PILOT}

Thumath, Meaghan A; Compton, Miranda; Bath, Misty;

Bolton, Shelley

Vancouver, BC

INTRODUCTION: As part of a four year HIV Treatment as Prevention (TasP) project in $\mathrm{BC}$ an ambitious pilot to screen over 5000 inner city residents using peers was initiated. The project was supported by a comprehensive social marketing campaign to encourage testing and increase community awareness of treatment as prevention.

METHODS: Preliminary evaluation using qualitative methods (key informant interviews with testing providers) as well as quantitative evaluation of rapid testing logs, HIV pre and post-test patient questionnaires and surveillance data of new HIV cases. Participants were offered a $\$ 5$ incentive for participating.

FINDINGS: Outcomes from June-October 2011 indicate over 4773 rapid HIV tests were conducted across 26 sites over 5 months (27\% female, $72.8 \%$ male and $2 \%$ transgendered). Of the 4773 tests the pilot detected 10 confirmed new HIV positives ( $0.2 \%$ positivity). Of the newly diagnosed HIV+ patients all had a current or previous history of injection drug use. 567 clients tested more than once over a 7 -month period. Of the 5,097 patients engaged at the testing events 324 were previously known $\mathrm{HIV}+$ clients who received an HIV primary care assessment and re-engagement in care by a registered nurse.

CONCLUSIONS: Peer HIV testing is feasible and acceptable for patients with $\mathrm{RN}$ support. Incentivizing testing contributed to high uptake and motivated people who felt they were not at risk to test, however improving $\mathrm{RN}$ triage and providing incentives to all participants (regardless of HIV status) helped to reduce unnecessary re-testing among known HIV positive clients. Community events are opportunities to link new and existing HIV positive clients to care and increased awareness of treatment as prevention in the inner city. Further analysis on cost effectiveness of this intervention is needed.

\section{O016}

CANADIAN PERINATAL HIV SURVEILLANCE PROGRAM (CPHSP): PERINATAL HIV TRANSMISSION, TREATMENT IN PREGNANCY AND DEMOGRAPHICS IN CANADA

Alimenti, Ariane ${ }^{1}$; Singer, Joel ${ }^{1}$; Samson, Lindy ${ }^{2}$; Lee, Terry ${ }^{1}$; Bitnun, Ari ${ }^{3}$; Money, Deborah'; Brophy, Jason'²; Lapointe, Normand ${ }^{4 ;}$ Canadian Pediatric and Perinatal HIV/AIDS Research Group (CPARG), The $^{3}$

${ }^{1}$ Vancouver, BC; ${ }^{2}$ Ottawa; ${ }^{3}$ Toronto, ON; ${ }^{4}$ Montreal, QC

OBJECTIVES: To describe vertical transmission (VT) rates, treatment during pregnancy and demographics of mother-infant pairs (MIP) in the Canadian perinatal HIV surveillance cohort from 1990 to 2010.

METHODS: Maternal and infant data are collected annually from 22 pediatric, obstetric and HIV centres across Canada. VT rates are obtained from the "perinatally identified cohort" defined as MIP delivered in Canada and identified within 3 months after birth. Data including maternal characteristics, pregnancy ART and infant outcome are submitted via a secure web-based system and analyzed by the Canadian HIV Trials Network.

RESULTS: Of the 235 identified HIV-positive women giving birth in Canada in 2010, 70\% had acquired HIV heterosexually, 18\% through IDU and $1 \%$ perinatally; $50 \%$ of mothers were black and $20 \%$ were aboriginal. 39\% of MIP were identified in Ontario, 20\% in Québec, 14\% in Alberta, $11 \%$ in Saskatchewan, $10 \%$ in BC and 7\% in Manitoba. Among the $92 \%$ of mothers who received HAART, there were 3 perinatal transmissions (1.4\%); all 3 mothers had initiated treatment $\leq 1$ week pre-delivery. Among the 16 (6.8\%) women who received no ART during pregnancy, there were 2 perinatal transmissions (12.5\%). Among 2297 MIP identified perinatally in the HAART era (1997-2010), the overall VT rate was $2.9 \%$ but only $1 \%$ in MIP receiving HAART and $0.4 \%$ in women receiving $>4$ weeks of HAART.

CONCLUSIONS: Although VT rates of HIV in Canada have remained low for over a decade, VT continues to occur due to incomplete implementation of preventive interventions. In 2010, 5 infected infants were born to women who received no ART or $<1$ week of ART before delivery. An increasing number of MIP are identified in the Prairie provinces. Efforts must continue to identify and support pregnant HIV-positive women to enhance their health and that of their infants.

\section{O017}

INCLUSION OF ONTARIO'S ENHANCED HIV SURVEILLANCE DATA: IMPACT ON NATIONAL DISTRIBUTIONS

Tomas, Kristina ${ }^{1}$; Remis, Robert $\mathrm{S}^{2}$; Gheorghe, Mihaela ${ }^{1}$;

Sullivan, Ashleigh ${ }^{1}$; Liu, Juan ${ }^{2}$; Swantee, $\mathrm{Carol}^{3}$;

Halverson, Jessica ${ }^{1}$; Archibald, Chris $\mathbf{P}^{1}$

${ }^{1}$ Ottawa; ${ }^{2}$ Toronto; ${ }^{3}$ Etobicoke, ON

OBJECTIVE: To examine the potential impact on national exposure category and race/ethnicity distributions of reported HIV cases of data from Ontario's Laboratory Enhancement Program.

BACKGROUND: National HIV/AIDS surveillance is coordinated by the Public Health Agency of Canada (PHAC). HIV is reportable in all provinces and territories, although the completeness of epidemiologic information collected and submitted varies by jurisdiction. Ontario reports HIV cases to PHAC from its laboratory based surveillance system, which contains incomplete exposure category and no race/ethnicity information. The large number of annual HIV cases in Ontario together with this missing information contributes to the large proportion of cases at the national level with unknown exposure category and/or race/ethnicity.

METHODS: Ontario's Laboratory Enhancement Program (LEP) collects additional epidemiologic information, including race/ethnicity (since January 2009) and risk factor information. Using LEP data, exposure category was assigned for cases without risk factor information and reassigned for low-risk heterosexual cases. The Ontario data was replaced with the enhanced data in the national surveillance tables and the two distributions compared, to assess the impact on exposure category and race/ethnicity distributions.

RESULTS: With inclusion of Ontario's enhanced HIV surveillance exposure category data for 2010 , the result was a $24.7 \%$ decrease in the 
proportion of national HIV cases with unknown exposure category. For exposure category, increases are seen among Het-endemic $(+9.3 \%)$ and MSM/IDU $(+0.6 \%)$ and decreases among IDU $(-3.5 \%)$ and MSM $(-3.2 \%)$. With inclusion of Ontario's LEP race/ethnicity data for 2010, there was a $26.0 \%$ decrease in the proportion of national HIV cases with unknown race/ethnicity. The race/ethnicity distribution changes were: decreases in the proportion of Aboriginal (-12.4\%) and Asian (-2.4\%) cases and increases in the proportion of Black $(+4.4 \%)$, White $(+2.3 \%)$ and Latin-American $(+1.6 \%)$ cases.

CONCLUSIONS: Inclusion of Ontario's enhanced exposure category and race/ethnicity data in the national HIV dataset is significant; the national dataset becomes more complete and the proportion of cases with unknown exposure category and/or race/ethnicity is significantly reduced.

\section{O018}

\section{POINT OF CARE HIV TESTING IN A TERTIARY CARE} EMERGENCY DEPARTMENT IN WINNIPEG, CANADA

Becker, Marissa $^{1}$; Thompson, Laura ${ }^{1}$; Bridger, Natalie ${ }^{2}$;

Pindera, Carla ${ }^{1}$ : Keynan, Yoav ${ }^{1}$; Bullard, Jared ${ }^{1}$; Van Caeseele, Paul ${ }^{1}$; Kasper, Ken ${ }^{1}$

${ }^{1}$ Winnipeg, MB; ${ }^{2}$ St John's, NL

INTRODUCTION: Approximately $26 \%$ of Canadians living with HIV are unaware of their status. In 2010, only 6\% of Manitobans over 16 years of age were tested for HIV. Point of care (POC) HIV tests have been introduced to simplify and improve HIV testing rates. This study evaluated the feasibility of POC testing in an Emergency Room (ER) setting in Winnipeg.

METHODS: This was a cross-sectional study of unselected adults presenting to the ER at the Health Sciences Centre Hospital, Winnipeg. Following informed consent, a research nurse conducted pre test counseling, administration of the INSTITM HIV-1/HIV-2 Antibody Test, post test counseling and administered a brief questionnaire.

Venous blood was collected on all reactive and indeterminate specimens for confirmatory Western Blot testing.

RESULTS: 501 adults participated in the study. The majority of participants were under 40 years of age and approximately half were women. About $53 \%$ of participants self-identified as Aboriginal. Half of the participants had received previous HIV testing although $63 \%$ of these tests were over a year earlier. Women were significantly more likely to have had a previous HIV test and high rates of testing were also seen among those who inject drugs and men who have sex with men.

A total of 7 persons tested reactive with the POC test and all confirmed positive by serologic testing (1.4\%). All 7 were linked to an HIV specialist within 24 hours.

$96 \%$ of participants reported satisfaction with the test and $93 \%$ felt it belonged in the ER.

CONCLUSIONS: $1.4 \%$ of participants tested reactive for HIV in this study which is much higher than the HIV prevalence within the general population in Manitoba. Further, all individuals were linked to timely care. We believe this demonstrates that this busy tertiary care ER is an important and feasible place for HIV POC testing.

Track D: Social Sciences: Positive Living: Exploring Realities

Volet D : Sciences sociales : Vivre avec le VIH : explorer les réalités

\section{O019}

\section{THE RIGHT TO FOOD: KEY CONSIDERATIONS FOR HIV SERVICE PROVISION IN CANADA}

\section{Fielden, Sarah ${ }^{1}$; Fergusson, Pamela $^{2}$}

\section{${ }^{1}$ Vancouver, BC; ${ }^{2}$ London, United Kingdom}

BACKGROUND: The right to food is considered a basic human right and Canada is one of many nations supporting this right through a commitment to the goals set forth in policy documents such as the Declaration on World Food Security. The objective of this presentation is to outline key ethical considerations related to the right to food, food security and HIV services in Canada.
METHODS: We present an interdisciplinary perspective informed by a literature review that utilized a comparative policy framework. The review used both academic and grey literature sources to summarize HIV and food security policy, locating Canada within the broader global arena. This was contextualized by social theory pertaining to human rights and vulnerability. Analysis was accomplished using content and comparative methods.

RESULTS: A number of ethical issues challenge the comprehensive integration of food security and HIV services within Canada. The ethical principle of Justice supports the right to food and Distributive Justice suggests that social systems should be in place to equitably allocate food resources. However, the development of HIV-specific food services in Canada creates additional layers of complexity - especially in the context of competing socio-economic marginalization. Key considerations include:

- Proliferation of charity-based models of food distribution for HIV such as food banks may contribute to the erosion of the Canadian social safety net and depend largely on the quantity, quality, and safety of donated food.

- Establishing selection criteria for those accessing food-related services poses ethical challenges such as prioritizing nutritional and/ or food security parameters and defining what is equitable for HIV. infected and HIV-uninfected people in a community.

CONCLUSIONS: Although the right to food is acknowledged and supported in Canada, providing services to address food insecurity among HIV-positive people is rife with ethical dilemmas that require careful consideration of structural factors such as income disparity, ethno-cultural perspectives, and HIV stigma. Policies and models of practice based on empowerment need further exploration.

\section{O020}

WHY HOUSING? WHY NOW? - A CRITICAL APPRAISAL OF THE SHIFT TOWARDS HOUSING AS A PRIORITY IN HIV RESEARCH, PROGRAMING, AND INTERVENTION Guta, Adrian ${ }^{1}$; Gagnon, Marilou ${ }^{2}$

${ }^{1}$ Toronto; ${ }^{2}$ Ottawa, ON

BACKGROUND: There has been a significant increase in interest about housing and HIV in recent years. This is evident in a growing body of research, academic and grey literature, conferences, funding opportunities, and programs addressing housing and HIV. This sudden and unprecedented shift towards housing is in need of critical appraisal. The objective of this paper is to problematize this shift and raises critical questions about the new emphasis of housing in HIV.

METHODS: Drawing on Foucauldian "biopolitics" and techniques of critical discourse analysis, this paper offers a re-articulation of North American HIV housing research and programmatic initiatives through a close reading of their textual production and broad impact. The authors examined relevant texts through an iterative inductive approach with an attention to how housing becomes framed as an important health issue, by whom, and for what purpose.

RESULTS: Our results demonstrate that homelessness and housing insecurity have become re-articulated through a biomedical, bioeconomic, and biocultural lens. What results is a discursive formation in which housing serves as a rubric for organizing and managing the health of people living with HIV through related, but sometimes contradictory, discourses about health, risk, treatment adherence, rights, and particular forms of evidence. Key concerns are raised about intersections between housing, treatment imperatives, new disciplinary mechanisms, social exclusion and the securitization of health.

CONCLUSIONS: As housing becomes taken up by researchers, ASOs, funders, and policy makers, this social justice issue risks being subsumed within a particular system that invests in health (as a bio-political object) without challenging the conditions that produce homelessness and housing insecurity. We call for greater theorising and empirical research to investigate intersections between housing and other established, and emerging, areas in HIV to prevent this important need from becoming a means of extending disciplinary control. 


\section{O021}

"WE ARE GETTING ALONG": IMPORTANT SOURCES OF SUPPORT FOR HIV-POSITIVE WOMEN DURING

\section{PREGNANCY}

Ion, Allyson' ${ }^{1}$; Greene, Saara ${ }^{1}$; Elston, Dawn'; Kwaramba, Gladys ${ }^{1}$; Smith, Stephanie ${ }^{1}$; Barry, Fatimatou${ }^{1}$; Carvalhal, Adriana ${ }^{2}$; Loutfy, Mona $\mathbf{R}^{2}$

${ }^{1}$ Hamilton; ${ }^{2}$ Toronto, ON

INTRODUCTION: HIV-positive women require multiple forms of support throughout pregnancy, however, the presence of HIV-related stigma complicates access to and experiences of HIV and/or pregnancy supports. This work highlights the types of support that are important to HIV-positive women during pregnancy and the role that stigma plays in determining access to appropriate pre-natal care.

METHODS: A narrative methodological approach was used to interview 27 HIV-positive pregnant women between March and December 2011. Women were asked to describe their overall experience of pregnancy in the context of living with HIV. Interviews were recorded and transcribed verbatim. Reflexive narrative analysis involving researchers and peer research assistants was used to understand key sources of support for HIV-positive pregnant women across Ontario.

RESULTS: HIV-positive pregnant women identified the complexity of accessing informational, emotional, spiritual and practical support in the context of the fear and reality of experiencing HIV-related stigma. Informational support was provided by healthcare professionals, particularly HIV specialists; emotional and spiritual support was provided by partners, close friends, and their faith-based community; practical support including childcare, transportation to appointments and access to formula was provided by family members and HIV/AIDS service organizations. Although most women were able to access these supports throughout pregnancy, fears of HIV-related stigma limited consistent receipt of support by either dichotomizing their HIV and pregnancy needs, or by "othering" HIV-positive mothers vis-à-vis separating their needs from the needs of pregnant women more generally.

CONCLUSIONS: HIV-positive women define support in multiple ways. Access to informational, emotional, spiritual and practical support is complicated by fear of HIV-related stigma, which determines where, if and by whom support needs are met. It is crucial that health and social care providers consider the role that HIV-related stigma plays in how support is provided and accessed, and develop policies and practices that systematically and individually meet the needs of HIV-positive women during pregnancy.

\section{2}

LIVING WITH A SLOW AND ASYMPTOMATIC INFECTION: A PSYCHOSOCIAL PORTRAIT OF A COHORT OF HIV ${ }^{+}$ NON-PROGRESSORS

Côté, José '; Rouleau, Geneviève'; Ramirez-Garcìa, Pilar';

Bourbonnais, Anne'; Tremblay, Cécile²; Massé, Brigitte ${ }^{1}$; Couture, Mélanie ${ }^{3}$; Nahas Chebli, Georgette ${ }^{1}$

${ }^{1}$ Montréal; ${ }^{2}$ Sainte-Anne-de-Bellevue; ${ }^{3}$ Sherbrooke, QC

INTRODUCTION: The cohort of non-progressors is composed of HIV-positive individuals who do not take antiretrovirals and whose infection develops slowly and asymptomatically. Few studies have examined the psychosocial factors and experience of these HIV-infected non-progressors.

OBJECTIVE: This study describes the psychosocial profile and experience of non-progressors. Method. A mixed method was used. Qualitative data was obtained from a written questionnaire of 11 open-ended, shortanswer questions. Quantitative data was collected through valid scales: social support (MOS-SSS), anxiety and depression (HADS), and coping strategies (COPE).

RESULTS: Data was collected from 50 participants, 42 men and 8 women whose average age was 46 years and average duration of infection was eight years. The mean score for full social support $(0-100)$ was $60.47(\mathrm{SD}=28.25)$ and for anxiety/depression (0-42) was $11.14(\mathrm{SD}$ $=8.57)$. The most frequently exercised coping strategies $(0-3)$ were acceptance $(M=2.51)$, positive reframing $(M=1.90)$, active coping $(M=1.73)$, and planning $(\mathrm{M}=1.63)$. The least used strategies were disengagement $(M=0.40)$, substance use $(M=0.47)$, and denial $(M=0.77)$. Four themes describe the experience of non-progressive HIV infection: 1-Reacting and coping with the diagnosis of seropositivity; 2-Valuing what is important and experiencing changes in life; 3-Attributing good health to specific beliefs; and 4-Living positively in spite of health status while dreading progression of the infection.

CONCLUSION: Results for this cohort show that symptoms of anxiety and depression are high. These individuals live well with their non-progressive status but they live in fearful anticipation of disease progression. They adapt by making good use of acceptance, positive reframing, and active coping strategies. These findings constitute a starting point in clarifying the psychosocial characteristics of HIV-positive non-progressors to health professionals.

\section{O023}

"WHAT DO I GET OUT OF IT": UNDERSTANDING AND IMPROVING RELATIONSHIPS BETWEEN RESEARCHERS AND WOMEN LIVING WITH HIV

Tharao, Wangari E; Logie, Carmen; James, LLana; Loutfy, Mona Toronto, ON

BACKGROUND: Historically, there has been limited participation of women in research. This has been likely due to the exclusion of women based on reproductive and hormonal concerns; limited research targeted to women, particularly in the field of HIV/AIDS; and women's/communities' historical experiences of bureaucratic institutions and research processes. One of the objectives of our study was to examine relationships between researchers and women living with HIV (WLWH) and how these can be improved to increase women's participation in research. METHODS: We conducted 15 focus groups with WLWH across Ontario including: Aboriginal, African/Caribbean, sex working, injection drug using (IDU), lesbian/bisexual, and transgender women. Four focus groups were implemented with HIV service providers and researchers in Ottawa, Hamilton and Toronto. A semi-structured interview guide was used. Focus groups were digitally recorded, transcribed, entered into NVivo 8 and examined with narrative thematic techniques from grounded theory.

RESULTS: Participants ( $\mathrm{n}=104$; mean age $=38$ years; $23 \%$ lesbian/bisexual; $22 \%$ transgender; $69 \%$ ethnic minority) and service providers $(n=48)$ described: 1a) power imbalances between WLWH and researchers including non-mutually supportive and exploitative relationships without acknowledging or crediting women, tokenistic involvement, no report back and feeling unqualified/not knowing enough to participate; 1b) mistrust of research and government (usually hidden) ranging from use of women as guinea pigs and a cure for HIV being available but kept away for profit purposes. 2) WLWH proposed reciprocity strengthening through: a) community (WLWH) engagement through use of community-based research (CBR) and peer researchers; researching issues of interest to women/communities; mixing groups of PLWH b) tailoring knowledge dissemination to meet needs of WLWH: centralization of knowledge and collaborations that bring people together; use of lay formats c) heightening awareness of research being conducted among WLWH.

CONCLUSION: Increasing women's participation in research requires the implementation of equitable and reciprocal research processes that mediate power dynamics and issues of intersectional stigma and discrimination. Developing respectful and mutually-responsive pathways of communication and recourse about research and emerging results can strengthen the relationship between researchers and WLWH.

\section{O024}

TRENDS IN ATTITUDES TOWARD PEOPLE WITH HIV: RESULTS FROM THE SRC-CANFAR 2011 CANADIAN NATIONAL SURVEY

Calzavara, Liviana ${ }^{1}$; Allman, Dan'; Worthington, Catherine²; Tyndall, Mark ${ }^{3}$; Adrien, Alix ${ }^{4}$; Walters, Melissa ${ }^{1}$; White, Samantha J1; Kelly, Christopher ${ }^{1}$

${ }^{1}$ Toronto, ON; ${ }^{2}$ Victoria, BC; ${ }^{3} \mathrm{Ottawa}, \mathrm{ON} ;{ }^{4}$ Montreal, QC

BACKGROUND: The impact of negative attitudes toward PHA on strategies aimed at preventing HIV is well documented. Understanding the social environment into which health promotion programs are implemented is critical. Five years have passed since behaviours, knowledge and attitudes toward HIV were last surveyed in Canada. 
METHODS: A bilingual survey of Canadians $(n=2,139)$ aged 16 years and older was conducted in May 2011. Survey participants were recruited from all provinces and territories using an online panel supplemented by random digit dial telephone interviewing. The margin of error was \pm 2.1 percentage points at the $95 \%$ confidence level.

RESULTS: $84 \%$ of Canadians reported positive attitudes toward PHA. A minority reported that they: felt afraid of PHA (16\%); felt PHA had only themselves to blame for their infection (11\%); and could not become friends with PHA (8\%). People more knowledgeable about HIV and those who had known someone who had died of AIDS were significantly more likely (p.>0.05) to report higher levels of comfort. Since 2006, greater levels of comfort in interacting with PHA were evident. Comfort with working in an office with a PHA increased from $74 \%$ to $77 \%(p=0.02)$, shopping at a store where the owner has HIV remained the same from $71 \%$ to $72 \%(\mathrm{p}=0.47)$, and having a child attend school where another child has HIV has increased from $56 \%$ to $60 \%(\mathrm{p}=0.04)$. Discomfort remains substantial, however, when considering a friend or family member dating someone with HIV (56\% in 2006 and 54\% in 2011, p=0.0003). Fear of transmission is the most cited reason for discomfort.

CONCLUSION: A substantial proportion of Canadians continue to report negative attitudes toward PHA. As participants most frequently cited fear of transmission as the reason for discomfort with PHA, and comfort level increases with HIV knowledge, more public discourse and education may lead to further reductions in stigma.

Track A: Basic Sciences: Innate and Adaptive Immune Responses to HIV Infection and Co-infection

Volet A : Sciences fondamentales : Réactions immunitaires innées et adaptatives à l'infection au VIH et à la co-infection VIH/VHC

\section{O025}

\section{SUPERIOR INHIBITION OF HIV REPLICATION IN AUTOLOGOUS CD4 ${ }^{+}$T CELLS BY NATURAL KILLER (NK) CELLS FROM INDIVIDUALS WHO ARE POSITIVE FOR HLA-BW4*80I AND KIR3DL $1 * H /{ }^{*} Y$}

Song, Rujun; Bruneau, Julie; Bernard, Nicole $\mathrm{F}$

Montreal, QC

BACKGROUND: NK cells function in early defences to viruses, such as HIV. NK activation state depends on the integration of signals received from receptors such as Killer Immunoglobulin-like Receptor (KIR) 3DL1. KIR3DL1 interacts with HLA-Bw4 antigens, particularly those with an isoleucine at aa80 (*80I), as a receptor-ligand pair (RLP). NK cells from individuals with the putative RLP, KIR3DS1+*80I, inhibit HIV replication in autologous CD4 $\mathrm{T}$ cells better than those from subjects without this RLP. We questioned whether NK cells from subjects with another RLP, KIR3DL1*h/*y (with high KIR3DL1 expression) and $* 80 \mathrm{I}\left(* \mathrm{~h} / *_{\mathrm{y}}+* 80 \mathrm{I}\right)$ inhibited HIV replication better than those from Bw6 homozygotes (hmz) who are negative for this RLP.

Hypothesis: NK cells from $* \mathrm{~h} / *_{\mathrm{y}}+* 80 \mathrm{I}$ carriers will inhibit HIV infection/ replication in autologous $\mathrm{CD}^{+}$cells better than those from Bw6hmz.

METHODS: $\mathrm{CD}^{+} \mathrm{T}$ cells from 23 HIV seronegative individuals were isolated, stimulated and infected with HIV-1JR-CSF. Purified autologous NK cells were co-cultured with infected T cells. P24 levels in supernatants were measured by ELISA. Viral inhibition was calculated by comparing p24 levels in wells with or without NK cells. Mann-Whitney tests assessed the significance of between-group differences.

RESULTS: NK cells from subjects with the KIR3DS1+*80I $(n=7)$ or $* \mathrm{~h} / \mathrm{y}^{+*} 80 \mathrm{I}$ RLPs $(\mathrm{n}=7)$ inhibited HIV replication more potently than those from Bw6hmz (n=9). Between-group differences in the inhibition of viral replication were significant for comparisons of KIR3DS1+*80I versus Bw6hmz and $* \mathrm{~h} / * \mathrm{y}^{+*} 80 \mathrm{I}$ versus Bw6hmz on days $3,7,10$ and 14 ( $\mathrm{p}<0.05$ for all comparisons). NK cells from subjects carrying KIR3DS1+*80I and $* \mathrm{~h} / *^{*} \mathrm{y}^{*} 80 \mathrm{I}$ inhibited HIV replication to a similar extent.

CONCLUSION: The $* \mathrm{~h} /{ }^{*} \mathrm{y}^{+*} 80 \mathrm{I}$ genotype encodes an RLP able to license NK cells for anti-HIV activity whereas the Bw6hmz genotype does not. Differential inhibition of HIV replication mediated by NK cells from

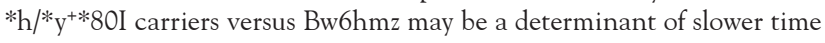
to AIDS observed in epidemiological studies.

\section{O026}

KIR3DS1 HOMOZYGOUS HIV-EXPOSED INDIVIDUALS HAVE A LONGER TIME TO HIV SEROCONVERSION THAN THOSE WITH OTHER GENERIC KIR3DL1/KIR3DS1 GENOTYPES

Tallon, Benjamin J; Bruneau, Julie; Tsoukas, Christos M;

Routy, Jean-Pierre; Tan, Xianming; Bernard, Nicole F

Montreal, QC

BACKGROUND: Natural killer (NK) cells play a key role in the clearance of viral infections and serve as a link between the innate and adaptive arms of the human immune system, representing a potential mechanism by which the host can block transmission of HIV. The highly polymorphic HLA family and the NK cell surface killer immunoglobulinlike receptors (KIR) have been implicated in both HIV pathogenesis and HIV susceptibility. We have previously demonstrated that homozygosity for KIR3DS1, an activating KIR, is more frequent in a cohort of HIV-exposed seronegative (HESN) subjects than HIV-susceptible individuals. Hypothesis: Time to HIV infection will be longer in exposed KIR3DS1 homozygotes (hmz) than in carriers of other KIR3DS1 genotypes.

METHODS: Longitudinal behavioural information for $172 \mathrm{HIV}$-exposed individuals allowed for the comparison of time-to-event (either seroconversion or last date of exposure while still seronegative) based on KIR3DS1 genotypes. Of these, 76 seroconverted (SC) and 96 were HESN. Survival analysis was used to compare time to event for KIR3DS1 hmz $(\mathrm{n}=15)$, KIR3DS1/L1 htz (n=58) and KIR3DL1 hmz (n=99). Average follow-up (FUP) years for HESN and SC were 8.65 \pm 5.1 and 3.77 \pm 3.28 (average \pm standard deviation), respectively.

RESULTS: KIR3DS1 hmz had a significantly longer time to HIV infection than KIR3DS1/KIR3DL1 htz, who carry only one copy of KIR3DS1 $(10.1 \pm 6.7$ versus $8.12 \pm 4.95$ seronegative years [average \pm standard deviation], $\mathrm{p}=0.012$, Log-rank test). Carriage of both KIR3DS1 and its putative ligand HLA-Bw4*80I, a combination shown previously to be associated with delayed progression to AIDS, did not confer protection from HIV infection when subjects who carried this genotype were compared to subjects who carried HLA-Bw6, which does not act as a ligand for KIR3DS1 ( $\mathrm{p}=0.43$, Log-rank test).

CONCLUSIONS: Carriage of the KIR3DS1 hmz genotype confers protection over time and this protection is independent of carriage of HLA-Bw4*80I.

\section{7}

HIV-1 VIRAL PROTEIN R (VPR) FROM PRIMARY VIRUS

ISOLATES MODULATES NATURAL KILLER (NK) CELL EFFECTOR FUNCTION THROUGH THE NKG2D PATHWAY: POTENTIAL IMPLICATIONS FOR NK CELL DYSFUNCTION IN IN VIVO HIV-1 INFECTION

Pham, Tram N; Routy, Jean-Pierre; Cohen, Eric A

\section{Montréal, QC}

Natural killer (NK) cells have been well documented to play a crucial role in containing viral propagation during acute HIV infection. Nevertheless, these cells are also known to progressively become dysfunctional in the chronic phase of infection. Although the underlying mechanisms remain elusive, several HIV-1 accessory proteins, including viral protein $\mathrm{R}(\mathrm{Vpr})$, have been shown in vitro to aberrantly modulate the pathway mediated by the centrally activating receptor NKG2D (natural killer group 2, member D). This in essence denotes the potential significance of NKG2D in HIV infection. Given that Vpr from laboratory-adapted virus strains upregulates ULBP2 - a NKG2D ligand - on $\mathrm{CD}^{+} \mathrm{T}$ cells and promotes their lysis by NK cells, our study initially focused on determining whether this activity could be extended to primary Vpr variants, with a subsequent broader objective of evaluating the relevance of the NKG2D pathway in NK cell dysfunction during HIV infection. Here, we document that Vpr variants from all 4 HIV-1 groups $(\mathrm{M}, \mathrm{N}, \mathrm{O}$ and $\mathrm{P})$ can promote $\mathrm{NK}$ cell killing of $\mathrm{CD}^{+} \mathrm{T}$ cells through the upregulation of ULBP2 on these target T cells. In addition, analysis of immune cells from patients with different stages of HIV-1 infection reveals evidence of: (1) ULBP-2 augmentation on infected CD4 ${ }^{+} \mathrm{T}$ cells, (2) progressive alteration of NKG2D expression on NK cells over time in the absence of antiretroviral therapy (HAART), and (3) partial recovery of the NKG2D receptor following HAART. Interestingly, we find that, 
in certain cases, reduced NKG2D expression may be correlated with decreased NKG2D-mediated NK cell effector function. Taken together, our results not only highlight the importance of HIV-1 Vpr protein in the modulation of the host's innate immune responses, but also shed a new light on the potential mechanism underlying the dysfunction of NK cells during chronic HIV-1 infection.

\section{O028 \\ PHENOTYPIC AND FUNCTIONAL CHARACTERIZATION OF A NEW TH17 SUBSET WITH DISTINCT CONTRIBUTIONS TO HIV PATHOGENESIS}

Wacleche, Vanessa S; Gosselin, Annie; Monteiro, Patricia; Boulassel, Mohamed-Rachid; Routy, Jean-Pierre; Ancuta, Petronela Montreal, QC

BACKGROUND: Th17 cells are critical for mucosal immunity against bacterial pathogens. Th17 cells are depleted from the gut-associated lymphoid tissues of HIV-infected individuals and their functional restoration under long-term antiretroviral therapy is only partial. We previously reported that Th17 cells with a CXCR $3^{-} \mathrm{CCR} 4^{+} \mathrm{CCR} 6{ }^{+}$phenotype ("classical" Th17) are permissive to HIV infection. However, Th17 cells are highly heterogeneous. We recently identified a new Th17 subset with a CXCR3-CCR4 ${ }^{-} \mathrm{CCR} 6{ }^{+}$phenotype (double negative, DN CCR6 ${ }^{+}$) and preserved frequency in the peripheral blood of HIV-infected subjects. Our hypothesis is that HIV infects some but not all Th17 subsets. Our goal is to determine the differentiation relationship between DN CCR6 ${ }^{+}$ and "classical" Th17 cells and their differential contribution to HIV pathogenesis.

METHODS: Memory CD4 ${ }^{+}$T-cell subsets were sorted by FACS and stimulated via CD3/CD28. Lineage-specific cytokines and transcription factors were quantified by ELISA and RT-PCR, respectively. Antigenic specificity and polarization profiles were determined by measuring T-cell proliferation and intracellular cytokine production, respectively. HIV replication and integration was measured by ELISA and real time PCR, respectively.

RESULTS: Similar to "classical" Th17, DN CCR6 ${ }^{+}$cells exhibited a Th17 differentiation profile and proliferated in response to C. albicans hyphaes (a Th17 antigen) but not CMV (a Th1 antigen). In contrast to "classical" Th17, DN CCR6 $6^{+}$cells produced the Th1 cytokine IFN- $\gamma$. DN CCR6 ${ }^{+}$T-cells were permissive to virulent R 5 HIV strains in vitro. Experiments are in progress to establish the differentiation relationship between DN R6 $6^{+}$and "classical" Th17 cells, their HIV-specificity, and their relative susceptibility to HIV infection in vivo.

CONCLUSION: The identification of molecular mechanisms explaining the preservation of certain Th17 subset during HIV disease progression is of paramount importance for the design of new therapeutic strategies aimed at restoring mucosal integrity in HIV-infected individuals and reducing target cell availability at the portal sites of viral entry.

\section{O029 \\ CYTOMEGALOVIRUS-RELATED CD8 ${ }^{+} \mathrm{CD} 57^{+}{ }^{+} \mathrm{CD}^{2} 8^{-} \mathrm{T}$ CELL EXPANSION IS ASSOCIATED WITH REDUCED IMMUNE RESPONSIVENESS IN HIV INFECTION}

Barrett, Lisa ${ }^{1}$; Stapleton, Staci ${ }^{2}$; Gallant, Maureen ${ }^{2}$;

Burt, Kimberley $^{2}$; Fowke, Keith ${ }^{3}$; Grant, Michael ${ }^{2}$

${ }^{1}$ Bethesda, MD, USA; ${ }^{2}$ St John's, NL; ${ }^{3}$ Winnipeg, MB

BACKGROUND: In the era of HAART, age associated chronic conditions (eg. poor vaccine response, cardiovascular disease, diabetes) cause significant morbidity in relatively young HIV-infected individuals. Very old, healthy, HIV negative individuals are often cytomegalovirus (CMV) seronegative, and lack the massive expansion of 'immune risk' $\mathrm{CD} 28^{-} \mathrm{CD} 57^{+} \mathrm{T}$ cells seen in CMV seropositive individuals of the same age. It is unknown whether chronic CMV infection contributes to immune senescence in HIV infection. Pandemic influenza A/California04/09 (H1N1) vaccination offered an opportunity to assess immune response in an aging HIV-infected population.

OBJECTIVE: Assess CMV-specific $\mathrm{CD}^{+} \mathrm{T}$ cell responses in pandemic H1N1 vaccinees, and define immune correlates of low H1N1 antibody response.

METHODS: PBMC from $45 \mathrm{HIV}$ positive individuals were stimulated with CMV peptides, and CMV-specific T cell responses assessed by IFN- gamma ELISPOT, intracellular and surface (CD28, CD57) flow cytometry. TNF-alpha, IL-6, and C-reactive protein (CRP) serum levels were determined pre-H1N1 vaccination. H1N1-specific antibody titers preand post-vaccination were measured by bead-based assay.

RESULTS: Nine of 45 individuals were CMV negative (20\%). Three of $9(33 \%)$ CMV negative individuals had H1N1 responses, while 21/36 (59\%) CMV positive individuals responded. CD28-CD57 ${ }^{+} \mathrm{T}$ cells were significantly higher in CMV positive than CMV negative individuals $(31.2 \% \pm 3.56 \%$ vs $19.5 \% \pm 5.4 \%$; $p<0.05)$, even in the CMV-nonspecific T cell compartment. The lowest vaccination-induced H1N1 titers were associated with the largest $\mathrm{CD} 28-\mathrm{CD} 57^{+} \mathrm{T}$ cell populations, and no individuals with $\mathrm{CD} 28-\mathrm{CD} 57^{+}$populations greater than $60 \%$ responded to $\mathrm{H} 1 \mathrm{~N} 1$ vaccination.

CONCLUSIONS: HIV/CMV coinfected individuals have an expanded $\mathrm{CD} 28^{-} \mathrm{CD} 57^{+} \mathrm{T}$ cell compartment that is associated with immune risk in very old HIV negative individuals. Expansion of this T cell population is associated with decreased influenza vaccine responses and strong CMVspecific $\mathrm{CD}^{+} \mathrm{T}$ cell responses. Even in the absence of CMV disease, chronic CMV infection may impair immunity and contribute to morbidity in HIV infection.

\section{O030 \\ THE NONCATALYTIC DOMAIN OF APOBEC3G MEDIATES THE INTENSITY OF DEOXYCYTIDINE DEAMINATION ON HIV-1 PROVIRAL DNA}

Feng, Yuqing; Chelico, Linda

Saskatoon, SK

APOBEC3G is a single-stranded (ss) DNA deaminase that restricts replication of Vif deficient HIV-1 by inducing viral genome mutagenesis through deamination of cytosine to uracil on HIV-1 cDNA. APOBEC3G has polydisperse oligomeric states and deaminates ssDNA processively through jumping and sliding. APOBEC $3 \mathrm{G}$ has a catalytically inactive $\mathrm{N}$-terminal CD1 domain that mediates processivity and an active Cterminal CD2 domain that catalyzes deaminations. There has been a lack of understanding of the key mechanistic properties of APOBEC3G DNA scanning that allow for efficient deamination during HIV replication. We have assessed the determinants of APOBEC3G deamination efficiency mediated by the $\mathrm{CD} 1$ domain by comparing native $\mathrm{APOB}$ EC3G and two CD1 mutants, a monomeric mutant (F126A/W127A) and a clinical mutant associated with high viral loads (H186R). Biochemical assays on ssDNA or partially dsDNA and with a reconstituted HIV replication system demonstrate that both mutants of APOBEC3G have altered DNA scanning properties in either jumping (F126A/W127A) or sliding (H186R), which results in decreased abilities to induce mutagenesis during reverse transcription. The data reveal a functionality for APOBEC3G oligomers in deamination and provide the first biochemical characterization of the clinical mutant H186R. We propose a model that accounts for the functionality of a dual sliding and jumping scanning mechanism in assuring APOBEC3G causes HIV-1 gene inactivation and avoids sublethal mutagenesis.

\section{O031}

MILK MATTERS: SOLUBLE TOLL-LIKE RECEPTOR 2 (STLR2) FORMS IN BREAST MILK PLAY A SIGNIFICANT ROLE IN INHIBITION OF HIV-1 AND INFLAMMATION

Henrick, Bethany $M$

Hamilton, $\mathrm{ON}$

The majority of infants who breastfeed from their HIV-positive mothers remain uninfected despite constant and repeated exposure to virus over weeks to years. This phenomenon is not fully understood but has been closely linked to innate factors in breast milk (BM). Most recently we have focused on one such innate factor, soluble Toll-like receptor 2 (sTLR2) for its significant contribution as an inhibitor of inflammation triggered by bacterial and viral antigens. We hypothesized that sTLR2 in BM inhibits immune activation/inflammation and directly inhibits HIV-1 infection. sTLR2 protein profiles were analyzed in HIV-uninfected BM from different women and showed variability in amounts and predominant sTLR2 forms between women. sTLR2 immunodepleted BM, versus mock-depleted BM, incubated with Pam3CSK4 lead to significant increases in IL-8 production in a TLR2-dependant fashion in U937, 
HEK293-TLR2, and Caco-2. Importantly, sTLR2-depletion in BM led to significant $(\mathrm{P}<0.001)$ increases in HIV-1 infection in TZM-bl cells. To confirm these findings, TLR2-specific antibody addition to BM prior to cell-free R5 HIV-1 addition led to significantly increased HIV infection in TZM-bl reporter cells. We present evidence linking predominant BM sTLR2 forms with decreased pro-inflammatory responses and inhibition of HIV infection. Thus, sTLR2 in BM may be critical to infant health and prove beneficial in decreasing vertical HIV transmission to infants.

\section{O032}

\section{SALIVARY BASIC PROLINE-RICH PROTEINS ARE ELEVATED IN HIV-EXPOSED SERONEGATIVE MEN WHO HAVE SEX WITH MEN}

Burgener, Adam ${ }^{1}$; Mogk, Kenzie ${ }^{1}$; Westmacott, Garrett ${ }^{1}$; Plummer, Francis'; Ball, Blake'; Broliden, Kristina ${ }^{2}$;

Hasselrot, $\mathrm{Klara}^{2}$

${ }^{1}$ Winnipeg, MB; ${ }^{2}$ Stockholm, Sweden

OBJECTIVE: Recent studies of HIV-exposed seronegative (HESN) individuals suggest that soluble mucosal factors are playing a role in HIV protection. However, studies of innate factors associated with HIV protection in men who have sex with men (MSM) have been very limited. Here we performed an unbiased comprehensive proteomic analysis of saliva from a well-defined HIV serodiscordant couple cohort of HESN MSM who have reported regular unprotected oral receptive intercourse with their HIV-infected male partner.

DESIGN AND METHODS: Saliva samples from 25 HESN individuals and 22 non-exposed (NE) male controls were analyzed by iTRAQ and 2D-LC mass spectrometry. Selected innate factors were further characterized by immunoblot and compared to CC-chemokine expression, HIV-neutralizing activity, and various clinical factors.

RESULTS: Of the 337 unique proteins identified, 7 were differentially abundant ( $>2$-fold cutoff) in the HESN group. The five overabundant proteins (Basic salivary proline-rich proteins (bPRP) 2, Histatin-3, Lysozyme C, bPRP3 and SLPI) are all known to have antimicrobial activity and be involved in host defense. bPRP2 showed the highest overabundance (>6-fold) in HESN individuals as compared with NE controls $(p=0.009)$. The presence and relative abundance of multiple isoforms of this protein were demonstrated by immunoblot. Salivary levels of bPRP2 correlated with levels of CC-chemokines in HESN individuals including RANTES ( $p=0.01)$, MIP-1-alpha $(p=0.005)$, MIP-1-beta $(p<0.0001)$, MCP-1 $(p=0.005)$ and Eotaxin $(p=0.001)$ but not with frequency of HIV neutralizing activity, oral sexual practices or viral load of the sexual partner.

CONCLUSION: This study identifies salivary bPRP2 as a novel soluble factor associated with oral HIV exposure in MSM.

This work was funded through the Canadian Institute for Health Research, the Public Health Agency of Canada, the Swedish Research Council and the Swedish Physicians against AIDS Foundation.

Track B: Clinical Sciences: Long-term Complications of HIV Treatment: Resistance and Comorbidities

Volet B : Sciences cliniques : Complications à long terme du traitement du VIH : résistance et comorbidités

\section{O033}

\section{EMERGENCE OF DRUG-RESISTANT MALE-SEX-MALE EPIDEMICS IN MONTREAL}

Brenner, Bluma G; Roger, Michel; Ibanescu, Ruxandra-Ilinca; Moisi, Daniela; Golubkov, Olga; Charest, Hugues; Legault, Mario; Routy, Jean-Perre; Hardy, Isabelle; Baril, Jean-Guy;

Wainberg, Mark

Montreal, QC

BACKGROUND: Primary/recent stage infection may account for $75 \%$ of spread of the Montreal male-sex-male (MSM) epidemic. This study demonstrates that transmission clustering may lead to the emergence of drug-resistant subepidemics.

METHODS: The Quebec genotyping program has offered genotyping for primary infection, antiretroviral-naïve, and treatment-experienced populations. The prevalence of mutations conferring resistance to nucleoside and non-nucleoside reverse transcriptase inhibitors (NRTIs and NNRTIs), and protease inhibitors (PIs) were assessed annually from 2002-2009, assessing first genotype of any given patient. Phylogenetic analysis was performed to identify clustering among primary infections. Montreal PHI cohort data was used to assess the effect of clustering on viral load and set-point.

RESULTS: There have been progressive declines in the frequencies of resistance to NRTIs, NNRTIs and PIs in antiretroviral-experienced population ( $\mathrm{n}=2443)$ from 2002 to 2009. Concomitant declines in the frequencies of transmitted resistance to PIs and NRTIs were observed among antiretroviral drug-naïve populations $(\mathrm{n}=2554)$. As an example, parallel declines in the frequencies of in the PI mutations (M46I/L and L90M) were observed in chronic treated $(44.7 \% \rightarrow 8.8 \%)$ and drug-naïve $(4.4 \% \rightarrow 1.0 \%)$ populations. In contrast, declines in NNRTI resistance $(\mathrm{K} 103 \mathrm{~N}, \mathrm{Y} 181 \mathrm{C}$, and $\mathrm{G} 190 \mathrm{~A})$ in treated persons $(36.7 \% \rightarrow 19.8 \%)$ did not lead to reductions in transmitted NNRTI resistance $(6.1 \% \rightarrow 8.6 \%)$. Phylogenetic surveillance shows $28 \%$ and $47 \%$ of PHI segregate into 107 small (2-5 PHI, n=318) and 40 large (6-60 PHI, n=530) clusters, respectively. Such analysis revealed six subepidemics harboring K103N $(\mathrm{n}=7, \mathrm{n}=8, \mathrm{n}=17, \mathrm{n}=6), \mathrm{K} 103 \mathrm{R} / \mathrm{V108I}(\mathrm{n}=16)$ and G190A $(\mathrm{n}=60)$ among drug-naïve persons. Longitudinal analysis of $\mathrm{PHI}$ cohort data ( 0 - 2 years) reveals viral load in the six NNRTI large clusters $(n=21)$ is significantly greater than other large clusters $(n=76)$ and small clusters $(n=57)$ which in turn are greater than unique transmissions $(n=70)$.

CONCLUSION: The emergence of NNRTI drug-resistant subepidemics has arisen because of low genetic barrier, transmission clustering and enhanced viral replicative fitness.

\section{4}

\section{LOW INCIDENCE OF INTEGRASE INHIBITOR FAILURE AND RESISTANCE IN HIV-1 INFECTED PATIENTS IN BRITISH COLUMBIA}

McGovern, Rachel A; Woods, Conan K; Brumme, Chanson J; Chan, Dennison; Hogg, Robert S; Montaner, Julio S;

Harrigan, Richard

Vancouver, BC

BACKGROUND: Raltegravir (RAL) is the first approved HIV-1 integrase inhibitor. In the BENCHMRK trial, $23 \%$ of patients receiving RAL experienced virologic failure. RAL resistance was conferred in $68 \%$ of those tested. We wished to characterize RAL usage, failure and resistance in a real-world clinical setting.

METHODS: As of January 6th, 2012, RAL has been prescribed to 876 patients in British Columbia. Most (61\%) started RAL while their viral load was $<250$ copies $/ \mathrm{mL}$. Viral rebound was defined as two or more consecutive $\mathrm{pVL}$ measurements $>250$ copies $/ \mathrm{mL}$ following a period of viral suppression (two or more consecutive $\mathrm{pVL}$ measurements $<250$ copies/mL). HIV RNA was extracted from patient plasma $(\mathrm{N}=146)$ after RAL exposure. HIV integrase was amplified using nested RT-PCR, and sequenced using population-based methods with automated basecalling ("ReCall"). Integrase mutations were determined using the Stanford HIV Drug Resistance Database.

RESULTS: Of the 876 patients exposed to RAL in British Columbia, 738 (84\%) had suppression while on RAL. Thirty seven patients had viral rebound while receiving RAL, with a median $\mathrm{pVL}$ increase to $3.9 \log$ copies $/ \mathrm{mL}$. 18 patients were tested for RAL resistance within six months of viral rebound but only one patient had developed known RAL resistance mutations (E92Q). Of the patients exposed to RAL with detectable HIV-1, 146 were tested for integrase drug resistance (median pVL 3.7 log copies/mL). RAL resistance mutation(s) at integrase codon 143,148 or 155 were found in a further nine patients before reaching confirmed virologic rebound. All patients with resistance had a high viral set point (>100,000 copies $/ \mathrm{mL})$ and four $(44 \%)$ did not achieve virologic suppression while on RAL.

CONCLUSION: Rates of RAL resistance mutations in British Columbia are lower than those reported in the BENCHMRK study. RAL resistance mutations were observed in $7 \%$ of patients tested, of whom many never achieved virological suppression. 


\section{O035}

INCREASE IN HIV DRUG RESISTANCE AMONG TREATMENT-NAÏVE PATIENTS IN ONTARIO, 2005-2011: GROUNDS FOR CONCERN

Sullivan, Ashleigh B ${ }^{1,2}$; Harrigan, Richard P3; Swantee, Carol'1; Wu, Keyi ${ }^{1}$; Rank, Claudia ${ }^{2}$; Halverson, Jessica ${ }^{2}$; Archibald, Chris ${ }^{2}$; Remis, Robert $\mathrm{S}^{4}$

\section{${ }^{1}$ Etobicoke; ${ }^{2}$ Ottawa, ON; ${ }^{3}$ Vancouver, BC; ${ }^{4}$ Toronto, ON}

BACKGROUND: In recent years, genotyping of treatment-naïve patients to guide decisions about initial antiretroviral therapy has become the standard of care. In Ontario, large-scale testing of treatment-naïve patients began in 2005.

METHODS: We examined genotyping results carried out for clinical purposes in Ontario from 2005 to 2011 for trends in drug resistance (DR) in treatment-naïve patients. Treatment-naïve status was determined by eliminating results from patients who had: 1) evidence of therapy on viral load (VL) requisitions 2) a $\mathrm{VL}<50$ (i.e. undetectable), or 3) a $1.5 \log$ decrease in viral load prior to the first genotype. DR was determined using genotypic testing with resistance mutations defined by the 2009 WHO transmitted resistance surveillance criteria. We analyzed trends in DR overall and for each drug class by year and HIV clade.

RESULTS: From January 2005 to September 2011, 5,550 patients had at least one genotype. 2,118 (38.2\%) were treatment-naïve. Overall, $13.0 \%$ $(275 / 2,118)$ were DR. By drug class, the proportions DR were: NRTI $8.6 \%$, NNRTI 5.6\%, and PI 2.6\%. We observed a significant increase in DR from $2005-2007$ to $2008-2011$ of $10.4 \%$ to $14.8 \%(\mathrm{p}=0.003)$. By drug class, DR increased as follows: NRTI $6.7 \%$ to $9.9 \%(\mathrm{p}=0.012)$ and NNRTI $4.1 \%$ to $6.7 \%$ ( $\mathrm{p}=0.014)$; no significant difference was observed for PI. Overall, DR was higher for B (16.0\%) than non-B strains (4.7\%) and significantly increased for $\mathrm{B}(12.8 \%$ to $18.1 \%, \mathrm{p}=0.006)$ but not for non-B strains over time.

CONCLUSIONS: We observed a significant increase in DR in B strains among HIV treatment naïve-patients in Ontario overall and for NRTI and NNRTI. While we cannot exclude selection bias related to demographic and clinical characteristics (eg, initial viral load or symptoms) or related to time from HIV infection to genotyping, these results have implications for clinical treatment and for the potential impact of treatment as prevention.

\section{6}

IMPACT OF THE DATA COLLECTION ON ADVERSE EVENTS OF ANTI-HIV DRUGS (DAD) COHORT STUDY ON ABACAVIR PRESCRIBING AMONG TREATMENT-NAÏVE HUMAN IMMUNODEFICIENCY VIRUS (HIV) INFECTED PATIENTS

Antoniou, Tony ${ }^{1}$; Gillis, Jennifer ${ }^{1}$; Loutfy, Mona R ${ }^{1}$; Cooper, Curtis ${ }^{2}$; Hogg, Robert $\mathbf{S}^{3,4}$; Klein, Marina $\mathrm{B}^{4}$; Machouf, Nima ${ }^{4}$;

Montaner, Julio $\mathbf{S}^{3}$; Rourke, Sean B ${ }^{1}$; Tsoukas, Christos ${ }^{4}$;

Raboud, Janet $\mathrm{M}^{1}$; Canadian Observational Cohort Collaboration, $\mathrm{CANOC}^{3}$,

${ }^{1}$ Toronto; ${ }^{2}$ Ottawa, ON; ${ }^{3}$ Vancouver; ${ }^{4}$ Burnaby, BC; ${ }^{4}$ Montreal, QC OBJECTIVE: The impact of observational studies on prescribing practice is not well documented, especially of antiretroviral agents. We sought to evaluate trends in abacavir prescribing among antiretroviral-naïve individuals in the Canadian Observational Cohort (CANOC) following the presentation of the Data Collection on Adverse Events of Anti-HIV Drugs (DAD) cohort study in February 2008.

DESIGN: We evaluated trends in new abacavir prescribing following the $\mathrm{DAD}$ in a retrospective cohort study using segmented regression analysis, structural break analysis and multivariable logistic regression analysis. RESULTS: Between January 1, 2000 and February 28, 2009, a total of 6673 antiretroviral-naïve patients were included in CANOC. In the segmented regression analysis, a significant change was not observed in the proportion of new abacavir prescriptions immediately following the release of DAD $(-2.5 \% ; \mathrm{p}=0.66)$, while a negative trend was observed in the months following the presentation of these data $(-2.3 \%$ per month; $\mathrm{p}=0.003)$. Structural break analysis identified a significant decrease in new prescriptions for abacavir in March 2008 (95\% confidence interval, January 2008 to January 2009). In multivariable logistic regression analysis, the presentation of the DAD was not associated with an immediate change in the odds of being prescribed abacavir versus tenofovir (adjusted 0.99, 95\% confidence interval: 0.71 to 1.38 ), while a significant post-presentation decrease was observed (adjusted OR, 0.41 per year, $95 \%$ confidence interval: 0.29 to 0.58 ).

CONCLUSIONS: Despite concerns about residual confounding, presentation of the DAD was associated with a significant decrease in abacavir use among antiretroviral-naïve HIV-positive patients initiating therapy.

\section{O037}

\section{HARMONIZATION OF PATIENT REPORT AND OBJECTIVE MEASURES OF COGNITIVE DEFICITS: A PROOF OF CONSTRUCT}

Brouillette, Marie-Josee; Palladini, Lisa; Koski, Lisa; Finch, Lois; Fellows, Lesley; Mayo, Nancy

Montreal, QC

BACKGROUND: Several studies have documented a high frequency of cognitive dysfunction associated with chronic HIV infection. Clinically, cognition is often documented using screening tests or questionnaires. Our group has developed a new measurement method that harmonizes the information obtained from both objective and self-report measures by calibrating these disparate items onto the same unidimensional, linear, scale. The purpose of the study is to provide evidence of the uniqueness of this construct by comparing the clinical factors that are associated with each assessment method.

METHODS: Seventy-six $\mathrm{HIV}^{+}$patients were evaluated with the Montreal Cognitive Assessment (MoCA), a brief computerized cognitive battery and two questionnaires (MSNQ and PDQ). All items were combined into a single dataset that was Rasch analyzed. The clinical factors studied were age, sex, education and depressive symptoms (Beck Depression Inventory-II, BDI-II). Stepwise linear regression analysis was used to evaluate the relative importance of the clinical variables to the scores on the MoCA, the PDQ, the MSNQ and the new harmonized tool.

RESULTS: The score on the MoCA was associated with language and education $\left(r^{2}: 0.15\right.$ and 0.06 , respectively). The PDQ and MSNQ were associated with the BDI score $\left(\mathrm{r}^{2}: 0.4\right.$ and 0.17 , respectively). The MSNQ score was additionally associated with age $\left(\mathrm{r}^{2}:+0.07\right)$. Performance on the new tool was associated only with BDI scores $\left(r^{2}: 0.19\right)$. Higher depression was associated with more reported symptoms.

CONCLUSION: This study provides evidence of the validity of the new construct in that it shares some but not all of the predictors of the MoCA alone, the PDQ or the MSNQ. It shows independence of age, language and education, and it is not as strongly associated with depression as the PDQ. As such, it may prove with further testing to be superior to either the MoCA alone or the PDQ and MSNQ.

\section{O038}

HIV AND CARDIOVASCULAR DISEASE: THE HIV QUIT SMOKING INTERVENTION PILOT STUDY

Balfour, Louise; Cameron, William; Love, Erin; Lawless, James; Keleher, Bethany; Pipe, Andrew; Reid, Bob; Aitken, Debbie; Corace, Kim; Tasca, George; Sandre, Daniella; Illing, Vanessa; Angel, Jonathan; Garber, Gary; Cooper, Curtis; Giguere, Pierre; Lee, Craig; la Porte, Charles; MacPherson, Paul

Ottawa, ON

BACKGROUND: Cardiovascular disease (CVD) is now a leading cause of morbidity and mortality among people living with HIV/AIDS (PHAs). After controlling for pre-existing disease, cigarette smoking is the most significant predictor of CVD among PHAs. In view of the alarmingly high rate of smoking (35\% to $70 \%$ ) among PHAs as compared to the national average $(17 \%)$ quitting smoking is likely the single most effective intervention to reduce mortality due to CVD among PHAs. Depression (40 to $60 \%$ ) and cigarette smoking (35 to $60 \%$ ) also commonly co-occur among PHAs and depression may contribute to smoking relapse rates. Unfortunately, smoking cessation programs are rarely offered in routine HIV care. The goal of this pilot study was to develop an "HIV quit smoking program" from the Ottawa Model for Smoking Cessation but tailored to the needs of PHAs, and to evaluate the quit smoking intervention outcomes.

METHOD: PHA smokers $(\mathrm{N}=49)$ were recruited during their regular HIV clinic visits at The Ottawa Hospital. Depression, levels of nicotine 
dependence, and smoking behaviours were assessed at study baseline and study follow-up visits. Participants received a 10-week supply of NRT patches, telephone and in-person smoking-cessation counselling, as well as relapse prevention and targeted interventions to reduce depressive symptoms in participants identified as depressed. Smoking status, psychological functioning, and immunological measures were assessed from baseline through 6 months post "quit date" follow-up.

RESULTS: All participants met their target "quit date." At 24-weeks post-quit date, $28 \%$ were still "smoke free" as verified by objective CO levels. In addition, at 24-week follow-up, those who were still "smoke free" had lower levels of nicotine dependence compared to those who had relapsed.

CONCLUSION: Results from this study may help inform health care policy by providing evidence of the benefits of incorporating an HIV tailored quit smoking program into routine HIV clinical care in HIV clinics across Canada.

\section{O039}

FACTORS ASSOCIATED WITH SHORTER LEUKOCYTE TELOMERE LENGTH IN HIV ${ }^{+}$AND HIV- ADULTS

Zanet, DeAnna L; Sattha, Beheroze; Maan, Evelyn J; Thorne, Anona; Gadawski, Izabelle; Burdge, David;

Murray, Melanie; Singer, Joel; Money, Deborah M; Pick, Neora; Cote, Helene C; CIHR Emerging Team in HIV Therapy and Aging, The

Vancouver, BC

BACKGROUND: Telomeres protect the ends of chromosomes and leukocyte telomere length (LTL) is a marker of aging. Oxidative stress due to HIV-induced immune activation/inflammation and/or NRTImediated telomerase inhibition could accelerate telomere shortening. We investigated predictors of shorter LTL in $\mathrm{HIV}^{+} / \mathrm{HIV}^{-}$adults.

MATERIAL \& METHODS: Blood was prospectively collected from CARMA cohort adult particpants. LTL was measured using qPCR. Uniand multivariable linear regression models examined the relationships between explanatory variables and LTL.

RESULTS: Data was obtained for $229 \mathrm{HIV}^{+}$subjects $(40.3 \pm 10.5$ years, $79 \%$ female) and $166 \mathrm{HIV}^{-}$controls ( $39.3 \pm 11.1$ years, $71 \%$ female). Shorter LTL were univariately associated with older age, younger parent age at birth, smoking, illicit drug use, HCV diagnosis ever, $\mathrm{HIV}^{+}$status, nonBlack/South Asian ethnicity, and income $<\$ 15 \mathrm{~K} /$ year. Parent's age, although important, was not included in the model as data was missing for $>55 \%$ of subjects. Various explanatory variables were correlated, including: drug use/ethnicity/HCV and income/smoking, increasing modeling complexity. There were also significant interactions whereby smoking, drug use, HCV and income were associated with shorter LTL only in the HIV-uninfected group. Age $(p<0.0001), \mathrm{HIV}^{+}$status $(\mathrm{p}=0.034), \mathrm{HCV}$ $(\mathrm{p}=0.005)$, income $(\mathrm{p}=0.07)$ and pack*years smoking $(\mathrm{p}=0.099)$ were associated with shorter LTL in a model adjusted for interactions $(\mathrm{R} 2=0.26)$. Among $\mathrm{HIV}^{+}$subjects, HIV duration, ART duration, current or nadir CD4, and HIV pVL showed no association with LTL.

CONCLUSIONS: These results suggest that beyond aging, $\mathrm{HIV}^{+}$status, $\mathrm{HCV}$ infection (or drug use) and low income are the strongest predictors of LTL and may therefore affect telomere maintenance and cellular aging. Lifestyle factors such as smoking, drug use (or HCV) and income are noticeably stronger predictors of LTL in uninfected subjects, suggesting that HIV related factor(s) may overwhelm their effects among infected subjects. Future work should attempt to further elucidate these factors and determine whether active vs. cleared HCV vs. drug use is most closely linked to LTL.

\section{O040}

\section{EFFECT OF OMEGA-3 FATTY ACID SUPPLEMENTATION ON HYPERTRIGLYCERIDEMIA IN HIV-INFECTED CHILDREN TAKING COMBINATION ANTIRETROVIRAL THERAPY}

Brophy, Jason C'; Read, Stanley²; Forbes, John ${ }^{3}$; Alimenti, Ariane ${ }^{3}$; Samson, Lindy'; Thorne, Anona ${ }^{3}$; Smieja, Marek ${ }^{4}$; Bitnun, Ari $^{2}$ ${ }^{1}$ Ottawa; ${ }^{2}$ Toronto, ON; ${ }^{3}$ Vancouver, $\mathrm{BC}^{4}{ }^{4}$ Hamilton, ON

INTRODUCTION: Hypertriglyceridemia (HTG) is a common complication of combination antiretroviral therapy (cART). Omega-3 fatty acid supplementation (O3FA) has shown benefit in HIV-infected adults with HTG, but no pediatric studies have been published.

METHODS: A clinical trial of O3FA was conducted in HIV-infected children $>4$ years of age with HTG (fasting triglyceride $[\mathrm{TG}]>1.4 \mathrm{mmol} / \mathrm{L}$ ) at 3 Canadian centres. Children received $600 \mathrm{mg}(5-11$ years) or 1200 mg (12-18 years) O3FA BID and identical placebo capsules for 3 months each in a randomized cross-over fashion separated by a one-month washout period. Fasting lipid profiles, adherence assessments, and tolerability questionnaires were completed at each study visit. The primary outcome was percentage difference in TG at 4 and 12 weeks of treatment.

RESULTS: Eleven children (8 male) completed the study. cART included 10 on PIs, 3 on NNRTIs, and 11 on NRTIs; all had CD4 $\geq 500$ cells/uL and 10 had undetectable VL at baseline. Five had BMI and 4 had weight $>90$ th percentile. Median TG at baseline was $2.0 \mathrm{mmol} / \mathrm{L}$. At week 4, median total and percentage changes in TG were $-0.4 \mathrm{mmol} / \mathrm{L}$ and $-20 \%$ on O3FA and $+0.1 \mathrm{mmol} / \mathrm{L}$ and $+3 \%$ on placebo. At week 12 , values were $-0.3 \mathrm{mmol} / \mathrm{L}$ and $-14 \%$ on O3FA and $-0.4 \mathrm{mmol} / \mathrm{L}$ and $-17 \%$ on placebo. Median percentage increase in LDL at 12 weeks was $30 \%$ on O3FA and $4 \%$ on placebo. All sign test p-values were non-significant. Burping/excess gas was reported by $36 \%$ of subjects on O3FA vs $18 \%$ on placebo; other side effects were similar between arms.

CONCLUSIONS: While insufficiently powered to demonstrate statistical significance, the $20 \%$ median reduction in TG at 4 weeks of O3FA therapy was similar to that seen in adult studies. O3FA was well tolerated. Further studies will be needed to determine the efficacy of O3FA for pediatric HTG.

Track C: Epidemiology and Public Health Sciences: Vulnerabilities and HIV Risk

Volet C : Épidémiologie et sciences de la santé publique : Vulnérabilités et risque lié au VIH

\section{O041}

\section{CRYSTAL METHAMPHETAMINE AND INJECTION DRUG USE INITIATION AMONG STREET-INVOLVED YOUTH IN A CANADIAN SETTING}

Werb, Dan; Kerr, Thomas; Montaner, Julio; Wood, Evan

Vancouver, BC

INTRODUCTION: The period surrounding initiation of injection drug use is associated with heightened risk of HIV transmission. Further, crystal methamphetamine has been hypothesized as a potential predictor of initiation of injecting. We sought to determine the impact of crystal methamphetamine use on risk of injecting initiation among street-involved youth in a Canadian setting.

METHODS: Cox regression analyses were used to identify predictors of injecting initiation among participants in the At-Risk Youth Study (ARYS), an open prospective cohort of street-involved youth aged 14 to 26 in Vancouver, Canada. Data on circumstances of first injection were obtained to explore the micro-setting characteristics of injecting initiation events.

RESULTS: Between October 2005 and December 2010, a total of 395 drug injecting naïve participants provided 1,434 observations, with 64 (16.2\%) participants reporting initiation of injection drug use. In multivariate analysis, non-injection crystal methamphetamine use (Adjusted Hazard Ratio $[\mathrm{AHR}]=1.91,95 \%$ Confidence Intervals $[\mathrm{CI}]: 1.34-2.73, \mathrm{p}=0.001)$ and non-injection heroin use (AHR $=1.50,95 \% \mathrm{CI}: 1.00-2.26, \mathrm{p}=0.049)$ were positively associated with injecting initiation. Older age (AHR = 0.91, 95\% CI: $0.85-0.97, p=0.003)$, non-injection cocaine use (AHR $=0.64,95 \%$ CI: $0.44-0.93, p=0.020)$, and marijuana use $(\mathrm{AHR}=0.58$, $95 \% \mathrm{CI}: 0.38-0.87, \mathrm{p}=0.009$ ) were negatively associated with injecting initiation. Among those reporting on circumstances of first injection (n $=25)$, over half $(13,52 \%)$ reported receiving the injected drug as a gift, while $17(68 \%)$ reported that they had not considered injecting prior to initiation. Finally, 14 (56\%) reported never becoming a regular injector throughout the study period after initiation.

CONCLUSION: Despite adjustment for potential confounders, noninjection crystal methamphetamine was strongly predictive of injecting initiation. These results have implications for the development of preventive interventions to reduce HIV transmission among street-involved youth. 


\section{2}

METHADONE SUBSTITUTION THERAPY (MST) ACCESS IN A POPULATION OF DRUG USERS INJECTING MULTIPLE TYPES OF DRUGS: DOES IT PROTECT AGAINST HIV?

Bruneau, Julie ${ }^{1}$; Zang, Geng ${ }^{1}$; Daniel, Mark²; Roy, Élise $^{3}$

${ }^{1}$ Montréal, QC; ${ }^{2}$ Adelaide, Australia; ${ }^{3}$ Longueuil, QC

RATIONALE: Opiate substitution therapy, mostly MST, is considered an efficient measure to prevent HIV infection in opiate user populations. It is unknown, however, whether MST is protective among injection drug users (IDUs) reporting high levels of poly-substance drug use, including cocaine and heroin.

OBJECTIVE: To examine the association between HIV incidence and exposure to MST, accounting for other known risk factors.

METHODS: IDUs were recruited into a prospective cohort between 1992 to 2008. At each semi-annual visit, IDUs completed interviewadministered questionnaires. Exposure to MST was defined as taking prescribed methadone in the past month. For comparability of results for participants enrolled at different periods, follow-up was censored at 48 months. Time-updated univariate and multivariable Cox regression models evaluated associations between incident HIV and MST, as well as other known risk factors.

RESULTS: Of 2,074 HIV-seronegative IDUs enrolled and with at least one follow-up visit ( $80 \%$ males, and $34 \%$ under 30 years of age, $65 \%$ IV cocaine and 31\% IV heroin use past month), 148 became HIV-positive within 48 months (incidence $=3.3$ per 100 p-y (95\% CI: 2.8,3.9). In univariate analyses MST was protective against HIV (Hazard Ratio (HR) $0.24(95 \%$ CI: $0.10,0.59))$ but this association did not persist after accounting for other variables (HR: 0.51 (95\% CI: 0.20, 1.28)). Risk factors associated with incident HIV in multivariable analyses included: female gender (HR: 0.53 (95\% CI: 0.29, 0.97)), unstable housing (HR: 2.02 (1.44, 2.83)), IV cocaine use (HR 3.75 (2.31, 6.09)), booting (HR:2.38 (1.60, $3.55))$, sharing syringes, (HR $2.97(2.04,4.33))$ and having sex with an HIV-infected partner (HR $2.46(1.34,4.52)$ No interaction was found with IV cocaine use.

CONCLUSIONS: Our data do not suggest that MST is, independent of other risk factors, protective effect against incident HIV among IDUs injecting multiple types of drugs.

\section{3}

THE CEDAR PROJECT: SEXUAL VIOLENCE RELATED VULNERABILITIES AMONG YOUNG ABORIGINAL PEOPLE WHO USE DRUGS IN TWO CANADIAN CITIES

Cedar Project Partnership, For the ${ }^{1}$; Pearce, Margo E²;

Teegee, Mary ${ }^{3}$; Zhang, Hongbin ${ }^{2}$; Blair, Alden $\mathrm{H}^{2}$; Christian, Chief $\mathrm{W}^{4}$;

Schechter, Martin T2; Spittal, Patricia $\mathbf{M}^{2}$

${ }^{1}$ Prince George; ${ }^{2}$ Vancouver; ${ }^{3}$ Vanderhoof; ${ }^{4}$ Enderby, BC

BACKGROUND: Little is understood regarding trends and risk for sexual assault among young Aboriginal people who use drugs.

METHODS: The Cedar Project is an ongoing cohort study of young Aboriginal people (aged 14 to 30) in Vancouver and Prince George, BC, who use injection and non-injection drugs. This study used generalized linear mixed models (GLIMM) to assess trends of sexual assault and associated risk factors between 2003-2010, adjusting for confounders. Predicted probability of sexual assault, adjusted odds ratios (AOR) and $95 \%$ confidence intervals $(\mathrm{CI})$ were estimated.

RESULTS: Among 498 participants ( $52 \%$ women) eligible, there were 137 incident cases of sexual assault reported among 94 participants (range: 1-4 events per-person) over the period. One-quarter (26\%) of the assaults had been by a boyfriend or known male. Overall, the chance of sexual assault increased over time but was not statistically significant. However, participants involved in sex work had a significant increased probability $(\mathrm{p}$-value $=0.031)$ of sexual assault from baseline $(\mathrm{AOR}=1.26$, 95\%CI: $0.57-2.78$ ) to the end of the study period (AOR=11.07, 95\% CI:8.34-14.69). Odds for sexual assault were significantly higher for participants who were involved in sex work $(\mathrm{AOR}=1.38,95 \% \mathrm{CI}: 1.11-1.65)$; injected drugs $(\mathrm{AOR}=1.93,95 \% \mathrm{CI}: 1.30-2.55)$, experienced childhood sexual abuse $(\mathrm{AOR}=3.25,95 \% \mathrm{CI}: 2.59-3.91)$, and smoked crack cocaine on a daily or more basis (AOR=1.64, 95\%CI: 1.00-2.27). Among participants who injected drugs, the odds of sexual assault were significantly higher for those who injected daily or more cocaine $(\mathrm{AOR}=2.53,95 \% \mathrm{CI}$ : 1.75-3.32) and daily or more heroin ( $\mathrm{AOR}=2.21,95 \% \mathrm{CI}: 1.51-2.92)$.

CONCLUSION: It is concerning that participants involved in sex work had an unparalleled odds of sexual assault over the study period. Sexual assault must be considered in the context of drug and sex related harms, and safe spaces for young Aboriginal people who use drugs must be made available 24-hours per day.

\section{O044}

\section{CORRELATES OF RECENT HIV TESTING AMONG} YOUNGER MSM IN NEW ZEALAND: RESULTS FROM NATIONAL HIV SOCIOBEHAVIOURAL SURVEILLANCE (2006-2011)

Lachowsky, Nathan J' ${ }^{1}$; Saxton, Peter $^{2}$; Dickson, Nigel ${ }^{2}$; Hughes, Anthony²; Dewey, Cate $\mathrm{E}^{1}$; Summerlee, Alastair $\mathrm{J}^{1}$ ${ }^{1}$ Guelph, ON; ${ }^{2}$ Auckland, New Zealand

INTRODUCTION: Recent research results from New Zealand recom mended increased HIV testing to encourage earlier diagnosis. New Zealand findings may provide some insight for Canada's HIV management. The objective was to conduct New Zealand's first investigation into recent HIV testing among YMSM.

METHODS: A pooled sample of YMSM (aged 16-29) was created from the 2006-2011 rounds of the Gay Auckland Periodic Sex Survey and Gay men's Online Sex Survey. Multivariate logistic regression, controlling for year, was used to investigate correlates of recent HIV testing (having reported an HIV test in $<12$ months or not). Only statistically significant findings of the final model are presented [adjusted OR (95\%CI)].

RESULTS: Of 3,352 eligible participants, 1,338 (39.9\%) reported having had a recent HIV test. In the final adjusted model, the odds of having a recent HIV test were higher for:

- a per year increase [1.06 (1.03-1.08)]

- spending at least some time with other gay men (1.44 [1.20-1.73])

- multiple sex partners versus one in the $<6$ months (eg, 1.73 [1.32-2.27] for 2-5 partners)

- having a regular partner for 6-12 months versus $<6$ months (1.69 [1.19-2.41])

- high condom use with casual partners (1.43 [1.11-1.86])

- disagreement that HIV is a less serious threat nowadays (1.27 [1.03. 1.57]) and disagreed that an HIV-positive man would disclose before $\operatorname{sex}(1.28$ [1.09-1.51])

The odds of having a recent HIV test were lower for:

- recruitment online (0.73 [0.60-0.89])

- bisexual identity $(0.70$ [0.57-0.86])

- Pacific Islander (0.61 [0.40-0.92]) or Asian (0.71 [0.54-0.94]) ethnicity

- no regular partner $(0.55$ [0.42-0.71]) or one for $>2$ years $(0.50$ [0.35-0.71])

- being insertive-only versus versatile with a regular partner $(0.61[0.44$ 0.85])

- not knowing that HIV cannot pass through an undamaged latex condom (0.74 [0.61-0.89])

CONCLUSIONS: Understanding factors related to lower HIV testing rates among specific YMSM should be used in conjunction with epidemiologic data to inform future prevention work.

\section{O045}

SEX FOR FOOD: FOOD INSECURITY AS A STRUCTURAL DRIVER OF HIV RISK AMONG STREET-BASED SEX WORKERS AND THEIR CLIENTS

Nesbitt, Ariel; Chettiar, Jill; Feng, Cindy; Montaner, Julio; Shannon, Kate

Vancouver, BC

OBJECTIVES: There is little understanding of food insecurity's impacts on sexual risks for HIV infection in the high resource context of Canada. We sought to examine the overall prevalence of food insecurity and associations with negotiation of sexual risk for HIV infection among a cohort of street and off-street sex workers (SWs) in Vancouver, Canada.

METHODS: Data were drawn from baseline interview questionnaires and HIV/STI testing (January 2010-present) in an ongoing, communitybased prospective cohort of street and off-street SWs: An Evaluation of Sex Workers Health Access (AESHA). Women and transgender individuals were recruited through time-location sampling and outreach to 
street and off-street (eg, brothels, massage parlours) sex venues. Bivariate and multivariate explanatory logic regression models were constructed to examine correlates of food insecurity and confounding models fit to examine the relationship between food insecurity and sexual HIV risks. RESULTS: Of a total of 510 SWS, 484 were include in the analyses with half $(n=256,50.5 \%)$ reporting food insecurity in the last 6 months, and 201 (39.4\%) relying on subsidized food for their primary food needs. In multivariate analysis, crack use $(\mathrm{aOR}=1.81,95 \%$ confidence intervals (95\% CI) 1.02-3.22), homelessness [aOR] = 1.61; 95\% CI 1.02-2.57), and reliance on subsidized food $(\mathrm{aOR}=1.57,95 \% \mathrm{CI} 1.00-2.47)$ were independently associated with increased odds of food insecurity, while working indoors was associated with reduced odds of food insecurity $(\mathrm{aOR}=0.10,95 \% \mathrm{CI} 0.05-0.23)$. In confounder models, food insecurity remained independently associated with agreeing to unprotected sex for more money $(\mathrm{aOR}=2.16 ; 95 \% \mathrm{CI} 1.19-3.93)$ and exchanging sex for food $(\mathrm{aOR}=5.61 ; 95 \%$ CI 2.35-13.37).

DISCUSSION/CONCLUSIONS: A high prevalence of food insecurity was documented and was associated with five-fold increased odds of exchanging sex for food and two-fold increased odds of agreeing for more money for unprotected sex by clients. Lack of adequate access to food appears to be a major structural barrier to negotiating sexual HIV risks among marginalized sex workers. Structural interventions, including onsite food access in safer indoor work-spaces, deserve attention.

\section{O046 \\ SEX-BASED ANALYSIS OF INJECTING, SEXUAL AND TESTING BEHAVIOURS AMONG PEOPLE WHO INJECT DRUGS: INTERIM RESULTS FROM A NATIONAL ENHANCED HIV SURVEILLANCE SYSTEM, I-TRACK PHASE 3 (2010-2012)}

Bennett, Rachel; Tarasuk, Jill; George, Diana B; Ogunnaike-Cooke, Susanna; Archibald, Chris P; I-Track Site Principal Investigators, The Ottawa, ON

BACKGROUND: I-Track, enhanced surveillance of HIV and hepatitis $\mathrm{C}$ risk behaviours among people who inject drugs in Canada, conducted Phase 3 data collection from 2010 to 2011 in 5 sites across Canada.

METHODS: Information regarding demographics, drug use, injecting and sexual risk behaviours, and HIV and hepatitis $\mathrm{C}$ testing patterns were collected in face-to-face interviews in 5 sentinel sites across Canada. A biological sample was collected for antibody testing for HIV and hepatitis C. Selected descriptive statistics were calculated and Chi-square tests assessed the differences between males and females.

RESULTS: A total of 997 participants (61\% male) were interviewed between April 2010 and October 2011 in Regina, Thunder Bay, Sudbury, Toronto, and Kingston. The average age was 39 years (males: 40 years; females: 36 years). Significant differences between the sexes were found in education completed (lower in females), Aboriginal ethnicity (higher in females), and housing stability (higher proportion of males living in unstable housing). A significantly higher proportion of males reported having ever spent time in jail.

Borrowing and passing on used needles and other injecting equipment were reported by a greater proportion of female participants. Cocaine was the most often injected drug in the previous 6 months (25\%). No significant difference between the sexes in age at initiation of injection was found.

No statistically significant differences between the sexes were found for selected sexual risk behaviours. Overall HIV seropositivity was 6\% (males $5 \%$ vs females $7 \%, p>0.05$ ). Awareness of HIV seropositivity was $69 \%$ overall and higher among females than males ( $81 \%$ vs. $59 \%, \mathrm{p}>0.05)$.

CONCLUSION: These analyses showed important sex-based differences among this population of people who inject drugs, particularly in the patterns of demographic characteristics and injecting risk behaviours. This supports the need for sex-specific prevention approaches, messages and programs.

\section{7}

LOST IN TRANSITION: HIV PREVALENCE AND RELATED VULNERABILITIES AMONG YOUNG PEOPLE SURVIVING ABDUCTION AND DISPLACEMENT IN POST-CONFLICT NORTHERN UGANDA

Patel, Sheetal $\mathbf{H}^{1}$; Schechter, Martin $\mathrm{T}^{\mathbf{1}}$; Muyinda, Herbert ${ }^{2}$;

Kiwanuka, Noah${ }^{2}$; Sewankambo, Nelson $\mathrm{K}^{2}$; Spittal, Patricia $\mathbf{M}^{1}$

${ }^{1}$ Vancouver, BC; ${ }^{2}$ Kampala, Uganda

OBJECTIVE: After more than two-decades of war and displacement in Northern Uganda, over one million Internally Displaced Peoples (IDPs) are returning to their home villages. However, thousands of IDPs are only halfway home, living in transit camps near their villages. This population in transition provided a unique opportunity to assess the influence of conflict on HIV infection among young people surviving displacement and abduction in post-conflict Northern Uganda.

METHODS: In 2010, a cross-sectional demographic and behavioral survey was conducted with 384 young people aged 15-29, residing in transit camps in one of two sub-counties in Gulu District. Biological specimens for HIV were collected for Rapid testing in-field and Confirmatory testing in-lab. Multivariable logistic regression identified factors significantly associated with HIV infection.

RESULTS: Of the 384 participants sampled, 192 (50\%) were female and $107(27.9 \%)$ were former child soldiers. Overall HIV prevalence was alarmingly high at $12.8 \%$. HIV prevalence among females was $15.6 \%$, $9.9 \%$ among males, and $12.1 \%$ among former child soldiers. In multivariable logistic regression, HIV infection was significantly associated with: non-consensual 1st sex (Adjusted Odds Ratio [AOR]: 9.9, 95\% Confidence Interval [CI]: 1.7, 18.06); sub-county (AOR: 2.9, 95\%CI: 1.3, 6.7); STI symptoms past 12 months (AOR: $2.4,95 \%$ CI: 1.4, 6.2); practicing dry sex (AOR: 2.3, 95\%CI: 1.0, 5.1); age (AOR: 1.2, 95\%CI: 1.1, 1.3); thinking you could protect yourself from HIV (AOR: .29, 95\%CI: .12, .69); No. of HIV tests in lifetime (AOR: .86, 95\%CI: .81, .91).

CONCLUSIONS: In post-conflict Northern Uganda it has been observed that NGOs focused on relief who previously supported HIV/AIDS prevention and treatment have shuttered operations, leaving gaps in care. This, coupled with other strains of post-conflict resettlement, has produced a generation of young people 'lost in transition', leaving them at heightened risk of contracting HIV/AIDS. Applicable post-conflict HIV/AIDS programming is urgently required.

\section{O048}

CHANGES IN SEXUAL BEHAVIOUR, STRUCTURAL VULNERABILITIES, AND HIV PREVALENCE AMONG FEMALE SEX WORKERS IN PAKISTAN

Mishra, Sharmistha ${ }^{1}$; Ahmad, Sajid ${ }^{2}$; Malik, Naeem ${ }^{4}$; Salim, Momina'; Thompson, Laura $\mathrm{H}^{3}$; Blanchard, James $\mathrm{F}^{3}$; Emmanuel, Faran 2,3

${ }^{1}$ Toronto, ON; ${ }^{2}$ Islamabad, Pakistan; ${ }^{3}$ Winnipeg, MB; ${ }^{4}$ Islamabad, Pakistan

OBJECTIVES: We sought to examine changes in individual and structural vulnerabilities among FSWs in Pakistan, and to characterize risk factors for inconsistent condom use and HIV prevalence in this population.

METHODS: To describe changes, we analyzed self-reported sexual behaviour data from serial cross-sectional biological and behavioural surveys of FSWs conducted across 9 cities in 2006 and 2011. Using data from 12 cities in 2011, we conducted logistic regression to examine associations with (a) inconsistent condom use in the past month, and (b) HIV, after adjusting for regional differences.

RESULTS: 6,987 FSWs were included for analysis between survey rounds, and 4,301 FSWs were included for risk factor analysis using 2011 data. FSWs in 2011 were significantly more likely to use cell-phones for soliciting clients (29.4\% vs. $19.5 \%$ in 2006$)$. Consistent condom use with clients had increased from $23.6 \%$ to $30.0 \%$. Program exposure had increased from 2.1 to $20.7 \%$ by 2011 , while experience of sexual violence, recent injection drug use, sex with an injection drug user (IDU), and anal sex with clients was more common in 2011. In 2011, risk factors for inconsistent condom use with clients included: recent sexual violence (AOR 1.4; 95\% CI: 1.1-1.7), recent sex with an IDU (AOR 1.7, 95\% CI: 1.2-2.3), and absence of program exposure (3.2; 95\% CI: 2.2-4.4). HIV 
prevalence was $0.63 \%$ (95\% CI: $0.43-0.92)$ in 2011, and was associated with a recent history of injection drug use (AOR 4.7; 95\% CI: 1.1-19.6) and absence of program exposure (AOR 7.5; 95\% CI: 1.5-39.1).

CONCLUSIONS: While condom use with clients has increased among FSWs, protective behaviours remain low and vulnerabilities related to sexual behaviour and structural factors have risen. HIV is emerging in this population and an adaptive HIV prevention response that addresses changing vulnerabilities and the intersection of sexual networks with IDU is needed.

\section{Track D: Social Sciences: Perspectives and Problematics} Relating to MSM Risk and Prevention

Volet D : Sciences sociales : Risque et prévention chez les HARSAH : perspectives et problématiques

\section{O049 \\ DOES SPOT REACH AND RETAIN MSM WHO ARE MOST AT RISK?}

Otis, Joanne; Haig, Thomas; Blais, Martin; Veillette, Ludivine;

Fadel, Riyas; Rousseau, Robert; Wainberg, Mark

Montréal, QC

BACKGROUND: SPOT is an on-going intervention-research project offering free, anonymous, rapid HIV testing to MSM in Montreal.

OBJECTIVE: Describe the profile of men who return to SPOT for a second HIV test.

METHOD: Between July 2009 and May 2011, 1184 MSM enrolled during a first visit (TO) to SPOT and 360 returned for a follow-up visit 3 months later (T1). Repeat testers (requesting an HIV test at T1) were compared to the other participants for socio-demographic, psychosexual and behavioural variables measured at $\mathrm{TO}$.

RESULTS: 264 (22\%) of T0 participants requested a second test at T1. Compared with those who didn't, repeat testers had a higher overall risk profile at TO. They were more likely to report UAI at least once with an HIV-positive partner or partner of unknown status in the last 3 months ( $41 \%$ vs. $29 \%, p \leq 0.0001$ ). However both groups reported coming to SPOT because they had taken a risk $(74.6 \%$ versus $76.7 \%$, ns) and did not differ on reported satisfaction with services received.

Multivariate analysis indicated that repeat testers were more likely to have had a positive partner in the last 3 months at TO (AOR: 2.19, CI95\%: 1.25-3.85), to have consumed poppers during sex (AOR: 1.66, CI95\%: $1.10-2.50$ ), and to report that personal problems were a barrier to condom use (AOR: 1.32, CI 95\%: 1.13-1.55). These results are confirmed by contrasting participants who did not have an HIV test at follow-up with those who requested a test.

CONCLUSION: SPOT has been able to reach and retain men who are more exposed to risk and for whom personal problems may interfere with condom use. Since these men seem motivated to access HIV testing at SPOT as part of a preventive routine, intervention workers at SPOT should intensify counselling on these issues.

\section{O050}

PROFILE OF PARTICIPANTS WHO REPORT HAVING HAD INTENTIONAL UNPROTECTED ANAL INTERCOURSE (IUAI): DATA FROM SPOT, A COMMUNITY-BASED RAPID HIV TESTING INTERVENTION IN MONTREAL

McFadyen, Amelie; Otis, Joanne; Veillette-Bourbeau, Ludivine; Blais, Martin; Wainberg, Mark; Haig, Thomas; Rousseau, Robert Montreal, QC

BACKGROUND: SPOT is an ongoing research-intervention project offering free, anonymous, rapid HIV testing to MSM in Montreal. OBJECTIVE: To describe the profile of SPOT participants who reported intentional unprotected anal intercourse with casual or one-night partners of unknown HIV status in the last three months.

METHOD: Cross-sectional data was used from 1184 MSM (T0), tested between July 2009 and May 2011. Data was gathered through structured interviews and self-administered questionnaires. Participants who reported IUAI were compared to other participants for socio-demographic, psychosexual and behavioural variables using multivariate logistic regression.
RESULTS: Among SPOT participants, $17.7 \%$ reported IUAI in the last three months. Those reporting IUAI were less educated (high school diploma or less: AOR: 0.42, CI 95\%: 0.27-0.67), and less likely to have had at least one regular partner in the last three months (AOR: 0.57, CI95\%: 0.37-0.88). They were less likely to avoid meeting sexual partners over the Internet as a risk-reduction strategy (AOR: 0.89, CI95\%:0.80-0.99). They were more likely to have had at least one anonymous partner (AOR: 1.94, CI95\%: $1.25-3.01$ ) or one-night stand (AOR: 2.29, CI95\%: $1.22-4.28$ ) and they were more likely to score higher on barriers with regards to condom use (AOR: 2.17, CI95\%: 1.75-2.70). When a condom was used, they were more likely to report having put it on after anal sex had begun (AOR: 2.04, CI95\%: 1.35-3.10) or having taken it off during sex (AOR: 2.91, CI95\%: 1.77-4.79).

CONCLUSIONS: SPOT reaches a high proportion of MSM who report intentional unprotected anal sex with casual partners of unknown HIV status. Counseling should be tailored to address barriers to condom use in the context of anonymous sexual encounters. Counseling based on motivational interviewing could allow exploration in identifying alternate approaches to risk-reduction.

\section{1}

\section{HIGH PREVALENCE OF SEROSORTING AS A DELIBERATE RISK REDUCTION STRATEGY IN A SAMPLE OF MEN WHO HAVE SEX WITH MEN AT SPOT, A RAPID HIV TESTING SITE IN MONTREAL: ASSUMPTIONS ABOUT PARTNER'S SEROSTATUS MAY UNDERMINE THE EFFECTIVENESS OF THE STRATEGY}

Fadel, Ghayas; Otis, Joanne; Veillette-Bourbeau, Ludivine;

Blais, Martin; Wainberg, Mark; Haig, Thomas; Rousseau, Robert Montreal, QC

BACKGROUND: Serosorting as a risk reduction practice has been documented among MSM however most research on this topic infers use of that strategy based on reported behaviour. We sought to characterise MSM who reported deliberate serosorting and compare them to men who accept sex with HIV positive partners on various sociodemographic and behavioural variables.

\section{METHODOLOGY:}

Cross-sectional data from 1184 MSM enrolled between July 2009 and May 2011 for a first visit in the Montreal SPOT project were used. Data were gathered through structured interviews. Respondents were categorized as serosorters based on responses to questions on deliberate risk reduction strategies, namely whether or not they would have sex with a partner who is known or believed to be HIV positive. Multivariate logistic regression was used.

RESULTS: 866 participants answered the serosorting question. Of these, $77 \%(n=667)$ said they would refuse sex with an HIV positive partner. Serosorters in the sample were more likely to report avoiding certain types of partners (AOR: 1.45, CI95\%: 1.29-1.63) and avoiding anonymous sex venues (AOR: 1.12, CI95\%: 1.01-1.25) as a prevention strategy. They were less likely to report having an HIV positive partner in the last 3 months (AOR: 0.11, CI95\%: 0.06-0.21) and less likely to think that HIV is now a manageable chronic disease because of treatments (AOR: 0.83, CI95\%: 0.73-0.93). However, they were more likely to make assumptions about their partner's serostatus such as assuming serocordance (AOR: 1.18, CI95\%: 1.02-1.36). More detailed results will be presented at the conference.

CONCLUSION: Serosorting as a deliberate risk reduction strategy is very prevalent within the sample and the characteristics of the men who report serosorting are consistent with that strategy. However, it is evident that prevention efforts must continue addressing the issue of making assumptions about a partner's serostatus among men who serosort. 


\section{O052}

THE IMPORTANCE OF MONITORING AND EVALUATING THE DEGREE OF IMPLEMENTATION OF PROVINCIAL POINT-OF-CARE RAPID HIV TESTING GUIDELINES: THE EXPERIENCE AT SPOT, A COMMUNITY-BASED RAPID HIV TESTING INTERVENTION FOR MSM IN MONTREAL

Veillette-Bourbeau, Ludivine; Otis, Joanne; Primeau, Marc-André; Haig, Thomas; Fadel, Ghayas; Blais, Martin; Wainberg, Mark A; Rousseau, Robert

Montreal, QC

BACKGROUND: The three components of Point-of-Care HIV testing (pre-test counseling, testing, and post-test counseling) at SPOT are performed by a community worker and a nurse following provincial guidelines. We sought to evaluate the degree of implementation and explain fluctuations in implementing various intervention tasks.

METHODS: From July 2009 to May 2011, intervention staff logged task completion for each participant $(\mathrm{N}=1181)$ on a three point scale (not at all, in part, or completely). Oneway ANOVA was used to analyse the implementation scores.

RESULTS: Overall implementation score is $88.76 \%$. Over a two-year period, a gradual but statistically significant decrease in scores can be measured $(95.2 \%$ to $84.5 \%, \mathrm{p}<0.0001)$. Tasks related to skills in exploring risk, safer-sex behaviour and explaining test results decreased while scores remained stable or increased slightly on research-related tasks and some tasks related to testing procedures (checking the health status of participants, determining an appropriate choice between the rapid or the standard HIV test). Tasks related to announcing a reactive test result have been implemented consistently throughout this period.

LESSONS LEARNED: Implementation scores decreased but remained high. Three main factors appear to have had an impact on implementation scores: staff members' concerns about being evaluated (social desirability); habituation due to repeatedly performing the intervention; and adaptation of the intervention to participants' profile. This hypothesis needs to be explored in future analysis. These results shed light on the complexity and importance of monitoring and analyzing degrees and fluctuations in implementation for a multiple step intervention involving various measures and quality controls. Decreases in the degree of implementation are not necessarily a sign of failure, but may instead reflect a flexibility to adapt the intervention to community realities.

\section{O053 \\ RÉDUCTION DES RISQUES SEXUELS CHEZ LES HOMOSEXUELS MASCULINS. LA MÉDICALISATION DE LA SEXUALITÉ EN QUESTION? \\ Girard, Gabriel \\ Paris, France}

En France, au cours de la décennie 2000-2010, les débats sur la prévention du VIH/sida chez les homosexuels masculins ont été marqués par une forte controverse autour de la notion de réduction des risques sexuels. Les lectures du risque - injonctif vs compréhensif - qui s'y sont opposées, mettent en jeu des conceptions différentes de l'ordre social et des relations communautaires. Après 2005, la montée en puissance d'approches biomédicales de prévention (notamment autour du concept de " traitement comme prévention ») a contribué à un déplacement global des débats. Les discours sur le phénomène de bareback laissent place à de nouvelles problématisations de la gestion du risque.

L'objet de cette intervention sera d'analyser comment ces évolutions, loin d'épuiser les options en présence, traduisent une reconfiguration des discours. Il s'agira d'interroger ce qui est en débat autour de la notion de médicalisation de la sexualité gaie. Ainsi, en visant à rationaliser les comportements, ces processus ne relèvent-ils pas d'une autre forme de moralisation du risque VIH ? Issue d'un travail de doctorat mené en France, cette intervention s'appuie sur une méthodologie qualitative (entretiens avec les acteurs, analyse de la littérature "grise " et de la presse). Cet éclairage sociologique sur le contexte français permettra d'ouvrir une discussion sur les enjeux sociaux et politiques des approches de réduction des risques, alors même que l'essai Ipergay (traitement en préexposition chez des gais séronégatifs exposés au risque VIH) est en cours de lancement en France et au Québec. Dans ce cadre, les sciences sociales peuvent apporter un regard utile à la compréhension des logiques de la prévention.

\section{O054 \\ SYNDEMIC AMONG YOUNG CANADIAN GAY MEN: UNCOVERING THE ASSOCIATIONS BETWEEN ANTI-GAY EXPERIENCES, PSYCHOSOCIAL DISORDERS, AND HIV RISKS}

Ferlatte, Olivier ${ }^{1,2}$; Trussler, Terry ${ }^{1}$; Marchand, Rick ${ }^{1}$;

Salway Hottes, Travis ${ }^{1}$

${ }^{1}$ Vancouver; ${ }^{2}$ Burnaby, BC

OBJECTIVES: We measured the extent to which anti-gay experiences are associated with psychosocial issues, which in turn have an additive effect on HIV risk, in a national sample of young gay men.

METHODS: We recruited a national sample of gay men through online promotion. Participants completed an anonymous online survey developed through extensive community consultation. We restricted this study to participants $<30$ years of age and explored relationships between lifetime anti-gay experience, psychosocial disorders, and unprotected anal intercourse (UAI). Analysis was informed by the syndemic model Stall (2008).

RESULTS: 7908 men completed the survey including a sub-sample of 1523 young gay men (YGM) $<30.69 \%$ of YGM reported at least one form of antigay experience such as verbal or physical harassment, child sexual abuse, forced sex or career affected by homophobia (27\% reported 1, 20\% 2 , and $23 \% \geq 3$ ). For each additional form of anti-gay experience, our data demonstrated increased likelihood of psychosocial disorder: emotional distress (OR 2.7[95\% CI 2.4-3.1]), social isolation (OR 1.8 [95\% CI 1.52.1]), substance abuse (OR 1.8 [95\% CI 1.6-2.2]), depression (OR 2.7 [95\% CI 2.3-3.2]) or other mental health problems (OR 2.0 [95\% CI 1.7-2.3]). UAI in the last 12 months was reported in $24.6 \%$ of those reporting no psychosocial problems, $28.2 \%$ of those who reported $1,32.8 \%$ among those who reported 2 , and $43.60 \%$ among those with $\geq 3$ psychosocial issues. Multivariate analysis to identify interactions and control for effect of covariates is pending.

CONCLUSIONS: HIV prevention among young gay men has overwhelmingly focused on sexual risk alone. Other health problems among young gay men are not only important in their own right, but also may interact to increase HIV risk. HIV prevention could be strategically enhanced by addressing the stigma faced by young gay men and their broader health concerns while aiming to reduce sexual risks.

0055

INFLUENCE DES CONTEXTES DE CONSOMMATION, DES SCÉNARIOS SEXUELS ET DES COMPORTEMENTS À RISQUE SUR L'INTENSITÉ DE LA CONSOMMATION ET LE CHOIX DE SUBSTANCES PSYCHOACTIVES DES HOMMES AYANT DES RELATIONS SEXUELLES AVEC D'AUTRES HOMMES (HARSAH) FRANÇAIS SELON LE NET GAY BAROMÈTRE 2009

Leobon, Alain ${ }^{1,2}$; Chicoine Brathwaite, Yannick ${ }^{2}$; Otis, Joanne ${ }^{2}$

${ }^{1}$ Angers, France; ${ }^{2}$ Montréal, QC

OBJECTIFS: caractériser les modes de consommation de substances psychoactives des répondants ayant au moins consommé une fois de l'alcool de manière intense ou une drogue au cours des 12 derniers mois (n=13 996) selon l'enquête en ligne Net Gay Baromètre, renouvelée en France en 2009 ( $\mathrm{n}=17$ 490).

MÉTHODE : Sur l'échantillon complet, l'analyse factorielle d'échelles d'intensité de consommation a dégagé trois facteurs. Le premier associe le cannabis et l'alcool, le second 5 drogues améliorant les performances sexuelles, le troisième 4 drogues plutôt récréatives. Des régressions linéaires multiples ont testé l'influence de diverses catégories de variables sur chacun des trois facteurs.

RÉSULTATS : La consommation d'alcool et de cannabis est plus intense chez les plus jeunes $\left(\beta=-0.15^{* * *}\right)$, chez ceux qui fréquentent les clubs ou bars pour rencontrer leurs partenaires $(\beta=0.07 *)$ et qui privilégient la consommation dans un contexte privé $(\beta=0.34 * * *)\left(\mathrm{R}^{2}: 0.25\right)$.

La consommation de drogues dites sexuelles est plus intense chez les HARSAH qui se déclarent gay $(\beta=0.03 * * *)$, séropositifs $((\beta=0.08 * * *)$, 
qui se protègent rarement $(\beta=0.09 * * *)$, souhaitent augmenter leurs performances sexuelles $(\beta=0.23 * * *)$, fréquentent les sites bareback $\left(\beta=0.07^{* * *}\right)$, déclarent plus de dix partenaires occasionnels dans l'année $\left(\beta=0.08^{* * *}\right)$, des pratiques sexuelles marginales $(\beta=0.07 * * *)$ ainsi que chez ceux qui rapportent une agression en raison de leur orientation sexuelle $\left(\beta=0.02^{* * *}\right)$ ou un score élevé du gout du risque $(\beta=0.02 * * *)$ $\left(\mathrm{R}^{2}: 0.61\right)$.

Une consommation plus intensive de drogues dites récréatives est associée à la consommation dans le night life gai $\left(\beta=0.15^{* * *}\right)$, à des relations sexuelles monnayées $\left(\beta=0.05^{* * *}\right)$ ainsi qu'à une consommation combinées de substances $(\beta=0.35 * * *)\left(R^{2}: 0.22\right)$. $(* * * p \leq 0.001 ; * * p \leq 0.01$; $\mathrm{p} \leq 0.05)$.

CONCLUSION : Ces résultats suggèrent la prédominance des contextes de consommation, des scénarios sexuels clairement associés à des comportements à risque, des types d'espaces fréquentés, des cultures de sexe dans la modulation de l'intensité et de la diversité des substances consommées.

Les intervenants auprès des HARSAH devraient tenir compte de la complexité de ces agencements particulièrement pour ceux qui privilégient les drogues sexuelles (poppers, ecstasy, cocaine, GHB, viagra).

\section{O056}

THE BURDEN AND OUTCOMES OF INTIMATE PARTNER VIOLENCE AMONG GAY AND BISEXUAL HIV-INFECTED

\section{MALES}

Siemieniuk, Reed A ${ }^{1,2}$; Miller, Patricia ${ }^{1}$; Woodman, Kate ${ }^{3}$;

Ko, Karen ${ }^{1}$; Krentz, Hartmut B ${ }^{1}$; Gill, M John ${ }^{1}$

${ }^{1}$ Calgary, $\mathrm{AB} ;{ }^{2}$ Hamilton, $\mathrm{ON} ;{ }^{3}$ Edmonton, $\mathrm{AB}$

INTRODUCTION: Intimate partner violence (IPV) among HIV-infected gay and bisexual men is poorly described but may have important clinical, social, and psychological implications. IPV was examined among gay and bisexual men in Southern Alberta.

METHODS: Routine screening for domestic violence was implemented in Southern Alberta. Self-reported gay and bisexual men screened for IPV between May 2009 and December 2011 were included. Multivariable analysis of clinical and demographic variables was conducted with a Poisson binomial model, adjusted for age, months with HIV, log of initial CD4, location of HIV diagnosis (locally or not locally), and when the specific variable was determined (if appropriate, same visit or prior visit).

RESULTS: $22.4 \%$ (154) of 687 gay and bisexual males disclosed past or present IPV. IPV was associated with younger age, Aboriginal ethnicity, abuse as a child, smoking, and psychological illness prior to HIV diagnosis (table). Victims also engaged in riskier sexual practices since last visit and reported poorer health-related quality of life. They were more likely to have an HIV-psychiatric consultation in the past year. Effectively treated patients more often disconnected from care, returning with viremia $>500 / \mathrm{mL}$ (APR: $2.0,95 \%$ CI: $1.2-3.1$ ). Of patients presenting with a CD4 $>200$, those with a history of IPV were more likely to progress to AIDS (APR: 2.0, 95\% CI: 1.2-3.7).

CONCLUSIONS: IPV is a prevalent and under-recognized co-morbidity among HIV-infected gay and bisexual men with important social, epidemiologic, and clinical consequences. Larger longitudinal studies are required to fully clarify the implications of IPV and identify evidencebased interventions.

\begin{tabular}{llc} 
& APR $(\mathbf{9 5} \% \mathbf{C l})$ & P-value \\
\hline Age (per year) & $0.97(0.95-0.99)$ & 0.01 \\
Years in care (per year) & $1.03(0.98-1.08)$ & 0.28 \\
Diagnosed elsewhere & $1.11(0.72-1.70)$ & 0.6 \\
Race/ethnicity & & \\
Aboriginal & $2.48(1.18-5.2)$ & 0.02 \\
Black & $0.81(0.12-3.79)$ & 0.8 \\
Other & $0.97(0.51-1.86)$ & 0.9 \\
Caucasian & Ref & \\
Childhood abuse & $4.27(2.84-6.41)$ & $<0.001$ \\
Lives alone & $0.91(0.59-1.41)$ & 0.7 \\
Housing & & \\
Homeless & $0.56(0.06-4.83)$ & 0.6 \\
Supported/temporary & $2.58(0.94-7.09)$ & 0.07
\end{tabular}

Stable

Current alcohol abuse

Ref

Current illicit drug use

Smoking history

Current

Former

Never

HIV-psychiatry appointment (past year)

HIV-psychiatry appointment (ever)*

Depression (prior to HIV)*

Anxiety disorder (prior to HIV)*

Suicide attempt (prior to HIV)*

Health-related quality of life (at screen)

Poor/fair

Good

Very good/excellent

Sexual activity since last visit

Unprotected sexual activity since last

visit

On ART (at screen)

$\mathrm{VL}>500 / \mathrm{mL}$ (at screen)

CD4 (/mm ${ }^{3}$, at screen)

$>500$

200-500

$<200$

Nadir CD4 $\left(/ \mathrm{mm}^{3}\right)$

$>500$

200-500

$<200$

CD4 at HIV diagnosis $\left(/ \mathrm{mm}^{3}\right)^{*}$

$>500$

200-500

$<200$

LTFU with undetectable VL, returned

with $\mathrm{VL}>500 / \mathrm{mL}$

History of AIDS

History of AIDS (of those presenting

with CD4>200)

*Of patients diagnosed locally. APR Adjusted prevalence ratio; Cl Confidence interval; LTFU Lost to follow-up; VL Viral load

Track A: Basic Sciences: Anti-retroviral Drugs, Microbicides and Vaccines (Preclinical)

Volet A : Sciences fondamentales : Médicaments antirétroviraux, microbicides et vaccins (précliniques)

\section{O057}

\section{A NOVEL HIV VACCINE TARGETS THE 12 PROTEASE CLEAVAGE SITES}

Luo, Ma ${ }^{1}$; Tang, David ${ }^{1}$; Capina, Rupert' ${ }^{1}$ Yuan, Xin-Yong ${ }^{1}$; Prego, Cecilia ${ }^{2}$; Pinto, Jorge $\mathrm{C}^{2}$; Alonso, Maria ${ }^{2}$; Barry, Christina ${ }^{1}$; Pilon, Richard ${ }^{3}$; Daniuk, Christina ${ }^{1}$; Tuff, Jeff'; Pillet, Stephane'; La, David'; Bielawny, Thomas'; Czarnecki, Chris' ${ }^{1}$; Lacap, Philip'; Peters, Harold ${ }^{1}$; Wong, Gary ${ }^{1}$; Kimani, Makubo ${ }^{4}$; Wachihi, Charles ${ }^{4}$; Kimani, Joshua ${ }^{4}$; Ball, Terry B ${ }^{1}$; Sandstrom, Paul ${ }^{3}$; Kobinger, Gary ${ }^{1}$; Plummer, Francis $A^{1}$

${ }^{1}$ Winnipeg, MB; ${ }^{2}$ Santiago de Compostela, Spain; ${ }^{3}$ Ottawa, ON; ${ }^{4}$ Nairobi, Kenya

A safe and effective preventative HIV-1 vaccine is the only way to stop the new infections and control the pandemic. However, developing an effective preventative HIV vaccine has proved to be a great challenge. The classical vaccine approach for combating other viruses has failed so far in dealing with HIV-1, a virus infecting a key component of immune 
system and with greater diversity and rapid mutation. New approaches are needed to develop a preventative vaccine.

The protease of HIV-1 is a small 99-amino acid aspartic enzyme mediating the cleavage of Gag, Gag-Pol and Nef precursor polyproteins. The process is highly specific, temporally regulated and essential for the production of infectious virions. A total of 12 proteolytic reactions are required to generate a viable virion. Therefore, a vaccine targeting the 12 protease cleavage sites(PCS) could be effective. The PCS of HIV-1 are highly conserved among major subtypes, direct immune responses against these sites would yield several advantages. First, the immune response could destroy the virus before its establishment in the host. Second, the vaccine could force the virus to accumulate mutations eliminating the normal function of the HIV protease. Third, restricting the immune responses to these sites can avoid distracting immune responses that often generate unwanted inflammatory responses, induce excess immune activation, and attract more targets for HIV-1 infection, establishment and spread.

We have conducted a pilot study to investigate the feasibility and effectiveness of this approach. The recombinant VSV-peptides were used to immunize cynomolgus macaques and nanopackaged peptides were used to boost the immune response to the 12 PCS of SIVmac239. The controls and immunized macaques were repeatedly challenged intrarectally with a increased dosage of SIVmac239. Results showed that antibody and $\mathrm{T}$ cell responses to the $12 \mathrm{PCS}$ can protect macaques against higher dosage of SIVmac239 challenge ( $\mathrm{p}=0.0005, \mathrm{R}=0.8005)$ and the vaccine group maintains significantly higher $\mathrm{CD}^{+}$counts $(\mathrm{p}=0.0002)$ than the controls weeks after being infected. Population coverage analysis showed that this approach can be applied to $>95 \%$ populations in the world

\section{O058 \\ ROLE OF INTESTINAL MEMBRANE TRANSPORTERS IN ANTIRETROVIRAL DRUG ABSORPTION AND DRUG- DRUG INTERACTIONS}

\section{Kis, Olena ${ }^{1}$; Sankaran, Sumathi ${ }^{2}$; Dandekar, Satya ${ }^{2}$;}

\section{Walmsley, Sharon ${ }^{1}$; Bendayan, Reina ${ }^{1}$}

${ }^{1}$ Toronto, ON; ${ }^{2}$ Davis, CA, USA

BACKGROUND: Several interactions can occur between antiretroviral drugs $(A R V s)$ in the gastro-intestinal tract. We investigated the role of drug carriers in facilitating or restricting the intestinal absorption of the HIV protease inhibitor (PI), atazanavir (ATV), and their potential contribution to ATV drug-drug interactions. We also examined the effect of HIV infection and antiretroviral therapy on the expression of drug transporters and metabolic enzymes in the human intestine.

METHODS: ATV intestinal permeability was measured across Caco-2 monolayers and in situ, by single-pass perfusion of rat intestine, in the absence or presence of specific transporter inhibitors and ARVs. Human intestinal biopsy tissues were obtained from i) HIV-infected patients, therapy-naïve; ii) HIV- infected patients, receiving HAART; and iii) uninfected, age-matched volunteers, and the expression of drug transporters and metabolic enzymes was analyzed by microarray analysis, real-time qPCR and immunoblotting.

RESULTS: ATV uptake by Caco-2 cells was stimulated by acidic extracellular $\mathrm{pH}$ and susceptible to inhibition by several OATP family inhibitors, suggesting that ATV cellular uptake is carrier-mediated. Inhibitors of efflux transporter P-glycoprotein (Pgp) significantly increased ATV permeability across Caco-2 monolayers and jejunum and ileum of rat intestine, suggesting that Pgp restricts ATV intestinal absorption. Several PIs and other ARVs significantly inhibited ATV efflux in Caco-2 cells at clinically relevant concentrations. Furthermore, tenofovir significantly lowered ATV intestinal permeability by $28 \%$ in the in situ perfusion model, mirroring the interaction observed clinically. The expression of several drug transporters and enzymes was significantly different in HIV-infected patients compared to uninfected individuals and was also altered in ARV-treated patients, suggesting that HIV infection and/or ARV therapy may alter drug absorption in HIV-infected populations. CONCLUSIONS: PIs can interact with several intestinal influx and efflux transporters at clinically relevant concentrations. Since many drugs are known to be substrates for these carriers, including statins and other ARVs, these transporters may be implicated in clinically significant drug-drug interactions at the intestinal mucosa. This study is funded by
CANFAR; OK received OHTN and CIHR studentships; RB and SW are OHTN career scientists.

\section{O059 \\ ANTI-GP41 ANTIBODIES INHIBIT INFECTION AND TRANSCYTOSIS OF HIV-1 INFECTIOUS MOLECULAR CLONES THAT EXPRESS TRANSMITTED/FOUNDER ENVELOPES}

Jain, Sumiti'; Ochsenbauer, Christina ${ }^{2}$; Kappes, John $C^{2}$;

Rosenthal, Kenneth $\mathrm{L}^{1}$

1Hamilton, ON; ${ }^{2}$ Birmingham, AL, USA

Prophylactic strategies against HIV must effectively prevent its transmission, infection and cell-to-cell spread during the earliest stages of acute infection. Since the genital mucosa is the primary site of entry, a mucosal defense is critical for early control of the virus. Recent identification of transmitted/ founder (T/F) HIV-1 genomes has demonstrated a consistent genetic bottleneck during mucosal transmission and suggests that $\mathrm{T} / \mathrm{F}$ viruses may exhibit distinct phenotypes. HIV-1 Env gp41-specific responses are among the first to be generated in natural HIV infection. Previously, we demonstrated that two highly conserved gp41 epitopes, ELDKWA and QARVLAVERY, that are critical for efficient virus entry, elicit protective antibodies against HIV-1. QARVLAVERY is of particular interest mucosally since it is a potent inducer of $\operatorname{IgA}$. In this study, we assessed the effectiveness of mucosal and systemic mouse antibodies elicited against these gp41 epitopes to inhibit T/F Env function. For this, we employed recombinant infectious molecular clones (Env-IMC) of HIV-1 that encode mucosally transmitted/ founder env genes. Our results show that the gp41-specific IgG and IgA fractions effectively prevented the infection of TZM-bl cells and inhibited HIV transcytosis in an assay measuring the passage of infectious virus across an epithelial monolayer. Interestingly, the T/F Env-IMC tested were more sensitive to the antibodies than the R5 lab-adapted strains included as controls. This highlights the potential of gp41-based immunogens to impart effective mucosal protection in the early stages of HIV transmission and infection.

\section{0 \\ STRUCTURAL CHANGES TO VIRAL DNA IMPACTS HIV INTEGRASE SUSCEPTIBILITY TO RALTEGRAVIR \\ $\underline{B i o n d i, ~ M i a ~ J 1 ~}^{\text {; }}$ Auger, Anick ${ }^{1}$; Kozak, Robert A ${ }^{1}$; Kvaratskhelia, Mamuka ${ }^{2}$; Gotte, Matthias ${ }^{1}$ \\ ${ }^{1}$ Montreal, QC; ${ }^{2}$ Columbus, $\mathrm{OH}$, USA}

The HIV integrase (IN) enzyme performs two distinct catalytic events: 3'-processing and strand transfer. Both activities are necessary to integrate the proviral DNA into the host chromosome. Raltegravir (RAL) is a potent, first-generation strand transfer inhibitor of HIV IN. The structure of the related gammaretroviral IN of Prototype Foamy Virus (PFV) bound to the DNA substrate, with and without RAL, sheds light on the mechanism of action. It appears that the inhibitor interacts specifically with the DNA and displaces the 3'-end of the primer, making it unavailable for strand transfer events. However, the validity of the PFV IN structure as a model for HIV IN remains to be established. Here, we rationally designed chemically modified DNA substrates and measured 3'-processing, strand transfer, and sensitivity toward RAL against HIV IN. We show that both enzymatic activities, and sensitivity to RAL are susceptible to subtle changes in the DNA substrate. These include the sequence context, specific base pairing, and the chemical nature of the backbone. We predominantly targeted nucleotides in close proximity to the active site. The addition of 2'-flouro groups at the terminal $\mathrm{C}$ and A of the 3'-end of the reactive strand significantly compromised activity. However, susceptibility to RAL was not affected. Methylphosphonate modifications to the backbone showed marginal effects on both activities. We then probed the complementary bases of the non-reactive strand and showed that a methylphosphonate bridging the $\mathrm{G}$ and $\mathrm{T}$ decreases sensitivity to RAL. This phenotype is amplified in the context of the RAL drug-resistant mutant, $\mathrm{N} 155 \mathrm{H}$. In conclusion, the structure of PFV IN in conjunction with our biochemical data obtained with the HIV enzyme provides novel insight into the role played by the DNA substrate in susceptibility to RAL. 


\section{1}

DNA VACCINES THAT ELICIT ANTIBODIES TARGETING THE MPER OF HIV-1 GP41

Gulzar, Naveed $^{1}$; Montero, Marinieve ${ }^{1}$; Klaric, Kristina-Ana ${ }^{1}$; Lepik, Christa'; Donald, Jason ${ }^{2}$; Tsai, Sue ${ }^{1}$; Wu, Sampson ${ }^{1}$;

Wang, Shixia ${ }^{3}$; DeGrado, William ${ }^{4}$; Lu, Shan ${ }^{3}$; Scott, Jamie K ${ }^{1}$

'Burnaby, BC; ${ }^{2}$ Philadelphia, PA; ${ }^{3}$ Worcester, MA, USA;

${ }^{4}$ San Francisco, CA, USA

The limited success of vaccines targeting the MPER, a target of five neutralizing $(\mathrm{Nt})$ monoclonal antibodies (MAbs), we hypothesize, reflects the difficulty of mimicking the neutralization-competent structure (NCS) of the MPER. We have developed DNA-vaccine candidates meant to mimic the NCS of the MPER, and report on their ability to elicit MPER-specific $\mathrm{NtAbs}$. Our vaccines encode gp41 ectodomain fragments fused to the transmembrane region (TM) of either the platelet-derived growth factor receptor (PGDFR), or gp41. Previous work with the protein products of these vaccines has shown they mimic the NCS by several criteria, including the ability to be bound tightly by well-characterized Nt MAbs, but weakly by their non-neutralizing mutant-MAb counterparts. In addition, analysis of a panel of 29 MPER mutants bearing single aa substitutions, has confirmed the critical binding residues for each Nt MAb, further validating these vaccines. Immunizations with constructs encoding the MPER tethered to the PDGFR-TM elicited MPER-specific Abs that targeted the epitope of the 2F5 NtMAb; these Abs did not neutralize HIV-1 pseudotyped virus. A second immunization with DNA vaccines encoding the MPER fused to the gp 41 TM, elicited low-titre Abs that crossreacted weakly with the MPER and regions outside the MPER. Both sets of immunizations failed to elicit Abs targeting the epitope of the 4E10 $\mathrm{NtMAb}$. We found that the presence of the PGDFR-TM significantly reduced MPER-binding 4E10 MAb, but not by 2F5. Putative models suggest that in the PDGFR-TM fusions, the 4E10 epitope points into the lipid bilayer, making it less accessible to 4E10, suggesting the importance of the TM in exposing the C-terminal portion of the MPER. While the gp41 TM better exposes epitopes for neutralizing Abs on the MPER, it also elicits Abs against sites outside the MPER. Current work is focused on engineering the gp41 TM to optimally expose MPER epitopes.

\section{O062 \\ DEVELOPMENT OF A NOVEL CLASS OF ANTI-HIV-1 INHIBITORS ALTERING VIRAL RNA PROCESSING: A NEW THERAPEUTIC STRATEGY}

Wong, Raymond W; Ostrowski, Mario; Lingwood, Clifford A; Cochrane, Alan W

\section{Toronto, ON}

The major rise of HIV-1 strains resistant to existing drugs requires the development of new and effective treatment strategies for continued success in combating HIV-1 infection. We aimed at developing inhibitors of HIV-1 replication that target viral RNA processing, a stage of the lifecycle dependent on the host cell. Through exploring compounds that could modulate alternative RNA splicing, we identified digoxin as a potent inhibitor of HIV-1 replication. Digoxin inhibited HIV-1 (LAI) gene expression in a stable HeLa cell line (IC50 $43 \mathrm{nM}$, IC80 $78 \mathrm{nM}$ ) and, also, HIV-1 (BaL) replication in a more natural context of infection in human CD4+ PBMCs (IC50 15 nM, IC80 25 nM). Digoxin achieved inhibition by two distinct mechanisms. Digoxin induces oversplicing of HIV-1 pre-mRNA, causing a shift in multiply spliced mRNA abundance (MS: Tat/Rev/Nef) to 300\% while decreasing both unspliced (US: Gag/ Gagpol) and singly spliced mRNA (SS: Env/Tat/Vpu/Vif/Vpr) by 6 - and 3 -fold, respectively, relative to control. Secondly, digoxin alters splice site usage of viral MS pre-mRNA leading to a 4-fold decrease in the mRNA encoding HIV-1 regulatory factor Rev. Reduction in Rev expression further impairs late phase synthesis of HIV-1 structural proteins by disrupting the export of incompletely-spliced RNAs (US/SS). In explaining the effects on RNA, subsequent analysis of host splice factors suggests that digoxin alters HIV-1 RNA processing through modulating SR proteins, indicated by blocked activity of CLK SR protein kinases and increased modification of SRp20, Tra2beta SRp54/55, and SRp75. Further studies of the signaling mechanisms and the effectiveness of digoxin and this family of drugs on clinical HIV strains and viral drug resistance are currently underway. Although widely used in the treatment of various heart conditions, this study demonstrates that digoxin can specifically inhibit HIV-1 replication by altering RNA processing - a stage not targeted by current antiretroviral therapies.

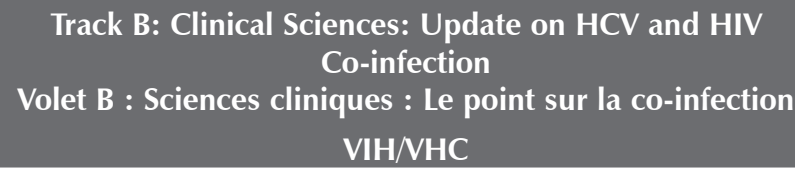

\section{3}

MARIJUANA SMOKING DOES NOT APPEAR TO CAUSE PROGRESSION OF LIVER FIBROSIS IN THE CANADIAN HIV/HCV CO-INFECTION COHORT STUDY

Brunet, Laurence ${ }^{1}$; Moodie, Erica $\mathrm{E}^{1}$; Rollet, Kathleen ${ }^{1}$; Tyndall, Mark2 ; Potter, Martin"1; Conway, Brian"4; Walmsley, Sharon ${ }^{3}$; Pick, Neora ${ }^{3}$; Cooper, Curtis ${ }^{2}$; Cox, Joseph ${ }^{1}$; Klein, Marina B ${ }^{1}$ ${ }^{1}$ Montreal, QC; ${ }^{2}$ Ottawa; ${ }^{3}$ Toronto, ON; ${ }^{4}$ Vancouver, BC

BACKGROUND: Literature on the effect of cannabis on liver diseases is conflicting. Cell cultures and animal model studies conclude that cannabidiol may have a positive effect on liver injuries. However, crosssectional studies of chronic HCV patients suggest that daily cannabis use is associated with fibrosis and steatosis. This study aims at estimating the causal effect of marijuana use on liver fibrosis progression in the Canadian Co-infection Cohort study.

METHODS: HIV/HCV co-infected individuals were followed-up every six month. At each visit, they provided information on marijuana use which was categorized as (1) did not use, (2) used occasionally, (3) used daily, $\leq 4$ joints/day, and (4) used daily, $>4$ joints/day. To account for timedependent confounding, marginal structural pooled logistic regression models were used to assess the effect of marijuana use on progression to significant fibrosis (APRI $>=1.5$ ). Baseline (age, sex, ethnicity, low income, duration of HCV infection) and updated characteristics (CD4 cell count, HIV viral load, antiretroviral therapy, alcohol use, illicit opioid use and other IDU) were included in the inverse probability of treatment weights calculation

RESULTS: A total of 843 patients contributed 3,914 person-visits and 161 progressed to significant fibrosis. At baseline, 52\% had smoked marijuana in the past 6 months (median: 2 joints/day [IQR: 1-4]), of whom 37\% smoked daily; $40 \%$ smoked to relieve symptoms, $42 \%$ to increase appetite, and $46 \%$ for recreational use. There was no causal association between progression to liver fibrosis and smoking occasionally (OR: 0.81 [95\% CI: 0.49-1.34]), smoking $\leq 4$ joints daily (OR: 0.47 [0.19-1.15]), or smoking $>4$ joints daily (OR: 1.26 [0.61-2.63]), compared to non-smokers.

CONCLUSION: Marijuana smoking does not appear to have a causal effect on progression to liver diseases in co-infected individuals. Self medication, causing time-dependent confounding, could have led to the association observed in cross-sectional studies. The next step involves modelling of cumulative exposure.

\section{4 \\ LONG-TERM FOLLOW-UP OF INNER CITY POPULATIONS TREATED FOR HCV INFECTION}

Tossonian, Harout; Gallagher, Lesley; Duncan, Fiona;

Alenezi, Osamah; Conway, Brian

Vancouver, BC

BACKGROUND: Injection drug users (IDUs) make up $>50 \%$ of prevalent and $>75 \%$ of incident cases of HCV infection in Canada. Despite this fact, only $1-2 \%$ has ever been offered treatment and only $20 \%$ of experts feel comfortable doing so. A comprehensive approach to IDUs will be an essential component of the global strategy to addressing the HCV epidemic.

METHODS: We conducted a retrospective analysis of patients receiving $\mathrm{HCV}$ treatment through the Pender Community Health Centre since 2005 and having completed treatment prior to July 2011. Care was delivered within a multidisciplinary program with all weekly interferon injections given at the clinic. Demographic and risk behavior data (prior to and during treatment) was collected, along with data on duration of therapy and virologic outcomes. 
RESULTS: A total of 182 patients were included in this analysis (26 women). All had a history of IDU ( $89.2 \%$ by injection), with $40 \%$ continuing drug use during HCV treatment. Only $12.4 \%$ patients were cirrhotic, and $30.8 \%$ had genotype 2 or 3 infection, $8 \%$ were co-infected with HIV. Overall, $72.9 \%$ completed assigned therapy. Of the total patient population, 19 genotype 1 patients discontinued due to lack of virologic response. Only 12 patients $(6.5 \%)$ had a relapse in IDU leading to non-adherence, and 12 patients $(6.5 \%)$ stopped due to drug toxicity. Overall sustained virologic response was $52.2 \%$ for genotype 1 and $73.7 \%$ for genotype 2 or 3 infection.

CONCLUSIONS: HCV infection can be treated successfully in IDUs with response rates, discontinuation rates and causes approaching those seen in other populations. As will be reflected in 2012 Canadian guidelines for the treatment of HCV infection, IDUs should be considered for therapy under many circumstances, especially within the context of multidisciplinary community-based models for the delivery of health care.

\section{5}

\section{MORTALITY IN HIV-HEPATITIS C VIRUS (HCV) CO-INFECTED PERSONS ENROLLED IN THE CANADIAN CO-INFECTION COHORT STUDY IN COMPARISON TO THE CANADIAN POPULATION}

Klein, Marina B ${ }^{1}$; Rollet, Kathleen ${ }^{1}$; Sahar, Saeed ${ }^{1}$; Cooper, Curtis ${ }^{2}$; Conway, Brian ${ }^{3}$; Cox, Joseph ${ }^{1}$; Gill, John ${ }^{4}$; Pick, Neora ${ }^{3}$;

Potter, Martin ${ }^{1}$; Walmsley, Sharon ${ }^{5}$

${ }^{1}$ Montreal, QC; ${ }^{2}$ Ottawa, ON; ${ }^{3}$ Vancouver, BC; ${ }^{4}$ Calgary, AB; ${ }^{5}$ Toronto, ON

BACKGROUND: Recent studies have suggested that all-cause mortality in HIV mono-infected patients might now approach that of similarly aged persons in the general population. We compared mortality rates in co-infected patients to those in the general Canadian population.

METHODS: A prospective multicentre cohort study followed 1020 $\mathrm{HIV} / \mathrm{HCV}$ co-infected persons from 16 sites (2003-2011). Linkage to provincial vital statistics was available in Quebec, Alberta and BC (representing 73\% of enrolled) to capture deaths among participants lost to follow-up. Standardized mortality ratios (SMRs) were calculated using age-, sex- and province- specific mortality rates from the Canadian $\mathrm{Hu}$ man Mortality Database, 2007.

RESULTS: Among 1020 participants followed for 2272 person-years we observed 89 deaths. Median follow-up time was 1.9 (IQR, 0.7-3.0) years; $9 \%$ of participants were lost to follow-up. Median age was 45 years (IQR, 39-50), 265(26\%) were women, 157(15\%) aboriginal, $348(34 \%)$ active IDU. End-stage liver disease (ESLD) was the main cause of death (29\%) followed by drug overdose (19\%). SMR (95\% CI) was: $13.17(10.44,15.91)$ overall; $9.50(7.17,11.83)$ for men and 24.92 $(15.15,34.69)$ for women.

SMRs according to behavioural and clinical characteristics at last visit are shown in the table. In all subgroups, women had higher SMRs than men.

CONCLUSIONS: SMRs for co-infected persons were substantially higher than the general population, particularly among women, aboriginals, IDUs and those with advanced, uncontrolled HIV and untreated HCV. Our results suggest interventions targeting modifiable risk factors such as substance use, adherence to ART and timely provision of HCV therapy could substantially reduce mortality in HIV-HCV co-infected persons.

\section{SMR $(95 \% \mathrm{Cl})$}

\section{Ethnicity}

Aboriginal

Other

IDU

Yes

No

\section{Cigarette smoking}

Smoker

Non-smoker
20.55 (8.92, 32.18)

$12.46(9.67,15.24)$

$19.97(12.58,27.37)$

$11.44(8.57,14.31)$

$15.48(11.93,19.04)$

$7.87(4.01,11.72)$

\begin{tabular}{|c|c|}
\hline \multicolumn{2}{|l|}{ cART } \\
\hline Yes & $12.01(9.29,14.72)$ \\
\hline No & $28.20(13.43,42.98)$ \\
\hline \multicolumn{2}{|l|}{ CD4, cells/uL } \\
\hline$<200$ & $33.30(20.96,45.63)$ \\
\hline $200-349$ & $15.19(9.24,21.14)$ \\
\hline $350-499$ & $8.58(3.73,13.43)$ \\
\hline$\geq 500$ & $7.12(3.92,10.32)$ \\
\hline \multicolumn{2}{|c|}{ HIV RNA (copies/mL) } \\
\hline$\leq 50$ & $10.86(7.96,13.75)$ \\
\hline$>50$ & $19.65(13.04,26.26)$ \\
\hline \multicolumn{2}{|l|}{ APRI } \\
\hline$\geq 1.5$ & $15.81(9.73,21.88)$ \\
\hline$<1.5$ & $11.43(8.43,14.42)$ \\
\hline \multicolumn{2}{|l|}{ ESLD } \\
\hline Yes & $18.82(11.72,25.91)$ \\
\hline No & $11.77(8.84,14.70)$ \\
\hline \multicolumn{2}{|c|}{ HCV treatment } \\
\hline Naive & $17.56(13.48,21.64)$ \\
\hline \multicolumn{2}{|l|}{ Experienced } \\
\hline PCR positive & $7.22(0.89,13.56)$ \\
\hline PCR negative & $5.65(0.70,10.60)$ \\
\hline \multicolumn{2}{|c|}{ Spontaneous HCV clearance } \\
\hline Yes & $4.14(-1.60,9.87)$ \\
\hline No & $8.29(5.32,11.25)$ \\
\hline
\end{tabular}

O066

SERUM BIOMARKERS PREDICTIVE OF FIBROSIS BY PROTEOMIC FINGERPRINTING IN HIV-1/HCV CO-INFECTION

Melendez-Pena, Carlos E ${ }^{1}$; Mazen, Habayeb ${ }^{1}$; Saeed, Sahar ${ }^{1}$; Conway, Brian $^{2}$; Cooper, Curtis ${ }^{3}$; Brian, Ward J ${ }^{1}$; Momar, Ndao ${ }^{1}$; Klein, Marina $\mathbf{B}^{1}$

${ }^{1}$ Montréal, QC; ${ }^{2}$ Vancouver, BC; ${ }^{3}$ Ottawa, ON

BACKGROUND: In developed countries, HCV co-infection affects more than $30 \%$ of HIV-infected patients. Hepatic complications of HCV, including fibrosis and cirrhosis are accelerated in HIV-infected individuals. Liver biopsy remains the gold standard for staging HCV-associated liver disease. This test is invasive and has major limitations such as serious complications and sampling error. These problems have prompted a search for non-invasive methods. To this end, we compared serum proteome profiles of HCV mono and HIV-1/HCV co-infected patients at different stages of fibrosis using SELDI-TOF MS.

METHODS: A panel of 76 sera was used, including $36 \mathrm{HCV}$ mono infected and $40 \mathrm{HIV} / \mathrm{HCV}$ co-infected subjects where in both group 10 were in each 4 stages of fibrosis $(0-1,2,3,4)$ except for stage 4 mono-infection where there was 6 subjects. Fibrosis stage was assessed by liver biopsy and only sera collected within a year of biopsy were profiled. Sera were fractionated, randomly applied to protein chip arrays (IMAC, CM10 and $\mathrm{H} 50$ ) and spectra were generated at low and high laser intensities. Each sample was analyzed using three $\mathrm{pH}$ fractions (F1, F3 and F6).

RESULTS: The 76 sera studied yield a total of 1368 spectra. More than a 100 peaks of interest were found by automated peak detection. After manual peak relabeling, 24 biomarkers achieved a p value $<0.01$ (ROC values $>0.75$ or $<0.25$ ) predictive of fibrosis status in co-infected individuals and 27 in mono infected subjects. Only 5 of these candidate biomarkers contributed in both mono and co-infected subjects. Using biomarker pattern software to generate candidate diagnostic algorithms, 4 biomarker peaks with good discriminatory power for co-infection were identified.

CONCLUSION: These preliminary data suggest that SELDI mass spectrometric profiling can identify diagnostic serum biomarkers for fibrosis not only in HCV mono infected individuals but also in HIV/HCV coinfected individuals. The 4 most promising biomarkers are currently being identified and validated. 


\section{7}

WHO NEEDS DIRECT ACTING ANTIVIRALS FOR HEPATITIS C VIRUS (HCV)? CHALLENGES FACED IN ADVANCING HCV THERAPY WITH DIRECT ACTING ANTIVIRALS (DAAS) IN HIV-HCV CO-INFECTED PERSONS Klein, Marina B ${ }^{1}$; Rollet, Kathleen ${ }^{1}$; Sahar, Saeed ${ }^{1}$; Moodie, Erica ${ }^{1}$; Cox, Joseph ${ }^{1}$; Cooper, Curtis²; Gill, John ${ }^{3}$; Hull, Mark ${ }^{4}$;

Potter, Martin ${ }^{1}$; Walmsley, Sharon ${ }^{5}$

${ }^{1}$ Montreal, QC; ${ }^{2}$ Ottawa, ON; ${ }^{3}$ Calgary, $A B$; ${ }^{4}$ Vancouver, $B C$; ${ }^{5}$ Toronto, ON

BACKGROUND: DAAs appear very promising for the treatment of co-infected patients with HCV genotype 1. Using DAAs, however, may be limited by important drug-drug interactions, the continued need for pegylated-interferon/ribavirin and increased rates of hematologic adverse events. We describe the characteristics of co-infected patients who could be eligible for DAAs.

METHODS: We evaluated the sociodemographic and clinical characteristics of 1020 co-infected participants enrolled in a Canadian prospective multicentre cohort study (2003-2011). 707 (85\%) had evidence of chronic HCV infection (HCV RNA positive) of whom 438(76\%) where infected with genotype 1 . Of these, 375 were HCV treatment naïve and 122 failed prior HCV treatment either at baseline or in follow-up.

RESULTS: A profile of the patients eligible for DAA treatment, at their most recent visit, is presented in the table.

The number of patients with no absolute contraindication to treatment (e.g. no alcohol abuse, compensated liver disease; no contraindicated medication, etc.) was 171 (46\%) for HCV treatment naive and 42 (34\%) for experienced patients. Furthermore, most were receiving other medications that could place them at risk for important drug-drug interactions requiring therapy adjustments.

CONCLUSIONS: Although the need is great, the actual number of patients eligible for DAAs may be quite low. In addition to medical and pharmacologic contraindications, low levels of income and education may represent further barriers to complex and expensive new $\mathrm{HCV}$ treatments. There remains an urgent need to develop safe, simple and interferon-sparing treatments for co-infected persons.

\begin{tabular}{lcc} 
& Naive (n=375) & $\begin{array}{c}\text { Prior treatment } \\
\text { failure }(\mathbf{n = 1 2 2})\end{array}$ \\
\hline Median Age (years) & $44(40,49)$ & $46(40,51)$ \\
Gender & $267(72 \%)$ & $99(82 \%)$ \\
Greater than high school education & $92(25 \%)$ & $50(41 \%)$ \\
Gross monthly income $\quad<\$ 1500$ & $294(78 \%)$ & $77(63 \%)$ \\
CAN & & \\
IDU in the last 6 months & $135(36 \%)$ & $13(11 \%)$ \\
Current alcohol abuse/dependence & $129(34 \%)$ & $33(27 \%)$ \\
Psychiatric diagnosis & $191(51 \%)$ & $67(55 \%)$ \\
Hemoglobin <120 g/L & $47(13 \%)$ & $12(10 \%)$ \\
Platelets $<90 x 10^{9}$ g/L & $29(8 \%)$ & $20(17 \%)$ \\
Neutrophils <1500/uL & $28(8 \%)$ & $24(20 \%)$ \\
Median CD4 count (cells/uL) & $420(263,580)$ & $485(270,655)$ \\
HIVRNA load <50 copies/mL & $241(64 \%)$ & $97(80 \%)$ \\
On cART & $321(86 \%)$ & $116(96 \%)$ \\
cART regimen & & \\
NNRTI & $82(26 \%)$ & $40(34 \%)$ \\
Efavirenz & $36(44 \%)$ & $21(53 \%)$ \\
PI/r & $222(69 \%)$ & $80(67 \%)$ \\
Atazanavir & $109(49 \%)$ & $34(43 \%)$ \\
Lopinavir & $32(14 \%)$ & $14(13 \%)$ \\
Raltegravir & $24(7 \%)$ & $13(11 \%)$ \\
Exposure to other contraindicated & $9(2 \%)$ & $2(2 \%)$ \\
$\quad$ medications & & \\
APRI $\geq 2.0$ (cirrhosis) & $51(14 \%)$ & $26(22 \%)$ \\
Decompensated liver disease & $43(11 \%)$ & $34(28 \%)$ \\
\hline
\end{tabular}

0068

\section{ESTIMATING VARIATIONS BETWEEN HEALTH CARE CENTRES IN THE UPTAKE OF HEPATITIS C VIRUS (HCV) TREATMENT IN HIV-HCV CO-INFECTED PATIENTS}

Potter, Martin ${ }^{1}$; Young, Jim ${ }^{1}$; Rollet, Kathleen ${ }^{1}$; Cox, Joe ${ }^{1}$; Walmsley, Sharon'; Cooper, Curtis ${ }^{3}$; Tyndall, Mark ${ }^{3}$; Montaner, Julio ${ }^{4}$; Conway, Brian ${ }^{4}$; Pick, Neora ${ }^{4}$; Côté, Pierre ${ }^{1}$; Rouleau, Danielle ${ }^{1}$; Klein, Marina B ${ }^{1}$

${ }^{1}$ Montreal, QC; ${ }^{2}$ Toronto; ${ }^{3}$ Ottawa, ON; ${ }^{4}$ Vancouver, BC

BACKGROUND: Despite effective and available treatment for chronic $\mathrm{HCV}$ infection, $\mathrm{HCV}$ treatment remains low in Canada. We investigated $\mathrm{HCV}$ treatment uptake in our cohort and explored whether some centres more frequently start patients on HCV treatment.

METHODS: Using data from the Canadian Coinfection Cohort, we modelled time to HCV treatment uptake using a Bayesian survival analysis model with random intercepts for each of the 16 cohort centres. To take into account variability in patient populations served at each centre (case-mix), models were adjusted for age, gender, ethnicity, HCV genotype; and at cohort enrolment, duration of $\mathrm{HCV}$ infection, receipt of combination antiretroviral therapy, history of psychiatric illness, CD4 cell count, and self-reports of homelessness, use of intravenous drugs, and current use of alcohol. Residual variation between centres in treatment uptake was then estimated and centres ranked according to according to their rate of starting patients on HCV treatment.

RESULTS: Among 996 participants, 390 were excluded (past HCV treatment $[\mathrm{n}=170]$, spontaneous clearance $[\mathrm{n}=50]$, enrolment data only $[\mathrm{n}=160]$ and missing baseline CD4 $[\mathrm{n}=10]$ ). Of the remaining 606 participants, 122 started HCV treatment. Patients with HCV genotypes 2 or 3 (hazard ratio [HR] 1.72, 95\% credible interval [CI] 1.10-2.56) and higher CD4 cell count at cohort entry (HR: 1.07, 95\% CI 1.0-1.15 per 100 cells $/ \mu \mathrm{L}$ ) were more likely to initiate treatment. Most centres' performances were indistinguishable from one another, but two centres more frequently initiated patients on $\mathrm{HCV}$ treatment (HR: 4.64, 95\%Cl 2.03-11.82 and HR: 4.57, 95\% CI 2.14-10.91) while one did so less often (HR: 0.17, 95\% CI 0.03-0.76).

CONCLUSIONS: After adjustment for case-mix, there was still variation in treatment uptake between centres. Although non-measured patients' characteristics may explain some of the differences observed, determining the factors associated with higher $\mathrm{HCV}$ treatment rates at two centres may inform efforts to improve access to HCV treatment.

Track C: Epidemiology and Public Health Sciences:

Detection, Evolution and Service Provision for HIV, AIDS and Co-morbidities

Volet $\mathrm{C}$ : Épidémiologie et sciences de la santé publique :

Détection et évolution du VIH, du sida et des comorbidités et prestation de services apparentés

\section{O069}

HIV TREATMENT OUTCOMES AMONG HIV-HCV CO-INFECTED INDIVIDUALS IN CANADA WITH AND WITHOUT INJECTION DRUG USE HISTORY

Cescon, Angela ${ }^{1}$; Chan, Keith'; Raboud, Janet M ${ }^{2}$; Samji, Hasina ${ }^{1,3}$; Klein, Marina $\mathrm{B}^{4}$; Loutfy, Mona $\mathrm{R}^{2}$; Machouf, Nima ${ }^{1}$;

Montaner, Julio S'; Rourke, Sean B2; Burchell, Ann N2;

Tsoukas, Christos ${ }^{1}$; Hogg, Robert S ${ }^{1}$; Cooper, Curtis ${ }^{5}$;

Canadian Observational Cohort Collaboration, CANOC $^{1}$

${ }^{1}$ Vancouver, BC; ${ }^{2}$ Toronto, ON; ${ }^{3}$ Baltimore, MD, USA; ${ }^{4}$ Montreal, QC; ${ }^{5}$ Ottawa, ON

BACKGROUND: Studies examining treatment outcomes of HIVhepatitis $\mathrm{C}(\mathrm{HCV})$ co-infected individuals rarely differentiate between those with and without a history of injection drug use (IDU). This approach may disregard individuals who acquired HCV through a non-IDU route and constitute a unique group of interest for $\mathrm{HCV}$ prevention and treatment. It is plausible that poorer clinical outcomes are due to factors associated with IDU, and not from HCV itself. This study compares treatment outcomes between HIV-HCV co-infected individuals with and without IDU history. 
METHODS: Participants enrolled in the Canadian Observational Cohort (CANOC) collaboration were included, a cohort of HIV-positive individuals $\geq 18$ years who initiated combination antiretroviral (ARV) therapy on or after January 1, 2000 in British Columbia, Ontario, or Quebec. To be included in this analysis, participants had to have documented HCV infection and known IDU history. Demographic and clinical variables were compared by IDU history (yes vs. no) using the Pearson $\chi^{2}$ test or Fisher's exact test for categorical variables and Wilcoxon rank-sum test for continuous variables. Cox proportional hazards regression was used to evaluate time to viral load suppression (two consecutive measures $<250$ copies $/ \mathrm{mL}$ ) and time to CD4 count recovery (increase of 100 cells $/ \mu \mathrm{L}$ ) after starting ARVs.

RESULTS: 1254 participants [31\% $(\mathrm{n}=387)$ female] were included. At baseline, those with IDU history ( $\mathrm{n}=1106,88 \%)$ were younger (41 vs.


IDU participants (both $\mathrm{p}<0.01$ ). The median follow-up time was 3.8 years (IQR 2.1-6.2). After adjustments for age, province, baseline viral load, viral load testing rate, initial third ARV agent, and ARV start year, individuals with IDU history were less likely to virologically suppress after ARV initiation $(\mathrm{AHR}=0.78,95 \% \mathrm{CI}=0.64-0.95 ; \mathrm{p}=0.012)$. Controlling for the same confounders, a marginal difference was observed between IDU and non-IDU in time to CD4 recovery $(\mathrm{AHR}=0.82,95 \% \mathrm{CI}=0.66$ $1.00 ; \mathrm{p}=0.055$ ).

CONCLUSIONS: Factors associated with IDU history impact clinical outcomes among co-infected individuals in CANOC. Further analyses are planned to compare additional key outcome measures between groups.

\section{O070 \\ EXTREMELY HIGH RISK OF SYPHILIS CO-INFECTION AND RE-DIAGNOSIS AMONG MEN WHO HAVE SEX WITH MEN LIVING WITH HIV IN ONTARIO}

Burchell, Ann N; Moravan, Veronika; Allen, Vanessa;

Bayoumi, Ahmed M; Collins, Evan; Gardner, Sandra; Kaul, Rupert;

Mazzulli, Tony; McGee, Frank; Millson, Peggy; Raboud, Janet;

Rourke, Sean B; OHTN Cohort Study Team, The

Toronto, ON

BACKGROUND: Since 2000, new syphilis cases increased ten-fold in Canada, particularly among men who have sex with men (MSM) living with HIV. We calculated the prevalence and incidence of syphilis in a cohort of HIV-positive MSM.

METHODS: We analysed data from MSM followed from 2000 to 2009 in the OHTN Cohort Study and linked data from the Ontario Public Health Laboratories. We classified reactive rapid plasma reagin results as acute $(\geq 16: 1)$ or non-acute $(\leq 8: 1)$ and calculated the lifetime and annual prevalence of syphilis and incidence of new syphilis diagnoses and re-diagnoses. Prevalence and incidence differences in subgroups were determined using logistic and Poisson regressions, respectively.

RESULTS: We linked 18,118 syphilis results for 2422/2903 (83\%) men, of whom $626 \mathrm{had} \geq 1$ specimens indicating past or current infection and 288 had $\geq 1$ specimens indicating acute infection. Lifetime prevalence was $23.6 \%(95 \%$ CI $21.8,25.3)$ by 2009 . The annual prevalence of acute syphilis increased from $0.1 \%(95 \% \mathrm{CI} 0.002,0.5)$ in 2000 to $3.8 \%(95 \% \mathrm{CI}$ $3.0,4.6)$ in 2009. Acute syphilis was most common among men aged $<30(\mathrm{OR}=7.6$; 95\%CI 4.9, 12) and in Toronto $(\mathrm{OR}=3.5 ; 95 \% \mathrm{CI} 2.2,5.5)$ and Ottawa (OR=3.6; 95\%CI 2.1, 6.2). Among 1505 men with a negative specimen, syphilis incidence was $1.3 / 100 \mathrm{PY}$ (95\%CI 1.0, 1.6), with higher rates in men $<30$ and in Toronto and Ottawa. Incidence rose in 2000-2004 ( $R R=2.1$ per year, 95\%CI 1.2-3.7) and in 2005-2009 ( $R R=1.2$ per year, $95 \%$ CI 1.0-1.5) peaking in 2009 at 3.3/100PY (95\%CI 2.2, 4.3). Among 626 men with past or current non-acute infection, incidence of re-diagnosis with acute syphilis was 4.6 per 100PY (95\% CI 3.7, 5.5), with multiple re-diagnoses in 35\%.

CONCLUSION: Many HIV ${ }^{+}$MSM in Ontario are at risk for syphilis, with incidence rates over 300 times greater than in the general male population. Re-diagnosis was common, suggesting re-infection or treatment failure.
O071

HIV PREVENTION MEASURES AND TESTING BEHAVIOUR BY HIV RISK CATEGORY AMONG A CROSS-SECTIONAL SURVEY OF MEN WHO HAVE SEX WITH MEN (MSM) IN VANCOUVER, CANADA

Moore, David ${ }^{1}$; Kantors, Steve ${ }^{1,2}$; Robert, Wayne ${ }^{1}$; Michelow, Warren ${ }^{1}$; McGuire, Marissa ${ }^{3}$; Hogg, Robert ${ }^{1,2}$; Gustafson, Reka ${ }^{1}$;

Trussler, Terry'; Gilbert, Mark'; Mancount Study Team, The ${ }^{1}$

${ }^{1}$ Vancouver; ${ }^{2}$ Burnaby, BC; ${ }^{3}$ Ottawa, ON

OBJECTIVE: We examined HIV testing behaviour and the use of HIV risk-reduction measures, other than condom use, among HIV-negative MSM in Vancouver with participants stratified on the basis of their selfreported HIV risk behaviour.

METHODS: Participants were recruited from August 1, 2008 to February 28, 2009 through community venues catering to MSM in Vancouver. Men aged $\geq 19$ years were asked to complete a self-administered questionnaire and provide a dried blood spot for HIV and other sexually transmitted and blood-borne infection testing. Self-reported HIV negative participants were categorized as low-, moderate-, or high-risk for HIV infection based on reported condom use, number of sexual partners, and/ or a history of STBBI in the previous six months.

RESULTS: Of 1169 participants, 949 self-reported as HIV-negative and were included in this analysis. $513(54.1 \%)$ were categorized as low-risk; $231(24.3 \%)$ as moderate-risk and $205(21.6 \%)$ as high-risk. HIV testing varied by risk category: $65.9 \%$ of high-risk and $44.5 \%$ of low-risk participants reported testing in the past year $(\mathrm{p}<0.001)$. Bivariate analysis by risk-category showed that sero-sorting was more commonly reported by high-risk men $(\mathrm{p}<0.001)$, as was having sex with HIV-positive men who have low viral loads or are on treatment $(\mathrm{p}=0.02)$. We found no differences in the proportion of participants reporting strategic positioning, withdrawal before ejaculation or asking about sero-status before sex between the risk categories. A total of $88.9 \%$ of men in the low-risk category reported they were unlikely or very unlikely to acquire HIV in their lifetime compared to $85.8 \%$ in the moderate- and $68.8 \%$ in the high-risk category $(\mathrm{p}<0.001)$.

CONCLUSION: In our study, the majority of men reported no or low risk, and HIV testing and perceived lifetime risk of HIV increased with greater reported risk behaviour. Prevention strategies were prevalent in our sample, and were either consistent regardless of reported risk or more common in high-risk men. These findings speak to the need to consider segmented health promotion messaging for MSM with different risk profiles.

\section{O072}

ASSOCIATION BETWEEN CONTACTS WITH HEALTHCARE SERVICES AND INITIATION OF HEPATITS C TREATMENT AMONG INJECTION DRUG USERS

Bégin, Marc-Antoine; Zang, Geng; Bamvita, Jean-Marie;

Bruneau, Julie

Montréal, QC

INTRODUCTION: In spite of comparable HCV and HIV treatment efficacy between injection drug users (IDUs) and non-IDUs, there are still important barriers impeding antiviral treatment access in this vulnerable population. Mistrust between IDUs and health care providers, along with IDU disorganised lifestyle, were shown to affect HCV and HIV treatment uptake. The objective of this study is to examine the association between HCV treatment initiation and the use of healthcare services among active IDUs.

METHODOLOGY: 758 active IDUs, seropositive for HCV antibody, were surveyed from November 2004 to March 2011 in Montreal. Interviewer-administered questionnaires elicited information on socio-demographic factors, drug use patterns and related behaviours, and health care service utilisation. Blood samples were collected and tested for HCV antibodies. Multivariate logistic regression adjusting for individual covariates was conducted to identify the health service correlates of treatment initiation.

RESULTS: Within the 758 subjects, 55 (7.3\%) had initiated an HCV treatment prior to enrolment. IDUs who initiated HCV treatment had a better education level and reported less alcohol use. The multivariate analysis included: older age (adjusted Odds Ratio (aOR): 1.04; CI: 
$1.03,1,07) ;>2$ years of lifetime addiction treatment exposure (aOR: 2.5 ; CI: 1.4,4.6); >3 medical visits in the last 6 months (aOR: 3.1; CI: 1.1, 8,3); having spent time in prison (aOR: 0.39; CI: 0.2,0.8) and HCV knowledge (aOR: 1.5; CI: 1.1,2.0)

CONCLUSION: Intensity of exposure to addiction and medical services is associated with HCV treatment initiation. These results suggest that such services efficiently play their role as entry points for HCV treatment. Alternatively, IDU having initiated HCV treatment, regardless of the viral response outcome, may have adopted a proactive stance towards improving their overall health, thereby reducing their risk of $\mathrm{HIV}$ acquisition and $\mathrm{HCV}$-reinfection. Incarceration on the other hand is an obstacle to $\mathrm{HCV}$ infection management.

\section{O073}

\section{TARGETED SCREENING OF LABORATORY SPECIMENS FOR ACUTE HIV INFECTION AMONG MEN WHO HAVE SEX WITH MEN (MSM)}

Cook, Darrel; Krajden, Mel; Kwag, Michael; Steinberg, Malcolm; Rekart, Michael; Gilbert, Mark

Vancouver, BC

OBJECTIVE: Individuals with acute HIV infection (AHI) play an important role in the ongoing transmission of HIV within populations. We assessed the performance of a diagnostic algorithm that includes pooled HIV RNA nucleic acid testing (NAT), targeted at laboratory specimens from clinic sites highly frequented by MSM, for the detection of AHI.

METHODS: Between January 2007 and June 2011, specimens submitted for HIV testing from males $\geq 19 \mathrm{yr}$ from clinic sites in Vancouver with a high proportion of MSM were tested. AHI was identified using a 3rd generation HIV EIA screen test (non-reactive or reactive), together with a non-reactive or indeterminate HIV-1 Western blot, and positive HIV-1 p24 antigen (Jan 2007-Mar 2009) or HIV-1 RNA (Apr 2009-Jun 2011). From Apr 2009 to Jun 2011, 3rd generation EIA non-reactive specimens were also tested for HIV-1 RNA in pools of 24 to identify pre-seroconversion $\mathrm{AHI}$

RESULTS:

\begin{tabular}{lccc} 
& $\begin{array}{c}\text { Jan 2007-Mar 2009 } \\
\text { (pre-pooled NAT) }\end{array}$ & $\begin{array}{c}\text { Apr 2009-Jun 2011 } \\
\text { (pooled NAT) }\end{array}$ & \% Change \\
\hline Test volume & 14,178 & 16,075 & $+13 \%$ \\
All new HIV cases & 173 & 155 & $-10 \%$ \\
AHI cases & 15 & 34 & $+127 \%$ \\
\hline
\end{tabular}

Of the AHI identified after implementing pooled NAT testing, 14/34 (41\%) would not have been detected by 3rd generation EIA screening. The study population accounts for $\sim 4 \%$ of all HIV tests in the province, while yielding $65 \%$ of all $\mathrm{AHI}$.

CONCLUSIONS: The use of targeted pooled NAT screening substantially increased the yield of AHI diagnoses among the study population. Targeted screening of high risk individuals with pooled NAT leads to earlier detection and significant yields of AHI, while avoiding the high cost of universal pooled NAT screening.

\section{O074}

\section{COMPARISON OF TIME TO AIDS BETWEEN ABORIGINALS AND NON-ABORIGINALS AMONG HIV HIGH RISK GROUP}

Mondal, Prosanta K; Konrad, Stephanie; Skinner, Stuart;

Hunt, Kelsey; Lim, Hyun J

Saskatoon, SK

BACKGROUND AND RATIONALE: Co-infection of HIV and Hepatitis- $\mathrm{C}$ virus (HCV) is a major problem among injection drug users (IDU). A high proportion of Saskatchewan's HIV and HCV co-infected population are IDU. In addition, Aboriginals are over-represented in the HIV population. Assessing ethnicity is critical to understanding and reducing ethnic disparities. The author acknowledges that precautions need to be undertaken while examining Aboriginal ethnicity.

METHODS: This is a retrospective cohort study with HIV infected adults between January 1, 2005 and June 1, 2010 from Royal University Hospital and West Side Community Clinic in Saskatoon. In our study,
AIDS was defined either clinically or immunologically (CD4 <200). Cox proportional hazards model was used.

RESULTS: Of a total 218 co-infected IDU patients, $112(51 \%)$ were female and $180(83 \%)$ were Aboriginals. The median age of Aboriginals was significantly lower than non-Aboriginals ( 32 vs. $41 ; \mathrm{p}=0.0004$ ). Ninety-two (59\%) Aboriginals and 15 (43\%) non-Aboriginals ever received ART; this difference was not statistically significant. Mean CD4 count at diagnosis was not significantly different between Aboriginals $(400 ; \mathrm{SD}= \pm 194)$ and non-Aboriginals $(440 ; \mathrm{SD}= \pm 175)$. Time to AIDS between these two groups was not significantly different $(p=0.31)$. In the multivariate Cox model, patients with ever ART (HR $=4.21, \mathrm{p}<0.0001)$, incarceration $(\mathrm{HR}=1.56, \mathrm{p}=0.048)$ and older age $(\mathrm{HR}=1.04, \mathrm{p}=0.003)$ had higher hazard of AIDS while early year of HIV diagnosis ( $\mathrm{HR}=0.25$, $\mathrm{p}<0.0001)$ had lower hazard. Ethnicity was not significant in the multivariate model $(\mathrm{HR}=1.73, \mathrm{p}=0.11)$.

CONCLUSION: Time to AIDS was not significantly different between ethnicities. However, Aboriginal people were diagnosed at an earlier age, suggesting an earlier exposure to HIV. Targeted and culturally appropriate programming is needed to reduce high risk behaviours.

\section{Track D: Social Sciences: HIV Criminalisation, Underlying Factors and Contexts \\ Volet D : Sciences sociales : Criminalisation du VIH facteurs sous-jacents et contextes}

\section{5}

\section{HIV AND CRIMINALIZATION: HOW PUBLIC HEALTH CAN} HELP THE CASE

Beaulieu, Marianne ${ }^{1}$; Joncas, Lucie ${ }^{1}$; Drouin, Marie-Claude ${ }^{1}$; Parent, Raymond²; Côté, François²; Dionne, Marc²; Beaupré-Bériau, Michèle ${ }^{2}$ ${ }^{1}$ Montréal; ${ }^{2}$ Québec, QC

HIV criminalization can have a significant impact within the fight against HIV infection, a priority of public health agencies.

In February 2012, the Supreme Court of Canada will hear two cases, from Manitoba (Her Majesty the Queen v. Mabior [33976]) and Quebec (Her Majesty the Queen v. D.C. [34094]), involving criminalization of exposure to HIV transmission from non-disclosure of the infection. Two major issues to be addressed are the obligation to disclose and the significant risk of injury.

In view of the potential impact on prevention, the Institut national de santé publique du Québec (INSPQ) submitted and was granted leave to intervene in these cases. This presentation will outline the process of intervention in Supreme Court and arguments defended by INSPQ.

A multidisciplinary working group composed of lawyers, sexologists, sociologist, epidemiologist, public health researcher and medical practionner was formed by INSPQ. First, an exhaustive literature review was conducted to inform the reflection. Then, using a delibarative process, arguments were developed based on the major findings that emerged from the literature. Throughout this process, the group has benefited from several collaborations in its efforts. Among other collaborators, they received the unanimous support of Quebec Regional Directors of Public Health board.

The group focused their work on the potential impact of criminalization on prevention and especially HIV testing. INSPQ believes that «overcriminalizing» non-disclosure (in the context of safe sex or undetectable viral load through antiretroviral therapy), will have negative effects on the HIV prevention and linkage to care of people living with HIV or at risk of contracting HIV.

\section{6}

\section{A SURVEY OF ORDERS ISSUED UNDER ONTARIO'S HEALTH PROTECTION AND PROMOTION ACT \\ French, Martin A \\ Kingston, $\mathrm{ON}$}

OBJECTIVE: In Ontario, the Health Protection and Promotion Act (HPPA) empowers Medical Officers of Health to issue, under certain conditions, written orders that require persons "to take or to refrain from taking any action that is specified in the order in respect of a communicable 
disease". The objective of this study was to gather up-to-date information from Ontario's 36 Local Public Health Agencies (LPHAs) about the frequency with which public health orders have been issued in the context of sexually transmitted infection case management.

METHOD: Using the Dillman tailored design method, a paper-based postal survey of LPHAs was undertaken between July-October 2011. Survey data were analyzed using SPSS (v. 20). Data are reported in aggregate format to ensure the anonymity of LPHAs.

RESULTS: Survey responses (32/36; response rate 88\%) indicated that, although used sparingly, LPHAs have issued public health orders in respect of STIs, including HIV, in every year during the period 2005-2009. An important related finding of this study is that a significant majority of respondents - 75\% (24/32) - would prefer the province to develop guidelines for determining when to issue a section 22 order with respect to STIs, including HIV. Indeed, no respondent indicated a preference for the status quo, whereby the province leaves it entirely to LPHAs to determine when to issue an order.

CONCLUSION: Increased provincial oversight and guidance, as well as more qualitative and quantitative research on public health orders, will help develop an understanding of how public health law and practice intersect and interact with the criminal law. Such research is especially urgent in the post-Cuerrier period (post-1998), which has witnessed an increasing trend in the criminal prosecution of prosecution of HIV nondisclosure, exposure and transmission.

\section{O077}

THE INFLUENCE OF PARTNER'S HIV STATUS ON SEXUAL BEHAVIOUR OF WOMEN LIVING WITH HIV: AN ANALYSIS OF SEXUAL BEHAVIOURS AMONG WOMEN IN THE OHTN COHORT STUDY

Robinson, Samantha ${ }^{1}$; Burchell, Ann N1; Light, Lucia ${ }^{1}$; Loutfy, Mona ${ }^{1}$; Gardner, Sandra ${ }^{1}$; Margolese, Shari ${ }^{1}$; Abelsohn, Kira ${ }^{1}$; Greene, Saara ${ }^{2}$; Tharao, Wangari';

Smith, Stephanie ${ }^{2}$; Brisebois, Cindy ${ }^{1}$; Kwaramba, Gladys ${ }^{2}$; Inoua, Haoua ${ }^{1}$; OHTN Cohort Study Research Team, The ${ }^{1}$

${ }^{1}$ Toronto; ${ }^{2}$ Hamilton, ON

OBJECTIVE: To explore the relationship status and sexual behaviours of women living with HIV in Ontario.

METHODS: We analyzed data from the OHTN Cohort Study (OCS), a province-wide, observational, longitudinal study of people living with HIV in Ontario. Participants were women recruited from specialized HIV clinics and primary care practices. Data were obtained from medical chart extractions, interviews, and data linkage with the Ontario Public Health Laboratories. We restricted the analysis to 404 women interviewed in 2010. We used descriptive and standard statistics to characterize relationship status and sexual behaviours reported in the past 3 months, and how these varied with partner's HIV status.

RESULTS: Women were predominantly heterosexual (92\%). 28\% $(113 / 404)$ reported a sexual partner. Sexually-active women were significantly younger (mean age $=38.5$ years; $\mathrm{CI}=36.8-40.3$ ) than women who were not (mean age $=43.4$ years; $\mathrm{CI}=42.3-44.6)(\mathrm{p}<0.0001)$. The majority $(81 \%)$ of sexually-active women $(n=113)$ had one regular partner, most of which were long-term relationships $(12+$ months). Only $19 \%$ reported casual partners. $9 \%$ did not report vaginal/anal intercourse. Among women who did ( $\mathrm{n}=91)$, for $36 \%$, her partner was also $\mathrm{HIV}^{+}$. Women were more likely to use a condom with casual partners $(\mathrm{p}=0.05)$, or with $\mathrm{HIV}^{-}$/ status unknown partners $(\mathrm{p}<0.01)$. Of those who had intercourse with a discordant HIV-status partner, 43\% (25/58) reported unprotected sex and of these, $71 \%$ had undetectable viral load (VL). Only $6 \%$ of sexuallyactive women with detectable VL reported unprotected sex with a discordant HIV-status partner.

CONCLUSION: These data constitute an integral first step to understand factors that affect sexual health choices of women living with HIV, for which there is little current data. Though a minority of $\mathrm{HIV}^{+}$women are sexually active, most who are have regular, long-term partnerships with HIV- men. Partner's HIV status was a strong determinant of condom use.
0078

«POUVOIR PARTAGER/POUVOIRS PARTAGÉS» (PP/PP): SUPPORTING WOMEN LIVING WITH HIV (WLWHIV) IN QUÉBEC IN DECIDING WHETHER OR NOT TO DISCLOSE THEIR HIV STATUS

Otis, Joanne $^{1}$; Massie, Lyne ${ }^{1}$; Fernet, Mylène ${ }^{1}$; Lévy, Joseph J1; Côté, Françoise ${ }^{2}$; Côté, José ${ }^{1}$; Mensah, Maria N ${ }^{1}$

${ }^{1}$ Montréal; ${ }^{2}$ Québec, QC

BACKGROUND: PP/PP is a program designed to empower WLWHIV with regards to the issue of disclosing their HIV status. The objective of this study is to evaluate the medium-term effects of PP/PP.

METHOD: In 2009-2010, the PP/PP program was implemented in 15 regions across Quebec. 84 WLWHIV (57.1\% in Montreal, $42.9 \%$ in other regions) completed a self-administered questionnaire one week before the program began (TO). The same questionnaire was completed by 67 women one month after the end of the program (T1), and by 59 women six months later (T2). The following variables were measured: ability to make an informed decision regarding the issue of disclosure ( 9 statements, $\alpha=0.78)$, perception of control over the decision not to disclose HIV status ( 3 set, $\alpha=0.75$ ), perception of control over the decision to disclose HIV status ( 5 items, $\alpha=0.92$ ), and general sense of control over one's life ( 4 items, $\alpha=0.74$ ).

RESULTS: Women who participated in the program felt more able : to make a free and informed decision about whether or not to disclose their HIV status (TO M = 3.37, T1 M = 3.99, T2 M = 4.05; p=0.0001), to develop and apply strategies for not disclosing their HIV status (TO M $=3.33, \mathrm{~T} 1 \mathrm{M}=4.20, \mathrm{~T} 2 \mathrm{M}=4.23, \mathrm{p}=0.0001)$, and to develop and apply strategies for disclosing their HIV status (TO M= 3.03, T1 M=3.91, T2 M $=4.09, \mathrm{p}=0.0001$ ). These effects were observed at short-term (T1) and were maintained six months later (T2). The program had no significant effect on participant's general sense of control over their lives (TO M = 3.23, $\mathrm{T} 1 \mathrm{M}=3.01, \mathrm{~T} 2 \mathrm{M}=3.37$, ns).

CONCLUSION: PP/PP met its objectives. Knowledge-application strategies are now underway to implement the program on a larger scale (via the dissemination of training and facilitation guides) and to develop a methodology suitable to other cultural contexts.

This study was funded by CIHR (grant \#180482).

\section{9}

DECISIONS, DECISIONS: INVESTIGATING THE EFFECT OF GENDER DYNAMICS IN SEXUAL DECISION-MAKING ON CONDOM USE AMONG HIV SERO-DISCORDANT COUPLES IN UGANDA

Muldoon, Katherine $A^{1}$; Duff, Putu $\mathrm{K}^{1}$; Birungi, Josephine ${ }^{2,3}$; Wang, Hong ${ }^{1}$; Chen, Yalin ${ }^{1}$; Ngolobe, Moses ${ }^{2,3}$; Shannon, Kate ${ }^{1}$; Moore, David $\mathbf{M}^{1}$

${ }^{1}$ Vancouver, BC; ${ }^{2}$ Kampala, Uganda; ${ }^{3}$ Manchester, United Kingdom OBJECTIVES: Given global concerns of gender inequities in sexual decision-making and HIV risk negotiations, research that independently collects information from both members of a sexual relationship provides an opportunity to better understand partner dynamics. We sought to investigate the relationship between couple's condom-use decision-making and reported condom use over time.

METHODS: Data were drawn from a cohort of HIV sero-discordant couples recruited through The AIDS Support Organization in Jinja, Uganda. Couples were assessed biannually over 18 months. The primary outcome was condom use at last sexual encounter. The sample was restricted to single-spouse couples. Sexual decision-making was categorized as follows: decides together, female decides, male decides, or they disagree. Multivariable logistic regression using generalized linear mixed modeling was used to investigate the influence of decision-making on condom use, adjusting for age, HIV status of each respondent, fertility desires and antiretroviral therapy status.

RESULTS: Out of 555 couples, 512 couples were eligible for this study, with a median age of 38 years (Interquartile range (IQR): 32-45 years). At baseline, $70.8 \%$ of couples reported condom use at last sexual encounter. This proportion ranged from $72.1 \%$ to $97.3 \%$ over 18 months of follow-up. Across time, $47.5 \%$ disagreed on which partner decided on condom-use, $40.8 \%$ reported they decided together, $8.1 \%$ reported the man decided and $3.6 \%$ reported the woman decided. Multivariable analysis revealed 
that compared to couples who decided on condom use together, significantly lower odds of condom use were found among couples that disagreed (adjusted Odds Ratios $[\mathrm{aOR}]=0.23 ; 95 \%$ Confidence Interval [95\% CI $0.12-0.44)$, where the female decided $(\mathrm{aOR}=0.23$; 95\% CI 0.07 $0.83)$, or where the male decided $(\mathrm{aOR}=0.20 ; 95 \% \mathrm{CI} 0.08-0.49)$.

DISCUSSION/CONCLUSIONS: We found that sero-discordant couples who decided on condom use together had elevated odds of reported condom use over time. Couple-and gender-focused interventions that encourage equitable sexual decision-making are an important HIV prevention strategy. This study design and analysis permits the future examination of the dynamic nature of decision-making over time and how it impacts important HIV prevention behaviour.

\section{O080 \\ ECONOMIC PRESSURES AND DEMANDS FOR CONDOM NON-USE, AND ASSOCIATED HIV VULNERABILITIES, AMONG SEX WORKERS AND CLIENTS IN VANCOUVER, CANADA}

Deering, Kathleen $\mathrm{N}^{1}$; Strathdee, Steffanie $\mathrm{A}^{2}$; Feng, Cindy $\mathbf{X}^{1}$;

Nosyk, Bohdan ${ }^{1}$; Shannon, Kate ${ }^{1}$

${ }^{1}$ Vancouver, BC; ${ }^{2}$ San Diego, CA, USA

BACKGROUND: Clients of sex workers (SWs) play a substantial role in determining the use of condoms for the prevention of HIV and other sexually transmitted infections (STIs) within commercial partnerships. The objective of this study was to understand the social, structural and environmental factors associated with accepting more money after clients' pressure for sex without a condom among SWs in Vancouver, Canada. METHODS: Baseline (Feb-July/10) data from a longitudinal cohort of street and off-street SWs in Vancouver, Canada (An Evaluation of Sex Workers' Health Access [AESHA]) were used. Multivariable logistic regression was used to identify factors associated with accepting more money for sex without a condom in the last six months. Multivariable (adjusted) odds ratios and 95\% confidence intervals were reported (AOR: [95\%Cls]).

RESULTS: Of a total of 510 SWs, the prevalence of being offered and accepting more money for sex without a condom was $65.7 \%$ (335) and $16.7 \%(85)$, respectively. In multivariable analysis, significantly higher odds of accepting more money for sex without a condom was found for SWs self-reporting as a sexual minority (eg, LGBTQ,Two-Spirited) (1.82 [1.02-3.23]), and in the last six months, experiencing client violence (2.17 [1.23-3.80]), exchanging sex while high (3.22 [1.42-7.28]), using crystal meth less than daily (vs. none) (2.55 [1.31-4.97]) and having higher numbers of clients per week (1.02 [1.00-1.03], per one-client increase).

CONCLUSIONS: These results highlight the social and economic pressures faced by SWs to not use condoms based on client demand. HIV prevention efforts should shift responsibility for condom use toward clients to reduce offers of more money for unsafe sex. Structural prevention programs aimed at mitigating the social and economic HIV risk environments of SWs through drug treatment, safer-environment interventions and the removal of criminal sanctions on safer indoor work spaces to enable condom use are urgently required to help reduce SW' vulnerability to HIV and STIs.

\section{Track A: Basic Sciences: Pathogenesis of HIV Infection and Co-infection \\ Volet A : Sciences fondamentales : Pathogénie de \\ I'infection au VIH et de la co-infection VIH/VHC}

\section{O081 \\ INSIGHTS INTO THE NATURE, DISTRIBUTION AND IMPLICATIONS OF HIV-1 IMMUNE ESCAPE REVEALED BY POPULATION-LEVEL ANALYSES}

Martin, Eric ${ }^{1}$; Carlson, Jonathan²; Brumme, Chanson ${ }^{3}$; Listgarten, Jennifer ${ }^{2}$; Le, Anh ${ }^{1}$; Chui, Cecilia ${ }^{3}$; Knapp, David ${ }^{3}$; Mo, Theresa ${ }^{3}$; Riddler, Sharon ${ }^{4}$; Haubrich, Richard ${ }^{5}$; Brockman, Mark'; Heckerman, David ${ }^{2}$; Carrington, Mary ${ }^{6}$; Mallal, Simon ${ }^{7}$; Brumme, Zabrina ${ }^{1}$

${ }^{1}$ Burnaby; ${ }^{2}$ Los Angeles, CA; ${ }^{3}$ Vancouver, BC; ${ }^{4}$ Pittsburg, PA, USA; ${ }^{5}$ San Diego, CA; ${ }^{6}$ Frederick, MD, USA; ${ }^{7}$ Murdoch, Australia

BACKGROUND: Population-level analyses of the distribution and characteristics of CTL escape in HIV-1 may offer insight into the mechanisms of HLA-associated "protective effects" on disease progression and other information relevant to vaccine design.

METHODS: In the largest study of its kind undertaken to date, we identify and analyze HLA-associated polymorphisms in HIV-1 in N=1888 treatment-naïve, chronically subtype B infected individuals from Canada, the USA and Australia. HLA associations were identified at allele group, protein and supertype-level resolution. Multiple tests were addressed using q-values.

RESULTS: Over 1600 unique HLA-restricted escape pathways were identified across HIV-1 $(\mathrm{q}<0.2)$. Of these, $45 \%$ occurred inside or within \pm 3 residues of an optimally described CTL epitope. Within 9-mer epitopes, the most frequently escaping sites were position 2 followed by the C-terminus and position $3\left(18 \% ; 14 \% ; 11 \%\right.$; binomial test $\left.\mathrm{p}<6 \times 10^{-7}\right)$; escape at other positions and flanking residues was less frequent $(<7.6 \%$ each). The strength of association was higher for HLA anchor residues (positions 2 and C-terminus) compared to other positions (Median Odds Ratio [OR] 5.5 vs. 4.5 p=0.003). Escape at anchor residues, but not other positions, was consistent with reduced binding affinity to the HLA molecule (NetMHC-3.0; $\mathrm{p}<0.0001)$. The strength of association was higher for escape mutations restricted by protective HLA alleles (B*13, B*27, $B * 57, B * 58)$ vs. non-protective alleles (Median $O R=4.6$ vs. $3.5 ; p<0.001$ ). After correction for HLA allele frequency, a significant inverse relationship was observed between the number of viral sites under active immune selection by a given HLA allele and published Hazard Ratios of AIDS progression (Spearman $\mathrm{R}=-0.33 ; \mathrm{p}=0.04$ ).

CONCLUSIONS: The most frequently observed immune escape occurs at anchor residues. Separately, the ability to mount stronger and broader immune selection pressures on HIV-1 appears to be a hallmark of HLAassociated "protective" effects on disease progression. Funding: supported by CIHR MOP-93536 (ZLB/MAB)

\section{2}

IDENTIFICATION OF POLYMORPHISMS IN HIV-1 ELITE CONTROLLER NEF SEQUENCES ASSOCIATED WITH IMPAIRED CD4 AND HLA DOWNREGULATION FUNCTION

Markle, Tristan ${ }^{1}$; Kuang, Tallie'; Martin, Eric ${ }^{1}$; Le, Anh ${ }^{1}$; Baraki, Bemuluyigza1; Mwimanzi, Philip²; Pereyra, Florencia ${ }^{3}$; Walker, Bruce ${ }^{3}$; Miura, Toshiyuki ${ }^{4}$; Ueno, Takamasa ${ }^{2}$; Brumme, Zabrina ${ }^{1}$; Brockman, Mark ${ }^{1}$

1Burnaby, BC; ${ }^{2}$ Kumamoto, Japan; ${ }^{3}$ Charlestown, MA, USA; ${ }^{4}$ Tokyo, Japan

BACKGROUND: Spontaneous control of HIV may result from impaired viral protein function. Gross deletions in the HIV regulatory protein Nef were previously associated with non-progression, but the role of Nef defects in HIV elite control remains poorly characterized.

METHODS: We examined the sequence and in vitro function of HIV plasma RNA-derived Nef genes from 52 elite controllers (EC) and 52 untreated chronic progressors $(\mathrm{CP})$. Representative clones were transfected into CEM-derived T cells expressing HLA-A*02, and surface CD4 and 
$\mathrm{A}^{* 02}$ protein levels were quantified using flow cytometry. Down-regulation activity of each patient-derived clone was normalized to a WT Nef control (strain SF2), such that function greater or less than WT was expressed as $>1$ or $<1$, respectively. We conducted a codon-by-codon analysis of Nef sequences to identify polymorphisms that were significantly associated with in vitro function.

RESULTS: Nef clones from EC did not encode large deletions or truncations, but nonetheless displayed significantly lower median CD4 and HLA down-regulation activity compared to Nef clones from CP (CD4: 0.91 [IQR 0.74-0.96] vs. 1.00 [0.92-1.02], p<0.0001; HLA: 0.89 [0.63-1.04] vs. 1.01 [0.89-1.05], $\mathrm{p}=0.05)$. In $\mathrm{EC}$, host expression of HLA-B*13, B*57, and/or C*06 was associated with a reduced ability to down-regulate CD4 (all $\mathrm{p}<0.05$ ), but HLA alleles were not associated with altered $\mathrm{A}^{*} 02$ down-regulation. CD4 and HLA down-regulation activity generally correlated with one another in EC-derived sequences $(R=0.61, p<0.0001)$. Polymorphisms R8S, E93K/K94E (which are associated with HLA-A*11 and $B * 08$ selection pressure), K105R ( $\left.B^{* 08}, B^{*} 49, C^{*} 07\right)$, and R188S ( $A * 74$ and $B * 27$ ) were independently linked with a diminished ability of EC-derived Nef to modulate both CD4 and HLA surface expression (all $\mathrm{p}<0.05$ ).

CONCLUSIONS: Despite a lack of gross Nef deletions, Nef clones from EC displayed significantly impaired CD4 and HLA down-regulation activity. Attenuated Nef activity may contribute to spontaneous HIV control observed in these rare individuals.

\section{3}

\section{A SYSTEMS BIOLOGY APPROACH TOWARD THE IDENTIFICATION OF NEW HIV PERMISSIVENESS FACTORS IN TH17 CELLS}

Cleret-Buhot, Aurélie ${ }^{1}$; Goulet, Jean-Philippe ${ }^{1}$; Monteiro, Patricia ${ }^{1}$; Gosselin, Annie'; Boulassel, Mohamed-Rachid ${ }^{1}$;

Routy, Jean-Pierre'; Sekaly, Rafick-Pierre²; Haddad, Elias²;

Ancuta, Petronela ${ }^{1}$

${ }^{1}$ Montréal, QC; ${ }^{2}$ Port St Lucie, FL, USA

Th17 cells are highly permissive to HIV replication and their frequency is significantly diminished in the gut-associated lymphoid tissues of HIVinfected subjects. Our hypothesis is that Th17 cells express a unique transcriptional profile compatible with HIV permissiveness.

To identify new HIV permissiveness factors in Th17 cells, genome-wide transcriptional profiles of Th17 cells (CCR $4^{+} \mathrm{CCR} 6^{+}$phenotype) were compared to those of Th1 cells (CXCR $3^{+} \mathrm{CCR}^{-}$phenotype), a T-cell subset we previously identified as being resistant to HIV. Cells were sorted by flow cytometry and stimulated via CD3/CD28. The expression of 46,000 probe sets was analyzed using the Illumina technology. Microarray data analysis identified 929 and 708 probe sets upregulated and downregulated, respectively, in Th17 versus Th1 cells $(\mathrm{p}<0.05$ and 1.3-fold change cutoff). Among differentially expressed transcripts, genes previously associated with T-cell differentiation (RORC), TCR signaling (ZAP-70, Lck) and HIV replication (PPARG) were preferentially expressed in Th17 cells. Other transcripts upregulated in Th17 versus Th1 cells with unknown role in HIV infection included PTPN13, a protein tyrosine phosphatase controlling apoptosis; KLF2, a transcription factor regulating T-cell migration; and RARRES3, a retinoic acid pathway responsive element. Differential expression of these transcripts was validated by RT-PCR, western blotting and/or fluorescence microscopy. Functional studies demonstrated that HIV permissiveness in Th17 was associated with lower TCR activation threshold and increased proliferation potential and NF- $\mathrm{KB}$ binding activity. RNA interference for RORC and KLF2 together with chemical activators of PPARG and RARRES3 pathways were used to determine the role of these molecules in regulating HIV permissiveness.

Our study identifies new molecular determinants regulating HIV replication in Th17 cells and suggests that interfering with certain Th17specific transcripts may help to design novel therapeutic strategies to limit HIV replication while maintaining the role of Th17 cells in mucosal immunity.

This work was supported by the CIHR grants \#82849 and \#114957 to PA.

\section{4 \\ EFFECT OF FEMALE SEX HORMONES ON HIV ENTRY AND REPLICATION WITHIN PRIMARY GENITAL EPITHELIAL CELLS.}

Ferreira, Victor $\mathbf{H}^{1}$; Kafka, Jessica $\mathbf{K}^{1}$; Nazli, Aisha ${ }^{1}$; Mueller, Kristen ${ }^{1}$; Tremblay, Michel' ${ }^{2}$; Cochrane, Alan ${ }^{3}$; Kaushic, Charu ${ }^{1}$

${ }^{1}$ Hamilton, ON; ${ }^{2}$ Laurier, QC; ${ }^{3}$ Toronto, ON

The early events of HIV-1 infection in the female genital tract (FGT), where $40 \%$ of all new HIV infections are established worldwide, remain incompletely understood. Genital epithelial cells (GECs) are the first barrier that HIV has to cross during heterosexual transmission. However, the contribution of GEC infection to HIV-1 acquisition in the FGT remains unknown, particularly in the context of female sex hormones which have been suggested to regulate susceptibility to HIV-1. To address this issue, primary GEC monolayers were prepared from human upper genital tract tissues and were grown in the presence or absence of physiological concentrations of estrogen (E2), progesterone (P4) or medroxyprogesterone acetate (MPA), the hormone in the controversial contraceptive formulation Depo-Provera, prior to HIV exposure. At 24 hours post-infection, significantly increased HIV p24 antigen was measured by ELISA inside HIV-infected cells grown in the presence of MPA and in basolateral supernatants of infected GECs grown in the presence of MPA and P4. Furthermore, entry of HIV-1 intro primary GECs was found to require heparan sulphate moieties and was significantly inhibited if the cells were treated with the endocytosis inhibitor dynasore prior to infection. Despite detection of p24 antigen and unspliced HIV RNA within infected GECs, no early or late reverse transcription products, spliced HIV RNA or integrated HIV DNA was measured at 24 and 48 hours post-infection, regardless of the viral strain used or the hormone conditioning of the cells. These results suggest that female sex hormones regulate HIV entry into, and shedding from primary GECs via noncanonical pathways. Furthermore, although HIV enters primary GECs, the virus undergoes a non-productive infection. Ongoing studies are investigating the mechanism and significance of HIV entry in the absence of productive infection as well as whether under certain conditions HIV replication does take place.

\section{5 \\ IL-7 INDUCES RAPID INTERNALIZATION OF CD127 BY CLATHRIN MEDIATED ENDOCYTOSIS, LEADING TO SUBSEQUENT DEGRADATION BY THE PROTEASOME Faller, Elliott M; Al-Ghazawi, Feras; MacPherson, Paul Ottawa, ON}

BACKGROUND: IL-7 signaling is important for CD8 T-cell homeostasis and function. Suppression of CD127(IL-7R) is mediated by both the HIV Tat protein and IL-7, both of which are elevated during HIV infection. The mechanisms by which IL-7 alone down regulates CD127 are currently under investigation in our lab and appear to occur at multiple levels. We show here by flow cytometry, western blot analysis and confocal microscopy that IL-7 induces rapid internalization of CD127 by endocytosis and subsequent degradation by the proteasome.

RESULTS: IL-7 decreases surface CD127 expression on CD8 T-cells in a dose- and time-dependent manner with initial suppression as early as 20 minutes and maximal suppression at 12 hours by inducing the internalization of surface CD127 through endocytic vesicles. Both Filipin, which disrupts lipid rafts, and Dynasore, which prevents fission of vesicles from the cell membrane, prevent IL-7 induced down regulation of CD127 at the cell membrane. Consistent with this we have shown by confocal microscopy that in resting CD8 T-cells CD127 is distributed evenly throughout the cell. After treatment with IL-7, CD127 forms multiple intracellular punctae with increased co-localization with clathrin by 5 minutes followed by co-localization with the early endosomal marker EEA1 after 30 minutes. By 2 hours CD127 staining associates with the late endosome marker RAB7 and the proteasomal $20 \mathrm{~S}$ subunit, while at the same time showing decreased colocalization with the lysosomal marker LAMP1. Interestingly, the proteasome inhibitors lactacystin and MG132 both blocked IL-7's ability to down regulate CD127 confirming IL-7 directs the receptor to the proteasome for degradation.

CONCLUSIONS: IL-7, a potent immunomodulatory cytokine, is currently being investigated as a potential therapy and vaccine adjuvant in 
the treatment of HIV infection. In order to maximize these therapeutic strategies, we must first understand how the IL-7 receptor is regulated. We show here that IL-7 down regulates CD127 protein on the surface of CD8 T-cells. IL-7 binding to its receptor induces aggregation of CD127 into lipid rafts, internalization through endosomes, and ultimately degradation via the proteasome.

\section{6 \\ IN VIVO EFFECT OF ANTI-INFLAMMATORY COMPOUNDS ON HIV-1 GP120-MEDIATED BRAIN INFLAMMATION}

Ashraf, Tamima; Jiang, Wenlei; Bendayan, Reina

Toronto, ON

Cognitive impairment remains highly prevalent in HIV-1 infected patients due to viral replication and associated inflammation in the brain. One obstacle to effective treatment is poor brain penetration of antiretrovirals due to functional expression of efflux transporters (P-glycoprotein [P-gp] and Multidrug-resistance associated proteins [MRPs]). Identifying therapeutic compounds that are not substrates of these transporters but exhibit anti-inflammatory properties may benefit treatment of HIV-associated neuroinflammation. Our laboratory has previously established that HIV-1 gp120 can trigger an inflammatory response and alter the expression of transporters in in vitro glial cell systems. In this study, by intracerebroventricular administration of gp120 in rats, we implemented an in vivo model of brain inflammation and investigated the regulation of transporters as well as the anti-inflammatory properties of minocycline and chloroquine.

Male Wistar rats were administered a single dose of gp120ADA (500 ng) daily for 7 consecutive days intracerebroventricularly with or without prior intraperitoneal administration of minocycline $(50 \mathrm{mg} / \mathrm{kg}$ loading dose followed by $25 \mathrm{mg} / \mathrm{kg} / \mathrm{day})$ or chloroquine $(25 \mathrm{mg} / \mathrm{kg} / \mathrm{day})$.

In gp120ADA- injected rats, transcripts of TNF- $\alpha$, IL-1 $1 \beta$ and iNOS were significantly elevated in frontal cortex and hippocampus. In cerebrospinal fluid (CSF), a significant increase in TNF- $\alpha$ and IL- $1 \beta$ was detected. In addition, gp120 administration resulted in a decrease in P-gp protein expression and an increase in Mrp1 in frontal cortex and hippocampus. Furthermore, minocycline or chloroquine completely attenuated the upregulation of IL-1 $\beta$ and iNOS transcripts in different brain regions and prevented IL-1 $\beta$ secretion in CSF. Minocycline was also successful in suppressing TNF- $\alpha$ in the brain tissues and CSF.

Our data demonstrate that administration of HIV-1 gp120ADA in rodents generates an inflammatory response and alters the expression of efflux transporters in several regions of the brain. Furthermore, minocycline and chloroquine can reverse gp120-associated brain inflammatory responses suggesting that these agents could potentially be considered in the prevention/treatment of HIV- associated cognitive disorders.

Supported by operating grants from OHTN and CIHR. Dr. Reina Bendayan is a Career Scientist from the OHTN.

\section{7}

HIV GP120 ACTIVATES PRO-INFLAMMATORY CYTOKINES IN FEMALE PRIMARY GENITAL EPITHELIAL CELLS VIA TLR2 AND 4 PATHWAYS FACILITATED BY HEPARAN SULPHATE Nazli, Aisha; Ferreira, Victor H; Anipindi, Varun; Mueller, Kristen; Kafka, Jessica; Chauvin, Sarah; Bowdish, Dawn M; Mossman, Karen; Mian, Firoz; Ashkar, Ali A; Kaushic, Charu

\section{Hamilton, ON}

Although women constitute more than $50 \%$ of HIV-1 infected population globally, the early events in heterosexual transmission of HIV-1 in the female genital tract are not well understood. Recently, we showed that HIV-1 could directly interact with genital epithelial cells (GECs) leading to impairment of mucosal barrier and microbial translocation. Subsequently, we have examined the signaling pathways that are activated in the primary GECs following exposure to HIV-1 to determine that mechanism involved in the early event of HIV pathogenesis in the female genital tract. Analysis of cytokines in supernatants from monolayers exposed to HIV- 1 showed that TNF- $\alpha$, IL-8, IL- 6 were significantly increased within 1-2 hours of HIV-1 exposure compared to mock-treated cultures. Gp120 was essential, as an env-deleted mutant of HIV-1 failed to induce any pro-inflammatory cytokines in GECs compared to wildtype HIV-1 or recombinant gp120 exposure. Cytokine induction was via activation of NFkB pathway in GECs, since NFkB nuclear translocation was observed within 1 hour of exposure and treatment of GEC with PDTC, an inhibitor of NFKB, blocked upregulation of pro-inflammatory cytokines after HIV and gp120 exposure. To examine the involvement of TLRs in the induction of pro-inflammatory cytokines production after gp120 exposure, GECs were pre-treated with neutralizing antibodies against TLR2, TLR4 and TLR5 or isotype antibodies, prior to gp120 treatment. TLR2 and TLR4 antibodies were found to prevent barrier breakdown after gp120 exposure and also blocked NFkB translocation and induction of cytokines. Further experiments indicated that heparan sulphate (HS) was required for TLR2 and TLR4 signaling via gp120, since removal of HS by Heparinase III blocked NFkB translocation, cytokine production and barrier impairment following exposure to gp120, even in the presence of TLR2 and TLR4. Antibodies against CD4 and CXCR4 receptors, TAK779, an inhibitor for CCR5 and removal of chondroitin sulphate did not have any effect.

\section{O088}

THE HUMAN IMMUNODEFICIENCY VIRUS TYPE 1 (HIV-1) CO-OPTS mTOR DURING THE LATE PHASE OF THE VIRUS LIFE CYCLE

\section{Miron, Marie-Joelle; Mouland, Andrew J}

Montreal, QC

The HIV-1 genomic RNA and Gag structural protein have been shown to associate with late endosomes and lysosomes. Movement of the latter towards the cell periphery during the late phase of the virus life cycle has been demonstrated to be important for virus egress and production.

The mammalian target of rapamycin (mTOR), a central regulator of cell growth nucleating two different protein complexes (mTORC1 and mTORC2) and modulating key aspects of cellular biology (protein and lipid synthesis, autophagy, cytoskeletal organization and cell survival), has recently been shown to relocalize to lysosomes for activation under nutrient-rich conditions. Relocalization and activation of mTORC1 has been demonstrated to depend on the presence of amino acids (AA) and on its association with the Rag Ragulator complex, a protein complex containing small GTPases.

As several viruses have been shown to co-opt other axes of mTORmediated signaling events and as mTOR pathway components are often mutated in cancer, we hypothesized that HIV-1 co-opts the mTOR/RagRagulator complex to promote virus egress and production in nutrientpoor conditions (AA starvation).

Our results show that, upon transfection and expression of the provirus genome in HeLa cells, 1) endogenous mTOR colocalizes with Gag in the cytoplasm and towards the cell periphery when nutrients are abundant; 2) endogenous mTOR localization towards the cell periphery is maintained under AA starvation conditions (75\% vs. control (13\%)); 3) AA starvation recovery does not affect mTOR peripheral localization; 4) Knocking down Rag proteins results in a 50\% decrease in Gag expression by western blotting, as well as in an accumulation of Gag protein in cytoplasmic patches and at the plasma membrane (PM) (increased number of cells with positive PM staining [KD: $68 \%$; control: $57 \%$ ] and increased general staining intensity) in immunofluorescence confocal microscopy studies.

Overall, these results suggest that HIV-1 is able to co-opt mTORC1 under stress conditions possibly by modulating the phosphorylation status of downstream effectors, thereby promoting protein synthesis and virus egress and production.

This work is supported by a grant from the CIHR (MOP-38111). 
Track B: Clinical Sciences: Treatment Strategies and HIV Infection in Women

Volet B : Sciences cliniques : Stratégies de traitement et infection au VIH chez les femmes

\section{O089 \\ PERINATAL OUTCOMES OF PREGNANCIES IN PERINATALLY INFECTED WOMEN}

Samson, Lindy ${ }^{1}$; Brophy, Jason ${ }^{1}$; Alimenti, Ariane ${ }^{2}$; Singer, Joel ${ }^{2}$; Lee, Terry ${ }^{2}$; Money, Deborah ${ }^{2}$; Boucher, Francois ${ }^{3}$; Lapointe, Normand ${ }^{4}$; Bitnun, $\mathrm{Ar}^{5}{ }^{5}$; Canadian Pediatric Perinatal AIDS Research Group, The ${ }^{1}$

${ }^{1}$ Ottawa, ON; ${ }^{2}$ Vancouver, BC; ${ }^{3}$ Quebec; ${ }^{4}$ Montreal, QC; ${ }^{5}$ Toronto, ON BACKGROUND: With the advent of cART, perinatally infected young women with HIV infection are entering their reproductive years and having children. Little is known about the fertility, reproductive health and infant outcome in this circumstance. This study describes HIV-related pregnancy and infant outcomes for women in Canada with perinatally acquired infection.

METHODS: Mother infant pairs (MIPs) were identified from the Canadian Perinatal HIV Surveillance Program (CPHSP), an active surveillance system with data submitted from 22 sites annually. All infants born after 2000 with maternal risk factor for HIV identified as "mother to child transmission" were included. Demographics, ARV exposure, pregnancy and infant outcome are described.

RESULTS: Twelve MIPs were identified. Median gestational age at birth was 39 weeks (28-41 weeks) with one premature birth. 95\% took ARVs during pregnancy, $25 \%$ required regimen changes and $51 \%$ received non-first line regimens. Five infants received postnatal prophylaxis with zidovudine alone and 7 with combination therapy. At the time of delivery, $58 \%$ of women had viral loads $<50$ copies $/ \mathrm{ml}, 17 \%$ between $51-100,000$ copies $/ \mathrm{ml}$ and $8 \%>100000$ copies $/ \mathrm{ml}$. 58\% delivered vaginally, $33 \%$ via elective c-section and $8 \%$ required emergent c-section. All infants were HIV negative. No major congenital anomalies were reported. $83 \%$ of infants were living with a biological parent.

CONCLUSIONS: Women living with perinatally acquired HIV infection in Canada are now having children. The full term gestations, absence of congenital anomalies and negative HIV status amongst the infants are reassuring. Of significant concern however, is the proportion of women who were not fully virologically suppressed at delivery. In addition, these infants are highly ARV exposed and their long term health outcomes must be monitored. Further studies are required to determine the reproductive health and long term outcomes of women with perinatally acquired HIV infection and their infants.

\section{O090}

\section{USE OF BOOSTED PROTEASE INHIBITORS IN} PREGNANCY AND RISK OF PREMATURITY AMONG HIV-EXPOSED UNINFECTED INFANTS IN MONTREAL

Kakkar, Fatima $^{1}$; Lamarre, Valerie ${ }^{1}$; Soudeyns, Hugo ${ }^{1}$;

Dahl, Cathrine $^{2}$; Lapointe, Normand ${ }^{1}$; Boucher, Marc ${ }^{1}$

${ }^{1}$ Montréal, QC; ${ }^{2}$ Oslo, Norway

OBJECTIVE: To compare the risk of preterm delivery among HIV-infected women treated with boosted versus non-boosted protease inhibitors (PI) in pregnancy.

METHODS: Study subjects were enrolled prospectively in the Centre maternel et infantile sur le sida mother-child cohort, CHU Sainte-Justine, Montreal, Canada, between 1987 and 2010 ( $n=585)$. Preterm was defined as delivery before 37 weeks gestation. Pearson's $\chi^{2}$ or Fisher's exact test was used to compare differences in proportion. Logistic regression was used to test the association between maternal PI use and preterm delivery.

RESULTS: 585 HIV exposed-uninfected infants and their mothers were included. 385 (64\%) mothers received 3 or more ARVS during pregnancy; $81 \%$ were PI based regimens. A total of 209 women received unboosted PIs (nelfinavir $\mathrm{n}=180$, other $\mathrm{n}=29$ ) and 102 received boosted PIs (lopinavir/ritonavir $[\mathrm{LPV} / \mathrm{r}], \mathrm{n}=85$; other $\mathrm{n}=37$ ). There was a significant difference in the proportion of preterm births among women receiving
LPV/r and other boosted PI regimens compared to women treated with nelfinavir-based HAART regimens ( $20 \%$ vs. $10.5 \%, \mathrm{p}<0.01 ; 29.7 \%$ vs. $10.5 \%, \mathrm{p}<0.01)$. The use of boosted PIs was the most significant variable associated with preterm delivery in univariate analysis (OR: 2.41, 95\% CI: 1.31-4.41, $\mathrm{p}<0.01$ ), which remained significant after adjustment for maternal age and delivery CD4 count (AOR: 1.90, 95\% CI: 0.99-3.64, $\mathrm{p}=0.05)$. There was no difference in the proportion of preterm births among women receiving nelfinavir or other non-boosted PI regimens compared to those receiving nevirapine $(10.5 \%$ versus $10.8 \%, \mathrm{p}=0.58$; $13.8 \%$ versus $10.8 \%, \mathrm{p}=0.71$ ) or other NRTI-based HAART regimens $(10.5 \%$ versus $8.1 \%, \mathrm{p}=0.149 ; 13.8 \%$ versus $8.1 \%, \mathrm{p}=0.50)$

CONCLUSIONS: PI boosting with ritonavir may explain the heterogeneity of results previously seen in studies of PIs and prematurity. These results support findings of increased frequency of preterm delivery with the use of boosted PIs and warrant further assessment of the use of ritonavir boosting during pregnancy.

\section{1 \\ DO PROTEASE INHIBITOR-CONTAINING HAART REGIMENS INCREASE RISK OF PRETERM BIRTH IN HIV-POSITIVE WOMEN?}

Wagner, Emily C; Chaworth-Musters, Tessa; Berg, Kyra B; Albert, Arianne Y; van Schalkwyk, Julianne E; Maan, Evelyn J; Ariane, Alimenti; Forbes, John C; Money, Deborah M Vancouver, BC

BACKGROUND: Highly active antiretroviral therapy (HAART) in pregnant women with HIV is standard-of-care for optimizing maternal health and preventing mother-to-child transmission of HIV, with a concomitant reduction in vertical transmission to $1-2 \%$. However, concerns have been raised about potential adverse effects of HAART; in particular, there have been conflicting reports of an association between protease inhibitor-containing regimens and preterm delivery.

METHODS: Demographics, HIV status, HAART type/duration, and pregnancy data were abstracted from a population-based database following all HIV-positive pregnancies in British Columbia (1994-2010). Multivariate logistic regression was used to test for an effect of HAART type on preterm birth.

RESULTS: From 542 total pregnancies, analysis was restricted to singleton live births with $>4$ weeks exposure to HAART $(\mathrm{n}=230)$. All women received an NRTI backbone and either NNRTI (58), PI (94), or boosted PI (bPI=78) -containing regimens. Mean maternal age was 30 years; ethnicity was $41 \%$ Caucasian, $25 \%$ Aboriginal, 22\% Black, and 12\% other/ mixed; $46 \%$ smoked cigarettes, $23 \%$ consumed alcohol, and 33\% reported drug use in pregnancy; and mean CD4 was 520 cells $/ \mathrm{mm}^{3}$. The overall preterm birth rate was $16 \%$ (37/221) compared to the provincial rate of $8 \%$, with rates of $19 \%(11 / 58)$ in NNRTI-treated, $12 \%(11 / 94)$ in PI treated, and 19\% (15/78) in bPI-treated women. Of the 37 preterm births, 4 were classified as iatrogenic, and 33 as spontaneous events. Receipt of HAART for less than 12 weeks (deviance $=9.92, p=0.007$ ), alcohol use in pregnancy (deviance $=7.30, \mathrm{p}=0.007$ ), and Hepatitis $\mathrm{C}$ antibody positivity (deviance $=8.05, p=0.005$ ) were independent predictors of preterm birth HAART regimen type was not a significant predictor of preterm birth (deviance $=2.14, \mathrm{p}=0.34$ ).

CONCLUSION: The preterm birth rate was high in this cohort, but was strongly influenced by suboptimal duration of HAART and substance use in pregnancy. This provides reassurance that HAART use and regimen type does not adversely affect perinatal outcomes.

\section{2}

METAGENOMIC CHARACTERIZATION OF THE VAGINAL MICROBIOME IN HIV-INFECTED WOMEN USING CULTURE INDEPENDENT METHODS

Mahal, Daljeet ${ }^{1}$; Diekema, Caroline ${ }^{2}$; Chaban, Bonnie ${ }^{3}$; Albert, Arianne $\mathrm{Y}^{1}$; Vicol, Laura ${ }^{1}$; Wagner, Emily $\mathrm{C}^{1}$; Hill, Janet $^{3}$; Pick, Neora ${ }^{1}$; Money, Deborah ${ }^{1}$

${ }^{1}$ Vancouver, BC; ${ }^{2}$ Utrecht, Netherlands; ${ }^{3}$ Saskatoon, SK

BACKGROUND: Imbalances in the vaginal microbiome can lead to negative reproductive health consequences for women. HIV-infected women, in particular, are vulnerable to microbiome disruptions. This 
study's objective was to explore and profile the vaginal microbiome in HIV-positive women utilizing novel cpn60 metagenomic profiling.

METHODS: 32 HIV-positive women were recruited, and demographic, clinical, and vaginal gram-stain information was obtained. Vaginal swabs were collected and DNA was extracted and amplified using cpn60 targeted primers. Cpn60 sequence libraries were generated with 454 GS FLX Titanium pyrosequencing. A total of 146 species were examined and subjected to phylogenetic analysis. Phylotypes were classified based on sequence similarity to known organisms.

RESULTS: Cohort characteristics: mean age $=35 \mathrm{y}$ (range $=16-49 \mathrm{y}$ ); ethnicity=16 Caucasian, 6 Black, 3 Aboriginal, 3 Asian, 1 South Asian, 3 mixed ethnicities; mean CD4 count $=547$ cells $/ \mathrm{mm}^{3}\left(\right.$ range $\left.=130-1180 \mathrm{cells} / \mathrm{mm}^{3}\right)$; $96 \%$ of women on combination antiretroviral therapy. Nugent's scored (gram stain scoring system for bacterial vaginosis diagnosis) swabs were abnormal in 34\% (11/32) of women, of which 19\% (6/32) had bacterial vaginosis and $16 \%(5 / 32)$ had intermediate scores. The mean number of bacterial species was 27 (range $=3-67$ ) with a Shannon Diversity Index of $0.90 \pm 0.62$. The most prevalent were Lactobacillus iners, $L$. jensenii, $L$. crispatus, and Gardnerella vaginalis. Low CD4 counts were significantly associated with higher proportions of Gardnerella to Lactobacilli using logistic regression analysis $(\mathrm{F}=5.56, \mathrm{p}=0.027)$.

CONCLUSIONS: Cpn60-based sequence data demonstrates substantial variation in the vaginal microbiome among HIV-infected women, with lowered immune status associated with bacterial population shifts from a Lactobacillus dominant (normal vaginal flora) to a Gardnerella dominant microbiome. Further study of this abnormal microbiome found in women with low CD4 counts may have important clinical implications.

\section{O093}

\section{HPV ANTIBODY RESPONSE IN HIV POSTIIVE GIRLS AND} WOMEN VACCINATED WITH AN HPV QUADRAVALENT 6/11/16/18 VACCINE

Money, Deborah $\mathrm{M}^{1}$; Blitz, Sandra2 ${ }^{2}$, Raboud, Janet ${ }^{2}$; Krajden, Mel'; Coutlee, Francois ${ }^{3}$; Loutfy, Mona ${ }^{2}$; Trottier, Sylvie ${ }^{4}$; Smaill, Fiona ${ }^{5}$; Bitnun, Ari²; Caddy, Sheila6; Walmsley, Sharon²; CTN 236 Study Group, The ${ }^{1}$

${ }^{1}$ Vancouver, BC; ${ }^{2}$ Toronto, ON; ${ }^{3}$ Montreal; ${ }^{4}$ Quebec City, QC; ${ }^{5}$ Hamilton, $\mathrm{ON} ;{ }^{6}$ Edmonton, $\mathrm{AB}$

BACKGROUND: HPV vaccines are immunogenic and efficacious in preventing HPV infection and cervical dysplasia in HIV- women, but despite high rates of HPV infection and cervical dysplasia there are no data in HIV+ women. This study assesses the seroresponsiveness of $\mathrm{HIV}^{+}$ females to the qHPV vaccine.

METHODS: An open labeled, multi-centered study of the seroresponsiveness of a $\mathrm{qHPV}$ in $\mathrm{HIV}^{+}$females. Clinical and immunologic data was collected with HPV infection determined by Linear blot assay, and antibody levels pre vaccine and 1 month post 3 rd dose, using the Merck cLIA assay to HPV 6,11,16,18 (cutoffs $>20 \mathrm{mMU6} / \mathrm{ml}$, 16mMU11/ $\mathrm{ml}, 20 \mathrm{mMU} 16 / \mathrm{ml}$ and $24 \mathrm{mMU} 18 / \mathrm{ml})$. Groups were G1: Age <18; G2: Age $>18$ seronegative at baseline; G3: Age $>18$ seropositive at baseline.

RESULTS: To date 350 women have been enrolled, with serologic data available on 112. Mean age 39 yrs (range: $10-60) ; 15.2 \%<18$ yrs; $39.3 \%$ white, $40.2 \%$ black and $7.1 \%$ aboriginal; $83,9 \%$ on ARV's; median (IQR) CD4 at screening was 502/. $\mathrm{mm}^{3}$ (360-630); $72.4 \%$ had HIV RNA $<50 / \mathrm{ml}$. All females under 19 were seronegative at baseline for all HPV types and women over age 19 had baseline seropositivity of $6-30 \%$ for any type. Seroconversion rates for genotypes $6,11,16$ and 18 at 7 months were $98.8 \%, 99.0 \%, 98.7 \%, 94.3 \%$, respectively. Geometric mean titres (GMTs) at 7 months were 451.4, 552.7, 2103.8 and 279.1. By group GMTs at 7 months were: HPV6 G1: 482, G2: 390, G3: 580; HPV11: G1: 540, G2: 544, G3: 637; HPV16: G1: 2679, G2: 1869, G3: 2297; HPV18: G1: 292, G2: 259, G3: 653 .

CONCLUSIONS: qHPV vaccinated $\mathrm{HIV}^{+}$females, show high, seroconversion rates, with the exception of response to HPV 18, which was lower than reported for HPV naïve, HIV uninfected females. Further evaluation of the antibody durability and efficacy is planned.

\section{O094 \\ NEUROCOGNITIVE IMPAIRMENT IN HIV POSITIVE WOMEN}

Pick, Neora

Vanocuver, BC

BACKGROUND: People infected with HIV are living longer, but there is increasing evidence for early aging, including neurocognitive impairment, which may affect quality of life and medication adherence. Current data on neurocognition status in $\mathrm{HIV}^{+}$women in Canada are lacking, which motivated this study.

OBJECTIVE: To examine the nature of cognitive impairment among HIV+ women $(n=75)$ compared to $\mathrm{HIV}^{-}$women $(n=42)$ in a Canadian cohort.

SETTING: $\mathrm{HIV}^{+}$women $>30$ years of age followed at Oak Tree Clinic were invited to participate. $\mathrm{HIV}^{-}$controls were recruited to match basic demographics of the $\mathrm{HIV}^{+}$participants.

MEASUREMENTS: Neurocognition was examined using both paper and pencil tasks, and a computerized battery of standardized tests (CANTAB). Domains examined included attention, processing speed, learning/memory, and executive functions.

RESULTS: Groups were well-matched on age (mean=45y) and education. HCV and IDU rates were similar in both groups. Only four (4/14) neurocognitive measures differed significantly between groups. As previously reported, $\mathrm{HIV}^{+}$participants had lower immediate and delayed memory recall (Hopkins Verbal Learning Test-Revised), and slower information processing speed (WAIS-IV-Symbol-Search). Additionally, $\mathrm{HIV}^{+}$ participants made more errors on select aspects of the Intra-dimensional/ Extra-dimensional-task (IDED), a measure of executive functioning. Specifically, the $\mathrm{HIV}^{+}$group made significantly more errors when reversal learning was required, but not on stages involving discrimination learning or attentional set-shifting. After pre-morbid ability (WTAR) and depression (CES-D scale) were statistically controlled for, only IDED impairment persisted in $\mathrm{HIV}^{+}$participants.

CONCLUSIONS: These preliminary findings indicate that after con trolling for pre-morbid ability and depression, the current sample of $\mathrm{HIV}^{+}$ women have persistent impairment limited to reversal learning, a component of executive functioning. This impairment may have significant functional implications that should be further investigated.

\section{O095 MICRONUTRIENT SUPPLEMENTATION AND DEFICIENCY IN HIV IN THE MAINTAIN TRIAL \\ Spaans, Johanna ${ }^{1,2}$; Singhal, Neera ${ }^{1}$; Lamoureux, Nancy ${ }^{1,2}$; Mallick, Ranjeeta ${ }^{1}$; Huff, Harold ${ }^{2}$; Walmsley, Sharon'; Mills, Edward ${ }^{1}$; Fergusson, Dean ${ }^{1}$; la Porte, Charles ${ }^{1,2}$; Rosenes, Ronald ${ }^{2}$; Balfour, Louise ${ }^{1,2}$; Cameron, Bill ${ }^{1,2}$ ${ }^{1}$ Ottawa, ON; ${ }^{2}$ Vancouver, BC}

INTRODUCTION: Micronutrient deficiencies are common in HIV/ AIDS and predict disease progression and death. Deficiency is due to low dietary micronutrient intake, malabsorption, and increased metabolic demand. Supplementation is common, in the absence of proven effectiveness. MAINTAIN is a randomized controlled trial to compare high-dose micronutrients and anti-oxidants versus recommended daily allowance multi-vitamin supplementation to delay HIV disease progression in ART-naïve asymptomatic individuals with CD4 $\geq 375 \mathrm{cells} / \mathrm{mm}^{3}$. OBJECTIVE: To determine prevalence of prior micronutrient supplementation, baseline serum micronutrient levels and correlates, and the prevalence of deficiencies among participants enrolled to the MAINTAIN study.

METHODS: Of the 165 patients screened, we present baseline serum levels of the 127 patients randomised. Population norms were taken from the Canadian Health Measures Survey 2007-2009 (Statistics Canada, 2010), and low level thresholds were set on established criteria (Table). Univariate linear regression was used to identify correlates of baseline micronutrient levels.

RESULTS: Prior supplementation was reported in $28 \%$ and was not correlated with baseline micronutrient levels in randomised patients. Mean micronutrient levels were comparable to population norms, and prevalence of low levels was common (Table). There was a significant 
reduction in baseline serum vitamin $B_{12}$ of $21 \mathrm{pmol} / \mathrm{L}$ per $100 \mathrm{cells} / \mu \mathrm{L}$ lower CD4 count $(\mathrm{p}=0.02)$.

CONCLUSION: Micronutrient levels in asymptomatic HIV infection are in keeping with population norms, but low micronutrient levels are frequent despite common supplementation. Given the association of low micronutrient levels with HIV/AIDS health risks, trials of effectiveness of supplementation and its role in HIV/ART are justified.

\begin{tabular}{lcc}
\hline $\begin{array}{c}\text { Serum } \\
\text { micronutrient level }\end{array}$ & Mean (SD), median (IQR) & $\%$ below threshold $<$ \\
\hline Carotene $(\mu \mathrm{mol} / \mathrm{L})$ & $1.85(1.36), 1.7(1.4)$ & $24 \%<1.0 \mu \mathrm{mol} / \mathrm{L}$ \\
25-OH vitamin D & $64.97(27.76), 66(44)$ & $68 \%<75 \mathrm{nmol} / \mathrm{L}$ \\
$(\mathrm{nmol} / \mathrm{L})$ & & $24 \%<40 \mathrm{nmol} / \mathrm{L}$ \\
& & $3.5 \%<20 \mathrm{nmol} / \mathrm{L}$ \\
Vitamin $\mathrm{B}_{12}(\mathrm{pmol} / \mathrm{L})$ & $319.02(153.77), 278(162)$ & $2.4 \%<133 \mathrm{pmol} / \mathrm{L}$ \\
Folate $(\mathrm{nmol} / \mathrm{L})$ & $104.51(328.43), 25.3(17.7)$ & $20 \%<15 \mathrm{nmol} / \mathrm{L}$ \\
\hline
\end{tabular}

\section{6}

SAFETY AND EFFICACY OF CHOP OR R-CHOP FOR TREATMENT OF DIFFUSE LARGE B-CELL LYMPHOMA WITH PROTEASE INHIBITOR OR NON-PROTEASE INHIBITOR BASED ANTIRETROVIRAL REGIMENS IN HIV-INFECTED PATIENTS: SCULPT STUDY

Wong, Alison Y'; Marcotte, Suzanne'; 'Laroche, Mathieu'; Sheehan, Nancy ${ }^{1}$; Kukreti, Vishal'2; Routy, Jean-Pierre'; Lemieux, Bernard'; Seki, Jack²; Rouleau, Danielle'; Tseng, Alice² ${ }^{1}$ Montreal, QC; ${ }^{2}$ Toronto, ON

BACKGROUND: Use of combination antiretroviral therapy (cART) and cyclophosphamide, doxorubicin, vincristine and prednisone with or without rituximab $(\mathrm{CHOP} \pm \mathrm{R})$ for treatment of diffuse large B-cell lymphoma (DLBCL) in HIV substantially increases response rates but may also increase toxicity, possibly due to antiretroviral-antineoplastic drug interactions.

METHODS: A retrospective multi-centered observational pilot study was conducted in HIV-infected patients on cART, treated for DLBCL with $\mathrm{CHOP} \pm \mathrm{R}$ between 2002-2010 in three academic hospitals. We evaluated the percentage of confirmed or unconfirmed complete remission $(\mathrm{CR} / \mathrm{CRu}), 2$-year overall survival (OS) and frequency of severe adverse events. Preliminary comparisons between patients receiving protease inhibitor (PI) and non-PI based cART were made using Fisher's exact and Wilcoxon's test. Possible predictors of CR/CRu between groups were evaluated by univariate logistic regressions.

RESULTS: Thirty-four patients were included, $65 \%$ and $35 \%$ of patients receiving a PI and non-PI based cART, respectively. Baseline characteristics were similar between groups; $85 \%$ of patients were male, median age 43 years, 50\% with International Prognostic Index (IPI) score 2-3, median 7 years since HIV diagnosis and median $\mathrm{CD}^{+}$of $225 \mathrm{cells} / \mathrm{mm}^{3}$. CR/CRu was achieved in $77 \%$ and $58 \%$ of patients in the PI and non-PI group, respectively $(\mathrm{p}=0.21$ ), with $65 \%$ and $63 \%$ of patients achieving 2 -year $\operatorname{OS}(\mathrm{p}=1.00)$. Univariate analyses showed that a lower IPI score and a higher total number of received chemotherapy cycles were significantly associated with higher CR/CRu rates ( $\mathrm{p}=0.02$ and 0.03 , respectively). Tolerability was similar between both groups except decreased frequency of anemia in the PI group ( $23 \%$ versus $37 \%$, $\mathrm{p}=0.04)$.

CONCLUSION: Similar efficacy and tolerability of $\mathrm{CHOP} \pm \mathrm{R}$ was observed with PI and non-PI based cART, though less anemia was observed in the PI group. Response rates appear to be higher in patients receiving a PI based cART but requires confirmation with larger studies.
0097

EFFICACY AND SAFETY OF AN ANTIRETROVIRAL REGIMEN CONTAINING SIX MONTHS OF STAVUDINE FOLLOWED BY LONG-TERM ZIDOVUDINE FOR FIRSTLINE HIV THERAPY IN RESOURCE-LIMITED SETTINGS: AN OPEN-LABEL, RANDOMIZED, MULTICENTER TRIAL IN CHINA

Li, Taisheng

Beijing, China

BACKGROUND: It is imperative to find accessible, well-tolerated first-line antiretroviral (ARV) regimens for HIV-1-infected patients in resource-limited settings.

METHODS: We conducted an open-label, randomized, multicenter, noninferiority study of 517 ARV-naïve, HIV-1-infected adults. Patients were randomized at a 2:1 ratio to receive a regimen of either stavudine (d4T) or zidovudine (AZT) plus lamivudine and nevirapine. At week 24, patients in the $\mathrm{d} 4 \mathrm{~T}$ group were re-randomized (1:1) to switch from $\mathrm{d} 4 \mathrm{~T}$ to AZT (AZT-substitution group) or to remain on the d4T regimen. The primary endpoints were the proportion of patients with HIV-1 RNA viral load $<40$ copies/ $\mathrm{ml}$ at week 48 , and the incidence of adverse events (AEs) leading to the premature discontinuation of treatment.

RESULTS: No statistical differences in the proportion of patients with $\mathrm{VL}<40$ copies $/ \mathrm{ml}$ and $\mathrm{CD} 4^{+} \mathrm{T}$ cell counts were found among the three groups (the AZT group, the d4T group, and the AZT-substitution group) after re-randomization. However, more patients in the AZT group experienced AEs that resulted in the premature discontinuation of treatment through week 24 than those in the d4T group $(31.8 \%$ vs. $17.6 \%$, respectively, $\mathrm{p}=0.001$ ). After week 24 , patients among the three groups had a similar prevalence of drug-related adverse events resulting in the discontinuation of treatment. From baseline through 48, bone marrow suppression was the most frequent reason for study withdrawal occurring at a rate of $3.1 \%$ in the AZT-substitution group and $14.7 \%$ in the AZT group $(\mathrm{p}=0.001)$. No bone marrow suppression resulting in the discontinuation of treatment occurred in the $\mathrm{d} 4 \mathrm{~T}$ group. The prevalence of lipodystrophy was $2.1 \%$ in the $\mathrm{d} 4 \mathrm{~T}$ group. No lipodystrophy was observed in either the AZT or the AZT-substitution groups.

CONCLUSIONS: Our results demonstrate the AZT-substitution regimen provides a safe and effective first-line ARV strategy in resourcelimited settings.

Track C: Epidemiology and Public Health Sciences: Current Societal and Methodological Issues

for HIV Research

Volet C : Épidémiologie et sciences de la santé publique :

Questions sociétales et méthodologiques actuelles en matière de recherche sur le VIH

0098

TOWARDS A SUPERVISED INJECTION SERVICE IN MONTREAL: WHAT DO PEOPLE WHO INJECT THINK ABOUT THE PROPOSED SIS?

Leclerc, Pascale; Gutierrez, Natalia; Morissette, Carole;

Larouche, Annie; Gagnon, Vincent

Montréal, QC

BACKGROUND: In 2011, the Montreal Public Health Department conducted a feasibility study on the implementation of a supervised injection service (SIS) in Montreal. As part of this study, a consultation was carried out with injection drug users (IDUs) to better know their expectations regarding a potential SIS.

METHOD: Active (past month) injectors were interviewed, mainly by members of the provincial drug user association (ADDICQ). The structured questionnaire, available in French and English, covered sociodemographics, injection behaviors, drug overdoses, intention to use a SIS and acceptability of various operating rules. Factors associated with intention to use a SIS were identified using logistic regression.

RESULTS: In 2011-08, 115 responders were recruited (77\% males, 46\% aged $30-44$ years). In the preceding month, $60 \%$ lived in their own apartment/house and $44 \%$ on the streets; $39 \%$ injected daily. A total of $59 \%$ 
ever overdosed. Intention to use a SIS was as follows: yes: $59 \%$, no: $21 \%$, maybe/don't know: 20\%. Mainly residing in his/her own apartment/ house $(A O R=0.32$ ) was the only variable associated with intention to use a SIS in the final multivariate model. Among potential users, projected frequency of use varied with injection frequency; $45 \%$ of daily injectors planned daily use. Most would use a SIS located in a community syringe distribution program (96\%) or a mobile SIS $(82 \%)$. Most would be comfortable with supervision of injection by peers $(78 \%)$ or nurses $(76 \%)$. Of the 18 proposed operating rules, 13 were considered acceptable by $\geq 70 \%$; the least acceptable were: registration with name and date of birth at every visit (53\%) and limit of one injection per visit (50\%). Following the interview, $70 \%$ intended to use a SIS.

DISCUSSION: The data collected suggest that the proposed SIS would be acceptable to Montreal IDUs and used by the most vulnerable of them. To further ensure that it meets their needs, IDUs should take part in its implantation.

\section{O099}

ATTITUDES ON THE CRIMINALIZATION OF HIV: RESULTS FROM THE SRC-CANFAR 2011 CANADIAN NATIONAL SURVEY

Tyndall, Mark W' ${ }^{1}$; Calzavara, Liviana ${ }^{2}$; Allman, Dan²; Worthington, Catherine ${ }^{3}$; Adrien, Alix ${ }^{4}$; Kelly, Chris ${ }^{1}$; Bunting, Chris ${ }^{1}$

10ttawa; ${ }^{2}$ Toronto, ON; ${ }^{3}$ Victoria, BC; ${ }^{4}$ Montreal, QC

BACKGROUND: The criminalization of HIV in Canada is driven largely by the criminal justice system with little attention to the public health implications. As the topic of criminalization is extremely important to HIV prevention and public health efforts we sought to gauge public attitudes as part of a large Canadian survey.

METHODS: A representative sample of people residing in Canada who were 16 years of age and older completed this survey in May 2011. The participants were recruited from an online panel supplemented by random digit dial telephone interviewing. For the total sample the margin of error was \pm 2.1 percentage points at the $95 \%$ confidence interval.

RESULTS: The analysis was based on 2,139 respondents. $87 \%$ were aware that people who are HIV positive can be criminally prosecuted for non-disclosure of their HIV positive status and $75 \%$ thought that it was "definitely" or "probably" appropriate to imprison a person found guilty of not disclosing their HIV positive status to a sexual partner. However, the perceived potential impact that criminalization had on public health and prevention was less clear. $46 \%$ believed that it was an effective way of reducing HIV transmission and $41 \%$ agreed that criminalization increased stigma and discrimination toward people living with HIV. Further, $31 \%$ believed criminalization could deter or stop people from seeking HIV testing.

CONCLUSION: This survey would indicate that while most respondents supported the criminalization of HIV and the overwhelming majority supported imprisonment for HIV non-disclosure, there was recognition also that criminalization results in negative consequences regarding discrimination, HIV testing and as a potential deterrent to transmission. As we await a landmark Supreme Court of Canada decision regarding the definition of reasonable risk and HIV disclosure in 2012, it is important to increase public discourse and measure the unintended consequences that criminalization has on HIV prevention.

\section{0}

\section{THE CEDAR PROJECT: HIV RELATED VULNERABILITIES AMONG LGTBQ ABORIGINAL YOUNG PEOPLE WHO USE DRUGS IN TWO CANADIAN CITIES}

Cedar Project Partnership, For the ${ }^{1}$; Blair, Alden $\mathrm{H}^{2}$; Louie, Mabel ${ }^{3}$; Pearce, Margo E²; Pan, Stephen W2; Zhang, Hongbin²; Christian, Chief W'; Schechter, Martin T' ${ }^{2}$; Spittal, Patricia $\mathbf{M}^{2}$ ${ }^{1}$ Prince George; ${ }^{2}$ Vancouver; ${ }^{3}$ Vanderhoof; ${ }^{4}$ Enderby, BC OBJECTIVE: This study explores HIV vulnerabilities among young Aboriginal people who use drugs. Aboriginal women and young people in Canada remain at increased risk for sex and drug-related harm, contributing to their overrepresentation in new cases of HIV. While this can be exacerbated by racism and discrimination based on sexual orientation there is a paucity of research on this subject.
METHODS: The Cedar Project is an ongoing cohort study of Aboriginal young people in Vancouver and Prince George who use illicit drugs. HIV vulnerability was examined through injection drug use (IDU), IDU related harms, and sexual vulnerability. Sexual orientation was based on self-identification. The data of 605 participants at baseline was analyzed with multivariate logistic regression to model associations between identifying as lesbian, bisexual, transgendered, gay, queer, or two-spirited (LGTBQ) and HIV risk after adjusting for confounders. Unadjusted and adjusted odds ratios (AOR) and 95\% confidence intervals (CI) were calculated.

RESULTS: Among Cedar participants, $7.7 \%$ of men $(n=24)$ and $14.6 \%$ of women $(n=43)$ self-identified as LGTBQ. Due to small sample size, multivariate models were run for women only, though of note, unadjusted models for GTBQ men showed significant rates of sex work $(\mathrm{p}<.0001)$ and suicide attempts $(\mathrm{p}=0.029)$. LGTBQ women had greater odds than straight identified women for having ever injected drugs (AOR: 2.87, 95\% CI: 1.32-6.83), reported ever rig sharing (AOR: 4.22, 95\% CI: 1.91 9.78), rig shared in the past six months (AOR: 3.16, 95\%CI: 1.37-7.27), having ever participated in sex work (AOR: 2.66, 95\% CI: 1.14-7.33), or participated in sex work in the past six months (AOR: $2.31,95 \% \mathrm{CI}$ : 1.14-5.02)

CONCLUSION: HIV vulnerability amongst LGTBQ Aboriginal women is distressing, suggesting current harm reduction programs are limited. LGTBQ young people must be involved in designing risk reduction programs that better accommodate their needs.

\section{1}

THE DYNAMIC EPIDEMIOLOGY OF HIV AMONG INJECTION DRUG USERS IN PAKISTAN

Shaw, Souradet $Y^{1}$; Ahmad, Sajid²; Malik, Naeem²; Thompson, Laura $\mathrm{H}^{1}$; Emmanuel, Faran²; Blanchard, James $\mathrm{F}^{1}$; Archibald, Chris ${ }^{3}$

${ }^{1}$ Winnipeg, MB; ${ }^{2}$ Islamabad, Pakistan; ${ }^{3}$ Ottawa, ON

INTRODUCTION: Globalization and conflict have resulted in injection drug users (IDU) being an important driver of HIV in Pakistan. Despite the potential for a large-scale HIV epidemic, little is known about the patterns of HIV among IDU in Pakistan. This study sought to describe the trends and correlates of HIV infection.

METHODS: Data were from serial cross-sectional biological and behavioural surveys of IDU across Pakistan. Data from two rounds of data (2006 and 2011) were analysed. Trends in HIV prevalence, risk and drug injection behaviours were examined. Adjusted odds ratios (AORs) and 95\% confidence intervals ( $95 \% \mathrm{CI}$ ) from separate multivariable logistic regression models examined the correlates of HIV infection from the two largest provinces in Pakistan (Sindh and Punjab).

RESULTS: Sample size was 3,167 and 3,509 in 2006 and 2011, respectively. HIV prevalence increased from 16\% to 31\% from 2006 to 2011. Frequency of injection 3+ times in the last day increased from $25 \%$ to $68 \%$ and use of an unclean syringe increased from $28 \%$ to $36 \%$. The proportion of heroin injectors increased from $45 \%$ to $73 \%$. HIV was associated with a longer duration of injection drug use in Punjab; those injecting for 5-9 years were 1.6 times more likely to be HIV-positive $(95 \%$ CI: $1.1-2.3 ; \mathrm{p}=0.007$ ), compared to those injecting for 1 year or less. HIV infection was associated with younger age in Sindh; IDU 40+ years were 0.2 times less likely to be HIV-positive, compared to those $18-20$ years of age (95\% CI: 0.1-0.5; $\mathrm{p}=.001$ ).

CONCLUSIONS: The epidemiology of HIV among Pakistani IDU continues to evolve. Results suggest different IDU subgroups are at highest risk of HIV infection, related to geography. Of particular concern is the association of HIV with younger IDUs in Sindh province. Results can be used to inform interventions tailored to the heterogeneity in HIV epidemics across Pakistan. 


\section{2}

THE NATURE OF MSM CONNECTIVITY IN VANCOUVER, BC AND THE IMPORTANCE OF FORMATIVE RESEARCH FOR HIGHLIGHTING POTENTIAL 'BOTTLENECKS' AMONG SUB-NETWORKS WHEN SELECTING OPTIMAL SEEDS FOR RESPONDENT DRIVEN SAMPLING (RDS)

Stevenson, Benjamin ${ }^{1}$; Forrest, Jamie I'; Pai, Jayaram ${ }^{1}$; Michelow, Warren ${ }^{1}$; Roth, Eric $\mathrm{A}^{2}$; Moore, David ${ }^{1}$; Hogg, Robert $\mathrm{S}^{1,3}$ ${ }^{1}$ Vancouver; ${ }^{2}$ Victoria; ${ }^{3}$ Burnaby, BC

BACKGROUND: Respondent Driven Sampling (RDS) has been criticised for vulnerability to biases in the chain-referral sampling method. RDS estimates are influenced by underlying network structure, distribution of traits and recruitment dynamics. 'Bottlenecks' occur when recruitment chains remain within highly connected clusters, missing other sub-networks. Formative research on social structure can aid in identifying potential bottlenecks and inform selection of recruitment seeds.

METHODS: We conducted a series of semi-structured key informant interviews (KIIs) with service providers and focus group discussions (FGDs) with local MSM. Key informants were asked about their knowledge of social, cultural and economic structures of the MSM community and the contexts in which MSM self-organize. FGDs used community mapping to explore participants' understanding of MSM community structure; post-mapping discussions probed the roles that reported substance use and sexual preferences play in structuring networks. We used a descriptive analysis and inductive approach to identify key features of the social and sexual network structure of the MSM community.

RESULTS: 24 KIIs and 6 FGDs with 48 participants were conducted. KIIs and FGDs were stratified by geography, HIV serostatus and age group. We found that MSM connect socially and sexually through online networks, organizations, clubs, bars, and cruising locales, despite increased geographic decentralization from the downtown gay neighbourhood. Clustering factors included age range/'generation', residence in urban vs suburban areas, social preference (i.e., 'scene' or 'non-scene'), sexual type (eg, 'twinks', 'bears'), socioeconomic status, HIV serostatus and substance use preferences. Young MSM reported higher degrees of overlap between sexual and social networks compared to older MSM who more commonly distinguished these as separate. 'Special connectors' between sub-groups include drag queens, sexually attractive guys, and 'daddies'.

DISCUSSION: Preliminary analyses of our data suggest special connectors, cruising locales and the Internet play a central role in connecting MSM networks in Vancouver.

\section{O103}

\section{NEW HIV PREVENTION TECHNOLOGIES: REGULATORY, POLICY, PROGRAMMING AND RESEARCH IMPLICATIONS FOR CANADA}

\section{LeBlanc, Marc-André1; Patten, San²; Broeckaert, Logan ${ }^{3}$;}

Buhler, Shayna ${ }^{3}$; Abraham, Lesley ${ }^{3}$; Peissel, Nicolas ${ }^{3}$; Rogers, Tim ${ }^{4}$; Wilton, James ${ }^{4}$

\section{${ }^{1}$ Gatineau, QC; ${ }^{2}$ Halifax, NS; ${ }^{3}$ Ottawa; ${ }^{4}$ Toronto, ON}

BACKGROUND: New HIV prevention technologies (NPTs) have recently begun to show great promise. Several large efficacy trials have demonstrated proof-of-concept or established efficacy for pre-exposure prophylaxis (PrEP), microbicides, vaccines and treatment-as-prevention. If their potential is to be harnessed, it is important to advance the dialogue on the research, policy and programmatic implications of these new technologies within the Canadian HIV prevention landscape. METHODS: In 2011, four Canadian partners (Canadian AIDS Society, CATIE, Interagency Coalition on AIDS and Development, Canadian Public Health Association) collaborated, with financial support from CIHR, to engage Canadian stakeholders in identifying NPT implementation issues and a research agenda for social, clinical and policy researchers. Policy-makers, community representatives, researchers, public health representatives and the private sector participated in identifying the knowledge gaps which should be filled for effective policy and programming decisions to be made in Canada, and to ensure that Canadian stakeholders are appropriately prepared for the introduction of NPTs. RESULTS: Participants identified six Priority Actions: 1) demonstration projects to determine the most effective way for delivering NPTs to different at-risk populations; 2) a Canadian version of the international
Mapping Pathways project,to fully understand the place of NPTs in the Canadian response to HIV; 3) national implementation guidelines for clinical and community practice; 4) a communication strategy for service providers to deliver NPTs as part of a comprehensive prevention approach; 5) an epidemiological monitoring system to monitor NPT reach and access, and resultant changes in HIV incidence; and 6) improved access to post-exposure prophylaxis (PEP) through renewed awareness, access and policies.

CONCLUSIONS: The six Priority Action Areas outline the research, regulatory, policy and programming barriers and issues that need to be addressed related to the introduction of NPTs in the Canadian context.

\section{4 \\ IMPACT OF STATISTICAL ADJUSTMENT FOR FREQUENCY OF VENUE ATTENDANCE IN THE VANCOUVER SITE OF THE M-TRACK HIV SURVEILLANCE SYSTEM}

Gilbert, Mark ${ }^{1}$; Gustafson, Paul'; Xia, Michelle ${ }^{1}$; Michelow, Warren ${ }^{1}$; Robert, Wayne ${ }^{1}$; Trussler, Terry ${ }^{1}$; McGuire, Marissa ${ }^{2}$;

Paquette, Dana ${ }^{2}$; Hogg, Robert ${ }^{1}{ }^{1}$; Gustafson, Reka ${ }^{1}$

1Vancouver, BC; ${ }^{2}$ Ottawa, ON

OBJECTIVE: Venue based sampling is common for surveys of gay, bisexual and other men who have sex with men (MSM) populations. However, results may be biased by frequency of venue attendance (FVA) by participants. While statistical methods to adjust for FVA have been proposed these are rarely applied. We developed a novel method for adjusting for FVA which we applied to data from the ManCount survey (the Vancouver site of the M-Track HIV surveillance system for MSM).

METHODS: ManCount used time-space sampling to recruit MSM aged $\geq 19$ years from August 2008 to February 2009 from community venues. Participants completed a self-administered questionnaire and provided a blood sample tested for HIV and other infections. Self-reported FVA was measured from an abbreviated list of venues in the sampling frame. We used a novel Bayesian model-based approach to adjust prevalence estimates for FVA for 55 health and behavioural indicators.

FINDINGS: We found little effect of FVA adjustment on biological prevalence or sexual behaviour indicators (primary outcomes) although where significant adjusted estimates were indicative of lower sexual risk. Adjustment resulted in lower estimates for the proportions: being tested for HIV/STI, vaccination for Hepatitis A and B, and having disclosed about male sex partners to a health care provider. Adjustment for FVA did increase the estimates for the proportions: identifying as "bisexual" or "heterosexual/straight", and income $<20 \mathrm{~K}$.

DISCUSSION: The lack of association between FVA and biological prevalence or sexual behaviour indicators is reassuring as these are the primary outcomes for monitoring within M-Track, and lends credence to unadjusted prevalence estimates. However, adjustment for FVA should be considered as our novel method proved informative about indicators of public health importance (ie, testing, vaccination), shed insights on under-represented populations of MSM (ie, bisexual men, low-income men), and accounts for a potential sampling bias.

\section{5}

\section{HOUSING STATUS AND THE HEALTH OF PEOPLE LIVING} WITH HIV/AIDS: A SYSTEMATIC REVIEW

Wilson, Michael G',2; Aidala, Angela ${ }^{3}$; Gogolishvili, David ${ }^{1}$;

Shubert, Virginia ${ }^{3}$; Rueda, Sergio ${ }^{1}$; Bozack, Anne $^{3}$; Chambers, Lori $\mathrm{A}^{1}$; Caban, Maria ${ }^{3}$; Rourke, Sean $B^{1}$

\section{${ }^{1}$ Toronto; ${ }^{2}$ Hamilton, ON; ${ }^{3}$ New York, NY, USA}

Issue: Homelessness and unstable housing contribute to HIV transmission and have been linked to inadequate HIV care and poor health outcomes. Improved housing stability appears to improve access to care and the health of people living with HIV, but resources often fall short of housing need. The aim of this review is to inform program and policy development and future research by examining the available empirical evidence on the effects of housing status on health-related outcomes in people living with HIV/AIDS.

METHODS: 10 electronic databases were searched from 1996 to April 2010. We also contacted key researchers in the area of housing and HIV. Search results were reviewed and data was extracted from included studies by two independent reviewers. We included studies conducted in 
high-income countries that included people with HIV, provided an analysis of empirical data with at least one measure of housing status as an independent variable and at least one quantitative health outcome (eg, physical, mental, behavioural, social and/or health mediating variables) as a dependent variable. We defined housing status as any measure of homelessness, marginal housing, stable housing, or quality of housing. RESULTS: After reviewing about 4000 references 112 studies have been selected for data analysis, which includes 4 randomized controlled trials, 30 longitudinal studies, 72 cross-sectional, 6 case control. Most studies were conducted in the United States or Canada. Findings from the included studies suggest that homelessness and unstable housing consistently contribute to significantly poorer health outcomes, anti-retroviral adherence, access to health and social services and increased HIV-risk behaviours.

CONCLUSIONS: This review demonstrates the importance of housing status and housing stability on access to care and the health of people living with HIV. Findings will contribute to a growing body of knowledge being used to inform local, provincial and national policy.

Track D: Social Sciences: From Social and Health Vulnerabilities to Action

Volet D : Sciences sociales : Des vulnérabilités sociales et de santé à l'action

\section{6}

THE 'STOLEN GENERATIONS' OF MOTHERS AND DAUGHTERS: CHILD APPREHENSION AND ENHANCED HIV VULNERABILITIES FOR ABORIGINAL SEX WORKERS

Duff, Putu; Bingham, Brittany; Simo, Annick; Montaner, Julio;

\section{Shannon, Kate}

Vancouver, BC

OBJECTIVES: The number of children in care of the state continues to grow in BC, Canada with a historical legacy of child apprehension among criminalized and marginalized populations, particularly Aboriginal women and sex workers (SWs). However, there is a paucity of research investigating child apprehension experiences among marginalized mothers, or how this may relate to HIV risks. The current analysis examines the prevalence and associations with child apprehension among women SWs in Vancouver, Canada.

METHODS: Analyses were drawn from the AESHA (An Evaluation of Sex Workers Health Access, 2010-present), a prospective cohort of indoor and outdoor SWs, including a detailed interview questionnaire and HIV/STI screening on a semi-annual basis. Bivariate and multivariate logistic regression were used to examine prevalence and associations with child apprehension.

RESULTS: Of a total of $510 \mathrm{HIV}^{+}$and seronegative SWs, 350 women who had given birth to 1+ children were included in the analyses (median age $=37 \mathrm{yrs}$ : IQR: $31-44 \mathrm{yrs}$ ). The prevalence of child apprehension among mothers was $36.5 \%$, with $38.0 \%$ reporting have been apprehended themselves by child welfare services. In multivariate logistic regression analysis, servicing clients in outdoor public spaces compared to indoor settings (adjusted odds ratio, $\mathrm{aOR}=2.74$; 95\%CI 1.28-5.90), previously injecting drugs $(\mathrm{aOR}=2.52 ; 95 \% \mathrm{CI} 1.42-4.47)$, Aboriginal ethnicity $(\mathrm{aOR}=1.66 ; 95 \% \mathrm{CI} 1.01-2.74)$, having been apprehended as children themselves ( $\mathrm{aOR}=1.48 ; 95 \% \mathrm{CI} 0.90-2.43)$, and previous lifetime incarceration $(\mathrm{aOR}=1.44 ; 95 \% \mathrm{CI} 0.77-2.69)$ were associated with increased odds of child apprehension.

Discussion/CONCLUSIONS: Cycles of child protection continue to fail marginalized and criminalized populations of sex workers and compound HIV sexual and drug risks, with a $50 \%$ increased odds of having your child apprehended among mothers who were forcibly removed from their own homes as a child. Structural reforms to child protection are urgently needed, and must address the historical legacy of residential school and racialized policies among Aboriginal peoples.

\section{7}

READING BETWEEN THE LINES: WHAT CAN WE LEARN FROM SPONTANEOUS COMMENTS IN A TELEPHONE SURVEY ON HIV STIGMA

Beaulieu, Marianne; Beaudry, Sylvain; Adrien, Alix; Potvin, Louise; Dassa, Clément; Comité consultatif sur les attitudes envers les PVVIH

Montréal, QC

OBJECTIVE: Analyze the spontaneous comments made by respondents during a population-based telephone survey examining HIV stigma. METHOD: Data were collected during a population-based telephone survey conducted between March 15 and April 2, 2010 in the province of Quebec to document HIV stigma. Of the 1500 people who completed the survey, a subsample of 900 respondents was randomly selected and their complete telephone interviews were transcribed. Respondents' spontaneous comments -supplementary to those required to complete the surveywere entered into a database. A thematic content analysis was performed in order to explore the rich information emerging from these comments. A descriptive analysis (weighted to represent Quebec's population) was then conducted.

RESULTS: Over $60 \%$ of respondents made at least one spontaneous comment during their interview, with $27.6 \%$ commenting on HIV knowledge and $59.0 \%$ on HIV stigma questions. Among those who offered a spontaneous comment, several $(40.9 \%)$ detailed their position regarding social support for people living with HIV (PLHIV), 33.4\% expressed that their position on HIV criminalization depends on different factors and $26.9 \%$ discussed the issue of personal responsibility of PLHIV for their own health status. Sexual liberalism (19.9\%), willingness to interact with (9.6\%) and tolerance to be physically close to a PLHIV (9.1\%) were also commented on. Finally, 14.5\% clarified their stance on HIV disclosure and $6.4 \%$ of respondents elaborated on their feelings towards PLHIV. In their comments, several respondents expressed their concern for the potential risk of transmission or the discomfort they would feel in an unlikely hypothetical situation. Surprisingly, the analysis also revealed that the population is very critical of criminalization, most stating that it should be limited to situations in which transmission is deliberate.

CONCLUSION: If results suggest that a part of Quebec's population is sensitized to PLHIV, they also suggest that HIV stigma has become more subtle, which may reflect an irrational fear associated with HIV. It is important to find ways to study this new form of stigma in order to address it more effectively.

\section{8 \\ CONTEXT COUNTS: FACTORS INFLUENCING NEIGHBOURHOOD PERCEPTION AMONGST WOMEN AND MEN ACCESSING ANTIRETROVIRAL THERAPY IN BRITISH COLUMBIA}

Parashar, Surita; Zhang, Wendy; Salters, Kate; Montaner, Julio S; Hogg, Robert S

Vancouver, BC

BACKGROUND: The physical and social environment play a crucial role in shaping health outcomes and quality of life of people living with HIV (PHA). Housing status is well-established as an important node on the causal pathway linking the broader environment, including neighbourhood perception, to individual health outcomes. Within the Longitudinal Investigations into Supportive and Ancillary Health Services (LISA) cohort, men and women do not differ in their likelihood to be stably housed; however, women report significantly worse neighbourhood perception than men. This analysis examines the factors that influence neighbourhood perception of PHA, and the extent to which the salient factors vary between men and women.

METHODS: The LISA cohort is a cross-sectional study of individuals on antiretroviral therapy in British Columbia. Interviewer-administered surveys collect socio-demographic information and social determinants of health data. Clinical variables are obtained through longitudinal linkages with the provincial Drug Treatment Program database. Bivariable and multivariable analyses identified factors that influence neighbourhood perception among men and women in LISA.

RESULTS: Between 2007 and 2010, 1,000 participants were interviewed. Of the 908 participants eligible for inclusion in this analysis, 
249 (27.4\%) were female. Amongst women, stable housing decreased perception of neighbourhood problems by approximately $20 \%(\mathrm{p}<0.001)$ and increased perceived neighbourhood cohesion by $7 \%(\mathrm{p}=0.001)$. Amongst men, being food secure was associated with an $11 \%$ decrease in perceived neighbourhood problems $(\mathrm{p}<0.001)$, and a $4 \%$ increase in neighbourhood cohesion $(\mathrm{p}=0.017)$.

CONCLUSION: The findings suggest that vulnerability to various aspects of the environment differ between men and women. In a context where ART is available free of charge and yet gendered disparities in treatment uptake and effectiveness persist, understanding the differing vulnerabilities experienced by men and women may help identify genderspecific barriers and facilitators to uptake of, and adherence to, therapy. Interventions targeting food insecurity and housing instability may help cultivate health-enabling environments and ultimately enhance treatment outcomes and quality of life of men and women living with HIV.

\section{9 \\ INTIMATE PARTNER VIOLENCE IS ASSOCIATED WITH LOW SEXUAL DECISION MAKING POWER AMONG SEX WORKERS: POTENTIAL NON-COMMERCIAL HIV RISK PATHWAYS \\ Muldoon, Katherine A; Deering, Kathleen N; Feng, Cindy X; \\ Shoveller, Jean S; Shannon, Kate \\ Vancouver, BC}

OBJECTIVES: Globally, intimate partner violence (IPV) against women has been demonstrated to be associated with increased HIV risk. However, very little is known about IPV among sex workers (SW) in Canada or globally, or potential pathways with sexual HIV risks. The objective of this analysis is to investigate the prevalence and correlates of IPV among a cohort of SWs in Vancouver, Canada.

METHODS: We conducted an analysis of baseline data (interview questionnaire and HIV/STI screening) of SW enrolled in a longitudinal cohort, AESHA (An Evaluation of Sex Workers Health Access) recruited through outreach to street and indoor sex venues (e.g massage parlours, brothels). IPV was measured by the World Health Organization's Intimate Partner Violence Scale, capturing physical, sexual and emotional violence. Descriptive statistics characterize the prevalence of IPV and bivariate and multivariate logistic regression modeled the correlates of IPV among SW.

RESULTS: Out of 510 SW, $49.80 \%(n=254)$ reported having an intimate partner and were included in this analysis, of whom $35.7 \%(\mathrm{n}=97)$ were Asian migrant/new immigrant workers, 34.2\% ( $\mathrm{n}=93)$, Aboriginal and $30.1 \%(n=82)$ Caucasian. Lifetime prevalence of IPV was $67.3 \%$ and IPV in the last 6 months was $32.8 \%(n=83)$. In bivariate analyses, older age, having dependent children, and working in primarily indoor locations, were associated with reduced likelihood of IPV, while homeless and noninjection drug use increased odds of IPV $(\mathrm{p}<0.05)$. In final multivariate logistic regression, SW were significantly less likely to experience IPV if they were Asian migrant/new immigrant workers (aOR: 0.25, 95\%CI: 0.10-0.67), and significantly more likely to experience IPV if they had low sexual relationship power (aOR: 6.55, 95\% CI: 2.58-16.65) after controlling for other covariates.

DISCUSSION/CONCLUSIONS: This analysis highlights the high burden of violence by intimate partners and reduced control over sexual decision-making among SWs in Canada. Our results suggest an important potential pathway of sexual risk for HIV infection through noncommercial intimate partners. Structural gender transformative violence prevention should be integrated within HIV prevention, treatment and care initiatives for sex workers and their intimate partners.

\section{0}

TAKING ACTION ! ABORIGINAL YOUTH (DE) COLONIZING HIV PREVENTION AND LEADERSHIP THROUGH THE ARTS

Yee, Jessica J; Youth, Taking Action; Konsmo, Erin M Toronto, ON

Taking Action! is a national project that worked with six Aboriginal communities across Canada. This presentation will showcase and centre arts-based and youth-led methods. Youth coordinators were key in connecting local Aboriginal youth to the Taking Action project and leading
HIV education in this national community-based project. Preliminary results from Taking Action show that youth feel both the process and product of arts-based methods are important. In particular, arts-based methods allow the ability for Aboriginal youth to connect with culture, build on their own sense of identity, and work through issues such as how colonization has affected them. Art allows for Aboriginal youth to express in a form that engages their culture and revitalizes spirit in a way that other methods do not allow. While traditional art forms allowed for specific cultural teachings (eg, talking sticks), other art forms allowed for new culture to be engaged (eg, graffiti \& hip-hop). In terms of the process, they identified several benefits including that arts-based methods are: Fun (engaging, not boring), Participatory (hands-on), Empowering (we get up and do it), Build self esteem (helps you feel good about yourself), Healing (through transformation), Draw on assets (youth are good at it), Teach cultural practices and skills (art teaches us new things). In addition, the process enhances recall and helps to facilitate dialogue about difficult topics. The products also: instill pride, communicate and transmit information, raise awareness, convey emotion, draw youth into learning about HIV, are tangible, long lasting, and can bridge, change and challenge traditional culture. Aboriginal youth, with the help of artists and community partners, shared their art with members of their communities to continue conversations around HIV and prevention. These lasting conversations sparked a movement among Aboriginal youth to continue using these methods to carry on HIV prevention in sustainable and meaningful ways.

\section{O111}

OPERATIONALIZING QUALITATIVE HIV RESEARCH FIELDWORK IN A RESOURCE-POOR COUNTRY: LESSONS LEARNED IN LUSAKA, ZAMBIA REGARDING RIGOUROUS AND ETHICAL RESEARCH PROCEDURES

Nixon, Stephanie ${ }^{1}$; Cameron, Cathy ${ }^{1}$; Hanass-Hancock, Jill ${ }^{2}$; Simwaba, Phillimon ${ }^{3}$; Solomon, Patty ${ }^{4}$

${ }^{1}$ Toronto, ON; ${ }^{2}$ Durban, South Africa; ${ }^{3}$ Harare, Zimbabwe; ${ }^{4}$ Hamilton, ON

BACKGROUND: The participation of Canadian researchers in qualitative HIV research conducted in resource-poor countries is growing, with a parallel increase in guidelines and practical tools related to key moral philosophical considerations (e.g., the Partnership Assessment Tool developed by the Canadian Coalition for Global Health Research). These contributions regarding ethical research, particularly as they relate to equitable partnership development, provide an essential foundation. A crucial next step involves the ethical and rigourous implementation of empirical research in the field. However, there are few practical guidelines regarding the "how-to" of implementation of qualitative research in resource-poor settings.

OBJECTIVE: To describe methodological challenges and practical solutions for operationalizing qualitative HIV research fieldwork in a resource-poor setting (Lusaka, Zambia).

METHODS: These lessons were derived from a series of reflective exercises conducting during and following the Sepo Study, a qualitative research project conducted by an international research team that explored the experiences of people living with HIV and disability in Lusaka, Zambia.

RESULTS: Lessons learned regarding the operationalization of qualitative research fieldwork include: (1) strategies for non-coercive participant recruitment, (2) protocols related to data collection, including issues related to transcription, translation and management of data, (3) conducting interviews with deaf/hard of hearing participants, (4) specific Internet and cell phone-based mechanisms for development of an audit trail, (5) clarifying expectations and processes for accessing data among team members, and (6) techniques for collaborative data analysis engaging multiple forms of expertise and knowing in multiple geographic settings.

CONCLUSION: It is crucial to reflect on and share procedural dimensions of qualitative HIV research fieldwork in resource-poor settings as a complement to the growing body of literature on ethical guidelines and ethical partnership development. The lessons learned here contribute to the effort to collectively develop more effective, efficient and ethical qualitative research processes. 
0112

TOWARDS A SUPERVISED INJECTION SERVICE IN MONTRÉAL: A FEASIBILITY STUDY THAT LED TO AN IMPORTANT MOBILIZATION OF LOCAL AND REGIONAL PARTNERS

Morissette, Carole; Larouche, Annie; Lessard, Richard;

Gutierrez, Natalia; Leclerc, Pascale; Labesse, Daniel;

Gagnon, François; Goyer, Marie-Ève; Mondou, Isabelle

Montréal, QC

BACKGROUND: In Montréal, HIV/AIDS, hepatitis C and drug overdoses are taking a toll on the injection drug user (IDU) population. These problems may be exacerbated by changes in drug consumption patterns, such as injecting prescription opioids. Therefore, we must rethink our interventions and define new strategies that fit IDUs' needs. A supervised injection service (SIS) is one of these strategies known to be effective in reducing harm. This is why Montréal's public health department conducted, in 2011, a feasibility study to conceptualize a regional SIS offer and identify the conditions that would allow its implementation. This presentation discusses the study approach and the recommendations that resulted.

METHOD: Based on the theory of social innovation, the study consisted of a participative approach led by a steering committee composed of 27 partner organizations. It included continuous and structured exchanges, use and sharing of knowledge and experiences from here and elsewhere, as well as numerous consultations with stakeholders (IDUs, caregivers from public and community organizations, police officers, professional orders, emergency services, legal departments of public institutions, political and institutional actors, resident and merchant associations).

RESULTS: Six recommendations result from the issues and conditions identified during the feasibility study: 1) create an SIS network in collaboration with the public health network and community organizations; 2) deliver the service through fixed facilities and a mobile component; 3) appoint a team of dedicated doctors and nurses to the region; 4) reinforce the capacity of involved community organizations; 5) put in place a regional coordinating committee; 6) develop a surveillance and monitoring framework.

CONCLUSION: The study demonstrates the legal and organizational feasibility of implementing an SIS in Montréal, and the acceptability and conditions for collaboration identified by the stakeholders. The outcome is extensive mobilization of all partners and support from relevant professional orders. Next steps include funding, applying for an exemption from the federal law and establishing collaboration agreements.

\section{3}

\section{NEGOTIATING IDENTITY AND SPACE: INSIGHTS FROM AN INVESTIGATION OF HARM REDUCTION IN A HOSPITAL SETTING}

\section{Strike, Carol; de Prinse, Karen; Guta, Adrian; Switzer, Sarah}

\section{Toronto, $\mathrm{ON}$}

BACKGROUND: Harm reduction services have been effective in improving various health outcomes for drug users, including reducing the transmission of HIV. Whereas most harm reduction services are offered through community-based programs, this presentation explores the impact of a formal harm reduction policy in a hospital-based setting. To date, little empirical research has been conducted on integrating harm reduction outside of community-based programming contexts.

METHODS: Data were collected as part of a capacity building grant to assess using arts-based approaches to engage people living with HIV in a discussion about harm reduction. This presentation reports on data from photo elicitation sessions with residential and community clients. Participants were provided with a disposable camera during a group information session and asked to take photos that represented their understanding of the harm reduction policy. Following the return of cameras and photo development, semi-structured interviews were conducted with individual participants $(\mathrm{n}=$ 13) to discuss the meanings they ascribed to their photos. Thematic analysis was conducted of transcribed audio and photographic data.

RESULTS: Our analysis surfaced rich insights into the ways drug use and identity are negotiated in a hospital setting. In particular, ambiguities emerged over what constitutes 'drug use' and who is a 'drug user'. Participants evoked characterisations of the 'good patient' to contrast with the 'drug user'. Participants offered accounts of how the harm reduction policy influences their individual health seeking, ability to access available services, and negotiating the use of private and communal space. Participants offered recommendations on how to create future spaces which address collective needs.

CONCLUSIONS: Overall, drug use and harm reduction approaches pose unique challenges in hospital-based care because of the restrictions placed by hospital protocols and regulations. These constraints pose barriers to effectively addressing the needs of drug-users and may result in tension between users and non-users housed together.

\section{Track D: Social Sciences: A Selection of the Best Abstracts Volet D : Sciences sociales : une sélection des meilleurs abrégés}

\section{O114}

BODY, AGING AND HIV

Wallach, Isabelle; Ducandas, Xuân; Martel, Michel; Trottier, Benoit; Thomas, Réjean

Montréal, QC

BACKGROUND: The population of people living with HIV aged 50 and over (PLHIV50+) is increasingly growing in western countries. In Canada, in 2008, 15.3\% of new HIV diagnoses and 21.6\% of AIDS cases occurred among the age group of 50 and over. People living with HIV face premature aging related to persistent immune dysfunction and inflammation. This aging process confronts (PLHIV50+) with premature physical changes.

OBJECTIVES: The premature aging induced by HIV infection confronts people living with HIV 50 years and over (PLHIV50+) in early bodily changes, both in appearance and health. The objectives of this presentation are to document these changes in the body, to examine the subjective experience of these changes and identify their impact on personal and social life of PLHIV50+.

METHODOLOGY: This qualitative research based on semi-structured interviews conducted with 38 participants living with HIV aged between 50 and 71 years. Data analysis is part of an ethnographic qualitative approach and is performed with the help of the software QDAMiner.

RESULTS: PLHIV50+ experiment many physical changes related to their pathology, drug side effects and premature aging (such as physiological changes and cognitive disorders). The intimate transformation of appearance, for instance, seems to affect the self-image and the love and life of the participants. In terms of the impact in social life, health problems and low energy related to HIV and aging may hinder participation in social activities or force PVVIH50 + to give up their work earlier, thus contributing to their isolation. The analysis of the speech participants showed different attitudes to these physical changes, ranging from acceptance to rejection. CONCLUSION: The bodily changes associated with the interaction of HIV and aging can create significant barriers to intimacy and social PVVIH50+ and affect their psychological well-being. It is essential for physicians and public health policies to take into account these physical changes and their psychosocial impact.

\section{5}

\section{SEXUAL HEALTH, RACISM AND BODY IMAGE AMONG ETHNORACIALIZED GAY AND BISEXUAL MEN IN TORONTO}

Brennan, David J ${ }^{1}$; Newman, Peter $A^{1}$; Ceranto, Andre ${ }^{1}$;

George, Clemon ${ }^{2}$; Hart, Trevor $\mathrm{A}^{1}$; Persad, Ishwar ${ }^{1}$

${ }^{1}$ Toronto; ${ }^{2}$ Oshawa, ON

BACKGROUND: Previous studies drawn from predominantly White samples have not adequately captured issues of body image among ethnoracialized gay and bisexual men (EGBM). The purpose of the present study was to examine the relationship between racism, body image and sexual health among EGBM in Toronto.

METHODS: A community advisory committee (CAC) of 9 EGBM advised the research team on study design, data collection and analysis. Eligibility criteria included being over age 18 , identifying as gay, bisexual or having had sex with another man in the past year, and identifying with one of four ethnoracial communities (Black/African/Caribbean, South Asian, East/ Southeast Asian, and Latino/Hispanic/Brazilian). Study participants were 
recruited through community agencies and list serves. Two to three focus groups for each ethnoracial community $(\mathrm{n}=58)$ and individual interviews $(n=3)$ were conducted, audio recorded and transcribed verbatim. Data analysis employed grounded theory techniques, aided by MAXQDA software. Member checking was conducted with the CAC for feedback.

RESULTS: Three major themes emerged across four ethnoracial groups: 1) The impact of White masculine body image ideals upon EGBM; 2) Negotiating a racialized body; and 3) The impact of body image on sexual health and relationships. EGBM actively negotiate pressures to conform to White masculine body ideals in the gay/bisexual culture and resisted against racialized body stereotypes. Furthermore, body image among EGBM impacted their sexual health and relationships. Analysis of between-group differences suggested the following: 1) Persistent race-specific stereotypes affect EGBM differentially depending on their ethnoracial identity; and 2) Experiences of racialization of bodies among EGBM were as diverse within ethnoracial groups as between groups. IMPLICATIONS: Our findings highlight the impact of racism on body image for EGBM navigating the White dominant GBM's socio-sexual culture. Future research should explore effective interventions to reduce racism and its impact on body image and sexual health among EBGM.

\section{6 \\ "FINDING OUR PLACE IN THE ENVIRONMENT": ART AND POSITIVE ABORIGINAL WOMEN'S PERCEPTIONS OF HEALTH}

Prentice, Tracey ${ }^{1}$; Peltier, Doris ${ }^{2}$

${ }^{1}$ Ottawa, ON; ${ }^{2}$ Vancouver, BC

BACKGROUND: Previous research on Positive Aboriginal Women (PAW) has focused primarily on their HIV-illness and the gaps and needs that arise from living with HIV. While this has allowed us to develop policies and services to meet these needs, it may have also contributed to dominant and disempowering representations of Aboriginal women living with HIV as troubled, vulnerable and in need of outside assistance. In this presentation, we explore PAWs perceptions of 'health' instead of illness, and we consider the individual and community strengths that some PAW use to create or maintain their health.

METHODS: We used a decolonizing methodology including strengthsbased, arts-informed, and community-based participatory action research methods. Local service providers helped to engage three small but diverse groups of PAW in three arts-informed group research processes $($ Toronto $=5$; Montreal $=4$; Skype $=4)$. Each group met for $40+$ hours over two weeks to six months and participated in two focus group discussions, data analysis and project evaluations. All groups came together for one in-person meeting to discuss policy and service implications.

RESULTS: 'Health' for PAW in this study is about much more than their HIV-status. Health is holistic and includes mental, physical, spiritual, emotional, social and environmental aspects. Health is about reclaiming and celebrating their culture and identity, healing and empowerment, spirituality, and finding their place in the environment. Health is also about connecting with families, friends and communities, finding and using their 'voice', and learning to believe in themselves.

CONCLUSION: This paper considers, for the first time, how PAW perceive and experience their 'health' as opposed to their illness. PAW's holistic and strength-based interpretation of health has implications for the kinds of, and the range of services that are provided. We conclude with a discussion of these implications.

\section{7 \\ FERTILITY DESIRES AMONG HIV POSITIVE AND SERONEGATIVE WOMEN: RECOGNIZING THE RIGHTS AND WANTS OF SEX WORKERS AS MOTHERS} Duff, Putu; Feng, Cindy; Chettiar, Jill; Shoveller, Jean; Montaner, Julio; Shannon, Kate

Vancouver, BC

OBJECTIVE: Public health research and programming in Canada among female sex workers (FSWs) who have children have almost exclusively focused on disease prevention and child protection, with limited consideration of their reproductive needs and rights. Furthermore, with HAART dramatically improving the life expectancy of HIV-positive women, there remains an important need to understand the reproductive health needs and desires of FSWs, both seronegative and those living with HIV/AIDS.

METHODS: Data were drawn from a baseline sample of an open prospective cohort of street and indoor sex workers in Vancouver, known as An Evaluation of Sex Workers' Health Access (AESHA). In partnership with local sex work and community agencies, FSWs are recruited through outreach to street and off-street locations (e.g., massage parlours, micro-brothels) and complete interview-administered questionnaires and HIV/STI testing. Bivariate and multivariate logistic regression were used to evaluate independent associations with current fertility desires and fertility desires, adjusted for potential confounders.

RESULTS: Of the 510 women at baseline, 394 (77.3\%) reported prior pregnancy, with 104 (27.5\%) reporting positive fertility desires. 38.4\% were of Aboriginal ancestry, 35.3\% were Caucasian, and 23.7\% were Asian new immigrant/migrant workers, with no significant differences in fertility desires by ethnicity. In our final multivariate model, FSWs with positive fertility desires had higher odds of being younger (median $=29$ years vs. 38years, adjusted odds ratio $(A O R)=0.89 ; 95 \%$ Confidence Interval (CI) 0.87-0.92), inconsistent condom use by commercial clients (AOR $=1.81 ; 95 \% \mathrm{CI} 1.06-3.10)$ and servicing clients in indoor locations(AOR=1.99; 95\% CI 1.07-3.71).

DISCUSSION/CONCLUSIONS: Over a quarter (27.5\%) reported a desire for (more) children, with no differences by HIV status. Of particular concern, FSWs reporting fertility desires were also more likely to report inconsistent condom use by clients, suggesting the critical need to marry HIV/STI and reproductive health/parenting services for this population, particularly for those working in hidden indoor settings.

\section{8 \\ THE WAY I SEE IT: A PHOTOGRAPHIC EXPLORATION OF HOUSING AND HEALTH AMONG PEOPLE LIVING WITH HIV IN VANCOUVER, BRITISH COLUMBIA}

Parashar, Surita $^{1}$; Salters, Kate ${ }^{1,2}$; Hogg, Robert $\mathrm{S}^{1,2}$

1Vancouver; ${ }^{2}$ Burnaby, BC

BACKGROUND: Vancouver, British Columbia has one of the highest HIV prevalence rates in Canada as well as a growing housing crisis fueled by rising housing costs, reduced income assistance, the lowest minimum wage and the highest poverty rate in the country. The limited data available on the impact of housing instability on the health of people living with HIV in $\mathrm{BC}$ has been generated without meaningfully involving community; consequently, it does not reflect the experiences of affected populations. This community based research initiative aims to develop an understanding of the housing-health nexus that is grounded in PHA's lived realities.

METHODS: Within a CBR framework this project adapts Photovoice, a research method used to assist people, often marginalized by socialstructural inequity, to reflect on their strengths, capacities and needs, engage with policymakers and encourage social change. Five men and four women were recruited through purposeful sampling at HIV/AIDS Service Organizations and trained as Community Researchers (CR). CR generated and analyzed over 300 photographs, as well as transcripts of group and one-on-one discussions in order to identify emergent themes. RESULTS: In the preliminary phases of data analysis, CR identified key conceptual categories, which were subsequently organized into two domains: resiliency promoted by healthy environments (ie, sense of belonging, emotional security) and the detrimental impact of inadequate housing on physical and mental health (ie, depression, addictions relapse). CR broadly conceptualized 'home' as any sanctuary where one is able to rest and momentarily forget challenges and trauma with which they concurrently cope. For individuals who had experienced homelessness, the services that provide low threshold support to PHA were consistently identified as a "home away from home." The most prevalent unmet need identified was transitional spaces and opportunities for individuals who are "post-street" to live, socialize, heal and rebuild.

CONCLUSIONS: Photos and accompanying narratives describing the living arrangements in which PHA must negotiate health-related decisions are a powerful tool in building a comprehensive, communityinformed definition of healthy housing as viewed through the eyes of PHA. 
BASIC SCIENCES/SCIENCES FONDAMENTALES

Anti-retroviral Drugs, Microbicides, and Vaccines (Preclinical)

Médicaments antirétroviraux, microbicides et vaccins (préclinique)

\section{P001}

\section{ENHANCING IMMUNOGENICITY OF HIV-1 ANTIGENS} USING B-CELL IMMUNOGENS

Clayton, Kiera L; Liu, Jun; Satkunarajah, Malathy; Rini, James; Ostrowski, Mario

Toronto, ON

BACKGROUND AND RATIONALE: Failure of envelope-based HIV vaccines to induce broadly neutralizing antibody responses can be attributed to the lack of gp160 immunogenicity. Studies have shown that immunogenicity can be improved by using antigens that mimic the native trimeric structure of gp160. Furthermore, linking HIV-1 antigens to Tumor Necrosis Factor Superfamily (TNFSF) molecules, such as CD40L, has also enhanced antibody responses in some studies. However, there are no reports of using antigen linked TNFSF members BAFF or APRIL as HIV vaccine adjuvants, both of which induce B-cell signalling, enhance isotype switching, affinity maturation, and development of antibodies directed against carbohydrates. We hypothesize that trimeric HIV-1 envelope fused to APRIL, BAFF or CD40L will enhance the immunogenicity of HIV-1 envelope based-vaccines and elicit a broadly neutralizing antibody response. Here we report the development and production of these fusion proteins for vaccine studies.

METHODS: Five fusion protein constructs were created, each with a protease resistant YU-2 gp140, four with a C-terminal Fibritin Foldon (F) trimerization domain, and three with $\mathrm{C}$-terminal fusions to either murine APRIL, BAFF or CD40L. Following expression in HEK293Ts, the constructs were assessed for proper expression, gp140 folding, and trimerization, via protein gel electrophoresis/western blotting, immunoprecipitation, and gel filtration/Blue Native-PAGE, respectively.

RESULTS: The trimerization domain significantly enhanced the stability of the trimeric form of the fusion protein. Furthermore, anti-envelope monoclonal antibodies F105, b12, 2G12, 447-52D, and 17b, confirmed that all proteins mimicked the natively folded state of the HIV-1 envelope protein. Endotoxin-free proteins are currently being prepared in milligram amounts for mouse studies.

CONCLUSIONS: We have successfully designed and developed five vaccine candidate antigens gp140-F-APRIL, gp140-F-BAFF, gp140-FCD40L, gp140-F, and gp140. Following TNFSF functional assays, these candidate proteins will be used in a mouse DNA-prime protein-boost vaccination strategy to assess their abilities to elicit broadly neutralizing, class-switched antibodies.

\section{P002 \\ GENE THERAPY USING SECRETED ANTI-HIV PROTEINS TO PROTECT UNMODIFIED TARGET CELLS}

Falkenhagen, Alexander; Ameli, Masoud; Asad, Sabah; Read, Stanley E; Joshi, Sadhna

Toronto, ON

The number of highly active anti-HIV proteins that efficiently prevent HIV entry into target cells is growing constantly. However, their lifelong application via injections is neither practical nor cost-effective. In contrast, delivery of these proteins via gene therapy has the potential to provide long-term control of HIV replication via a single treatment. We assess here the feasibility of developing a gene therapy using secreted soluble $\mathrm{CD} 4$ (sCD4) and $\mathrm{sCD} 4$ fusion proteins (sCD4 connected to a fusion inhibitor, a single chain antibody, or both), for the systemic delivery of anti-HIV proteins that prevent HIV entry not only in gene-modified, but also unmodified target cells.
The anti-HIV proteins were efficiently secreted into the cell culture medium of gene-modified $293 \mathrm{~T}$ cells and significantly reduced infection of unmodified MAGI-CCR 5 cells at nanomolar concentrations in single-round-infection assays with HIV-1(JRFL). The effect was reversed when the proteins were specifically removed from the medium via affinity chromatography, showing that the proteins and not another secreted factor mediated the entry reduction. We also observed that the purified proteins effectively neutralized entry of HIV-1(JRFL) in a dose-dependent manner, while entry of VSV-G pseudotyped particles remained unaltered. When tested at the same concentration, the potency of the proteins was superior to the commercially available fusion inhibitor T20. We could also show that gene-modified MAGI-CCR5 cells themselves were not only highly resistant, but also protected neighbouring unmodified cells from infection. This significant protective bystander effect was further observed when we used HIV-1(NL4-3) to infect unmodified peripheral blood mononuclear cells co-cultured with gene-modified 293T cells. Overall, this study highlights that anti-HIV proteins secreted from genemodified cells can protect unmodified HIV target cells. Therefore, other cell types, such as hematopoietic stem cells, B cells, fibroblasts, or epithelial cells, could be genetically modified to allow a systemic delivery of novel therapeutic proteins for HIV-1 treatment.

\section{P003}

\section{SIRNA DELIVERY USING NANOSYSTEMS TO PREVENT HIV} INFECTION

Lavigne, Carole ${ }^{1}$; Kell, Arnold J1 ; Thierry, Alain R ${ }^{2}$; Slater, Kathryn ${ }^{1}$; Barnes, Michael $^{1}$; Jasinghe, Viraj J ${ }^{1}$; Gajayanaka, Niranjala ${ }^{1}$;

Stals, Sandra ${ }^{1}$

${ }^{1}$ Ottawa, ON; ${ }^{2}$ Montpellier, France

The development of non-viral DNA delivery systems using nanomaterials has attracted much research interest for its potential in biomedicine. However, for these new nanocarriers to be successfully used in therapeutic applications they have to overcome many barriers. One major limitation is the stability of the complexes in the presence of serum. Here, we report the development and characterization of two different nanocarriers: the Neutraplex delivery system and functionalized silica nanoparticles. We compared their efficiency to deliver active anti-HIV oligonucleotide-based therapeutic agents in the presence of serum to the commonly used cationic commercial transfection reagent Lipofectamine RNAiMAX.

Intracellular localization of a fluorescent siRNA was monitored in live cells using fluorescence microscopy and cellular uptake was quantified by flow cytometry. Silencing activity was measured by flow cytometry, RT PCR and Western Blot analysis. Inhibition of HIV infection was mon itored using the TZM-bl indicator cell line and cell cytotoxicity using the CellTiter Blue assay. We found that both nanocarriers were able to deliver active siRNA in the presence of serum with high level of efficiency comparable to the commercial cationic formulation. In addition, their low cytotoxicity presents an advantage for in vivo RNAi therapeutics. When Neutraplex was used to deliver a siRNA targeting the co-receptor CXCR4, reduction of HIV infection was observed in TZM-bl cells showing its potential as a vehicle for delivering anti-HIV therapeutic agents. Altogether, our findings support further investigation for in vivo delivery of therapeutic siRNAs using nanomaterials.

\section{P004}

\section{THE DEVELOPMENT OF CYNOMOLGUS MACAQUE CYTOMEGALOVIRUS (CYCMV) AS AN HIV/SIV VACCINE VIRAL VECTOR}

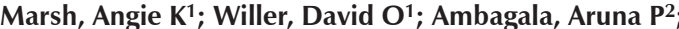

Dzamba, Misko ${ }^{1}$; Chan, Jacqueline $\mathrm{K}^{1}$; Pilon, Richard ${ }^{3}$,

Fournier, Jocelyn ${ }^{3}$; Sandstrom, Paul ${ }^{3}$; Brudno, Michael' ${ }^{1}$;

MacDonald, Kelly $\mathbf{S}^{1}$

${ }^{1}$ Toronto, ON; ${ }^{2}$ Lethbridge, AB; ${ }^{3}$ Ottawa, ON

Within the field of HIV vaccine design there is a real need to diversify the pool of vectors undergoing testing. Recent studies by Picker et al. using rhesus macaque cytomegalovirus (RhCMV) as an SIV vaccine 
vector have shown much promise. In an effort to examine RhCMV-based viral vectors in cynomolgus macaques, we have discovered that CMV strains are highly species-specific between even closely related NHPs and that cynomolgus macaques are not readily infected with RhCMV. To overcome this species-specificity, we have recently isolated and described the complete genomic sequence and organization of a novel cynomolgus macaque CMV (CyCMV; Ottawa strain) for its development as an alternative NHP model to evaluate CyCMV as an HIV/SIV viral vector. The CyCMV genome is 218,041 bp in length, with $49.5 \% \mathrm{G}+\mathrm{C}$ content and $84 \%$ protein-coding density. We have identified 262 putative open reading frames (ORFs) with an average coding length of $789 \mathrm{bp}$. The genomic organization of CyCMV is largely collinear with that of RhC$\mathrm{MV}$. We compared and contrasted the structural and functional genes of CyCMV to HCMV as well as RhCMV with respect to pathogenesis, immune evasion, and species-specificity. Similar to HCMV, CyCMV does not contain the RhCMV-specific viral homologue of COX-2. In order to maximize the ability to manipulate the virus for vaccine purposes, we are cloning CyCMV as a BAC. To initiate cloning of CyCMV-BAC, we have generated a recombinant CyCMV-GFP virus. This newly characterized CMV may provide a novel model in which to study HIV/SIV CMV-based vaccines and HCMV vaccine development in hopes of directing forthcoming clinical trials in humans.

\section{P005 \\ RESISTANCE PATHWAYS TO THE SECOND-GENERATION INTEGRASE INHIBITOR DOLUTEGRAVIR}

Mesplede, Thibault ${ }^{1}$; Quashie, Peter $\mathbf{K}^{1}$; Han, Yingshan ${ }^{1}$; Singhroy, Diane N1; Osman, Nathan ${ }^{1}$; Oliveira, Maureen ${ }^{1}$; Fujiwara, Tamio ${ }^{2}$; Underwood, Mark $\mathbf{R}^{3}$; Wainberg, Mark $\mathbf{A}^{1}$ ${ }^{1}$ Montreal, QC; ${ }^{2}$ Osaka, Japan; ${ }^{3}$ Research Triangle Park, NC, USA HIV-1 integrase strand transfer inhibitors (INSTIs) specifically target the strand transfer step of integration. Although INSTIs have demonstrated their efficacy against HIV-1 in vitro and in patients, being particularly useful against viruses resistant to other drug classes, first-generation INSTI-based therapies might be threatened by the emergence of resistance mutations. Second-generation drugs such as MK-2048 and dolutegravir (DTG) are INSTIs designed with a higher genetic barrier to resistance. Being the latest class of antiviral drugs to have been developed against HIV, INSTIs are deemed to become an important part of the arsenal against the pandemic. It is therefore essential to gain a better understanding of the resistance pathways that can emerge against these new drugs.

Through selection studies with DTG in primary human cells, we have demonstrated that $\mathrm{R} 263 \mathrm{~K}$ is a resistance mutation against this drug. In addition, we identified the frequent emergence of the secondary mutation H51Y. We have now investigated the role of this secondary mutation on viral fitness and resistance to DTG. Wild-type and mutated recombinant HIV-1 integrases were purified and in vitro biochemical assays were used to measure the effect of these mutations on strand transfer activity and susceptibility to DTG. In addition, the H51Y mutation was introduced into HIV-1 proviral DNA by site-directed mutagenesis and the corresponding viruses were produced. We then used cell-based infectivity and replication assays to further characterize the effects of the secondary mutation $\mathrm{H} 51 \mathrm{Y}$ on fitness and resistance of the resulting virus. Altogether, our results reveal for the first time the existence of a new resistance pathway against DTG, in which $\mathrm{H} 51 \mathrm{Y}$ is a secondary mutation that augments the effects of the primary R263K mutation.

\section{P006 \\ ANGIOGENIC FACTOR IMBALANCE IN CART EXPOSED TROPHOBLAST CELLS}

Papp, Eszter ${ }^{1}$; Serghides, Lena ${ }^{2}$

${ }^{1}$ Vancouver, BC; ${ }^{2}$ Toronto, ON

BACKGROUND: Having small for gestational age (SGA) babies is a common complication for HIV/ cART exposed pregnant women. The proper formation of blood vessels (angiogenesis) is crucial for optimal placental function. A well-maintained balance of multiple angiogenic factors is necessary for placental angiogenesis. Important placental angiogenic factors include vascular endothelial growth factor (VEGF), placental growth factor (PlGF), Angiopoietin-1 (Ang-1) and Angiopoietin-2 (Ang-2) and their receptor Tie-2. Antiretroviral (ARV) drugs, especially protease inhibitors can alter the levels of these factors, possibly leading to abnormal development of placental blood vessels.

METHODS: Placental chorioblastoma (HTR-8/Svneo) cells were incubated in the presence of ARV drugs; AZT/3TC was used in combination with either ritonavir-boosted Atazanavir (ATV/r), Darunavir (DRV/r) or Lopinavir (LPV/r) for 24 hours in concentrations representing 10, 100, 1000 and 10000-times of minimum essential plasma concentrations. Levels of secreted VEGF, PIGF, Ang-1, Ang-2 and Tie-2 were quantified by ELISA. Hypoxia ( $1 \%$ oxigen) was used as a positive control. Pearson correlation was performed for statistical analysis.

RESULTS: ARV drug exposure resulted in concentration-dependent changes in angiogenic factors. VEGF levels increased with increasing drug concentrations (Pearson coefficients: ATV/r: r=0.93, DRV/r: r=1.00, LPV/r: $r=0.99$ ). PlGF levels showed no change in ATV/r exposure, but decreased with the other drug combinations (Pearson coefficients: ATV/r: $\mathrm{r}=0.222, \mathrm{DRV} / \mathrm{r}: \mathrm{r}=-0.583, \mathrm{LPV} / \mathrm{r}: \mathrm{r}=-0.954)$. Ang-1 levels decreased with ARV exposure (ATV/r: $\mathrm{r}=-0.451$, DRV/r $\mathrm{r}=-0.468, \mathrm{LPV} / \mathrm{r} \mathrm{r}=-0.872$ ). There was a slight change in Ang-2 levels (Pearson coefficients: ATV/r: $\mathrm{r}=-0.484$, DRV/r: $\mathrm{r}=-0.432, \mathrm{LPV} / \mathrm{r}: \mathrm{r}=0.119$ ). The secretion of soluble Tie-2 was also decreased as a result of ARV exposure (Pearson coefficients: ATV/r: $r=-0.506$, DRV/r: $r=-0.460$, LPV/r: $r=-0.476)$

CONCLUSION: Our data suggest that cART may alter the angiogenic balance in the placenta, potentially playing role in adverse birth outcomes in HIV/cART exposed pregnancies.

\section{P007 \\ DETERMINATION OF THE CRITERIA TO DESIGN EFFECTIVE HEPATITIS DELTA VIRUS RIBOZYMES TARGETING HIV RNA FOR INHIBITION OF HIV REPLICATION IN GENE THERAPY}

Scarborough, Robert ${ }^{1}$; Motard, Julie' ${ }^{2}$; Levesque, Michel$^{2}$; Daniels, Sylvanne'; Shaw, Eileen ${ }^{1}$; Daher, Aicha ${ }^{1}$;

Perreault, Jean-Pierre ${ }^{2}$; Gatignol, Anne ${ }^{1}$

${ }^{1}$ Montreal; ${ }^{2}$ Sherbrooke, QC

Nucleic acid therapies targeting HIV replication have the potential to be used in conjunction with, or in place of the standard combination therapies. Improvements in the delivery of nucleic acids to HIV target cells could lead to the eventual use of these agents in drug therapy, and several studies have supported the possibility of using them in a gene therapy setting.

Among the different classes of nucleic acid therapies, several hammerhead and hairpin ribozymes (Rzs, RNA enzymes) have been designed to target HIV RNA, and some have advanced to clinical trials. Results have demonstrated that $\mathrm{R} z$ therapy is safe, however improvements in efficacy are required. In an attempt to improve upon the stability and activity of available Rzs targeting HIV RNA, we have designed and evaluated several Specific On/oFf Adaptor, Hepatitis Delta Virus (SOFA-HDV) Rzs. We have developed an algorithm to identify SOFA-HDV Rzs that have the ability to target the majority of HIV clinical isolates published on the Los Alamos HIV database. Rzs were evaluated for their ability to cleave HIV RNA in vitro and for their ability to restrict HIV virus production in cell culture. In combination with an anti HIV drug (Atazanavir) or with a short hairpin RNA, selected Rzs showed an additive effect on the inhibition of HIV virus production, suggesting that they could be used effectively in a combination gene therapy approach.

Our results suggest that the target site for SOFA-HDV Rzs needs to be accessible, highlighting the importance of target site selection in the design of Rzs targeting HIV RNA. We have identified Rzs that have a high potential to be used effectively in a combination gene therapy approach for the treatment of HIV infection, and may be able to be used in drug therapy with appropriate delivery vehicles.

\section{P008 \\ MITOCHONDRIAL DNA OXIDATIVE DAMAGE IN NRTI- EXPOSED CULTURED HUMAN CELLS}

Sohi, Gurmeet K; Hukezalie, Kyle; Wong, Judy; Côté, Hélène Vancouver, BC

BACKGROUND: NRTIs can affect mitochondrial DNA (mtDNA) quantity and quality. This may lead to mitochondrial dysfunction which 
is associated with degenerative diseases and aging. We previously determined that treatment with NRTIs (d4T, ddI, AZT, ABC, TDF but not 3TC) accelerated telomere shortening in cultured human colorectal adenocarcinoma cells (HT29), through telomerase inhibition and/or oxidative stress. We sought to quantify and compare mtDNA oxidative damage in cultured cells exposed to these NRTIs.

METHODS: HT29 were continuously exposed to various concentrations of d4T, ddI, AZT, ABC, TDF, and a single concentration of 3TC for $\sim 30$ days. Relative mtDNA oxidative damage was quantified using a qPCR-based assay. This assay exploits the fact that PCR amplification by Taq polymerase is slowed by DNA oxidative damage and this can be quantified relative to undamaged control DNA. Cellular mtDNA content was also quantified by qPCR.

RESULTS: d4T and ddI treatments were accompanied by a concentration-dependent decrease in cellular mtDNA content while exposures to AZT, ABC, and 3TC conversely showed mild to moderate concentration-dependent increases in mtDNA content. TDF was cytotoxic to HT29 cells before day 20. There was no evidence of increased oxidative damage to mtDNA with any of the NRTIs tested

CONCLUSIONS: The observed mtDNA depletion with $\mathrm{d} 4 \mathrm{~T}$ and ddI confirms that these agents cause mitochondrial toxicity in HT29 cells. Assuming that this depletion should lead to mitochondrial dysfunction, compensatory mechanism(s) may protect HT29 cells against detectable oxidative stress-induced mtDNA damage. The increase in mtDNA content following AZT, ABC, and 3TC exposure may reflect mtDNA proliferation following mitochondrial stress. Such expansion of mtDNA may mask or compensate oxidative damage to mtDNA.

\section{P009 \\ EFFECT OF COBICISTAT AND RITONAVIR ON PROXIMAL RENAL TUBULAR CELL UPTAKE AND EFFLUX TRANSPORTERS}

Lepist, Irene; Wedgwood, Orson M; Murray, Bernard; Tong, Leah; Bannister, Roy; Roy, Anupma; Ray, Adrian

\section{Mississauga, ON}

BACKGROUND: Increases in serum creatinine $(\mathrm{Cr})$ and a corresponding reduction in estimated glomerular filtration rate (eGFR) without actual changes in GFR (aGFR) have been reported with a number of approved and investigational drugs. Since eGFR is commonly used to monitor renal function, further understanding of the mechanism affecting tubular $\mathrm{Cr}$ secretion is important. In this study, in vitro models were used to evaluate the effect of multiple drugs on proximal renal tubular cell transporters thought to mediate tubular $\mathrm{Cr}$ secretion.

METHODS: Inhibition of OCT2, OCTN1, MATE1, MATE2-K, Pgp, MRP2 and BCRP transporters was studied in transfected cell-lines. Known inhibitors were used as positive controls in each independent assay. Studies were conducted with drugs known to reduce eGFR including cimetidine, trimethoprim, dolutegravir (DTG), cobicistat (COBI) and ritonavir (RTV).

RESULTS: Cimetidine, trimethoprim, COBI and RTV all markedly inhibited the apical efflux transporter MATE1 (IC50 values of 0.95, 3.31, 1.87 and $1.34 \mu \mathrm{M}$, respectively) and were relatively weaker inhibitors of the basolateral uptake transporter OCT2 (IC50 values of 135, 68, 14 and $\sim 20 \mu \mathrm{M}$, respectively). In contrast, DTG was a more potent inhibitor of OCT2 (IC50 of $0.07 \mu \mathrm{M}$ ) relative to MATE1 (IC50 of $4.67 \mu \mathrm{M}$ ). Of the other transporters, COBI and RTV inhibited the apical efflux transporter OCT;N1 (IC50 values of 2.49 and $2.08 \mu \mathrm{M}$, respectively) and trimethoprim inhibited the apical efflux transporter MATE2-K (IC50 of $1.87 \mu \mathrm{M})$. All of the compounds tested were only weak inhibitors of the apical efflux pumps Pgp, BCRP or MRP2 (IC50 >20 $\mu$ M).

CONCLUSIONS: Inhibition of the active tubular secretion of creatinine facilitated by uptake from plasma into the proximal tubule by OCT2 (DTG) and efflux into the urine by MATE1 (cimetidine, trimethoprim, COBI and RTV) may explain the effects of these agents on $\mathrm{Cr}$ and eGFR.
HIV Structure, Function and Genetics

Structure, fonctionnement et génétique du VIH

\section{P010}

\section{A NOVEL MUTATION IN HIV-1 VIF FOUND IN A LONG-TERM SURVIVOR FROM NAIROBI SIGNIFICANTLY ATTENUATED A3G DEGRADATION AND VIRAL REPLICATION}

Peng, Jinyu; Ao, Zhujun; Wang, Xiaoxia; Ramdahin, Sue; Ball, Blake; Fowke, Keith; Plummer, Frank; Embree, Joanne; Yao, Xiaojian Winnipeg, MB

BACKGROUND: A small proportion of HIV-1 positive individuals who tend to prolong disease progression in absence of antiretroviral therapy are defined as long-term survivors (LTSs). The mechanisms of this natural control of HIV-1 infection are based on viral, genetic and immunological components. With respect to virological features, the polymorphisms of the accessory genes of HIV-1 are the possible contributors to the retarded disease progression.

RESULTS: To investigate the genetic diversity of HIV-1 Vif and its activity against host protein APOBEC3G (A3G), the vif genes of proviral DNAs isolated from PBMCs of four Nairobi HIV-1 infected women (two typical progressors, two LTSs) and one reported LTS of Nairobi were cloned into an expression vector. The Vif's ability to counteract the host antiviral molecule A3G was analyzed. Results revealed that the Vifs derived from two LTSs (Vif15 and Vif30) displayed significantly reduced ability to mediate $\mathrm{A} 3 \mathrm{G}$ degradation. In the presence of A3G, these two Vif proteins failed to restore HIV-1 infectivity. The sequence analysis of vif genes found that the two Vif genes harbored more frequent amino acid (aa.) mutations than did those with typical progressive AIDs. To determine the aa. substitution responsible for functional inactivation of Vif of LTSs, the single or multiple aa. mutation in the functional domain of Vif15 or Vif30 was reverted to highly conserved residue. The results suggested that the functional defect of Vif30 might be an accumulated effect through multiple mutations. Interestingly, when T107 in Vif15 was converted back to the wild type residue (I), the resulting Vif protein restored its ability to interact, degrade and exclude $\mathrm{A} 3 \mathrm{G}$ from incorporating into virions. To further test the effect of mutation (I107T) on HIV-1 replication, we introduced vif107T into an HIV-1 provirus and revealed that the viral replication was significantly attenuated in $\mathrm{A} 3 \mathrm{G}^{+} \mathrm{T}$ cells.

CONCLUSION: The study identified a novel HIV-1 vif gene mutant (I107T) present naturally in HIV-1 infected LTS and provide useful information for the understanding of mechanisms involved in the progression of HIV disease.

\section{P011}

\section{HIV-1 ESCAPES FROM IFITM1 RESTRICTION BY} MUTATING VPU AND ENV

\section{Ding, Shilei}

\section{Montreal, QC}

Interferon inducible transmembrane (IFITM) proteins are a small protein family. They were recently shown to inhibit a number of viruses including influenza A viruses, flaviviruses, filoviruses, SARS coronaviruses, vesicular stomatitis virus and HIV-1. This indicates the important role of IFITMs in the interferon-mediated host antiviral defence. Our group previously reported that IFITM1, IFITM2 and IFITM3 inhibited HIV-1 replication in the SupT1 CD4 ${ }^{+} \mathrm{T}$ cells. In order to understand the mechanisms behind the anti-HIV-1 activity of IFITM proteins, we grew HIV-1 in IFITM1-expressing SupT1 cells over a long period of time and obtained an HIV-1 revertant that exhibited wild-type replication capacity in the presence of IFITM1. Sequencing the entire viral genome revealed four mutations, including Vpu36, EnvR311K, EnvG367E and Envg7823a. Further mutagenesis studies showed that the Vpu36 and EnvG367E mutations together were sufficient to confer resistance to IFITM1. In contrast to the parental virus, the revertant virus was resistant to the inhibition by soluble CD4 (sCD4) and the broadly neutralizing antibody VRC03 that targets the CD4 binding site on gp120, which suggests that the revertant virus has become CD4-independent. Collectively, our work indicates that IFITM1 poses a strong inhibitory pressure 
on HIV-1 replication, which drives the virus to evolve and escape from IFITM1 restriction.

\section{P012}

\section{ANALYSIS OF SELECTIVE PRESSURES EXERTED ON THE ENVELOPE GENE IN PREGNANT WOMEN INFECTED WITH HIV-1}

Ransy, Doris G; Lord, Etienne; Motorina, Alena; Merindol, Natacha; Akouamba, Bertine S; Samson, Johanne; Lapointe, Normand; Boucher, Marc; Diallo, Abdoulaye B; Soudeyns, Hugo Montreal, QC

BACKGROUND: Pregnancy is associated with a down-regulation of Th1 and up-regulation of Th2 responses. These changes may reflect on the selective pressure exerted on viral genes. HIV-1 envelope is the target of both humoral and cell-mediated immune responses. The aim of this study was to document the selective regimen acting on env during pregnancy.

METHODS: Plasma env sequences (V1-V3 region) were amplified, cloned, and sequenced at each trimester of pregnancy in 19 subjects infected with HIV-1 of different clades. Sequences from 18 pregnant and 31 non-pregnant women were also retrieved from GenBank for analysis. Overall rates of synonymous (ds) and non-synonymous substitutions (dn) were estimated and the $\omega$ ratio $(\mathrm{dn} / \mathrm{ds})$ representing the selective pressure was calculated. Site-by-site selection was assessed using a maximum likelihood method.

RESULTS: In every subject, hypervariable regions were subjected to significantly higher selective pressures than constant region $\mathrm{C} 2$. The overall $\omega$ in env and in individual loops did not differ between pregnant and non-pregnant subjects. However, in $\mathrm{C} 2, \omega$ was significantly lower in both pregnant groups as compared to non-pregnant controls $(\mathrm{p}<0.007)$. In agreement with what is known for env in non-pregnant subjects, siteby-site selection analysis showed positively selected sites localized in potential antibody and CTL epitopes, and that were mainly focused on variable regions and on potential N-linked glycosylation sites. Subjects in whom X4-capable sequences were detected had higher selective pressure in V3 that focused on sites associated with coreceptor tropism. Overall, $\omega$ remained unchanged throughout pregnancy despite important decreases in viral loads following initiation of antiretroviral therapy. Selected sites remained analogous between the 1st and 3rd trimesters. However, the number of selected sites decreased significantly with the progression of pregnancy independent of the decrease in viral load.

CONCLUSION: These results indicate that pregnancy is associated with subtle changes in selective pressures exerted on HIV-1.

\section{P013}

\section{THE TRANSCRIPTION OF HIV-1 PROMOTER IS INCREASED BY PROTEINS OF THE RNA INTERFERENCE PATHWAY TRBP AND PACT}

Sinck, Lucile; Daniels, Sylvanne; Lainé, Sébastien; Daher, Aïcha; Gatignol, Anne

Montréal, QC

The cellular proteins TRBP (TAR-RNA Binding Protein) and PACT (PKR-Activator) are two RNA binding proteins with 40\% homology. They both bind to PKR but while TRBP inhibits PKR activity, and enhances translation in cell, PACT enhances PKR activity and inhibits translation. In addition, TRBP is an integral part of the RNA-induced silencing complex (RISC) which mediates RNA interference (RNAi) and PACT has been found to be associated with the RISC and to increase RNAi activity. In HIV-1 infected cells, TRBP is an essential protein for viral replication and surprisingly, PACT has recently been shown to also enhance expression of viral genes.

To get further insight into the mechanism of HIV-1 gene expression, we examined the effect of TRBP and PACT on the RNA expression of reporter genes that are under the control of HIV-1 LTR and SV-40 viral promoters. In addition to the known translational activity of TRBP and PACT, we identified that these proteins also increase RNA levels produced from these viral promoters. To characterize the transcriptional activation domain of TRBP and PACT, we expressed different fragments of the proteins fused to a DNA binding domain and tested their ability to activate transcription in yeast and in mammalian cells. In these assays, we mapped their transcriptional component to a 26aa C-terminal fragment of both proteins. Finally, in the context of HIV infected cells, we show that siRNAs against TRBP decrease the production of HIV RNA. This work describes a surprising regulation of HIV transcription by TRBP and PACT, and suggests an intriguing new mechanism by which these RNA-binding proteins increase viral RNA production. It opens the way to interconnections between viral transcription, RNAi and PKR regulation.

Innate and Adaptive Immune Responses to HIV Infection
and Co-Infection
Réponse immunitaire naturelle et acquise à l'infection et à
la co-infection au VIH

\section{P014}

\section{ACTIVATED PLATELETS CONSTITUTE THE MAIN SOURCE OF IL-18 IN THE CIRCULATION OF HIV-INFECTED INDIVIDUALS}

Allam, Ossama; Boulassel, Mohamed R; Samarani, Suzanne; Marzouk, Rim; Routy, Jean-Pierre; Ahmed, Ali Montreal, QC

IL-18, a member of the IL-1 family, is a multifunctional pro-inflammatory cytokine. It is known to be produced by monocytes, macrophages, dendritic cells, keratinocytes and adrenal cortex in the human body. The cytokine is produced as an inactive precursor protein, which is cleaved into mature form by activated caspase 1 . We show here for the first time that IL-18 is also produced and secreted by human platelets upon activation. The platelets also contain components of the inflammasome, which activate caspase 1 and process the precursor IL-18 into its mature form during the platelet activation process. Different activating agents activate platelets and release IL-18 to variable extent, the maximum being observed with thrombin. Contrary to IL-18, the platelets constitutively express pre-formed IL-18-binding protein (IL-18BP), and release it upon activation. Cycloheximide does not affect expression of IL-18BP in these cells. The platelet-released IL-18 constitutes the main source of this cytokine in the human circulation. We found decreased amounts of this cytokine in the platelet lysates in HIV-infected individuals as compared to the healthy ones. On the other hand, its concentrations were increased in the serum and platelet-poor plasma in the infected individuals. Similar findings were obtained with respect to IL-18BP in platelet lysates from healthy and HIV-infected individuals. However, lower amounts of this IL-18 antagonist were found in the serum and platelet-poor plasma from HIV-infected individuals compared with the healthy ones. Our findings signify the role of platelets in the dysregulated production of IL-18 and its antagonist in HIV-infections. They also have important implications for other chronic inflammatory disease conditions in which increased IL-18 activities play a pathogenic role.

\section{P015}

ROLE OF IMMUNOSUPPRESSIVE TRYPTOPHAN

METABOLITES IN HIV DISEASE PROGRESSION AND CD4 T CELL RECOVERY BY ANTIRETROVIRAL THERAPY

Boulassel, Mohamed-Rachid ${ }^{1}$; Jenabian, Mohammad-Ali ${ }^{1}$; Patel, Mital'; Lebouché, Bertrand'; Brouillette, Marie-Josée'; Tremblay, Cécile'; Kema, Ido P2 ; Routy, Jean-Pierre ${ }^{1}$

${ }^{1}$ Montreal, QC; ${ }^{2}$ Groningen, Netherlands

BACKGROUND: Increased Tryptophan (Trp) catabolism into kynurenine (Kyn) and/or 3-hydroxykynurenine (3OH-kyn) by indoleamine 2,3 dioxygenase (IDO), plays a detrimental role in $\mathrm{T}$ cell responses in advanced AIDS, contributes to the innate immune activation and has neurotoxic catabolites. Effect of prolonged suppressive anti-retroviral therapy (ART) on Trp catabolism remains unknown. We evaluated whether ART impacts Trp catabolism and its immunosuppressive function in groups of patients with different disease outcomes.

METHODS: Plasma samples from ART-naive ( $n=96)$, ART-successfully treated $(\mathrm{ST})(\mathrm{n}=17$, mean duration of treatment $=7$ years), elite controllers (EC) $(n=20)$ and healthy controls $(n=45)$ were collected. All these groups were standardized for nutritional status (albumin levels and body mass 
index). Levels of Trp, Kyn and 3OH-kyn were measured using isotope dilution tandem mass spectrometry and the markers of Trp catabolism (Kyn/Trp and 3OH-kyn/Trp ratios) were correlated to clinical data.

RESULTS: In ART-naive patients a strong positive correlation was observed between viral load and Kyn and 3OH-kyn levels as well as the markers of Trp catabolism $(\mathrm{p}<0.0001)$. In addition an inverse correlation was observed in Kyn levels and Kyn/Trp ratio with absolute CD4 cell count $(\mathrm{p}<0.05$ and $\mathrm{p}<0.01$ respectively). Aged matched ART-naive patients (median $=38$ years, $n=60$ ) had significantly lower plasma Trp levels compared to controls $(\mathrm{p}<0.001)$ and to ST $(\mathrm{p}<0.05)$. Similarly, an increase in Trp metabolites such as Kyn and $3 \mathrm{OH}-\mathrm{kyn}$ was observed in ART-naive vs ST $(\mathrm{p}<0.001, \mathrm{p}<0.05$, respectively $)$ and vs controls $(p<0.0001, p<0.01$, respectively). Accordingly, the markers of Trp catabolism were significantly higher in ART-naive patients. ST had similar Trp levels to EC and Control subjects. Interestingly, EC had significantly lowered Kyn and 3OH-kyn levels compared to ART-naive patients but not for Trp, suggesting a unique Trp metabolism associated with suppressed viral replication.

CONCLUSION: Tryptophan metabolites may be considered as inflammation-related markers for disease progression and CD4 recovery after prolonged successful ART.

\section{P016 \\ INVOLVEMENT OF HIV NEF IN DRIVING BLYS/BAFF OVER-EXPRESSION BY DENDRITIC CELLS}

Chagnon-Choquet, Josiane; Fontaine, Julie; Poudrier, Johanne;

Roger, Michel

Montréal, QC

INTRODUCTION: Dendritic cells (DC) are known to influence $B$ cell survival and differentiation through production of factors such as B lymphocyte stimulator (BLyS/BAFF) and may therefore be involved in driving B cell disorders found in the context of HIV, which are not fully restaured by therapy. As such, previous work with the HIV-transgenic mouse model demonstrated that B cell dysregulations, in these animals, involved DC and was dependant on Nef. In recent longitudinal studies involving HIV-infected individuals with different rates of disease progression, we have shown that B cell dysregulations were associated with increased BLyS expression in serum and by blood myeloid DC (mDC), as soon as in the acute phase and persisting despite successful therapy. Aim: to investigate the impact of HIV Nef in modulating DC phenotype and $B$ cell dysregulations.

METHODS: Plasma Nef was measured by ELISA in 42 HIV-infected individuals (13 rapid progressors, 17 normal progressors and 12 slow progressors) and 8 healthy donors. In vitro $\mathrm{mDC}$ were derived from CD14 ${ }^{+}$ monocytes, which were magnetically enriched from PBMC of human healthy donors, and cultured with or without soluble recombinant Nef, in presence of human recombinant IL-4 and GM-CSF.

RESULTS: Soluble Nef was measured in the blood of all viremic HIV infected patients, throughout follow-up and beyond therapy. Levels correlated with those of BLyS in the blood of acutely infected individuals. In vitro, Nef drives monocyte-derived $\mathrm{mDC}$ towards a pro-inflammatory phenotype by increasing the expression of BLyS and TNF- $\alpha$.

CONCLUSION: These data suggest that HIV Nef may have an importance in the modulation of the B cell status in HIV-infected individuals by favouring a DC pro-inflammatory profile, as soon as in the acute phase of infection and despite therapy.

\section{P017}

\section{THE IMPACT OF HIV INFECTION AND ITS TREATMENT} WITH HAART ON IMMUNE RESPONSES TO MALARIA

Finney, Constance A ${ }^{1}$; Ayi, Kodjo ${ }^{1}$; Wasmuth, James ${ }^{2}$;

Sheth, Prameet ${ }^{1}$; Kaul, Rupert ${ }^{1}$; Loutfy, Mona ${ }^{1}$; Kain, Kevin ${ }^{1}$;

Serghides, Lena ${ }^{1}$

\section{${ }^{1}$ Toronto, ON; ${ }^{2}$ Calgary, AB}

Plasmodium falciparum malaria and HIV-1 adversely interact, with $\mathrm{HIV}(+)$ individuals suffering more frequent malaria infections, higher parasitemia, and greater malaria disease severity. However, the underlying mechanisms responsible have yet to be fully investigated.

OBJECTIVES: Our aim was to examine the innate inflammatory response of NK, NKT and $\gamma \delta \mathrm{T}$ cells isolated from the peripheral blood of therapy-naive $\mathrm{HIV}(+)$ donors to malaria parasites, and determine the effect of HAART on these responses.

DESIGN: Severe malaria is characterized by robust pro-inflammatory host immune responses to infection. We hypothesized that HIV co-infection compromises innate immune cell function in response to malaria, and that HAART may be able to restore these responses.

METHOD: Freshly isolated PBMCs from therapy-naïve HIV(-) and $\mathrm{HIV}(+)$ individuals were cultured in the presence of $\mathrm{P}$. falciparum over the course of four days. Supernatants and cells were collected on days 1 , 2 and 4 to assess cytokine production. The experiments were repeated 3 and 6 months post-HAART.

RESULTS: Compared to HIV(-) participants, NKT, NK and $\gamma \delta$ T cell subsets from patients with chronic HIV infection showed marked differences, including decreased production of TNF and IFN $\gamma$ in response to malaria parasites. Six months of HAART provided partial cellular reconstitution but had no effect on cytokine production.

CONCLUSION: We suggest that HIV infection impairs the inflammatory response of innate effector cells to P. falciparum malaria, and that the impairment is not fully restored within six months of HAART. This may contribute to higher parasite burdens and ineffective immune responses in co-infected individuals.

\section{P018}

\section{CHRONIC HIV INFECTION IMPAIRS PHAGOCYTOSIS OF MALARIA PARASITES}

Finney, Constance ${ }^{1}$; Ayi, Kodjo ${ }^{1}$; Wasmuth, James ${ }^{2}$; Sheth, Prameet ${ }^{1}$; Kaul, Rupert ${ }^{1}$; Loutfy, Mona ${ }^{1}$; Kain, Kevin ${ }^{1}$; Serghides, Lena ${ }^{1}$

${ }^{1}$ Toronto, $\mathrm{ON}$; ${ }^{2}$ Calgary, $\mathrm{AB}$

A detailed understanding of the effect of HIV on the pathogenesis of Plasmodium falciparum malaria may facilitate the identification of novel and effective interventions. Co-infected individuals have higher parasite burdens and ineffective immune responses to malaria infection. Our aim was to examine the phagocytic ability of macrophages to malaria parasites, in the context of HIV infection, as well as determining the effect of highly active anti-retroviral therapy (HAART) on this process. We hypothesized that HIV co-infection compromises the function of macrophages, and that HAART may be able to restore these responses.

METHODS: Freshly isolated peripheral blood mononuclear cells from therapy-naïve $\operatorname{HIV}(-)$ and $\operatorname{HIV}(+)$ individuals were cultured to obtain monocyte-derived macrophages, which were subsequently used in opsonic and non-opsonic phagocytosis assays. Isolated cells were also characterised for monocyte surface markers by flow cytometry. Experiments were repeated 3 and 6 months post-HAART initiation.

RESULTS: Compared to HIV(-) participants, macrophages from patients with chronic HIV infection were impaired in both opsonic and non-opsonic phagocytosis. Functional impairment could not be attributed to differences in activation (HLA-DR), co-stimulation (CD40), or phagocytic (CD36) markers. Three months of HAART restored monocyte function, emphasising the importance of HIV treatment in the context of co-infections like malaria.

CONCLUSIONS: We suggest that HIV infection alters the innate inflammatory response to $P$. falciparum malaria, which contributes to worsened pathology in co-infected individuals.

\section{P019}

\section{INFLUENCE OF THE SEX WORK ON IMMUNE} ACTIVATION IN THE FEMALE GENITAL TRACT

Lajoie, Julie ${ }^{1}$; Kimani, Josuha ${ }^{2}$; Kimani, Makubo ${ }^{2}$; Plummer, Frank ${ }^{1}$; Fowke, Keith $\mathbf{R}^{1}$

${ }^{1}$ Winnipeg, MB; ${ }^{2}$ Nairobi, Kenya

INTRODUCTION: Sexual activities leading to discharge of semen and sperm are known to activate the female genital tract (FGT) immune system. Compared to low risk women, female commercial sex worker (CSW) are exposed to a wide variety of sperm and we hypothesize that their mucosal immune system will be more activated. Knowing the basic level of immune activation in low risk women is important as they are often used as control group in HIV study.

METHODS: Participants for this study were derived from two longstanding cohorts based in the Pumwani district of Nairobi, Kenya. The 
Majengo cohort is a group of commercial sex workers, while the $\mathrm{MCH}$ cohort is low risk women attending an antenatal clinic. Cervico vaginal lavage (CVL) and cervico mononuclear cells (CMC) from $120 \mathrm{HIV}^{-}$ $\mathrm{CSW}$ (Majengo) and $44 \mathrm{HIV}^{-}$low risk women ( $\mathrm{MCH}$ cohort) were analyzed for the presence of 22 chemokines/cytokines by Milliplex and by cytometry (CCR5-FITC, CXCR3-PE, HLA-DR-PE-Cy7, CD69-PE Cy5, Live dead-ECD, CD16-APC H7, CD4-ALEXA 700, CD56-APC, CD8V500, CD3-V450) respectively.

RESULTS: In low risk women, we observed higher level of MIP-3a, ITAC, MIG, IL-1a, IL-1b, IL-1Ra, Il-6, IL-8, IL-10, IP-10, MDC, MIP-1a, MIP-1b, MCP-1 and TNF-a compared to the level observed in the CSW, while the level of IL-15 and sIL-2Ra were higher in CSW.

We observed that CSW had a higher frequency of CD3-CD56dim, whereas low risk women have higher level of $\mathrm{CD}^{+}, \mathrm{CD} 4^{+} \mathrm{CCR} 5^{+}, \mathrm{CD} 8^{+}$, CD $8{ }^{+} \mathrm{CCR}^{+}$and $\mathrm{CD} 8{ }^{+} \mathrm{CD} 69^{+}$cells.

CONCLUSION: Even if preliminary, these results show an important difference in the mucosal milieu of the FGT due to sex work. Surprisingly, low risk women have a more activated milieu than the CSW. Our data supports the view that in CSW, due to constant immune stimulation due to sex, the immune system develops a certain tolerance, which decreased the immune activation.

\section{P020 \\ ANALYSIS OF EXON ARRAY DATA BASED ON CD4 AND CD8 T CELL COUNTS TO IDENTIFY GENES/GENE NETWORKS DIFFERENTIALLY EXPRESSED IN THE HIV RESISTANT WOMEN IN THE PUMWANI SEX WORKER COHORT}

Liu, Lewis R; Sainsbury, James; Liang, Binhua; Ball, Blake;

Plummer, Frank A; Luo, Ma

Winnipeg, MB

BACKGROUND: Despite repeated exposure through high-risk sex work, a subset of enrolees in the Pumwani cohort remains uninfected by HIV-1. Identification of genes that are differentially expressed in these highly exposed seronegative individuals can provide clues to how these women are protected from infection. In this study, we incorporate CD4 ${ }^{+}$ and $\mathrm{CD} 8^{+}$T-cell counts in Affymetrix exon array data analysis. By comparing genes highly correlated to T-cell counts in HIV resistant women (HESN), HIV-1 uninfected new enrolees (NSN) and HIV-1 infected individuals $\left(\mathrm{HIV}^{+}\right)$, we identified genes that are differentially regulated in the HESN subpopulation.

METHODS: PBMC Exon Array data from 44 women (8 NSN, $19 \mathrm{HIV}^{+}$, 17 HESN) were matched with CD4/CD8 T-cell counts from annual resurveys. The CEL files were analyzed using Partek ${ }^{\circledR}$ genomic suite (6.5). The expression value was correlated with T-cell counts by linear regression for each gene-probe. The $\mathrm{r}^{2}$ of linear fit was used as selection criteria for the gene lists, which were analyzed using Pathway Studio® 8.0 to identify significantly enriched pathways.

RESULTS: Among 5004 genes with expression level correlated well with CD8+ T-cell counts $\left(r^{2} \geq 0.60\right)$, a numbers of genes are unique in each group: 63(HESN), $4880(\mathrm{NSN})$ and $6\left(\mathrm{HIV}^{+}\right)$. While there is no overlap between HESN and $\mathrm{HIV}^{+}, 49$ were shared between HESN and NSN, and 6 overlaps between NSN and $\mathrm{HIV}^{+}$. Gene expression level correlated less well with CD4 ${ }^{+}$T-cell counts. Of 1110 genes identified $\left(\mathrm{r}^{2} \geq 0.50\right), 216,46$ and 838 genes were unique in HESN, HIV ${ }^{+}$and NSN respectively. Only 5 shared between HESN and NSN, 4 between NSN and $\mathrm{HIV}^{+}$, and 1 was common in three groups.

CONCLUSION: Correlating the gene expression level with $\mathrm{T}$ cell counts is an alternative approach to analyze gene array data and the results showed that HESN women have a gene expression profile that is different from NSN and $\mathrm{HIV}^{+}$women.

\section{P021}

\section{DETERMINANTS OF EFFICIENT HIV RESTRICTION BY DEOXYCYTIDINE DEAMINASES APOBEC3G AND APOBEC3A}

\section{Love, Robin P; Xu, Huixin; Chelico, Linda}

Saskatoon, SK

The study of the APOBEC3 family of single-stranded (ss) DNA cytosine deaminases has revealed members with restriction activity of HIV replication. The flagship enzyme demonstrating this activity, APOBEC3G, is antagonized by the HIV encoded Vif protein. Vif is able to circumvent APOBEC3G restriction by facilitating its ubiquitination and degradation. APOBEC3G appears to be the most effective restrictor of HIV and the most easily degraded through Vif-mediated action. We have investigated the reason for APOBEC3G being an efficient restrictor of HIV through comparison with APOBEC3A, a cytosine deaminase that does not normally restrict HIV through hypermutation. APOBEC3G is a processive enzyme that exhibits a preference to deaminate cytosines toward the 5'-end of ssDNA. In contrast, we find that APOBEC3A is a non-processive enzyme with no spatial deamination preference, but is still able to deaminate actively reverse-transcribing DNA in a reconstituted HIV replication assay. The number of deaminations induced by APOBEC3A was less than APOBEC3G and resulting mutations were less likely to inactivate the protease gene in comparison to APOBEC3G. Coupled with in vitro deamination assays on ssDNA and DNA binding assays we present a model of the biochemical determinants of an efficient APOBEC3 enzyme as relating to HIV restriction.

\section{P022}

\section{CD56BRIGHT/DIM NATURAL KILLER CELLS OF HIGHLY EXPOSED SERONEGATIVE COMMERCIAL SEX WORKERS UP-REGULATE CD69 MORE THAN NEW NEGATIVES AFTER TOLL-LIKE RECEPTOR STIMULATION}

\section{Omange, Were $\mathbf{R}^{1}$; Su, Ruey $\mathrm{C}^{1}$; Meyers, Adrienne ${ }^{1}$;}

Kimani, Makobu ${ }^{2}$; Kimani, Joshua ${ }^{1,2}$; Plummer, Francis $A^{1}$;

Ball, Terry $\mathbf{B}^{1}$

${ }^{1}$ Winnipeg, MB; ${ }^{2}$ Nairobi, Kenya

Natural killer cells are important innate cells that play a critical role in antiviral and antitumor immunity. They have been shown to express Toll-like receptors and respond to stimulation by TLR ligands resulting in up-regulation of activation markers, production of nitric oxide intermediates and interferons. The role of NK cells in relation to TLR function is largely unknown. In the current study we hypothesised that highly exposed seronegative (HESN) commercial sex workers have a higher responsiveness to TLR stimulation in NK cells that might contribute to their protection against the HIV virus. In our experiments we compared the responses of PBMCs to TLR stimulation with Escherichia coli LPS (TLR4), single strand RNA (TLR7/8) and Imiquimod (TLR7) in two groups of HIV negative commercial sex workers. The groups have been previously defined using epidemiological studies and are now divided into 'HESN' who are HIV negative, more than 7 years of clinical follow-up with continuous sex work and a comparison group we call 'new negatives' (NN) who are HIV negative, less than 7 years of clinical follow-up with continuous sex work. The results of this study showed that $\mathrm{CD}^{+} 6^{+}$and CD16- CD56Bright NK cells of HESN had significantly higher CD69 expression after ssRNA (TLR7/8) stimulation $(\mathrm{p}=0.0480 ; \mathrm{p}=0.0378$ respectively) and only CD16 ${ }^{+} \mathrm{CD} 56 \mathrm{dim}$ NK cells also showed a difference in the surface expression of CD69 after LPS and ssRNA stimulation ( $\mathrm{p}=0.0496$ and 0.0023 respectively). The expression of HLA-DR was significantly higher in CD16- CD56dim NK cells and not in CD16 ${ }^{+}$CD56dim NK cells of HESN compared to NN after TLR stimulation. It is important to mention that prior to TLR stimulation there was no differences observed in the expression of CD69 and HLA-DR on CD56bright and CD56dim NK cells. These results point to a difference in TLR responsiveness in NK cells of HESN women when compared to those of NN women. This implies that difference in innate function that could have a major influence in acquisition of HIV virus. 
P023

THE COMMON THREAD: INFECTIONS WITH MULTIPLE HIV TYPES AND SUBTYPES INDUCE ANTIBODIES SHARING THE 1F7-IDIOTYPE

Parsons, Matthew $\mathrm{S}^{1}$; Center, Robert J ${ }^{2}$; Routy, Jean-Pierre ${ }^{1}$; Rouleau, Danielle' ${ }^{1}$; LeBlanc, Roger ${ }^{1}$; Wainberg, Mark $A^{1}$; Tremblay, Cecile ${ }^{1}$; Kent, Stephen $\mathbf{J}^{2}$; Grant, Michael $\mathrm{D}^{3}$; Bernard, Nicole $\mathrm{F}^{1}$

${ }^{1}$ Montreal, QC; ${ }^{2}$ Melbourne, VIC, Australia; ${ }^{3}$ St John's, NL

BACKGROUND: HIV infection induces virus-specific antibodies (Abs). Abs against HIV envelope (Env) control replication of early viral variants through several effector functions, including neutralization and ADCC. Humoral responses can exert pressure that drives the emergence of immune escape variants. Subsequently, anti-viral Ab responses are locked into a form of original antigenic sin, known as repertoire freeze, characterized by maintenance of $\mathrm{Ab}$ responses against initial viral variants, and a failure to induce novel Abs against escape variants. Evidence for this phenomenon includes the early appearance and maintenance of a common idiotype, designated $1 \mathrm{~F} 7$, on anti-viral Abs during human subtype B HIV-1 and Rhesus macaque SIV/SHIV infections. Suppression of 1F7-idiotypic Abs in macaques allows new Abs to emerge, which can neutralize autologous contemporaneous viruses. It is unknown whether 1F7-idiotypic anti-HIV Abs emerge in infections with other HIV types and subtypes.

HYPOTHESIS: 1F7-idiotypic anti-HIV Abs emerge in HIV-2 and multiple HIV-1 subtype infections.

METHODS: Plasma from 28 HIV-1 subtype B-infected, 19 HIV-1 nonsubtype B-infected, and 2 HIV-2-infected individuals were tested. ELISA plates were coated with HIV-1 gp41, gp120, or HIV-2 gp32. Plasma samples were added, followed by a murine anti-1F7-idiotypic IgM Ab or an isotype control. Binding of the $1 \mathrm{~F} 7$ anti-idiotypic $\mathrm{Ab}$ was detected using an HRP-conjugated goat-anti-mouse IgM.

RESULTS: The 1F7 idiotype was detected on $82 \%$ and $97 \%$ of antigp120 and anti-gp41 Abs from HIV-1 subtype B-infected samples, on 83\% and $100 \%$ of Abs with these specificities from non-subtype B-infected samples and on anti-gp32 Abs from both HIV-2-infected samples.

CONCLUSIONS: The 1F7-idiotype characterizes anti-Env Abs induced by infections with diverse HIV types and subtypes. This may reflect the ability of 1F7-idiotypic Abs to recognize conserved regions on HIV Env. $1 \mathrm{~F} 7$-idiotypic repertoire freeze may contribute to the inability of $\mathrm{Ab}$ responses to keep pace with viral escape.

\section{P024}

ROLE OF THE CYTOSKELETON IN TRIM5 $\alpha$-MEDIATED RESTRICTION

Pawlica, Paulina; Berthoux, Lionel

Trois-Rivières, QC

TRIM5 $\alpha$ is a host factor playing an important role in the control of retroviruses. This protein acts in the cytoplasm shortly after virus entry into the host cell and employs several mechanisms to restrict retroviruses: decreased stability of the viral core, a diminution of reverse transcription products and impairment of transport to the nucleus. In addition, TRIM5 $\alpha$ self-ubiquitinates and its stability decrease in the course of retroviral restriction. Little is known about other cellular proteins involved in the restriction process. In this study we are investigating functional interactions between TRIM $5 \alpha$ and components of the cytoskeleton, with a focus on microtubules and the dynein complex. Microtubules are essential in maintaining cell structure, for intracellular transport and cell division. Dyneins are molecular motors that transport specific cargos toward the microtubule organizing center (MTOC) through binding to microtubules. Human or feline cells endogenously or exogenously expressing TRIM5 $\alpha$ were treated with nocodazole, a drug preventing microtubule polymerization, and with EHNA, which blocks dynein ATPase activity. Expression of the dynein complex heavy chain was decreased by siRNA transfection. These treatments reduced restriction mediated by TRIM $5 \alpha$ of two distinct retroviruses (HIV-1 and N-tropic MLV). The associated increase in infectivity was very significant (up to 80 -fold). These experiments suggest that preventing polymerization of microtubules impairs the ability of TRIM $5 \alpha$ to block reverse transcription. Additionally, preliminary data show that disruption of the dynein complex might decrease the stability of TRIM $5 \alpha$. Current and future efforts will allow us to precisely characterize the functional relationships between TRIM5 $\alpha$, post-entry retroviral cores, and the microtubules/dynein network.

\section{P025}

INFLUENCE OF DENDRITIC CELLS ON B CELL RESPONSES DURING HIV INFECTION

Poudrier, Johanne

Montréal, QC

Mechanisms underlying HIV-mediated B cell dysregulations, which can evolve towards autoimmune manifestations and malignancies, remain poorly understood. Previous work with the HIV-Tg mouse model demonstrated that such disorders may involve dendritic cells (DC) and derived B cell growth factors such as B lymphocyte stimulator (BLyS/BAFF). In recent longitudinal studies involving HIV-infected individuals with different rates of disease progression, we have shown that myeloid DC $(\mathrm{mDC})$ levels were reduced in the blood of rapid and normal progressors, as soon as in the acute phase and beyond successful therapy. The low blood levels of mDC correlated with increased serum levels of DC-tissue tropic chemokines CCL2, CCL19 and CCL20, suggesting drainage to peripheral sites. Importantly, these $\mathrm{mDC}$ over-expressed BLyS, and this was concomitant with polyclonal B cell activation. Importantly, blood $\mathrm{mDC}$ levels and their BLyS expression status were unaltered in elite controllers, consistent with the absence of polyclonal B cell activation in these individuals. Suggesting that the extent to which HIV disease progression is controlled may be linked to the integrity of the DC compartment and to its capacity to orchestrate B cell population dynamics and responses.

\section{P026}

THE REGULATION OF PKR ACTIVATION BY PACT DURING HIV-1 REPLICATION

Shaw, Eileen; Clerzius, Guerline; Daher, Aicha;

Boulassel, Mohamed-Rachid; Routy, Jean-Pierre;

Mouland, Andrew; Gatignol, Anne

Montreal, QC

Interferon (IFN) production in response to viral infection plays a critical role in host innate immunity due to its ability to upregulate genes that inhibit viral replication in host cells. IFN has been effective in decreasing HIV replication in vitro; however, the IFN response may also contribute to persistent immune activation and to a decline in $\mathrm{CD} 4^{+} \mathrm{T}$-cell count. The IFN-induced dsRNA-activated protein kinase (PKR) plays a critical role in the anti-viral response and cell apoptosis by preventing translation initiation through the phosphorylation of translation initiation factor 2 (eIF-2 $\alpha$ ). PKR is regulated by various viral and cellular factors. During HIV-1 replication, the viral TAR RNA element activates PKR while the viral protein Tat and cellular proteins ADAR1 (Adenosine Deaminase Acting on RNA) and TRBP (TAR RNA Binding Protein) inhibit PKR function. PACT (PKR Activator) is a known cellular activator of PKR under stress conditions. We have assessed the role of PACT during HIV-1 replication.

We observe that PKR is transiently activated at the beginning of HIV infection of peripheral blood mononuclear cells, but not when the virus is actively replicating. Our results indicate that there is an increased association between PACT and PKR at the peak of HIV infection. In addition, we show that PACT inhibits PKR activation and increases HIV production and protein expression in 293T cells. Conversely, shRNA knockdown of PACT decreases HIV production and protein expression. We have observed that PACT inhibits PKR activation and increases HIV production in infected cells, but the intimate mechanism of this change of function remains to be elucidated. Deciphering PKR regulation by PACT will contribute to a better understanding of the innate immune system against HIV-1 and its role in the control of disease progression. 


\section{P027}

\section{PRMT6 AUTOMETHYLATION REGULATES ITS HIV-1 ANTIVIRAL ACTIVITY}

Singhroy, Diane N; Mesplede, Thibault; Quashie, Peter;

Sabbah, Arielle; Wainberg, Mark A

Montréal, QC

HIV-1 infection may be controlled in vivo by innate immune responses for a number of years prior to clinical manifestation of disease. Host restriction factors such as APOBEC3G, SAMHD1, Trim $5 \alpha$ and Tetherin have been found to play a role in the control of HIV replication. Other host factors like the protein arginine methyltransferase PRMT6 may also contribute to restraining HIV-1 infection.

Protein arginine methyltransferase 6 (PRMT6), is a nuclear enzyme that methylates histones, HMGA1 and DNA polymerase $\beta$. Additionally, we have previously shown that PRMT6 directly methylates and interferes with the functions of HIV-1 proteins, namely Tat, Rev and NC. PRMT6 can dimerize and displays automethylation capabilities. Through liquid chromatography mass-spectrometry, we have identified putative automethylation sites on PRMT6 and have identified an arginine at position 35 that is dimethylated. We have verified this dimethylated residue to be the bona fide PRMT6 automethylation site through site-directed mutagenesis and automethylation assays. We have also found that methylation of PRMT6 is important for its HIV-1 antiviral activity as demonstrated in single-cycle TZM-bl cell infectivity assays. Arginine methylation has shown to be an important regulatory post-transcriptional modification. This is the first characterization of the automethylation capability of PRMT6 and its role in HIV-1 replication.

\section{P028 \\ TRACKING THE IMMUNE RESPONSE IN PERIPHERAL BLOOD MONONUCLEAR CELLS BY QUANTITATIVE PROTEOMICS.}

Stein, Derek R; Westmacott, Garrett; McCorrister, Stuart;

Plummer, Frank; Ball, Terry B

Winnipeg, MB

BACKGROUND: It is becoming increasingly evident that HIV vaccine development is in critical need of simple and unbiased techniques for monitoring immune responses in vaccine trails. We have developed a new approach using mass spectrometry to monitor immune responses in vaccine trials generated at the systemic as well as mucosal level.

METHODS: Peripheral Blood Mononuclear Cells (PBMCs) were collected from four healthy donors and stimulated with PHA followed by protein collection at various time-points ( $0,1,3$ and 24 hours). Using a mass spectrometry approach called iTRAQ, we were able to identify and quantify differentially expressed proteins within study participants. Cluster analysis (Perseus) was performed to visualize the global proteome changes over time, and Ingenuity Pathway Analysis software was used to identify significantly enriched immune pathways.

RESULTS: In total 3075 unique proteins were identified and relatively quantified across all donors. Clustering of the respective time points between patients was expected to occur, however clustering occurred within patients during early time points ( 1 and 3 hours), and it was only until the 24 hour time point that significant clustering between patients was evident. Also, at the 24 hour time point, networks associated with viral infection and cellular growth were increased, while pathways related to cell communication and adhesion were decreased.

CONCLUSION: This data suggests that PHA stimulation drives PBMCs into a specific proteomic profile once the 24 hour time point is reached. Earlier time points do not cluster together suggesting that patients may use different pathways to arrive at a similar cellular state by 24 hours. iTRAQ technology is a high through-put and effective means of monitoring patient specific immune responses to PHA stimulation. This method could easily be adapted to follow patient responses in a variety of conditions as well as immunogens over several different time points during a vaccine trial. Further, this approach requires as little as $10 \mathrm{e} 4$ cells and could become an attractive approach for comprehensive immune monitoring during vaccine trials.
P029

GENITAL INTERFERON-ALPHA PRODUCTION IS ASSOCIATED WITH HIV-1 INFECTION IN BENINESE COMMERCIAL SEX WORKERS

Thibodeau, Valérie $^{1}$; Lajoie, Julie ${ }^{2}$; Labbé, Annie-Claude ${ }^{1}$; Alary, Michel $^{3}$; Poudrier, Johanne ${ }^{1}$; Roger, Michel ${ }^{1}$

${ }^{1}$ Montréal, QC; ${ }^{2}$ Winnipeg, MB; ${ }^{3}$ Québec, QC

Interferon-alpha (IFN-a) is an important antiviral molecule.

AIMS: To determine the association between genital level of IFN-a and risk of HIV-1 infection in commercial sex workers (CSWs) from Cotonou (Benin) and to investigate whether IFN-a expression is modulated by the inflammatory microenvironment and/or the gene polymorphism implicated in it signalling pathways.

METHODOLOGY: Cervico-vaginal lavages (CVLs) from 56 HIV-uninfected CSWs, 45 HIV-1-infected CSWs and 69 HIV-uninfected nonCSWs were analysed. Cytokine/chemokine levels were measured in CVL supernatants by commercial quantitative Milliplex array kits. Genital epithelial cells TLR protein expression patterns were assessed by FACS analyses. TLR7, TLR8, TLR9 and IRF-7 polymorphisms involved in IFNa signalling pathways will be genotyped by direct DNA sequencing. RESULTS: Genital IFN-a levels were significantly $(\mathrm{p}=0.0055)$ higher in HIV-1-infected CSWs when compared to those found in HIV-uninfected CSW and non-CSW groups. Levels of IFN-a did not correlate with viral load. Two IRF-7 genetic variants (rs1061502 and rs12290989) were significantly ( $\mathrm{p}=0.041)$ associated with IFN-a expression in the HIV-1-infected group. Polymorphisms in the TLR7, 8 and 9 gene did not influence IFN-a expression.

CONCLUSION: IFN-a expression in the genital mucosa is associated with HIV-1 infection and its production could be genetically-determined in CSWs from Benin.

This work is supported by the Canadian Institutes of Health Research (CIHR)

\section{P030}

PHENOTYPIC CHANGES OF THE HIV-1 CAPSID CORE ASSOCIATED WITH RESISTANCE TO HUMAN TRIM5 $\alpha$ MUTANTS

Veillette, Maxime ${ }^{1}$; Pawlica, Paulina ${ }^{1}$; Shah, Vaibhav B ${ }^{2}$;

Pham, Quang Toan ${ }^{1}$; Plourde, Mélodie B1'; Aiken, Christopher²; Berthoux, Lionel ${ }^{1}$

${ }^{1}$ Trois-Rivières, QC; ${ }^{2}$ Nashville, TN, USA

Highly specific restriction factors targeting different viruses play an important role in innate immunity. TRIM $5 \alpha$ is such a factor and was found to be responsible for the block of some non-human retroviruses in humans. Previous work in our laboratory and others has led to the identification of mutants of the human TRIM $5 \alpha$ protein with increased activity against HIV-1. The viral target of TRIM $5 \alpha$ is the viral capsid (CA) protein and interaction between TRIM5 $\alpha$ and incoming viral cores is known to cause different functional blocks to the viral cycle. Human TRIM5 $\alpha$ mutants-based gene therapy represents a possible clinical application against HIV. We hypothesized that a previously described mutation on the viral capsid protein (V86M) linked to partial resistance to TRIM5 $\alpha$-Rh would also confer resistance to the restriction by the human mutants of TRIM5 $\alpha$. We showed that it was indeed the case, and our next objective was to explore the mechanisms by which this resistance is acquired. One of the effector mechanisms of TRIM5 $\alpha$ is acceleration of viral uncoating. Surprisingly, fate-of-capsid assays with the V86M-mutated virus showed that the capsid mutation accelerated the uncoating process in human non-restrictive cells without affecting either infectivity or in vitro stability of the virion-associated CA-V86M cores. The host cyclophilin A (CypA) protein stimulates HIV-1 infection of human cells but also promotes TRIM $5 \alpha$-mediated restriction of HIV-1. Our results show that the CA-V86M virus is still dependent on functional CypA for its infectivity of human cells while it is not dependent on CypA for restriction by TRIM $5 \alpha$. In conclusion, the TRIM $5 \alpha$ resistance mutation V86M modifies functional interactions between the CA core and CypA, possibly affecting the uncoating process. These results have important implications for the development of TRIM5 $\alpha$-based gene therapy applications and highlight functional interactions between the viral capsid, TRIM5 $\alpha$ and CypA. 


\section{P031}

\section{INFLAMMASOME EXPRESSION WITHIN THE BRAIN}

Walsh, John G; Power, Christopher

\section{Edmonton, $\mathbf{A B}$}

The chronic inflammation observed in neurodegenerative diseases such as multiple sclerosis (MS) and HIV-associated neuro-cognitive disorders (HAND) has long been associated with the expression of the pro-inflammatory molecules Interleukin- $1 \beta$ and Interleukin-18. The activation and release of these molecules depends on the formation of the inflammasome complex. However, the presence of the inflammasome in the brains of persons with neuro-inflammatory diseases remains to be fully explored. Herein, we investigated the expression of the inflammasome components within the frontal cerebral white matter from HAND, MS and Other Disease Control (ODC) patients, as well as within primary human fetal neural cell cultures representing the principle cells of the brain (ie. neurons, astrocytes and microglia). Transcriptomic deep sequencing with qRT-PCR confirmation disclosed that all of the inflammasome components (IL-1 $\beta$, IL-18, NALP1, NLRP3, NLRC4, ASC, and AIM2) were present in the brain but with differing expression profiles depending on the specific disease. The principal neural cell type expressing the inflammasome machinery were microglial cells, although inflammasome components were detected in astrocytes and neurons. Human fetal microglia exhibited caspase-1 dependent activation and release of IL-1 $\beta$ in response to LPS and ATP. The present observations emphasize the differential expression of the infammasome in the human brain and highlight the role of microglia as the chief cellular effector of the brain's inflammasome.

\section{P032}

\section{THE ROLE OF SUPPRESSOR OF CYTOKINE SIGNALING (SOCS) PROTEINS IN REGULATING IL-7 SIGNALING AND FUNCTION IN CD8 ${ }^{+}$T-cells}

\section{Woit, Cassandra; Crawley, Angela M; Angel, Jonathan B}

\section{Ottawa, ON}

BACKGROUND: IL-7 is an essential cytokine for early T-cell development and is important for the function of $\mathrm{CD} 8^{+}$T-cells. The IL-7 signaling pathway occurs mainly through the activation of Jak/STAT proteins and is regulated by negative feedback inhibitors, such as suppressors of cytokine signaling (SOCS) proteins. In progressive HIV infection, the activity of CD8 ${ }^{+}$T-cells is impaired and unable to control viral replication. Recent reports indicate defects in $\mathrm{CD}^{+}$T-cell-associated IL-7 signaling in HIV infection. Further reports demonstrate that HIV infection interferes with SOCS1/3 protein expression, resulting in the induction of the Jak/STAT pathway. One study reported that murine SOCS3 was elevated in viral infection and that IL-7 represses SOCS3 to clear chronic infection. Otherwise, the role of SOCS proteins in regulating IL-7 activity in $\mathrm{CD}^{+} \mathrm{T}$-cells has not been well described.

METHODS: The effect of SOCS proteins on IL-7 signaling and function was assessed by determining basal protein and mRNA expression in resting $\mathrm{CD}^{+}$T-cells and PBMCs by western blot and quantitative RT-PCR. Small inhibitory RNAs (siRNA) were used to knockdown SOCS1-3 gene expression. Optimization of gene knockdown will be assessed using intracellular staining for SOCS proteins and RT-qPCR. The effect of SOCS knockdown on IL-7 signaling and activity will then be determined in HIV infection, complimenting our initial studies. Activities such as Bcl-2 and perforin production, glucose uptake, and cell proliferation will be assessed.

RESULTS: Expression of SOCS1-3 proteins and mRNA transcripts have been detected in unstimulated CD $8^{+} \mathrm{T}$-cells and PBMCs. Preliminary data suggests IL-7 induces SOCS1 expression. The effects on SOCS 2 and 3 are pending. After transfection with SOCS-specific siRNAs, partial knockdown of these genes was detected in unstimulated $\mathrm{CD} 8^{+}$ T-cells and PBMCs.

CONCLUSION: Determining the role of SOCS proteins in HIV- individuals may provide insight towards future studies investigating the mechanism of impaired $\mathrm{CD} 8^{+} \mathrm{T}$-cell function in HIV infection.

\section{P033}

ANTI-MAJOR HISTOCOMPATIBILITY COMPLEX CLASS I AND II ANTIBODIES IN HIV-EXPOSED SERONEGATIVE INJECTION DRUG USERS: AN EXPLORATORY ANALYSIS Zanoni, Pilar; Bruneau, Julie; Parsons, Matthew; Bernard, Nicole $F$ Montreal, QC

INTRODUCTION: Some HIV-exposed seronegative (HESN) individuals remain HIV seronegative despite multiple exposures to HIV. When HIV buds from infected targets, cell surface molecules such as HLA-DR and major histocompatibility complex (MHC) class I antigens are incorporated into its virion envelope. Injection drug users (IDU) who share needles with others may develop allo-antibodies $(\mathrm{Ab})$ to non-self $\mathrm{MHC}$ antigens. Previous work from our lab implicates Natural Killer (NK) cells in protection from infection. NK cells contribute to the host immune response through cytokine release, direct and indirect cytolysis or Antibody Dependent Cell-mediated Cytotoxicity (ADCC).

HYPOTHESIS: IDU who share needles with others have Abs specific for MHC class I and II antigens.

METHODS: Plasma from 54 HESN IDU and 16 low risk non-IDU healthy controls $(\mathrm{HC})$ were screened for $\mathrm{Abs}$ and $\mathrm{Ab}$ specificities to $\mathrm{MHC}$ Class I and II using commercially available LabScreen ${ }^{\circledR}$ (OneLambda Inc) $\mathrm{Ab}$ detection kits.

RESULTS: Anti-MHC Class I Abs were observed in 15 (28\%) HESN IDU and $2(12.5 \%) \mathrm{HC}(\mathrm{p}=0.3231)$. Anti-HLA Class II Abs were observed in 14 (26\%) HESN IDU and $8(50 \%)$ HC $(\mathrm{p}=0.1227)$. HESN IDU displayed from 1 to $36 \mathrm{Ab}$ specificities and $\mathrm{HC}$ from 8 to 15 specificities ( $\mathrm{p}=0.7858)$.

DISCUSSION: A proportion of IDU who share needles with others develop anti-MHC class I and II Abs. The observation that non-IDU HC also develop anti-MHC Abs was unexpected and needs to be further explored. If these Abs recognize infected allogeneic cells or virus carrying allogeneic MHC class I and/or II antigens, they may be able to mediate ADCC, whereby anti-HLA Abs could bridge NK cells with HIV-infected allogeneic targets leading to cytolysis or inhibition of HIV replication in infected cells. We are interested in exploring whether these anti-MHC Abs play a role in protection from infection in multiply exposed HESN using such a mechanism.

Pathogenesis and Cell Biology of HIV Infection and Co-infection

Pathogénie et biologie cellulaire de l'infection et de la co-infection au VIH

\section{P035}

\section{SUPPRESSOR OF CYTOKINE SIGNALLING (SOCS)} PROTEINS ARE INDUCED BY IL-7 AND MAY PLAY A ROLE IN REGULATING CD127 EXPRESSION IN HUMAN CD8 T-CELLS

Al-Ghazawi, Feras $\mathrm{M}^{1}$; Parmar, Parmvir ${ }^{1}$; Sugden, Scott ${ }^{1}$; Faller, Elliott ${ }^{1}$; Sant, Nadia ${ }^{1}$; MacPherson, Paul A ${ }^{1}$ 1 Ottawa, ON

BACKGROUND: Interleukin (IL)-7 plays essential roles in T-cell development, homeostasis and activation. Disruption of this cytokine pathway likely contributes to HIV-induced immune deficiency. We previously showed that IL-7 and the HIV Tat protein reduce the half-life of the IL-7 receptor alpha-chain (CD127) in human CD8 T-cells but the mechanism directing CD127 to the proteasome is not yet understood. In this study we examined roles of SOCS proteins in regulating CD127 expression. METHODS: CD8 T-cells isolated from healthy HIV-negative volunteers were treated with IL-7 $(0.1-10 \mathrm{ng} / \mathrm{ml})$ in the presence or absence of various inhibitors. SOCS1-7 and CIS transcripts were examined by qPCR and protein expression was measured by Western. The interaction of SOCS proteins with CD127 was examined by Co-IP. Surface CD127 protein expression was measured by flow cytometry. Intracellular localization of SOCS and CD127 protein was examined by confocal microscopy.

RESULTS: IL-7 induces the expression of SOCS1-3 and CIS transcripts in CD8 T-cells via the JAK/STAT-5 signaling pathway in a time- and dosedependent manner with SOCS2 transcripts increasing 300-fold within 3 hours. While induction of SOCS2 and SOCS3 mRNA was transient, 
SOCS1 and CIS transcripts remained elevated over baseline for at least 48hours. Western blot analysis confirmed increased protein expression of the induced SOCS genes. Preliminary data on CD8 T-cells isolated from $\mathrm{HIV}+$ patients indicate that the IL-7-mediated up-regulation of SOCS transcripts is significantly decreased compared to healthy controls. IL-7 induces rapid phosphorylation and internalization of CD127 followed by proteasomal degradation. By Co-IP we show SOCS proteins induced by IL-7 physically interact with CD127 and study their cellular localization by confocal microscopy. We hypothesize this interaction directs the receptor to the proteasome.

CONCLUSIONS: IL-7 induces the expression of SOCS1-3 and CIS genes through the JAK/STAT-5 pathway. Through physical interaction with CD127, SOCS proteins may direct CD127 to the proteasome for degradation.

\section{P036}

\section{IL-7 SUPPRESSES CD127 GENE TRANSCRIPTION IN} HUMAN CD8 T-CELLS VIA A STAT5-INDUCED REPRESSOR Al-Ghazawi, Feras M; Faller, Elliott; Parmar, Parmvir; Kakal, Juzer; MacPherson, Paul A

\section{Ottawa, ON}

BACKGROUND: In view of the role interleukin (IL)-7 plays in T-cell survival, homeostasis and function it is no surprise expression of the IL-7 receptor alpha-chain (CD127) is tightly regulated. We previously showed that expression of CD127 is suppressed on CD8 T-cells in $\mathrm{HIV}^{+}$patients and that this suppression is mediated by both IL-7 and the HIV Tat protein. IL-7 down-regulates CD127 transcripts and surface protein through two distinct mechanisms. In this study we examine the mechanism by which IL-7 down-regulates the CD127 gene at the level of transcription. METHODS: CD8 T-cells from HIV-negative volunteers were treated with IL-7 $(0.1-10 \mathrm{ng} / \mathrm{ml})$ in the presence or absence of various inhibitors. CD127 transcripts were quantified by qPCR. STAT-5 phosphorylation was measured by flow cytometry. Nuclear run-on assays were utilized to measure the rate of CD127 gene transcription. Candidate CD127 repressors were identified using PCR arrays, qPCR and Western.

RESULTS: IL-7 attenuates levels of CD127 transcripts in CD8 T-cells in a time- and dose-dependent manner. Both the full-length transcript and the splice-variant encoding the secreted isoform of CD127 are suppressed by IL-7. We show by nuclear run-on assay that IL-7 suppresses the rate of transcription of the CD127 gene and found no evidence that IL-7 affects the stability of CD127 mRNA. Further, the suppression of CD127 transcripts is dependent on JAK kinase activity and phosphorylation of STAT-5 but not STAT-3. Notably, cycloheximide blocked IL-7's ability to down-regulate CD127 transcripts suggesting IL-7 stimulates the de novo synthesis of a transcriptional repressor which in turn suppresses CD127 gene transcription. We recently identified several candidate repressors using PCR arrays and are currently examining their involvement in the transcriptional suppression by siRNA-mediated knockout experiments. CONCLUSIONS: Upon binding to its receptor, IL-7 activates the JAK/ STAT-5 signaling and induces the expression of a transcriptional repressor which suppresses CD127 gene transcription.

\section{P037 \\ TRANSCRIPTIONAL PROFILING IN HIV PERMISSIVE VERSUS RESISTANT CD4 ${ }^{+}$T-CELL SUBSETS IDENTIFIED PPARG AS A NEGATIVE REGULATOR OF HIV REPLICATION \\ Bernier, Annie ${ }^{1}$; Monteiro, Patricia ${ }^{1}$; Goulet, Jean-Philippe ${ }^{1}$; \\ Gosselin, Annie' ${ }^{1}$; Sekaly, Rafick-Pierre²; Haddad, Elias'; \\ Ancuta, Petronela ${ }^{1}$ \\ ${ }^{1}$ Montreal, QC; ${ }^{2}$ Port St Lucie, FL, USA \\ BACKGROUND: We previously demonstrated that CXCR $3^{+} \mathrm{CCR} 6^{+}$ (Th1Th17) and CXCR3 ${ }^{+} \mathrm{CCR}^{-}{ }^{-}$(Th1) T-cells are permissive and resist- ant to HIV, respectively. A systems biology approach was used to iden- tify molecular mechanisms regulating HIV permissiveness in primary Th1Th17 versus Th1 cells. \\ METHODS: The Affymetrix technology was used to identify genes dif- ferentially expressed in Th1 versus Th1Th17 cells. One-way ANOVA analysis identified differentially expressed genes. Gene expression was validated by SYBR Green real-time RT-PCR, FACS, and western blot- ting. Specific activators/inhibitors and RNA interference were used to}

assess the role of identified transcripts in HIV regulation. ELISA and real-time PCR were used to quantify HIV replication and integration, respectively. Viability (Vivid) and proliferation assays (CFSE dilution) were performed in parallel.

RESULTS: Among 38,114 present calls, 780 probe sets were differentially expressed in Th1Th17 versus Th1 cells $(\mathrm{p}<0.05)$, with 265 and 235 genes being upregulated and downregulated, respectively. PPARG (peroxisome proliferator-activated receptor gamma), a known negative regulator of Th17 polarization, was upregulated in Th1Th17 versus Th1 cells. Activation of PPARG pathway using Rosiglitazone significantly decreased HIV replication and integration in Th1Th17 cells, without interfering with cell viability, proliferation, nor CD4 expression. RNA interference studies confirmed the role of PPARG in the negative regulation of HIV replication.

CONCLUSION: This is the first genome-wide characterization of gene expression in CD4 ${ }^{+}$T-cell subsets permissive (Th1Th17) versus resistant (Th1) to HIV infection. We identified the PPARG pathway as a negative regulator of HIV replication, likely by interfering with Th17 polarization. These studies have the potential to suggest new therapeutic strategies to interfere with HIV replication in primary T-cells.

\section{P038}

EFFECT OF PAMPS ON DENDRITIC CELL MATURATION AND THEIR SUBSEQUENT ABILITY TO TRANSFER HIV-1 TO RESTING CD4+T CELLS

\section{Cote, Sandra C}

Ottawa, ON

Microbial translocation and opportunistic infections are key components of HIV-1 pathogenesis. The recognition of microbial compounds by dendritic cells (DC) leads to their maturation, a process that allows DC to activate $T$ cells. Our knowledge of the impact of many microbial components on DC maturation of and DC-mediated HIV-1 transfer is limited. We evaluated the effect of different agonists of Pathogen Associated Molecular Patterns (PAMPs) on the maturation of monocyte-derived DC and their subsequent ability to activate resting $\mathrm{CD} 4^{+} \mathrm{T}$ cells. Moreover, we measured the DC-dependent propagation of HIV-1 to CD4 $4^{+} \mathrm{T}$ cells. Our results demonstrate that treatment of DC with PGN, zymosan and Pam3Csk4 increases HIV-1 transmission in trans and to a lesser extent in cis. This phenomenon is associated with increased susceptibility of DC to infection with an X4-using virus, which is linked with an increased surface expression of CXCR4. This study demonstrates the involvement of DC at different stages of the HIV-1 infection process and the importance of treatment of opportunistic infections in HIV-1 positive patients.

\section{P039}

\section{HIV-1 SUBVERTS ENDOSOME-ASSOCIATED FACTOR} ORP1L TO PROMOTE HIV-1 GENOMIC RNA EGRESS

\section{Crossie, Christina L; Mouland, Andrew J}

\section{Montreal, QC}

Rab7-interacting lysosomal protein (RILP) interacts with Rab7 GTPase, found exclusively on late endosomes (LEs) and a subunit of the dynein motor protein. We have previously determined RILP over-expression results in the clustering of Gag and vRNA at the microtubule organizing center (MTOC). Oxysterol binding protein (ORP1L) interacts with RILP and Rab7 on LEs and undergoes conformational changes upon sensing cholesterol-rich membranes resulting in RILP recruitment of dynein and movement of LEs to the MTOC.

As cholesterol-rich membranes have been determined to promote HIV1 Gag membrane association, we hypothesized that HIV-1 subverts ORP1L function to promote egress of cholesterol-rich LEs occupied by Gag and vRNA. To investigate the relationship between ORP1L and HIV-1, we utilized two ORP1L sensor mutants. The first, ORP1L $\triangle$ ORD, that lacks the cholesterol sensing domain, prevents RILP recruitment of dynein to result in the peripheral dispersal of LEs. The second, ORP1LAORDPHDPHD, contains two PHD domains that anchor the molecule to LEs and allows RILP to recruit dynein to result in LE clustering at juxtanuclear domains. We co-expressed pNL4.3 proviral DNA with wildtype and mutant ORP1L DNAs and determined the localization of vRNA, Gag, and LEs by combined immunofluorescence/fluorescence in situ hybridization and laser scanning confocal microscopy. 
As expected, co-expression of pNL4.3 and ORP1LAORD resulted in peripheral co-localization of LEs and ORP1L $\triangle O R D$. Interestingly, LEs and ORP1LAORDPHDPHD co-localized at the cell periphery when pNL4.3 was co-expressed with ORP1L $\triangle$ ORDPHDPHD. Similar results were obtained when ORP1L and HIV-1 were co-expressed. In all three conditions, Gag and vRNA were located peripherally.

These results demonstrate that HIV-1 subverts ORP1L signalling to influence LE egress and that HIV-1 co-opts host membranes and motor complexes to promote egress of vRNA and Gag to assembly sites.

This work is supported by a grant from the Canadian Institutes of Health Research (MOP-56974)

\section{P040 \\ A ROLE FOR NEGATIVE REGULATORS OF T CELL ACTIVATION IN THE ESTABLISHMENT AND MAINTENANCE OF THE HIV RESERVOIR}

Da Fonseca, Sandrina ${ }^{1,2}$; El-Far, Mohamed ${ }^{1}$; Boulassel, Rachid ${ }^{1}$; Routy, Jean-Pierre'; Sékaly, Rafick-Pierre ${ }^{2}$; Chomont, Nicolas ${ }^{2}$

${ }^{1}$ Montréal, QC; ${ }^{2}$ Port St Lucie, FL, USA

BACKGROUND: In spite of advances in the treatment of HIV infection, HAART does not eradicate HIV. A small pool of latently infected memory $\mathrm{CD}^{+} \mathrm{T}$ cells persists in virally suppressed subjects. Characterization of the phenotype of these cells is a prerequisite to the design of novel targeted strategies aimed at eliminating them. Receptors at the cell surface known as negative regulators inhibit cell proliferation and contribute to the induction of cellular quiescence. The engagement of these receptors could inhibit viral production and induce viral latency. Here we investigate a possible role for these receptors in the establishment and maintenance of a cellular reservoir for HIV.

METHODS: PBMCs were obtained by leukapheresis from HAART naïve chronically HIV-1 infected subjects, HAART treated subjects and uninfected controls. The level of expression of seven negative regulators (including PD-1, CTLA-4, TIM-3, CD85j, LAG-3, LAIR-1 and CD160) were measured in $\mathrm{CD}^{+}{ }^{+} \mathrm{T}$ cell subsets by flow cytometry. In addition, the frequency of $\mathrm{CD}^{+} \mathrm{T}$ cells harboring HIV DNA was determined in sorted cells expressing high and low levels of these receptors by Q-PCR. RESULTS: Flow cytometry analysis showed high frequencies of $\mathrm{CD}^{+} \mathrm{T}$ cells expressing PD-1, TIM-3 and CTLA-4 in viremic donors, suggesting that these receptors may contribute to the establishment of the viral reservoir during untreated HIV infection. Cell sorting and Q-PCR experiments showed that PD-1high cells from viremic donors preferentially harbor HIV-1 integrated DNA when compared to their PD-1low counterparts, indicating that these cells constitute a preferential reservoir for the virus. Similar results were obtained when viral DNA was quantified in CTLA-4high versus CTLA-4low cells.

CONCLUSIONS: Our results demonstrate that $\mathrm{CD}^{+} \mathrm{T}$ cells expressing negative regulators are enriched for HIV DNA in HIV infection and suggest a crucial role for these molecules in the establishment and maintenance of HIV latency.

\section{P041}

HIV-1 TAR AND RRE RNAS COMPETE FOR BINDING TO TRBP AND INHIBIT LET7 FUNCTION: IMPLICATIONS FOR PATHOGENESIS

Daniels, Sylvanne M; Sinck, Lucile; Ward, Natalie J;

Scarborough, Robert; Melendez-Pena, Carlos E; Azar, Ibrahim;

Gatignol, Anne

Montreal, QC

RNA interference (RNAi) is an essential mechanism of post-transcriptional regulation in eukaryotic cells. Plants and invertebrates rely on RNAi for their antiviral defence, and many plant and insect viruses encode suppressors of RNAi (VSRs). RNAi activity requires the assembly of the RNA induced silencing complex (RISC), composed of Dicer, Argonaute 2 (Ago2) and the TAR-RNA Binding Protein (TRBP). The presence of TRBP in the RISC is essential for proper RNAi function. Studies have identified the HIV TAR RNA as a potential suppressor of RNAi, and suggested that it may act by sequestering TRBP away from the RISC. We evaluated whether HIV expresses other structured RNAs that act as VSRs and whether they contribute to viral replication and changes cellular functions.
We have shown that the structured RNA elements TAR and the Rev-Response Element (RRE) inhibit RNAi activity. We have identified RRE as a novel VSR and have shown that it binds to TRBP. TAR and RRE block the binding of siRNAs to TRBP, but do not prevent TRBP from binding to other RISC proteins. In the presence of the pNL4-3 provirus, RNAi activity remains functional in cells. This suggests that TAR and RRE act at discrete steps in the replication cycle by competing with cellular si/ miRNAs rather than by disrupting the cellular RNAi machinery.

Our work shows that in functional assays, RRE acts as a VSR and binds to TRBP, thereby preventing the recruitment of siRNAs. TAR and RRE may contribute to HIV pathogenesis by changing the incorporation of endogenous miRNAs into the RISC, thereby modifying cellular gene expression.

\section{P042 \\ IN VITRO HIV INFECTION REDUCES IL-17 EXPRESSION BY HUMAN TH17 CELLS}

Fernandes, Jason R ${ }^{1}$; Angel, Jonathan $\mathbf{B}^{\mathbf{1}}$

1Ottawa, ON

BACKGROUND: $\mathrm{T}$ cell dysfunction persists in $\mathrm{HIV}^{+}$individuals despite restoration of $\mathrm{CD}^{+} \mathrm{T}$ cell counts to near normal levels with HAART. Selective loss and deficiency of Th17 cells in the gut was recently described and may contribute to microbial translocation and persistent inflammation observed in HIV infection. It is unclear whether this deficiency results from HIV inhibiting Th17 cell differentiation orinhibition of Th17 cell function. Th17 cell development and IL-17 production require the STAT3 intracellular signaling pathway. The known interaction of HIV regulatory proteins with STAT3 may explain the ongoing deficiency in Th17 cells in HAART-treated patients even after $\mathrm{CD}^{+} \mathrm{T}$ cell recovery.

We hypothesize that Th17 differentiation and function is impaired in HIV infection due to altered STAT3 signalling.

METHODS: $\mathrm{CD}^{+} \mathrm{T}$ cells were isolated from peripheral blood using established methods and activated with anti-CD3 and anti-CD28 in the presence of IL-1 $\beta$, IL-6, and IL-23 for 7 days. The resulting T cell blasts were infected with a dual tropic HIV strain (HIVCS204) for 24 hours. Following infection these cells were stimulated with PMA and ionomycin for 4 hours in the presence of brefeldin A. Th17 function was assessed through flow cytometric staining of intracellular IL-17.

RESULTS: Restimulation of control, uninfected CD4 ${ }^{+} \mathrm{T}$ cells activated with IL-1 $\beta$, IL- 6 and IL-23 induced IL-17 production in a small subset of cells. IL-17 induction was inhibited when differentiated cells were infected in vitro with HIVCS204 prior to activation with PMA and ionomycin.

CONCLUSION: In vitro HIV infection inhibits production of IL-17 by in vitro differentiated Th17 cells suggesting that HIV may play a role in altering Th17 responses through inhibition of upstream intracellular signaling pathways. Understanding the mechanism of this inhibition may provide insight into therapies that will correct immune homeostasis in the gut of patients on HAART.

\section{P043}

\section{THE ROLE OF CELLULAR DNA SINGLE-STRAND BREAK REPAIR PROTEINS IN HIV-1 REPLICATION}

Flores-Soto, Rodrigo A; Plourde, Mélodie B; Berthoux, Lionel

Trois-Rivières, QC

BACKGROUND: The life cycle of retroviruses, including HIV-1, comprises an integration step which consists of the insertion of the virus DNA into the host DNA. Although integration itself is carried out by the viral protein integrase, the integrated DNA bears defects and needs to be repaired by specialized cellular proteins. The type of DNA damage resulting from retroviral integration, called "single strand break" (SSB), is the most common one in the cell and is often repaired by pathways called SSB repair (SSBR) or "base excision repair" (BER).

HYPOTHESIS and OBJECTIVES: We hypothesize that components of the SSBR or BER pathways are critical to HIV-1 post-integration DNA repair. We are investigating this possibility with the aim of evaluating the potential of some of these proteins as targets for AIDS drugs. METHODOLOGY and RESULTS: We are taking a "loss-of-function" approach to this problem, using several techniques: (i) knockdown by 
RNAi, (ii) expression of negative-dominant versions, (iii) use of pharmacological inhibitors. Our initial observations with FEN1 (flap endonuclease 1) are not conclusive, with siRNAs showing no effect on transduction of HIV-1 vectors while, on the other hand, the FEN1 smallmolecule inhibitor PTPD decreased retroviral transduction. We are presently testing siRNAs inhibiting other targets such as PolB, PCNA, ERCC1 and ERCC5. Some of these proteins (ERCC1 and PolB) have been found to be important for HIV replication in large-scale screens in recent years.

Future directions: Impaired DNA reparation could lead to mutations at viral:cellular DNA junctions. In addition to quantifying the effects of knocking down the candidate proteins on transduction, we will perform sequencing analyses of these junctions to test this hypothesis.

\section{P044 \\ EXAMINING THE ROLE OF TGF- $\beta$ AND SEMINAL PLASMA FROM HIV-1 INFECTED AND UNINFECTED MEN ON FEMALE GENITAL TRACT BARRIER FUNCTION}

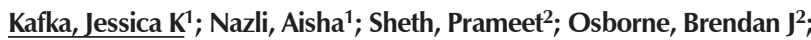
Kovacs, Colin ${ }^{2}$; Kaul, Rupert ${ }^{2}$; Kaushic, Charu ${ }^{1}$

${ }^{1}$ Hamilton; ${ }^{2}$ Toronto, ON

Greater than $60 \%$ of HIV transmission occurs via seminal plasma (SP), however little is known about the immune impact of SP on HIV susceptibility. We have shown that SP from HIV-infected men in chronic stages of infection contains high levels of TGF- $\beta 1$. TGF- $\beta 1$ has been found to strengthen intestinal epithelial barrier (EB) and prevent EB dysfunction mediated by bacterial pathogens. Previous studies from our lab have shown that HIV-1 can directly impair mucosal EB integrity; however the role of SP on this process is unknown. This study was undertaken to characterize the effects of TGF- $\beta$ and SP on EB function in the female genital tract (FGT) and determine whether TGF- $\beta$ and SP treatment prevents HIV-mediated increase in EB permeability. Confluent primary genital epithelial cell (GEC) monolayers were incubated with $5 \mathrm{ng} / \mathrm{ml}$ rhTGF- $\beta 1$ alone, $1 \times 10 \wedge 5$ IU of HIV- 1 alone or both for 24 hours. To measure the strength of GEC tight junctions, trans-epithelial electrical resistance (TER) values were taken prior to and 24 hours post-treatment. Treatment with TGF- $\beta$ enhanced the TERs of GEC monolayers. This was accompanied with increase in ZO-1 and occludin expression following TGF- $\beta$ treatment. When TGF- $\beta$ was added with HIV-1, no decrease in TER of GEC monolayers was observed suggesting that the presence of TGF- $\beta$ completely prevented the HIV-mediated disruption of EB integrity. The results so far show that TGF- $\beta$ can strengthen EB integrity by increasing expression of tight junction proteins as well as preventing HIV-mediated barrier impairment in GECs suggesting that the presence of TGF- $\beta$ can regulate HIV transmission in the FGT. Further studies will examine the role of SP on HIV-mediated EB permeability in the FGT. By understanding the role of TGF- $\beta$ and SP on EB function in the FGT, therapeutic strategies can be developed to increase EB-strengthening factors present in the FGT during HIV transmission.

\section{P045}

THE PYK2 AND C-SRC PROTEIN TYROSINE KINASES PLAY PROTECTIVE ROLES IN EARLY HIV-1 INFECTION OF CD4 ${ }^{+}$

T-cells

McCarthy, Stephen $\mathrm{D}^{1}$; Sakac, Darinka ${ }^{1}$; Xue-Zhong, $\mathrm{Ma}^{1}$;

Jung, Daniel'2; Branch, Donald R ${ }^{1}$

${ }^{1}$ Toronto, ON; ${ }^{2}$ Quebec, QC

BACKGROUND: During early infection of $\mathrm{CD}^{+}$T-lymphocytes, a variety of protein tyrosine kinases (PTK) become activated within minutes of HIV-1 infection, including the phosphoprotein pp60c-src (c-Src) and the focal adhesion kinase family member, proline-rich tyrosine kinase 2 (Pyk2). Whether their activation facilitates or impedes infection remains to be determined.

METHODS: c-Src kinase inhibitors SU6656, PP1 and PP2 were used to reduce c-Src activity in Jurkat $\mathrm{C}$ and Jurkat E6-1 T-cells. Adenovirus vectors containing wild-type (WT) or dominant-negative (DN) c-src genes were used to transduce Jurkat E6-1, HuT78 or KIT225 T-cells. Small interfering RNA's (siRNA) specific for c-src or pyk2 mRNA were used to knockdown their respective protein levels in Jurkat E6-1. In all three approaches, cells were then infected with HIV-1 luciferase reporter viruses containing either VSV-G or HXB2(X4) envelopes and luciferase activity was measured after 2 days.

RESULTS: Pre-treating Jurkat T-cells with SU6656, PP1 or PP2 led to an increase in luciferase activity using the VSV-G reporter virus. PP3 used as a control did not have this effect. In the adenovirus-vector experiments, Jurkat, HuT78 and KIT225 T-cells overexpressing DN c-Src, but not WT c-Src, showed increased luciferase activity after VSV-G infection. siRNA knockdown of both c-Src and Pyk2 in Jurkat T-cells lead to increased luciferase activity when using the HXB2 reporter virus. CONCLUSIONS: Pyk2 is activated following HIV infection, and this coincides with increased activation of c-Src. Pyk2 is also known to interact with c-Src. Our findings show that reducing c-Src or Pyk2 activity/ protein decreases the luciferase activity of $\mathrm{CD}^{+} \mathrm{T}$-cells infected with VSV or HXB2 enveloped HIV-1. This suggests an inhibitory effect on HIV-1 entry, integration and/or transcription. Thus, the increased c-Src and Pyk2 activity seen early in HIV-1 infection may be a cellular response to slow or prevent infection in $\mathrm{CD}^{+} \mathrm{T}$-cell lines.

\section{P046}

RÔLE DU DCIR DANS LA LIBÉRATION D'EXOSOMES

Mfunyi, Claude M; St Pierre, Christian; Lambert, Alexandra;

Gilbert, Caroline

Québec, QC

INTRODUCTION : Les exosomes sont des microvésicules d'origine endosomale produites principalement par les cellules hématopoïétiques et épithéliales qui facilitent la communication intercellulaire. Leur voie de dissémination converge avec celle de la capture et du transfert du virus d'immunodéficience humaine (VIH) par les cellules dendritiques. Dans l'infection au VIH-1, le récepteur lectine de type C, DCIR, joue un rôle majeur dans l'attachement des virions aux cellules dendritiques et leurs transferts aux cellules T CD4. Nous avons étudié l'impact de la liaison du DCIR avec le VIH-1 afin d'en dégager le rôle que jouent ce récepteur et ses voies de signalisation dans la libération d'exosomes.

MÉTHODES : Premièrement, nous avons évalué le niveau d'expression de DCIR sur les RajiCD4DCIR en culture dans du RPMI avec sérum contenant ou non des exosomes bovins, et déterminer l'impact de ces exosomes sur la prolifération cellulaire. Ensuite, nous avons déterminé le rôle de DCIR dans la production d'exosomes après stimulation avec, des anticorps monoclonaux anti-DCIR spécifiques, et avec le VIH-1. Finalement, nous avons étudié le rôle du domaine de signalisation ITIM du récepteur DCIR dans la production d'exosomes.

RÉSULTATS : Les exosomes bovins favorisent l'internalisation de DCIR et diminuent la prolifération des cellules en culture. La stimulation de DCIR sur la lignée cellulaire RajiCD4DCIR avec les anticorps monoclonaux entraîne une production d'exosomes plus importante à 48 heures par rapport au contrôle. Alors que l'infection de la même lignée cellulaire avec le VIH-1 indique également une augmentation de la production d'exosomes. Par contre la stimulation anti-DCIR de la lignée mutante, RajiCD4DCIRY7F, qui possède une modification dans son domaine de signalisation intracellulaire (ITIM), libère peu d'exosome. Ce qui montre l'implication de ce motif ITIM dans la libération d'exosomes.

CONCLUSION : Notre étude met donc en évidence le rôle de DCIR et l'importance de sa signalisation dans la production d'exosomes.

\section{P047}

INDUCTION OF TIM-3 BY THE COMMON GAMMA-CHAIN CYTOKINES IL-2, IL-7, IL-15 AND IL-21

Mujib, Shariq; Jones, Brad; Lo, Calvin; Aidarus, Nasra; Clayton, Kiera; Ostrowski, Mario

Toronto, ON

Chronic HIV-1 infection is established as a result of the host immune system's inability to control viral load. Previous work from our lab has shown that virus-specific CD8 killer T-cells in the PBMC of chronically HIV-1-infected individuals express greater levels of the exhaustion marker Tim-3, on their surface (Jones et al. J Exp Med, 2008). Tim-3 expressing T-cells are dysfunctional as measured by their failure to proliferate and secrete effector cytokines, such as IFN-g, in response to infected cells. Acute and chronic HIV infection is also marked by a global upregulation of Tim-3 on T cells, the mechanisms behind which are poorly understood. Here we show that the HIV virus itself is unable to upregulate 
Tim-3 expression on $\mathrm{T}$ cells and it is rather the common gamma-chain cytokines IL-2, IL-7, IL-15 and IL-21, also found to be elevated in the blood plasma of HIV infected individuals, that are implicated in the over expression of Tim-3. These cytokines are associated with $\mathrm{T}$ cell survival, proliferation and improving effector function; however, we show here that they also play a counter-regulatory role in dampening the immune response by upregulating Tim-3. We also show that cytokine induced Tim-3+ $3^{+}$-cells are more prone to death by Tim-3's binding to its ligand, galectin-9, and are unable to secrete IFN-g upon TCR stimulation and are thereby 'exhausted'. Finally, we were able to abrogate Tim-3 induction by the gamma-chain cytokines on $T$ cells in the presence of a phosphoinositide 3-kinase (PI3K) pathway inhibitor, LY 294002. Overall, this study suggests that Tim-3 expression is associated with elevated cytokine production and is dependent on the PI3K pathway.

\section{P048}

\section{IL-22 PREVENTS BARRIER IMPAIRMENT CAUSED BY HIV-1 EXPOSURE IN FEMALE PRIMARY UPPER GENITAL TRACT EPITHELIAL CELLS.}

Nazli, Aisha ${ }^{1}$; Kim, Connie J²; Kaul, Rupert ${ }^{2}$; Smaill, Fiona ${ }^{1}$;

\section{Kaushic, Charu ${ }^{1}$}

\section{${ }^{1}$ Hamilton; ${ }^{2}$ Toronto, ON}

Our recent data indicates that HIV-1 exposure leads to induction of proinflammatory cytokines that cause impairment of mucosal barrier in the female genital tract, possibly resulting in viral and bacterial translocation. IL-22 is an important cytokine that can prevent tissue damage and epithelial barrier breakdown in mouse model of IBD and also enhance wound healing in an in vitro injury model of primary human keratinocytes. Therefore we investigated the effect of IL-22 in preventing HIV-1 induced mucosal barrier breakdown in primary upper genital epithelial cells (GECs). Transepithelial resistance (TER) measurements of epithelial monolayers pre-treated with recombinant IL-22 prior to HIV-1 exposure showed that IL-22 completely prevented barrier impairment in presence of HIV-1. Examination of tight junction protein ZO-1 staining in IL-22 pre-treated, HIV-1 exposed monolayers revealed completely intact ZO-1 staining by confocal microscopy compared to HIV-1 exposed monolayers where ZO-1 staining was profoundly disrupted. These results indicate that primary GECs are exquisitely responsive to IL-22. However, the presence or source of IL-22 in female genital tract is not known. To investigate, IL-22 was measured by quantitative ELISA in vaginal and cervical lavages (CVL) collected from $40 \mathrm{HIV}$-infected, HSV-1 or 2 co-infected women attending Hamilton SIS Clinic. Vaginal lavages were also collected from normal healthy women. IL-22 was detected in vaginal and CVL of most women. It was present in lower concentrations in vaginal lavages compared to CVL and lowest levels were present in healthy uninfected controls. CVLs collected from HIV-1 positive women, co-infected with HSV-1 and 2 contained significantly higher quantities of IL-22 compared to HIV-1 alone or HIV-1 co-infected with either HSV1 or 2 groups. These studies indicate that IL-22 is present and regulated by the local milieu in the female genital tract. IL-22 may play an important role in regulating the barrier functions in the female genital tract.

\section{P049 \\ DELAY IN CART INITIATION RESULTS IN PERSISTENT IMMUNE DYSREGULATION AND POOR RECOVERY OF T-CELL HOMEOSTASIS DESPITE A DECADE OF SUCCESSFUL HIV SUPPRESSION \\ Ndumbi, Patricia; Falutz, Julian; Tsoukas, Christos M Montreal, QC}

BACKGROUND: Combination antiretroviral therapy (cART) increases levels of $\mathrm{CD}^{+}{ }^{+} \mathrm{T}$-cells but this increase may not accurately reflect long-term immune recovery as HIV-mediated T-cell dysregulation and loss of T-cell homeostasis often persist despite successful cART. We assessed the impact of a decade of effective cART on immune regulation, T-cell homeostasis and overall T-cell phenotype (TCP).

METHODS: Within a large observational cohort, we conducted a retrospective study of a subset of $288 \mathrm{HIV}^{+}$individuals that were cARTnaïve prior to study baseline. We identified 86 study participants receiving cART for at least a decade, of which 44 maintained undetectable plasma HIV RNA levels for almost the entirety of their treatment time
(95.53\%, [95\%CI: 93.48, 97.59]). At baseline, participants were classified into three groups according to pre-treatment $\mathrm{CD} 4^{+} \mathrm{T}$-cell counts: Group I (CD4 <200 cells $/ \mathrm{mm}^{3}$ ), Group II (CD4: 200-350 cells/mm3) and Group III (CD4 $>350$ cells $\left./ \mathrm{mm}^{3}\right)$. A normal CD4:CD8 T-cell ratio was defined as a ratio within 1.2-3.3, and $\mathrm{CD}^{+}{ }^{+} \mathrm{T}$-cell homeostasis was defined as the maintenance of a dynamic equilibrium of peripheral blood T-lymphocytes with $\mathrm{CD}^{+}$T-cell percentages between $65 \%-85 \%$ of peripheral blood lymphocytes.

RESULTS: Despite a decade of sustained suppressive cART, patients initiating therapy at an advanced stage of the disease exhibited the slowest recovery rate in terms of CD4 T-cell counts and CD4:CD8 ratio. Though there was no difference in the change in $\mathrm{CD}^{+} \mathrm{T}$-cell percentages across the groups, patients with CD4 ${ }^{+} \mathrm{T}$-cell counts $<200$ cells $/ \mathrm{mm}^{3}$ exhibited the lowest level of $\mathrm{CD}^{+} \mathrm{T}$-cells at baseline and through out the follow-up. Overall complete TCP normalization only occurred in 13\% of patients, most of which initiated therapy at higher $\mathrm{CD} 4^{+} \mathrm{T}$-cell counts.

CONCLUSIONS: Poor T-cell phenotype normalization was most apparent in patients who initiated cART with baseline CD4+ T-cell count $<200$ cells $/ \mathrm{mm}^{3}$. It is unclear how this persistently impaired T-cell phenotype will relate to life-long immune function and potential comorbidities.

\section{P050 \\ RÔLE DE LA PROTÉINE S100A9 SUR LA LIBÉRATION D'EXOSOMES PAR LES LYMPHOCYTES T CD4}

Subra, Caroline; Tessier, Philippe; Gilbert, Caroline

Québec, QC

La présence du complexe protéique nommé calprotectine, composé d'un hétérodimère des protéines S100A8 et S100A9 est étroitement reliée à une activité inflammatoire. Chez les patients séropositifs pour le virus de l'immunodéficience humaine (VIH-1), leur présence est aussi corrélée avec la gravité de la maladie. De plus, il a récemment été observé que des ARNs double brins induisaient l'augmentation des protéines S100A8/ A9 dans des monocytes/macrophages. Les lymphocytes T CD4 (LTCD4) des patients séropositifs sont donc susceptibles d'être en contact avec les protéines S100A8/A9, médiateurs de l'inflammation. D'autre part, plusieurs types de leucocytes libèrent constitutivement ou sous l'action d'agoniste, dans le milieu extracellulaire, des nanovésicules très semblables au virus: les exosomes. Ces exosomes participent entre autre à la communication intercellulaire et pourraient jouer un rôle important dans l'infection par le VIH-1. La protéine S100A9 pourrait favoriser la libération des exosomes par les LTCD4 infectées ou non. Dans notre laboratoire, nous avons observé que les particules de VIH-1 favorisent leurs libérations par les cellules dendritiques et que ces exosomes peuvent provoquer l'apoptose des LTCD4. Nos résultats indiquent aussi une augmentation de la quantité d'exosomes dans le milieu extracellulaire des LTCD4 infectés. De plus la protéine S100A9, augmente également la sécrétion d'exosomes par les LTCD4. Enfin grâce aux méthodes que nous avons développées, il est possible de séparer les particules virales des exosomes. Ces méthodes nous ont permis de caractériser séparément ces deux types de vésicules à la suite d'une stimulation avec la protéine S100A9. La sécrétion d'exosomes apparait donc être un mécanisme régulé par différents médiateurs de l'inflammation et pourrait contribuer à l'inactivation des LTCD4, un phénomène décrit dans la pathogenèse de l'infection à VIH-1.

\section{P051}

HIV TAT PROTEIN BINDS THE CYTOPLASMIC TAIL OF CD127 VIA TAT'S N-TERMINAL DOMAIN RESULTING IN DOWN REGULATION OF CD127 FROM THE SURFACE OF CD8 T CELLS

\section{Sugden, Scott M; MacPherson, Paul A}

Ottawa, ON

HIV Tat protein down regulates surface expression of the interleukin-7 receptor alpha-chain (CD127) on CD8 T-cells resulting in impaired Tcell proliferation and cytolytic capacity. Once taken up by CD8 T-cells, Tat binds directly with the cytoplasmic tail of CD127 inducing receptor internalization and degradation. Given CD127's important roles in proper immune function, we characterized which domains of Tat are required to interact with CD127 and induce receptor loss from the surface of CD8 T cells. 
His-tagged Tat deletion mutations mutants were generated, expressed in bacteria and purified over nickel. Wild-type Tat and Tat mutants were incubated with CD8 T-cells isolated from HIV-negative volunteers and surface CD127 expression was analyzed by flow cytometry. In parallel, a CD8 T-cells and a Jurkat-CD127 line were transduced with wildtype or mutant Tat expressing DNA vectors. To characterize Tat-CD127 physical interactions, wild-type Tat and Tat mutants were incubated with JurkatCD127 lysates followed by CD127 immunoprecipitation.

Removal of amino acids 22-26, core (aa. 38-46), glutamine-rich (60-72), or carboxyl-terminal (aa. 72-86) domains did not effect Tat's ability to suppress CD127 expression. Substitution of the first $10 \mathrm{~N}$-terminal residues or deletion of residues 17-21 prevented both exogenous and endogenous Tat from down regulating CD127. These mutants also failed to bind CD127 via immunoprecipitation. Deletion of the basic domain (aa. 48-59) prevented exogenous Tat from down regulating CD127. This is likely a result of Tat losing its ability to enter the cell. Deletion of the basic domain did not prevent Tat from interacting physically via immunoprecipitation. Strikingly, endogenously expressed $\Delta$ Basic Tat did not down regulate CD127 but rather acted as a dominant negative mutant, causing an accumulation of CD127 at the cell surface.

We show here that Tat binds CD127 via its amino-terminal region and then recruits a trafficking protein via its basic domain which targets CD127 for degradation.

\section{P052}

NEW ANTI-HIV-1 INHIBITORS, 8-AZA AND 150, TARGET HIV-1 RNA PROCESSING BY ALTERNATE MECHANISMS Wong, Raymond W; Balachandran, Ahalya; Haaland, Matthew; Cochrane, Alan W

\section{Toronto, ON}

We have recently demonstrated that HIV-1 RNA processing is a viable therapeutic target by two small molecule inhibitors. However, the properties of these drugs preclude their direct clinical use. In broadening our approach, we focused on identifying new inhibitors for alternate mechanisms of impairing HIV-1 RNA processing. Using cells stably transduced with a modified Tet-On HIV-1 provirus, we screened a library of approximately 30 small molecules modulating alternative RNA splicing for antiretroviral activity. We identified the compounds 8-Aza and 150 to have strong, dose-dependent inhibition of HIV-1 structural (Gag) protein expression (IC80: $\sim 26 \mathrm{uM}$ and $\sim 2 \mathrm{uM}$, respectively) without significant effects on cell viability. Notably, the mechanisms by which these compounds alter HIV-1 RNA processing were distinct from drugs that we previously described. In fact, 8-Aza and 150 treatments of cells demonstrated no effect on HIV-1 regulatory factor Rev abundance in contrast to effects seen upon treatment with digoxin/chlorohexidine. To explain how these molecules inhibit the virus without affecting Rev accumulation, analysis of the subcellular localization of Rev revealed both compounds caused cytoplasmic relocalization of Rev. Further assessment with nuclear import/export inhibitors confirmed that 150 treatment of cells blocked Rev nuclear import. In characterizing the effects on HIV-1 RNA metabolism, these compounds induced only moderate oversplicing of viral pre-mRNA compared to digoxin. In addition, 8-Aza treatment altered use of pre-mRNA splice sites in HIV-1 MS RNA distinct from digoxin. In understanding the effects on RNA, we assayed for changes in phospho-SR protein levels and revealed that 8-Aza treatment increased SRp54/55 compared to digoxin's effect on multiple members of this splice factor family. In this study, we have successfully identified two potent compounds that inhibit HIV-1 by new mechanisms modulating viral RNA processing, improving our odds of developing a novel class of antiHIV-1 inhibitors targeting this stage of the virus lifecycle.

\section{CLINICAL SCIENCES/SCIENCES CLINIQUES}

Adherence

Adhérence

\section{P053}

ADHERENCE TO HIGH-DOSE LONG-TERM

MICRONUTRIENT AND ANTIOXIDANT SUPPLEMENTATION IN ASYMPTOMATIC HIV INFECTION IN THE MAINTAIN TRIAL

Balfour, Louise

Ottawa, ON

BACKGROUND: Broad-spectrum and high-dose micronutrient supplement use in HIV/AIDS is common but unproven for effectiveness. This blinded analysis of tolerance, adherence and follow-up in a randomized double-blind active matched control trial is to determine the acceptability and long-term adherence of a high pill-burden regimen.

METHODS: Asymptomatic HIV-infected adults with CD4 count $>375$ cells $/ \mu \mathrm{L}$ and without prior anti-HIV drug treatment were recruited to the MAINTAIN trial during routine HIV clinic visits at 19 CTN centres. Participants were randomly assigned to a regimen of broad spectrum high-dose micronutrients and antioxidants or a recommended daily allowance multivitamin supplement, each comprising eight capsules to be taken twice daily. This blinded analysis assessed treatment adherence at study weeks $12,24,36$, and 48 by measures of residual pill count and by subjective self-reported adherence, as measured by two validated adherence scales (Global Treatment Score, GTS and the HIV Treatment Adherence Scale, HATS).

RESULTS: Of 150 randomized volunteers, adherence data on 125 was analyzed for a median follow-up time of 10.8 months, for over 100-patient years of treatment. Thirteen (10.4\%) discontinued study medication. Adherence to treatment with study medication was high: Mean percentage of capsules taken in 3-month intervals for 48 weeks of treatment was between 79 and $85 \%$. Similar results were found for self-reported adherence, with means ranging between $78 \%$ and $82 \%$. Overall, there was a moderate but highly significant correlation between pill count and questionnaire adherence measures $(\mathrm{p}<0.001, \mathrm{r}=0.31)$.

CONCLUSIONS: Adherence to broad-spectrum micronutrient and antioxidant supplementation appears to remain relatively high during 48 weeks of follow-up, despite a significant burden of capsules and twice daily dosing. This degree of adherence to medication and protocol follow-up will permit a valid evaluation of the effects of supplementation on CD4 decline and treatment initiation in the MAINTAIN trial.

\section{P054}

THE EXPERIENCE OF ACTIVE INJECTION DRUG USE ON ADHERENCE TO ANTIRETROVIRAL TREATMENT IN ABORIGINAL PEOPLE LIVING IN PRINCE GEORGE

Milligan, Robert S; Lavoie, Josee

Prince George, BC

In Canada the incidence rates of HIV in Aboriginal populations continue to grow, despite an overall reduction in HIV prevalence of the Canadian population as a whole. With this context in mind, it is not surprising that the difficulties of managing HIV - a disease which involves complex treatment regimens and wide variations in health status - can create unbearable burdens on people infected (and affected) by HIV/AIDS. The complicated treatment regimens associated with HIV can pose great problems for Aboriginal people, but are exacerbated when active injection drug use is factored in. The purpose of this study is to gain insight into how active injection drug use impacts adherence to antiretroviral treatment in Aboriginal people living in Prince George, British Columbia. The study asked participants what they felt could be done by healthcare providers to better support them in their struggles to maintain the high levels of adherence needed for maximal HIV viral suppression. Nine interviews were conducted and analyzed using a Grounded Theory methodology. Analysis and interpretation revealed that healthcare providers need to be more caring and consistent in their approach. Adherence is 
facilitated by the knowledge of the health consequences of non-adherence; trust in healthcare providers; incorporating HAART into daily routines; receiving support from AIDS services organizations, friends, and family; and that combining HAART administration with methadone is an effective pathway to increase adherence. On the contrary, the most common barrier to adherence identified by the participants was "being high." Finally the study found that the majority of participants expressed a feeling of disconnection from Aboriginal culture.

\section{P055}

HIV TREATMENT ADHERENCE AND DEPRESSION AMONG HIV PATIENTS IN GUYANA, SOUTH AMERICA

Oulanova, Olga; Balfour, Louise; MacPherson, Paul; Tasca, Giorgio A; Raghunauth, Jadunauth; Best Plummer, Wallis; La Fleur, Curtis;

Ramotar, Karam; Corace, Kim; Benedict, Roumyana;

Cameron, William D

Ottawa, ON

BACKGROUND: Excellent HIV medication adherence is required to achieve treatment success. Barriers to adherence often differ by country, availability of resources, and socio-cultural factors. While much research has examined factors impacting HIV medication adherence in developed countries such as Canada, to date, very little adherence research has been conducted in very poor developing countries such as Guyana, South America. Guyana is the second poorest country in the Americas after Haiti and Guyana is one of the most HIV affected countries in the western hemisphere.

METHODS: The goal of this study was to examine psycho-social factors affecting HIV treatment adherence among HIV patients in Guyana, South America. During regular HIV hospital clinic visits, 144 HIV patients were recruited to complete psychological questionnaires administered via interview format. Measures included socio-demographics, health behaviours, HIV treatment knowledge and adherence measures, and the CES-D depression scale. To further examine cultural and gender factors, the same questionnaire package was also administered to $155 \mathrm{HIV}$ patients at The Ottawa Hospital HIV clinic. CD4 counts were obtained from HIV medical chart reviews.

RESULTS: Preliminary analyses indicate high levels of treatment uptake in both countries with over $80 \%$ of HIV patients being on HIV medications. However, suboptimal adherence rates were common, important gaps in HIV patients' knowledge levels were found, and levels of depression were high (43\%) in both countries. From a gender perspective, in Guyana, $\mathrm{HIV}^{+}$women reported higher levels of depression than $\mathrm{HIV}^{+}$ men.

CONCLUSIONS: Results will be discussed in the context of developing culturally relevant and gender tailored interventions for optimizing mental and physical health and treatment adherence in developing countries such as Guyana, South America.

ARV Clinical Trials and Other ARV Studies

Essais cliniques et autres études sur les ARV

\section{P056}

\section{ARE KNOWN TRADITIONAL RISK FACTORS FOR} THROMBOEMBOLIC EVENTS THE SAME IN HIV PATIENTS? Berthoumieux, Sylvie; Madeleine, Durand; Tremblay, Cecile L Montreal, QC

BACKGROUND: Patients infected with HIV are more likely to suffer from a deep vein thrombosis and pulmonary embolism (DVT/PE) than non-infected patients. A pro-thrombotic state caused by increased inflammation, concurrent malignancy, coagulopathy, and antiretrovirals have been suggested as contributing risk factors. We aimed to study the prevalence of traditional risk factors for thromboembolism in HIV patients with DVT/PE.

METHODS: We retrospectively identified all HIV patients with DVT/ PE from January 1990 to March 2011 at the Centre Hospitalier de l'Université de Montréal. Cases of DVT/PE were identified using hospital discharge summary and a local database, and confirmed with imaging or at autopsy. Catheter or intravenous drug use-related events were excluded. Baseline characteristics, classical risk factors and HIV status at the time of the event were collected from medical records. Appropriate descriptive statistics were used.

RESULTS: We identified 71 events in 64 HIV-infected patients, 52 DVT, $12 \mathrm{PE}$ and $7 \mathrm{DVT} / \mathrm{PE}$. The median age of occurrence of first DVT/PE was 45,5 (SD 9.5). Sixty (93.8\%) of patients were male. A history of trauma was found in $2(3.1 \%)$, hospitalization or post-surgery status in $22(34.4 \%)$, neoplasia in $19(29.7 \%)$ and thrombophilia in 17 (26.6\%) of patients. Thirty-two (46.9 \%) of DVT/PE were unprovoked/idiopathic. Of note, $18 / 22$ immobilized or post-surgery patients did not receive thromboprophylaxis before the event. Median duration of HIV infection was 6 years (IQR 2-12). Median CD4 count was 200 (IQR 53-400). Thirty (43.8\%) had a current or past opportunistic infections and 40 (59.4\%) of patients were on antiretroviral treatment. Only 13 (19.1\%) patients had undetectable viral loads.

CONCLUSIONS: A large proportion of events were considered idiopathic in regards to traditional risk factors. Patients presented with advanced HIV disease: low CD4 counts, high prevalence of opportunistic infections, and few were virologically controlled.

\section{P057}

SWITCHING FROM EFAVIRENZ/EMTRICITABINE/ TENOFOVIR DISOPROXIL FUMARATE (EFV/FTC/TDF) SINGLE TABLET REGIMEN (STR) TO EMTRICITABINE/ RILPIVIRINE/TENOFOVIR DISOPROXIL FUMARATE (FTC/ RPV/TDF) STR IN VIROLOGICALLY SUPPRESSED, HIV-1 INFECTED SUBJECTS

Mills, $\mathrm{A}^{1}$; Cohen, $\mathrm{C}^{2}$; dejesus, $\mathrm{E}^{3}$; Rashbaum, $\mathrm{B}^{4}$; Brinson, $\mathrm{C}^{5}$; Borg, $\mathbf{P}^{6}$; Yale, $\mathbf{K}^{7}$; Ramanathan, $\mathrm{S}^{7}$; Wang, $\mathbf{H}^{7}$; Jandourek, $\mathrm{A}^{7}$; Cheng, $\mathrm{A}^{7}$

${ }^{1}$ Los Angeles, CA; ${ }^{2}$ Boston, MA; ${ }^{3}$ Orlando, FL; ${ }^{4}$ Washington, DC; ${ }^{5}$ Austin, TX, USA; ${ }^{6}$ Mississauga, ON; ${ }^{7}$ Foster City, CA, USA

BACKGROUND: A pharmacokinetic (PK) study in healthy volunteers demonstrated that switching from EFV reduced RPV Cmin up to $25 \%$ for $\sim 4$ weeks due to EFV-mediated CYP3A induction. The clinical implications of reduced RPV exposures in the setting of switching suppressed HIV-1 infected patients from EFV to RPV require evaluation.

METHODS: This open-label study enrolled virologically suppressed HIV-infected adult subjects on treatment with EFV/FTC/TDF STR for $\geq 3$ months, who decided on a regimen change due to EFV intolerance. The primary objective was to evaluate the efficacy of the RPV/FTC/TDF STR after switching in maintaining HIV RNA $<50$ copies/mL at week 12; the study is continuing through 48 weeks. HIV-1 RNA and PK were assessed at week 1, 2, 4, 6, 8 and 12. Safety and tolerability were also assessed.

RESULTS: Fifty subjects were enrolled. Of these, 49 were dosed and completed the study and 1 subject withdrew consent before dosing. The median age was 39 years, median CD4 count 653, median time on EFV/ FTC/TDF 2.5 years, 92\% (45/49) were males, and 80\% (39/49) were white. All subjects maintained HIV-1 RNA $<50$ copies/mL at 12 weeks. RPV/FTC/TDF STR was well tolerated with no adverse events that led to study drug discontinuation. Compared with Phase 3 mean $\mathrm{C}$ trough ( $50-80 \mathrm{ng} / \mathrm{ml}$ ), RPV mean Ctrough was $55 \mathrm{ng} / \mathrm{mL}$ at week 2 post-switch, and $68-85 \mathrm{ng} / \mathrm{ml}$ between Week 4 to 12 ; no subject had RPV below quantifiable levels at any visit. Mean (\%CV) EFV baseline Ctrough was $2.2(56) \mu \mathrm{g} / \mathrm{mL}$. There were measurable EFV levels in $~ 50 \%$ of patients 4 weeks post-switch.

CONCLUSIONS: All subjects switching from EFV/FTC/TDF to RPV/ FTC/TDF remained virologically suppressed at 12 weeks, suggesting that the PK interaction observed when switching from EFV to RPV in virologically suppressed subjects is not associated with adverse clinical outcomes. The study is continuing through 48 weeks 


\section{P058}

SUSTAINED EFFICACY WITH LOW AND SIMILAR RATES OF VIROLOGIC FAILURES IN SECOND YEAR OBSERVED WITH RILPIVIRINE (RPV) VERSUS EFAVIRENZ (EFV) PLUS EMTRICITABINE/TENOFOVIR DF (FTC/TDF) IN TREATMENT-NAÏVE, HIV-1 INFECTED ADULTS - POOLED 96-WEEK ECHO AND THRIVE ANALYSIS

Cohen, $\mathrm{C}^{1}$; Rachlis, $\mathrm{A}^{2}$; Walmsley, $\mathrm{S}^{2}$; Boissonnault, $\mathrm{M}^{3}$; Brunetta, J²; Kasper, $\mathbf{K}^{4}$; Boven, $\mathbf{K}^{5}$; Borg, ${ }^{6}$; Zhong, $\mathbf{L}^{7}$; Guyer, $\mathbf{B}^{7}$; Chuck, SK $^{7}$

${ }^{1}$ Boston, MA, USA; ${ }^{2}$ Toronto, ON; ${ }^{3}$ Montreal, QC; ${ }^{4}$ Winnipeg, MB; ${ }^{5}$ Titusville, USA; ${ }^{6}$ Mississauga, ON; ${ }^{7}$ Foster City, CA, USA OBJECTIVES: Pooled analysis of ECHO-THRIVE, two Phase III trials in treatment-naïve HIV-1-infected adults, demonstrated $\mathrm{RPV}+\mathrm{FTC} / \mathrm{TDF}$ to have non-inferior efficacy to EFV+FTC/TDF at Week 48 with a more favourable tolerability/safety profile. The present analysis reports pooled 96-week efficacy, virologic failure (VF), resistance, and safety data for subjects also receiving FTC/TDF. A single-tablet regimen formulation of FTC/RPV/TDF was Health Canada approved Sep-2011.

METHODS: 1,096 subjects that received FTC/TDF with either RPV $(\mathrm{N}=550)$ or $\mathrm{EFV}(\mathrm{N}=546)$ were included in this analysis.

RESULTS: Similar response rates were observed; $77 \%$ of subjects achieved HIV-1 RNA $<50$ copies/mL (ITT-Snapshot) at Week 96 for $\mathrm{RPV}^{+} \mathrm{FTC} / \mathrm{TDF}$ and $\mathrm{EFV}^{+} \mathrm{FTC} / \mathrm{TDF}$.

Virologic/lmmunologic outcomes at week 96

\begin{tabular}{|c|c|c|c|}
\hline & $\begin{array}{l}\mathrm{RPV}^{+} \mathrm{FTC} / \\
\text { TDF }\end{array}$ & $\begin{array}{l}\mathrm{EFV}^{+} \mathrm{FTCl} \\
\mathrm{TDF}\end{array}$ & $\begin{array}{c}\text { Delta } \\
(95 \% \mathrm{Cl})\end{array}$ \\
\hline \multicolumn{4}{|l|}{$\begin{array}{l}\text { Virologic efficacy (HIV-1 RNA } \\
<50 \text { copies/mL, \%) }\end{array}$} \\
\hline ITT-TLOVR (Overall) & 76.9 & 77.3 & $-0.4(-5.4,4.6)$ \\
\hline \multicolumn{4}{|l|}{ ITT-snapshot } \\
\hline Overall & 77.3 & 76.7 & 0.6 (ND) \\
\hline $\begin{array}{l}\text { Baseline HIV-1 RNA } \leq \\
\text { 100,000 c/mL }\end{array}$ & 83.0 & 80.4 & 2.7 (ND) \\
\hline $\begin{array}{l}\text { Baseline HIV-1 RNA > } \\
100,000 \mathrm{c} / \mathrm{mL}\end{array}$ & 71.0 & 73.5 & -2.5 (ND) \\
\hline $\begin{array}{l}\text { Mean increase from baseline } \\
\text { in } \mathrm{CD} 4 \text { count }\left(\mathrm{NC}=\mathrm{F}^{*}\right) \text { cells/ } \\
\mathrm{mm}<\text { sup }>3</ \text { sup }>\end{array}$ & 226 & 222 & 4 (ND) \\
\hline
\end{tabular}

In Year 1, VFs in the resistance analysis population (VFres) were greater with RPV+FTC/TDF (11.5\%) vs. EFV+FTC/TDF (4.2\%). In Year 2, total VFres including proportion of rebounders were low and similar for $\mathrm{RPV}+\mathrm{FTC} / \mathrm{TDF}(2.7 \%)$ and EFV+FTC/TDF (2.6\%). The proportions of these subjects developing NNRTI and/or N(t)RTI mutations in Year 2 was similar between groups ( $58 \%$ vs. $50 \%$, respectively).

The overall safety profile at Year 2 was superior for RPV+FTC/TDF with less subjects discontinuing due to AEs (3.8\% RPV+FTC/TDF vs. $8.6 \%$ $\left.\mathrm{EFV}^{+} \mathrm{FTC} / \mathrm{TDF} ; \mathrm{p}=0.0011\right)$. Grade 2-4 rash, dizziness, abnormal dreams/ nightmares and lipid abnormalities were all significantly lower in the RPV+FTC/TDF arm. In Year 2 the incidence of at least possibly treatment-related Grade 2-4 AEs increased by only $1 \%$ with RPV FTC/TDF and $2 \%$ with $\mathrm{EFV}^{+} \mathrm{FTC} / \mathrm{TDF}$.

CONCLUSIONS: At Week 96, RPV+FTC/TDF showed sustained antiviral efficacy that was non-inferior to EFV+FTC/TDF. While the VF in resistance analysis population was higher with RPV, there were similar small increases for both groups in Year 2. RPV+FTC/TDF had a superior tolerability profile.
P059

ELVITEGRAVIR/COBICISTAT/EMTRICITABINE/TENOFOVIR DF (QUAD) HAS NONINFERIOR EFFICACY AND FAVORABLE SAFETY COMPARED TO EFAVIRENZ/ EMTRICITABINE/TENOFOVIR DF IN TREATMENT NAÏVE HIV-1 INFECTED SUBJECTS

Sax, $\mathrm{P}^{1}$; deJesus, $\mathrm{E}^{2}$; Mills, $\mathrm{A}^{3}$; Zolopa, $\mathrm{A}^{4}$; Cohen, $\mathrm{C}^{2}$; Wohl, $\mathrm{D}^{5}$; Gallant, J6; Borg, $\mathbf{P}^{7}$; White, $\mathrm{K}^{8}$; Liu, $\mathrm{C}^{8}$; Plummer, $\mathrm{A}^{8}$; Kearney, ${ }^{8}$; Quirk, $\mathrm{E}^{8}$; Cheng, $\mathrm{A}^{8}$

${ }^{1}$ Boston, MA; ${ }^{2}$ Orlando, $\mathrm{FL} ;{ }^{3}$ Los Angeles, CA; ${ }^{4}$ Palo Alto, CA; ${ }^{5}$ Chapel Hill, NC; ${ }^{6}$ Baltimore, MD, USA; ${ }^{7}$ Mississauga, ON; ${ }^{8}$ Foster City, CA, USA

BACKGROUND: The integrase inhibitor elvitegravir (EVG) is coformulated with the pharmacoenhancer cobicistat (COBI), emtricitabine (FTC), and tenofovir DF (TDF) in a single-tablet regimen (STR) known as Quad. We report Week 48 results of a prospective, randomized, double blind, active-controlled, ongoing Phase 3 trial comparing two STRs Quad and co-formulated efavirenz/emtricitabine/tenofovir DF (EFV/ FTC/TDF) as initial HIV therapy.

METHODS: Treatment-naïve subjects with HIV were randomized 1:1 to Quad or EFV/FTC/TDF once daily plus matching placebos, stratified by HIV-1 RNA $>$ or $\leq 100,000 \mathrm{c} / \mathrm{mL}$. Eligibility criteria included screening HIV RNA $\geq 5,000$ copies $/ \mathrm{mL}(\mathrm{c} / \mathrm{mL})$, and $\mathrm{CLCr}>70 \mathrm{~mL} / \mathrm{min}$. The primary endpoint was the proportion of subjects with HIV RNA $<50 \mathrm{c} / \mathrm{mL}$ at week 48 per the FDA snapshot algorithm (12\% prespecified noninferiority margin). RESULTS: 700 subjects ( $89 \%$ male, $33 \%$ with $\mathrm{VL}>100,000 \mathrm{c} / \mathrm{mL}$ ) were randomized and treated. Quad was noninferior to EFV/FTC/TDF with $88 \%$ and $84 \%$ respectively meeting the week 48 primary endpoint (difference $+3.6 \%$, $95 \% \mathrm{CI}-1.6 \%$, $+8.8 \%$ ). Among subjects with baseline HIV RNA $>100,000 \mathrm{c} / \mathrm{mL}$, response rates were similar (Quad 84\%, EFV/ FTC/TDF $82 \%$ ). Virologic failure rates at Week 48 were $7 \%$ in both arms. Mean CD4 cell increase was 239 cells $/ \mu \mathrm{L}$ in Quad and 206 cells $/ \mu \mathrm{L}$ in EFV/FTC/TDF ( $\mathrm{p}=0.009)$. Drug discontinuation rates for adverse events were similar (Quad 3\%, EFV/FTC/TDF 5\%). Among AEs occurring in $>10 \%$ of subjects (all grades), nausea was significantly more frequent in Quad than EFV/FTC/TDF (21\% vs. 14\%) while dizziness (7\% vs. 24\%), abnormal dreams ( $15 \%$ vs. $27 \%$ ), insomnia ( $9 \%$ vs. $14 \%$ ) and rash ( $6 \%$ vs. $12 \%)$ were significantly less common in Quad. CLCr decrease occurred by Week 2 of Quad therapy and was significantly greater than with EFV/ FTC/TDF by Week 48 ( -14.3 vs. $-3.0 \mathrm{~mL} / \mathrm{min})$. Total cholesterol and LDL increases were significantly lower for Quad than EFV/FTC/TDF. CONCLUSIONS: Quad demonstrated similarly high response rates compared to EFV/FTC/TDF at Week 48 with favorable CNS, rash, and fasting lipid results. These results suggest that Quad could become an important new option for initial HIV therapy.

\section{P060}

SWIFT STUDY: SWITCHING FROM LAMIVUDINE/

ABACAVIR (3TC/ABC) TO EMTRICITABINE/TENOFOVIR DF (FTC/TDF) IMPROVED FASTING LIPID PARAMETERS WHILE MAINTAINING VIROLOGIC SUPPRESSION Logue, $\mathrm{K}^{1}$; deJesus, $\mathrm{E}^{2}$; Smith, $\mathrm{G}^{1}$; Baril, JG ${ }^{3}$; Campo, $\mathbf{R}^{4}$; Bredeek, UF ${ }^{5}$ Popovic, $\mathbf{V}^{6}$; Wang, $\mathrm{H}^{7}$; Guyer, $\mathrm{B}^{7}$; Piontkowsky, $\mathrm{D}^{7}$ ${ }^{1}$ Toronto, ON; ${ }^{2}$ Orlando, FL, USA; ${ }^{3}$ Montreal, QC; ${ }^{4}$ Miami, FL; ${ }^{5}$ San Francisco, CA, USA; ${ }^{6}$ Mississauga, ON; ${ }^{7}$ Foster City, CA, USA BACKGROUND: In prior treatment naïve and experienced studies, use of TDF has been associated with more favorable fasting lipid profile. There are limited data on the impact of switching from fixed-dose 3TC/ABC to FTC/TDF. METHODS: The SWIFT study is a prospective, multicentre, randomized 48 week study to evaluate the safety and efficacy of switching virologically suppressed subjects from 3TC/ABC to FTC/TDF. Subjects receiving $3 \mathrm{TC} / \mathrm{ABC}+\mathrm{PI} / \mathrm{r}$ with $\mathrm{HIV} \mathrm{RNA}<200 \mathrm{c} / \mathrm{mL} \geq 3$ months were randomized (1:1) to either continue 3TC/ABC or switch to FTC/TDF, with $\mathrm{PI} / \mathrm{r}$ unchanged. Primary endpoint was time to loss of virologic response (TLOVR, premature discontinuation, or ARV modification = failure). Fasting lipid profile and 10 year Framingham scores were evaluated at baseline and every 12 weeks though Week 48 .

RESULTS: 311 subjects were treated (FTC/TDF 155, 3TC/ABC 156). Baseline characteristics were similar between arms: $85 \%$ males, median age 46 years, $72 \%$ with comorbidities, and $47 \%$ taking lipid-lowering agents. There were no significant differences in adverse events between 
arms. Through Week 48, FTC/TDF was non-inferior to $3 \mathrm{TC} / \mathrm{ABC}$ by TLOVR ( $86.5 \%$ vs $83.3 \%$ HIV RNA $<200 \mathrm{c} / \mathrm{mL}$ ), with lower rate of VF for FTC/TDF vs 3TC/ABC (2\% vs 8\%; $=0.034$ ). At Week 48, FTC/TDF compared to $3 \mathrm{TC} / \mathrm{ABC}$ showed greater declines in fasting LDL (median change -0.18 vs. $0.05 \mathrm{mmol} / \mathrm{L} ; \mathrm{p}=0.007)$, TC $(-0.54$ vs. $-0.08 \mathrm{mmol} / \mathrm{L}$; $\mathrm{p}<0.001)$, and TG $(-0.20$ vs. $-0.10 \mathrm{mmol} / \mathrm{L} ; \mathrm{p}=0.074)$. Significant declines in LDL and TC were observed by Week 12. According to NCEP thresholds, subjects who switched to FTC/TDF had improved TC $(p=0.004)$ and TG (p <0.001); $61.5 \%$ on FTC/TDF vs $44.6 \%$ on 3 TC/ABC had TC $<5.17 \mathrm{mmol} / \mathrm{L}$ and $55.6 \%$ vs. $41.0 \%$ had $\mathrm{TG}<1.69 \mathrm{mmol} / \mathrm{L}$. Mean (SD) change in 10 year Framingham scores were 1.2 (4.4) and 0.3 (4.0) for FTC/TDF vs 3TC/ABC (between arms $\mathrm{p}=0.22$; within arms $\mathrm{p}=0.006$ for FTC/TDF and $\mathrm{p}=0.40$ for $3 \mathrm{TC} / \mathrm{ABC}$ ).

CONCLUSION: Switching subjects from 3TC/ABC to FTC/TDF maintained virologic suppression with a lower rate of VF and improved lipid parameters and Framingham scores.

\section{P061}

WEEK 48 RESULTS OF AN ONGOING GLOBAL PHASE 3 STUDY COMPARING ELVITEGRAVIR/COBICISTAT/ EMTRICITABINE/TENOFOVIR DF (QUAD) WITH RITONAVIRBOOSTED ATAZANAVIR PLUS EMTRICITABINE/TENOFOVIR DF IN TREATMENT NAÏVE HIV-1 INFECTED SUBJECTS SHOWING EFFICACY, SAFETY, AND PHARMACOKINETICS dejesus, $\mathrm{E}^{1}$; Walmsley, $\mathrm{S}^{2}$; Chang, $\mathrm{B}^{2}$; Laplante, $\mathrm{F}^{3}$; Rachlis, $\mathrm{A}^{2}$; Henry, $\mathrm{K}^{4}$; Gathe, $\mathrm{J}^{5}$; Popovic, $\mathrm{V}^{6}$; White, $\mathrm{K}^{7}$; Wei, $\mathrm{X}^{7}$; Plummer, $\mathrm{A}^{7}$; Kearney, ${ }^{7}$; Cheng, $\mathrm{A}^{7}$

${ }^{1}$ Orlando, FL, USA; ${ }^{2}$ Toronto, ON; ${ }^{3}$ Montreal, QC; ${ }^{4}$ Minneapolis, MN; ${ }^{5}$ Houston, TX, USA; ${ }^{6}$ Mississauga, ON; ${ }^{6}$ Foster City, CA, USA

BACKGROUND: Quad is a single tablet regimen (STR) in development composed of an integrase inhibitor elvitegravir (EVG), a pharmacoenhancer cobicistat, emtricitabine (FTC) and tenofovir DF (TDF). Week 48 results of a Phase 3 study comparing Quad with ritonavir-boosted atazanavir (ATV/r) plus fixed dose FTC/TDF in treatment naive HIVinfected subjects are described.

METHODS: Subjects with HIV RNA $\geq 5000 \mathrm{c} / \mathrm{mL}, \mathrm{CLCr}>70 \mathrm{~mL} / \mathrm{min}$, no prior HIV therapy, and no resistance to ATV, FTC, or TDF were randomized 1:1 to receive either Quad or ATV/r+FTC/TDF (stratified by baseline HIV RNA $\geq$ and $<100,000 \mathrm{c} / \mathrm{mL}$ ) in an ongoing, blinded, activecontrolled study. Primary objectives included the proportion of subjects with HIV RNA $<50 \mathrm{c} / \mathrm{mL}$ at Week 48 (FDA snapshot algorithm, 12\% prespecified noninferiority margin) in the intent to treat population and assessment of safety. Secondary objectives included pharmacokinetic/ dynamic (PK-PD) analyses.

RESULTS: 708 subjects were randomized: 90\% male, 39\% with VL $\geq 100,000 \mathrm{c} / \mathrm{mL}$. Quad was noninferior to ATV/r+FTC/TDF with $90 \%$ and $87 \%$, respectively, having HIV RNA of $<50 \mathrm{c} / \mathrm{mL}$ at Week 48 (difference $+3.0 \%, 95 \%$ CI $[-1.9 \%,+7.8 \%])$. Among subjects with HIV RNA $>100,000$ copies $/ \mathrm{mL}$, response rates were similar (Quad 85\%, ATV/ r+FTC/TDF 82\%). Virologic failure (FDA snapshot algorithm) was infrequent, 5\%, in both arms. Median CD4 increases were similar (Quad 207 cells $/ \mu \mathrm{L}, \mathrm{ATV} / \mathrm{r}+\mathrm{FTC} / \mathrm{TDF} 211$ cells $/ \mu \mathrm{L})$. Discontinuation rates for adverse events (AE) were similar (Quad 4\%, ATV/r+FTC/TDF 5\%). Median change in CLCr from baseline was $-12.7 \mathrm{~mL} / \mathrm{min}$ in Quad and -9.5 $\mathrm{mL} / \mathrm{min}$ in ATV/r+FTC/TDF $(\mathrm{p}<0.001)$. Median triglyceride increases were $0.12 \mathrm{mmol} / \mathrm{L}$ in Quad and $0.33 \mathrm{mmol} / \mathrm{L}$ in $\mathrm{ATV} / \mathrm{r}+\mathrm{FTC} / \mathrm{TDF}(\mathrm{p}=$ 0.006). PK-PD analyses showed $~ \geq 90 \%$ efficacy across all quartiles or octiles for EVG C trough.

CONCLUSIONS: Quad demonstrated noninferior efficacy and was well tolerated at 48 weeks in this Phase 3 blinded active-controlled study in treatment naïve HIV infected subjects. The efficacy of Quad was confirmed by robust PK analyses. These data support the use of Quad as a potential new STR option for initial HIV treatment.
P062

NATURAL HISTORY AND RESPONSE TO COMBINATION ANTIRETROVIRAL THERAPY (CART) IN PATIENTS WITH ACUTE/EARLY HIV INFECTION AS A FUNCTION OF THE ROUTE OF TRANSMISSION (CTN 214 STUDY)

Tossonian, Harout ${ }^{1}$; Margolick, Joe ${ }^{2}$; Apuzzo, Linda ${ }^{2}$; Singer, Joel ${ }^{1}$; Fraser, Chris ${ }^{3}$; Loutfy, Mona ${ }^{4}$; Rachlis, Anita ${ }^{4}$; Kasper, Ken ${ }^{5}$; El-Helou, Philippe ${ }^{6}$; Conway, Brian ${ }^{1}$

${ }^{1}$ Vancouver; ${ }^{2}$ Baltimore, MD, USA; ${ }^{3}$ Victoria, BC; ${ }^{4}$ Toronto, ON; ${ }^{5}$ Winnipeg, MB; ${ }^{6}$ Hamilton, ON

BACKGROUND: Injection drug users (IDUs) make up 20-25\% of new cases of HIV infection diagnosed in North America. Little is known about the natural history of acute/early HIV infection in this group or the response to cART if initiated at this stage of disease.

METHODS: Within the context of a randomized controlled clinical trial (CTN 214) of a 12-month course of cART versus observation in patients diagnosed within the first year of HIV infection, an analysis was performed based on the risk factor for disease acquisition to examine key baseline characteristics, natural history, and response to cART if initiated. Comparison was made between IDUs and men who have sex with men (MSMs) enrolled in the study; all subjects reporting IDU as a risk behaviour were assigned to the IDU group.

RESULTS: We enrolled 24 IDUs ( 5 female) and 80 MSMs, with $12.5 \%$ versus $18.3 \%$ having been infected within the previous 2 months. Baseline CD4 cell counts and plasma viral loads did not differ significantly between the groups (643 vs. 532 cells/ul, 4.54 vs. 4.57 logs, respectively). Of those assigned to immediate treatment, full virologic suppression was achieved in $46 \%$ IDUs versus $73 \%$ MSMs. Of those receiving immediate treatment, $88.8 \%$ IDUs and $62.7 \%$ MSMs did not require re-initiation of cART within 24 months of stopping therapy. Of those not receiving immediate treatment, $42.4 \%$ IDUs versus $44.4 \%$ MSMs did not need to start cART within 24 months of randomization into the study.

CONCLUSIONS: In patients with acute/early HIV infection, the need to re-initiate or delay cART were similar in both IDUs and MSMs with better virologic responses reported with MSMs.

\section{P063}

\section{REMOVING AN INACTIVE NRTI FROM AN EFFECTIVE} SALVAGE REGIMEN IS SAFE AND MAINTAINS VIROLOGIC SUPPRESSION: FINAL (WEEK 48) RESULTS FROM THE VERITAS TRIAL

Trottier, Benoit; Machouf, Nimâ; Longpré, Danièle; Dion, Harold; Vézina, Sylvie; Gallant, Serge; Lavoie, Stéphane; Murphy, Daniel; Nguyen, Vinh-Kim; Thomas, Réjean

Montréal, QC

BACKGROUND: In HIV-positive patients exhibiting multi-drug resistance (MDR), nucleoside reverse transcriptase inhibitor (NRTIs) often have little activity, increase toxicity, and interact with other drugs. A simplification treatment strategy of removing inactive NRTIs, as determined by genotypic data, was implemented and virological, immunological, clinical and financial outcomes were evaluated.

METHODS: MDR patients with an undetectable viral load (VL) on a stable regimen containing $\geq 4 \mathrm{ARV}$ were enrolled in a single-arm, openlabel, prospective trial of simplification, where one inactive NRTI was removed at baseline (BL). A second inactive NRTI could be removed at week 24 if the patient's regimen contained $\geq 5$ ARV at enrolment. At week 48 , the proportion of patients maintaining viral suppression (VS) was evaluated, along with immunological response and toxicity. Matched-pair t-tests were used to evaluate differences between $\mathrm{BL}$ and week 48

RESULTS: 31 male patients with a mean age of 50 years (IQR:46-56) were enrolled. $94 \%$ were MSM. Average length of treatment was 14 years (IQR:12-16), with a median CD4 of 525 cells/mm3 (IQR:360-680) at BL and nadir of 158 cells/mm3 (IQR:69-210). The BL regimen comprised of four ARV in 22 patients (71\%) and five ARV in nine patients (29\%). $3 \mathrm{TC}$ or FTC was removed in 29 patients $(94 \%)$, and either AZT or TDF was removed in two other patients. Three patients had a second NRTI removed at week 24 . One patient was withdrawn from the study at week 26 for toxicity reasons. At week 48 , all remaining 30 patients maintained a VL<50 (ITT:97\%, OT:100\%). No deaths or serious adverse events were 
observed. After 48 weeks, a mean change of $-6.8 \mathrm{U} / \mathrm{L}$ of ALT was observed (95\% CI: -15.5 to $+1.8, \mathrm{p}=0.116$ ).

CONCLUSIONS: Removing inactive NRTI(s) from a regimen in patients taking $\geq$ four ARV with controlled VL appears to be safe, and maintains virologic suppression through 48 weeks.

\section{P064}

\section{ABACAVIR/LAMIVUDINE FIXED-DOSE COMBINATION WITH RITONAVIR-BOOSTED DARUNAVIR, A NOVEL REGIMEN FOR HIV THERAPY}

Trottier, Benoit; Machouf, Nimâ; Longpré, Danièle; Vézina, Sylvie; Boissonault, Michel; Lavoie, Stéphane; Huchet, Emanuelle; Gallant, Serge; Legault, Danielle; Charest, Louise; Dion, Harold; Thomas, Réjean

Montréal, QC

BACKGROUND: Data which led to the licensing of DRV/r as a firstline treatment for HIV comes from the ARTEMIS trial, where DRV/r was combined with TDF/FTC. The use of DRV/r with a fixed-dose of Abacavir/ Lamivudine (ABC/3TC) has not been studied. Until recently, this combination was classified only as an "acceptable regimen" in first-line therapy by the DHHS guidelines. However, based on our preliminary 24-week results, it was upgraded to "alternative regimen". Here, we present the results from week 48 where we evaluated the combination of ABC/3TC/ $\mathrm{DRV} / \mathrm{r}$.

METHODS: Prospective study of HIV-infected adults who received an open-label combination of $\mathrm{ABC} / 3 \mathrm{TC} / \mathrm{DRV} / \mathrm{r}$ were followed in a community clinic in Montreal. Patients were either treatment-naïve or experienced, and had no resistance to any of the compounds in their regimen. Viral load (VL), CD4 cell count, and safety parameters were examined after 48-weeks of follow-up. T-tests were used to compare differences between groups and differences between baseline (BL) and the 48-week endpoint.

RESULTS: Sixty-six patients with a mean age of 43 years were enrolled in the study. Thirty-five (53\%) were treatment-experienced and the remaining were treatment-naïve. HLA-B*5701 test results were available for 56 patients and none were positive. At BL, mean VL was 4.8 logs for treatment-naïve and 2.2 logs for experienced patients. Twelve D/C: 8 for side effects and 4 for other reasons. At w48, 79\% had a $\mathrm{VL}<50$ and $81 \%$ $<400$ copies/ml (ITT; $98 \%$ and 100\%: OT) The only person with a VL $>50$ became undetectable after the $48 \mathrm{w}$ endpoint. CD4 increased to 273 cells (naïve) and 91 cells (experienced), $\mathrm{p}=0.002$.

CONCLUSIONS: The new combination of ABC/3TC/DRV/r demonstrates a high rate of antiviral activity with no major toxicity. The drug combination appears to be generally safe and well tolerated.

\section{P065 \\ TREATMENT TOLERABILITY, SATISFACTION AND LABORATORY PARAMETERS IN HIV ${ }^{+}$PATIENTS SWITCHING FROM RITONAVIR CAPSULE TO TABLET FORMULATION}

Mtambo, Andy; Harris, Marianne; Toy, Junine; Guillemi, Silvia; Zhang, Wendy; Lima, Vivian D; Montaner, Julio; Hull, Mark Vancouver, BC

BACKGROUND: The objective of this study was to evaluate treatment satisfaction, gastrointestinal tolerability, depressive symptoms and alterations in HIV and laboratory parameters after the switch from ritonavir capsule to tablet formulation.

METHODS: HIV+ adults ( $>19$ years of age) beginning routine switch from ritonavir capsules to tablets were recruited when they attended the pharmacy for antiretroviral prescription refill. Before switching and 3-4months after switching, 3 questionnaires were self-administered: HIV Treatment Satisfaction Questionnaire (HIVTSQ), Gastrointestinal Symptom Rating Scale (GSRS) and the CES-D Depression inventory. Results of CD4, plasma viral load (pVL) and routine blood work within three months of each questionnaire were also collected. Wilcoxon signed rank sum test was used for continuous variables and chi-squared test for categorical variables.

RESULTS: 82 participants completed the questionnaires, 80 (97.6\%) males, $79(96.4 \%)$ Caucasian and median age 51 years (interquartile range $[\mathrm{IQR}] 47$, 58). Most participants were taking ritonavir $100 \mathrm{mg}$
[62 (75.6\%)]. Ritonavir was taken in combination with atazanavir in $57(69.5 \%)$, darunavir in 20 (24.4\%), saquinavir in $3(3.7 \%)$ and fosamprenavir in $2(2.4 \%)$. Multivariate analysis for factors associated with having better scores after the switch did not show any significant differences on both demographic and clinical variables. A comparison of clinical parameters before and after the switch is shown in the table below:

\section{TABLE 1}

Comparison of clinical parameters in participants before and after the switch $(\mathrm{N}=82)$

\begin{tabular}{lccc}
\hline Variable * & Before (capsule) & After (tablet) & p-value \\
\hline CD4 count (cells/mm $)$ & $530(400-715)$ & $515(400-690)$ & 0.48 \\
Plasma viral load & $67(88 \%)$ & $70(92 \%)$ & 0.59 \\
$\quad<40$ copies/mL, N (\%) & & & \\
Total cholesterol (mmol/L) & $4.63(4.11-5.43)$ & $4.63(4.04-5.58)$ & 0.83 \\
LDL cholesterol (mmol/L) & $2.89(2.18-3.34)$ & $2.55(2.02-3.61)$ & 0.43 \\
HDL cholesterol (mmol/L) & $1.09(0.94-1.31)$ & $1.18(1.02-1.39)$ & 0.002 \\
Triglycerides (mmol/L) & $1.5(1.30-2.02)$ & $1.41(1.26-2.14)$ & 0.68 \\
HIVTSQ score & $49(45-54)$ & $53(48-57)$ & $<0.0001$ \\
GSRS Total score & $7(3-13)$ & $3(0-9)$ & $<0.0001$ \\
GSRS- Abdominal pain & $0(0-1)$ & $0(0-1)$ & 0.21 \\
$\quad$ syndrome & & & \\
GSRS- Dyspeptic syndrome & $1(0-3)$ & $1(0-2)$ & 0.064 \\
GSRS- Indigestion & $2(0-4)$ & $2(0-3)$ & 0.065 \\
syndrome & & & \\
GSRS- Bowel syndrome & $3(1-5)$ & $2(0-4)$ & 0.016 \\
CES-D Score & $11(4,18)$ & $12(3,19)$ & 0.798 \\
\hline
\end{tabular}

${ }^{*}$ Results shown are median and interquartile range unless otherwise indicated CONCLUSION: Results of this study suggest that the newer tablet formulation of ritonavir is better tolerated and has fewer gastrointestinal side effects than the older capsule formulation.

Co-infections (including HCV, HBV, HPV, syphilis, TB) Co-infections (y compris VHC, VHB, VPH, syphilis, TB)

\section{P066}

RESPIRATORY HEALTH STATUS PERFORMANCE AMONG HIV $^{+}$PATIENTS WITH RISK FACTORS FOR CHRONIC OBSTRUCTIVE PULMONARY DISEASE (COPD)

Mtambo, Andy; Harris, Marianne; Guillemi, Silvia;

Shaipanich, Tawimas; Chan, Keith; Tan, Wan; Lima, Vivian D;

Montaner, Julio; Sin, Don; Man, Paul

Vancouver, BC

BACKGROUND: It is unknown whether HIV control with HAART is accompanied by improvements in general health status in $\mathrm{HIV}^{+}$patients with comorbid COPD. The objective of this study was to describe respiratory disease-specific health status and its associations in this population.

METHODS: $\mathrm{HIV}^{+}$adults with risk factors for COPD, but no acute respiratory symptoms, provided consent and completed the St. George's Respiratory Questionnaire (SGRQ), which assesses symptoms, activities and impacts of respiratory disease. SGRQ total score $>6$ indicates significantly impaired health status. Component scores $>12,>9$, and $>2$ indicate significant symptoms, reduced activity, and impact on quality of life (QoL) related to respiratory disease, respectively. Baseline sociodemographic and medical information, blood tests and spirometry were obtained within 1 month of SGRQ. Categorical variables were compared using Fisher's Exact test and continuous variables using Wilcoxon's rank sum test. Logistic regression was used to generate adjusted odds ratios (AOR).

RESULTS: Between 08/2009 and 11/2011, 213 participants (193 [90.6\%] male) were enrolled: median age 48 (interquartile range [IQR] 42-55) years; $88.8 \%$ past or present smokers; $37(17.4 \%)$ prior COPD diagnosis; 92\% receiving HAART; median CD4 490 (IQR 360-650) cells/ $\mathrm{mm}^{3} ; 161(75.6 \%) \mathrm{pVL}<40$ copies/mL. Median total SGRQ score was 34/100 (IQR 15-48). Median (IQR) symptom, activity, and impact scores were 47/100 (24-70), 41/100 (17-59), and 22/100 (8-41), respectively. After 
adjusting for demographics, smoking, respiratory disease history, respiratory medications, and spirometry results, SGRQ symptom score $>12$ was associated with lower current CD4 count (AOR 0.83 per 100 cell increase, $95 \%$ CI $0.70-0.98)$. Neither total score nor any other component score was associated with HIV duration, AIDS diagnosis, current or nadir CD4, viral load, or current HAART use.

CONCLUSION: Despite good HIV control and respiratory medications, the QoL of $\mathrm{HIV}^{+}$patients with risk factors for COPD is impaired by poor respiratory disease-specific health status.

\section{P067 \\ TUBERCULOSIS SCREENING IN AN HIV POSITIVE POPULATION IN KINGSTON, ONTARIO}

Bach, Paxton J; Rumman, Amir; Wobeser, Wendy L; Liu, Theresa T Kingston, ON

Approximately 5.6\% of HIV positive patients in Canada are co-infected with tuberculosis (TB). Due to their greatly increased risk of infection, assessment for active TB and a tuberculin skin test (TST) are recommended as soon as possible in all newly diagnosed HIV positive patients in whom active TB has been ruled out. Despite these recommendations, published TST uptake at North American HIV clinics is suboptimal, ranging from $53.8-69.0 \%$. Multiple patient, clinic, and care provider factors have been proposed as variables affecting TST uptake.

We set out to ascertain TST uptake in 155 active $\mathrm{HIV}^{+}$patients at the Clinical Immunology Outpatient Clinic (CIOC) in Kingston, Ontario, and to determine whether any factors are predictive of patients going untested. Demographics, risk factors, measurements of HIV progression, and histories of substance use were also collected. Data was gathered by retrospective chart review. 116/155 (75.8\%) of patients had a documented TST, with a mean time between HIV diagnosis and TST of 5.0 years $(\mathrm{SD}=5.3) .84(72.4 \%)$ of these tests were performed at the CIOC. The median CD4 count at the time of TST was $462 / \mathrm{uL}(\mathrm{IQR}=300)$, and the mean log viral load was $1.40(\mathrm{SD}=0.20)$. A total of 9 patients $(7.8 \%)$ had a positive TST and all were subsequently treated for latent TB infections (LTBI). No predictors of receiving/not receiving a TST could be identified.

Our results demonstrate that TB screening has not been meeting the published recommendations at the CIOC, although no systemic biases are detectable. Consistently reinforcing the importance of testing all patients in keeping with Canadian recommendations is a necessary step in improving internal TB screening practices. The advent of new bloodbased diagnostic assays for TB may provide a significant opportunity to improve TB screening coverage for all our patients.

\section{P068}

\section{EVOLUTION OF PANDEMIC H1N1 INFLUENZA VACCINE} ANTIBODY AFFINITY IN HIV-INFECTED CHILDREN

Yam, Karen K ${ }^{1}$; Brophy, Jason $\mathrm{C}^{2}$; Bitnun, Ari ${ }^{3}$; Samson, Lindy ${ }^{2}$; Lamarre, Valérie ${ }^{1}$; Karatzios, Chris ${ }^{1}$; Ward, Brian ${ }^{1}$

${ }^{1}$ Montreal, QC; ${ }^{2}$ Ottawa; ${ }^{3}$ Toronto, ON

INTRODUCTION: While influenza vaccine immunogenicity in HIVinfected children is generally poor relative to healthy children, somewhat better responses were achieved with the ASO3-adjuvanted pandemic H1N1 vaccine (pH1N1-v). This study describes antibody $(\mathrm{Ab})$ avidity patterns post-immunization with $\mathrm{pH} 1 \mathrm{~N} 1-\mathrm{v}$ in this population.

METHODS: Vaccine responses of HIV-infected children at 3 Canadian centres were assessed. Sera were collected prior to, 2 months, and 6 months after $\mathrm{pH} 1 \mathrm{~N} 1-\mathrm{v}$ immunization for assessment of hemagglutination inhibition titres (HAI). An avidity ELISA was used to assess affinity/ avidity maturation. An avidity index (AI) was calculated as the concentration of urea needed to displace $50 \%$ of influenza-specific IgG Abs.

RESULTS: Samples from all 3 timepoints were available for 88 subjects (43 females). Median age, CD4 count, and VL at baseline were 13.3 years (27<10 years), 643 cells/uL, and $<50$ copies/mL, respectively; 32 had detectable VL (median 3041 copies/mL). 62 (70\%) achieved seroprotection (HAI titre $>/=40)$ and $39(44 \%)$ seroconverted $(>/=4$-fold rise in HAI). Overall, the expected pattern of $\mathrm{Ab}$ maturation was observed with increase in $\mathrm{AI}$ from low at baseline to high at 2 months, and maintenance in $\mathrm{AI}$ at 6 months. 46 subjects were $\mathrm{pH} 1 \mathrm{~N} 1$ naïve $(\mathrm{HAI}<40)$ at baseline, with females more likely to be naïve (Mann-Whitney $U$ test $p=0.01$ ).
Of these, 21 (46\%) had low AI while $26(54 \%)$ had high AI prior to immunization. 15 (71\%) low AI and 6 (24\%) high AI subjects had the expected increase in $\mathrm{AI}$ at 2 months. Factors associated with decreased likelihood of AI increase included older age $(\mathrm{p}=0.03)$ and lower baseline CD4 ( $\mathrm{p}=0.06)$ and CD4\% $(\mathrm{p}=0.04)$.

CONCLUSIONS: Ab avidity maturation after $\mathrm{pH} 1 \mathrm{N1} 1 \mathrm{v}$ was not uniform in HIV-infected children. Without accounting for the role played by prior infection with $\mathrm{pH} 1 \mathrm{~N} 1$ in these results, $\mathrm{CD} 4$ suppression related to HIV may impair the normal progression of Ab avidity.

\section{P069}

MORTALITY AMONG CANADIAN HIV-HCV CO-INFECTED INDIVIDUALS WITH AND WITHOUT INJECTION DRUG USE HISTORY: A COMPETING RISKS ANALYSIS

Cescon, Angela ${ }^{1}$; Chan, Keith ${ }^{1}$; Raboud, Janet $\mathbf{M}^{2}$; Samji, Hasina ${ }^{1,3}$; Klein, Marina B ${ }^{3}$; Loutfy, Mona $\mathbf{R}^{2}$; Machouf, Nima ${ }^{4}$; Montaner,

Julio S1; Burchell, Ann N2; Rourke, Sean B ${ }^{2}$; Tsoukas, Christos ${ }^{4}$

Hogg, Robert S ${ }^{1,5}$; Cooper, Curtis ${ }^{6}$; Canadian

Observational Cohort Collaboration, CANOC $^{1}$

${ }^{1}$ Vancouver, BC; ${ }^{2}$ Toronto, ON; ${ }^{3}$ Baltimore, MD, USA; ${ }^{4}$ Montreal, QC; ${ }^{5}$ Burnaby, BC; ${ }^{6}$ Ottawa, ON

BACKGROUND: Studies focusing on HIV-HCV co-infected individuals without a history of injection drug use (IDU) are largely absent from the HIV literature. In fact, cohort studies may use HCV and IDU as markers for each other due to collinearity of variables. This approach may disregard individuals who acquired HCV through a non-IDU route and constitute a unique group of interest for $\mathrm{HCV}$ prevention and treatment. This study compares mortality between HIV-HCV co-infected individuals with and without IDU history, using competing risks methods to take into account loss to follow-up (LTFU).

METHODS: Participants enrolled in the Canadian Observational Cohort (CANOC) collaboration with documented HCV infection and known IDU history were included. CANOC is a cohort of HIV-positive individuals $18+$ years who initiated combination antiretroviral (ARV) therapy on or after January 1, 2000. Eight sites from British Columbia, Ontario, and Quebec contribute data. Demographic and clinical variables were compared by IDU history status using the Pearson $\chi^{2}$ test or Fisher's exact test for categorical variables and Wilcoxon rank-sum test for continuous variables. In order to account for LTFU, competing risk analysis was used to evaluate time to death (all-cause). LTFU was defined as no contact for $1+$ years.

RESULTS: In total, 1254 participants (31\% female) were included. At baseline, those with IDU history $(n=1106,88 \%)$ were younger

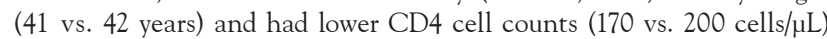
than non-IDU participants (both $\mathrm{p}<0.01$ ). During median follow-up time of 3.8 years (IQR 2.1-6.2), 217 deaths were reported and 148 participants were LTFU. After adjustments for age, province, and ARV start year, significant differences were observed between IDU and non-IDU in the time to death analysis $(A H R=2.13,95 \% \mathrm{CI}=1.23-3.71 ; \mathrm{p}=0.007)$. In the competing risk analysis adjusted for the same confounders, significant differences were also observed between IDU and non-IDU (AHR=2.17, 95\% CI=1.26-3.72; $\mathrm{p}=0.005$ ).

CONCLUSIONS: Factors associated with IDU history impact mortality among HIV-HCV co-infected individuals in CANOC, with similar results when accounting for LTFU.

\section{P070}

UPTAKE AND OUTCOMES OF ANAL CANCER SCREENING IN HIV PRIMARY CARE

Collins, Evan; Tinmouth, Jill; Aggarwal, Banita; Blitz, Sandra;

Raboud, Janet; Kovacs, Colin; Salit, Irving

Toronto, ON

BACKGROUND: $\mathrm{HIV}^{+} \mathrm{MSM}$ are at high risk for anal cancer. Anal cytology (Pap tests), plus high-resolution anoscopy (HRA) as required, are felt to be the best screening approach, although this practice is generally restricted to research/specialty settings. We present preliminary results from STANDOUT, a study of acceptability and outcomes of anal cancer screening in this target population receiving usual care.

METHODS: All HIV+ MSM from one HIV primary care practice, previously unscreened, were invited by e-mail to receive anal cancer screening. 
Responders had anal cytology done at specially arranged clinics. Those with high-grade squamous intraepithelial lesions (HSIL) on cytology were offered high-resolution anoscopy to assess anal intra-epithelial neoplasia (AIN). Invitation response rates, as well as demographic, clinical, smoking and HIV data by cytology result, were calculated. Medians [inter-quartile range] or counts (\%) are used depending on data.

RESULTS: 169 (46\%) of the 364 subjects responded and received anal Pap tests; 195 did not respond, refused or accepted but did not attend screening. Among responders, 1 had an inadequate sample; 104 (62\%) had normal cytology, 49 (29\%) low-grade squamous intraepithelial lesions (LSIL) and 15(9\%) HSIL. Of the 8 HRA results available, 6 had AIN2 and 2 had AIN3. Those with HSIL were younger (HSIL: 36 years [IQR: 33-49]; LSIL: 46 [37-53]; Normal: 48 [43-55]) and more likely to be smokers (HSIL:47\%; LSIL:35\%; Normal: 20\%). CD4 count was lowest in the LSIL group (HSIL:570 cells/mm ${ }^{3}$ [300-740]; LSIL:480 [370-620]; Normal: 560 [475-700]). There were no differences in symptoms, HIV duration, viral load or anti-retroviral use.

DISCUSSION: In our sample of $\mathrm{HIV}^{+} \mathrm{MSM}$, almost half of those invited for anal cancer screening responded. Those with HSIL were more likely smokers, and were younger (possibly reflecting higher screening acceptance among younger patients at high risk of disease). Next steps will include enrolling subjects in other practices and assessing reasons for refusal.

\section{P071}

\section{OPTIMIZING RIBAVIRIN DOSE IN HIV/HCV CO-INFECTED INDIVIDUALS TREATED FOR HEPATITIS C VIRUS INFECTION IN REAL-LIFE CLINICAL SETTINGS} Farley, John D; Truong, Andy; Nguyen, Trang; Shum, Wendy Vancouver, BC

BACKGROUND: Hepatitis C (HCV) and HIV share common transmission pathways; the acquisition of both viruses are relatively common. Concurrent treatment for HCV with HAART therapy should be considered in HIV co-infected individuals to decrease the progression of liver damage. Hepatotoxity and less satisfactory treatment outcomes are often concerns when treating co-infected individuals. The objective of this study is to evaluate the difference in response rates of HCV treatment in HIV co-infected inmates with varying doses of Ribavirin.

METHODOLOGY: Retrospective medical chart reviews of $53 \mathrm{HCV} /$ HIV co-infected inmates who underwent HCV therapy between 2003 and 2010. All received standard doses of pegylated interferon alpha $2 \mathrm{a}$ or $2 \mathrm{~b}$ and $800 \mathrm{mg}-1600 \mathrm{mg}$ of ribavirin depending on weight. The recommended dosage for genotypes 2 and 3 is $800 \mathrm{mg} /$ day. For other genotypes, if weight is $<75 \mathrm{~kg}$, the recommended ribavirin dose is $1000 \mathrm{mg} /$ day or $1200 \mathrm{mg} /$ day if $>75 \mathrm{~kg}$. Efficacy was defined as attaining sustained virological response (SVR). Univariate analyses was performed using SPSS18; Chi-square test with $\mathrm{p}$-value $<0.05$ was defined significant.

RESULTS: 52 co-infected ( 3 females \& 49 males) were identified. Mean age was $40 \pm 7$ years. Caucasians accounted for $83.0 \%$; First Nations for $13.2 \%$ and Asians $1.9 \% .36$ were concurrently on HAART. The genotype distribution was: geno $1,66.0 \%$; geno $2,7.5 \%$; geno $3,26.4 \%$. SVR by ribavirin dosage ratio (actual dosage/recommended dosage): $\leq 1.0$; $42.4 \%(14 / 33),>1.0 ; 57.6 \%(19 / 33)$.

DISCUSSION: Suboptimal doses of weight-based ribavirin may be contributing to a lower treatment response in HCV/HIV co-infectants. Our results show a substantial and significant (15\%) increase in SVR if the dose of ribavirin used is greater than the currently recommend dose. We recommend that ribavirin dose be increased in the co-infected, (and that the hemoglobin be monitored more regularly), to increase the likelihood of achieving SVR.

\section{P072}

\section{WHITE LESIONS OF THE TONGUE IN AN HIV ${ }^{+}$PATIENT;} IN DISGUISE AS CANDIDA?

\section{Fletcher, Susan}

Montreal, QC

CASE REPORT: A 53 year old, $\mathrm{HIV}^{+}$white male presented complaining of a broken upper tooth and painful lesion on the left side of the tongue. Patient had been diagnosed $\mathrm{HIV}^{+}$nineteen years previously. Treated for over ten years, and was very compliant taking current combination.
Medical history: Allergy to Penicillin, past Hep A+B, remote syphilis, and genital herpes. Non smoker, no alcohol, past drug use. Viral load was $<50$, CD4 330

Dental history: Previous oral hairy leukoplakia, oral candidiasis, xerostomia, rampant caries tooth extractions.

On examination:

Extra-oral: Lipodystrophy. No swelling left submandibular lymphadenopahy.

Intra-oral: Missing teeth. Generalised gingival recession. Palate, shiny. Scant saliva. Tongue dry, sticky, a few mottled white patches.Upper tooth broken, jagged. Left lateral border seemed ulcerated. On inspection, left lateral border showed two whitish thick patches, largest $1.5 \mathrm{~cm}$ $\mathrm{x} 1 \mathrm{~cm}$ with surface ulceration and second smaller whitish lesion more posteriorly placed.

Differential diagnosis:

1. Traumatic ulcer, candidal overgrowth, HPV lesion

2. HPV lesion, ulcer, hyperkatosis

3. Possible malignancy

Treatment: Removed jagged edge, excisional biopsy under local anaesthesia, sent to pathology.

DIAGNOSIS: Ulcerated squamous papilloma, no evidence of dysplasia or malignancy.

Staining for spirochetes, showed abundant numbers and infection by trponema pallidum was strongly suggested.

Referral to infectious diseases confirmed syphilis past RPR was 1:2 now $\mathrm{RPR}$ was $1: 128$. Treatment was doxycycline $100 \mathrm{mg}$ bid $\times 28$ days.

DISCUSSION: Syphilis has been described as the great imitator! This patient had history of allergy to penicillin, which might have affected previous treatment. It is more likely that this was a case of reinfection due to the patients active sexual life style.

\section{P073}

FAILURE TO NORMALIZE ALT AT WEEK 12 OF HCV THERAPY IS ASSOCIATED WITH LOWER RATES OF SVR IN HIV/HCV COINFECTED PATIENTS

Kelly, Erin M; Cooper, Curtis

Ottawa, ON

Aims: In many centres, IL28B status, liver histology and frequent, rapid HCV RNA testing are not readily available. Alanine aminotransferase (ALT) is an inexpensive, convenient test that may be underutilized in determining treatment efficacy. Accordingly, we evaluated the utility of baseline, and on-therapy ALT decline in predicting antiviral virologic response in co-infected patients.

METHODS: A retrospective analysis of consenting, adult (18+), $\mathrm{HIV}^{-}$ $\mathrm{HCV}$ co-infected patients receiving HCV antiviral therapy at our site between January 1st, 2000 and December 31st 2009 was performed.

RESULTS: 46 patients met inclusion criteria. Patients were predominantly GT1 (65.2\%), male (87.0\%) and white (92.9\%). 90.5\% of patients were on highly active antiretroviral therapy (HAART), $83.8 \%$ had undetectable viral loads and mean CD4 counts were 537 cells $/ \mathrm{mm}^{3}$. Baseline ALT were similar between SVR and non-SVR patient $[95 \mathrm{mmol} / \mathrm{l} \mathrm{vs}$ $79 \mathrm{mmol} / \mathrm{l}(\mathrm{p}=0.35)]$. SVR rates were similar in those with baseline ALT above ULN (63 mmol/l) [39.3\% (11/28)] and those with normal baseline ALT [33.3\% (6/18)] $(\mathrm{p}=0.89)$. Mean ALT levels were lower in patients achieving SVR from week 4 onwards [week 4: $39 \mathrm{mmol} / \mathrm{l}$ vs $63 \mathrm{mmol} / \mathrm{l}$ ( $\mathrm{p}=0.029)$; week 12: $27 \mathrm{mmol} / \mathrm{l}$ vs $57 \mathrm{mmol} / \mathrm{l}(\mathrm{p}=0.006)$ ], with greatest decay rates in the first four weeks. Absolute and relative declines in ALT at week 4 did not predict SVR [AD $63 \mathrm{mmol} / \mathrm{l}$ vs $30 \mathrm{mmol} / \mathrm{l}(\mathrm{p}=0.21$ ); RD: $39.7 \%$ vs $22.4 \%(\mathrm{p}=0.32)]$. Normalization of ALT at week 12 was a sensitive [100\%] but not specific [33.3\%] marker for treatment success $(\mathrm{p}=0.009)$. No ALT cutoff value was sufficiently specific in predicting treatment outcomes (range $33.3 \%-77.8 \%$ for ALT $20-63 \mathrm{mmol} / \mathrm{l}$ ).

CONCLUSIONS: In HIV-HCV co-infected patients, baseline ALT values do not predict SVR. SVR patients achieve lower on-treatment ALT levels. Week 4 ALT does not predict treatment response, however failure to normalize ALT by week 12 may identify patients unlikely to achieve SVR. 


\section{P074}

\section{EXPLORING PERCEIVED HEALTH BY HIV-HCV CO-INFECTED PERSONS}

Maurais, Emilie ${ }^{1}$; Cox, Joseph ${ }^{1}$; Rollet, Kathleen ${ }^{1}$; Potter, Martin ${ }^{1}$; Pick, Neora²; Walmsley, Sharon ${ }^{3}$; Moodie, Erica $E^{1}$;

Rouleau, Danielle ${ }^{1}$; Klein, Marina B $^{1}$; Canadian Co-Infection

Cohort Study (CTN 222), For the ${ }^{1}$

${ }^{1}$ Montreal, QC; ${ }^{2}$ Vancouver, BC; ${ }^{3}$ Toronto, ON

INTRODUCTION: The majority of HIV-HCV co-infected individuals remain HCV treatment-naive. Health-related quality of life (HRQOL) may be an important factor to consider when initiating HCV treatment. Exploring the perceived HRQOL of HCV-treatment naive patients may offer clues for preparing patients for treatment. For example, it has been observed that $\mathrm{HCV}$-infected females have lower perceived HRQOL.

METHODS: This descriptive analysis examined baseline HRQOL data for $521 \mathrm{HCV}$ treatment-naive participants in a prospective Canadian, multisite cohort of $1052 \mathrm{HIV} / \mathrm{HCV}$ co-infected patients. Data were collected using the EuroQol EQ-5D-3L scale. Participants rated perceived problems, regarding five HRQOL dimensions, as no, moderate and severe problems. The proportions of participants with moderate and severe problems in each of the dimensions were determined. Differences in proportions between men and women were evaluated using Fisher's exact tests.

RESULTS: A total of 375 (72\%) men and 145 (28\%) women were HCV treatment-naive at baseline. The median (IQR) age was $44.3(38.8,49.6)$. Approximately two-thirds of participants reported problems in the pain/ discomfort and anxiety/depression dimensions. No significant changes in HRQOL were observed between men and women within each of the dimensions.

\section{TABLE}

Profile and perceived HRQOL of HCV treatment-naive participants $(\mathrm{N}=521)$

\begin{tabular}{|c|c|c|}
\hline \multicolumn{2}{|l|}{ Characteristics } & n (\%) \\
\hline \multicolumn{2}{|c|}{ Injection drug use (past 6 months) } & $209(40)$ \\
\hline \multicolumn{2}{|c|}{ Receiving antiretroviral therapy } & $406(78)$ \\
\hline \multicolumn{2}{|c|}{ History of AIDS diagnosis } & $146(28)$ \\
\hline \multicolumn{2}{|c|}{ History of end-stage liver diseases } & $40(7)$ \\
\hline \multirow{2}{*}{\multicolumn{2}{|c|}{$\begin{array}{l}\text { History of psychiatric diagnoses (depression, bipolar } \\
\text { disorder, schizophrenia, personality disorder) }\end{array}$}} & $228(44)$ \\
\hline & & Moderate/severe \\
\hline HRQOL dimensions & No problem & problems \\
\hline Mobility & $360(69)$ & $158(31)$ \\
\hline Self-care & $484(93)$ & $34(7)$ \\
\hline Usual activity & $360(70)$ & $157(30)$ \\
\hline Pain/discomfort & $199(38)$ & $319(62)$ \\
\hline Anxiety/depression & $192(37)$ & $325(63)$ \\
\hline
\end{tabular}

Percentages are based on the number of question answered

CONCLUSION: The pain/discomfort and anxiety/depression HRQOL dimensions stand out. The absence of gender differences in HRQOL was unexpected. Behavioural and clinical characteristics of this sample offer some understanding. Given the well-known side effects of HCV treatment, further analyses are needed to understand how problems in these dimensions affect $\mathrm{HCV}$ treatment initiation and change relative to treatment success. A longitudinal analysis of within individual transitions in HRQOL dimensions while considering sociodemographics, behavioural and clinical factors is needed.

\section{P075}

\section{SUBCLINICAL CMV CO-INFECTION IS ASSOCIATED} WITH THE DEVELOPMENT OF AN IMMUNE SENESCENT PHENOTYPE IN TREATED HIV(+) PATIENTS WITH UNDETECTABLE VIRAL LOAD

Ndumbi, Patricia; Gilbert, Louise; Tsoukas, Christos M

Montreal, QC

BACKGROUND: Chronic viral infections including HIV have been associated with the development of an immune senescent $\mathrm{T}$ cell phenotype. Cytomegalovirus (CMV) is the virus most frequently associated with the expansion of circulating $\mathrm{CD}_{28}{ }^{-}$T-cells. These cells define the phenotype and correlate with immune system aging in HIV seronegative individuals. High frequencies of $\mathrm{CD}^{+}{ }^{+} \mathrm{CD} 28^{-} \mathrm{T}$ cells have also been associated with acute coronary syndromes. In this study we investigated the impact of HIV-CMV co-infection on the immune senescent profile of treated HIV+ patients with undetectable viral load.

METHODS: Within the Immunodeficiency Treatment Center (IDTC) of the Montreal General Hospital, we studied a subset of $145 \mathrm{HIV}(+)$ individuals receiving cART and 50 healthy controls. CMV-seropositivity status was assessed by anti-CMV immunoglobulin $G$ antibody detection. Patients and controls were classified into 4 groups according to both HIV and CMV serologic status. Fluorescence staining and flow cytometric analysis were used to determine expression of CD4, CD8 and CD28 surface markers on T-cells. All comparisons between groups were done using the Mann Whitney test and $\mathrm{p}$-values $<0.05$ were considered significant. RESULTS: The prevalence of CMV seropositivity was higher among HIV patients than among controls $(86 \%$ vs. $52 \%, \mathrm{p}<0.0001)$. $\mathrm{HIV}(+)$ yet $\mathrm{CMV}(-)$ individuals had a higher frequency of CD28-T cells, than controls. The frequency of $\mathrm{CD} 4^{+} \mathrm{CD} 28^{-}$and $\mathrm{CD} 8^{+} \mathrm{CD} 28^{-} \mathrm{T}$-cells was significantly higher in HIV-CMV co-infected patients compared to controls and CMV $(-)$ patients infected with HIV $(\mathrm{p}<0.0001)$.

CONCLUSIONS: Overall the data suggest that the immune senescence profile of $\mathrm{HIV}(+)$ patients is exacerbated by the presence of subclinical chronic CMV co-infection. The relevance of this profile to atheroclerosis in those infected with HIV remains to be determined.

CVD and Other Issues in the Aging Population Maladie cardiovasculaire et autres affections touchant la population âgée

\section{P076 \\ CLINICAL CHARACTERISTICS AND SYNTAX SCORE IN HIV-INFECTED PATIENTS UNDERGOING CARDIAC CATHETERIZATION, COMPARED TO NON-INFECTED CONTROLS}

Carrier, Marc; Mansour, Samer; Potter, Brian; Riahi, Mounir; Tremblay, Cécile; Durand, Madeleine

Montreal, QC

BACKGROUND: HIV infection is associated with increased risk for coronary artery disease (CAD). This study aimed to describe the patient characteristics associated with CAD and the angiographic complexity in HIV-positive patients in comparison to non-infected controls.

METHODS: From 2006 to 2010 inclusively, 20684 patients underwent a cardiac catheterization at the CHUM in Montreal. Of these, $71 \mathrm{HIV}^{-}$ infected adults fit the selection criteria. 71 year-matched, randomly selected, non-infected, adult controls were compared. Data was obtained through the UHRESS database, the cardiac catheterization laboratory database, the CHUM's computerized chart database and manual chart review. Each catheterization was also analyzed for a SYNTAX score to evaluate the complexity of CAD.

RESULTS: HIV+ patients were younger (mean age of 55 vs 66 years) and more frequently of male gender ( 86 vs $59 \%$ ). Smoking, cocaine use and intravenous-drug use was more frequent in the $\mathrm{HIV}^{+}$group (48 vs $31 \% ; 13$ vs $6 \%$ and 9 vs $1 \%$ respectively), while hypertension, diabetes and a higher body mass index were more frequent in the control group (44 vs $59 \%, 18$ vs $33 \%$ and 24 vs 26 respectively). $43 \%$ of $\mathrm{HIV}^{+}$patients had an undetectable viral load. SYNTAX score was positive in $48 \mathrm{HIV}^{+}$ compared to 40 in the control group. The difference in SYNTAX score was not statistically significant between the two groups.

CONCLUSION: HIV-positive patients with CAD, undergoing cardiac catheterization, possess significantly different clinical characteristics and risk factors compared to the regular CAD patient. Furthermore, SYNTAX scores showed no statistically significant difference in angiographic complexity between HIV-infected patients and controls. 


\section{P077}

\section{HARMONIZATION OF PATIENT REPORT AND OBJECTIVE} MEASURES OF COGNITIVE DEFICITS: A RASCH ANALYSIS Palladini, Lisa; Brouillette, Marie-Josee; Fellows, Lesley; Mayo, Nancy

Montreal, QC

BACKGROUND: Combination antiretroviral therapies have changed the prognosis of the Human Immunodeficiency Virus (HIV). However, neurological disorders, particularly cognitive manifestations, remain a concern. Unless routine cognitive screening is in place in HIV clinics, clinicians are usually first confronted by patient report, of which the latter has been questioned in terms of clinical significance. Consequently, the objective of this study is to improve precision of measurement of cognitive functioning in $\mathrm{HIV}^{+}$individuals by creating a calibrated measure of cognition that includes items from neuropsychological testing, as well as items of patient self-report. More specifically, the goal is to estimate the extent to which items of patient reported cognitive difficulties and items from direct measures of cognition form a hierarchical uni-dimensional construct by using Rasch modeling.

METHODS: A convenience sample of 76 patients drawn from the MUHC Immunodeficiency Clinic was measured cross-sectionally. Patients completed paper and pencil tests, a computerized battery, and self-report questionnaire, all aimed at measuring cognition.

RESULTS: Preliminary analyses show that a measure of the latent construct that we labeled "cognitive ability", which combines patient self-report as well as objective measures, was created with good statistical fit to the Rasch model with inclusion of 27 of the original 78 items. The measure covers 6 logits of the theoretical construct range, with an excess of items too easy for this sample, where items range from -2.5 to +3.9 (target is -4 to +4 ). The measure is now uni-dimensional, and items are ordered hierarchically in terms of ability of cognitive functioning.

CONCLUSION: A measure such as this is the first of its' kind, and can have a profound impact on the field of HIV and the measurement of cognition. We have the opportunity to understand mathematical relationships, in terms of severity of cognitive impairment, between patient reported and directly measured deficits.



\section{P078}

\section{PATIENT ELIGIBILITY, BARRIERS TO UPTAKE AND CLINICAL OUTCOMES OF A NON-OCCUPATIONAL POST-EXPOSURE PROPHYLAXIS PROGRAM}

Chan, Alvin C; Gough, Kevin; Yoong, Deborah; Dimeo, Mary;

\section{Tan, Darrell H \\ Toronto, ON}

Non-occupational post-exposure prophylaxis (nPEP) using ARVs has become the standard of care for minimizing risk of acquiring HIV after high-risk sex. However, stringent eligibility criteria, high drug costs and antiretroviral toxicities are challenges to successful nPEP delivery. We reviewed patients' nPEP eligibility, barriers to treatment, and key outcomes at St. Michael's Hospital (SMH) to identify opportunities to improve service provision. We performed a retrospective chart review of all patients presenting to SMH between January 1, 2008 and December 31, 2010 with a recent potential exposure to HIV. Descriptive statistics were used to characterize the patient population, healthcare interventions and follow-up. Overall, 241 patients were identified, of which 87\% were eligible for nPEP. Fifty-six percent, 33\% and 9\% presented for high (receptive anal/vaginal, blood), medium (insertive anal/vaginal) and lowrisk (oral) sexual exposure, respectively. Nearly all (99\%) presented to ED within 72 hours. Seventeen patients discontinued nPEP prematurely; at least 3 due to cost, but data was missing on how ARVs were obtained for $82 \%$ of patients. Importantly, no patients discontinued nPEP due to adverse effects. Follow-up was completed at SMH for 46\%, 28\% and 15\% of patients at 2-6 weeks, 3-4 months, and 6 months respectively. Five cases of syphilis were diagnosed. Two HIV seroconversions were noted in patients with ongoing potential HIV exposure. Most patients presenting to hospital for nPEP do so under appropriate circumstances, ie. within 72 hours and after medium/high risk exposures. Barriers to nPEP completion were few, though the extent to which cost was a barrier was unclear. HIV and other STI diagnoses were rare, though STI testing was limited. Our findings demonstrate overall success of the nPEP program, though further work is needed to improve patient nPEP completion, follow-up and STI testing rates.

\section{P079 \\ EFFICACY OF BOOSTED AND UNBOOSTED ATAZANAVIR- BASED THERAPY IN PATIENTS WITHOUT PRIOR ANTIRETROVIRAL TREATMENT FAILURE}

Wentzell, Jason; Kravcik, Stephen; Giguere, Pierre Ottawa, ON

Context: Atazanavir (ATV) may be administered alone or with ritonavir. Both regimens are appropriate, although there has been a trend toward higher efficacy rate and more metabolic adverse effects with atazanavir/ ritonavir $(\mathrm{ATV})$.

OBJECTIVES: The objective of this study is to compare the efficacy of ATV and ATVr in two patient populations: patients with no prior history of treatment failure (Tx-naïve), and those who have switched from effective antiretroviral therapy (switch). Secondary objectives include the comparison of rates of treatment discontinuation between patients receiving boosted and unboosted ATV.

METHODS: A retrospective chart review with current or remote use of ATV was completed. Patients with a documented genotypic resistance or who had failed a prior antiretroviral therapy were excluded. Virologic failure was defined as a viral load above 50 copies $/ \mathrm{mL}$ on two consecutive measurements. In the case of detectable viral loads followed by virologic suppression on the same regimen, data were censored but not counted as failure. Efficacy and discontinuation rates were compared by Cox regression analyses.

RESULTS: 175 patients were eligible (89 Tx-naïve, 86 switch) for a median [IQR] follow-up of 27.6 [11.9, 43.4] months. Of those, 5 experienced virologic failures (ARV-naïve: 3 and 0; Switch: 1 and 1 for ATV and $A T V r$ respectively; $p=0.333)$ ). Younger age was associated with increased treatment failure $(\mathrm{p}=0.04)$. In treatment naïve patients, ATV $(\mathrm{n}=41)$ was associated with fewer discontinuations [HR 0.583 (95\% CI: 0.255, 1.330) $\mathrm{p}=0.154]$. Similarly, in switch patients, unboosted atazanavir $(\mathrm{n}=50)$ was associated with fewer discontinuations [HR 0.299 (95\% CI: 0.092, 0.979), $\mathrm{p}=0.046]$. Higher CD4 count at baseline was associated with ATV use but was not associated with outcomes.

CONCLUSIONS: In this cohort of patients with not prior antiretroviral failure, ATV and ATVr were equally efficacious. Unboosted ATV was better tolerated and is an option in patients intolerant to ritonavir.

\section{P080 \\ DESCRIPTION OF FACTORS ASSOCIATED WITH MEDICATION ERRORS IN AN HIV AMBULATORY CARE SETTING: THE DEFEAT STUDY}

Higgins, Niamh $\mathrm{M}^{1,2}$; Moodie, Erica $\mathrm{E}^{1}$; Gauthier, Josianne';

Wong, Leala ${ }^{1,3}$; Potter, Martin ${ }^{1}$; Klein, Marina $\mathbf{B}^{1}$

${ }^{1}$ Montreal, QC; ${ }^{2}$ Boston, MA, USA; ${ }^{3}$ Vancouver, BC

BACKGROUND: In the HIV population, medication errors are common and can result in virological failure or drug toxicity. The purpose of the DEFEAT study was to characterize medication errors in an HIV outpatient population and identify the risk factors associated with these errors.

METHODS: Pharmacists conducted patient interviews to obtain best possible medication histories (BPMH). Two pharmacists compared the $\mathrm{BPMH}$ to the clinic and community pharmacy medication records and documented any discrepancies. The medication profiles were evaluated for therapeutic appropriateness. Medication errors were classified by severity using the National Coordinating Council for Medication Error Reporting and Prevention Index. Risk factors for the number of errors per patient were identified using Poisson regression. Risk factors for error severity were identified using a proportional odds model.

RESULTS: Pharmacists interviewed 151 patients who took 1,717 medications and identified a total of 1,699 discrepancies or errors. 224 errors reached patients and 133 required interventions. Among study patients, $89(58.9 \%)$ experienced at least one error that reached the patient. 
Possible risk factors included the use of certain medication classes, such as chemotherapy, erectile dysfunction drugs, inhalers, and anti-infectives; the number of concomitant medications; the number of over-the-counter products; years since HIV diagnosis; history of cardiovascular disease; and obtaining prescription drugs from more than one pharmacy.

CONCLUSIONS: In this sample of ambulatory patients with HIV, pharmacists identified a high number of medication errors that reached patients. These findings may guide healthcare providers in identifying patients who may be at a higher risk of experiencing medication errors.

\begin{tabular}{lrr} 
Characteristic & Count (\%) & $\begin{array}{c}\text { Overall } \\
\text { frequency }\end{array}$ \\
\hline Any error & $1144(66.6)$ & 1699 \\
Errors that reached patients & $209(12.2)$ & 224 \\
$\quad$ (ie, Category C or higher) & $995(58.0)$ & 1007 \\
Database discrepancy & $435(25.3)$ & 447 \\
Community pharmacy discrepancy & & \\
Error types & $645(37.6)$ & 1001 \\
\hline Omission error & $215(12.5)$ & 238 \\
Inappropriate dosing & $180(10.5)$ & 186 \\
Insertion error & $139(8.1)$ & 156 \\
Inappropriate frequency & $47(2.7)$ & 55 \\
Clinically significant drug-drug interaction & $37(2.2)$ & 39 \\
Wrong drug & $11(0.6)$ & 11 \\
Therapeutic duplication & $9(0.5)$ & 9 \\
Inappropriate dosing for renal function & $4(0.2)$ & 4 \\
No indication & & \\
Error severity & $1049(61.1)$ & 1454 \\
\hline Category A & $21(1.2)$ & 21 \\
Category B & $88(5.1)$ & 91 \\
Category C & $119(6.9)$ & 128 \\
Category D & $5(0.3)$ & 5 \\
Category E & & \\
\hline
\end{tabular}

\section{P081 \\ POCKET PEP: DEVELOPMENT OF A CLINICAL TOOL TO PROMOTE BEST PRACTICE}

Yoong, Deborah M; Gough, Kevin A Toronto, ON

It is the policy of St.Michael's Hospital (SMH) to minimize transmission of hepatitis B virus (HBV), hepatitis C virus (HCV), and human immunodeficiency virus (HIV). In order to appropriately manage a potential occupational or non-occupational exposure, clinicians must assess the exposure risk considering factors such as the type of injury/ exposure and characteristics of the source patient or material. No major centre in Ontario has published such a protocol. The objectives of this project were to 1) review and update the post-exposure prophylaxis (PEP) protocol at $\mathrm{SMH}$ to align with current guidelines and best practices; 2) create a convenient pocket reference for clinicians to assist with managing potential occupational or non-occupational exposures to HBV, HCV and/or HIV; and 3) to promote safe and appropriate prescribing of PEP medications.

METHODS: A MEDLINE search was conducted using terms post-exposure prophylaxis, human immunodeficiency virus, hepatitis $\mathrm{C}$ virus and hepatitis $B$ virus. Current guidelines were reviewed and an internet search for pocket guides for post-exposure prophylaxis was performed.

RESULTS: An evidenced-based protocol for the clinical management of non-occupational and occupational exposures to blood borne pathogens was developed and a pocket reference, "Pocket PEP" was then created from this document to provide clinicians a convenient tool to use in practice. "Pocket PEP" contains a concise, best practice, 5-step process for managing non-occupational and occupational exposures to blood borne pathogens and has been distributed to key stakeholders at SMH. Next steps include sharing this tool with key partners both internal and external to SMH, obtain feedback from users of "Pocket PEP" to improve usability, and evaluate the impact of this tool on both the appropriate management of potential exposures to HBV, HCV and/or HIV and the appropriate prescribing of PEP medications.

Issues in the Developing World and Vulnerable Populations

Problématique dans les pays vulnérables et en voie de développement

\section{P082}

\section{INTER AND INTRA-PROFESSIONAL CARE IN THE CARE OF VULNERABLE POPULATIONS}

Meili, Ryan; Skinner, Stuart; Stewart, Kris

Saskatoon, SK

Over the past 5 years, Saskatoon has experienced an explosion in new HIV cases with incident rates of 30/100,000 people, the highest in Canada. Despite the availability of therapy, many patients in Saskatoon are not accessing the care and treatment that will prolong survival.

Saskatoon's HIV-positive population is unique as it is concentrated in the inner city, disproportionately affecting injection drug users, young women and Aboriginal peoples. This population faces numerous barriers to accessing services, including racism, distance from services, addictions and the challenges of daily living in poverty.

The WestSide Community Clinic is a community health centre that has been located in the inner city since 1975, contributing to the development of trust between the clinic and the local people. As a result of this community presence, patients who might not seek services elsewhere are more likely to do so at WestSide. This led to the co-location of many services, including Family Medicine, Nurse Practitioners, physiotherapy, needle exchange, parenting classes, nutrition counselling, community kitchens, an after-hours student-run clinic and much more

There are over $300 \mathrm{HIV}^{+}$patients who attend WestSide, and many more with hepatitis C. Many of these patients require specialist services but struggle with attending appointments that are located far from their home or in unfamiliar settings.

In response to the epidemic, specialists now offer clinics at WestSide. This started with Infectious Disease, but has expanded to Obstetrics and Gynaecology, Psychiatry, Internal Medicine, and Dermatology. The multidisciplinary approach has increased attendance and contributed to greater access to care for a high need population. Efforts are underway to move from simple co-location to real collaboration through inter and intra-professional case conferences. The innovative measures being taken to locate services where needed and enhancing communication and collaboration between health care-providers are a promising element in addressing Saskatoon's HIV epidemic.

\section{P083}

ASSESSING HEALTHCARE PROVIDER PERCEPTIONS OF BARRIERS TO MEDICATION RECONCILIATION IN HOMELESS PATIENTS WITH HIV/AIDS AT CASEY HOUSE

Sharma, Tanu; Carusone, Soo C; de Prinse, Karen

Toronto, ON

INTRODUCTION: Medication-related errors are a major cause of potentially fatal and disabling adverse drug events and are common in hospital and community settings. High rates of medication-related errors have been documented in homeless HIV/AIDS patients who are commonly on complicated medication regimens and have additional co-morbidities. Medication reconciliation is the process of preventing these medication-related errors, which occur predominantly at admission, transfer and discharge. Casey House is a small community-based hospital in Toronto providing sub-acute and end-of-life care to people living with HIV/AIDS. Approximately twenty percent of Casey House patients are homeless or unstably housed. This qualitative study explored healthcare provider's perceptions of barriers to medication reconciliation in homeless patients at Casey House.

METHODS: Thirteen healthcare workers from Casey House participated in one-on-one, audio-recorded, semi-structured interviews. Interviews were transcribed and analyzed for common themes. 
RESULTS: Thematic analysis identified four barriers to medication management of homeless HIV/AIDS patients. These included 1) the inability to store medications 2) competing priorities such as addiction, food insecurity and co-morbid physical and mental illness, including cognitive impairment 3) challenges with community follow-up due to a mobile lifestyle and 4) stigma around HIV/AIDS resulting in difficulties finding and maintaining housing.

DISCUSSION: Homelessness poses challenges for medication access, storage and adherence. Limitations in obtaining accurate medication histories, accessing up-to-date pharmacy medication lists and following patients in the community after discharge are all challenges faced by Casey House healthcare workers. For these reasons, established medication reconciliation processes require modification to meet the needs of the homeless HIV/AIDS population. Establishing ties with homeless shelters, social worker assistance in finding housing, encouraging single pharmacy access and decreasing the pill burden are all proposed strategies to help facilitate medication reconciliation in this population.

Issues in Women and Children

Problématique chez les femmes et les enfants

\section{P084 \\ TRANSITION FROM PEDIATRIC TO ADULT HIV CARE: PATIENT PERCEPTION OF TRANSITION READINESS AND HEALTHCARE PROVIDER ASSESSMENT OF HIV-RELATED KNOWLEDGE}

Bitnun, Ari ${ }^{1}$; Kerrison, Caroline ${ }^{2}$; MacDougall, Georgina ${ }^{1}$;

Louch, Debra ${ }^{1}$; Read, Stanley $\mathrm{E}^{1}$

${ }^{1}$ Toronto, ON; ${ }^{2}$ Ormskirk, United Kingdom

BACKGROUND: With improved survival afforded by combination antiretroviral therapy (cART) most HIV-infected children are reaching adulthood and transitioning from a pediatric to an adult care setting. Failure of effective transition is associated with health care drop out, discontinuation of cART and disease progression. This project describes adolescents' perceptions of transition readiness and healthcare provider (HCP) assessment of their HIV-related knowledge in the context of a transition program.

METHODS: A prospective "good-to-go" transition program was implemented in the pediatric HIV clinic at SickKids in March 2011. It includes educational materials (posters providing developmental timelines, patient oriented website) as well as a transition readiness checklist for patients and a knowledge assessment checklist for caregivers, both completed at 6 month intervals during regular clinic visits.

RESULTS: Thirty youths completed both baseline and 6 months assessments. Median age was 15 years (IQR 14-17); 50\% were female. At baseline, 22 were on cART, 4 on lamivudine monotherapy and 4 on no antiretroviral therapy; median viral load (VL) and CD4 counts were $<50$ copies/mL (IQR <50-601) and 632 cells/microliter (IQR 446-838), respectively. Baseline transition readiness checklist score correlated positively with age $(\mathrm{p}=0.03)$ and number of attended clinic appointments $(p=0.05)$. HIV-related knowledge score correlated positively with age $(\mathrm{p}<0.01)$. Neither measure correlated with VL, CD4 count, caregiver attendance in clinic, extent of antiretroviral medication experience or medication adherence. Transition readiness checklist score did not change significantly over the study period, whereas HCP-assessed HIVrelated knowledge improved $(\mathrm{p}=0.04)$. The proportion judged to have, on average, partial HIV-related knowledge or better increased from $53 \%$ $(16 / 30)$ at baseline to $70 \%(21 / 30)$ after 6 months.

CONCLUSIONS: Patient self assessment of transition readiness and HCP-assessed HIV-related knowledge improve with age. A longer duration of follow-up will be needed to determine if transition readiness and HIV-related knowledge can be enhanced by this formal transition program.

\section{P085}

DEMOGRAPHIC AND MANAGEMENT TRENDS AMONG HIV-POSITIVE PREGNANT WOMEN OVER TEN YEARS AT A CANADIAN URBAN HOSPITAL

Caprara, Daniela; Shah, Rajiv R; MacGillivray, Jay; Urquia, Marcelo; Yudin, Mark H

Toronto, ON

OBJECTIVE: To review the experience of one Canadian urban hospital in the demographics and management trends of HIV-positive pregnant women over a ten-year period.

STUDY METHODS: Retrospective chart review of all HIV-positive pregnant women delivered at St. Michael's Hospital in Toronto, ON from March 2000 - March 2010. Demographic, pregnancy, and intrapartum data were collected and analyzed.

RESULTS: There were 141 singleton pregnancies during the study period. The mean age of the cohort was 30.4 (range 16-43). A significantly increasing number of women were seen over time $(\mathrm{P}<0.0001)$ with $63 \%(\mathrm{n}=89)$ of cases in care from 2007 to 2010. The majority of women (66\%) were of African descent with recent immigration to Canada. There was a statistically significant increase in the number of Afro-Caribbean women over the study period $(\mathrm{P}=0.03)$. Only $4 \%(\mathrm{n}=5)$ reported illicit drug use in their current pregnancy. Although the majority of women had a known diagnosis of HIV prior to pregnancy, 30 (22\%) were diagnosed on antepartum testing. Most women were compliant with their antiretroviral therapy (94\%) and had undetectable viral loads at time of delivery (76\%). Protease inhibitors were used significantly more as the study period progressed $(\mathrm{P}<0.0001)$. Mean gestational age at delivery was 38.1 weeks (range 22-41.4). Mode of delivery included 84 (61\%) vaginal deliveries (3 vacuums) and 54 (39\%) cesarean sections. All neonates received AZT after delivery and there were no cases of mother to child transmission of HIV.

CONCLUSIONS: An increasing number of HIV-positive pregnant women have entered care over the past ten years with the majority being Afro-Caribbean. Most women are healthy with well-managed disease, and have favorable pregnancy outcomes. There were no infected children born during the study period.

\section{P086}

HEMATOLOGICAL TOXICITY FROM COMBINATION NEONATAL ANTIRETROVIRAL THERAPY FOR THE PREVENTION OF MOTHER-TO-CHILD TRANSMISSION OF HIV: A REVIEW OF 20 YEARS OF PMTCT AT ONE CANADIAN CENTER

Kakkar, Fatima; Lapointe, Normand; Soudeyns, Hugo;

Lamarre, Valerie

Montréal, QC

OBJECTIVE: To describe and compare the incidence of antiretroviral (ARV) therapy-related hematological toxicity among HIV-exposed uninfected infants treated with different ARV regimens at birth.

METHODS: Study subjects were enrolled prospectively in the Centre maternel et infantile sur le sida (CMIS) mother-child cohort, CHU Sainte-Justine, Montreal, Canada, between 1987 and 2010 (n=585). Pearson's $\chi^{2}$ or Fisher's exact test were used to compare differences in proportion of anemia and neutropenia, defined according to the Division of AIDS table for grading the severity of pediatric adverse events.

RESULTS: $12.9 \%$ of infants did not receive any treatment, $11.5 \%$ received AZT monotherapy, $28.7 \%$ received double therapy, and $46.9 \%$ received 3 or more agents for 6 weeks after birth. The incidence proportion of anemia and neutropenia at different ages is described below. The difference in incidence between ARV groups was not statistically significant at any time point.

Table 1: Incidence proportion of Grade 2 or higher anemia

\begin{tabular}{|c|c|c|c|c|}
\hline $\begin{array}{l}\text { Infant ARV } \\
\text { regimen }\end{array}$ & $\begin{array}{c}2 \text { weeks } \\
(\mathrm{Hg}<120 \mathrm{~g} / \mathrm{L})\end{array}$ & $\begin{array}{c}4 \text { weeks } \\
(\mathrm{Hg}<95 \mathrm{~g} / \mathrm{L})\end{array}$ & $\begin{array}{c}8 \text { weeks } \\
(\mathrm{Hg}<84 \mathrm{~g} / \mathrm{L})\end{array}$ & $\begin{array}{c}26 \text { weeks } \\
(\mathrm{Hg}<99 \mathrm{~g} / \mathrm{L})\end{array}$ \\
\hline None & $24 \%$ & $42 \%$ & $2.4 \%$ & $5.1 \%$ \\
\hline Monotherapy & $47 \%$ & $42 \%$ & $5.4 \%$ & $3.5 \%$ \\
\hline $2 \mathrm{ARV}$ & $50 \%$ & $70 \%$ & $2.6 \%$ & $4.3 \%$ \\
\hline $3^{+} \mathrm{ARV}$ & $42 \%$ & $58 \%$ & $4.3 \%$ & $3.6 \%$ \\
\hline Overall & $39 \%$ & $50 \%$ & $3.6 \%$ & $4.2 \%$ \\
\hline $\mathrm{n}$ & 93 & 113 & 492 & 482 \\
\hline
\end{tabular}


Table 2: Incidence proportion of Grade 2 or higher neutropenia

\begin{tabular}{lcccc}
\hline $\begin{array}{l}\text { Infant ARV } \\
\text { regimen }\end{array}$ & $\begin{array}{c}\text { 2 weeks } \\
(\mathbf{A N C}<999 / \\
\left.\mathbf{m m}^{3}\right)\end{array}$ & $\begin{array}{c}\text { 4 weeks } \\
(\mathbf{A N C}<999 / \\
\left.\mathbf{m m}^{3}\right)\end{array}$ & $\begin{array}{c}\mathbf{8} \text { weeks } \\
(\mathbf{A N C}<999 / \\
\left.\mathbf{m m}^{3}\right)\end{array}$ & $\begin{array}{c}\mathbf{2 6} \text { weeks } \\
(\mathbf{A N C}<999 / \\
\left.\mathbf{m m}^{3}\right)\end{array}$ \\
\hline None & $0 \%$ & $10 \%$ & $4.8 \%$ & $12 \%$ \\
Monotherapy & $0 \%$ & $15 \%$ & $16 \%$ & $13 \%$ \\
$2 \mathrm{ARV}$ & $8.3 \%$ & $20 \%$ & $20 \%$ & $8.6 \%$ \\
$3^{+} \mathrm{ARV}$ & $5.2 \%$ & $11 \%$ & $15 \%$ & $13 \%$ \\
Overall & $3.2 \%$ & $14 \%$ & $16 \%$ & $11 \%$ \\
$\mathrm{n}$ & 93 & 113 & 492 & 482 \\
\hline
\end{tabular}

CONCLUSIONS: There were no significant differences in the incidence of anemia and neutropenia according to type of neonatal ARV regimen used, although there was a high overall incidence of transient anemia, and evidence persistent neutropenia to 6 months of age among treated infants. Further work needs to be done to understand the clinical implications of these findings.

\section{P087}

\section{MATERNAL AND CORD BLOOD VITAMIN D LEVELS IN HIGHLY ACTIVE ANTIRETROVIRAL THERAPY (HAART) EXPOSED PREGNANCIES.}

van Schalkwyk, Julie E; Murray, Melanie C; Wingate, Kirstin; Albert, Arianne; Côté, Hélène C; Money, Deborah M; Pick, Neora; Green, Timothy

Vancouver, BC

INTRODUCTION: Vitamin D deficiency may be associated with adverse pregnancy outcomes. Lower vitamin D (herein defined as plasma 25-hydroxy vitamin D (25OHD)) levels are reported in HIV infection, and are reportedly associated with increased mother-to-child transmission in the developing world. We hypothesized that clinical factors and HAART would be associated with vitamin D concentrations in an HIV. infected Canadian pregnant cohort.

METHODS: From 2005-2009, we enrolled HIV-infected pregnant women in a prospective study of HAART toxicity. On enrollment at $\sim 18$ weeks gestation, demographic data were recorded. Maternal blood samples were collected at 34 weeks and cord blood at delivery. Women received prenatal supplements during pregnancy ( 400 IU/d vitamin D). Deficiency was defined as a $25 \mathrm{OHD}$ of $<25 \mathrm{nmol} / \mathrm{L}$, and insufficient as $<50 \mathrm{nmol} / \mathrm{l}$ or $<75 \mathrm{nmol} / \mathrm{l}$. Effect of demographics and days of HAART on maternal and cord blood vitamin D was assessed with ANOVA.

RESULTS: Vitamin D levels were determined for 57 maternal and 35 cord blood samples. Mean \pm SD maternal serum $25 \mathrm{OHD}$ was $64 \pm 29 \mathrm{nmol} / \mathrm{L}$. One woman $(1.8 \%)$ was vitamin D-deficient while 20 $(35.1 \%)$ and $40(70.2 \%)$ were insufficient at $<50 \mathrm{nmol} / \mathrm{L}$ and $<75 \mathrm{nmol} / \mathrm{L}$ respectively. Only $5 / 57$ received $<8$ weeks of HAART prior to 34 -week blood work. Ethnicity, BMI, smoking and Hepatitis B or C virus co-infection were not associated with vitamin D levels. Duration of HAART tended towards a positive association with maternal 25OHD (F1,54=3.57, $\mathrm{p}=0.06$ ). Cord blood samples showed no association. There was no mother-to-child transmission in the cohort.

CONCLUSIONS: Many women had vitamin D levels insufficient for optimal health. There was a trend to higher vitamin D levels with longer HAART duration in pregnancy. Larger studies investigating current and higher dosing of vitamin D are needed to define the impact of HAART on plasma $25 \mathrm{OHD}$ levels in pregnancy, and their association with adverse maternal or pediatric outcomes.

\section{P088}

\section{A SURVEY EXAMINING CELL PHONE ACCESS AND} ACCEPTABILITY OF A MOBILE HEALTH (MHEALTH) INTERVENTION TO SUPPORT PATIENTS WITH HIV/AIDS IN BRITISH COLUMBIA

Murray, Melanie C; van Borek, Natasha; van der Kop, Mia L;

Pick, Neora; Maan, Evelyn J; Maginley, Juanita J; Rebecca, Graham J; Money, Deborah M; Ogilvie, Gina S; Lester, Richard T; WelTel BC Study Team, and the

Vancouver, BC

INTRODUCTION: Despite widespread availability of HAART, many HIV positive individuals, especially women and youth, struggle with adherence issues that may not respond to conventional interventions. Mobile health (mHealth), the use of mobile phone technology to deliver health care, is an emerging area of interest for disease management and prevention. We conducted a survey among patients attending clinic for HIV care to examine cell phone access and receptiveness to an mHealth intervention in the form of SMS (short message system) text messaging to improve clinical care.

METHODS: Between October 12th and December 23th 2011, patients attending the Oak Tree Clinic in Vancouver, British Columbia were offered a survey examining their access and attitudes toward cellular phones and text messaging. Consent was implied if patients filled out the survey and returned it to clinic staff. Data was assessed using descriptive analysis.

RESULTS: 354 patients attended clinic during the study period and were offered the survey if time permitted. 180 (51\%) completed the survey. The median age of participants was 39 (range 11-63); 111 identified as female. Of patients polled, $81 \%$ owned a cell phone, $87 \%$ of whom used text messaging, and 39\% of whom access the internet from their phone. $71 \%$ text message at least weekly. $83 \%$ believed the intervention could assist in clinical care and follow up. Perceived potential benefits of the intervention included easier access to care/communication with physicians, and reminders for medication and appointments. The main concern cited was privacy.

CONCLUSIONS: Overall, a majority of patients had cell phone and text messaging access, and believed the WelTel intervention could benefit them, providing confidentiality was considered. This data will inform a currently enrolling pilot study to test the feasibility and acceptability of an mHealth intervention in HIV positive patients in British Columbia.

\section{P090}

ORAL EPITHELIAL CELL TELOMERE LENGTHS (TL) ARE CORRELATED WITH PERIPHERAL BLOOD LEUKOCYTE TL IN HIV POSITIVE $\left(\right.$ HIV $\left.^{+}\right)$AND HIV-EXPOSED UNINFECTED (HEU) CHILDREN, AND ARE NOT AFFECTED BY HIV VIREMIA

Zanet, DeAnna L ${ }^{1}$; Soudeyns, Hugo ${ }^{2}$; Alimenti, Ariane ${ }^{1}$; Lamarre, Valerie ${ }^{2}$; Lapointe, Normand ${ }^{2}$; Brophy, Jason ${ }^{3}$; Samson, Lindy ${ }^{3}$; Money, Deborah $\mathrm{M}^{1}$; Thorne, Anona ${ }^{1}$; Forbes, John $\mathrm{C}^{1}$; Cote, Helene $\mathrm{C}^{1}$; CIHR Emerging Team in HIV Therapy and Aging, The

${ }^{1}$ Vancouver, BC; ${ }^{2}$ Montreal, QC; ${ }^{3}$ Ottawa, ON

BACKGROUND: NRTIs given to $\mathrm{HIV}^{+}$pregnant women and their HEU infants may inhibit telomerase while HIV-induced inflammation/ oxidative stress can also adversely affect telomere length (TL), and possibly influence tissue aging. We previously showed that although peripheral blood leukocyte (PBL) TL was similar between HEU and $\mathrm{HIV}^{+}$ children, the latter demonstrated shorter TL if they had a detectable HIV $\mathrm{pVL}$. This study examined whether similar TL dynamics are observed in epithelial cells.

METHODS: Mouth swabs and PB were prospectively collected from $\mathrm{HIV}^{+}$and HEU children in the CARMA cohort. TL was measured by qPCR. Univariate and multivariate linear regression models examined relationships of predictor variables with TL.

RESULTS: Oral epithelial cell (OEC) TL data was obtained for $101 \mathrm{HIV}^{+}$ (median [IQR] age (years) 13.3 [9.9-15.8] and $247 \mathrm{HEU}$ (1.6 [0.5-3.9]) children. All HEU children were exposed to NRTI in utero and/or neonatally. OEC TL was $\sim 70 \%$ longer ( $\mathrm{p}<0.0001$, paired $\mathrm{t}$-test) than and correlated with PBL TL (Pearson's $\mathrm{r}^{2}=0.26, \mathrm{p}<0.0001$ ). In multivariate analysis 
that included age, gender, HIV status, parental ages and ethnicity, older children had shorter OEC TL $(\mathrm{p}<0.001)$. Compared to Caucasians, children of Aboriginal ethnicity had shorter TL $(p=0.05)$ while Blacks had longer TL ( $p=0.027)$. Among HEU, OEC TL was not associated with duration of in utero ART. Among $\mathrm{HIV}^{+}$children, although immune parameters were predictive univariately, only older age $(\mathrm{p}=0.003)$ was predictive multivariately.

CONCLUSIONS: Our preliminary results did not detect differences in OEC TL between $\mathrm{HIV}^{+}$and HEU children but this reassuring observation is limited by the age imbalance between the groups. OEC TL was unrelated to antenatal NRTI exposure in HEU. Furthermore, unlike PBL $\mathrm{TL}, \mathrm{OEC} \mathrm{TL}$ was not related to HIV pVL in $\mathrm{HIV}^{+}$children. This suggests that immune activation rather than systemic inflammation may explain the association between short PBL TL and HIV viremia.

Mental Health Topics

Sujets portant sur la santé mentale

\section{P091}

VALIDITY OF MONTREAL COGNITIVE ASSESSMENT AND HIV DEMENTIA SCALE DOMAINS IN AN HIV ${ }^{+}$SAMPLE

Gil, Diana M; Yamamoto, Aiko; Harris, Marianne; Guillemi, Silvia; Hull, Mark; Montaner, Julio; Hsiung, G-Y Robin

Vancouver, BC

BACKGROUND: The Montreal Cognitive Assessment (MoCA) and HIV Dementia Scale (HDS) are cognitive screening tests frequently used with $\mathrm{HIV}^{+}$populations. However, no research has examined the cognitive subdomains purportedly assessed by these tests. The purpose of this study was to examine the construct validity of the MoCA and HDS.

METHODS: Thirty-six $\mathrm{HIV}^{+}$adults within an urban hospital immunodeficiency clinic gave informed consent to participate in this study and had MoCA and HDS testing. MoCA and HDS domains were compared with Pearson correlation coefficients.

RESULTS: Subjects were predominantly male (33/36) and all but 2 had at least a high school education (22 had university/college education). Median age was 53 years (interquartile range $[\mathrm{IQR}]=48-57$ ), median time since HIV diagnosis 15 years $(\mathrm{IQR}=7-21)$, median $\mathrm{CD} 4$ count 505 (IQR=358-658) cells $/ \mathrm{mm} 3$, plasma viral load was $<50$ copies $/ \mathrm{ml}$ for 31 subjects (range <50-157427), and all subjects were on antiretroviral medication. Median MoCA score was 23/30 (IQR=21-26) and HDS score was $12 / 16(\mathrm{IQR}=8.3-13.4)$.

HDS Memory was only correlated with MoCA Memory $(r=0.46, \mathrm{p}<0.01)$. HDS Attention was only correlated with MoCA Visuospatial Function $(\mathrm{r}=0.42, \mathrm{p}<.05)$. HDS Psychomotor Speed was correlated with MoCA Visuospatial Function $(r=0.432, p<0.01)$, Attention $(r=0.55, p<0.01)$, and Language $(r=0.041, p<0.05)$ domains. HDS construction was most strongly related to Visuospatial Function $(r=0.074, p<0.001)$ but was correlated with all MoCA domains ( $\mathrm{r}=0.38$ to $.46, \mathrm{p}<0.05)$ except for Attention.

CONCLUSION: Construct validity was demonstrated for memory and construction/visuospatial MoCA and HDS domains. Processing speed and construction/visuospatial measures appear to reflect less unique cognitive skills and may require more widespread cognitive contributions. MoCA and HDS Attention domains were uncorrelated; these tests appear to assess distinct areas (MoCA focuses on sustained attention and auditory-verbal working memory while HDS is based on inhibitory control of eye movements). Caution is required when interpreting results for specific cognitive domains within these screening tests.

\section{P092 \\ ELEVATED C-REACTIVE PROTEIN IS ASSOCIATED WITH NEUROCOGNITIVE IMPAIRMENT IN HIV}

Gil, Diana M; Yamamoto, Aiko; Harris, Marianne; Guillemi, Silvia; Hull, Mark; Montaner, Julio; Hsiung, G-Y Robin

Vancouver, BC

BACKGROUND: HIV results in chronic immune system activation and possible systemic inflammation. Elevated C-reactive protein (CRP) is a biomarker of inflammation and tissue damage, and has been demonstrated to be associated with morbidity and mortality within HIV populations. The relationship between CRP and cognitive functioning has not yet been examined and is the focus of this study.

METHODS: Nineteen $\mathrm{HIV}^{+}$adults referred for a neurocognitive evaluation within an urban hospital immunodeficiency clinic gave informed consent to participate in this study and had CRP obtained within 3 months of neurocognitive testing. Only subjects with concomitant undetectable plasma viral load $(<40$ copies $/ \mathrm{mL})$ were selected for inclusion in the study as elevated viral load levels may be a confounding factor. A logarithmic transformation of CRP values was performed to normalize the data. Relationships between $\log _{10} \mathrm{CRP}$ and Montreal Cognitive Assessment (MoCA) and HIV Dementia Scale (HDS) scores were determined using Pearson correlation coefficients.

RESULTS: Subjects were all males, median age 56 years (interquartile range [IQR] 49, 60), and all but one had at least a high school education (11 had college or university). Subjects had been diagnosed with HIV for a median of 16 yrs (IQR 10,21), were all on antiretroviral medication, and had median CD4 counts of 450 (IQR 350, 650) cells/ $/ \mathrm{mm}^{3}$ and median CRP values of 1.4 (IQR 0.9, 3.5) $\mathrm{ml} / \mathrm{L}$. The $\log _{10}$ CRP was significantly correlated with HDS score (Pearson correlation coefficient $[\mathrm{r}]=-0.583$, $\mathrm{p}<0.005)$ but not with MoCA score $(\mathrm{r}=-0.280, \mathrm{p}=0.123)$.

CONCLUSION: Higher CRP values were associated with poorer performance on the HDS but were unrelated to MoCA scores. The HDS includes unique psychomotor speed and saccadic eye movement components which may be more sensitive to inflammatory changes. Overall results suggest a relationship between inflammatory changes and neurocognitive functioning, even in the presence of plasma viral load suppression.

\section{P093 \\ NEW HORIZONS IN PSYCHOSOCIAL SUPPORT FOR PEOPLE LIVING WITH HIV: EMDR AND HIV-RELATED TRAUMA}

Rivas, Carlos

Toronto, ON

In a context where resources are limited, living longer with HIV means that psychosocial challenges -the constant struggle for PHA's in Canada- need to be addressed in the most effective way possible. This presentation introduces an evidence-based form of psychotherapy designed to reduce trauma-related stress, anxiety, and depression: Eye Movement Desensitization and Reprocessing (EMDR). Participants will know what it is, the rational behind this approach (eg, the Adaptative Information Processing Model), and some clinical evidence showing how EMDR is successfully applied to support PHA's in dealing with the condition. The discussion includes specific protocols for HIV-related trauma, disclosure, compliance to treatment and expectations of wellbeing, as well as considerations about incorporating the EMDR methodology into research/ intervention projects.

\section{P094}

BRIEF COGNITIVE SCREENING TOOLS FOR DETECTION OF HIV-ASSOCIATED NEUROCOGNITIVE DISORDERS AND IMPAIRMENT: A SYSTEMATIC REVIEW OF THE LITERATURE

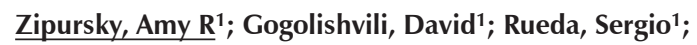

Carvalhal, Adriana ${ }^{1}$; Brunetta, Jason ${ }^{1}$; Gill, MJ ${ }^{2}$; Rachlis, Anita ${ }^{1}$; Rosenes, Ron ${ }^{1}$; Atkinson, Maggie ${ }^{1}$; McCombe, Jennifer $A^{2,3}$;

Collins, Evan ${ }^{1}$; Rourke, Sean $\mathbf{B}^{1}$

${ }^{1}$ Toronto, ON; ${ }^{2}$ Calgary; ${ }^{3}$ Edmonton, AB

BACKGROUND: HIV-associated neurocognitive disorders (HAND) persist in the HAART era. These have been shown to have a significant impact on everyday functioning and survival. Recent evidence suggests that milder forms of HAND are increasing. As such, there is need for accurate screening and diagnosis in clinic settings. The aim of this study is to systematically review the literature on brief screening tools for HAND.

METHODS: We conducted a systematic review of electronic databases of national and international literature in the fields of medical/health sciences and psychology. We included studies that: (1) focused on people living with HIV; (2) included individuals with neurocognitive impairment associated with HIV; (3) addressed screening of neurocognitive or 
neuropsychological issues by evaluating the utility of, validating, or comparing brief ( $\leq 20$ minutes) screening tools; and (4) reported findings from empirical research. Two reviewers independently assessed journal articles for inclusion and extracted data from the selected papers. Disagreements between reviewers were resolved by a third party reviewer.

RESULTS: Our findings from 43 articles suggest that there are effective brief screening tools for the detection of HIV-associated dementia (HAD), but that a gap in literature exists regarding brief screening tools for more mild forms of HAND, ie, mild neurocognitive disorder (MND). However, the articles included in the review employed a variety of methods that presented challenges in comparing tools for clinical utility. CONCLUSION: The review provides evidence supporting further need for development and evaluation of brief screening tools that are sensitive to mild neurocognitive impairment associated with HIV. In addition, our results highlight the need for more stringent and standardized methods for evaluating screening tools for HAND.

Other Complications of HAART

Autres complications du traitement antirétroviral hautement actif (HAART)

\section{P095 \\ DXA-DIAGNOSED LIPODYSTROPHY IN A COHORT OF HIV-INFECTED CHILDREN IN CANADA}

Leifso, Kirk ${ }^{1}$; Brophy, Jason $C^{1}$;Samson, Lindy ${ }^{1}$; Alimenti, Ariane ${ }^{2}$; Cote, Helene ${ }^{2}$; Kelly, Thomas $\mathrm{L}^{3}$

\section{${ }^{1}$ Ottawa, ON; ${ }^{2}$ Vancouver, BC; ${ }^{3}$ Boston, MA, USA}

INTRODUCTION: Lipodystrophy (LD) is a common treatment complication in HIV-infected adults, but is less frequently diagnosed in children. There is a paucity of published normative fat body distribution data in children. We describe fat redistribution patterns in HIV-infected children using normative data from the National Health and Nutrition Examination Survey (NHANES).

METHODS: A single-centre retrospective review of children with perinatal HIV infection followed at the Children's Hospital of Eastern Ontario was performed. All children $>7$ years with DXA scans ordered clinically for bone health assessment were included. Regional fat, lean, and bone mass data from DXA reports were collected, along with subjects' demographic and clinical HIV parameters. Age, race/ethnicity, and gender-specific NHANES data were used to calculate Z-scores for trunk:limbs fat mass ratio (T:L), \%trunk:\%leg fat ratio (\%T:\%L), and \%total fat (\%TF).

RESULTS: DXA scans were available for 24 children aged 8-18 years (14 females, 21 black). Median age, CD4 count, and HIV pVL were 15.7 years, 500 cells/uL, and $<50$ copies $/ \mathrm{mL}$, respectively. Twenty-three (96\%) were currently on PI-based cART, and median ART duration was 12.0 years. Median Z-scores for T:L, \%T:\%L, and \%TF were 2.15, 1.50, and -0.15 respectively. Correlation coefficients between $Z$-scores and years on any ART (T:L r $=0.459, p=0.023 ; \% \mathrm{~T}: \% \mathrm{~L} r=0.503, \mathrm{p}=0.012 ; \% \mathrm{TF} r=-0.431$, $\mathrm{p}=0.036)$ and years on $\mathrm{LD}$-associated drugs stavudine and/or didanosine ( T:L r $=0.475, p=0.019 ; \% \mathrm{~T}: \% \mathrm{~L} r=0.570, \mathrm{p}=0.004 ; \% \mathrm{TF} r=-0.391, \mathrm{p}=0.059$ ) were statistically significant except $\% \mathrm{TF}$ for LD-associated drugs.

CONCLUSIONS: High Z-scores for T:L and \%T:\%L, which suggest redistribution of fat from limbs to trunk, were common in this cohort of heavily treated children. This novel use of normative population data on pediatric body fat composition from DXA could allow earlier recognition of fat redistribution in HIV-infected children. Future studies of a larger cohort are planned to validate this method and compare it to other modalities.

\section{P096 \\ ÉVALUATION DE LA TOXICITÉ RÉNALE À LONG TERME EN RELATION AVEC L'EXPOSITION AU TÉNOFOVIR CHEZ LES PATIENTS INFECTÉS PAR LE VIH DE LA COHORTE DE LA CLINIQUE MÉDICALE QUARTIER LATIN, MONTRÉAL, CANADA}

Laprise, Claudie; Baril, Jean-Guy; Dufresne, Serge; Côté, Pierre; Trottier, Helen

Montréal, QC

OBJECTIF DE L'ÉTUDE : Évaluer l'ampleur de la toxicité rénale à long terme du ténofovir chez les patients VIH positifs.

MÉTHODES : Dans le cadre d'une étude de cohorte prospective, l'ampleur de l'atteinte rénale à long terme (mesurée à l'aide de la formule d'estimation du débit de filtration glomérulaire CKD-EPI) ainsi que de ses déterminants, ont été analysés à partir de courbes Kaplan-Meier, d'une ANOVA et d'un modèle de régression de Cox, en stratifiant selon l'âge (50 ans et plus vs 50 ans et moins). L'exposition au ténofovir, seul ou en combinaison, a été comparée à l'exposition d'autres antirétroviraux. RÉSULTATS OBTENUS : La cohorte compte 2352 patients VIH positifs, recrutés à la première mesure de charge virale, depuis 1996, et suivis généralement tous les 3 à 6 mois. Lâge moyen au recrutement était de 39 ans (écart type=9), et 92,4\% des patients (2 173) sont des hommes. Soixante-deux pour cent des patients (1431) étaient toujours actifs en 2011. La durée moyenne de suivi est de 6,1 ans (343,2 semaines, écart type $=254,7)$, et $54,4 \%(978 / 1798)$ des patients prennent ou ont pris du ténofovir en cours de suivi, comparativement à ceux qui ont pris d'autres antirétroviraux. Le segment de suivi des patients sous ténofovir a été comparé à celui des patients sous autres thérapies antirétrovirales, soit du 11 novembre 1998 au 8 septembre 2011. Une augmentation de l'incidence cumulative de la perte de fonction rénale ainsi qu'une perte de débit moyen de fonction rénale sont attendues chez les patients exposés au ténofovir par rapport aux patients non exposés. Les déterminants de la perte de fonction rénale associés au ténofovir, dont l'âge, seront considérés après un contrôle de CKD EPI de départ.

CONCLUSION : Les résultats influeront sur la prise en charge des patients vivant avec le $\mathrm{VIH}$, particulièrement sur le suivi à long terme des patients âgés, de plus en plus nombreux en raison de la meilleure espérance de vie attribuable à la prise d'antirétroviraux.

\section{P097}

\section{THE DEVELOPMENT OF A CLINICAL TOOL FOR MONITORING AND DETECTION OF TENOFOVIR- ASSOCIATED NEPHROTOXICITY}

Foisy, Michelle M; Hughes, Christine; Cooper, Ryan

\section{Edmonton, AB}

OBJECTIVES: Tenofovir used as part of antiretroviral therapy to treat HIV infection has been associated with nephrotoxicity. This can present as acute renal insufficiency with rapid reduction in estimated glomerular filtration rate (eGFR) or as frank Fanconi's syndrome. Tenofovir associated-nephrotoxicity can also present more subtly with proximal renal tubular dysfunction and smaller reductions in eGFR. Currently there is a lack of clear guidelines on the optimal approach to detecting this druginduced toxicity. The objective is to describe the development of a practical tool to assist clinicians in monitoring for and detecting tenofovirrelated nephrotoxicity.

METHODS: Development of the tool involved a group of local clinicians with expertise in HIV (pharmacists, Infectious Diseases physicians and nephrologists). The content was based on published literature, an educational workshop facilitated by an experienced HIV nephrologist and clinician experience with the use of tenofovir.

RESULTS: The tool had four main sections: 1) Baseline screening prior to starting tenofovir to identify patients potentially at increased risk of tenofovir associated-nephrotoxicity, with a focus on screening for nephrotoxic comorbidities and medications. 2) Routine monitoring for patients taking tenofovir, including the role of serum creatinine, eGFR, urinalysis, and phosphate. 3) Clinical patient assessment when tenofovir nephrotoxicity is suspected. Details on the clinical presentation, the onset, and other potential causes of renal toxicity to consider were included to assist the clinician in determining causality. 4) Laboratory investigations to further explore the potential diagnosis of tenofovir-associated 
nephrotoxicity. Suggested testing included serum findings (creatinine, eGFR, potassium, chloride, phosphate, $\mathrm{HCO}_{3}$, anion gap, fasting glucose); urinalysis (protein, glucose); urine (total protein to creatinine ratio, fractional urinary phosphate excretion and protein electrophoresis). The role of other measures such as renal ultrasound, renal biopsy and referral to nephrology was also addressed.

CONCLUSIONS: A clinical tool for monitoring and detecting tenofovir-related nephrotoxicity was developed in our clinic for the purpose of standardizing our approach to patient care. Follow-up study is needed to evaluate the utility of the tool and impact on antiretroviral use patterns.

Pharmacology and Pharmacokinetics

Pharmacologie et pharmacocinétique

\section{P098}

\section{MANAGING COMPLEX ANTICONVULSANT- ANTIRETROVIRAL DRUG INTERACTIONS}

Foisy, Michelle M; Ahmed, Rabia; Chiu, Isabelle; Cooper, Ryan; Joffe, A Mark

\section{Edmonton, $\mathrm{AB}$}

OBJECTIVES: To report a case series describing the clinical management of complex dual drug interactions between hepatic cytochrome p450 enzyme-inducing anticonvulsants and protease inhibitors, which are both substrates and inhibitors of certain CYP450 isoenzymes such as CYP4503A4.

METHODS: A case series is described. Data on therapeutic drug monitoring for both drug classes, required dosage adjustments, drug toxicity and efficacy, and patient outcome is presented.

\section{RESULTS:}

\begin{tabular}{|c|c|c|}
\hline $\begin{array}{c}\text { Baseline } \\
\text { Demographics }\end{array}$ & $\begin{array}{c}\text { Antiretrovirals/ } \\
\mathrm{Cmin}(\mathrm{mg} / \mathrm{L})\end{array}$ & $\begin{array}{c}\text { Anticonvulsants } \\
\text { Cmin (umol/L) }\end{array}$ \\
\hline $\begin{array}{l}39 \text { y.o. African } \\
\text { male; CD4 } \\
210, \mathrm{VL} \\
110,000\end{array}$ & $\begin{array}{l}\text { LPV/r 600/150 mg } \\
\text { BID; LPV Cmin: } \\
11.1 \text { (1 week), } \\
5.3 \text { (4 months) }\end{array}$ & $\begin{array}{l}\text { CBZ } 400 \text { mg BID; } \\
\text { CBZ Cmin: } 31 \\
\text { (pre-ARVs), } 53 \text { (1 } \\
\text { week), } 38 \text { ( } \downarrow \text { CBZ } \\
\text { to } 200 / 400 \mathrm{mg} \\
\text { BID) }\end{array}$ \\
\hline $\begin{array}{l}52 \text { y.o. Cau- } \\
\text { casian male; } \\
\text { CD4 160, VL } \\
30,000\end{array}$ & $\begin{array}{l}\text { DRV/r 900/100 mg } \\
\text { QD- DRV Cmin: } \\
0.41 ; \uparrow \text { to DRV/r } \\
600 / 100 \mathrm{mg} \text { BID- } \\
\text { DRV Cmin: } 2.7\end{array}$ & $\begin{array}{l}\text { PHT } 450 \text { mg QD; } \\
\text { PHT Cmin: } 54 \\
\text { (pre-ARVs), } 50 \text { (1 } \\
\text { month) }\end{array}$ \\
\hline
\end{tabular}

36 y.o. African LPV/r 600/150 mg PHT $400 \mathrm{mg}$ QD; $\quad 50 \% \uparrow \mathrm{LPV} / \mathrm{r}$ dose;

female; CD4 BID; LPV Cmin: PHT Cmin: 25-32 12.5\% $\downarrow$ PHT 250, VL $\quad 5.0 \quad$ (pre-ARVs), $33(\uparrow \quad$ dose; Switch to VA $36,000 \quad$ PHT to $450 \mathrm{mg}$ QD) allowed $\downarrow$ LPV/r dose; Well-tolerated; Seizure-free and $\mathrm{VL}<40(3$ years)

34 y.o. African LPV/r 600/150 mg PHT 250 QD; PHT 50\% $\uparrow$ LPV/r dose; male; CD4 40, BID; LPV Cmin: Cmin: 35 (on 20\% $\uparrow$ PHT dose; $\begin{array}{lll}\text { VL } 150,000 & 5.8 & \text { ARVs), } 61(\uparrow \mathrm{PHT} \quad \text { Well-tolerated; }\end{array}$ to $300 \mathrm{mg} \mathrm{QD)} \quad$ Seizure-free and VL $<40$ (3 years)

CBZ: carbamazepine (target Cmin 20-50 umol/L); Cmin: serum trough concentrations; CNS: central nervous system; DRV: darunavir (target Cmin $>2.2 \mathrm{mg} / \mathrm{L}$ ); LPV: lopinavir (target Cmin >1-5mg/L); PHT: phenytoin (target Cmin 40-80umolL); r: ritonavir; VA: valproate; VL: viral load

CONCLUSIONS: Concurrent use of protease inhibitors and enzymeinducing anticonvulsants should generally be avoided due to the risk of virologic failure and anticonvulsant toxicity or failure. However, through therapeutic drug monitoring and careful dose-titration of both agents, patients may be successfully stabilized on these complex regimens.

\section{P099}

IATROGENIC CUSHING SYNDROME AFTER INTRAARTICULAR INJECTION OF TRIAMCINOLONE IN A PATIENT TREATED WITH RITONAVIR BOOSTED DARUNAVIR

Hall, Jill; Hughes, Christine ; Foisy, Michelle; Shafran, Stephen;

Houston, Stan

Edmonton, $\mathrm{AB}$

OBJECTIVE: To describe a case of Cushing syndrome due to an interaction between intra-articular triamcinolone and ritonavir boosted darunavir.

METHODS: A case report.

RESULTS: A 53-year-old African woman, stabilized on a regimen of ritonavir-boosted darunavir and raltegravir (HIV RNA 110 copies/mL, CD4 290 cells $/ \mu \mathrm{L}$ ), presented to the HIV clinic complaining of: anxiety, insomnia, emotional lability, xerostomia, polydipsia and polyuria, elevated self-monitored blood glucose and blood pressure, nausea, increased appetite, and facial puffiness, which she attributed to her protease inhibitor. Upon history and physical exam, it was noted that she was experiencing heart palpitations and had florid moon facies and fat redistribution to her upper thighs. Her hemoglobin $\mathrm{A}_{1 \mathrm{C}}$ had increased to $9.8 \%$ ( $<7 \%$ over the previous year). The patient denied any inhaled or other corticosteroid use. The patient was referred urgently to endocrinology at which point she gave a history of receiving an intra-articular triamcinolone injection 4 weeks earlier. Eight weeks following the injection the patient reported normalization of her sleep, appetite and thirst; her facial swelling had begun to subside, however she still complained of fat redistribution to her upper thighs and poorly controlled blood glucose. Lab work at 11- and 12-weeks was found to be normal (24-hour urine free cortisol, serum morning cortisol, ACTH-stimulation test) due to delayed recognition of the drug interaction. There have been seven published case reports of Cushing syndrome due to an interaction between ritonavir and triamcinolone with similar timing of onset and resolution as our patient.

CONCLUSION: This experience highlights a potentially serious and under-recognized drug interaction. Concomitant triamcinolone and ritonavir-containing regimens should be avoided where possible. Education of patients, physicians and other health care providers is required. This case also stresses the importance of timely communication between HIV specialists and other prescribers caring for these patients.

\section{P100 \\ THE QUÉBEC ANTIRETROVIRAL THERAPEUTIC DRUG MONITORING PRACTICE GUIDE: DETERMINATION OF THE SPECIFIC INDICATIONS FOR ARV TDM USING AN EVIDENCE-BASED GUIDELINE APPROACH}

Sheehan, Nancy L.; Baril, Jean-Guy; Gauthier, Josianne;

Higgins, Niamh M.; Therrien, Rachel; Labbé, Line;

Lapointe, Normand; Tsarevsky, Irina

Montréal, QC

BACKGROUND: Québec's ministry of health mandated a working group to develop a practice guide on antiretroviral (ARV) therapeutic drug monitoring (TDM). The primary objective was to recommend the specific indications for ARV TDM.

METHODS: A thorough review of the literature was done using PubMed and MEDLINE (until March 2011). Studies presented at HIV conferences were also included. Specific indications for TDM for each ARV (except NRTIs and enfuvirtide) were recommended using a classification code for the strength of the recommendation (A: strongly recommended, B: moderately recommended, C: optional, D: not recommended) and for the quality of the evidence (recommendation supported by: I, at least one prospective clinical study based on clinical or biological parameters; II, retrospective, observational or pharmacokinetic studies; III, expert opinion). Consensus was reached amongst the working group members.

RESULTS: TDM is strongly recommended for the following indications and ARVs: routine controls in patients without past treatment failure (nelfinavir, indinavir $\mathrm{AI}$ ); routine controls in patients with resistant 
virus (atazanavir, indinavir/ritonavir(r) AII); central nervous system side effects (efavirenz AII); hyperbilirubinemia (atazanavir/r AI); nephrotoxicity (indinavir/r AI); drug-drug interactions (all ARVs AII); dialysis (atazanavir, atazanavir/r, darunavir/r AII); moderate or severe hepatic impairment (atazanavir $/ \mathrm{r}$, fosamprenavir $/ \mathrm{r}$, indinavir $/ \mathrm{r}$ AII); pediatrics (lopinavir/ $\mathrm{r}$ and efavirenz AII, other ARVs AIII); validating nontraditional doses (all ARVs, AIII), validating dose adjustments (nelfinavir and indinavir $\mathrm{AI}$, other ARVs AIII). In general, TDM is moderately recommended (B) for virologic failure, pregnancy, dialysis, moderate or severe hepatic impairment, suspected malabsorption and nonadherence to dosing requirements.

CONCLUSIONS: ARV TDM is strongly or moderately recommended for numerous indications based on scientific evidence and on expert opinion. ARV TDM could be beneficial for patient care when used for these specific indications.

Prevention, Natural History and Monitoring Prévention, histoire naturelle et surveillance

\section{P102}

\section{ATTITUDES AND EXPECTATIONS OF CANADIAN WOMEN IN LABOUR TOWARDS POINT OF CARE HIV TESTING ON THE LABOUR AND DELIVERY UNIT}

\section{Iqbal, Salikah; De Souza, Leanne R; Yudin, Mark H}

\section{Toronto, ON}

OBJECTIVE: To assess attitudes and opinions surrounding point of care HIV testing among Canadian women, and to determine predictors for acceptance of testing.

STUDY DESIGN: A survey assessing acceptability and attitudes towards rapid HIV testing was distributed on the Labour and Delivery unit in an academic hospital in Toronto, Canada during the summer of 2011. Information collected included demographics, health and pregnancy history, willingness to undergo rapid HIV testing while in labour, and barriers to testing. HIV testing was not performed.

RESULTS: Responses for 92 completed questionnaires were analyzed. The average age of respondents was 32 years and all were HIV-negative. $12 \%$ of patients reported having at least 1 risk factor for HIV transmission. 59\% of women were willing to be tested at the time of survey completion, and these women stated that they would accept any of saliva, urine, or serum testing. If found to be positive, $98 \%$ would accept antiretroviral treatment and $96 \%$ would formula feed their infants. Of the women who were not willing to be tested ( $41 \%$ of respondents), their reasons for refusal included "don't want to know" (39\%) and being in "too much labour pain" (29\%). Regardless of willingness to be tested, social stigma and reaction from partners were the most frequently cited barriers to testing (64\% and 69\% respectively).

CONCLUSIONS: Canadian women in labour are willing to undergo rapid HIV testing via urine, saliva or serum. If found to be positive, women are willing to undergo treatment as well as formula feed in order to prevent mother to child transmission of HIV.

\section{P103}

\section{HIV DISEASE PROGRESSION IN SASKATOON, SASKATCHEWAN}

Konrad, Stephanie ${ }^{1}$; Skinner, Stuart ${ }^{1}$; Lim, Hyun J1;

Bukassa Kazadi, Germain 2; Gartner, Kali ${ }^{1}$

${ }^{1}$ Saskatoon; ${ }^{2}$ Regina, SK

OBJECTIVE: This study aimed to characterize and identify determinants of HIV disease progression to immunological AIDS or death in Saskatoon, Saskatchewan.

METHODS: This is a retrospective cohort study of 343 seroprevalent HIV positive patients diagnosed between January 2005 and December 2010. Of these, $73 \mathrm{had}$ an estimated seroconversion date. Data was extracted from medical charts at two clinics specialized in HIV/AIDS care. Disease progression was measured as time from HIV diagnosis (or seroconversion) to immunological AIDS (ie, CD4 <200 cells/ $\mu$ l) and death. The Cox proportional hazards model was used.

RESULTS: The 3-year and 5-year immunological AIDS free probability was $53 \%$ and $33 \%$, respectively. The 3 -year and 5 -year survival probability was $89 \%$ and $77 \%$, respectively. Among the seroconversion cohort, the 3-year immunological AIDS free probability was $76 \%$.

Multicollinearity between injection drug use (IDU), hepatitis C (HCV) coinfection, and ethnicity was observed and thus separate models were built. A history of IDU ( $\mathrm{HR}=3.0,95 \% \mathrm{CI}$ : 1.2-7.1, $\mathrm{p}=0.016)$ and $\mathrm{HCV}$ coinfection $(\mathrm{HR}=2.9,95 \% \mathrm{CI}: 1.2-6.9, \mathrm{p}=0.016)$ were significant predictors of progression to immunological AIDS when controlling for baseline CD4 counts, treatment, age at diagnosis, and year of diagnosis among the seroprevalent cohort. Sex and ethnicity were not found to be significant predictors.

For survival, only treatment use was a significant predictor (HR, 0.34; $95 \% \mathrm{CI}, 0.1-0.8)$. HCV was marginally significant $(\mathrm{p}=0.067)$, while a history of IDU, ethnicity, gender, age at diagnosis, and year of diagnosis were not.

Among the seroconversion cohort, no predictors of progression to immunological AIDS were identified. Ethnicity, HCV and history of IDU could not be assessed.

CONCLUSION: Our study found that baseline CD4 counts, IDU, $\mathrm{HCV}$, year of diagnosis and treatment use were significant predictors of disease progression. This study highlights the importance of treatment and the need for targeted interventions for these particularly vulnerable populations to slow disease progression.

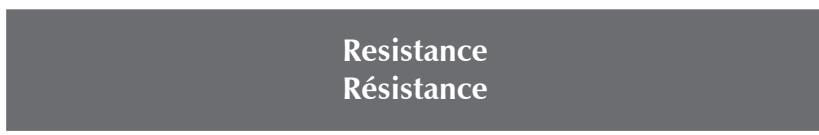

\section{P104}

TRANSMITTED HIV RESISTANCE IN ART-NAÏVE PATIENTS, CALI, COLOMBIA, 2010

Galindo, Jaime ${ }^{1}$; Galindo, Pablo ${ }^{1}$; Mueses, Héctor $\mathrm{F}^{1}$; Agudelo, Juan $\mathrm{F}^{1}$; Martinez, Jorge $\mathbf{L}^{2}$

${ }^{1}$ Cali, Colombia; ${ }^{2}$ Kingston, ON

INTRODUCTION: In Colombia, limited data on primary HIV drug resistance has been published. Genotype resistance testing (GART) is not recommended for ART-naïve patients by the Colombian HIV guidelines. The Canada-Colombia collaboration for HIV research conducted a study to determine the frequency of transmitted HIV drug resistance from a sample of antiretroviral therapy (ART) naïve patients attending the CLS clinic in Cali, Colombia.

METHODOLOGY: All adult patients with confirmed HIV infection never exposed to ART and having an HIV GART were eligible for this study. The recruitment period was from 2008 to 2010. The resistance mutations included in the analysis are those defined by Bennet, 2009 and Jonhson, 2010. Additional data was collected including socio-demographic characteristics, HIV-related conditions, CD4-cell count, viral load and estimated time of infection.

RESULTS: 156 patients were eligible for the study and agreed to participate. $152 \mathrm{HIV}$ sequences were available for analysis. Four poor-quality sequences were excluded. The mean age was $32 \pm 10.2$ years, $75.7 \%$ were men. The frequency of primary resistance was $11.8 \%$. The most common mutations were V108I (2.6\%), F77L (2.0\%), M46L (2.0\%) y K103N (1.3\%). NNRTI mutations were found in $5.3 \%$, NRTI mutations in $5.3 \%$ and PI mutations in $2.7 \%$ of the sequences. The factors associated with primary resistance were estimated time of infection $<1$ year (odds ratio $[\mathrm{OR}]=7.26)$ and a $\mathrm{CD} 4 / \mathrm{CD} 8$ ratio $<0.5$ at the time genotyping $(\mathrm{OR}=9)$.

CONCLUSIONS: The frequency of primary resistance in this sample is higher than that found in previous studies in Colombia and similar to those of countries with broad ART use. In Colombia, a GART seems favoured for ART-naïve patients whose HIV infection occurred within a year prior or have a CD4/CD8 ratio $<0.5$. However, more sensitive tests are needed to detect archived resistance in patients whose HIV infection occurred longer than one year prior. 


\section{P105}

\section{DEVELOPMENT OF AN ALLELE-SPECIFIC PCR METHOD} FOR RAPID AND SENSITIVE DETECTION OF E138 MUTATIONS IN HIV-1 REVERSE TRANSCRIPTASE

\section{McCallum, Matthew; Kramer, Victor G; Oliveira, Maureen;}

Moisi, Daniela; Xu, Hongtao; Wainberg, Mark A

Montreal, QC

Despite second-generation non-nucleoside reverse transcriptase inhibitors (NNRTI's), etravirine (ETR) and rilpivirine (RPV), having a higher genetic barrier for HIV drug resistance than first generation NNRTI's, drug resistance continues to be a significant concern. During the phase III clinical trials of ETR and RPV, and also during our cell culture selection experiments, E138 mutations_E138A/G/K/Q/R/V—have emerged as signature mutations of second generation NNRTIs, frequently in tandem with M184I. Subsequently, our laboratory found that E138K and M184I display mutual compensation: E138K restores enzyme processivity at low dNTP concentrations to the M184I mutant, and M184I mutant restores the enzyme polymerization rate of the E138K mutant. However, if E138K is indeed a compensatory mutation for M184I, it is unclear why E138K has not been observed in tandem with M184I previously. We hypothesized that compensation from E138K mutation has long been occurring; however, due to the low sensitivity of many bulk-sequencing methods (>20\%) it was simply not detected until recently. Moreover, we hypothesized other E138 mutations may be appearing in tandem with M184I. In this report, we developed an allele specific PCR (AS-PCR) method for detecting of the E138 mutations with sensitivity below $1 \%$. The AS-PCR was then used to screen patients for position 138 mutations that were not previously detected by bulk sequencing. To gain further insight into the observed prevalence and the potential transmission dynamics of E138 mutants, viral fitness was assessed by co-infecting cells with multiple viral mutants and following them over time using AS-PCR. We found position 138 mutations at a higher than expected proportion in patients. Furthermore, the viral fitness of the 138 mutants with and without the M184I mutation is more complex than previously anticipated. In conclusion, position 138 mutations are of clinical relevance, and the AS-PCR method shown here may find its place in clinical practice.

\section{P106 \\ PREFERENTIAL CELL CULTURE SELECTION OF M184I \\ AND E138K WHEN ETRAVIRINE IS COMBINED WITH EMTRICITABINE (FTC) OR LAMIVUDINE (3TC)}

Oliveira, Maureen; Brenner, Bluma G; Moisi, Daniela; Spira, Bonnie; Wainberg, Mark

Montreal, QC

BACKGROUND: Our laboratory has identified the importance of the E138K mutation in the development of resistance to etravirine (ETV). Recent clinical studies that have used rilpivirine-based regimens have shown the selective emergence of $138 \mathrm{~K}$ with M184I rather than M184V in treatment failures. In this study, we have used tissue culture models to shed light on the resistance pathways favored by treatment with ETV in tandem with emtricitabine (FTC) or lamivudine (3TC).

METHODS: To gain a more detailed insight into pathway development, cord blood mononuclear cells (CBMCs) and clinical isolates from drug-naive persons were selected with ETV, FTC, and 3TC alone or in combination. To further expand this study, we used MT-2 cell lines to test single, double and triple combinations of ETV, FTC and tenofovir (TDF). PNL4.3 wild-type recombinant clones with 138K, 184V and 184I site-directed mutations were treated with respective drug combinations. RESULTS: The 138K mutation was the first mutation to emerge with double combination (ETV and 3TC or ETV and FTC) drug pressure In CBMCs and three subtype B. In subtype C clinical isolate, E138K developed followed by M184I with FTC/ETV while K101E developed followed M184I with 3TC/ETV. In contrast, in MT-2 cells the M184I mutation was the first mutation to arise in 3TC/ETV, FTC/ETV, FTC/ TDF amd FTC/ETV/TDF selections. When starting with the $138 \mathrm{~K}$ clone, the 184I rather than $184 \mathrm{~V}$ arose with 3TC or FTC pressure. Also as expected, both $184 \mathrm{I}$ and $184 \mathrm{~V}$ clones acquired the $138 \mathrm{~K}$ mutation with ETV pressure.

CONCLUSION: Our data shows that in our tissue culture models, E138K is a favored pathway for ETV resistance. In addition, in CBMCs and clinical isolates, E138K precedes M184I. In MT-2 cells, M184I precedes E138K. These findings demonstrate the low genetic barrier to E138K, as well as the favored backbone of E138K and M184I.

\section{P107}

HOW WOULD THE OLIGONUCLEOTIDE LIGATION ASSAY (OLA) PERFORM IF USED IN COLOMBIA FOR DETECTION OF HIV DRUG RESISTANCE

Rumman, Amir ${ }^{1}$; Mueses, Hector ${ }^{2}$; Galindo, Jaime²;

Agudelo, Juan $\mathrm{F}^{2}$; Martinez-Cajas, Jorge $\mathrm{L}^{1}$

${ }^{1}$ Kingston, $\mathrm{ON}$; ${ }^{2}$ Cali, Colombia

BACKGROUND: HIV drug resistance is a major factor that limits the effectiveness of antiretroviral therapy (ART). Conventional HIV RNA drug resistance genotyping is prohibitively expensive in resource-limited settings. An affordable alternative to this could be the Oligonucleotide Ligation Assay (OLA), which is a rapid, specific and sensitive assay for detection of known point mutations. However, it is unknown if OLA could be suited as a tool to detect HIV drug resistance for clinical decision making.

METHODS: HIV genotype sequences from patients experiencing virologic failure (VF) to a first-line ART regimen were obtained from the Corporación de Lucha Contra el SIDA, Colombia. OLA primers for NRTI mutations were run in silico at $90 \%$ and $80 \%$ percentage identity (PI) primer hybridization and the performance was estimated in the nucleotide sequences from this sample. The expected sensitivity of OLA for nevirapine, efavirenz, zidovudine and tenofovir was calculated using the HIVdb Program from the Stanford HIV database as comparator for levels of drug resistance.

RESULTS: 100 HIV-1 genotypes from eligible patients were included in the study. Eighty-eight percent of patients were taking zidovudine/ lamivudine and $60 \%$ were taking efavirenz or nevirapine at the time of VF. The frequency of OLA-detectable mutations was $\mathrm{M} 184 \mathrm{~V}=78 \%$, $\mathrm{K} 103 \mathrm{~N}=46 \%, \mathrm{~T} 251 \mathrm{Y}=11 \%, \mathrm{~T} 215 \mathrm{~F}=8 \%$ and $\mathrm{Y} 181 \mathrm{C}=5 \%$. In silico simulation with this sample suggests that OLA would be able to detect all such mutations except $215 \mathrm{~F}$ in $>95 \%$ at $\mathrm{PI}=80 \%$ and $>80 \%$ at $\mathrm{PI}=90 \%$. The detection rate for $\mathrm{T} 215 \mathrm{~F}$ was $81.8 \%$ at $\mathrm{PI}=80 \%$ and $27.2 \%$ at $\mathrm{PI}=90 \%$. Overall sensitivity of OLA would approach $79 \%$ for nevirapine, $77 \%$ for efavirenz, $95 \%$ for zidovudine and $100 \%$ for tenofovir.

CONCLUSIONS: OLA has good potential to detect relevant resistance mutations in patients failing first-line ART regimens. Developing primers to detect additional NRTI and NNRTI mutations could increase its sensitivity and might allow its clinical use in resource-limited settings.

\section{P108}

ROUTINE DEEP SEQUENCING USING A 454 GS-JUNIOR INSTRUMENT FOR HIV TROPISM DETERMINATION IN THE CLINICAL SETTING

Swenson, Luke C; Dong, Winnie; Mo, Theresa; Taylor, Jeremy J; Barrios, Liliana; Woods, Conan; Chan, Dennison;

Harrigan, P Richard

Vancouver, BC

BACKGROUND: "Deep" sequencing of the V3 loop of HIV gp120 using next-generation sequencing (Roche/454-GS-FLX) is more sensitive to minority CXCR4-using variants than standard, population-based sequencing. New instruments such as the 454 GS-Jr allow for smaller sample batches, allowing more cost-effective and timely tropism testing than the original 454 sequencer. The laboratory at our Centre has initiated this approach in routine screening for patients where maraviroc is being considered.

METHODS: Plasma samples $(\mathrm{N}=150)$ were amplified with triplicate RT-PCR from extracted RNA, and sequenced by both the ABI 3730XL (standard) and GS-Jr (deep) sequencers. V3 sequences were analyzed with the bioinformatic algorithm geno2pheno with cutoffs previously optimized for each method. Samples were classified as R5 or non-R5 based on their geno2pheno values and/or the percentage of non-R 5 variants, respectively.

RESULTS: Overall concordance between methods was $76 \%$ (114/150 concordant classifications). Where tropism results were discordant, the majority were classified as non-R 5 by deep sequencing but $\mathrm{R} 5$ by standard sequencing ( 29 of 36 samples; $81 \%$ ). The median percentage of 
non-R 5 variants by deep sequencing in those samples was $7.3 \%$ (interquartile range: 4.4-25.5) - below the reported sensitivity of standard sequencing. Concordant R5 and non-R5 sample groups had medians of $0 \%$ and $44.6 \%$ non-R 5 variants, respectively.

A subset of 24 samples was additionally sequenced with the original 454 GS-FLX instrument. The GS-Jr gave very similar results to the GSFLX $(\mathrm{R}=0.998)$, with a mean absolute difference of $1.5 \%$ non-R5 variants. Classifications were concordant in $21 / 24$ samples (88\%), with the differences due to chance variation around the $2 \%$ cutoff used to classify samples.

CONCLUSIONS: The ability to sensitively screen for HIV tropism with smaller batches using the 454 GS-Jr has allowed routine clinical use of deep sequencing and confirmed its advantages over standard sequencing. The utility of the 454-GS-Jr in routine resistance testing remains to be established.

\section{EPIDEMIOLOGY AND PUBLIC HEALTH/} ÉPIDÉMIOLOGIE ET SANTÉ PUBLIQUE

Biomedical and Behavioral Interventions (evaluation, interventional, service delivery models)

Recherche interventionnelles biomédicale et

comportementale (évaluation, intervention, soins et services)

\section{P109}

\section{RESULTS FROM A QUALITY IMPROVEMENT INITIATIVE TO IMPROVE ANTIRETROVIRAL THERAPY ADHERENCE AT NORTH AMERICAN'S ONLY HIV/AIDS HEALTH CARE FACILITY THAT INCORPORATES SUPERVISED INJECTION SERVICES INTO AN INTEGRATED HIV/AIDS HEALTH CARE PROGRAM}

Baltzer Turje, Rosalind; Barrios, Rolando; Payne, Martin;

Simpson, Dianne; Jamal, Rosa; Clarke, Christina;

McDougall, Patrick

Vancouver, BC

The Dr. Peter Centre (DPC) is a not-for-profit health care facility located in Vancouver, BC that provides health care and support services to mitigate the risks faced by marginalized persons living with HIV/ AIDS. Among the inherent risks these individuals face are unsafe illicit drug injection practices, homelessness, mental illness, food insecurity, unemployment, drug criminalization, and a reluctance of health professionals to provide health care due to negative attitudes towards this population.

In 2010, the DPC joined the STOP HIV/AIDS Structured Learning Collaborative ("the Collaborative"), a quality improvement initiative aiming to improve care and treatment for better adherence and client outcomes. Aligned with this, the DPC aimed to increase ARV adherence by $15 \%$ and develop a measure for client engagement as a proxy for health outcomes.

While the DPC initially faced challenges in obtaining data that the Collaborative required for some of its monthly health outcome measures, the DPC responded with creative solutions that included medication administration records, virological results and self-reports using a customized validated survey to ascertain client-provider engagement and satisfaction as a proxy for health outcomes. The DPC applied quality improvement frameworks to test, measure and apply interventions in each area.

After 10 months, ARV adherence increased by 14\% [an increase from $78 \%$ to $89 \%$, median $=83 \%, n=44]$ and incidence of undetectable viral loads (pVL less than 200 copies $/ \mathrm{ml}$ ) increased 9\% [an increase from $88 \%$ to $97 \%$, median $=89 \%, n=44]$. Surveys were administered twice and demonstrated positive client-provider engagement scores of $92 \%$ [ $n=13$, July 2011] and 93\% [ $\mathrm{n}=30$, Nov 2011]. Client satisfaction increased from $84 \%$ [n=37, July 2011] to $87 \%$ [n=30, Nov 2011].

\section{P110}

AWARENESS TO MAKE CHANGE (ATOMC): A PROFILE OF RECRUITERS PARTICIPATING IN A SOCIAL NETWORK (SN) HIV TESTING INTERVENTION AMONG MEN WHO HAVE SEX WITH MEN (MSM) IN MONTREAL

Cox, Joseph; Maurais, Emilie; Lacombe, Élysabeth; Buccitelli, Anthony; McKye, James; Lambert, Gilles; Bernier, Mélina; Monteith, Ken; Llamas, Rodrigo; Hapanowicz, Mark

Montreal, QC

INTRODUCTION: The US Centers for Disease Control recommends the use of SN strategies for improving HIV testing programs. These programs enlist HIV-positive and high-risk HIV-negative persons to encourage social, sexual and drug using network members to be HIV tested. This improves access to HIV-infected unaware and other at-risk individuals. No similar strategy has been reported in Canada. ATOMc is a community-based $\mathrm{SN}$ initiative using incentive-based peer recruitment to identify HIV at-risk MSM. A community-based research project was implemented to evaluate the feasibility of ATOMc. We report on the first recruiters of ATOMc.

METHODS: Since September 2010, ATOMc recruiters were enlisted using a variety of promotional strategies. Each recruiter was evaluated for the presence of past and present HIV transmission risks. Consenting recruiters completed a self-administered questionnaire on socio-demographics, risk behaviours and HIV testing practices. A profile of recruiters' HIV risk behaviours is provided.

RESULTS: As of the end of 2011, ATOMc had engaged 50 recruiters. Data were available for 35 recruiters: 28 males, 4 females and 3 transgendered males. The mean age was 30 years.

Table: Risk behaviours among HIV-positive and -negative ATOMc male recruiters $(\mathrm{n}=28)$

\begin{tabular}{|c|c|c|}
\hline & $\begin{array}{l}\text { HIV-positive } \\
\quad(n=8)\end{array}$ & $\begin{array}{l}\text { HIV-negative } \\
\quad(n=20)\end{array}$ \\
\hline STI diagnosis (past 12 months) & 4 & 2 \\
\hline Shared injection materials* & 0 & 0 \\
\hline Number of sexual partners* & $\begin{array}{l}31 \text { (mean); } \\
20 \text { (median) }\end{array}$ & $\begin{array}{l}14 \text { (mean); } \\
5 \text { (median) }\end{array}$ \\
\hline Any unprotected anal inter course* & 7 & 10 \\
\hline with a "one-night stand" partner** & 4 & 4 \\
\hline with a partner of unknown status ${ }^{* *}$ & 3 & 8 \\
\hline with a HIV-positive partner** & 5 & 2 \\
\hline At least one "at-risk partner"* & Not applicable & 9 \\
\hline At least one of the risk categories ${ }^{\star, * * *}$ & 7 & 12 \\
\hline
\end{tabular}

"past 6 months; **not exclusive; "at-risk partner"=unprotected anal sex with a one-night stand" or sex buddy or couple [HIV-positive or unknown status] partner; ${ }^{* *} S T I$ diagnosis, shared injection materials, unprotected anal intercourse or at least one at-risk partner.

CONCLUSION: This mid-term evaluation revealed $68 \%$ of male recruiters with a risk for HIV transmission or acquisition and none who inject. Given the goal of SN HIV testing initiatives, efforts to engage appropriate recruiters will be developed. Interestingly, several female recruiters were enlisted because many of their peers were MSM. Evaluations of this type are useful in monitoring project implementation.

\section{P111}

SUCCESSES AND GAPS IN REGULAR HIV TESTING FOR HIDDEN STREET- AND OFF-STREET SEX WORKERS IN VANCOUVER, CANADA

Deering, Kathleen N${ }^{1}$; Montaner, Julio $\mathbf{S}^{1}$; Feng, Cindy $\mathrm{X}^{\mathbf{1}}$; Chettiar, Jill ${ }^{1}$; Strathdee, Steffanie $\mathrm{A}^{2}$; Shannon, Kate ${ }^{1}$

${ }^{1}$ Vancouver, BC; ${ }^{2}$ San Diego, CA, USA

BACKGROUND: Given extensive efforts to scale-up HIV testing and access to care in Vancouver, British Columbia as part of governmentsponsored pilot initiative of 'treatment as prevention', there remains a critical need to evaluate use of HIV testing and inform barriers to care among vulnerable sub-populations. This study assessed the prevalence 
and associations with having a recent HIV test among sex workers (SWs) in Vancouver.

METHODS: Baseline (Feb-July/10) data were used, including an interviewer-administered questionnaire, HIV/STI testing and geographic location data, from an open prospective cohort of street and off-street SWs in Vancouver (An Evaluation of Sex Workers' Health Access[AESHA]). Multivariable logistic regression was used to identify social, environmental and structural factors associated with recent HIV testing (in the last year). Adjusted odds ratios and $95 \%$ confidence intervals were reported (AOR [95\%CIs]).

RESULTS: Overall, 435 seronegative SWs were included in this analyses with $67.1 \%$ (292) reporting having a recent HIV test. Inconsistent condom use (2.51 [1.13-5.55]) and injecting drugs within the last 6 months (2.61 [1.48-4.58]) were associated with a higher probability of having a recent HIV test, while Asian migrant/new immigrant SWs (0.36 [0.20-0.65], versus Caucasian) and SWs reporting a language barrier (0.23 [0.08-0.65]) had a lower probability of having a recent HIV test. Strong bivariate associations between the distance from places of residence and solicitation to places of recent HIV testing were observed $(\mathrm{p}<0.001)$.

CONCLUSIONS: Our results highlight the successes of HIV testing in reaching SWs most vulnerable to HIV infection through sexual and injection drug pathways. Increased mobile and safer-environment interventions that facilitate access to HIV testing at sex work locations and SWs' place of residence remain a critical priority, in addition to culturally safe services with language support for lower-risk Asian migrant/new immigrant SWs. Removal of criminal sanctions on safer indoor work spaces with onsite access to health and testing services warrants immediate attention.

\section{P112}

"WHAT ARE YOU WAITING FOR?": EVALUATION OF A HEALTH COMMUNICATION CAMPAIGN PROMOTING THE UPTAKE OF NEW HIV TESTING TECHNOLOGIES AMONG GAY MEN IN VANCOUVER, BRITISH COLUMBIA Ferlatte, Olivier $^{1,2}$; Trussler, Terry ${ }^{1}$; Marchand, Rick'; Kwag, Michael ${ }^{1}$; Jollimore, Jody ${ }^{1}$; Steinberg, Malcolm ${ }^{1}$; Gilbert, Mark ${ }^{1}$

${ }^{1}$ Vancouver; ${ }^{2}$ Burnaby, BC

OBJECTIVE: As options for HIV testing increase, communication of their relative advantages may be a helpful motivation for HIV testing in gay men. We conducted a community-based evaluation of a health communication campaign for gay men that aimed to promote adoption of point-of-care (POC) and nucleic acid amplification HIV tests (NAAT), through increasing knowledge about the rapidity of results and reduced window period (earlier detection) with these tests, respectively.

METHODS: A cross-sectional survey was conducted online, in gay venues and at testing clinics in Vancouver. Data was analyzed through descriptive and bivariate analysis.

FINDINGS: 599 gay men completed the survey (23\% in venues, $27 \%$ in clinics, $49 \%$ online). 214 (35.4\%) recalled seeing the campaign, of whom $44.8 \%$ reported the campaign reminded them to get tested and $16 \%$ reported getting tested as a result. Those who had seen the campaign were significantly more likely to be knowledgeable about POC and NAAT testing than those who had not. They were also more likely to choose POC as a preferred HIV test; however, exposure to the campaign was not associated with preference for NAAT.

DISCUSSION: The campaign achieved its primary objective to increase community knowledge of new HIV testing technologies and did lead to men getting tested as a result. Preference for POC testing may be explained by its observable advantage to the individual: offering results within minutes. While knowledge of the benefits of NAAT did increase in men exposed to the campaign these appear to be less persuasive for individual adoption, and are perhaps more abstract or perceived as less relevant. Future HIV test promotion campaigns may need new approaches to appeal to the advantages from the point of view of potential users.
P113

A TURNING POINT IN HIV TESTING? PERCEPTIONS OF ACCEPTABILITY OF POINT OF CARE TESTING IN HALIFAX, NOVA SCOTIA

Gahagan, Jacqueline; Stein, Carlye; Campbell, Angus

Halifax, NS

BACKGROUND: Rapid point of care (POC) testing has been available in Canada since 2000, although not currently available in Nova Scotia. Studies over the last 10 years indicate POC testing has led to an increase in the number of individuals who have been screened and received their results, as well as to a decrease in the number of individuals not returning for results.

PURPOSE: This study aimed at offering current service users of a sexual health centre in Halifax the opportunity to respond to a survey on whether they would access a Rapid POC Test if it were available in Nova Scotia, and whether they would be willing to pay for the test if they could obtain their results in one appointment.

METHODS: All participants were from an anonymous HIV testing programme at a sexual health centre and were invited to voluntarily complete a closed-ended survey consisting of 13 questions. Ten questions related to the current HIV testing service provided and 3 to the availability and potential cost of a Rapid POC Test.

FINDINGS: Of the 411 surveys distributed, 258 were completed and available for analysis. Ninety percent stated they would access a Rapid POC test if it were available. Only 8 respondents expressed concern about the accuracy of the rapid test. Of the $90 \%$ in favour of a Rapid POC test, $26 \%$ would not be willing to pay for it. Comments, however, were varied: some noted they would rather wait than pay; others felt the suggested fee of $\$ 20$ was appropriate, but would only pay if finances allowed, or if it were an emergency.

CONCLUSIONS: This study offers important new knowledge regarding perceptions among Nova Scotians of Rapid POC testing and its potential cost. Potential benefits of using this test, such as an increase in the number of individuals in Nova Scotia who are tested and receive their results, and the impact this can have on health care access, should also be considered.

\section{P114}

NATIONAL HIV INTEGRASE INHIBITOR RESISTANCE SURVEILLANCE IN CANADA 2002-2011

Ji, Hezhao; Li, Yang; Merks, Harriet; Halverson, Jessica; Archibald, Chris; Sandstrom, Paul; Brooks, James Ottawa, ON

BACKGROUND: In late 2007, Raltegravir (RAL) was approved for use by Health Canada as the first integrase strand transfer inhibitor (INSTI). Consistent with other antiretroviral therapy (ART), treatment failure and associated genotypic patterns of drug resistance have been reported for RAL. This study was designed to assess the prevalence of a priori and secondary INSTI drug resistance (DR) among ART-naïve subjects in Canada.

METHODS: We analyzed a stratified sample of 782 ART-naïve HIV specimens collected during the years 2002-2011 from the Canadian HIV Strain and Drug Resistance Surveillance Program. HIV Integrase gene sequences from all subjects were analysed using bidirectional populationbased sequencing. INSTI DR mutations (DRM) were identified using the Stanford HIV database. The prevalence of major and minor INSTI DRMs was determined and analyzed by year of specimen draw. The potential linkage of the INSTI DRMs with protease (PR) and reverse transcriptase (RT) DRMs was also evaluated.

RESULTS: A single major mutation, S147G was detected in a subtype C specimen from 2008. Consistent with previous results L74M, T97A and V151I were detected as polymorphisms at rates of $>0.6 \%$. However, accessory DRM E138K, V54I, L68V, R263K and T66S were also present in $\geq 0.5 \%$ of the specimens. No association between INSTI and PR and RT DRMs was observed.

CONCLUSIONS: The prevalence of transmitted major INSTI DRMs in Canada is extremely low. Although previously identified polymorphisms were detected, accessory DRMs E138K, V54I, L68V, R263K and T66S were also identified as polymorphic existing in up to $1.0 \%$ of this ART-naïve cohort. This data suggests that although INSTI therapy in 
ART-naïve patients in Canada will be successful, ongoing INSTI surveillance is required to track the novel DRM polymorphisms identified in this study.

\section{P115}

HEALTH WORKERS VIEWS ON THE USE OF TEXT MESSAGES TO IMPROVE ADHERENCE TO ART: A CROSSSECTIONAL SURVEY OF HEALTH WORKERS INVOLVED IN THE CAMPS TRIAL

Mbuagbaw, Lawrence $\mathrm{C}^{1}$; Thabane, Lehana ${ }^{1}$;

Ongolo-Zogo, Pierre ${ }^{1}$; Karanja, Sarah ${ }^{2}$; Morfaw, Frederick $\mathrm{IL}^{3}$

${ }^{1}$ Hamilton, ON; ${ }^{2}$ Nairobi, Kenya; ${ }^{3}$ Yaoundé, Cameroon

BACKGROUND: Close to 30 million people are infected with HIV. Antiretroviral therapy (ART) has reduced the morbidity and mortality associated with HIV infection. However, poor adherence to medication is a major limitation of the potential of ART. Recent evidence suggests that mobile phone text messages may improve adherence to ART, but there's limited information on the views of health workers.

OBJECTIVES: To determine the views of health workers at the Yaoundé Central Hospital Accredited Treatment Centre (YCH ATC) on the use of text messages to improve adherence to medication immediately after the Cameroon mobile phone SMS randomized controlled trial and before the results of the trial were published.

SETTING: YCH ATC in Cameroon - the largest HIV clinic serving about 6500 patients.

METHODS: A cross-sectional anonymous self administered questionnaire was offered to all the staff of the YCH ACT in August 2011, based on their interactions with patients who received text messages during the trial.

RESULTS: Twenty-nine out of 40 (72.5\% response rate) staff participated in the study. Sixty-nine percent (20/29) were females. Physicians, nurses and social workers represented $24.1 \%, 41.4 \%$ and $24.1 \%$ respectively. Two-thirds reported observing benefits to patient care $(69.0 \%)$ such as better adherence to medication, better compliance with appointments and follow-up tests. Negative effects such as reduced compliance with appointments and follow-up tests were reported by $3.4 \%(1 / 29)$ of the respondents. Up to $62.1 \%(18 / 29)$ declared that the intervention increased their workload. Over three quarters (22/29) would like to participate in the up-scaling of the text messaging programme.

CONCLUSIONS: Overall, health workers at the YCH ATC are supportive of using mobile phone text messages to improve adherence to ARTs and willing to participate in the scale-up of the service despite the increase in workload.

\section{P116}

\section{KNOW YOUR STATUS: OUTCOMES OF A COMMUNITY-} BASED MULTI-DISCIPLINARY HIV TESTING AND CARE PROJECT IN A SASKATCHEWAN FIRST NATIONS COMMUNITY

Khan, Ibrahim ${ }^{1}$; Smith, Leslie Ann ${ }^{1}$; Bukassa Kazadi, Germain²; Dow, Brett ${ }^{1}$; Rushowick, Bonnie ${ }^{1}$; Andrews, Jocelyn ${ }^{1}$;

Bowen, Colleen ${ }^{1}$; Skinner, Stuart ${ }^{3}$

\section{${ }^{1}$ Regina, SK; ${ }^{2}$ Ottawa, ON; ${ }^{3}$ Saskatoon, SK}

BACKGROUND: Saskatchewan has experienced a significant increase in the numbers of newly diagnosed HIV cases. The most significant burden has been among First Nations and Metis people. Injection drug use, hepatitis $\mathrm{C}$ co-infection, multiple social barriers to treatment, and a lack culturally competent HIV services have complicated care. The highest burden has been focused in Saskatoon and Regina, however, there is limited access to care and testing in smaller communities. In January 2011, with the support of all partners, Big River First Nations community initiated a robust project, titled "Know Your status" (KYS).

METHODS: After informed consent, blood specimens were collected for HIV testing, screened under KYS, and sent to the Provincial laboratory for HIV confirmatory tests. Additional demographic information was collected on tested individuals and their contacts. The First Nations and Inuit Health multi-disciplinary team designed and managed the KYS project. Community-based HIV clinics were developed to support linkage and engage patients and provide support for community-health workers.
RESULTS: From January to December 2011, 190 blood specimens were tested. The average age of individuals tested was 24 years. Ten new HIV positive cases were diagnosed (incidence $5.3 \%$ ). The average time to treatment for newly diagnosed HIV cases was three months. Infectious Disease clinics maintained contact with all newly diagnosed HIV cases, ensured ongoing support to the clients and families, and provided timely public health follow up. KYS currently manages 17 cases with good health outcomes.

CONCLUSION: Increased HIV testing and harm reduction is required for smaller communities in Saskatchewan. Strong partnerships and community leadership, including nursing, can facilitate HIV testing and care for hard-to-reach populations. Access to a multidisciplinary team and case management facilitates HIV treatment and improved health outcomes.

\section{P117 \\ HIV TESTING DURING THE CANADIAN IMMIGRATION MEDICAL EXAMINATION: A NATIONAL SURVEY OF DESIGNATED MEDICAL PRACTITIONERS}

Tran, Jennifer M; Li, Alan; Owino, Maureen; Tan, Darrell Toronto, ON

BACKGROUND: Since 2002, HIV testing has been mandatory for individuals aged 15 or older wishing to immigrate to Canada. Since the Designated Medical Practitioners (DMPs) who perform these tests may have varying experience in HIV care and significant time constraints in performing their clinical duties, there may be variability in the quality of pre- and post-test counselling provided. We surveyed Canadian DMPs regarding HIV testing, counselling, and immigration inadmissibility. METHODS: A 16-item survey divided into five domains (Demographics, Knowledge, Attitudes/Beliefs, Practices, and Needs) was pilot-tested and mailed to all DMPs across Canada $(\mathrm{N}=203)$. The survey inquired about DMP practice characteristics, knowledge of HIV infection, attitudes and practices regarding medical inadmissibility and HIV counselling, and interest in continuing medical education on related topics. Responses were coded and analyzed using descriptive statistics.

RESULTS: There were 76 respondents (37.4\%) from 9 of 11 possible provinces/territories. Most (65\%) had been practicing as a DMP for 1120 years. The number of Immigration Medical Exams performed was bimodal, with most physicians either performing $<20(52 \%)$ or $>51(17 \%)$ per month. Participants frequently rated their knowledge of HIV services, infection, diagnostics, counselling, and immigration criteria as "fair". $31 \%, 58 \%$, and $12 \%$ of respondents agreed/strongly agreed with the statements "HIV infected individuals pose a danger to public health and safety", "HIV-positive immigrants cause excessive demand on the healthcare system", and "HIV seropositivity is a reasonable grounds for denial into Canada", respectively. Although participants felt prepared to perform counselling, language was cited as a barrier. Counselling tended to focus on transmission risks (50\% reported discussing this "always" or "often") more so than coping and social support (36\%). There was a high level of interest $(60 \%)$ in continuing medical education in this area. CONCLUSIONS: There are areas for improvement regarding DMPs' knowledge, attitudes, and practices about HIV infection, counselling, and immigration criteria. Continuing medical education and other support for DMPs to facilitate practice changes could benefit newcomers who test positive through the immigration process.

\section{P118}

IN-DEPTH ANALYSIS OF PATIENT-CLINICIAN CELL PHONE COMMUNICATION DURING THE WELTEL KENYA ANTIRETROVIRAL ADHERENCE TRIAL

van der Kop, Mia L ${ }^{1}$; Karanja, Sarah ${ }^{2}$; Kariri, Anthony ${ }^{2}$; Thabane, Lehana ${ }^{3}$; Lester, Richard $\mathrm{T}^{1}$;

${ }^{1}$ Vancouver, BC; ${ }^{2}$ Nairobi, Kenya; ${ }^{3}$ Hamilton, ON

BACKGROUND: The WelTel Kenyal trial demonstrated that text message support improved antiretroviral adherence and viral suppression. The intervention involved weekly messages sent to patients inquiring how they were doing; participants were required to respond either that they were well or that there was a problem. This study's objectives were to: (1) describe problems identified by participants and reasons for non-response; (2) investigate factors associated with problem and non- 
response; and (3) examine participant-perceived barriers and benefits to the intervention.

\section{METHODS AND FINDINGS:}

During the 52 weeks participants received the intervention, all patientclinician cell phone communication and non-response were recorded in a study log. Logistic regression, with generalized estimating equations, was used to determine predictors of problem and non-response. Patientperceived barriers and benefits recorded at the final follow-up visit were described.

Between June 2007 and November 2009, 271 participants generated 11,873 responses; 377 of which indicated a problem. Health issues were the primary reason for problem responses $(n=272)$. Living in a rural area (OR 1.89; 95\%CI 0.20-0.79; p=0.01), older age, and time were independently associated with indicating a problem. Participants did not respond on 3,730 occasions; cell phone problems were commonly cited as the reason for not responding $(\mathrm{n}=814)$. Non-response was more common among females (OR 1.49; 95\%CI 1.03-2.18; $\mathrm{p}=0.04$ ) and inversely associated with educational level. Most participants believed there were no barriers to the intervention $(n=141)$, while its benefits included reminding patients to take medication and promoting a feeling that 'someone cares'. Limitations include the inability to determine the reason for non-response in the majority of instances $(n=2,314)$ and possible social desirability bias when ascertaining patient perceptions.

CONCLUSIONS: Participant response was associated with time and various socio-demographic characteristics. The intervention, well-received by participants, provided valued patient support. This in-depth investigation of patient-clinician cell phone communication informs future applications of WelTel and other mHealth interventions.

Epidemiology of HIV/AIDS Among At-Risk Populations (IDU, MSM, CSW, etc.)

Épidémiologie du VIH/SIDA dans les populations vulnérables

\section{P119}

\section{HIV RISK PERCEPTIONS AND THE DISTRIBUTION OF HIV RISK AMONG AFRICAN, CARIBBEAN AND OTHER BLACK PEOPLE: MIXED-METHODS RESULTS FROM THE BLACCH STUDY}

\section{Baidoobonso, Shamara; Bauer, Greta; BLACCH Study Team, The} London, ON

BACKGROUND: African, Caribbean and other Black people (ACBP) are a priority group for HIV prevention efforts in Canada. ACBP and service providers' (SP) perceptions about HIV risk impact the uptake and delivery of prevention messages. These perceptions may not reflect actual risk among ACBP, so it is important to assess them and identify groups for which they may be valid. Emerging evidence from Sub-Saharan Africa shows that social determinants of health (SDOH) impact the distribution of HIV risk. However, SDOH are context-specific, and to date, virtually no research has explored their impact on HIV risk among ACBP in North America.

OBJECTIVE: Compare ACBP's and SP's perceptions about HIV risk among ACBP to a corresponding quantitative risk profile.

METHODS: Using a community-based approach, a purposive sample of eight SPs and 23 ACBP were recruited for qualitative interviews. They were asked questions about HIV, and their responses are being analyzed using qualitative content analysis to identify themes. Following the interviews, a convenience sample of 188 ACBP completed a quantitative questionnaire covering HIV-related risk behaviours. Quantitative data are being analyzed to describe the distribution of HIV risk behaviours according to the following $\mathrm{SDOH}$ : gender, poverty status, educational attainment, immigration experience, ethnicity and employment status. Quantitative results will be weighted based on 2006 Census data for ACBP in London, Ontario (the research setting). Weighted and unweighted prevalences and prevalence ratios will be presented. Qualitative and quantitative data will be combined using concurrent triangulation. RESULTS: Analyses are underway, so results are not currently available. Key qualitative results will be presented using thematic summaries and illustrative quotes. Quantitative results will be presented in tables with key results highlighted.

DISCUSSION: Areas in which qualitative and quantitative data converge and fail to converge will be noted. This analysis' strengths and limitations, and the findings' generalizeability will be discussed.

\section{P120}

HIGH HETEROGENEITY OF HIV-RELATED SEXUAL RISK AMONG TRANSGENDER PEOPLE IN ONTARIO: A PROVINCE-WIDE RESPONDENT-DRIVEN SAMPLING SURVEY

Bauer, Greta $^{1}$; Travers, Robb ${ }^{2}$; Scanlon, Kyle ${ }^{3}$; Coleman, Todd ${ }^{1}$ ${ }^{1}$ London; ${ }^{2}$ Waterloo; ${ }^{3}$ Toronto, ON

BACKGROUND: HIV-related risk in trans (transgender, transsexual, or transitioned) people has most often been studied using urban convenience samples of male-to-female (MTF) spectrum individuals. These studies detected high prevalences of HIV-related risk behaviours, self-reported HIV, and HIV seropositivity.

METHODS: The Trans PULSE Project conducted a respondent-driven sampling survey from 2009-2010 to recruit trans Ontarians age 16 and over $(n=433)$. Weighted estimates were calculated for HIV-related risk measures, HIV testing and self-reported HIV, including subgroup estimates by gender spectrum and ethno-racial groups.

RESULTS: A wide range of sexual behaviours with a full range of partner types were reported. The largest behavioural contributors to risk were sexual behaviours some may assume trans people do not engage in: unprotected receptive genital sex for FTMs and insertive genital sex for MTFs. High proportions $-24.8 \%$ of female-to-male (FTM) and $50.3 \%$ of MTF individuals - had not had a sex partner within the past year. Of MTFs, $19.1 \%$ had a past-year high-risk sexual experience, versus $6.7 \%$ of FTMs. Overall, 46.4\% had never been tested for HIV; lifetime testing was highest in Aboriginal trans people and lowest among non-Aboriginal racialized people. Approximately $15 \%$ of both FTM and MTF participants had engaged in sex work or exchange sex and $2 \%$ currently worked in the sex trade. While self-report of HIV prevalence was 10 -times that expected for Ontario, given wide confidence intervals and the high proportion who had never been tested for HIV, estimating the actual prevalence was not possible.

CONCLUSIONS: Results suggest higher than baseline levels of HIV. Explicit inclusion of trans people in surveillance statistics would provide much-needed information on incidence and prevalence. Given the wide range of sexual behaviours and partner types reported, HIV prevention programs and materials should not make assumptions regarding types of behaviours trans people do or do not engage in.

\section{P121}

OPIOID SUBSTITUTION THERAPY AMONG INJECTION DRUG USERS IN THE SURVUDI NETWORK

Blouin, Karine $^{1}$; Leclerc, Pascale ${ }^{2}$; Noël, Lina ${ }^{1}$; Alary, Michel ${ }^{1}$; Morissette, Carole'; Roy, Élise $^{3}$; Blanchette, Caty ${ }^{1}$; Parent, Raymond ${ }^{1}$; Gagnon, Dominique ${ }^{1}$; Serhir, Bouchra ${ }^{4}$ ${ }^{1}$ Québec; ${ }^{2}$ Montréal; ${ }^{3}$ Longueuil; ${ }^{4}$ Sainte-Anne de Bellevue, QC OBJECTIVE: This analysis aimed at describing the extent of opioid substitution therapy (OST), examining its temporal trend and identifying its correlates among injection drug users (IDUs) in the SurvUDI network.

METHODS: Participants who had injected in the past 6 months were recruited across the Province of Quebec and Ottawa, Canada, mainly in harm reduction programs. They completed a questionnaire and provided saliva for anti-HIV and anti-HCV antibody testing. Data from 2004 to 2010 were analysed. For IDUs with multiple participations, the last interview was selected. OST was defined as non-injection use of prescribed methadone or Suboxone in the last six months. Participants who reported OST ( $n=771)$ were compared to non-users ( $n=3$ 907). Correlates of OST were identified using multivariate logistic regression. Trend analyses were performed with the bootstrap method.

RESULTS: Compared to non-users, participants who reported OST were significantly younger (mean age: 33.5 vs. 37.0 years, $\mathrm{p}<0.001$ ) and more of them were women ( $33.9 \%$ vs. $22.0 \%, \mathrm{p}<0.001)$. The use of OST increased significantly between 2004 and 2010 (from $14.7 \%$ to $22.2 \%$, 
$\mathrm{p}<0.001)$. The final multivariate model showed significant association between OST and age (adjusted odds ratio $(A O R)=1.89$ for $<37$ vs. $\geq 37$ years), female sex $(A O R=1.52)$, year $(A O R=1.58,1.63$ and 1.85 , respectively, for 2008, 2009 and 2010 vs. 2004), region (AOR=0.67 and 0.52 , respectively, for semi-urban and Ottawa/Outaouais vs. Montréal), heroin $(\mathrm{AOR}=4.49)$, prescription opioid $(\mathrm{AOR}=2.80)$ and other drugs $(\mathrm{AOR}=1.79)$ as most often injected drugs (vs. cocaine), non-injection use of crack/freebase ( $\mathrm{AOR}=0.71)$ and HCV seropositivity $(\mathrm{AOR}=1.73)$.

CONCLUSION: The use of OST increased over time and was associated with age, sex, region of recruitment, opioid injection and HCV seroprevalence. Further analyses should be performed to understand if the observed increase in OST is related to the increased popularity of prescription opioids or better access.

\section{P122 \\ PHYLOGENETIC ANALYSIS OF THE SPOT COHORT REVEALS RISK DETERMINANTS IMPLICATED IN THE SPREAD OF THE MONTREAL MALE-SEX-MALE (MSM) EPIDEMIC}

Brenner, Bluma G; Moodie, Erica; Otis, Joanne; Ohnona, Frédéric; Wainberg, Mark; Rousseau, Robert; Cox, Joseph; Lambert, Gilles; Roger, Michel

Montreal, QC

BACKGROUND: Primary/recent stage infection (PHI) is driving onward spread of the Montreal male-sex-male (MSM) epidemic. Provincial surveillance shows $25 \%, 28 \%$ and $47 \%$ of $\mathrm{PHI}$ segregate as unique $(1 \mathrm{PHI}$, $\mathrm{n}=392), 107$ small (2-5 PHI, $\mathrm{n}=318)$ and 40 large (6-60 PHI, $\mathrm{n}=530)$ clusters, respectively. SPOT was opened (2009) to promote testing, increase awareness of status and avert transmission chains.

METHODS: SPOT offered free and anonymous rapid testing at flexible hours to at-risk MSM. Testing, questionnaire completion, and counseling were performed by MSM nurse/interveners. Finger-prick dry blood spots were collected for PCR-based antigen testing and anonymized genotypic/ phylogenetic analysis. Testing habits and risk behaviours were compared in $\mathrm{HIV}^{-}(\mathrm{n}=1158)$ and $\mathrm{HIV}^{+}(\mathrm{n}=31)$ persons using Fisher's exact tests and quasi-Poisson models.

RESULTS: High (2.5\%) seropositivity was observed with ten infections identified as unique transmissions (eight from persons born outside Quebec), three infections associated with small clusters and 16 infections belonging to ten large clusters. HIV status was significantly associated with reported numbers of one-night partnerships $(\mathrm{p}=0.0005)$ and observed to be significantly elevated in those reporting 5-9 partners as compared to $<2$ partners $(\mathrm{OR}=4.545)$. A significant association was observed between cluster size and having reported 5-9 partnerships $(\mathrm{p}=0.0017, \mathrm{O}=6.731)$. A negative association was observed between numbers of one-night partnerships and times of reported testing $(\mathrm{p}=0.0005)$. Numbers of onenight partnerships were significantly elevated in reported last test 3 and 4-6 months ago (mean 6.1 and 6.4), twice that in those tested 12-24 and +24 months ago (mean 2.9 and 3.5) and 4X that in those never tested (mean 1.4). Average cluster size of participants reporting frequent testing ( $2+$ tests in last two years) was 0.16 times those reporting no tests in the last two years.

DISCUSSION: Targeted/frequent testing and counselng (or early treatment intervention) may prevent HIV spread among MSM engaging in episodic high-risk behaviour.

\section{P123}

\section{A POPULATION-BASED STUDY INFORMING "HIV TREATMENT AS PREVENTION": EPIDEMIOLOGICAL AND CLINICAL CHARACTERISTICS OF VIROLOGICALLY DETECTABLE HIV-POSITIVE INDIVIDUALS IN BRITISH COLUMBIA}

Cescon, Angela ${ }^{1}$; Kanters, Steve ${ }^{2}$; Samji, Hasina ${ }^{1,3}$; Milan, David ${ }^{1}$; Lepik, Katherine ${ }^{1}$; Hull, Mark'; Forrest, Jamie I'; Moore, David ${ }^{1}$; Hogg, Robert $\mathrm{S}^{1,2}$; Montaner, Julio $\mathrm{S}^{1}$

${ }^{1}$ Vancouver; ${ }^{2}$ Burnaby, BC; ${ }^{3}$ Baltimore, MD, USA

BACKGROUND: In February 2010, the province of BC and the BC Centre for Excellence in HIV/AIDS (BC-CfE) announced the "Seek and Treat for Optimal Prevention of HIV/AIDS" pilot program, which aims to expand access to antiretroviral therapy (ART) to reduce HIV/AIDSrelated morbidity and mortality, as well as HIV transmission. This analysis aims to compare characteristics of virologically unsuppressed persons in 2010 to those with suppression, and document HIV-RNA ranges of all unsuppressed individuals.

METHODS: HIV-positive individuals in the provincial BC-CfE database were included. Virologic suppression was defined as two consecutive HIV-RNA measures $<500$ copies/mL in 2010. Groups were compared using the Pearson $\chi^{2}$ or Fisher's exact test for categorical variables and the Wilcoxon rank-sum test for continuous variables, with participants in the unsuppressed group only included if they were on ART or if treatment was clinically indicated, defined as a CD4 count $<350$ cells $/ \mu \mathrm{L}$. A sensitivity analysis then raised this threshold to $<500 \mathrm{cells} / \mu \mathrm{L}$.

RESULTS: 5,870 participants (18\% female) were included: 4,979 (85\%) suppressed and 891 (15\%) unsuppressed. Virologically unsuppressed (compared to suppressed) individuals were more likely (all $\mathrm{p}<.001$ ) to be female (31\% vs. $16 \%$ ), younger (median 42 vs. 48 years), have injection drug use (IDU) history ( $37 \%$ vs. $31 \%$ ), report Aboriginal ancestry $(31 \%$ vs. $16 \%$ ), and have hepatitis C (HCV) ( $58 \%$ vs. $34 \%$, among $n=2,766$ of known HCV status). They were less likely $(\mathrm{p}<.001)$ to have had a baseline AIDS-defining illness (6\% vs. $13 \%)$. Similar patterns were observed using the CD4 cut-off of $<500$ cells/ $\mu \mathrm{L}$, with exception of IDU $(p=0.619)$. Of all unsuppressed individuals, the median HIV-RNA measure in 2010 was 12,896 copies/mL (IQR 1,495-47,763), with 21\% having measures between 500-999; 25\% 1,000-9,999; 40\% 10,000-99,999; and 14\% $>100,000$.

CONCLUSIONS: In 2010, virologically unsuppressed individuals on or eligible for $\mathrm{ART}$ in $\mathrm{BC}$ were more likely to be female, younger, report Aboriginal ancestry, and have HCV. Over half had HIV-RNA $\geq 10,000$ copies $/ \mathrm{mL}$. Further efforts are needed to ensure the maximal benefits of available therapies are accessible.

\section{P124}

THE CEDAR PROJECT: CONCERNING PREVALENCE OF SEXUALLY TRANSMITTED INFECTIONS AMONG A COHORT OF YOUNG ABORIGINAL PEOPLE WHO USE DRUGS IN BRITISH COLUMBIA

Cedar Project Partnership, For the ${ }^{1}$; Chavoshi, Negar ${ }^{2}$;

Waters, Shannon T2; Zhang, Hongbin'²; Richardson, Chris G²; Spittal, Patricia $\mathrm{M}^{2}$; Schechter, Martin $\mathrm{T}^{2}$

${ }^{1}$ Prince George; ${ }^{2}$ Vancouver, BC

INTRODUCTION: International research has established that sexually transmitted infections (STIs) are markers for sexual vulnerability that enhance opportunity for HIV infection. Existing geographic bridges in many Aboriginal communities continually facilitate the entrance of STIs in even the most geographically isolated areas. In order to intervene in pathways of HIV infection, the independent lifetime experiences that increase susceptibility to contracting STIs must be better explored.

METHODS: The Cedar Project is an ongoing prospective study of Aboriginal young people in Vancouver and Prince George who use injection and non-injection drugs. This analysis includes data from October 2003 and April 2005. Participants were asked to self-report if they had ever contracted an STI. Univariate and multivariate modeling identified independent risk factors associated with self-reported STI positivity.

RESULTS: Of the 605 participants at baseline, prevalence of selfreported STI positivity was $42 \%$. In univariate analysis, STI positivity was significantly associated with being a woman, ever attempting suicide, having a regular partner who injects drugs, sharing rigs, and ever accessing drug or alcohol treatment. Being engaged in recent sex work and injection drug use were marginally associated with STI positivity. In multivariate modeling, after controlling for confounders, participants who ever attempted suicide were 1.77 times $(95 \% \mathrm{CI}$ : 0.98, 3.19) more likely to report having an STI, and participants with a regular partner who injects drugs were 2.18 times $(95 \% \mathrm{CI}: 1.04,4.57)$ more likely to report having an STI

CONCLUSION: The inequitable access to health services experienced by Aboriginal people renders individuals with STIs, along with their partners and neonates in pregnant women, particularly vulnerable to the physical and emotional consequences of untreated infections. It is critical to examine the disproportionate burden of STIs carried by young 
Aboriginal people within a gendered socio-historic framework that accommodates concepts of multigenerational trauma.

\section{P125}

HIV-RELATED SEXUAL RISK BEHAVIOUR IN GAY, BISEXUAL, AND OTHER MEN WHO HAVE SEX WITH MEN IN LONDON, ONTARIO: PRELIMINARY FINDINGS FROM THE HEALTH IN MIDDLESEX MEN MATTERS (HIMMM) PROJECT SURVEY

Coleman, Todd A ${ }^{1}$; Pugh, Daniel ${ }^{2}$; Aykroyd, Gloria ${ }^{1}$;

Bauer, Greta $\mathbf{R}^{1}$; Newman, Rob ${ }^{1}$; Pierre Pitman, Lyn ${ }^{1}$;

Powell, Leanne ${ }^{1}$

${ }^{1}$ London; ${ }^{2}$ Toronto, ON

BACKGROUND: The Health in Middlesex Men Matters (HiMMM) Project is a community-based study of factors (homophobia, social exclusion, and communication) identified within the LGBT2SQ community of London-Middlesex, Ontario and their impact on HIV-related risk, HIV testing, and health services access. Outside of major metropolitan areas (such as Toronto, Vancouver, and Montreal), Canadian information on the health of gay, bisexual and other men who have sex with men (GB-MSM) and information on HIV are lacking. A city of approximately 350,000 situated amidst smaller rural communities and farmland, London is similar to other mid-sized Canadian cities in its non-abundance of resources for LGBT2SQ communities, continuation of public displays of homophobia, and meeting of HIV-related needs through a limited number of organizations and a single AIDS service organization.

METHODS: HiMMM is currently collecting data from an online survey - containing original variables, and variables adapted from the M-Track questionnaire on sexual experiences and HIV-related risk - of local GBMSM, 18 years or older, via respondent-driven sampling. Data collection began in November, 2011.

RESULTS: Preliminary unweighted results from participants will be presented. Results will include demographic characteristics, HIV prevalence, past six-month sexual behaviour (including condom use), and HIV testing.

CONCLUSION: Combined with previously-gathered qualitative data, results from the HiMMM Project survey will: 1) provide information on current levels of sexual risk-taking; 2) allow us to compare these levels in a mid-size Canadian city with those in major cities; 3 ) describe processes through which communication, homophobia, and social exclusion impact the health of GB-MSM, and; 4) quantify these factors' effects on sexual risk, HIV testing, and access to GB-MSM-supportive physical and mental health services. Findings will be available through local town hall meetings, open-access peer-reviewed publications, fact sheets, and videos. Documents (paper/electronic) and video will be distributed through networking, conferences, our website, and knowledge exchange organizations.

\section{P126}

I-TRACK: ENHANCED SURVEILLANCE OF HIV AND HEPATITIS C RISK BEHAVIOURS AMONG PEOPLE WHO INJECT DRUGS IN CANADA, 2002 TO 2012

\section{George, Diana B; Tarasuk, Jill; Bennett, Rachel;}

Ogunnaike-Cooke, Susanna; Archibald, Chris P;

I-Track Site Principal Investigators, The

Ottawa, ON

BACKGROUND: I-Track, an enhanced surveillance system that monitors HIV and hepatitis $\mathrm{C}$ risk behaviours among people who inject drugs, was conducted in sentinel sites across Canada in 2002-2003 (Pilot), 20032005 (Phase 1 - P1), 2005-2008 (Phase 2 - P2), and 2010-2012 (Phase 3 - P3).

METHODS: Information regarding demographics, drug use, injecting and sexual risk behaviours, and HIV and hepatitis C testing patterns were collected in confidential, anonymous face-to-face interviews in four sites in the Pilot, seven sites in P1, 10 sites in P2 and seven sites to date in P3. A biological sample was collected for HIV antibody testing. Selected descriptive statistics were compared across time.

RESULTS: A total of 794, 3030, 3089 and 859 (as of April 2012) participants were interviewed in the Pilot, P1, P2, and P3 surveys, respectively.
Demographic characteristics varied slightly across phases: the majority of participants were male $(61.8 \%-69.0 \%)$, the average age ranged from 35.5 to 39.4 years and the proportion self-identified as Aboriginal ranged from $26.3 \%$ to $44.1 \%$. The drug most often injected was cocaine (26.1\%-54.3\%) and recent increases in opiate use, including oxycodone, were noted. Less than a quarter of participants borrowed needles $(14.1 \%-23.6 \%)$, mostly from sex partners $(34.8 \%-65.5 \%)$ or friends/family $(30.3 \%-43.8 \%)$. The majority reported ever testing for HIV (90.0\%-94.9\%) and 80.9\%-87.6\% tested within the previous 2 years. Overall HIV prevalence ranged from 5.9\%-15.0\%: $20.9 \%-33.3 \%$ of HIV positive participants were unaware of their HIV positive status and $86.0 \%-93.3 \%$ reported being under the care of a doctor for their HIV infection.

CONCLUSIONS: This comparison across I-Track phases presents a national profile of risk behaviours over a 10 -year period. Noteworthy trends include: the emergence of prescribed and non-prescribed opioids as drug of choice; a decline in HIV prevalence since P1; and an increase in the proportion unaware of HIV status among HIV seropositive participants.

\section{P127}

INCREASING TEST FREQUENCY AS A STRATEGY FOR EARLIER HIV DETECTION: TIME SINCE LAST NEGATIVE TEST AMONG THOSE NEWLY DIAGNOSED IN BRITISH COLUMBIA, 2006-10

Salway Hottes, Travis; Kim, Paul; Gilbert, Mark; Krajden, Mel Vancouver, BC

BACKGROUND: Canadian community surveys of gay/bisexual men having sex with men (MSM) and people using injection drugs (IDU) demonstrate that most have HIV-tested at least once, but half or fewer in the past year. To better inform population HIV testing guidelines, we derived estimates of inter-test interval (ITI) for persons newly diagnosed with $\mathrm{HIV}$ in $\mathrm{BC}$ during the past five years, stratified by exposure category, year, age, and region of residence.

METHODS: Provincial HIV case report and testing data were linked by deterministic (personal health number) and probabilistic (based on name/date of birth) matching. ITI was defined as time from last recorded negative to first positive HIV result; those with ITI $\leq 30$ days or $>10$ years (y) were excluded.

RESULTS: 1735 persons were newly diagnosed with HIV in BC during 2006-10, and 957 (55\%) had a recorded negative result in the previous $10 \mathrm{y}$. Overall median ITI was $1.8 \mathrm{y}$ with a right-skewed distribution (mean 2.6y; inter-quartile range: 0.8-3.9); median ITI was 1.4y for MSM, 1.7y for IDU, and 2.7y for heterosexual exposure. Median ITI increased with year of diagnosis (1.3y in 2006, 1.8y in 2010), age (1.4y among those 20-39 yoa versus $2.4 \mathrm{y} \geq 40$ yoa), and non-Vancouver residence ( $1.4 \mathrm{y}$ versus $2.1 \mathrm{y}$ ). Overall, 5\% had tested within 3 months $(\mathrm{m})$ of diagnosis, $15 \%$ within $6 \mathrm{~m}$, and $33 \%$ within $1 \mathrm{y} ; 6 \% / 17 \% / 38 \%$ of newly diagnosed MSM and $3 \% / 13 \% / 32 \%$ of IDU had tested within $3 \mathrm{~m} / 6 \mathrm{~m} / 1 \mathrm{y}$.

CONCLUSIONS: Interval since last test was $>1.8 \mathrm{y}$ for half and $>3.9$ for a quarter of persons recently diagnosed with HIV in BC, highlighting missed opportunities for earlier detection of HIV among those already engaged with testing. ITI was long among MSM and IDU, populations engaged in testing and with generally high awareness of HIV. New strategies to increase test frequency in these populations are needed.

\section{P128}

LONGITUDINAL ANALYSIS OF CD4 ${ }^{+}$CHANGES AMONG HIV INFECTED PATIENTS

Hunt, Kelsey; Konrad, Stephanie; Mondal, Prosanta; Gartner, Kali; Lim, Hyun-Ja; Skinner, Stuart

Saskatoon, SK

BACKGROUND: Saskatchewan has the highest incidence of HIV among all Canadian provinces. There is some evidence of rapid progression to immunological AIDS in Saskatchewan. The objectives of this study were to (i) describe rates of change in CD4 count among a cohort in Saskatoon and to (ii) assess factors associated with faster and slower rates of decline.

METHODS: This is a retrospective longitudinal analysis of HIV positive patients diagnosed between January 1st 2003 and September 30th 2011, who accessed care at Royal University Hospital (RUH) and the West Side Community Clinic (WSCC) in Saskatoon. The rate of change 
in CD4 count was determined among gender, history of injection drug use (IDU), hepatitis $\mathrm{C}$ co-infection and ethnicity using mixed effects models.

RESULTS: Of a total 453 patients, 423 (93.4\%) patients had at least one recorded CD4 count. Among these, 219 (51.9\%) were male, 271 (71.9\%) were of First Nations or Métis ethnicity, 87 (23.1\%) were Caucasian, $210(49.7 \%)$ were treated at RUH and125 (29.6\%) were treated at WSCC. 209 (62.0\%) were ever prescribed HAART. 318 (78.1\%) were Hepatitis $\mathrm{C}$ antibody positive and $316(78.0 \%)$ had a history of IDU. $27(7.6 \%)$ were diagnosed clinically with AIDS and 29 (6.9 \%) were deceased from any cause. The mean $\mathrm{CD}^{+}$count and log viral load at diagnosis were $389.1( \pm 28.3)$ cells/mm3 and $4.4(\mathrm{Q} 1=4.0, \mathrm{Q} 3=4.9)$, respectively. There was a significant difference in CD4 rate of change between gender ( $p$-value $<0.0001$ ), aboriginal versus non-aboriginal status (p-value=0.047), Hepatitis C antibody status ( $p$-value=0.031) and history of IDU (p-value=0.0003).

CONCLUSION: Multiple factors including gender, ethnicity, IDU and Hepatitis $\mathrm{C}$ are associated with rate of CD4 decline. Further analysis is underway to assess the co-linearity of the results. Individuals with these characteristics have a more rapid CD4 decline and could be considered for earlier treatment.

\section{P129}

\section{THE ADDITIVE EFFECTS OF MULTIPLE PSYCHOSOCIAL PROBLEMS PREDICT RISKY SEX AMONG MSM}

Ing, Stanley; Hart, Trevor A; Loutfy, Mona; Calzavara, Liviana; Myers, Ted

Toronto, ON

BACKGROUND: Although HIV prevention initiatives have contributed to the decline of HIV incidence rates, MSM still bear a disproportionate disease burden of the HIV epidemic in Canada. Syndemic theory delineates a social epidemiologic framework examining the interplay of multiple disease interactions in creating and maintaining epidemics. The current study employs the syndemic theory framework to measure the extent to which four psychosocial problems have an additive effect on HIV vulnerability among MSM in Toronto.

METHODS: A sample of $220 \mathrm{HIV}$-seronegative and HIV-seropositive MSM completed self-administered questionnaires at baseline and at 6-month follow-up. Four psychosocial variables measured at baseline (i.e., depression, social anxiety, internalized homophobia, and anti-gay physical assault) were examined. Logistic regressions examined how each psychosocial problem was associated with the other 3 psychosocial problems. Using logistic regressions, analyses then examined if a greater number of psychosocial problems was associated with two outcome variables measured at 6-month follow-up: being HIV-seropositive and engaging in unprotected anal intercourse with partners of unknown HIV-status or serodiscordant partners (high risk UAI).

RESULTS: The psychosocial problems measured were significantly intercorrelated (all ORs $>2.30$ except for the relationship between antigay physical assault and internalized homophobia). A greater number of psychosocial problems was not associated with HIV-seropositive status. However, a greater number of psychosocial problems was associated with later high risk UAI $(\mathrm{OR}=1.47 ; 95 \% \mathrm{CI}=1.08,2.00)$.

CONCLUSIONS: A greater number of psychosocial problems is associated with increased prevalence of high risk UAI among MSM. HIV prevention efforts may be more effective when programs focusing on sexual risk are coupled with initiatives addressing multiple psychosocial health problems experienced among MSM. These data extend upon previous findings from the US, and show that a syndemic of psychosocial problems temporally predict high risk sexual behaviour among Canadian MSM.
P130

THE CEDAR PROJECT: HIV VULNERABILITY ASSOCIATED WITH YOUNGER VERSUS OLDER AGE AMONG ABORIGINAL ADOLESCENTS WHO USE ILLICIT DRUGS

Cedar Project Partnership, For the ${ }^{1}$; Jongbloed, Kate ${ }^{2}$;

Miller, Cari²; Thomas, Vicky ${ }^{1}$; Pearce, Margo E²;

Spittal, Patricia $\mathrm{M}^{2}$; Schechter, Martin $\mathrm{T}^{2}$

1 Prince George; ${ }^{2}$ Vancouver, $\mathrm{BC}$

OBJECTIVE: To compare differences in HIV vulnerability among younger versus older Aboriginal people who use illicit drugs in Vancouver and Prince George, British Columbia.

METHODS: The Cedar Project is an ongoing prospective study of young Aboriginal people in Vancouver and Prince George who use illicit drugs. Venous blood samples were tested for HIV and HCV antibodies. Participants were included in the analysis if they had at least 2 follow-up interviews. Unadjusted and adjusted generalized estimating equation (GEE) models examined HIV vulnerabilities associated with being an adolescent ( $\leq 19$ vs. $>19$ years) over the study period (2003-2008). Odds ratios (OR) and $95 \%$ confidence intervals $(\mathrm{CI})$ were calculated.

RESULTS: Overall, 22\% ( $\mathrm{n}=133)$ participants were $<19$ years of age. In adjusted analysis, younger participants had significantly elevated odds of ever having been in foster care (AOR: 1.64, 95\% CI: 1.01, 2.68), having an STI in the past six months (AOR: 1.58, 95\% CI: 1.08, 2.33), and daily methamphetamine smoking in the past six months (AOR: $2.22,95 \% \mathrm{CI}$ : $1.55,3.18)$. Younger participants had marginally significant odds for having been involved in sex work in the past six months (AOR: 1.35, 95\% CI: 0.99, 1.85).

CONCLUSIONS: Vulnerable Aboriginal adolescents are distinct in comparison to their older counterparts and require a tailored public health response. Injection drug prevention and treatment interventions combining traditional healing opportunities and western medical interventions must be developed in collaboration with Aboriginal adolescents that address vulnerability including methamphetamine use, sex work and the trauma associated with removal from families into the foster care system.

\section{P131}

\section{HIV RISK AMONG SUSCEPTIBLE AFRICAN-CARIBBEAN} WOMEN IN TORONTO, ONTARIO

Kesler, Maya A; Kaul, Rupert; Liu, Juan; Tharao, Wangari;

Loutfy, Mona; Green-Walker, LoriAnn; Saunders, Megan;

Remis, Robert $\mathrm{S}$

Toronto, ON

BACKGROUND: We examined sexual behaviour among AfricanCaribbean women in Toronto.

METHODS: In 2009-2010, HIV-positive and HIV-negative participants were recruited through Women's Health in Women's Hands, a community clinic. Women were eligible if they, a parent or grandparent were born in Africa or the Caribbean. Women completed a questionnaire using ACASI, including demographic and sexual behaviour questions. We defined HIV risk as unprotected sex in the previous 6 months with a man who was HIVpositive or of unknown HIV status, or with a casual partner.

RESULTS: 291 HIV-negative women were recruited. Median age was 31 years. 58 (23\%) of HIV-negative women reported HIV risk in the previous 6 months. Of these, 4 (7\%) had unprotected sex with an HIV-positive regular partner, $23(40 \%)$ with a regular partner of unknown HIV status, and 18 (31\%) with a casual partner; 13 (22\%) had a casual partner and a positive or unknown status regular partner. In a multivariate regression model, younger age was significantly associated with increased HIV risk. Compared to African-born women, women born in Canada were 3.4 times more likely to report HIV risk and Caribbean born women were almost twice as likely. Among those not born in Canada, women arriving at a younger age reported significantly more HIV risk.

CONCLUSIONS: Unprotected sex with partners who were HIVpositive or unknown HIV status or with casual partners put one in five susceptible African-Caribbean women at potential risk for HIV infection. Much of this exposure was in regular sexual relationships where condoms are rarely used. Although this is not very different from other heterosexual populations, this is of particular concern in this population with a higher HIV prevalence. HIV prevention including promotion of 
condom use and HIV testing, needs to be reinforced in this population. In particular, systematic HIV testing early in a relationship may help to reduce the risk of HIV transmission.

\section{P132}

\section{MODELING THE IMPACT OF SEROSORTING ON THE} SPREAD OF HIV IN MEN WHO HAVE SEX WITH MEN

Kok, Sarah ${ }^{1}$; Tupper, Paul ${ }^{1}$; Vasarhelyi, Krisztina ${ }^{1,2}$;

Rutherford, Alexander ${ }^{1}$

${ }^{1}$ Burnaby; ${ }^{2}$ Vancouver, BC

BACKGROUND: Serosorting is the practice of restricting unprotected sexual contacts to partners of the same serostatus. It is often assumed that serosorting limits the spread of HIV among men who have sex with men (MSM). If this is not the case, then serosorting could potentially contribute to the spread of HIV. We utilize mathematical modeling to explore how serosorting may impact HIV prevalence in MSM.

METHODS: Using a deterministic HIV transmission model we studied the impact of serosorting on the course of the epidemic. It was determined when serosorting leads to prevalence decreasing towards zero versus prevalence increasing to endemic HIV. Transmission probabilities per unprotected anal intercourse (UAI) act were $0.0069,0.0023$ and 0.0032, respectively, if the infected partner is believed to be $\mathrm{HIV}^{-}, \mathrm{HIV}^{+}$, or of unknown status (Wilson et al., STDs, 2010). To quantify serosorting, we allowed $0-100 \%$ of the population to serosort for all UAI acts. Treatment efficiency was varied from $85-100 \%$, and we assumed an average of 60 UAI acts per year.

RESULTS: When mean time to diagnosis was less than 3.5 years, serosorting decreased HIV prevalence in the model over time, reaching $0 \%$ to $20 \%$ at equilibrium, assuming $95 \%$ treatment efficiency. If time to diagnosis was longer, serosorting increased equilibrium prevalence, reaching $20-80 \%$.

CONCLUSIONS: Our model suggests that the impact of serosorting is directly linked to time until diagnosis. Therefore, serosorting may be an effective preventive measure at the population level only once a critical time to diagnosis has been achieved.

\section{P133}

\section{HIV AND HCV PREVALENCE AMONG COCAINE USERS IN MONTREAL}

Leclerc, Pascale'1; Roy, Elise ${ }^{2}$; Morissette, Carole";

Vaillancourt, Éric ${ }^{2}$

${ }^{1}$ Montréal; ${ }^{2}$ Longueuil, QC

BACKGROUND: A multimethod project, including epidemiologic and ethnographic components, on the interface between crack smoking and drug injection in Montréal has been funded by CIHR. Additional funding was provided by the Montreal Public Health Department to collect saliva samples from participants in the epidemiologic component for anti-HCV and anti-HIV testing.

OBJECTIVE: To document HIV and HCV prevalence among cocaine users, stratifying by history of injection.

METHODS: Individuals having used cocaine in the past month, and aged 14 years and older, were recruited in harm reduction programs in Montréal. Three groups were created based on injection history: currentinjectors (injected any drug in past six months), past-injectors (ever injected but not in past six months) and never-injectors. An intervieweradministered questionnaire was completed and saliva samples were collected. Due to budget constraints, samples of only a random selection of current-injectors were tested.

RESULTS: From June 2008 to October 2010, 886 cocaine users were recruited: 652 current-injectors, 85 past-injectors and 149 never-injectors. Mean age of participants in these groups was 37.0, 37.8 and 29.8 years respectively and proportions of male participants were $82.2 \%, 87.1 \%$ and $87.2 \%$. Samples were available for 636 participants: 422 current-injectors, 77 past-injectors and 137 never-injectors. Observed HIV prevalence $(95 \%$ confidence interval) was as follows: current-injectors: $19.7 \%$ (16.0-23.9\%); past-injectors: $15.6 \%$ (8.7-26.0\%); never-injectors: $4.4 \%$ (1.8-9.7\%). Observed $\mathrm{HCV}$ prevalence (95\% confidence interval): current-injectors: $64.8 \%$ (60.0-69.4\%); past-injectors: $27.3 \%$ (18.0-38.8\%); never-injectors: $2.9 \%(0.9-7.8 \%)$.
CONCLUSIONS: HIV and HCV prevalence are significantly lower among never-injectors than among past- and current-injectors, but higher than in the general population. Injection drug use is clearly associated with HIV and HCV infections among cocaine users. However, our findings indicate significant HIV and HCV risks among cocaine users having never injected. Interventions specific to this population are needed, including interventions targeting unsafe sexual behaviours. Interventions aimed at preventing initiation of drug injection among never-injectors and relapse into drug injection among past-injectors should be high priorities.

\section{P135}

\section{HIV-POSITIVE SOLVENT USERS: A VOLATILE} COMBINATION

McClarty, Leigh M; Shaw, Souradet; Kasper, Ken; Keynan, Yoav; Pindera, Carla; Becker, Marissa

Winnipeg, MB

BACKGROUND: Volatile solvent inhalation is a form of substance abuse prevalent among marginalized and vulnerable populations globally, however, it continues to receive little attention from both the public and research communities. In 2011, the Manitoba HIV Program (MHP) estimated that approximately $2 \%$ of HIV-positive individuals enrolled in the Program inhale solvents. These addictions, in addition to their compromised immune system, make HIV-positive solvent users a particularly vulnerable population.

METHODS: Retrospective chart reviews on HIV-positive solvent users currently in care were performed at both sites of the MHP in Winnipeg (Health Sciences Centre Hospital and Nine Circles Community Health Centre). Focus was placed on examining the effects of solvent use on clinical presentation of HIV infection and the prevalence of comorbid conditions among solvent users. This was a matched case-control analysis where $17 \mathrm{HIV}$-positive patients who used solvents were ethnicity-, age- and sex-matched to $17 \mathrm{HIV}$-positive controls who did not report solvent use. Clinical features at presentation, such as viral load, CD4 counts, co-infections, and risk behaviours were compared between cases and controls. Odds ratios were calculated using Mantel-Haenszel methods, and McNemar's test for matched pairs was used for statistical testing. All analyses were performed using Stata 11 .

RESULTS: The average age of cases and controls was 37.3 years, with males comprising $53 \%$ of each sample. Solvent users were more likely to present with CD4 counts of 350 or less (69\% vs. $56 \%$ ) although this was not significant at the $\mathrm{p}<0.05$ level. Additionally, solvent users were more likely to be HCV positive (59\% vs. $18 \%$; OR: 3.3, 95\%CI: 1.1-9.8; $\mathrm{p}=0.02$ ). Otherwise, prevalence of STI and non-STI co-morbidities were not found to be significantly different between solvent users and non-users.

IMPLICATIONS: This study highlights the importance of developing better methods for engaging HIV-positive solvent users earlier into care. Furthermore, there is a need to better understand the effects solvent use has on disease progression and the immunologic mechanisms behind this phenomenon.

\section{P136}

ASSESSMENT OF M-TRACK: AN ENHANCED SURVEILLANCE SYSTEM OF HIV AND OTHER SEXUALLY TRANSMITTED AND BLOOD BORNE INFECTIONS (STBBI) AMONG MEN WHO HAVE SEX WITH MEN (MSM) IN CANADA

McGuire, Marissa; Nahmias, Sherine; Ogunnaike-Cooke, Susanna; Totten, Stephanie; Archibald, Chris P; Tarasuk, Jill;

M-Track Study Team, The

Ottawa, ON

OBJECTIVES: In 2005, the Public Health Agency of Canada implemented an enhanced surveillance system of HIV and other STBBI among MSM in Canada (M-Track). In 2010, an assessment of M-Track was undertaken; the objectives were to identify the most relevant strengths/ weaknesses of the system and key opportunities for future phases.

METHODOLOGY: The US Centers for Disease Control and Prevention guidelines for evaluating public health surveillance systems were used to direct the assessment. The assessment examined the overall usefulness of the system and the following system attributes: flexibility, data quality, 
acceptability, timeliness, stability, representativeness, sensitivity and simplicity. The assessment analysed information from multiple sources (eg, implementation reports, data processing documents). Stakeholders validated the findings and provided feedback from a sentinel site perspective. RESULTS: The M-Track assessment demonstrated the usefulness and acceptability of the surveillance system. It entailed collaboration with over 50 partners and resulted in the implementation of seven surveys in six sentinel sites. M-Track data have contributed to the development of national HIV/AIDS estimates in Canada, to international reports and have been presented in more than 50 publications and presentations. However, implementation demands and human resource issues were identified as barriers to optimal data use. The system's flexibility served local needs but to some extent resulted in methodological inconsistencies. The complexity and high resource needs of the system adversely affected overall timeliness and may hinder the stability of the system over time.

CONCLUSION: M-Track is successful in monitoring HIV, other STBBI and related risk behaviours in MSM in Canada. While the system will continue to support sentinel site priorities, further standardization will be needed. Improvements across several attributes would allow the system to better meet its primary objectives and further contribute to its overall usefulness.

\section{P137}

DEVELOPMENT AND PILOTING THE A-TRACK, A

SENTINEL SURVEILLANCE SYSTEM DESIGNED TO MONITOR TRENDS IN THE PREVALENCE OF HIV AND ASSOCIATED BEHAVIOURS AND SOCIO-DEMOGRAPHIC FACTORS AMONG ABORIGINAL POPULATIONS IN

\section{CANADA}

Ogunnaike-Cooke, Susanna $\mathrm{O}^{1}$; Hennink, Maurice ${ }^{2}$;

Poitras, Margaret ${ }^{2}$; Lloyd, Kathy²; Abbas, Zahid²;

Bourassa, Carrie ${ }^{2}$; Masching, Renée ${ }^{3}$; Nurbhai, Munira ${ }^{1}$;

Zylak, Candace ${ }^{1}$; Paquette, Dana ${ }^{1}$; Tarasuk, Jill' ;

Archibald, Chris $\mathbf{P}^{1}$

${ }^{1}$ Ottawa, ON; ${ }^{2}$ Regina, SK; ${ }^{3}$ Dartmouth, NS

BACKGROUND: Aboriginal people continue to be over-represented in Canada's HIV epidemic. In 2008, Aboriginal people comprised an estimated $12.5 \%$ of new HIV infections and $8.0 \%$ of prevalent HIV infections. Available data from routine surveillance inadequately explain HIV infection trends. The A-Track surveillance system aims to monitor trends in HIV infection, behavioural, and socio-demographic risk factors in Aboriginal populations in Canada.

METHODS: Over the period 2006-2009, the framework for the A-Track surveillance system was developed with input from the National A-Track Working Group and community consultations. The system recognizes Aboriginal peoples' shared control over data, respects Aboriginal customs, and is based on the tenants of mutual respect between all stakeholders, recognition of shared responsibility, Aboriginal community involvement, and utilization of existing local expertise. In order to pilot the system, a survey was launched in Regina, Saskatchewan in December 2011.

RESULTS: The A-Track framework defines core objectives, principles, target population, survey methodology, and implementation tools. The core questionnaire includes: socio-demographic/social determinants of health; sexual/drug-use practices; diagnostic testing/treatment for HIV, tuberculosis, and other infections; access to health services; and knowledge on HIV/STBBI transmission modes. Strategies have been developed to ensure the meaningful involvement of the Aboriginal community at all stages of development and implementation. A pilot survey being conducted in Regina aims to determine the feasibility (methodological, logistic and cost) of conducting an enhanced surveillance among Aboriginal populations in Canada including optimal strategies towards obtaining a sample that is representative of the sentinel site population. CONCLUSIONS: A-Track will provide valuable information on prevalence of HIV, tuberculosis and other important STBBI among Aboriginal peoples in Canada. Regina's pilot survey results will help inform existing interventions designed to decrease infection risk among Aboriginal peoples. Lessons learned will also provide guidance for possible A-Track implementation in other urban and/or reserve locations in Canada.

\section{P138}

A COMPREHENSIVE REVIEW OF HIV/AIDS RESEARCH AMONG MEN WHO HAVE SEX WITH MEN (MSM) IN BRITISH COLUMBIA, CANADA

Pai, Jayaram; Michelow, Warren; Hogg, Robert

Vancouver, BC

BACKGROUND: MSM continue to be a primary focus population for HIV/AIDS studies, both nationally and internationally. Research in British Columbia (BC) has contributed substantially to this literature since the early 1980's, largely due to the rapid and sustained epidemic of HIV among resident MSM. To date, there has not been an analysis of patterns and trends in HIV/AIDS research concerning MSM studies from BC. To resolve this knowledge gap, a comprehensive review of clinical and behavioural literature was performed.

METHODS: Peer-reviewed abstracts and full-text articles were retrieved from three scientific databases (Pubmed, EMBASE and ISI Web of Science). Search queries included all spelled variants and abbreviations of the following terms: ("men who have sex with men", "gay", "bisexual", "homosexual", "transgender") AND ("HIV", "AIDS"), while locationspecific terms were restricted to "BC" and locations within (e.g., "Vancouver", "Victoria"). All original articles meeting the following criteria were included: (1) Study population within BC (entirely or as a component of a multi-site study); (2) Analysis exclusive to or mostly concerning gay men, bisexual, transgender or MSM; (3) Clinical or behavioural focus on HIV-positive MSM, comparing HIV-positive and HIV-negative MSM, or measuring social or behavioural factors that predispose MSM to contracting HIV.

RESULTS: Of 213 original articles initially retrieved, 106 articles from 1985-2011 satisfied the inclusion criteria for this review. Over the 26year period, the number of annual publications from $\mathrm{BC}$ has not fluctuated drastically (median 4 per year). Approximately $62 \%$ of the studies are almost equally distributed between two prospective cohort studies of MSM in Vancouver: the Vancouver Lymphadenopathy-AIDS Study and the Vanguard Project. Thematically, research until 1988 focused almost exclusively on clinical and laboratory correlates of AIDS, and the serioepidemiology of HIV. The frequency of clinical HIV/AIDS studies among MSM in BC declined after 1993, while socio-behavioural focused studies began to emerge. From 1998 onwards, socio-behavioural studies predominate and highlight topics in sexual risk behaviours for HIV, sex-trade involvement, mental health correlates, and more recently substance use and violence/abuse among MSM.

\section{P139}

THE CEDAR PROJECT: EFFECTS OF POLICING ON INJECTION SAFETY FOR YOUNG ABORIGINAL PEOPLE WHO USE DRUGS IN TWO CANADIAN CITIES

Cedar Project Partnership, For the ${ }^{1}$; Pan, Stephen $W^{2}$;

Christian, Chief $\mathrm{W}^{3}$; Zhang, Hongbin ${ }^{2}$; Pearce, Margo $\mathrm{E}^{2}$;

Blair, Alden $\mathrm{H}^{2}$; Jongbloed, Kate ${ }^{2}$; Teegee, Mary ${ }^{4}$; Thomas, Vicky ${ }^{1}$; Schechter, Martin ${ }^{2}$; Spittal, Patricia $\mathrm{M}^{2}$

${ }^{1}$ Prince George; ${ }^{2}$ Vancouver; ${ }^{3}$ Enderby; ${ }^{4}$ Vanderhoof, BC

INTRODUCTION: Policing activities may inadvertently heighten HIV-related vulnerabilities among people who inject drugs. However, little is known about how policing activities impact HIV-related risks among Aboriginal people who inject drugs. This exploratory study examines the prevalence of select policing activities and its effects on risky injection practices among Aboriginal youth who inject drugs in Vancouver and Prince George, British Columbia.

METHODS: The Cedar Project is a cohort study of Aboriginal young people in Vancouver and Prince George who use drugs. Using generalized linear mixed effects models, we analyze longitudinal Cedar cohort data from 2003-2010 restricted to those who inject drugs. Three HIV/health risk factors were examined: syringe sharing, rushed injection, and reuse of syringe. For each risk factor, we analyze the effects of three policing activities: police presence that affected location of drug use or purchase, being stopped by police, and had drug equipment confiscated by police. RESULTS: Analytic sample sizes varied by policing activity, and ranged between 125 and 301 participants. At baseline, 40\% reported being affected by police presence, $61 \%$ reported being stopped by police, and $4 \%$ reported having drug equipment confiscated by police. Odds of reporting 
being affected by police presence was higher among Vancouver participants (UOR: 3.11, 95\% CI: 2.20 to 4.40).

Police presence was associated with rushed injection (AOR: 2.91; 95\% CI: 1.82 to 4.65 ) and reusing syringe (AOR: $1.56,95 \%$ CI: 1.06 to 2.30 ). Being stopped by police was associated with syringe sharing (AOR: 5.06 , 95\% CI: 1.01 to 25.23$)$. Data sparsity precluded multivariable analysis of drug equipment confiscation.

CONCLUSIONS: Aboriginal young people who inject drugs are negatively impacted by policing activities in both cities. However, police do not appear to be confiscating safe injection equipment. Expansion of safe injection sites may increase injection safety among Aboriginal young people who inject drugs.

\section{P140}

PARTNERSHIP BETWEEN COMMUNITY AND PUBLIC HEALTH FOR SURVEILLANCE: RECOGNITION OF SHARED RESPONSIBILITY, ABORIGINAL COMMUNITY INVOLVEMENT, AND UTILIZATION OF EXISTING LOCAL EXPERTISE AND INFRASTRUCTURE IN THE IMPLEMENTATION OF THE A-TRACK SURVEILLANCE

\section{SYSTEM}

Poitras, Margaret $^{2}$; Hennink, Maurice ${ }^{2}$;

Ogunnaike-Cooke, Susanna $\mathrm{O}^{1}$; Abbas, Zahid ${ }^{2}$; Lloyd, Kathy ${ }^{2}$;

Bourassa, Carrie'2; Masching, Renée ${ }^{3}$; Faye, Rachel²;

Paquette, Dana'; Zylak, Candace ${ }^{1}$; Tarasuk, Jill' ${ }^{1}$; Archibald, Chris P1

${ }^{1}$ Ottawa, ON; ${ }^{2}$ Regina, SK; ${ }^{3}$ Dartmouth, NS

BACKGROUND: Enhanced behavioural and biological surveillance surveys are an integral part of surveillance systems that enable monitoring of HIV trends. Given the disproportionate burden of HIV among Aboriginal peoples in Canada, population-based HIV prevalence estimates and behavioural risk factor information for the Canadian Aboriginal population are needed.

OBJECTIVE: To reflect on the partnerships established to conduct an A Track survey in the city of Regina. Partners included All Nations Hope AIDS Network (ANHAN), other community-based organizations/ members, Regina Qu'Appelle Health Region (RQHR), Public Health Agency of Canada (PHAC), Canadian Aboriginal AIDS Network and academia.

METHODS: In 2009, the National A-Track working group recommended the city of Regina as the pilot A-Track site. In response, PHAC consulted with Aboriginal Elders, community-based organizations and RQHR. A study team was established. The survey was promoted at local events and to First Nations and Métis nation leadership. Strategies were developed to ensure that the Regina A-Track survey would be implemented in a manner that is inclusive, culturally appropriate and respectful of the principles of ownership, control access and possession (OCAP) of data on Aboriginal people.

RESULTS: The Regina A-Track survey is led by a local study team, with advice from a community advisory group. A formal agreement was developed between PHAC, ANHAN and RQHR which served to define roles and responsibilities around survey implementation, data storage, sharing and use. From its inception, the project has received guidance from an Elder. In order to honour community ownership, a symbol was developed by a local Cree artist for use in promotional material. Inclusion of Aboriginal interviewers, Elders and ceremonies has brought life to the method of administering the survey.

LESSONS LEARNED: The successes of A-Track are the principles of OCAP and inclusion of the Aboriginal community at every stage of development and implementation of the enhanced surveillance system.

\section{P141}

INVESTIGATING THE IMPACT OF VARYING PATTERNS OF ILLICIT DRUG USE ON SUBOPTIMAL ADHERENCE TO HAART IN BRITISH COLUMBIA, CANADA

Salters, Kate $\mathrm{A}^{\mathbf{1}}$; Milloy, M-J'; Puskas, Cathy ${ }^{2}$; Min, Jeong $\mathrm{E}^{\mathbf{1}}$; Parashar, Surita ${ }^{1,2}$; Montaner, Julio S1; Hogg, Robert S $^{1,2}$ ${ }^{1}$ Vancouver; ${ }^{2}$ Burnaby, BC

BACKGROUND: Although studies have found that illicit drug use can be an individual-level barrier to optimal adherence to HAART, the effect of patterns of drug use (i.e. type, frequency and concurrency) on HAART adherence have not been well documented. The objective of this study was to examine the impact of illicit drug use type, frequency of use and poly-substance use (using $>2$ substances concurrently) on suboptimal adherence to HAART.

METHODS: The Longitudinal Investigation into Supportive and Ancillary health services (LISA) cohort is a cross-sectional study of treatmentexperienced persons PHA in BC. Between 2007 and 2010, participants completed an interviewer-administered questionnaire. Clinical variables were obtained through longitudinal linkages with the provincial Drug Treatment Program. LISA is not representative of all people living with HIV/AIDS in BC as women and Aboriginal peoples were oversampled. Multivariable analyses identified factors associated with suboptimaladherence (defined as $<95 \%$ adherence within 12 months) among illicit drug users (defined as crack, cocaine, heroin and/or crystal meth use in the three months prior to the interview).

RESULTS: Among 1000 participants, 284 (28\%) reported current illicit drug use, of which 222 (78.2\%) reported using crack, 146 (51.4\%) cocaine, $69(24.3 \%)$ heroin and $145(51.1 \%)$ participants reported poly-substance use. Nearly half of the participants (46\%) had suboptimal adherence to HAART. In the multivariable analysis, individuals using heroin $<1 \mathrm{x}$ per week were more likely to have suboptimal adherence than those using heroin $>1 \mathrm{x}$ per week (AOR 3.57, 95\% CI 1.18-11.11). Significant differences in adherence between drug type and frequencies were not noted for crack, cocaine, or crystal meth.

CONCLUSION: While no significant differences in adherence were found based on drug type or concurrent substance use, individuals who use heroin $<1 \mathrm{x}$ per week were more likely to have suboptimal adherence to HAART. These findings underline the complexity in addressing adherence to HAART among illicit drug users. This suggests there is a need for adherence support informed by a comprehensive assessment of patterns of individual drug use.

\section{P142}

SEXUAL POSITIONING WITH DIFFERENT PARTNERSHIP TYPES AMONG MEN WHO HAVE SEX WITH MEN IN SHANGHAI, CHINA

Steele (nee Taleski), Sarah J ${ }^{1}$; Myers, Ted ${ }^{1}$; Ning, Zhen ${ }^{2}$;

Allman, Dan'; Calzavara, Liviana'; Kang, Laiyi ${ }^{2}$

${ }^{1}$ Toronto, ON; ${ }^{2}$ Shanghai, China

OBJECTIVE: To describe sexual positioning with different partnership types among non-monogamous, regularly sexual active men who have sex with men (MSM) in Shanghai, China.

METHODS: The Men's Health and Lifestyle Study is a longitudinal, event-based, quantitative web-diary of MSM in Shanghai. The diary collected information about partnership dynamics; social conditions, and; the sexual act. Daily data were entered by participant via online portal. Socio-demographic characteristics were collected using an intervieweradministered questionnaire. Here, SAS is used to explore sexual positioning by partnership type.

RESULTS: 178 men completed 28 diary days each and reported at least 1 sexual event during this time. $67.4 \%(n=120 / 178)$ had at least one sexual event with a primary partner, $51.1 \%(n=91 / 178)$ with a regular partner, 53.4\% ( $n=95 / 178)$ with a casual partner. Among those reporting anal sex, the proportion of participants who were versatile (had both insertive (IAS) and receptive (RAS) anal sex) was highest with primary partners ( $\mathrm{n}=52 / 104,50.0 \%, 95 \% \mathrm{CI}: 40.4,59.6)$. The proportion who were versatile was $35.1 \%(\mathrm{n}=26 / 74,95 \% \mathrm{CI}: 24.3,46.0)$ with regular partners and $42.7 \%$ ( $n=35 / 82,95 \% \mathrm{CI}: 32.0,53.4)$ with casual. The proportion of men who only had IAS with their primary partners was higher $32.7 \%$ ( $n=34 / 104 ; 95 \%$ CI: $23.7,41.7)$ than the proportion who only had RAS $(n=18 / 104,17.3 \% ; 95 \% C I: 10.0,24.6)(M c N e m a r ' s$ test $p=0.03)$. Although not significant, data suggest that the proportion of men only having IAS with regular and casual partners was also higher than the proportion only having RAS.

CONCLUSIONS: In this study, men were more likely to adhere to only a single position with casual and regular partners than with primary partners. IAS was more commonly reported than RAS. Sexual positioning is an important area for sexual health education among this population. Future multivariable analyses will explore this whilst considering issues of identity, masculinity and HIV status. 


\section{P143}

\section{COMPARISON OF HIV TESTING BEHAVIOURS IN INJECTION DRUG USERS AND NON-INJECTING USERS OF CRACK-COCAINE}

White, Samantha J; Hopkins, Shaun; Shahin, Rita; Millson, Peggy M Toronto, ON

BACKGROUND: Like users of injection drugs, non-injecting oral users of crack-cocaine are also at risk for HIV infection and transmission. Since public health efforts have previously focused on injection drug users (IDUs) rather than users of crack, it is possible that HIV testing behaviours vary between the groups. This analysis examined differences in testing behaviours between IDUs and users of crack.

METHODS: Data were collected as part of the national I-Track surveillance system (Phase 3 Cycle). Interviewer-administered surveys were conducted at several service sites in Toronto between January 25 and April 6, 2011.

RESULTS: Of the overall sample $(\mathrm{n}=498), 93.62 \%$ reported previous HIV testing, with greater numbers of IDUs $(95.77 \%)$ compared to users of crack $(91.15 \%)$ having done so $(\mathrm{p}=0.038)$. Both groups sought tests most frequently from doctor's offices $(35.49 \%)$ and anonymous testing sites $(27.23 \%)$. Numbers of IDUs and users of crack recently tested for HIV differed, with $57.26 \%$ of IDUs reporting testing within $<6$ months compared to $44.56 \%$ of users of crack $(\mathrm{p}=0.021)$. Similarly, more IDUs (39.67\%) than users of crack (25.64\%) had $\geq 3$ tests in the past two years $(p=0.012)$. The difference in numbers of IDUs and users of crack seeking HIV testing due to drug-related risks approached significance $(\mathrm{p}=0.080)$, with IDUs (20.88\%) more frequently seeking testing for this reason compared to crack users (14.15\%). Comparisons of reported HIV status and lab results determined 6/19 HIV positive participants were unaware of his/her status ( $n=4$ reported negative status, $n=1$ waiting for test result, $\mathrm{n}=1$ never tested).

DISCUSSION: IDUs in Toronto reported more frequent and more recent HIV testing, as well as more testing due to drug-related risk behaviours, compared to non-injecting users of crack. Given that fewer users of crack sought testing due to drug-related exposure, efforts encouraging these users to seek testing should be increased.

\section{P144}

INCIDENCE OF ACUTE HEPATITIS C VIRUS INFECTION

AMONG MEN WHO HAVE SEX WITH MEN WITH AND WITHOUT HIV INFECTION: A SYSTEMATIC REVIEW

Yaphe, Sean; Bozinoff, Nikki; Kyle, Rachel; Shivkumar, Sushmita; Pant Pai, Nitika; Klein, Marina

Montreal, QC

BACKGROUND: A recent increase in reports of acute hepatitis $C$ viral infection $(\mathrm{HCV})$ in HIV-infected and uninfected men who have sex with men (MSM) with the sole risk factor being sexual exposure has led to routine screening and targeted prevention requests for this population; however, current evidence for this necessity is unclear.

METHODS: A systematic review was conducted to justify such a need. The MEDLINE, EMBASE, and BIOSYS databases were searched for the period January 2000 to June 2010. Additionally, 6 conferences were hand-searched for the same period. Data was divided into HIV-/MSM and $\mathrm{HIV}^{+} / \mathrm{MSM}$ subgroups and pooled incidence density measurements were used to compare HCV incidence using a DerSimian-Laird random effects model.

RESULTS: A total of 14 articles and 5 abstracts were included in this study. The incidence densities of HCV seroconversions in the $\mathrm{HIV}^{-} /$ MSM subgroup ranged from $0.038 / 100$ person-years to $0.810 / 100$ personyears. The pooled HCV incidence was $0.166 / 100$ person-years $(95 \% \mathrm{CI}$, 0.089-0.243). In the $\mathrm{HIV}^{+} / \mathrm{MSM}$ subgroup, incidence densities of $\mathrm{HCV}$ seroconversions ranged from $0.068 / 100$ person-years to $1.23 / 100$ personyears. The pooled incidence density was $0.537 / 100$ person-years $(95 \%$ CI, 0.437-0.636 ), 3.2-times higher than the HIV-/MSM subgroup. Three studies provided a head to head comparison of incidence rates in the $\mathrm{HIV}^{-}$and $\mathrm{HIV}^{+}$populations, resulting in a combined incidence difference of 0.36 per 100 person-years (95\% CI, 0.05-0.67).

CONCLUSION: Incidence of $\mathrm{HCV}$ in the $\mathrm{HIV}^{+} / \mathrm{MSM}$ population was high and, substantiates the need for routine screening of the $\mathrm{HIV}^{+} / \mathrm{MSM}^{2}$ population. The incidence of $\mathrm{HCV}$ in the $\mathrm{HIV}^{-} / \mathrm{MSM}$ however is similar to that reported in the general population therefore routine screening may not be warranted except on case by case basis, such as in high risk sexual exposures.

Epidemiology of HIV/AIDS, Co-infections \& Blood Borne Infections

Épidémiologie du VIH/SIDA, des co-infections et autres infections transmise par le sang

\section{P145}

CLUSTER ANALYSIS OF RECENTLY HCV INFECTED INJECTION DRUG USERS (IDUS) LIVING IN MONTREAL: PRELIMINARY DATA ON A NOVEL METHOD TO BETTER UNDERSTAND TREATMENT UPTAKE

Bamvita, Jean-Marie; Bruneau, Julie Montréal, QC

RATIONALE: HCV infection occurs mostly in the first year of HCV infection. Early HCV treating may help prevent potential HIV infection by modifying health related behaviors. Despite free access to HCV treatment and no waiting list, high proportion of HCV infected IDUs don't initiate treatment. Knowing the profiles of those who accept treatment and those who don't may help improve health outcomes and prevent HIV infection in susceptible individuals by building more tailored intervention programs.

OBJECTIVES: The purpose of this study was to classify recently seroconverted IDUs in distinctive and homogeneous clusters according to treatment initiation and socio-demographic, behavioral and clinical characteristics.

STUDY METHODS: From 2007 to December 2011, 53 participants have been selected in this ongoing cohort study of IDUs newly infected with HCV, living in Montreal. A baseline questionnaire was administered and laboratory analyses were performed. From the baseline data, a cluster analysis was carried out.

RESULTS: The mean age was 34 years old. Males represented $72 \%$. Only $28 \%$ have initiated treatment. The cluster analysis yielded 4 classes as shown in Table 1 below.

\begin{tabular}{|c|c|c|c|c|c|c|}
\hline & & $\begin{array}{l}\text { Class } 1 \\
(\mathrm{n}=16 ; \\
31.4)\end{array}$ & $\begin{array}{l}\text { Class 2 } \\
(n=8 ; \\
15.7)\end{array}$ & $\begin{array}{l}\text { Class } 3 \\
(n=9 ; \\
17.6)\end{array}$ & $\begin{array}{l}\text { Class } 4 \\
(n=18 \\
35.3)\end{array}$ & $\begin{array}{l}\text { Combined } \\
(N=51)\end{array}$ \\
\hline \multicolumn{2}{|c|}{ CES-D score ([Mean (SD)] } & $22.7(11.2)$ & $25.3(11.5)$ & $31.4(17.1)$ & $20.4(13.9)$ & 23.8 \\
\hline \multicolumn{2}{|c|}{$\begin{array}{l}\text { Self-rating of one's health status (1-7 } \\
\text { scale) ([Mean (SD)] }\end{array}$} & $4.9(2.0)$ & $5.0(2.1)$ & $4.1(2.3)$ & $5.9(2.1)$ & $5.1(2.1)$ \\
\hline \multicolumn{2}{|c|}{$\begin{array}{l}\text { Physical component of quality of life } \\
\text { (QoL) ([Mean (SD)] }\end{array}$} & $47.8(11.9)$ & $46.9(7.9)$ & $40.0(8.2)$ & $48.5(9.0)$ & $46.5(9.9)$ \\
\hline \multicolumn{2}{|c|}{ Mental component of QoL ([Mean (SD)] } & $34.0(13.1)$ & $30.5(11.3)$ & $32.0(16.1)$ & $40.6(13.6)$ & $35.4(13.8)$ \\
\hline \multirow{3}{*}{$\begin{array}{l}\text { Age categories [n } \\
(\%)]\end{array}$} & $<30$ y.o. & $0(0.0)$ & $4(16.0)$ & $3(12.0)$ & $18(72.0)$ & $25(100.0)$ \\
\hline & 30-39 у.о. & $6(60.0)$ & $4(40.0)$ & $0(0.0)$ & $0(0.0)$ & $10(100.0)$ \\
\hline & $>40$ y.o. & $10(62.5)$ & $0(0.0)$ & $6(37.5)$ & $0(0.0)$ & $16(100.0)$ \\
\hline \multirow[t]{2}{*}{ Gender [n (\%)] } & Females & $2(20.0)$ & $1(10.0)$ & $0(0.0)$ & $7(70.0)$ & $10(100.0)$ \\
\hline & Males & $14(34.1)$ & $7(17.1)$ & $9(22.0)$ & $11(26.8)$ & $41(100.0)$ \\
\hline \multirow[t]{2}{*}{ Education [n (\%)] } & $\begin{array}{l}\text { Elementary or sec- } \\
\text { ondary }\end{array}$ & $13(35.1)$ & $5(13.5)$ & $1(2.7)$ & $18(48.6)$ & $37(100.0)$ \\
\hline & College or over & $3(21.4)$ & $3(21.4)$ & $8(57.1)$ & $0(0.0)$ & $14(100.0)$ \\
\hline \multicolumn{2}{|l|}{ Living alone [n (\%)] } & $6(50.0)$ & $2(16.7)$ & $0(0.0)$ & $4(33.3)$ & $12(100.0)$ \\
\hline \multicolumn{2}{|c|}{ IV cocaine consumption [n (\%)] } & $15(32.6)$ & $8(17.4)$ & $9(19.6)$ & $14(30.4)$ & $46(100.0)$ \\
\hline \multicolumn{2}{|c|}{ IV heroine consumption [n (\%)] } & $2(6.9)$ & $6(20.7)$ & $6(20.7)$ & $15(51.7)$ & $29(100.0)$ \\
\hline \multicolumn{2}{|c|}{ Alcohol consumption [n (\%)] } & $8(28.6)$ & $6(21.4)$ & $4(14.3)$ & $10(35.7)$ & $28(100.0)$ \\
\hline \multicolumn{2}{|c|}{$\begin{array}{l}\text { Having been followed-up by a family } \\
\text { physician }[\mathrm{n}(\%)]\end{array}$} & $4(44.4)$ & $1(11.1)$ & $1(11.1)$ & $3(33.3)$ & $9(100.0)$ \\
\hline \multicolumn{2}{|c|}{$\begin{array}{l}\text { Having been followed-up by a drug } \\
\text { addiction treatment doctor [n (\%)] }\end{array}$} & $1(5.9)$ & $7(41.2)$ & $2(11.8)$ & $7(41.2)$ & $17(100.0)$ \\
\hline \multicolumn{2}{|c|}{ Having initiated treatment $[\mathrm{n}(\%)]$} & $9(25.0)$ & $0(0.0)$ & $9(25.0)$ & $18(50.0)$ & $36(100.0)$ \\
\hline
\end{tabular}


CONCLUSION: Treatment initiation seems to be influenced by favorable rating of one's health and high scores of quality of life. Profiles of these latent classes could be a first step to understanding health related behaviors of IDUs recently infected with HCV. This natural experiment represent a novel approach to understanding how specific patient characteristics could help put forward more targeted prevention programs to fight against $\mathrm{HIV} / \mathrm{HCV}$ infection.

\section{P146 \\ MORTALITY IN A LARGE COMMUNITY-BASED COHORT OF INNER CITY RESIDENTS IN VANCOUVER, CANADA}

Deans, Gregory D ${ }^{1}$; Raffa, Jesse $D^{2}$; Lai, Calvin ${ }^{1}$; Fischer, Benedikt ${ }^{3}$; Krajden, Mel ${ }^{1}$; Dore, Gregory J4; Grebely, Jason ${ }^{4}$; Tyndall, Mark W ${ }^{5}$ ${ }^{1}$ Vancouver, BC; ${ }^{2}$ Waterloo, ON; ${ }^{3}$ Burnaby, BC; ${ }^{4}$ Sydney, NSW, Australia; ${ }^{5}$ Ottawa, $\mathrm{ON}$

BACKGROUND: Inner city populations with endemic injection drug use are characterized by high mortality rates. The aim of this study was to evaluate causes of mortality among inner city residents in Vancouver.

METHODS: CHASE is a community-based cohort recruited from the Vancouver Downtown Eastside from January 2003 to June 2004. Interviews were completed at recruitment. Linkages were performed with provincial virology and mortality databases; ICD-10 codes determined causes of death.

RESULTS: Among 2,913 participants (mean age 43; 29\% female), $80 \%(\mathrm{n}=2,337)$ had used illicit drugs in the previous 6 months. Among those with HCV and HIV testing, HCV and HIV prevalence were $64 \%$ $(n=1,533)$ and $24 \%(n=534)$. Death occurred in $13 \%(n=387$; median follow-up 6.5 years; 17,631 person-years). Causes of death were HIV-related in $21 \%$, drug-related in $14 \%$, liver-related in $6 \%$, and other cause-related in $59 \%$. All-cause mortality rate was $219 / 10,000$ person-years [ $95 \%$ confidence interval (95\% CI): 199, 242] overall and 262/10,000 person-years (95\% CI: 231, 297) in those with HCV. Overall, HIV-, drug- and liver-related mortality rates were 45, 32 and 12/10,000 person-years. Among HCV-infected participants, HIV-, drug- and liver-related mortality rates were 72, 50, and 20/10,000 person-years. Liver-related mortality rates among HCV-infected participants $\leq 35$ and $>50$ years of age were $5(95 \%$ CI 1, 29) and 59/10,000 person-years $(95 \%$ CI 30,116$)$. This 12 -fold increase in liver-related mortality between the $\leq 35$ and $>50$ year groups contrasts with overall mortality rate in the same groups, which increased 3-fold from 151 (95\% CI 105, 216) to $448 / 10,000$ person-years $(95 \%$ CI 349,576$)$.

CONCLUSIONS: Mortality rates attributed to HIV, illicit drugs and liver-related causes among inner city residents were high, particularly among those with HCV. In those with HCV, liver-related mortality was higher in those $>45$ years. Strategies are needed to address HCV-related liver disease among aging inner city residents.

\section{P147}

\section{EFFECT OF HIV COINFECTION ON GENOTYPIC} DISTRIBUTION OF HCV INFECTED PERSONS ATTENDING CLINICAL IMMUNOLOGY OUTPATIENT CLINIC (CIOC), HOTEL DIEU HOSPITAL (HDH), KINGSTON, ONTARIO

\section{Medford, Richard; Newman, Adam; Martinez-Cajas, Jorge;}

Hopman, Wilma; Wobeser, Wendy

Kingston, ON

Kingston, Ontario is characterized by the highest prevalence of HCV in the province with a three-fold higher estimate rate of infection compared to the next jurisdiction, whereas this region ranks third for HIV. CIOC has cared for and treated those with HIV coinfection since the early 1990s and recently expanded its role to treat monoinfection in partnership with Street Health. We undertook this analysis to compare the genotypic diversity within these two populations. This analysis focuses only on patients seen at $\mathrm{HDH}$.

A comparative analysis was performed of the 35 coinfected persons with the first 24 monoinfected cases under our care. We found that those coinfected were more likely to be male (70\% [28] vs. 36.8\% [7], $\mathrm{p}=0.015)$. There was an age difference of 5.97 years between the two groups with an average age of 45.57 (coinfected) vs. 51.54 (monoinfected), $\mathrm{p}=0.015$. The coinfected persons were more likely to be infected with a genotype $3(71.4 \%$ [10] vs. $28.6 \%$ [4], p=0.410). Relatively few demonstrated spontaneous clearance $(0.02 \%$ [1] vs. $4.2 \%$ [2], $\mathrm{p}=0.410)$. Comparing the coinfected group to the monoinfected, $70 \%$ had no alcohol use, $52.0 \%$ drank socially and $57.1 \%$ had a history of alcohol abuse, $p=0.466$. Lastly, $62.1 \%$ vs. $56.7 \%$ of the coinfected persons demonstrated evidence of a positive psychiatric history, $\mathrm{p}=0.673$.

As the partnership between CIOC and Street Health grows, it is important to understand the local genotypic variation of our monoinfected and coinfected population because of its morbidity, mortality and treatment implications. Future research will be looking at other potential variables that may influence genotype such as history of incarceration, tobacco use and mode of transmission.

\section{P148}

DISTRIBUTION AND CORRELATES OF RECENT HIV INFECTION IN FOUR CANADIAN PROVINCES, 2000-2008 Rank, Claudia ${ }^{1}$; Bangura, Helen²; Gilbert, Mark ${ }^{3}$;

Zahariadis, George ${ }^{4}$; Van Caeseele, Paul ${ }^{5}$; Halverson, Jessica ${ }^{1}$; Kim, John ${ }^{1}$; Archibald, Chris P1 ${ }^{1}$; Sandstrom, Paul ${ }^{1}$; Brooks, James ${ }^{1}$ ${ }^{1}$ Ottawa, ON; ${ }^{2}$ Regina, SK; ${ }^{3}$ Vancouver, BC; ${ }^{4}$ Edmonton, $\mathrm{AB}$; ${ }^{5}$ Winnipeg, MB

BACKGROUND: Testing for recent HIV seroconversion is one component of the laboratory assessment of diagnostic specimens submitted to the Canadian HIV Strain and Drug Resistance Surveillance Program (SDR). We aimed to characterize recent HIV infections in four provinces participating in the SDR program from 2000-2008.

METHODS: Recent infections (within 170 days prior to sample collection) were identified among persons newly diagnosed with HIV in British Columbia, Alberta, Saskatchewan or Manitoba from 2000-2008 using the Abbott 3A11 LS, bioMerieux Vironostika HIV-1-LS or Calypte BED assays. Using routine HIV surveillance data, we determined correlates of recent infection by multivariate logistic regression.

RESULTS: Of 4,505 HIV diagnostic specimens, 1,417 (31.5\%) were classified as recent; this proportion varied from $20.3 \%$ to $42.5 \%$ by province and increased over time $(28.0 \%$ to $39.8 \%, \mathrm{p}<0.0001)$, with the greatest increase in Saskatchewan (range $10.3 \%$ to $54.2 \%$ ). Overall, a substantial proportion of recent infections were among persons $<30$ years $(40.5 \%)$, Aboriginal peoples (40.4\%), people who use injection drugs (IDU, 38.3\%) and women (36.2\%); few (8.7\%) persons from HIV-endemic countries were recently infected. In multivariate analysis adjusted for province and year (AOR [95\% CI]), recent HIV infection was more likely among females (1.46 [1.22, $1.74])$, persons $<35$ years (1.49 [1.29, 1.72] vs $\geq 35$ years), Aboriginal peoples $(1.25[1.04,1.51]$ vs Caucasians), IDU $(1.45[1.20,1.75]$ vs heterosexual) and men who have sex with men (MSM, $1.51[1.23,1.86]$ vs heterosexual). CONCLUSIONS: Although dependent on HIV testing patterns, assessment of recent infections compliments HIV surveillance by characterizing leading edges of the HIV epidemic. While the dataset was complete only to 2008, we found a greater proportion of recent HIV infections over time and among women, younger persons, Aboriginal peoples, MSM and IDU. This may represent an improvement in timely HIV diagnosis, but warrants further investigation as some groups may be more likely to seek HIV testing.

\section{P149}

SUBTYPES AND TRANSMITTED DRUG RESISTANCE AMONG RECENT HIV INFECTIONS IN FOUR CANADIAN PROVINCES, 2000-2008

Rank, Claudia ${ }^{1}$; Bangura, Helen²; Krajden, $\mathrm{Mel}^{3}$; Zahariadis, George ${ }^{4}$; Bullard, Jared ${ }^{5}$; Halverson, Jessica ${ }^{1}$; Kim, John ${ }^{1}$; Archibald, Chris $\mathbf{P}^{1}$; Sandstrom, Paul ${ }^{1}$; Brooks, James ${ }^{1}$ ${ }^{1}$ Ottawa, ON; ${ }^{2}$ Regina, SK; ${ }^{3}$ Vancouver, BC; ${ }^{4}$ Edmonton, $A B$; ${ }^{5}$ Winnipeg, MB

BACKGROUND: The Canadian HIV Strain and Drug Resistance Surveillance Program (SDR) monitors HIV subtypes and transmitted drug resistance (TDR) among antiretroviral treatment-naïve persons newly diagnosed with HIV. We aimed to describe subtypes and TDR among recent HIV infections in a historical sample of SDR data.

METHODS: Recent HIV infections (within 170 days prior to sample collection) were identified using Abbott 3A11 LS, bioMerieux Vironostika HIV-1-LS or Calypte BED assays among diagnostic HIV specimens submitted from 2000-2008 by British Columbia, Alberta, Saskatchewan and Manitoba. Subtype and TDR mutations from genotyped speci- 
mens were identified using the Stanford CPR Tool. TDR and subtype distribution were analyzed by province and time.

RESULTS: $1,417(31.5 \%)$ of 4,505 specimens tested were classified as recent HIV infections; among these the distribution of subtypes was as follows: B (92.2\%), C (3.7\%), A (1.7\%), others (2.4\%). The proportion of non-B subtypes was higher among established compared to recent HIV infections (13.1\% vs $7.8 \%$ respectively, $\mathrm{p}<0.0001$ ); in Saskatchewan non$B$ subtypes among recent infections decreased over time. Prevalence of TDR among recent infections was $12.8 \%$ (vs $8.4 \%$ among established infections, $\mathrm{p}<0.0001)$ and greater among $\mathrm{B}(13.4 \%)$ compared to non-B subtypes $(5.4 \%, \mathrm{p}<0.0001)$. Overall, the proportion with TDR varied from $7.9 \%$ to $18.5 \%$ over time and $9.3 \%$ to $28.9 \%$ by province. TDR prevalence was $6.3 \%, 5.6 \%$ and $2.0 \%$ for NNRTI, NRTI and PI drug classes, respectively; $1.2 \%$ had resistance to at least two drug classes.

CONCLUSIONS: Examination of TDR among recent HIV infections minimizes potential bias due to elapsed time from infection to HIV diagnosis. DR among recent infections likely reflects HIV transmission from persons already diagnosed and under care. Although not capturing the most recent trends, this analysis provides baseline estimates for further examination of TDR among sub-populations defined by region, risk factors and antiretroviral exposure.

\section{P150}

MONTREAL COHORT: RETROSPECTIVE DATABASE ON HIV Rollet, Kathleen C; Vassal, Anne; Machouf, Nima; Thomas, Rejean; Dufresne, Serge; Klein, Marina B; Tremblay, Cecile;

Durand, Madeleine; Roger, Michel; Sheehan, Nancy;

Trottier, Benoit; Baril, Jean-Guy

Montreal, QC

BACKGROUND: In Montreal, HIV care is concentrated in few facilities: around 78\% of HIV patients in Quebec are treated in the four facilities included in this project. The Montreal Cohort was created to develop an epidemiological infrastructure for HIV/AIDS on the basis of the various databases of AIDS and infectious diseases network researchers. The infrastructure was also established to develop subsequent research to improve knowledge on the clinical, epidemiological and behavioral profile of people living with HIV/AIDS.

METHODS: The Montreal Cohort includes 20 researchers and is based on four sites: two hospitals (MUHC, CHUM) and two private clinics (Quartier latin, Actuel). The database includes only active cases defined as patients who had at least one medical appointment with one viral load result between April 2008 and May 2010. We therefore evaluated the socio-demographic and clinical characteristics of the Montreal Cohort patients.

RESULTS: Currently, the Montreal Cohort includes 5525 individuals of whom 5322 (98\%) are from one site only; 199 from 2 sites; 4 from 3 sites. $4718(85 \%)$ patients are men, $3229(58 \%)$ are homosexual, $213(4 \%)$ are bisexual and $676(14 \%)$ are UDIs. Mean age is 47 years old. Among the participants, $4729(86 \%)$ are treated and $616(11 \%)$ remain ARV naïve. $654(12 \%)$ are co-infected with HCV and $330(6 \%)$ with HBV. Median values of last viral load and CD4 measures are respectively 49 (IQR: 49-50) copies $/ \mathrm{mL}$ and 473 (IQR:332-650) cells/ $\mu \mathrm{L}$. Women are more frequently followed in hospital centers representing $25 \%$ of their patients compared to $7 \%$ in community centers.

CONCLUSIONS: The Montreal HIV Cohort will allow a better description of the needs in care for HIV in Montreal. It will become a unique epidemiological surveillance tool and a research infrastructure with good statistical power to detect new markers of the epidemic or natural history of the infection.

\section{P151}

SOCIAL NETWORK CHARACTERISTICS ASSOCIATED WITH HCV INFECTION AMONG INJECTION DRUG USERS WITH AND WITHOUT SOLVENT USE HISTORIES IN WINNIPEG, MANITOBA

Shaw, Souradet $\mathrm{Y}^{1}$; Jolly, Ann $\mathrm{M}^{2}$; Wylie, John $\mathrm{L}^{1}$

${ }^{1}$ Winnipeg, MB; ${ }^{2}$ Ottawa, ON

INTRODUCTION: In Winnipeg, Manitoba, high risk for HIV and hepatitis $\mathrm{C}(\mathrm{HCV})$ has been observed in injection drug users (IDUs) with a history of solvent use (S-IDUs). It is thought that higher infection risk among S-IDUs is a result of interactions between structural, environmental and individual factors unique to chronic solvent users. The purpose of this study was to further examine environmental correlates of $\mathrm{HCV}$ infection by comparing egocentric social network characteristics between IDU and S-IDU who were positive for $\mathrm{HCV}$.

METHODS: Data were from a 2008-2009 cross-sectional study of Winnipeg IDU which captured both individual-level and egocentric network data. Differences in network characteristics of HCV-positive S-IDU and IDU were assessed with multivariable logistic regression models, using generalized estimating equations to account for clustering within individuals. Adjusted odds ratios (AOR) and their $95 \%$ confidence intervals $(95 \% \mathrm{CI})$ are reported; Stata 11 was used for all analyses.

RESULTS: Network data were from $134 \mathrm{HCV}$-positive individuals, of which $74 \%$ were S-IDU. IDU named an average of 4.8 network members, while S-IDU 5.8; for a total of 744 risk network members identified. The risk network members of S-IDU (compared to IDU) were more likely to include: individuals residing in the same house, apartment, or rooming house ( $27 \%$ vs. $21 \%$ ), other IDU ( $40 \%$ vs. $25 \%$ ), and those whom S-IDU had known for a longer duration. In multivariable models controlling for age, gender, education and ethnicity, and compared to HCV-positive IDU, HCV-positive S-IDUs were more likely to have an IDU in their risk network (AOR: 2.0, 95\%CI:1.3-3.3; $\mathrm{p}=0.002$ ), and to have known network members for 5 years or more (AOR: 2.9, 95\%CI:1.7-5.2; $\mathrm{p}<0.001$ ). CONCLUSIONS: The risk networks of HCV-positive S-IDU differ from IDU. S-IDU networks were more homogeneous with respect to injection drug use, and were characterised by longer relationship duration.

Methodological Issues and Meta-analyses (new models, applications, improvements)

Aspects méthodologiques et méta-analyses (nouveaux modèles, améliorations, applications)

\section{P152}

\section{ANALYSIS OF ATTRITION FROM THE ONTARIO HIV} TREATMENT NETWORK COHORT STUDY (OCS)

\section{Gardner, Sandra; Burchell, Ann; Raboud, Janet; Manno, Michael;} Zhao, Xiu Yan; Rourke, Sean B

Toronto, ON

BACKGROUND: As of December 2010, 5,644 participants have enrolled in the OCS. In Phase I of the OCS (1995-2006) clinical data was collected. Additional psychosocial and behavioural data was captured in Phase II (2007-2010). This study describes factors associated with attrition for each phase of enrollment.

METHODS: Length of follow up was calculated from the enrollment date until last visit date or the death date for participants who had died. Participants were considered lost to follow up if reported lost by the site or if no data was available in the 18 months prior to the site's last submission. Time to loss of follow up was analyzed using cumulative incidence functions and Cox proportional hazards $(\mathrm{PH})$ regression with death as a competing risk, stratified by phase.

RESULTS: Phase I: 3,176 participants with 6.1 median years of follow up. There was a cumulative incidence of $10.3 \%$ loss to follow up and $8.4 \%$ death at 1.5 years. Multivariate $\mathrm{PH}$ indicated that age $<50(\mathrm{HR}=1.6$, $\mathrm{p}<0.0001), \mathrm{CD} 4$ count $>200(\mathrm{HR}=1.5, \mathrm{p}<0.0001)$, white race $(\mathrm{HR}=1.2$, $\mathrm{p}=0.024)$, and recent HIV diagnosis $(<1$ vs $1-5$ years, $H R=1.5, p<0.0001)$ were associated with higher attrition. Unknown viral load was associated with lower attrition (reference detectable, $\mathrm{p}=0.002$ ) and undetectable viral load was not significant $(\mathrm{p}=0.2)$. Attrition also varied significantly by site. Phase II: 2,468 participants with 2.1 median years of follow up. There was a cumulative incidence of $4.0 \%$ loss to follow up and $1.3 \%$ death at 1.5 years. Having children $(\mathrm{HR}=2.5, \mathrm{p}<0.0001)$, and not being employed $\mathrm{FT} / \mathrm{PT}(\mathrm{HR}=1.8, \mathrm{p}=0.04)$ were associated with higher attrition.

CONCLUSION: Less attrition and death were observed by 1.5 years of follow up in Phase II. Different patient characteristics were associated with higher attrition in each phase. Attrition varied significantly by site in Phase I. 


\section{P153}

\section{METHODOLOGICAL CHALLENGES IN AN ATLANTIC CANADIAN SCOPING REVIEW OF YOUTH-FOCUSED PRIMARY AND SECONDARY HIV/HCV PREVENTION POLICIES}

Hudson, Pamela L' ${ }^{1}$; Gahagan, Jacqueline ${ }^{1}$; Walker, Sally ${ }^{1}$; Hare, Kathleen ${ }^{1}$; Dube, Anik²; Maurice, Jocelyn²;

Tucker, Maryanne ${ }^{3}$; Harris, Greg $^{3}$

${ }^{1}$ Halifax, NS; ${ }^{2}$ Moncton, NB; ${ }^{3}$ St John's, NL

BACKGROUND: This study sought to complete an inter-provincial, coordinated scoping review of existing HIV/HCV primary and secondary prevention policies focusing on youth (15-24 years) in Atlantic Canada within a social determinants of health framework.

METHODOLOGY: Policy mentors and trainees in the four Atlantic Provinces collaborated to identify search terms, data collection strategies, and database in order to scan both academic and grey literature. Searches were continued until saturation was reached. A record of databases, search terms, and number of results was maintained. Representatives of stakeholder organizations were also contacted and organizational websites were scanned to collect policies, program guidelines, and organizational documents. All documents were categorized by type (formal policy, strategy, programs); target group (youth, women, men, prison, minorities); sector from which the document came (health, education, justice, community); and type of prevention addressed (HIV $1^{\circ}$; HIV 2 $2^{\circ}$; $\mathrm{HEPCl}^{\circ}$; $\mathrm{HEPC}^{\circ}$; Blood Borne Pathogens).

RESULTS: Challenges included the variation and fragmentation across provinces, sectors, and organizations with respect to the parameters of program planning and service delivery, the nature of documents yielded, the effectiveness of search terms/databases, and the definition of youth by age group. These variations posed challenges in both data collection and interpretation. In order to address the methodological challenges encountered, it was necessary to create an adaptable, but highly focused, search strategy. Through an iterative process, a search framework and findings classification system were developed and utilized. The importance of the early identification of the individual structure of HIV/HCV prevention efforts within each province was highlighted.

DISCUSSION: The highly fragmented policy approach to addressing HIV/HCV prevention among youth in the Atlantic Provinces posed significant methodological challenges in this comprehensive policy review. Addressing such challenges required a collaborative and iterative approach to identifying search terms, databases, strategies, and systems of policy classification.

\section{P154 \\ VARIATION IN THE COSTS OF ANTIRETROVIRAL THERAPY AT DIFFERENT TIMEFRAMES OF INITIATION IN BRITISH COLUMBIA, CANADA}

\section{Nosyk, Bohdan; Lima, Viviane D; Yip, Benita; Hogg, Robert S;}

Montaner, Julio S

Vancouver, BC

INTRODUCTION: Treatment options and therapeutic guidelines have evolved since the onset of highly active antiretroviral treatment (HAART), resulting in substantial variation in direct treatment costs. We aimed to identify the determinants of the direct costs of HAART for individuals initiating therapy at different stages of the evolution of HAART.

METHODS: We considered all individuals who had ever initiated treatment after 1996 and received antiretroviral treatment from 2008 to 2011, as observed in the BC HIV Drug Treatment Program. Quarterly costs of drug treatment were presented in 2010\$CDN. Multivariable linear mixed effects regression models were constructed to determine the effects of demographic indicators, clinical stage (as indicated by initial and current CD4 cell counts and HIV-1 RNA) and treatment characteristics ( 1 year adherence, therapy switches and current therapy type: traditional, contemporary, and salvage therapy for multi-drug resistance) on quarterly costs of HAART overall, with sub-analyses based on the years of initiation.

RESULTS: Longitudinal data on N=6901 HAART recipients (17.6\% female, mean age of ARV initiation=40.5) was analyzed. Those on salvage therapy initiated primary between 1996 and 2000; within this cohort, annual costs of treatment were $\$ 16,737$, whereas those initiating after 2008 had mean annual costs of $\$ 15,502$. Injection drug use, male gender, better treatment adherence and lower initial CD4 were independently associated with higher costs. Current CD4 cell counts of $50-200$ cells $/ \mu \mathrm{L}$ and $<50$ cells $/ \mu \mathrm{L}$ were independently associated with increments of $\$ 100(\mathrm{p}=0.002)$ and $\$ 258(\mathrm{p}<0.001)$ in quarterly costs. Finally, compared to traditional HAART, the costs of contemporary treatment regimens were $\$ 773$ greater $(p<0.001)$, while salvage therapy was $\$ 2468$ greater per quarter $(\mathrm{p}<0.001)$

CONCLUSIONS: Early identification and effective management of HIV reduce the direct costs of HAART. Further study is required to accurately estimate and project the direct costs of treatment given the evolution in treatment guidelines and medications available.

\section{P155}

SELF-TESTING FOR HIV: A SYSTEMATIC REVIEW (2004-2011)

Pant Pai, Nitika ${ }^{1}$; Sharma, Jigyasa ${ }^{1}$; Shivkumar, Sushmita ${ }^{1}$; Gill, John $\mathrm{M}^{2}$; Peeling, Rosanna ${ }^{3}$

${ }^{1}$ Montreal, QC; ${ }^{2}$ Calgary, $\mathrm{AB} ;{ }^{3}$ London, United Kingdom

BACKGROUND: Worldwide, stigma, loss of privacy and confidentiality deter uptake of HIV testing in public settings. HIV self-testing (HST) with rapid point-of-care tests offers a potential proactive private screening solution; a critical review of this topic is much needed.

OBJECTIVE: We synthesized global evidence on all outcomes linked with HST, along with a critique of study quality.

METHODS: For the period 2004-2011, two reviewers independently searched two databases and five key conferences, abstracted data, and critiqued quality using the STROBE checklists. Outcomes assessed included uptake, concordance, accuracy, acceptability, feasibility, preference, cost, counselling and linkages.

RESULTS: About 13 moderate to high quality observational studies, were reviewed of which 8 (61.5\%) were conducted in developed settings. About $46 \%$ evaluated HST in high risk; while $54 \%$ in low risk populations. All studies evaluated oral Oraquick rapid /advance HIV1/2 test (Orasure Technologies, PA, USA)

Across studies, accuracy and acceptability of HST was high (99-100\%). In 5/13(38.4\%), studies, participants stressed convenience, speed, and privacy of HST as factors central to increasing its acceptability. Difficulties in conduct and interpretation of self-test were reportedly low (5\%-10\%). Uptake was reportedly high in low risk general population (85\%-91\%), compared to $(20-30 \%)$ in high risk marginalized populations. Cost preference was up-to $\$ 20$. About (75\%-90\%) participants stressed the importance of pre- and post-test counselling and favoured alternatives such as telephone-based counselling. Major barriers enumerated were perceived social stigma, and a lack of trust in the accuracy of results.

CONCLUSION: Self-test with an oral HIV self test was found to offer a convenient, proactive, feasible alternative to public health screening. Data on linkages to counselling and referral with HST in future will help establish the scalability of this option for global settings.

\section{P156}

\section{MIXING QUALITATIVE AND QUANTITATIVE METHODS IN HIV RESEARCH: A METHODOLOGICALLY-FOCUSED REVIEW OF THE LITERATURE}

Antoniou, Tony; Strike, Carol; Loutfy, Mona; Glazier, Richard H Toronto, ON

BACKGROUND: We undertook a critical review examining the quality of HIV-related studies that combine both qualitative and quantitative methods.

METHODS: We searched the major bibliographic databases for HIV-related studies using both qualitative and quantitative methods within the same publication or self-identifying as "mixed-method" or "multi-method". Studies were included if the participants were living with or affected by HIV-infection. We made no exclusions based on language, country of publication or substantive matter. Building upon existing instruments and frameworks, a coding scheme was developed and formulated into checklists designed to assess the reporting adequacy and quality of the quantitative, qualitative and integrative components of studies meeting our inclusion criteria. 
RESULTS: Among 12,426 citations identified from our literature review, 135 studies met our inclusion criteria, of which 53 (39.3\%) were convergent design, $13(9.6 \%)$ were sequential qualitative to quantitative design, $22(16.3 \%)$ were sequential quantitative to qualitative design and $11(8.1 \%)$ were multi-strand designs. For the remaining $36(26.7 \%)$ studies, the sequence could not be determined. An explicit reason for gathering both qualitative and quantitative data was not provided in $71(52.6 \%)$ studies. Qualitative and quantitative data were generally analyzed separately $(\mathrm{n}=118 ; \mathrm{n}=87.4 \%)$, and were most commonly mixed in the Discussion/Interpretation section of the publications ( $n=49 ; 36.3 \%$ ). In $25(18.5 \%)$ of the studies, evidence of integration was not apparent. Rather, the findings were presented in parallel and not mixed in any manner.

DISCUSSION: We observed substantial variability in the quality of studies using both qualitative and quantitative methods in the field of HIV, with significant reporting deficiencies among the individual threads of the studies. We conclude by providing recommendations for the reporting and evaluation of mixed-methods studies.

Prevention to Policies: Epidemiology that Matters De la prévention aux politiques : une épidémiologie qui compte

\section{P157}

SOCIOECONOMIC- AND GENDER-BASED DISPARITIES IN RATES OF HOSPITALIZATION AMONG HIV-INFECTED PATIENTS IN ONTARIO: A POPULATION-BASED STUDY

Antoniou, Tony; Zagorski, Brandon; Loutfy, Mona; Strike, Carol; Glazier, Richard H

Toronto, ON

BACKGROUND: Because hospitalization of persons living with HIVinfection (PLWH) in the era of combination antiretroviral therapy (cART) may be an indicator of inadequate community-based care, population-based assessments of inpatient service utilization are a necessary component of the evaluation of the quality of HIV-related care.

METHODS: Using a validated algorithm, we identified all PLWH in Ontario's health-care databases aged 18 years and older between 1992 and 2008, and conducted a population-based study using ecologic and longitudinal individual-level analyses to quantify the immediate impact of cART on hospitalization rates and analyze recent trends in rates of total and HIV-related hospitalizations.

RESULTS: The introduction of cART in 1996/97 was associated with more pronounced reductions in rates of total ( -94.8 vs. -63.3 per 1000 PLWH; $p=0.002$ ) and HIV-related hospitalizations (-59.5 vs. -37.1 per 1000 PLWH; $\mathrm{p}=0.0002$ ) among men relative to women. Between 2002 and 2008, higher rates of total hospitalization were associated with female sex [adjusted relative rate (aRR) 1.13; $95 \% \mathrm{CI}: 1.02$ to 1.24 ] and low socioeconomic status (aRR 1.22; 95\% CI: 1.14 to 1.30 ). Higher rates of HIV-related hospitalizations were associated with low socioeconomic status (aRR 1.25; 95\% CI: 1.12 to 1.40). Recent immigrants had lower rates of both total (aRR 0.70; 95\% CI 0.61 to 0.80 ) and HIV-related hospitalizations (aRR 0.76; $95 \%$ CI 0.60 to 0.96 ).

CONCLUSIONS: Despite universal access to health care and overall declines in hospitalizations, we observed important gender- and socioeconomic-based disparities in hospitalization rates among PLWH living in Ontario, Canada.

\section{P158 \\ INSTITUTIONAL POLICIES AND PHYSICIAN PRESCRIBING PRACTICES OF NON-OCCUPATIONAL POST-EXPOSURE PROPHYLAXIS (NPEP) IN ONTARIO}

Barnes, Stephanie; Loutfy, Mona; Prasad, Roger; Tan, Darrell Toronto, ON

BACKGROUND: Non-occupational post-exposure prophylaxis (nPEP) may prevent HIV transmission when started within 72 hours of high-risk exposures such as unprotected sexual intercourse and injection drug use, according to published guidelines. We assessed institutional policies and physician prescribing practices regarding $\mathrm{nPEP}$ in Ontario.
METHODS: We distributed an online 26-item questionnaire to lead physicians of Ontario emergency departments and sexual health clinics. Questions assessed institutional policies, prescribing practices, barriers to providing nPEP, and attitudes towards nPEP. Results were summarized using descriptive statistics.

RESULTS: Of 158 physicians identified from 229 institutions, 33 (21\%) responded. Only $18 \%$ of institutions had formal nPEP policies for consensual unprotected sex, $18 \%$ for injection drug use, and $55 \%$ for sexual assault. Common clinical indications for PEP included needle-stick injury $(66.7 \%)$, sexual assault $(60.6 \%)$, receptive anal sex $(36.4 \%)$, receptive vaginal sex $(36.4 \%)$, insertive anal sex $(33.3 \%)$, injection drug use (33.3\%), and insertive vaginal sex (27.3\%). While 10/24 (41.7\%) respondents appropriately identified a 72 hour post-exposure time window for determining PEP eligibility, 6/24 (25\%) provided shorter windows and $5 / 24(20.8 \%)$ said there was no timeline; $3 / 24$ (12.5\%) were unsure. Respondents who answered this question used PEP regimens consistent with published guidelines. Barriers to providing PEP included lack of familiarity with clinical guidelines (48.5\%), lack of experience prescribing (45.5\%), patients' lack of drug coverage (42.4\%), and lack of starter packs $(30.3 \%)$. Physicians were more comfortable prescribing PEP for occupational than non-occupational exposures. Most respondents felt the community would benefit from increased nPEP availability $(67.7 \%)$, and that funding (81.2\%) and clinical guidelines (93.7\%) are needed in Ontario.

CONCLUSIONS: Few Ontario institutions have formal nPEP policies, and familiarity with guideline recommendations was modest. Our findings suggest the need for improved awareness of best practices in nPEP among front-line physicians in emergency departments and sexual health clinics, and funding for nPEP medication.

\section{P159}

\section{IS LOW CONDOM USE ATTRIBUTABLE TO ATTITUDES AROUND THE EFFECTIVENESS OF CONDOM USE?: RESULTS OF THE SRC-CANFAR 2011 CANADIAN NATIONAL SURVEY}

Calzavara, Liviana ${ }^{1}$; Allman, Dan ${ }^{1}$; Worthington, Catherine' ${ }^{2}$; Tyndall, Mark'; Adrien, Alix ${ }^{4}$; White, Samantha J';

Kelly, Christopher ${ }^{1}$

${ }^{1}$ Toronto, ON; ${ }^{2}$ Victoria, BC; ${ }^{3}$ Ottawa, ON; ${ }^{4}$ Montreal, QC

OBJECTIVES: Condoms were the first HIV prevention technology. Studies show that condom use among HIV discordant couples reduces HIV transmission by $80 \%$. The objectives were to estimate the prevalence of condom use among Canadians and perceived effectiveness. METHODS: A bilingual survey of Canadians $(\mathrm{N}=2,139), 16$ years of age or older was conducted in May 2011. All provinces and territories were represented. The survey used a recruited online-panel supplemented by random digit dial telephone interviewing. The margin of error is $\pm 2.1 \%$. Descriptive statistics and logistic regression were used to examine relationships between perceived effectiveness of condoms and sociodemographic characteristics (ie, age, sex, income, education, marital status, number of children), sexual behaviour (ie,, number of partners, casual partners, condom use at last intercourse, sexual minority), ever tested for HIV, perceived effectiveness of HIV treatment, and level of HIV knowledge.

RESULTS: 94\% reported ever having had sexual intercourse and $74 \%$ had sex in the past 12 months. $22 \%$ used a condom at last sexual intercourse. Condom use at last intercourse among those with multiple partners or with casual partners was $41 \%$ and $47 \%$ respectively. Only $50 \%$ viewed condoms as "very effective" in reducing sexual transmission/spread of HIV. Logistic regression indicates those who perceived condoms to be very effective had high levels of education $(\mathrm{OR}=1.42$, $\mathrm{p}=0.028)$ and high perceived HIV-knowledge $(\mathrm{OR}=1.13, \mathrm{p}=0.007)$. Those who perceived condoms to be "somewhat effective/not effective" included: those who have casual sex partners $(O R=0.70, p=0.038)$, females $(\mathrm{OR}=0.79, \mathrm{p}=0.04)$, and those who believe that HIV treatments are less than"very effective" $(\mathrm{OR}=0.37, \mathrm{p}<0.001)$.

CONCLUSIONS: Condom use among Canadians with multiple sexual partners and casual partners was low. Only half of the population viewed condoms as very effective and those that did were better edu- 
cated, informed about HIV and reported no casual partners. Renewed and innovative condom promotion efforts are urgently required.

\section{P160 \\ HIV DIAGNOSES AND DEATHS DECLINING MORE RAPIDLY IN BRITISH COLUMBIA THAN IN ONTARIO AND QUEBEC}

Hogg, Robert S; Lima, Viviane D; Nosyk, Bohdan; Wood, Evan; Kerr, Thomas; Harrigan, Richard; Shannon, Kate; Montaner, Julio Vancouver, BC

BACKGROUND: We characterized changes in regional patterns for new HIV diagnoses, HAART use, mortality and averted cases in British Columbia, Ontario and Quebec from 1996 to 2008.

METHODS: Data on new HIV diagnoses were obtained from Health Canada, HIV-related morality statistics were obtained from Statistics Canada, and information on the number of people on HAART was obtained from Intercontinental Marketing Services (IMS) Health. Averted cases were calculated by using rates in 1996 as a standard. Trends of new HIV-positive tests were assessed using spearman rank correlations and the association between the number of individuals on HAART and new HIV diagnoses were estimated using generalized additive models.

RESULTS: 28,622 new HIV diagnoses were observed. BC was the only province with a statistically significant decrease $(\mathrm{p}<0.0001)$ in new HIV diagnoses from a rate of 18.12 to 7.94 new diagnoses per 100,000 population. If 1996 rates of new HIV diagnoses applied over the whole study period, we would expect 3,988 averted cases in BC in comparison with 1,120 in Ontario and 1,422 in Quebec. Rates of death in BC are now approaching those in Ontario and Quebec with the rate being 2.14 versus 1.06 and 1.15 per 100,000 population, respectively. BC has seen the largest decrease in deaths since 1996 with a 5.2 fold decrease versus 4.0 and 2.9 , respectively for the other two provinces. The number on HAART increased from 5,400 in 1996 to 21,400 in 2008 in these three provinces with highest rates being in $\mathrm{BC}$. Our analysis showed that for each $1 \%$ increment in HAART coverage the odds ratio for reducing the rate of new HIV diagnoses was 1.08 (95\% CI: 1.05, 1.10)

CONCLUSION: In comparison with Quebec and Ontario, BC stands alone as the only jurisdiction showing a steady decline in the rate of HIV new diagnoses and deaths.

\section{P161}

\section{FACTORS ASSOCIATED WITH MENTAL DISORDERS IN A CANADIAN COHORT OF TREATMENT-EXPERIENCED HIV-POSITIVE INDIVIDUALS}

Anema, Aranka ${ }^{1}$; Salters, Kate $A^{1}$; Somers, Julian ${ }^{3}$; Patterson, Thomas L1,2; Zhang, Wendy'; Montaner, Julio S1; Hogg, Robert $\mathrm{S}^{1,3}$

1Vancouver; ${ }^{2}$ Burnaby, BC; ${ }^{3}$ San Diego, CA, USA

BACKGROUND: A growing body of evidence shows that people living with HIV/AIDS (PHA) often concurrently cope with mental disorders. There remain gaps in our understanding of the experiences of dual diagnosed PHA in British Columbia (BC). The objective of this study is to examine the socio-demographic, behavioural and clinical characteristics of PHA ever diagnosed with a mental disorder in BC.

METHODS: The Longitudinal Investigation into Supportive and Ancillary health services (LISA) cohort is a cross-sectional study of treatment-experienced PHA in BC. Between 2007 and 2010, participants completed an interviewer-administered questionnaire and clinical variables were obtained through longitudinal linkages with the provincial Drug Treatment Program. LISA is not representative of all PHA in BC as women and Aboriginal peoples were oversampled. Logistic regression was used identify independent variables associated with self-reported mental disorder including anxiety disorder (i.e. obsessive compulsive disorder, post-traumatic stress disorder), mood disorder (i.e. depression, bipolar), psychotic disorder (i.e. schizophrenia), eating disorder (i.e. anorexia nervosa, bulimia nervosa), personality disorder and/or dementia.

RESULTS: Of the 916 participants included in this analysis, 494 (54\%) reported ever having a mental disorder. The two most prevalent disorders were depression (82\%) and anxiety disorder (60\%). Mental disorder diagnosis was independently associated with decreased overall functioning $[\mathrm{AOR}=0.90,95 \% \mathrm{CI}=0.82-0.99)$, life satisfaction $(\mathrm{AOR}=0.84$,
95\% CI=0.76-0.93), and health worries $(\mathrm{AOR}=0.89,95 \% \mathrm{CI}=0.81-0.98)$. People with mental disorder diagnoses had 2 times increased odds of a history of sexual assault ( $\mathrm{AOR}=2.04,95 \% \mathrm{CI}=1.39-3.00)$, and 10-times increased odds of reporting psychiatric care (AOR=10.11, 95\%CI: 6.9914.61).

CONCLUSION: In a large sample of PHA, the majority of participants have been diagnosed with a mental disorder. Self-reported mental disorder diagnosis was strongly associated with history of sexual assault, reduced functioning and health worries. Clinical outcomes were not significantly different between participants with and without history of mental disorders suggesting that while treatment is effective in improving clinical outcomes, PHA with mental disorders have disproportionately poorer socio-economic and behavioural outcomes.

\section{P162 \\ HIV TREATMENT AS PREVENTION: A COMPARISON OF FOUR GROUPS OF VIROLOGICALLY UNSUPPRESSED HIV-POSITIVE INDIVIDUALS IN BRITISH COLUMBIA, CANADA}

Samji, Hasina ${ }^{1,2}$; Cescon, Angela ${ }^{1}$; Kanters, Steve ${ }^{2}$; Milan, David ${ }^{1}$; Lepik, Katherine $^{1}$; Hull, Mark ${ }^{1}$; Zhang, Wendy ${ }^{1}$; Forrest, Jamie I';

Moore, David ${ }^{1}$; Hogg, Robert $\mathbf{S}^{1,2}$; Montaner, Julio $\mathbf{S}^{\mathbf{1}}$

${ }^{1}$ Vancouver, BC; ${ }^{2}$ Baltimore, MD, USA; ${ }^{3}$ Burnaby, BC

BACKGROUND: HIV viral suppression is important for optimizing the health of HIV-positive individuals and for preventing HIV transmission at the population level. Many individuals who initiate treatment are subsequently lost to care or interrupt treatment, leading to viral rebound. This study seeks to characterize unsuppressed individuals in the British Columbia Centre for Excellence in HIV/AIDS (BC-CfE) province-wide database and to examine differences among unsuppressed individuals over a one-year period.

METHODS: We defined virological suppression as $\geq 2$ consecutive HIV plasma viral load measures $<500$ copies $/ \mathrm{mL}$ in 2010 . We compared characteristics of unsuppressed individuals in the BC-CfE database by four strata: never been on ART ("No ART"); not on ART in 2010 ("Off ART"); on ART with $\geq 1$ treatment interruption of $\geq 3$ months in 2010 ("ART Gap"); and continuously on ART ("ART"). Differences among groups were tested using the Pearson $\chi^{2}$ test for categorical variables and the Wilcoxon rank-sum test for continuous variables.

RESULTS: Of 5889 HIV-positive individuals, 918 (16\%) were virologically unsuppressed in 2010: No ART 361(39\%), Off ART 141 (15\%), ART Gap 199 (22\%) and ART 127 (24\%). 31\% were women, 34\% had a reported history of injection drug use and $12 \%$ were MSM. The proportion with baseline AIDS-defining illnesses differed significantly $(p<0.001)$ : No ART 1\%, Off ART 3\%, ART Gap 9\% and ART 13\%. CD4 counts were lowest for the ART Gap group (median 220 cells/mL, [Interquartile range:100-355] vs. No ART 290 [200-350], Off ART 340 [200-485], and ART 250 [130-410]. Adherence $\geq 95 \%$ for Group 4 was 23\% in 2010 . Of individuals on treatment, the ART group had the shortest treatment duration: 3.3 years [0-10.6] vs. ART GAP 7.3 [3.2-12.3] and Off ART 7.5 [2.6-12.3].

CONCLUSIONS: Virologically unsuppressed individuals are a nonuniform group who vary in their characteristics based on whether they are interrupted, not started or continuously on ART. As such, targeted efforts to enhance access to ART in each of these groups are needed to optimize the roll out of Treatment as Prevention in BC.

Social Epidemiology (evaluations, methods) Épidémiologie sociale (évaluations, méthodes)

\section{P163}

\section{TREATMENTUPDATE READERSHIP SURVEY}

Challacombe, Laurel A

Toronto, ON

BACKGROUND: CATIE's TreatmentUpdate (TU) is a regularly published digest that covers cutting-edge developments in the research, prevention and treatment of HIV, hepatitis $\mathrm{C}$ virus (HCV) and sexually transmitted infections. TU synthesizes and translates this scientific information into a language that is more accessible to people living with HIV, 
their caregivers, and others working in HIV. Approximately 185 issues of TU have been published since its initial launch in January 1989.

METHODS: In the summer of 2011, CATIE launched a survey to gather information on the readership, their needs and to determine the current impact of the digest. The survey was disseminated through multiple channels in a paper and online format. 304 people responded to the survey.

RESULTS: TU readers come from a variety of stakeholder groups across the country: people living with HIV (PHAs) (57\%), community intermediaries (46\%), and healthcare intermediaries (21\%). Eighty-five percent of respondents have read TU for at least one year.

Readers of TU reported very high satisfaction (92\%) and utility (85\%) ratings for TU. Overall, $99 \%$ of respondents feel TU provides relevant and up-to-date developments in HIV-related research and treatment, 96\% feel they have a greater understanding of treatment issues from reading TU, 97\% feel that TU is helpful for PHAs looking to improve their health and quality of life and $98 \%$ feel that it increases knowledge or HIV treatment and care for people working in HIV.

CONCLUSION: CATIE's TU is a well-regarded and important tool used by a wide range of stakeholders. The evaluation provided important information to move this publication forward and to ensure that it continues to be relevant and meets the needs of its readership.

\section{P164}

A DESCRIPTIVE EVALUATION OF HIV-INFECTED MALES AND FEMALES SEEKING CARE IN ST PETERSBURG, RUSSIA Lioznov, Dmitry ${ }^{1}$; Nikolaenko, Svetlana ${ }^{1}$; Yatsishin, Sergey ${ }^{1}$; Lesnikova, Darya ${ }^{1}$; Cox, David²; Pankovich, Jim²; Rosenes, Ron ${ }^{3}$; Wobeser, Wendy ${ }^{4}$; Cooper, Curtis ${ }^{5}$

${ }^{1}$ St Petersburg, Russia; ${ }^{2}$ Vancouver, BC; ${ }^{3}$ Toronto; ${ }^{4}$ Kingston; ${ }^{5}$ Ottawa, ON;

INTRODUCTION: St Petersburg, Russia is home to over 40,000 HIVinfected individuals. In an effort to understand patient characteristics and barriers to care, a questionnaire was developed.

METHODS: HIV patients at 3 St Petersburg sites self completed a questionnaire capturing demographic information, opinions and barriers related to HIV care between March 28 and May 20, 2011. Staff assisted with specific questions upon request. Patient responses were not verified by clinic data. Results were compared by gender.

RESULTS: 152 surveys were completed. Mean age (SD) [male=32.4 (5.0), female $=31.0$ (5.8)], education level, income, residence type and marital status were similar. Males were more likely to live alone $(16 \%$ vs $4 \%$; $\mathrm{p}=0.02)$. Females were more likely to have had a child $(63 \%$ vs $32 \%$; $\mathrm{p}<0.001)$ and be currently raising that child within their residence $(47 \%$ vs $15 \%$; $<0.001)$. History of HCV and HBV rates were similarly high ( $74 \%$ vs $58 \%$; $=0.11)$ and ( $32 \%$ vs $28 \%$; $=0.24)$. TB history was more common in males ( $17 \%$ vs $1 \% ; \mathrm{p}=0.002)$ and sexual transmitted infection history more common in females ( $29 \%$ vs $18 \%$; $\mathrm{p}=0.02)$. Unprotected sex was reported more frequently in females $(58 \%$ vs $15 \%$; $<<0.01)$. Males reported IDU history ( $78 \%$ vs $43 \%$; $<<0.001)$ and IDU within the last 30 days $(15 \%$ vs $8 \%$; $=0.008)$ with greater frequency. Current alcohol use $(47 \%$ vs $48 \% ; \mathrm{p}=0.80)$ and frequency of use were similar by gender. There was a high level of knowledge that HIV treatment exists ( $96 \%$ vs $96 \% ; \mathrm{p}=\mathrm{NS}$ ) but less certainty among women regarding ARV efficacy ( $85 \%$ vs $94 \%$; $p=0.06$ ). Rates of ARV uptake were similar $67 \%$ and $52 \%$ $(\mathrm{p}=0.11)$. More females accessed mental health support $(45 \%$ vs $29 \%$; $\mathrm{p}=0.05)$ and social services ( $49 \%$ vs $25 \%$; $p<0.01$ )

CONCLUSION: There is a heavy burden of concurrent infectious disease (including viral hepatitis), substance use and abuse, mental health illness and need for social service support. Important differences exist between genders which may be important to consider in the allocation of limited resources in this high HIV prevalence region of Russia.

\section{P165}

THE FREQUENCY OF TREATMENT INTERRUPTION EPISODES ARE PREDICTIVE OF AN INCREASE IN MEDIAN TIME TO MORTALITY IN A COHORT OF HIV POSITIVE INDIVIDUALS IN BRITISH COLUMBIA, CANADA

Humphreys, Suzanne; Yip, Benita; Samji, Hasina; Hogg, Robert; Lima, Viviane; Colley, Guillaume

Vancouver, BC

Previous studies have investigated therapy interruption (TI) durations but not potential effects of the number of TIs. This population based group of 2,164 adult antiretroviral (ARV) naïve $\mathrm{HIV}^{+}$British Columbians started triple therapy between Jan/2000 and Aug/2009 with minimum two years of follow-up. Outcome is all-cause mortality, with nonevent individuals censored at the last contact date on/before August 31, 2010. Kaplan Meier curves and a failure time survival model were used to compare patients who ever had TI $(\mathrm{N}=1378)$ with those who did not $(\mathrm{N}=786)$ (each interruption $>90$ days).

Patients were $80.9 \%$ male, with 180 cells $/ \mathrm{mm}^{3}$ as median CD4, and median viral load of $5.0 \log \mathrm{c} / \mathrm{mL}$ at baseline. During follow-up (median 4.8 years) there were 62 deaths in the non-TI group and 130 deaths in the TI group (crude mortality of $5 \%$ and $17 \%$ respectively; $\mathrm{p}<0.001$ ).

When comparing ever-TI patients with those in the non-TI group, probability of mortality at three years was $0.0567( \pm 0.0085$, standard error) and $0.0151( \pm 0.0036)$, at seven years was $0.1944( \pm 0.0170)$ and $0.0723( \pm 0.0102)$. Our confounding model estimated hazard of mortality as $2.90(95 \% \mathrm{CI} 2.09-4.02 ; \mathrm{p}<0.001)$ in the TI group compared to the nonTI group. Comparison is also made between those with 1 TI and those with $>1$ TI with both having increased risks for mortality.

Patients with any episodes of TIs had increased risk of mortality, as seen in previous studies, and highlights that a single TI can have the same detrimental effect on survival as multiple interruptions.

\section{SOCIAL SCIENCES/SCIENCES HUMAINES}

An Anthropological Perspective Un regard antropologique

\section{P166}

\section{GAY DOCTORS TRANSFORM WHILE TAKING CARE OF} HIV $^{+}$GAY PATIENTS? IN WHAT WAYS AND HOW?

Jacob, Carl G

Montréal, QC

CONTEXT: More than any other epidemic, HIV has transformed the way doctors see themselves and their medical practice as they are continuously confronted with a complex and ever-changing work environment.

OBJECTIVE: To identify how six gay doctors transformed while caring for $\mathrm{HIV}^{+}$gay patients as well as the process by which these transformations were brought about, using the theory of experiential learning. METHODS: A qualitative approach was used to interpret data from case studies. The data was collected in 2008-09 from six gay doctors having an average of 20 years' experience caring for $\mathrm{HIV}^{+}$gay patients. These doctors participated in a semi-structured interview in which they talked about both what they transformed in their medical practice as well as how they transformed as a result of their $\mathrm{HIV}^{+}$gay patients. The analysis was carried out using qualitative software called ATLAS-ti.

RESULTS: The six gay doctors transformed in at least three areas as a result of reflexive experiences with active and informed patients - experiential learning. They transformed their personal and professional identities (e.g., their perception of life, death, time, euthanasia); they transformed the relationship with their patients (eg, eliminating therapeutic distance); and they transformed the relationship with their professional and non-professional networks (eg, their relationship with the pharmaceutical industry). They used learning-combined processes: mostly informal (eg, between colleagues), sometimes more formal (eg, specialized courses), but almost always outside basic medical training or institutionalized continuous medical training. 
CONCLUSION: These results show the importance of recognizing the value of the patient in the transformation of the medical practice of doctors, without denying the value of constituted and institutionalized knowledge. This learning method should be recognized and integrated into the context of basic and continuous medical training. Finally, the question of HIV "chronicity" in the continued experiential learning of these doctors is asked.

\section{P167}

\section{RISK FOR HIV AND STIS IN THE MONTREAL BONDAGE/} DISCIPLINE, DOMINATION/SUBMISSION AND SADOMASOCHISM [BDSM] COMMUNITY

\section{Caruso, Jessica; Martin, Blais; Joanne, Otis}

Montréal, QC

BACKGROUND: Although the Montreal BDSM community has become increasingly visible, quite little is known about its members and their practices. The predominant social conception regarding BDSM practices suggests that they are deviant, pathological and unsafe, but upon further investigation, the community appears to be structured, selfregulated and knowledgeable. However, limited information is available regarding HIV and STIs risk behaviors.

OBJECTIVES: To describe community members attending the studied BDSM events and to describe sexual and blood-related practices potentially at risk for HIV and STIs in the Montreal BDSM community.

METHOD: Ethnographic observation was undertaken in a number of public BDSM dungeons in the Montreal area. A Montreal-based Internet forum was also scrutinized to describe community members who followed a series of BDSM events.

RESULTS: 272 member profiles were examined. $51.1 \%$ identified as male and $44.5 \%$ identified as female (mean age $=38.77$ ). Regarding sexual orientation, $45,6 \%$ identified as heterosexual, $22,6 \%$ as heteroflexible and $20,4 \%$ as bisexual. Analysis of the codes and protocols involved in the BDSM culture shows that rules regarding the exchange of bodily fluids are strict. Most dungeons prohibit all sexually explicit contacts, while others only prohibit contacts involving genital fluids. Digital penetration, masturbation and oral sex were observed in a few instances, but overall, genital contacts were uncommon. Moreover, most dungeons prohibit practices that involve or might involve exposure to blood. For the few dungeons that tolerate these practices, experienced participants provide supervision and sterile medical equipment.

CONCLUSION: Sexual contacts and bodily fluids exchange among BDSM community members are very uncommon during the BDSM events, thus minimizing the risk for STIs. When games involving blood exposure are allowed, the strict conditions in which they take place render the transmission of blood-borne infections unlikely. However, HIV and STIs prevalence still needs to be estimated in the Montreal BDSM community.

\section{P168}

THINKING EQUITABLY: A CRITICAL EXAMINATION OF THE MEDICALIZATION OF HIV PREVENTION

\section{Chown, Sarah A; Ferlatte, Olivier}

Vancouver, BC

BACKGROUND: Recent developments in HIV prevention have renewed attention on biomedical solutions to the epidemic in Canada. While research provides evidence of the efficacy of treatment as prevention (TasP) and pre-exposure prophylaxis (PrEP), there is little discussion of their impacts on health equity. Given that vulnerabilities to HIV are shaped by social causes, including interlocking systems of oppression, these must be addressed in HIV prevention work. By addressing the inequities resulting from these systems of oppression, vulnerabilities to HIV can be reduced amongst individuals in one or more of the populations most affected by, and at greatest risk of, HIV: Aboriginal peoples, gay men and men who have sex with men, injection drug users and sex workers.

METHODS: Medicalization theory makes visible the ways medicine and preventive medicine can be used to regulate human behaviour. Through this critical perspective, we consider the equity implications of emerging approaches to HIV prevention based on a review of academic writing, grey literature and popular media on this topic.
FINDINGS: Biomedical solutions such as TasP and pharmaceutical PrEP locate the solution to HIV infection at the level of the individual and do not address social causes. The potential of these biomedical interventions diverts attention from upstream prevention strategies that address causal structures and maintain heightened vulnerabilities amongst some populations. Further, biomedical solutions often fail to acknowledge the unique needs within and between populations, which may influence their success or failure. Biomedical solutions also create and reinforce individuals' dependence on the pharmaceuticals and health care practitioners.

CONCLUSION: Medicalization provides a framework that highlights the health equity impacts of biomedical interventions, which must be addressed in order to reduce new HIV infections in Canada. This is a crucial conversation for researchers and organizations engaged in HIV prevention.

\section{P169}

SUPPORTED HOUSING AS A COMPONENT OF A TREATMENT AS PREVENTION (TASP) PILOT INITIATIVE

Compton, Miranda; Thumath, Meaghan; Boyd, Sandra;

McKinney, Megan

Vancouver, BC

INTRODUCTION: The Seek and Treat for Optimal Prevention of HIV/ AIDS (STOP) Project is a 3 year TasP pilot initiative aiming to expand access to HIV medications among vulnerable populations in Vancouver, BC. Three housing interventions have been implemented as components of an integrated approach to increasing client engagement in care and capacity for treatment adherence: 1) Stabilization Housing - Temporary housing with clinical support to stabilize clients where homelessness is a barrier to engagement in care and treatment re/initiation; 2) Supported Housing - Longer term housing in a congregate setting, to increase client treatment engagement and adherence 3) Supported Independent Living Subsidies (SILS) - Market rental subsidies, with peripheral support to maintain client engagement in treatment and transition to greater independence.

METHODS: A formative project evaluation employing a mixed methods approach including chart review and quantitative analysis of adherence markers $(\mathrm{Cd} 4, \mathrm{VL})$ was applied to evaluate the efficacy of supported housing in improving clinical outcomes. All participants enrolled in the intervention were assessed to have had a detectable viral load in the 6 months prior to admission. Adherence monitoring was performed every 3 months.

RESULTS: In the first 8 months of the pilot intervention, 26 clients were housed in supported housing units. $12 \%$ of this cohort had suppressed viral load at intake, $71 \%$ were suppressed at 8 months. 19 clients were provided Stabilization Housing, and 14 clients were enrolled in the SIL program, adherence markers not yet available. Chart reviews indicate clients are reporting decreased substance use, improved adherence and engagement in care.

CONCLUSION: Given the small sample size these results are not generalizable beyond the cohort. Preliminary results indicate that provision of stable, supported housing with integrated clinical and social supports can have a positive impact viral on suppression and increased capacity for sustained HIV self-management.

\section{P170}

\section{THE CONSTRUCTION OF HIV AS A CHRONIC ILLNESS: A CRITICAL ANALYSIS OF BIOCULTURAL AND RACE DISCOURSES}

\section{Gagnon, Marilou ${ }^{1}$; Manning, Elizabeth (Eli) ${ }^{2}$}

${ }^{1}$ Ottawa, ON; ${ }^{2}$ Burnaby, BC

It became possible to speak of HIV as a chronic illness in the first decade of the epidemic, as a result of social and professional movements, scientific and medical discoveries, epidemiological surveillance, and a greater understanding of the natural course of HIV infection and the illness experience. At the time, the construction of HIV as a chronic illness was part of a broader counter-discourse and a strategic response to government inaction. And so, much has been written about the reasons for framing HIV as a chronic illness and the implications for policy, research, resource allocation as well as program development. However, 
we contend that the process of framing HIV as a chronic illness has been largely overlooked since the introduction of antiretroviral therapy. In this presentation, we will critically examine the ways in which we currently speak of HIV as a chronic illness and disturb the notions that we all take for granted. Drawing on poststructuralist thought, we will pursue three main arguments: i) that chronic illness is constructed within a diffused network of power relations fundamental to the deployment of health and various technologies of government; ii) that biocultural discourses work together to inform wider perceptions of chronicity and manageability in the context of HIV; and (iii) that HIV as a chronic illness enacts race in a way that centres whiteness. We will conclude with a discussion on how the construction of HIV as a chronic illness works within and against the modern and neoliberal times of antiretroviral therapy.

\section{P171}

COLLIDING EPISTEMOLOGIES: A CRITICAL RETHINKING OF SAFER ORAL SEX AND HIV PREVENTION

\section{Gaspar, Mark A}

\section{Montreal, QC}

In Canada, oral sex is normally classified as a "low risk" activity for HIV transmission. While this categorisation aims to help individuals make their safer sex choices, its interpretation varies considerably from expert to expert and thus it is not necessarily always a useful framework for those negotiating HIV and STI risks. This presentation thus examines some of the conflicting messages about oral sex and HIV risk to elaborate on the broader relationship between the field of HIV/AIDS research and services, and the realities of those most at-risk of infection, especially (though not necessarily exclusively) men who have sex with men.

Particularly, this presentation critically examines recent literature about oral sex and HIV risk from the fields of biomedicine, Public Health and epidemiology, sociology and legal studies, as well as looking at some recent prevention campaigns, to show just how disparate opinions are on this issue. To interpret these various findings, this presentation draws on sociological literature from the fields of epistemology, risk and health management.

This presentation thus uses oral sex to demonstrate the particular ways in which knowledge in this epidemic is highly contextual, ambiguous and often contradictory, even when we are dealing with "the basic" elements of prevention-that is, safer sex. This presentation thus seeks to complicate our ideas about safer sex and suggest that as a field we must begin to rethink how to perform research that best responds to the biosocial and ethical realities of the everyday.

\section{P172}

"I'M NOT AFRAID OF DEATH BUT I'M AFRAID OF AIDS": A SOCIOLOGICAL ANALYSIS OF HIV/AIDS-RELATED STIGMA 30 YEARS LATER

\section{Hiltz, Erica $R$}

\section{Hamilton, ON}

30 years has passed since the on-set of the HIV/AIDS epidemic. For this reason, it is important to determine the level of stigmatization people living with HIV/AIDS (PLA) experience today in Western society. This research focused on whether stigma is still a problem for PLA. A symbolic interactionist perspective was used to frame this analysis. This theory focuses on the meaning-making process through social interactions. Data was collected for this study by analyzing blogs and forums specifically geared toward PLA. The sample for this study consisted of people primarily from the United States and Canada and included men, women, heterosexuals, homosexuals, bisexuals, as well as people of various races and ethnicities. Narratives provided by PLA illustrate that HIV/AIDSrelated stigma is still a common problem for those infected with the virus. The findings of this study are broken down into three categories; Experienced Stigma (stigma that is experienced through social interaction), Felt Stigma (stigma that is perceived but not directly experienced), and Layered Stigma. Layered stigma is an important finding for understanding HIV/AIDS-related stigma holistically. This type of stigma not only results from pre-existing stigmatized identities, but also from the moral assumptions and associations that come with an HIV-positive diagnosis.
P173

REPRÉSENTATIONS SOCIALES DU VIH-SIDA DANS L'ENVIRONNEMENT DES SERVICES SOCIAUX ET DE SANTÉ DE LA RÉGION DU MAULE, AU CHILI

\section{Labra, Oscar}

Rouy-Noranda, QC

Recherche doctorale que s'intéresse aux représentations sociales du VIHsida telles qu'elles sont culturellement construites et partagées dans le milieu des services sociaux et de santé de la région du Maule, au Chili. Son objectif est de décrire, à partir des discours de deux types d'acteurs (soignants/soignés), les éléments constitutifs des représentations sociales du VIH-sida. Une étude qualitative fondée sur la théorie des représentations sociales a été privilégiée et des entrevues semi-structurées ont été menées auprès de onze soignants et de vingt-huit soignés des provinces de Talca, de Curico et de Linares.

Les résultats de la recherche mettent en évidence que les représentations sociales du VIH-sida dans le Maule s'élaborent et se structurent sous l'influence de processus socioculturels au sein des communautés et que celles-ci demeurent préjudiciables pour les personnes atteintes du VIH. Toutefois, le discours des soignants et des soignés révèle des changements positifs observés au niveau des équipes de soins de première ligne où la pratique auprès de cette population vulnérable serait davantage valorisée et moins stigmatisée. Ceux qui y reçoivent des soins expriment s'y sentir bien accueillis, alors que les attitudes à leur endroit seraient davantage respectueuses des différentes formes de souffrance qu'ils éprouvent. Conclusion, un changement d'attitude de la part des équipes de soins en VIHsida envers les personnes soignées commence à avoir lieu dans les services de santé de la région du Maule au Chili. Cela nous conduit à penser qu'il y a une conception dynamique qui évolue avec le temps dans la représentation sociale du VIH-sida. Nonobstant cela, ce changement d'attitude n'est pas généralisé dans le milieu hospitalier, qui n'est pas adapté pour faciliter l'intégration des soignés, l'exception à la règle étant les équipes qui constituent les services spécialisés en VIH, distincts des autres services disponibles dans l'hôpital.

\section{P174 \\ REPRESENTATION OF MEN WHO HAVE SEX WITH MEN IN CAHR CONFERENCE ABSTRACTS: 2007-2011}

Tooley, Len

Toronto, ON

BACKGROUND: Understanding and critically assessing research and evidence is an important task for health promoters working to lessen the burden that HIV/AIDS places on gay, bisexual, and other men who have sex with men. Given that the Canadian Association for HIV Research (CAHR) is considered by some to be "synonymous with the history of HIV research in Canada" (CAHR, 2011), it is a logical place to evaluate the representation of gay/bi/MSM men in Canadian HIV/AIDS research.

METHODS: Using keyword searches, five years of abstracts (2007-2011, totalling 1,605 abstracts) from annual CAHR conferences were evaluated for mention \& representation of gay/bi/MSM men. Subsequently, following the general inductive approach, a thematic analysis of the 45 gay/bi/ MSM-exclusive abstracts from the two most recent CAHR conferences (2010/2011) was conducted using NVivo 9.

RESULTS: $7 \%(\mathrm{n}=119)$ of all abstracts over five years of CAHR conferences focused exclusively on gay/bi/MSM populations, $3 \%(n=41)$ addressed gay/bi/MSM men amongst other populations, and 2\% $(n=40)$ made passing demographic mention of gay/bi/MSM men. The thematic analysis of CAHR 2010 and 2011 abstracts revealed that the majority focused on risk- and deficit-based perspectives that often highlighted what could be considered 'failures' of gay/bi/MSM men and their communities to halt the spread of HIV. In general authors were not detailed in describing the characteristics of the population(s) they researched: for instance, only $38 \%(n=17)$ of abstracts mentioned participants' age and $29 \%(\mathrm{n}=13)$ race or ethnicity.

CONCLUSIONS: This analysis highlighted a potential disparity between the proportion of the HIV epidemic comprised by gay/bi/MSM men in Canada ( $51 \%$ ) (PHAC 2010) and the dearth of representation of this population throughout Canadian HIV/AIDS research. This prevents 
a nuanced understanding of the dynamics of HIV in gay/bi/MSM men's communities and blurs the diversity found among gay/bi/MSM men.

Communication, Disclosure and Criminalization Issues Communication, dévoilement et enjeux de criminalisation

\section{P176}

\section{THE IMPACT OF HIV/AIDS CRIMINALIZATION ON} AWARENESS, PREVENTION AND STIGMA IN THE GREATER TORONTO AREA: A QUALITATIVE ANALYSIS OF STAKEHOLDERS' PERSPECTIVES

Chung Tun Wong, Karen Y; Greene, Brittany D; Lax-Vanek, Jessica; Rans, Sofija; Shorkey, Allyson; Wilson, Michael G

\section{Hamilton, ON}

OBJECTIVE: To conduct a scoping review and examine the views and experiences of stakeholders in the Greater Toronto Area, Ontario about the impact of HIV/AIDS criminalization on awareness, prevention, and stigma.

METHODS: For the scoping review, we conducted searches of 11 databases and reviewed (in duplicate) the results for any articles addressing topics related to HIV criminalization. We then identified stakeholders (policy/content experts, executive directors and frontline workers from community-based HIV/AIDS organizations) and invited a purposive sample to participate in one-on-one, semi-structured interviews.

RESULTS: Our searches yielded 1301 results from which we included 147 articles. The majority of literature published is in the form of case reports, editorials, commentaries and essays $(n=136)$, while primary research is limited with only 11 studies. The literature highlights widespread confusion regarding what behaviours constitute "significant risk", resulting in difficulties in the application of legal precedent, as well as uncertainty regarding HIV knowledge in the general public. The available research evidence is unclear about the behavioural changes attributable to criminalization but there is some evidence to suggest that it contributes to disincentives for testing and disclosure, fears of secondary disclosure and false accusation; strained therapeutic relationships; HIVrelated stigma, and barriers for promoting shared responsibility for safer sex. The interviews explored perspectives on criminalization and its impact on prevention efforts, community awareness of prosecutions, and stigmatization of people living with HIV/AIDS. Participants discussed recommendations regarding optimal use of criminal law as it pertains to HIV/AIDS non-disclosure.

CONCLUSION: The findings from the scoping review and analysis of stakeholder interviews provide a meaningful contribution to the growing body of locally applicable primary research evidence that assesses the impact of HIV/AIDS criminalization in the community. Further research is necessary to characterize the impact from the perspective of people living with HIV/AIDS and to inform ongoing policy discussions.

\section{P177 \\ "I DON'T HAVE CHOICE...BUT WHAT CAN I DO, IT'S PART OF LIFE": EXPLORING THE BOUNDARIES AND CONTRADICTIONS OF BREASTFEEDING FOR HIV- POSITIVE MOTHERS}

Greene, Saara ${ }^{1}$; Ion, Allyson ${ }^{1}$; Elston, Dawn ${ }^{1}$; Kwaramba, Gladys ${ }^{1}$; Smith, Stephanie'; Barry, Fatimatou'; Kennedy, Logan²; Carvalhal, Adriana ${ }^{2}$; Loutfy, Mona $\mathrm{R}^{2}$

${ }^{1}$ Hamilton; ${ }^{2}$ Toronto, ON

INTRODUCTION: The WHO HIV and infant feeding guidelines recommend exclusive breastfeeding in low- and middle-income countries as it may provide a greater chance of survival even when antiretrovirals are unavailable. UK guidelines, however, recommend that HIV-positive mothers refrain from breastfeeding and be supported to formula-feed their infants, but also to breastfeed if required under rare circumstances. Canadian guidelines recommend avoiding breastfeeding regardless of HIV treatment and plasma viral load. Consequently, in Canada where culturally constituted social norms assert that "breast is best," HIV-positive mothers are precariously positioned as villains if they want to breastfeed. This work highlights the conflicting emotions related to breastfeeding and raises key questions about the impact of global vs. local breastfeeding guidelines, and messaging around breastfeeding more generally, for HIVpositive women in Canada.

METHODS: A narrative methodological approach was used to interview 27 HIV-positive pregnant women between March and December 2011. Women were asked to describe their overall experience of pregnancy in the context of living with HIV. Interviews were recorded and transcribed verbatim. Narrative analysis involving researchers and peer research assistants was used to understand HIV-positive pregnant women's perspectives of breastfeeding.

RESULTS: Participants expressed a range of feelings regarding not breastfeeding as a result of their desire to prioritize their baby's wellbeing. Women balanced feelings of loss and self-blame about being prevented from breastfeeding with their view of responsibility and "good mothering" under the current Canadian breastfeeding guidelines. Acknowledging responsibility to put their child's health first, women revealed that their choices were influenced by societal norms and guidelines, and that these choices were complicated by alternative messaging and variations in breastfeeding guidelines across geographical and cultural contexts.

CONCLUSIONS: Women's narratives about breastfeeding highlight a complex range of emotions that are affected by cultural, social and politicized messages and guidelines about breastfeeding with HIV suggesting the need to more effectively address this complex and highly emotional area of social and emotional care.

\section{P178}

THE REALITIES OF MAKING INFANT FEEDING CHOICES FOR MOTHERS LIVING WITH HIV

\section{Kapiriri, Lydia'; Siegel Sandy ${ }^{1}$; Tharao, Wangari $^{2}$}

${ }^{1}$ Hamilton; ${ }^{2}$ Toronto, ON

BACKGROUND: Breastfeeding is integral to motherhood and involves psychological, emotional, and social dynamics between the mother, her body, the baby and society at large. However, in the era of the HIV where there is a risk of mother to child HIV transmission through breast milk, breast feeding is not advised. The policies with regards to whether or not a mother is advised against breast feeding vary depending on whether or not the mother can afford replacement feeding. As such, women in low income countries, where the risks of not breast feeding outweigh the risks of HIV transmission, are encouraged to exclusively breastfeed their infants exclusively until they are 6 months old and then stop. This is in line with most of the African cultures where breastfeeding is still upheld. What are the implications of these practices for women from these contexts who are living with HIV and have children in contexts where breastfeeding is not encouraged? There is lack of documentation of the experiences of HIV positive mothers with choices related to infant feeding and the social, cultural, and ethical issues that maybe related to these mothers' making infant feeding choices in Canada.

We conducted 16 in-depth interviews and 3 group discussions with women who are living with HIV to determine their cultural beliefs with regards to infant feeding, their knowledge, attitudes and practices with regards to infant feeding and their experiences with making infant feeding choices within the Canadian context. The findings provide insight into the complexities of making infant feeding choices for mothers living with HIV in Canada. These findings also have potential to foster shared understanding between the health care providers and their clients. We will present preliminary findings from this study.

\section{P179}

'DO AS I SAY, NOT AS I DO': FAMILY COMMUNICATION ABOUT SEX AND RISKS FOR HIV-STI AMONG VULNERABLE YOUNG QUEBECERS OF HAITIAN ORIGIN Lebounga Vouma, Jenny Ingrid; Beaulieu, Marianne; Adrien, Alix Montréal, QC

OBJECTIVE: To document how parent-adolescent communication about sex impacts HIV and sexually transmitted infections (STI) risktaking among vulnerable young Quebecers of Haitian origin (VYQHO) in Montreal.

METHOD: From May to October 2008, we conducted in-depth interviews with 15 males and 15 females aged 15 to 25, born in Haiti or having 
at least one parent born in Haiti. Participants were recruited by staff members from community groups doing HIV prevention interventions among vulnerable youth in Montreal. A content analysis of transcribed and coded material was performed based on the Watzlawick theoretical communication model.

RESULTS: All the VYQHO spoke about the importance of family influence on their HIV-STI behavioural risks-taking. Despite a parental message content that promotes the adoption of healthy sexual behaviours, parents' nonverbal communication sends a contradictory message much stronger than the content itself. The VYQHO's comments reflect three components of nonverbal communication, namely: parenting model, sexual taboo and parenting style. The VYQHO have few parental models they can identify with, since many have absent fathers and mothers who are both strong and vulnerable. The sexual taboo is manifested by the lack of sex education. Finally, several VYQHO indicated that the predominant parenting style in their family is authoritarian, which contributes to a climate of mistrust within the family. Consequently, some VYQHO live their sexuality in secret.

CONCLUSION: To end vulnerabilities of young Quebecers of Haitian origin, interventions and HIV-STI prevention programs should aim to emphasize nonverbal aspects of parent-adolescent communication such as the parenting model, the sexual taboo and parenting style, rather than verbal content only, and should involve family, including parents, which through their attitudes, behaviours and values play a decisive role in the HIV-STI risk-taking among VYQHO.

\section{P180 \\ DO AS I SAY, NOT AS I DO? HOW KNOWING SOMEONE WITH HIV/AIDS INFLUENCES RISKY SEXUAL BEHAVIOUR Patocs, Audrey E \\ Hamilton, ON}

HIV/AIDS continues to exact a heavy toll of disease and deaths in many countries. In countries with generalised epidemics, the bulk of transmission is due to heterosexual contact in the general population. Thus, understanding the factors that underlie sexual risk behaviour is important in understanding disease spread and in building effective intervention programs. Here we use data from national population-based surveys conducted by Demographic and Health Surveys (DHS) in several subSaharan African countries. We use multi-level logistic regression to investigate how specific predictors are related to HIV-risk behaviours. Predictors include basic socio-demographic characteristics, and HIV-related beliefs, knowledge and experience. Response variables describe HIVrelated behaviours: condom use, and the number and type of partners. The analysis finds some patterns that are consistent across countries as well as others that vary drastically by region. In particular, we investigate the complex relationship between personal exposure to the epidemic and risk behaviours. Previous research has suggested that knowing someone with HIV/AIDS might contribute to increased safe-sex behaviours. We use multivariate analysis to disentangle the confounding effects of fear-motivated risk-reducing behaviour and the tendency for individuals who associate to share (risky) behaviours and beliefs. The trends and interactions associated with these factors are shown to vary by country, and underline the importance of targeted, region-specific approaches to behaviour-based interventions.

\section{P181}

\section{QUALITY OF RELATIONSHIPS WITHIN FAMILIES OF ADOLESCENTS LIVING WITH HIV SINCE BIRTH: A QUALITATIVE PERSPECTIVE}

Proulx-Boucher, Karène ${ }^{1}$; Fernet, Mylène ${ }^{1}$; Martin, Nancy ${ }^{1}$; Otis, Joanne $^{1}$; Lévy, Joseph J1; Lapointe, Normand ${ }^{1}$; Samson, Johanne ${ }^{1}$; Morin, Guylaine ${ }^{1}$; Thériault, Jocelyne ${ }^{1}$; Trottier, Germain ${ }^{2}$

${ }^{1}$ Montréal; ${ }^{2}$ Québec, QC

INTRODUCTION: Adolescents Living with HIV (ALHIV) since birth often face family instability that is likely to impede the management of their physical and mental health. The objective is to explore the quality of relationships within the family from the perspective of ALHIV and their evolution over time.
METHODOLOGY: Within a qualitative chronological (longitudinal) research framework, 18 youths aged 13 to 22 years old (11 girls and 7 boys) participated twice in individual semi-directed interviews within a three year interval at the Centre maternel et infantile sur le sida du CHU Sainte-Justine in Montreal.

RESULTS: All participants used strategies to keep the silence surrounding HIV in order to maintain a balance within their family environment. Two types of relational dynamics emerged over time. The first one is characterized by an investment in a close and trusting relationship with at least one member of the immediate family, often the mother or the sister. The second one is characterized by disengagement and seems punctuated by conflicts within the immediate family and the occurrence of dramatics events such as a death, a placement or a sexual abuse. These events require youth to reorganise their daily lives, adapting to a new context of life, often without family support. Conflicting family relationships may place ALHIV in situations where they must meet their needs themselves (material, physical, psychological) and rapidly develop a sense of autonomy. These challenges are compounded by the heavy responsibilities associated with their treatments.

DISCUSSION: It appears necessary to develop interventions to support adolescents in their efforts to become more autonomous and their need for social support.

\section{P183}

LET'S TALK ABOUT SEX: EXPLORING THE FACTORS THAT INFLUENCE THE SEXUAL DECISIONS OF YOUNG ACB WOMEN

Wilson, Ciann L

Brampton, ON

The Jane-Finch neighbourhood is considered one of Toronto's largest Black communities. Social inequality and poor policy decisions impose negative, oppressive conditions on the inhabitants of Jane- Finch, which inhibit the opportunities available to youth to achieve educational, social and economic success. This context of inequality manifests in poor sexual health outcomes in the young inhabitants of the community, as youth from Jane-Finch exhibit some of the highest STI rates in the city. The situation is worsened for some of the young African, Caribbean and Black - Canadian (ACB) women in the community who are reported to develop low self esteem and are left socially and economically dependent on their male partners, which hinders their ability to negotiate safe sex, putting them at increased risk for HIV. Importantly, due to the social unacceptability within the Black community of engaging in discussion about sexual health and HIV, alternative strategies are needed to address sexual health promotion and HIV prevention with young ACB women. Data collection for the Let's Talk About Sex project occurred during a photovoice workshop held once a week for 9 consecutive weeks. This workshop was completed by 14 young ACB women ages 14-18. These young women used photography and creative writing to express their opinions on the barriers and facilitators to making healthy sexual decisions. From the themes identified in the transcripts of the workshop as well as the photo-narratives created by the youth, four interconnected factors were identified as influencing the sexual decisions and realities young women were confronted with in the Jane-Finch community. These factors included negative stereotypes associated with being a young woman from the Jane-Finch community; the lack of intergenerational communication and support; the influence of men in interpersonal relationships; and the lack of financial resources and the resulting trade of sex for money and material resources amongst some young women. This presentation will analyze these factors and suggests future directions for policy, research and community-based interventions. 
First Nations

Communautés autochtones

\section{P184}

TOOLS FOR ACCESS (TFA): ADDRESSING TREATMENT

ACCESS FOR ABORIGINAL PEOPLES IN CANADA

Boucher, Rene ${ }^{1,2}$; Haight, Jack ${ }^{1,3}$; Vincent, Howard ${ }^{1,4}$;

Peltier, Doris $^{1,5}$; Stephenson, Leah $\mathbf{M}^{1}$

${ }^{1}$ Toronto; ${ }^{2}$ Thunder Bay; ${ }^{3}$ Chatham, ON; ${ }^{4}$ Lake Babine; ${ }^{5}$ Vancouver, BC

ISSUES: Data demonstrates that Aboriginal peoples in Canada continue to be over-represented in Canada's HIV/AIDS and co-infection epidemics, with many diagnosed late in the virus' progression, revealing that many are not accessing care and treatment in a timely fashion. The pressing need to understand and overcome these treatment access barriers led CTAC, Canada's only PHA and co-infected-driven NGO, to form the Aboriginal Working Group (AWG), run by positive Aboriginal people and engaging Aboriginal service providers. AWG members revised and disseminated the TFA to both share information with community while simultaneously consulting them.

DESCRIPTION: The TFA uses participatory, culturally relevant facilitation approaches to share information and consult on treatment access issues faced by positive Aboriginal people and those co-infected. The TFA gives an overview of major treatment access issues: stigma and discrimination, broader determinants of health, health care delivery (systems like First Nations Inuit Health). The interactive session includes key questions to elicit emerging and priority treatment access issues and effective actions to address them. The TFA has been presented by positive and co-infected Aboriginal facilitators at the Alberta Positive Voices Conference and the Canadian Aboriginal AIDS Network's Skillsbuilding.

LESSONS LEARNED: Central to the TFA's effectiveness in meaningfully engaging participants in identifying treatment access issues is its experiential, participatory facilitation approaches, including using indigenous methodologies of incorporating visual components, role modelling, storytelling about lived experiences, using a circle setting to avoid perceived hierarchies. Importantly, the TFA goes beyond treatment access barriers by facilitating the identification of actions, strategies, approaches to effectively address them. Finally, the TFA's development and facilitation by positive Aboriginal people and those co-infected is vital to its success, as is the meaningful engagement of positive Aboriginal people as workshop participants.

\section{NEXT STEPS:}

- Create versions of different lengths;

- Tailor for geographic perspectives and remote communities;

- Explore virtual dissemination;

- Tailor for different stakeholders, like front-line service providers.

\section{P186}

\section{SHARE: SASKATOON HIV/AIDS RESEARCH ENDEAVOUR}

\section{Hagen, Briana; Meili, Ryan; Stewart, Kris; Skinner, Stuart}

\section{Saskatoon, SK}

Over the past 5 years, Saskatoon has experienced an explosion in new human immunodeficiency virus (HIV) cases with incident rates of $30 / 100,000$ people, the highest in the nation. Patients are presenting late in the progression of the disease, often with acquired immunodeficiency syndrome (AIDS) defining illnesses. Despite the availability of antiretroviral therapy that has revolutionized HIV care, many patients in Saskatoon are not accessing the care and treatment that will prolong survival. In addition, in Saskatoon, rates of maternal-fetal transmission are 5 times the national average.

Saskatoon's HIV-positive population is unique as it is concentrated in the inner city, disproportionately affecting injection drug users, young women and Aboriginal peoples.

A coordinated and strategic response is required to address the epidemic being witnessed in Saskatoon. In 2010, the Saskatchewan Ministry of Health released a four year HIV strategy which identifies four strategic pillars: 1) community engagement and education; 2) prevention and harm reduction; 3) clinical management; and 4) surveillance and research.
As part of the provincial response to the epidemic, Drs. Skinner, Meili and Stewart, have led the development of the Saskatoon HIV/AIDS Research Endeavour (SHARE) - an HIV-specific, multi-disciplinary and multi-sectoral community-based research team and agenda.

The group that has come together to form SHARE includes a unique combination of community leaders from health services (Medicine, Nursing, Pharmacy etc), community-based organizations (AIDS Saskatoon), Aboriginal leadership (Saskatoon Tribal Council), community members and university researchers to collaborate on a research agenda that combines the best of academic processes with strong, respectful community connections.

SHARE has gained significant momentum over the past year and with continued support, has the potential to bring Saskatchewan's HIV/AIDS research agenda to the forefront in Canada.

The purpose of this presentation is to discuss the development of SHARE, highlight its current research capacity, and explore future research partnerships and projects across Canada.

\section{P187}

"THEY HAVE TO WANT TO BE THERE": NEW APPROACHES TO MEANINGFULLY ENGAGE HIV-POSITIVE ABORIGINAL WOMEN IN COMMUNITY-BASED RESEARCH Prentice, Tracey $^{1}$; Peltier, Doris ${ }^{2}$

${ }^{1}$ Ottawa, ON; ${ }^{2}$ Vancouver, BC

BACKGROUND: We have seen increased calls for meaningful and inclusive community-based research with HIV-Positive Aboriginal Women (PAW). When research is initiated, however, it is often complicated and sometimes terminated by recruitment and retention challenges that include small numbers of PAW in any given city, even smaller numbers of PAW who are known to service providers, isolation and fear of disclosure, disinterest, and other barriers to participation such as transportation, childcare costs, etc. In this presentation, we present the experience of one research project that successfully and meaningfully engaged PAW in community-based research.

DESCRIPTION: In this academic-community partnership, we used a 'women-centred' decolonizing methodology guided by the principles of Ownership, Control, Access and Possession and the Meaningful Involvement of People Living with HIV. Our approach included strengths-based, arts-informed, and community-based participatory action research methods and was built around the primary notion that the needs of the women participants came first. We engaged three small but diverse groups of PAW in three arts-informed group research processes (Toronto $=5$; Montreal $=4$; Skype $=4$ ). Depending on the needs and desires of each group, we met for $40^{+}$hours over two weeks to six months to carry out the research, but equally important, to build trust and community between participants and between participants and project personnel.

CONCLUSION: Meaningfully engaging Positive Aboriginal Women in community-based research can be challenging, time consuming and expensive. Creating a safe and inviting research environment, in addition to attending to the barriers to participation in research that Positive Aboriginal Women face, is essential to engaging women in the research process and to producing meaningful and high-quality research results that can translate to action. In the context of Aboriginal research, it also contributes to the project of decolonization that must be on-going.

\section{P190}

TAKING ACTION: USING ARTS-BASED APPROACHES TO DEVELOP ABORIGINAL YOUTH LEADERSHIP IN HIV PREVENTION

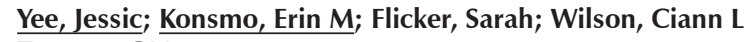
Toronto, $\mathrm{ON}$

Aboriginals are disproportionately represented in the Canadian HIV epidemic, with an infection rate 3.6-times higher than for non-Aboriginals. Additionally, the age at HIV diagnosis for Aboriginal people tends to be lower than for people of other ethnicities. A national review of HIV prevention programming targeted to Aboriginal youth reveals significant gaps in services, capacity and prevention resources. The national Taking Action was aimed at (a) examining the efficacy of engaging Aboriginal youth in HIV prevention leadership using art-based approaches and (b) exploring how Aboriginal youth linked structural inequality with 
individual risk. The project has partnered with 6 different Aboriginal communities, hosting workshops facilitated and organized "by youth, for youth." Accordingly, the project has built a national network of young people who have begun taking action on HIV in their local communities. We have also collected data from nearly 100 young people. During our workshops, youth created different forms of art-based outputs such as songs and murals. Some talked about HIV as a grim reaper wrecking havoc on their communities. Others situated HIV in larger discussions of exclusion, harassment, racism and inequality. Many of the youth connected everything in their lives to colonization: they lamented loss of land, culture and language. These youth linked HIV with the elevated rates of sexual abuse and substance use in their communities that they associated with the ongoing detrimental impacts of residential schooling. While bereavement held some youth back, others talked about the importance of their communities needing to "move on" and invest more heavily in the future. By drawing the links between individual vulnerability to HIV and larger structural inequalities, youth began conversations amongst themselves and their larger communities that challenged dominant HIV prevention strategies. They were able to see the connections between historical structural violence and current inequities, and used humour, fear, resilience and strength to imagine new prevention possibilities. This presentation will explore the successes, challenges and findings of the Taking Action project.

Global Methodologies of Intervention, Research and Policies

Méthodes d'intervention globales, recherche et politiques

\section{P191}

PREVIOUS INVOLVEMENT WITH AND FUTURE

INTENTIONS TOWARD CHARITABLE GIVING FOR HIV AND AIDS: RESULTS FROM THE SRC-CANFAR 2011 CANADIAN NATIONAL SURVEY

Allman, Dan ${ }^{1}$; Calzavara, Liviana ${ }^{1}$; Worthington, Catherine ${ }^{2}$; Tyndall, Mark ${ }^{3}$; Adrien, Alix ${ }^{4}$; White, Samantha ${ }^{1}$;

Kelly, Christopher ${ }^{1}$

${ }^{1}$ Toronto, ON; ${ }^{2}$ Victoria, BC; ${ }^{3}$ Ottawa, ON; ${ }^{4}$ Montreal, QC

BACKGROUND: Five years have passed since Canadians' behaviours, knowledge and attitudes to HIV and AIDS were surveyed last. To address this, a 2011 national survey was developed. For the first time the survey included questions about charitable giving for HIV and AIDS.

METHODS: In May 2011 a bilingual survey of people in Canada 16 years of age or older was conducted. All provinces and territories were represented. The survey recruited an online panel supplemented by random digit dial telephone interviewing. The margin of error for the total sample was \pm 2.1 percentage points at the $95 \%$ confidence interval.

RESULTS: 2,139 participated. $82.9 \%$ had donated to any charitable or not-for-profit organization in the previous year. Cancer, heart and stroke, MS and diabetes were the diseases most frequently donated to. $22.1 \%$ of contributors had ever made a donation to an organization dealing specifically with HIV and AIDS, and $13.3 \%$ had done so in the past year. $47.4 \%$ expressed a willingness to consider donations to HIV and AIDS in the future. Bivariate comparisons $\left(\chi^{2 *}\right.$, wald test**) suggest these potential future contributors tended to be younger $(\mathrm{p}<0.062 *)$, single $\left(\mathrm{p}<0.003^{*}\right)$, members of a sexual minority $(\mathrm{p}<0.001 *)$ and knowledgeable of HIV and AIDS ( $\left.<<0.001^{* *}\right)$. Social media represented an emerging element in the charitable giving sphere, with $13.2 \%$ who had donated reporting changed giving habits due to social media options. CONCLUSION: The research revealed that less than one-quarter of Canadians had ever been involved in charitable giving for HIV and AIDS. For this important minority, however, the demographic characteristics of contributors were similar to those whose donation habits were most likely to be influenced by social media, a finding that justifies efforts by those involved in HIV and AIDS fundraising to continue to incorporate social media into their work.

\section{P192}

\section{SOCIAL NETWORK TESTING INITIATIVES: A CROSS- COMPARATIVE ANALYSIS OF THE CDC AND ATOM-C PROJECTS}

Buccitelli, Anthony A; McKye, James A; Cox, Joe; Maurais, Emilie; Hapanowicz, Mark; Lacombe, Élysabeth; Lambert, Gilles; Monteith, Ken; Bernier, Mélina; Diaz Llamas, Rodrigo Montreal, QC

INTRODUCTION: Awareness To Make change (ATOM-c), a prevention project developed by AIDS Community Care Montreal (ACCM), uses incentive-based peer recruitment to promote HIV testing among men who have sex with men (MSM) in Montreal. ATOM-c was modelled after the social network testing (SNT) demonstration project created by the US Centers for Disease Control (CDC). The CDC project enlisted HIV-positive and HIV at-risk persons to refer social, sexual, or drug-using network members (ie, network associates (NAs)) for HIV testing. OBJECTIVE: To report on the implementation of a SNT initiative in a community setting.

METHOD: The ATOM-c implementation process was compared to each of the SNT phases of the CDC model project: 1. recruiter enlistment, 2. engagement (orientation, interview, and coaching), 3. recruitment of network associates, and 4. counselling, testing, and referral (CTR).

RESULTS: Differences between the CDC and ATOM-c projects are presented.

\begin{tabular}{ll}
\hline SNT phases & CDC Model \\
$\begin{array}{c}\text { 1. Recruiter } \\
\text { enlistmen }\end{array}$ & $\begin{array}{c}\text { Recruiters identified and } \\
\text { enlisted through clinics. }\end{array}$
\end{tabular}

ATOM-c Model

- Similar recruiter eligibility requirements were used.

- ACCM does not offer onsite testing services. A promotional campaign was developed to attract recruiters (eg, posters, videos, presentations at events and community organizations, etc.)

2. Engagement Recruiters were provided a project orientation which included tips on how to: approach NAs; make proper CTR referrals, etc.

3. Recruitment Recruiters referred their of NAs NAs for CTR. Agency staff followed up with all recruiters on a regular basis to discuss progress.

4. CTR Agencies running the project $•$ NAs required to seek CTR had on-site CTR services. services elsewhere.

- Partnerships were established with clinics offering CTR services on-site and off-site.

- ATOM-c now hosts "testing parties" at recruiters' homes for their NAs.

CONCLUSION: Agencies with on-site CTR services seem to encoun ter fewer barriers in the implementation of SNT initiatives. Nevertheless, there are innovative strategies community-based organizations can adopt to circumvent such challenges.

\section{P193}

THE THRILL OF THE DRILL: MIGRATORY WORKERS AND HIV/AIDS ASSOCIATED RISK FACTORS

Bulman, Donna $E^{1}$; Dykeman, Margaret ${ }^{1}$; Mathews, Maria ${ }^{2}$; Gustafson, Diana ${ }^{2}$

${ }^{1}$ Fredericton, NB; ${ }^{2}$ St John's, NL

OBJECTIVE: This poster describes a qualitative community-based research project that explored lifestyle behaviours of migratory workers, 
specifically in regard to the transmission of HIV/AIDS and other communicable diseases.

METHODOLOGY: Sixteen people who regularly travel between Newfoundland, New Brunswick, and the oil sands in Northern Alberta (Fort McMurray) were interviewed using a semi-structured interview process. These interviews occurred between 2008 and 2011 and focused on HIV/AIDS related risk factors. A thematic analysis was used to interpret the data.

FINDINGS: Some emerging themes from the research suggest that 1) a sense of alienation and isolation is prevalent in the camps (shadow community), thus contributing to the "party culture" and HIV/AIDS associated risk behaviours that may occur when on leave from the camps; 2) different types of violence exists within the work camps and in order to avoid harassment many people try to build a "macho image" thus contributing to risky behaviours such as unsafe sex or substance use 3) employees living in work camps/hotels tend to shift their drug of choice from marijuana to cocaine, so if a surprise drug test is completed his/ her test is more likely to come back "clean."; and 4) some participants indicate they are reluctant to speak about life in the oil sands when at home in Atlantic Canada as they experience a "disconnect" between their two lives and need to keep them separate in order to maintain their emotional health.

CONCLUSION: Additional qualitative research is required in order to gain a full understanding of the complex social and economic factors that may be contributing to the spread of HIV/AIDS among migratory workers.

\section{P194 \\ "ONTARIO AIDS NETWORK COMMUNITY-BASED RESEARCH PROGRAM: PROGRAM PLANNING LOGIC MODEL"}

Cameron, Ruth; McEwen, Owen

Toronto, ON

BACKGROUND/OBJECTIVES: The Community Based Research (CBR) Program in Ontario is situated at the Ontario AIDS Network (OAN). Funded by the Canadian Institutes of Health Research (CIHR), the program fosters CBR capacity through developing skills building and learning opportunities with OAN members. Areas of focus include creating opportunities for partnership and research development, and facilitating project implementation and knowledge exchange. To efficiently and effectively meet these multiple goals, programming has shifted in focus from intensive work with interested ASO's to service delivery within a framework targeting stakeholders linked to the main Ontario populations impacted by HIV. Given current restructuring of the CIHR CBR funding tool, an environmental scan was completed to inform a framework consisting of a program plan and evaluation matrix supporting future Ontario HIV/AIDS CBR development.

METHODS: An environmental scan of data from agencies, OCHART, CIHR and the OAN was compiled to inform a program planning logic model and evaluation matrix focused on the integration of meaningful engagement of persons living with HIV/AIDS (MIPA) into all facets of CBR, strategy-level capacity building, comprehensive CBR curriculum development and monitoring and tracking tools facilitating more productive ASO CBR consultation.

RESULTS: Data from 2009-2011 CIHR and 2003 OAN consultations on the CBR program, strategic plans of key Ontario stakeholders, and 2009/2010 OCHART reporting were used to develop a program plan emphasizing systemic approaches to increasing CBR capacity, collaboration with key stakeholders within and across the sector to maximize impact and engage and identify new CBR research networks. The framework highlights needs for greater sector-wide impact identified in various CIHR consultations and the desire for practical tools supporting partnership development, research development and implementation noted in the OAN consultation.

CONCLUSION: An evaluation framework with short-term and intermediate objectives will be implemented over four years. Ontario CBR capacity will be assessed through a variety of indicators linked to identification and operationalization of strategy-specific CBR goals, development of new partnerships and research initiatives and more extensive assessment and monitoring of CBR consultations and CBR curriculum uptake.
P195

UNDERSTANDING THE CONCEPT OF WOMEN-SPECIFIC HIV/AIDS SERVICES: A LITERATURE REVIEW

Carter, Allison J'; Bourgeois, Sonya ${ }^{2}$; O'Brien, $\mathrm{Nadia}^{3}$;

Abelsohn, Kira ${ }^{2}$; Tharao, Wangari²; Greene, Saara ${ }^{4}$;

Margolese, Shari²; Kaida, Angela ${ }^{1}$; Sanchez, Margarite ${ }^{5}$;

Palmer, Alexis $\mathrm{K}^{1}$; Cescon, Angela ${ }^{1}$; de Pokomandy, Alexandra ${ }^{3}$;

Loutfy, Mona $\mathbf{R}^{2}$

${ }^{1}$ Vancouver, BC; ${ }^{2}$ Toronto, ON; ${ }^{3}$ Montreal, QC; ${ }^{4}$ Hamilton, ON;

${ }^{5}$ Salt Spring Island, BC

BACKGROUND: The increasing prevalence of HIV among women globally and the gendered realities driving the shift in the epidemic has evoked calls for tailored services that respond to women's specific needs. We explored the concept of women-specific HIV/AIDS services to identify and define what key elements underlie this approach to care.

METHODS: We conducted a comprehensive literature search using online databases (CSA Social Service Abstracts, OvidSP, Proquest, Psycinfo, PubMed, and the CINAHL), augmented with an internet search for grey literature. Articles were reviewed and included in this analysis if they had women-specific services as their central focus and made a contribution to exploring the concept of women-specific HIV/ AIDS services. Articles were restricted to English language publications with no limit set on the date or place of publication. Owing to limited literature on this topic, we considered articles from various subject areas if they explored women-specific services in general and we discussed implications for HIV. In total, 84 articles were retrieved and 30 included for full review. Fifteen were specific to HIV/AIDS, 11 mental health and addictions, and 4 came from other disciplines.

RESULTS: A consensual definition for women-specific HIV/AIDS services was absent from the literature. This concept was distilled into its main characteristic and 12 additional dimensions. The defining feature of women-specific HIV/AIDS services was the gender of clientele and staff. Other key elements included: (1) creating a safe atmosphere; (2) facilitating interaction among peers; (3) involving women in service planning and delivery; (4) providing empowering opportunities; (5) offering tailored programming for women; (6) providing social and ancillary services; (7) facilitating access to information on women- and HIV-specific issues; (8) considering family as the unit of intervention; (9) integrating an array of services; (10) meeting women "where they are at"; (11) providing gender- and HIV-sensitive training; and (12) conducting gendered HIV/AIDS research.

CONCLUSION: Further research on women-specific HIV/AIDS services is needed to better understand this emerging concept, and ultimately assess the effectiveness of these services on HIV-positive women's health outcomes.

\section{P196 \\ "UNCOMFORTABLE SILENCES." TEACHER'S EXPERIENCES WITH HIV/AIDS EDUCATION: NARRATIVES OF FOUR TEACHERS IN A MONTREAL SCHOOL}

Cobbler, Melissa-Anne

Montréal, QC

From 2005, the Quebec Ministry of Education cut what was five hours of sex education (STIs, HIV/AIDS, gender, sexual diversity, etc.) per year from the secondary school curriculum. High schools are highly sexualized sites and teachers can be the catalyst to providing valuable and life altering information around HIV/AIDS and sexual health to their students. In the context of the Québec education reform, teachers holding specializations in English, Science \& Technology and Art Education were persuaded to integrate sexuality in their course. Through a qualitative case study, teacher narratives were collected to identify their classroom structure; strategies; awareness of HIV/AIDS; and the challenges that might have been encountered when discussing HIV/AIDS in their classroom.

Common themes from the data showed a surface-level understanding around HIV/AIDS, and a need for consistent training and access to accurate resources. Likewise, the study uncovered the subtle yet, uncomfortable silences that are included in the experience of discussing HIV/ AIDS in their classroom. Identifying how teachers understand and value 
information related to HIV/AIDS will ultimately influence how their students also comprehend the virus.

\section{P198 \\ SUBSTANCE USE AND SEXUAL PREFERENCE STRUCTURE SOCIAL NETWORKS FOR MSM IN VANCOUVER, BC: IMPLICATIONS FOR RESPONDENT DRIVEN SAMPLING (RDS)}

Forrest, Jamie I1; Stevenson, Benjamin ${ }^{1}$; Pai, Jayaram ${ }^{1}$; Michelow, Warren ${ }^{1}$; Roth, Eric $A^{2}$; Moore, David ${ }^{1}$; Hogg, Robert $\mathrm{S}^{1}$ ${ }^{1}$ Vancouver; ${ }^{2}$ Victoria, BC

BACKGROUND: In the context of conducting formative Respondent Driven Sampling (RDS) research, it can be informative to look at the interacting roles substance use and sexual preferences (ie, the intent to engage in particular sexual behaviours or with particular types of men) play in differentially structuring social networks among MSM.

METHODS: A series of semi-structured key informant interviews (KIIs) with service providers and focus group discussions (FGDs) were conducted with MSM in Vancouver. KII participants were asked about their knowledge of social, cultural and economic structures of the MSM community and the contexts in which MSM self-organize. FGDs used community mapping to explore participants' understanding of MSM community structure; post-mapping discussions probed the roles substance use and sexual preferences play in structuring MSM social and sexual networks. Descriptive analysis and an inductive approach were used to identify important features of the social and sexual network structure of the MSM community.

RESULTS: 24 KIIs and 6 FGDs with 48 participants were conducted. KIIs and FGDs were stratified by geography, HIV serostatus and age group. Participants described different social scenes in which different substance use norms were acceptable and particular substances were preferred. Important connections were identified between the substance use patterns, sexual preferences and certain venues or locales in which different behavioural norms are permitted. Participants noted that MSM interact differentially within these overlapping social scenes depending on their preferences, with examples highlighting movement between parties-bars-clubs-baths-parks/trails in pursuit of sexual satisfaction and influenced by substance use. Scenes predominantly structured by 'professional' MSM or MSM of higher socioeconomic status were less linked to overt substance use patterns.

DISCUSSION: Our preliminary analysis indicates that researchers employing RDS methodology would benefit from exploring how substance use and sexual preferences structure and influence the social and sexual networks of MSM.

\section{P199}

\section{MEETING NEW CHALLENGES ACROSS STAKEHOLDER AND DISCIPLINARY BOUNDARIES: TOWARDS A COORDINATED RESEARCH AGENDA FOR WOMEN AND HIV/AIDS IN CANADA}

Gahagan, Jacqueline'; Wertheimer, Sophie ${ }^{2}$; Binder, Louise ${ }^{3}$; Liddell, Michael'2; Duff, Ashley²; Davidson, Marnie $^{2}$

${ }^{1}$ Halifax, NS; ${ }^{2}$ Ottawa; ${ }^{3}$ Toronto, ON

BACKGROUND: The 3rd decade of the HIV epidemic has brought about a reframing of the challenges in the fight against HIV for women. A growing number of Canadian researchers, community members and other stakeholders have been examining the multifaceted issues that affect women in relation to HIV. Despite these efforts, women as a population remain under-represented in many types of HIV research in Canada. Additionally, the tendency to work within disciplinary and stakeholder silos has limited the development of a national, coordinated approach to research with and for women.

METHODS: Partners from diverse academic, community and government affiliations have been collaborating to develop a coordinated research agenda for women and HIV in Canada. Ancillary events were held in conjunction with CAHR 2009 and CAHR 2011, bringing together diverse stakeholders to identify gaps and priorities in research. The "Gathering of Spirits: Canadian Women, Transpeople and Girls' HIV Research Collaborative" was formed in April 2011. Members of the collaborative are encouraged to share resources and remain involved in a pan-Canadian dialogue on research. Working groups in the areas of knowledge translation and exchange; fundraising; research gaps and priorities; and ethics and capacity building, have been formed to devise tools and implement activities to further the work of the collaborative. CONCLUSIONS: While the development of the coordinated research agenda for women and HIV in Canada is still in its formative stages, this project has allowed stakeholders of varied disciplinary and experiential backgrounds to work collaboratively to identify key issues and gaps in research, develop research and action items, and establish new partnerships. By creating opportunities to merge theoretical knowledge and lived experience, and to increase the capacity of different stakeholders to meaningfully participate in research, this initiative seeks to ensure that research effectively includes and responds to women's realities.

\section{P200}

THE UTILITY OF THE ASSESSMENT OF MOTOR PROCESS SKILLS (AMPS) IN EXAMINING THE EVERYDAY FUNCTIONAL ASPECTS OF LIVING WITH HIV/AIDS Merritt, Brenda ${ }^{1}$; Gahagan, Jacqueline ${ }^{1}$; Kottorp, Anders ${ }^{2}$ ${ }^{1}$ Halifax, NS; ${ }^{2}$ Stockholm, Sweden

INTRODUCTION: While it has been well documented that people living with HIV/AIDS (PHAs) have higher health risks and accelerated aging, limited research has been conducted to explore the everyday functional consequences of living with HIV/AIDS from occupational and health promotion perspectives.

OBJECTIVES: To explore the validity of the Assessment of Motor and Process Skills (AMPS), a standardized performance-based evaluation of the quality of ADL task performance, for use with PHAs; and to determine if PHAs, who have been evaluated using the AMPS, demonstrate significantly lower ADL motor and/or ADL process ability measures than their healthy, age matched peers.

METHODS: Trained occupational therapists utilized the AMPS to evaluate all individuals in the data set. The sample $(n=137)$ included preexisting data records from the International AMPS database, and included men and women from diverse geographic regions who had a diagnosis of HIV/AIDS. Descriptive analyses (eg, frequencies) of the demographic factors of the data records were conducted. To evaluate the validity of using the AMPS with PHAs, the data were subjected to Rasch analysis and the resulting person goodness-of-fit-statistics were analyzed. Finally, ADL motor and process ability measures for each data record were compared to normative expectations.

RESULTS: The preliminary findings suggest that the AMPS can be validly used to evaluate the ADL ability of PHAs. Individuals in the AMPS data set had significant ADL performance limitations; the majority of whom had ADL ability measures that were well below age expectations and indicative of the need for assistance to manage community living. These findings imply that occupational therapy services within the continuum of HIV healthcare may not occur until significant health and/or functional challenges occur. From a health promotion perspective, this raises the question of the potential role of occupational therapy and access to other rehabilitation services within HIV care.

\section{P201}

HIV, CHRONIC DISEASE, AND REHABILITATION IN CANADA: A SCOPING REVIEW OF HIV POLICY APPROACHES.

Gahagan, Jacqueline; Ross, Eric; Hill-Mann, Alexandra; Walker, Sally; Lewellyn, Denver; HIV and Rehabilitation Policy Research Team, The

Halifax, NS

OBJECTIVES: With the advent of combination antiretroviral therapy, people with HIV/AIDS (PHAs) are living longer. However, health policies, including those related to eligibility for rehabilitation programs and services differ depending on federal, provincial, or regional differences and which may impact the health outcomes of PHAs. The purpose of this study was to explore policies on HIV and rehabilitation in Canada in light of the reframing of HIV as a chronic illness.

METHODS: Our search included CINAHL, PubMed, MEDLINE, ERIC, PsycInfo, and Cochrane Library for published policy documents 
related to rehabilitation service delivery, as well as grey literature sourced from AIDS service organizations, non-governmental organizations, and discipline-specific professional organization websites. Documents were reviewed by at least 1 reviewer to determine study inclusion and confirmed by 5 team members.

RESULTS: Initially 71 citations were found and determined to be relevant to our research. Eighty-two percent $(n=58)$ met our defined inclusion criteria and of these, 28 (48\%) were specific to HIV. These citations were then classified based on policies related to a) traditional rehabilitation service delivery (occupational therapy, physical therapy) and b) rehabilitation policies related to employment and income support. While policies regarding the provision of rehabilitation services were evident for chronic and episodic illness, few policies promoting or targeting the inclusion of rehabilitation as a component of HIV healthcare service provision were evident.

CONCLUSIONS: Our research suggests the need for HIV-specific considerations to be maintained in the provision of rehabilitation services due to the myriad of unique issues faced by PHAs living longer with HIV. Additionally, a need exists for rehabilitation policy considerations to move toward healthy aging across the life course, thereby taking into consideration the key deteminants of health for PHAs.

\section{P202}

\section{METHODOLOGICAL CHALLENGES IN AN ATLANTIC} CANADIAN SCAN OF YOUTH-FOCUSED PRIMARY AND SECONDARY HIV/HCV PREVENTION POLICIES

Hudson, Pamela ${ }^{1}$; Gahagan, Jacqueline ${ }^{1}$; Hare, Kathleen ${ }^{1}$;

Tucker, Ruth'; Walker, Sally'; Harris, Greg'; Dube, Anik ${ }^{3}$

${ }^{1}$ Halifax, NS; ${ }^{2}$ St John's, NL; ${ }^{3}$ Moncton, NB

BACKGROUND: The need for policy frameworks and policies related specifically to youth is well documented. As a response, this study sought to explore the existence of policies that address both primary and secondary prevention of $\mathrm{HIV} / \mathrm{HCV}$ among youth (15-24 years) in Atlantic Canada.

METHODOLOGY: Policy research trainees in the 4 Atlantic provinces identified search terms and databases in order to scan both academic and grey literature. Searches were continued until saturation was reached, and a record of databases, search terms, and number of results was maintained. Representatives of HIV/HCV-related organizations were also contacted to collect policies and program guidelines. All documents were categorized by type (formal policy, strategy, or programs); target group (youth, women, men, prison, minorities); sector from which the document came (health, education, justice, community); and type of prevention addressed (HIV $1^{\circ}$; HIV $2^{\circ}$; $\mathrm{HEPC1}^{\circ}$; HEPC $2^{\circ}$; Blood Borne Pathogens). Documents were retained on the basis of relevance, determined by independent classification by two researchers.

Methodological Challenges: A first methodological challenge involved variations across provinces, sectors, and organizations with respect to the nature of documents yielded and the effectiveness of search terms/ databases. The definition of youth also varied highly, posing difficulty in identifying policies specifically targeting the study's $16-25$ year age bracket.

RESULTS: In reviewing findings, emphasis was placed on grey literature due to limited academic documents yielded in the Prince Edward Island and Newfoundland scans. The majority of youth specific policies were found in the community sector, pertaining to programs/practices as well as primary prevention. Relatively few strategic documents were yielded, and very few policies targeted secondary prevention. Our results suggest youth prevention policies in the Atlantic region are highly fragmented. CONCLUSIONS: In order to address the methodological challenges encountered, an iterative approach in developing search terms and identifying databases is recommended. Additionally, this study exposes the need for a collaborative and cohesive policy approach to addressing HIV/ $\mathrm{HCV}$ prevention among youth in the Atlantic Provinces of Canada.
P203

WHEN IS A GOOD TIME? NEGOTIATING ETHICAL TENSIONS IN AN HIV COMMUNITY-BASED RESEARCH STUDY CONDUCTED IN A HOSPITAL SETTING

Guta, Adrian; Switzer, Sarah; de Prinse, Karen;

Chan Carusone, Soo; Strike, Carol

Toronto, ON

BACKGROUND: Conducting community-based research with people living with HIV requires maintaining both individual and collective ethical standards. Research teams need to be cognisant of individual and community conceptions of informed consent, risks and benefits, and confidentiality. Although much has been written about challenges to obtaining ethics approval for HIV CBR, there have been fewer opportunities to describe ethical issues which emerge over the 'life' of these projects. This presentation addresses this gap by describing the ethical tensions which emerged during an HIV CBR study conducted in a hospital setting.

METHODS: Using a case study analysis, this presentation draws on field notes, minutes from team meetings, and reflexive memos to offer a critical chronology of decisions made to improve ethical practice in one HIV CBR study. Various disciplinary (nursing, social work, public health) and stakeholder (clinician, coordinator, researcher, participant) perspectives are highlighted and contrasted to show how 'ethics' was understood and negotiated.

RESULTS: We discuss the range of issues that emerged at the intersections of HIV, drug use, negotiating clinical space, and piloting innovative arts-based methods. Issues of cognition and HIV required the use of a multi-step informed consent process. Research team members balanced the need to support participants with reminders while being conscious of not creating undue pressure to participate. Maintaining confidentiality proved challenging because of the way space and time are managed in a clinical setting. The sensitive nature of the study and proposed group data collection necessitated changing the study design from group to individual data collection techniques to protect confidentiality. Interestingly, this happened despite having undertaken preliminary community consultations to determine the best approach.

CONCLUSIONS: Our findings highlight the importance of understanding and integrating both individualistic and communitarian ethical concerns, remaining flexible about design issues, and maintaining clear and open lines of communication between all project stakeholders.

\section{P204}

\section{EVALUATION RESULTS OF TRAINING AN EMERGING GENERATION OF HIV RESEARCHERS IN CANADA: UNIVERSITIES WITHOUT WALLS}

Ibanez-Carrasco, Francisco ${ }^{1}$; Rourke, Sean ${ }^{1}$; Worthington, Catherine'; Bacon, Jean ${ }^{2}$; Fisher, Mark²

${ }^{1}$ Victoria, BC; ${ }^{2}$ Toronto, ON

This presentation describes a national training program for HIV researchers and summarizes the evaluation results from the evaluation of 3 cohorts since 2009 .

a) Context

Universities Without Walls (UWW) is funded by a Strategic Training in Health Research Initiative (STIHR) grant from the CIHR, and housed a the Ontario HIV Treatment Network (OHTN). It was created to design and implement a curriculum that focuses on HIV community based research, interdisciplinarity, ethics, knowledge transfer and exchange and professional development skills such as grant crafting, conference presentation skills, peer-review skills. The curriculum is individualized to each cohort depending on their needs and expectations. UWW is closely linked with the CIHR Centre for Research Evidence into Action for Community Health (REACH) in HIV/AIDS and with other HIV related training cohorts.

b) Tools and Educational Intervention

In a 9 months period, UWW offers a training stipend and provides to up to 15 Fellows per cohort ( $\mathrm{n}=41$ since 2009), a blend of up to 18 synchronous online graduate seminars and skills building sessions, 2 in-person meetings (including a 1 week long intensive retreat), field mentoring in an academic, policy or practice environment, team writing, and up to 
60 total monthly hours of individualized mentoring to current and past Fellows.

c)Evaluation and Results

The impact of the program is evaluated as academic outcomes (e.g., peer reviewed articles, academic presentations, abstracts submitted), pedagogical outcomes (e.g., changes in knowledge, skills and experience), and environmental impact over time (e.g. to faculty, mentors, HIV community leaders). In key content areas such as "ethics applied to HIV research" consistently more than $50 \%$ of Fellows progress from "novice" and "advance beginner" to "competent" and "proficient" using an assessment tool adapted from Brocklehurst \& Rowe (2003) and Kirkpatrick's evaluation framework. UWW is contributing to the practice of training emerging researchers and their research by pioneering eLearning of HIV community based research online; our results also indicate increased learning, networking (for Fellows and collaborators) and maintained involvement in HIV research post-UWW tenure.

\section{P206}

PRÉDOMINANCE DES CONTEXTES DE CONSOMMATION, DES ESPACES FRÉQUENTÉS ET DES SCÉNARIOS SEXUELS DANS L'INTENSITÉ DE LA CONSOMMATION ET LE CHOIX DES SUBSTANCES PSYCHOACTIVES CHEZ LES HARSAH AU QUÉBEC

Leobon, Alain ${ }^{1,2}$; Otis, Joanne ${ }^{2}$; Chicoine Brathwaite, Yannick ${ }^{2}$

${ }^{1}$ Angers, France; ${ }^{2}$ Montreal, QC

OBJECTIFS : caractériser les modes de consommation de substances psychoactives des répondants ayant au moins consommé une fois de l'alcool de manière intense ou une drogue au cours des 12 derniers mois $(\mathrm{n}=2606)$ selon l'enquête en ligne Net Gay Baromètre, renouvelée en 2008 au Québec ( $\mathrm{n}=3718)$.

MÉTHODE : Sur l'échantillon complet, l'analyse factorielle d'échelles d'intensité de consommation a dégagé trois facteurs de consommation. Le premier associe le cannabis et l'alcool, le second le viagra et le poppers, le troisième un ensemble plus vaste de 7 drogues récréatives.

Des régressions linéaires multiples ont testé l'influence de diverses catégories de variables sur chacun des trois facteurs.

RÉSULTATS : La consommation d'alcool et de cannabis est plus intense chez les plus jeunes $\left(\beta=-0.21^{* * *}\right)$ et chez ceux qui fréquenter les clubs ou bars conviviaux pour rencontrer leurs partenaires $\left(\beta=0.06^{*}\right)\left(R^{2}\right.$ : 0.13).

La consommation de drogues dites sexuelles est plus intense chez les plus âgés $((\beta=0.20 * * *)$, chez ceux qui souhaitent augmenter leurs performances sexuelles $\left(\beta=0.39^{* * *}\right)$ qui fréquentent les saunas pour des rencontres occasionnelles $(\beta=0.12 * * *)$, qui déclarent plus de dix partenaires occasionnels $\left(\beta=0.09^{* * *}\right)$ ou une pratique sexuelle marginale dans l'année $(\beta=0.09 * * *)$ ( $R^{2}$ ajusté : 0.44$)$.

Une consommation plus intensive de drogues dites récréatives est associée à un plus jeune âge $\left(\beta=-0.15^{* * *}\right)$, à la consommation dans le contexte de "night life" gai $(\beta=0.38 * * *)$ ou privé $(\beta=0.19 * * *)$, à une consommation combinées de drogues $\left(\beta=0.17^{* * *}\right)$ ainsi qu'à la fréquentation de la communauté gaie $(\beta=0.04 *)$ (R2: 0.38).

$(* * * \mathrm{p} \leq 0.001 ; * * \mathrm{p} \leq 0.01 ; \mathrm{p} \leq 0.05)$

CONCLUSION : Ces résultats suggèrent la prédominance des contextes de consommation, du choix des espaces fréquentés et des scénarios sexuels possiblement à risque dans la modulation de l'intensité et de la diversité des substances consommées.

Les intervenants auprès des HARSAH devraient tenir compte de la complexité de ces agencements variant selon le parcours et les préférences de ces hommes consommateurs de substances.

\section{P207}

DO SOCIO-CULTURAL VARIABLES PREDICT YOUNG ADULT'S PREFERENCES FOR THE VARIOUS TYPES AND COMPONENTS OF HIV PREVENTION INTERVENTIONS?

\section{Miranda, Joyal}

Toronto, ON

BACKGROUND: Individuals' preferences for various interventions may be reflective of their socio-cultural characteristics. Few studies have identified socio-structural characteristics, such as gender, age and education, as relating to individuals' preferences for interventions within various clinical areas. To date, few empirical studies have explored the relationship between socio-cultural characteristics and individuals' preferences for different types (educational versus cognitive/behavioral) and components of HIV prevention interventions (education, communication skills, goal attainment). The purpose of this study was to determine what socio-cultural factors predict individuals' preferences for the various types of HIV prevention interventions and components.

METHODS: The study used a cross-sectional mixed methods design. The data were collected using a face-to-face interview. Structured and open-ended questions were used to inquire about participants' preferences for intervention.

RESULTS: A total of 150 individuals participated in the study. Of the socio-structural factors hypothesized to be associated with participants' preferences for intervention type, only age had a significant association with preference for an education informational only type of intervention $(\mathrm{p}<0.05)$. Older participants were more likely than younger participants to select education only as the intervention of their choice. In examining preferences for intervention components, age and gender were significantly related to express preferences for two treatment components: education and goal attainment. Participants who were older chose the education component over combined components $(\mathrm{OR}=0.15, \mathrm{p}<0.05)$. Females preferred the goal attainment component over combined components $(\mathrm{OR}=3.8, \mathrm{p}<0.05)$.

CONCLUSION: In the past, clinicians have sought to understand and account for patients' preferences. Such an understanding, allows clinicians to select interventions that are consistent with individuals' preferences and socio-cultural characteristics. The contributions in providing interventions that match individual's socio-cultural characteristics and preferences, have shown to increase individuals adherence and satisfaction with the intervention in addition to increasing the interventions effectiveness.

\section{P208}

BARRIERS TO TREATMENT AS PREVENTION: THE NEED FOR A UNIVERSAL DRUG COVERAGE PLAN IN CANADA Reinhard, Robert J; Binder, Louise; Stephenson, Leah Toronto, ON

"Treatment as prevention" (TasP) is a promising approach to curb the incidence of HIV in Canada and avert costs resulting from lifetime treatment of individuals who become infected with the virus. Averted lifetime care and treatment costs per individual are substantial, according to published estimates. TasP uses antiretroviral treatment to reduce the amount of infectious virus in infected individuals capable of being transmitted to others. Prevention success with TasP has been suggested by recent reports of a reduced number of HIV infections in British Columbia. Informed individual patient choice of time to initiate and continue on treatment as well as broad access and coverage are necessary to yield beneficial prevention results on a population wide basis.

In Canada the decision to have a publicly funded drug plan and its terms remain a provincial/territorial decision except for federally covered plan holders. A universal drug coverage (UDC) plan across the country for PLWHA mitigates inconsistent conditions for coverage and inequities that may cause many individuals to experience suboptimal care affecting their own health. Suboptimal care includes situations of inadequately controlled or uncontrolled virus reproduction during periods of disease progression or due to late diagnosis and care.

Introducing a UDC plan for Canada faces significant obstacles in design and acceptance. To secure UDC, the plan must also be included in proposals towards the structure of the Health Accord of 2014.

In addition to achieving equitable treatment and care benefits for PLWHA in need of essential medicines for their own health, a UDC plan stands to add new tools to implement cost effective means to reduce HIV incidence in Canada. 


\section{P209}

\section{WHAT IS THE DOMAIN STRUCTURE OF THE HIV} DISABILITY QUESTIONNAIRE (HDQ)? AN EXPLORATORY

\section{FACTOR ANALYSIS}

$\mathrm{O}^{\prime}$ Brien, Kelly K${ }^{1}$; Solomon, Patricia ${ }^{1}$; Bayoumi, Ahmed $\mathbf{M}^{2}$;

\section{${ }^{1}$ Hamilton; ${ }^{2}$ Toronto, ON}

OBJECTIVE: To assess the domain structure of the HIV Disability Questionnaire (HDQ), a 72 item self-administered questionnaire developed to describe the presence, severity and episodic nature of disability experienced by people living with HIV (PHAs).

METHODS: We recruited PHAs from hospital clinics, AIDS service organizations and a specialty hospital and administered the HDQ followed by a demographic questionnaire. We conducted an exploratory factor analysis using disability severity scores to determine the domain structure of the HDQ using the following steps: a) We assessed the correlation matrix of item scores for correlations $\geq 0.30$ and $\leq 0.80$; b) conducted a principal components analysis to extract factors; c) used the Scree Test and Kaiser Criterion (eigenvalues $>1.5$ ) to determine the number of factors to retain; and d) oblique rotation to simplify the factor loading matrix. We defined factor loadings $\geq 0.30$ as loading on a given factor.

RESULTS: Of the 361 participants who completed the HDQ, 80\% were men and $77 \%$ reported living with at least two concurrent health conditions in addition to HIV. One item, (gaining weight) did not correlate with any other items $(\geq 0.30)$ and was removed from subsequent analysis. The exploratory factor analysis suggested retaining six factors. Two items (constipation and housing) did not load on any factor $\geq 0.30$ and one item (afraid for my future) cross-loaded $(\geq 0.40)$ on two factors. Items related to symptoms and impairments tended to load on three distinct factors (physical, cognitive, emotional and mental health impairments) and items related to worrying about the future, daily activities, and personal relationships loaded on three additional factors (uncertainty, difficulties with day-to-day activities, social inclusion). The HDQ appeared to have six domains: physical symptoms and impairments (20 items); cognitive symptoms and impairments ( 3 items); emotional and mental health symptoms and impairments (11 items); uncertainty (14 items); difficulties with day-to-day activities ( 9 items); and challenges to social inclusion (12 items).

CONCLUSIONS: The HDQ has six domains. These domains indicate the scoring structure of the HDQ. Next steps include item reduction and HDQ revision.

Health Care and Services to PWHIV Soins et services aux PVIH

\section{P210}

DEVELOPING EVIDENCE-INFORMED

\section{RECOMMENDATIONS ON REHABILITATION FOR} OLDER ADULTS LIVING WITH HIV: LESSONS LEARNED SYNTHESIZING THE EVIDENCE

$\mathrm{O}^{\prime}$ Brien, Kelly K${ }^{1}$; Solomon, Patricia ${ }^{1}$; Trentham, Barry ${ }^{2}$;

Baxter, Larry²; Chegwidden, Will3; Robinson, Greg²;

Wu, Janet ${ }^{2}$; Tynan, Anne-Marie ${ }^{2}$; Zack, Elisse ${ }^{2}$

${ }^{1}$ Hamilton; ${ }^{2}$ Toronto, ON; ${ }^{3}$ London, United Kingdom

OBJECTIVE: To describe the lessons learned developing evidenceinformed recommendations on rehabilitation for older adults living with HIV.

METHODS: We conducted a knowledge synthesis, combining research evidence specific to HIV, rehabilitation and aging, with evidence on rehabilitation interventions for common comorbidities experienced by older adults with HIV. We searched for and included: a) published evidence on HIV, rehabilitation and aging and b) high-quality evidence (systematic reviews and meta-analyses) on the effectiveness of rehabilitation interventions for comorbidities commonly experienced by older adults aging with HIV (bone and joint disorders, cancer, cardiovascular disease, mental health, neurocognitive decline, cardiopulmonary disease, diabetes). We extracted relevant data from included studies and synthesized the available evidence to draft evidence-informed recommendations on rehabilitation for older adults aging with HIV. Draft recommendations were circulated to an interprofessional team for GRADE rating and suggestions for refinement. We reviewed the strengths and challenges of our process developing these recommendations.

RESULTS: The synthesis included 165 studies (50 specific to HIV rehabilitation and aging and 115 studies on rehabilitation interventions for common comorbidities). Strengths of our approach included: incorporating PHA and clinician values and preferences in the process, incorporating high level evidence on rehabilitation interventions, and implementing the GRADE rating process to facilitate revision. Challenges included: defining 'common' comorbidities experienced by older adults with HIV for inclusion; the paucity and low level of research evidence on rehabilitation specific to older adults with HIV, our uncertainty of how to weight research evidence with PHA and clinician values and preferences, combining two areas of research evidence that differ in quality and context, and determining the format of the recommendations that will be most useful to clinicians and older adults with HIV.

CONCLUSIONS: Developing evidence-informed recommendations on rehabilitation for older adults with HIV is challenging, particularly synthesizing research evidence from two different areas of literature, while incorporating PHA and clinician values and preferences. These findings have applicability to others developing recommendations in areas where research evidence is still emerging. Next steps include recommendation refinement and endorsement.

\section{P211}

CLIENT INVOLVEMENT AS VOLUNTEERS: A NEEDS ASSESSMENT ASSESSING THE DIMENSIONS OF GREATER AND MEANINGFUL INVOLVEMENT OF PEOPLE LIVING WITH HIV/AIDS

Ahluwalia, Amrita; Blair, Michael; Campbell, Connor; Ndyanabo, Emmanuel ; Ndungu, Jacqueline; Byles, Mark; Allan, David; Lindsay, Joanne; McGiffin, Chris Toronto, ON

BACKGROUND: Peer involvement has been recognized to positively impact self-care, access to services, social support, and self-efficacy. However, issues of confidentiality and boundaries are often reasons preventing involvement of clients as volunteers. To enhance the greater and meaningful involvement of people living with HIV/AIDS (PHA), the objective of this needs assessment study was to explore the meaningful ways and opportunities to engage PHA clients as volunteers and to develop an understanding of the benefits and challenges of such involvement.

METHODS: Eight focus groups were conducted over a period of four months with 40 participants: thirty clients; two current PHA volunteers and eight staff members of Fife House, the largest provider of supportive housing services to PHAs in Canada. A 'bottom-up' approach is central to this assessment. Peers were involved in all stages of the assessment. The assessment included two-phases: first, training of peers in focus group facilitation skills and recruitment of participants; second, conducting the focus groups and analysis of data. The focus groups were tape recorded and transcribed. The data were analyzed using thematic analysis.

FINDINGS AND RECOMMENDATIONS: The results reveal that while volunteer engagement provides opportunity for personal growth, enhances self-worth and reduces social isolation, volunteer work assigned is generally incongruent with the level of skills and experience. The probability of volunteer engagement is higher if the opportunity existed within the agency providing services. This kind of engagement however, requires developing consistent structures of support. Challenges identified included incongruent placement, inadequate trainings, policies preventing such engagement, issues of confidentiality and boundaries in dual role structure, conflicting expectations and varying work ethics. Recommendations include development of relevant policies and structures to protect client confidentiality, developing appropriate communication strategies, formal complaint procedures, structured review process, and trainings. 
P212

A QUALITATIVE STUDY OF THE HELP-SEEKING EXPERIENCES OF HETEROSEXUAL MEN LIVING WITH HIV Antoniou, Tony; Loutfy, Mona; Glazier, Richard H; Strike, Carol Toronto, ON

BACKGROUND: Despite the increasing importance of heterosexual transmission in sustaining the HIV epidemic in developed countries, very little empirical research exists describing the health and help-seeking experiences of heterosexual men living with the illness. We sought to characterize the help seeking experiences of heterosexual men living with HIV and explain these experiences in relation to the broader social relations in which they are embedded.

METHODS: We conducted a qualitative study using focus groups and theoretically informed constructivist grounded theory. A total of 40 men aged 18 years and older participated in the study. With one exception, focus groups were conducted in the offices of community-based AIDS service organizations across Ontario, Canada.

RESULTS: Heterosexual men living with HIV are relegated to the margins of a health care and service field that was developed historically within a context that privileges the priorities of gay men and heterosexual women living with the virus. Specifically, gay men are better positioned than heterosexual men when vying for the services and recognition of AIDS service organizations due to their social capital within these agencies, thereby benefiting by virtue of their membership with the group perceived to control the decision making apparatuses when resource allocation and program development are at stake. Relative to women, heterosexual men are poorly positioned due to their negative symbolic capital, derived from being perceived as the 'guilty' parties in the context of heterosexual HIV transmission. As a result, the material and support needs of women have been prioritized, while those of heterosexual men living with HIV remain largely unaddressed.

CONCLUSIONS: Heterosexual men living with HIV are operating within a health and service field that has not kept pace with their increased representation among the population of persons living with the virus. The implications of these findings for researchers and policy-makers are discussed.

\section{P213}

SPANISH-SPEAKING INTERVENTION FOR LATINO PHAS IN ONTARIO: A FINAL EVALUATION (WWW.TURADIOPLUS. PODOMATIC.COM) OF THE PILOT PROJECT

Betancourt, Gerardo

Toronto, ON

PLAIN LANGUAGE SUMMARY: There is a lack of Spanish HIV/ AIDS services in rural-small urban settings in Ontario for Latino People Living with HIV (PHAs) due to geographic access, language barriers and the effect of stigma and discrimination. An intervention to overcome those services delivering and access barriers was conducted with 12 internet pod-cast radio shows in Spanish as a way to connect with Latino PHAs in Ontario.

ISSUES: In rural and small cities in Ontario Latino immigrants who do not speak the official languages are in disadvantage when seeking counselling, information and services in Spanish. This situation is aggravated for Latino PHAs due to the social stigma attached to living with HIV and the multiple axis of discrimination within the intersection of race, sexual orientation, immigration status and HIV serostatus.

DESCRIPTION: Shire laboratories provided funding in order to develop a series of 12 radio -internet- pod-cast programs that would be recorded and uploaded on www.turadioplus.podomatic.com The intervention's objectives were: a) Reduce experiences of isolation among Latino PHAs in Ontario. b) Provide information relevant for this community such as: medical referrals, social services, legal (immigration and HIV criminalization), recreational, etc. c) Promote a space for critical discussion of post-prevention condom use. d) Reduce the impact of discrimination and stigma. e) Provide opportunities for contact with Spanishspeaking counselling and education services.

RESULTS: 12 shows in Spanish. Total of visits to www.turadioplus. podomatic.com ( $n=276)$, distribution per city: Hamilton, $\mathrm{ON}(\mathrm{n}=220)$; Toronto, ON ( $\mathrm{n}=48)$; Montreal, QC ( $\mathrm{n}=5)$; Winnipeg, MB ( $\mathrm{n}=2)$; Edmonton, $\mathrm{AB}(\mathrm{n}=1)$.
LESSONS LEARNED: - Limited resources are difficult barriers at producing and editing pod cast radio shows.

- Despite the fact that the intervention was designed for Latino PHAs living in Ontario, it is interesting to observe visits from other geographic locations, which shows the great need of cultural/language oriented interventions for minority groups in other regions of Canada.

RECOMMENDATIONS: - In intervention with more resources and better theoretical frameworks should be planned and evaluated with better quantitative and qualitative approaches to measure real impact at the community level.

\section{P214}

FACTORS ASSOCIATED WITH MENTAL HEALTH RELATED QUALITY OF LIFE AMONG OLDER ADULTS LIVING WITH HIV

Brennan, David J1 ; Emlet, Charles A2 ; Brennenstuhl, Sarah'; Rueda, Sergio ${ }^{1}$; Hart, Trevor A $^{1}$; Karapita, Stephanie ${ }^{1}$;

Ibanez-Carrasco, Francisco ${ }^{1}$; Rourke, Sean ${ }^{1}$

${ }^{1}$ Toronto, ON; ${ }^{2}$ Tacoma, WA, USA

BACKGROUND: While HIV treatments have extended the lives of those living with HIV, there has been a concurrent increase in new infections among adults over age 50 . Older adults are less likely to be screened and often are diagnosed at a much later point in the spectrum of HIV disease. Recent evidence suggests that older adults living with HIV face a myriad of physical and mental health challenges. However, factors associated with resiliency among this population are rarely examined. This study sought to understand factors associated with better mental health-related quality of life (MHQOL) among older adults living with HIV in Ontario.

METHODS: This cross-sectional study analyzed data on 383 older adults (age 50-86) who participated in the Ontario HIV Treatment Network Cohort Study (OCS) and completed extensive interviewer assisted questionnaires between September 2007 and March 2010. Using multiple linear regression, differences between heterosexual men, gay/ bisexual men (GBM) and women in the total standardized score of the SF-36 Mental Component Scale (MCS) were analyzed, while accounting for socio-demographic (age, income, education, race, marital status), positive (social support, mastery) and deleterious (maladaptive coping, stigma, cigarette smoking) psychosocial and health-related factors.

RESULTS: Heterosexual men reported comparable MCS scores $(\mathrm{M}=45.90)$ as compared to GBM (46.52); however, women reported significantly lower scores $(\mathrm{M}=44.24, \mathrm{p}<0.01)$, even after controlling for socio-demographic, psychosocial and health-related variables. Variables predicting better MCS scores included Black/African racial identity $(\mathrm{p}=0.05)$, Emotional-Informational social support $(\mathrm{p}<0.001)$, and mastery $(\mathrm{p}<0.001)$; the latter two variables accounted for $25 \%$ of the variance in the fully adjusted model.

IMPLICATIONS: These results advance our knowledge of the needs and assets of older PHAs. Further research is needed to understand the mechanisms of how these factors enhance mental health-related quality of life among older adults living with HIV.

\section{P215}

WHAT MAKES SOMEONE A LONG-TERM SURVIVOR?: DECIPHERING THE MULTIPLE MEANINGS OF THE LONG-TERM SURVIVORSHIP OF HIV

Cattaneo, Jessica $S^{1}$; Husbands, Winston $\mathrm{C}^{1}$; Karapita, Stephanie ${ }^{1}$; Murzin, Kate'; King, Kenneth ${ }^{1}$; Carusone, Soo $\mathrm{C}^{1,2}$

${ }^{1}$ Toronto; ${ }^{2}$ Hamilton, ON

The "Living with HIV over the Long Term" catalyst study examines issues related to long-term survivorship, including the experiences and priorities of people living long term with HIV, and the impact of the long-term use of health and social services. Led by community-based researchers, this study addresses the challenges that services providers and clinicians face in understanding the changing needs of this diverse population.

The concept of long-term survivorship of HIV/AIDS emerged early in the epidemic. Though it is ambiguously defined, "long-term survivor" remains part of the lexicon of HIV care, support, and lived experience. In 
this presentation we explore the multiple uses and meanings attributed to the term long-term survivor.

We conducted 4 focus groups with people who identified as living long term with HIV. 31 people participated, ranging from 24 to 70 years old. We also carried out 14 interviews with a range of services providers working in AIDS service organizations, hospitals, and clinics. We ask all of our participants to define long-term survivor and if and how they use the term.

Many participants began with what they thought was a clear definition, to have it unravel through discussion and debate. In working through their understandings and experiences of long-term survivorship, participants would work to situate the place of HIV on their or their clients' lives. By examining the differences, disagreements, and associations that emerged in our discussions about long-term survivorship, this research draws attention to assumptions regarding HIV as a chronic and episodic illness.

\section{P216}

NEED FOR ORGANIZATIONAL TOOLS AND PROCESSES IDENTIFIED THROUGH PARTICIPANTS IN TURNING TO ONE ANOTHER PHA ENGAGEMENT PROJECT IN

\section{ONTARIO}

Demetrakopoulos, Anna S; Perreault, Yvette; Samuels, Claudette;

\section{Leaver, Chad}

Toronto, ON

OBJECTIVES: The Greater Involvement of People Living with HIV/ AIDS (GIPA) Principle, adopted by the UNAIDS in 1999, affirms that PHAs be fully involved and integrated in the development of policies and programs providing HIV care, prevention, treatment and research. In an effort to facilitate and accelerate the GIPA principle in the Ontario context, ABRPO has coordinated 'Turning to One Another' (TTOA) as an annual PHA facilitator training program and collaborative community development initiative involving 13 pilot site ASOs.

METHODS: Both PHA Facilitator trainees and ASO frontline staff $(n=35)$ participate in an annual Joint Planning Meeting for 2.5 days where successes and challenges, and planning for the next project stages takes place. Structured feedback, event evaluation and a post-1 questionnaire at three months through survey monkey were used to gather input from participants.

RESULTS: In June, 2011, post-1 qualitative data was received from almost $50 \%$ of participants. Their input addresses participant perception of priorities, goals and possibilities at both an individual and community level. Participants have concrete goals around program and event development, with ten follow-up projects identified. Feedback highlights key challenges of managing the flow of information and creating stronger, more effective relationships within the organizations at all levels.

CONCLUSIONS: In response to verbal and written feedback from the participants, new tools and processes have been and will continue to be created to support practical planning and greater clarity of working relationships at the local level. On-going dialogue and evaluation of this pilot is needed in order to address unique challenges with distinct communities. All materials generated by this project, including funding proposals will be shared with those pursuing similar provincial or local initiatives.

\section{P217}

ESSENTIAL ELEMENTS OF SUCCESSFUL ADULT LEARNING TECHNIQUES UTILIZED IN PHA FACILITATOR TRAINING: LEARNING STYLES, LEADERSHIP AND DIALOG IN A SAFE ENVIRONMENT

Demetrakopoulos, Anna S; Perreault, Yvette; Samuels, Claudette; Leaver, Chad

Toronto, ON

OBJECTIVES: In 2009, the AIDS Bereavement and Resiliency Program of Ontario (ABRPO) and the Ontario AIDS Network (OAN) initiated a 10-day training program for people living with HIV/AIDS (PHAs). The program, entitled PHA Facilitator Training, is a collaborative initiative with PHAs and ASOs, and includes defined training objectives, competencies, and evaluation. This initiative aims to facilitate and accelerate the GIPA principle in the Ontario context and to enhance the leadership and community facilitation skills of PHAs.

METHODS: The three-part, 10-day training program was free to participants and open to PHAs who were actively involved in their local ASO. Two series were conducted between 2009-2011, with each beginning with 22 participants. All sessions included longitudinal qualitative and quantitative evaluation using Structured Feedback Forms rating all aspects of the training (facilitators, learning tools and practical resources, process and overall contextual factors eg, achievement of learning objectives, overall learning outcomes and components of the learning setting).

RESULTS: PHA community facilitators confirmed the benefit and action-oriented impact of a holistic, learner-centred process that was designed to foster multiple opportunities for experiential learning, resources, and feedback from peers. Learning tools/resources, training format, and achievement of learning goals were consistently rated: 'good or excellent'. Participants identified key elements of the training as: learning styles (Kolb, 1984); small group work, where they demonstrated leadership through co-accountability; and dialog in a safe environment through direct peer support and structured feedback supported by the Code of Conduct.

CONCLUSIONS: These identified elements played a critical role in the success of these trainings. This comprehensive support is imperative to help PHA community facilitators in Ontario's ASOs integrate into new roles. Emerging leaders need support to maintain and develop skills in these areas though ongoing engagement, training and leadership development for PHA community facilitators.

\section{P218 \\ GAY POZ SEX (GPS): QUALITATIVE FINDINGS OF A COMMUNITY BASED COUNSELLING INTERVENTION FOR HIV-POSITIVE GAY AND BISEXUAL MEN}

Hart, Trevor ${ }^{1}$; Adam, Barry ${ }^{2}$; Stratton, Natalie ${ }^{1}$; Maxwell, Johnn'; MacKay, Robert ${ }^{1}$; Hoe, David ${ }^{1}$; Leahy, Robert ${ }^{1}$; Murray, James ${ }^{1}$; Loutfy, Mona ${ }^{1}$

${ }^{1}$ Toronto; ${ }^{2}$ Windsor, ON

INTRODUCTION: Despite high HIV prevalence among gay and bisexual (GB) men, there is a lack of empirically supported programs targeting GB men, and even fewer targeting HIV-positive GB men. Gay Poz Sex (GPS) is a peer-facilitated sexual health program for HIV-positive GB men, which aims to decrease HIV and STI transmission by empowering HIV-positive GB men to make positive changes to their sexual health. Previous results showed GPS participants were less likely to engage in unprotected anal intercourse from baseline to 3-month follow-up $(p=0.008)$. The present study assesses the acceptability and effectiveness of the program from the perspective of the participants.

METHODS: The GPS program, administered by the AIDS Committee of Toronto, delivers Motivational Enhancement Counselling to groups of 5-8 GB men. Over the course of seven 2-hour weekly sessions, GPS guides participants through the development and identification of individualized sexual health behaviour goals, strategies and potential supports. Two men from each GPS group $(n=8)$ were randomly selected to complete a semi-structured interview at post-intervention regarding their experiences in the program. Interviews were transcribed and examined using thematic analysis.

RESULTS: In qualitative interviews, participants reported high satisfaction with the GPS program. Participants noted several strengths, the most common including having the opportunity to interact with other men with common experiences as HIV-positive GB men, feeling safe discussing difficult topics, and feeling supported by the peer facilitators. Most participants recommended that the GPS program be longer than 7 sessions. When asked about GPS outcomes, participants reported learning new strategies that helped them improve their sex lives. All men endorsed GPS and had already referred a peer to GPS.

DISCUSSION: The present study shows that peer facilitators can be effective at promoting sexual health among HIV-positive GB men, making this program implementable in community organizations. The program was also effective at improving participants' sexual health. 
P219

\section{GRASSROOTS TO HIV CHAMPIONS: MEANINGFUL ENGAGEMENT OF PHA IN COMMUNITY-BASED ACTION RESEARCH}

Wong, Josephine P; Luyombya, Henry; Li, Alan; Bisignano, Alex; Hui, Christian; Corroy, Francisco; Maitlan, Dale; Poon, Kenneth Toronto, ON

Although the GIPA principle was formalized in 1994 at the Paris AIDS Submit, PHAs continued to experience barriers to meaningful participation in HIV research and policy development. Common factors contributing to these barriers include resources constraints, organizational commitments, gaps in knowledge and skills, HIV stigma and lack of engagement opportunities. To address these barriers, the Committee for Accessible AIDS Treatment (CAAT) developed the Grassroots to HIV Champions capacity building approach to engage PHAs in HIV community-based research since 2006. Challenging the common practice of contracting PHAs as research assistants to conduct recruitment and data collection, CAAT engages PHAs in the roles of project coordinators and research associates who function as active research team members throughout all phases of the research projects. PHAs research capacity building was achieved through implementation of an intensive multi-phase research training, which integrated three domains of learning - cognitive, emotive and experiential learning. Over the past 5 years, CAAT has engaged over 35 PHA research associates and knowledge translation and exchange (KTE) ambassadors. Lessons learned from the Grassroots to HIV Champions approach include: (1) collaborative learning that takes into account of PHA lived experiences and transferrable skills is key to program success; (2) competing priorities at the individual and organizational levels affect program outcomes; (3) meaningful engagement of PHAs in CBR strengthens the outcomes of every phase of a study; (4) effective implementation of GIPA principle in HIV research requires equitable community-campus partnerships; and (5) research capacity building is an effective strategy to develop and mobilize HIV championship among PHAs.

\section{P220}

ADDRESSING THE HIV STIGMA CYCLE THROUGH EDUCATION AND PHA INVOLVEMENT

Mahood, Gail $^{1}$; Donatelli, Jolene ${ }^{2}$

${ }^{1}$ Toronto, ON; ${ }^{2}$ Montreal, QC

THE CONTEXT: HIV stigma is experienced on both institutional and personal levels. As a result of stigma, people living with HIV/Aids do not always receive consistent care or lack health care, community and family support, their basic human rights are violated and they become increasingly marginalized. Research demonstrates how stigma is cyclical and the cycle of stigma leads to increased HIV infection rates within the community at large.

THE CHALLENGE: In order to break the stigma cycle and decrease the risk of increased infection rates, education campaigns are needed in targeting the Canadian government and legal system, Canadian Health Care system, employment environments, human rights organizations and the general public. There remains a critical need to address the stigma cycle and reduce the high risk factors through education and raising awareness of how stigma increases the rate of HIV infection and decreases effective treatment, care and support.

THE APPROACH: CTAC is creating Tools for Access workshops to address systemic access to treatment issues, including HIV-related stigma and discrimination. The workshops target PHAs and people co-infected with HIV and viral Hepatitis and health care and service providers working with PHAs and co-infected people, including those from specific populations such as Aboriginal peoples and young people. These workshops are facilitated by PHAs and co-infected people.

\section{KEY FINDINGS:}

- encourage increased involvement, visibility of PHAs, and disclosure of status to break silence

- encourage self care, effective treatment and ongoing adherence to medication by facilitating access to health care

- address stigma before a reported incident, rather than after the fact

- increase HIV education, training, publicity through HR departments in the work place, among health care providers, in community centers, general public, media campaigns, presentations of new HIV research findings to key groups

- engage in community outreach

- place emphasis on cultural aspects of HIV education

- take legal action; write letters to ombudsmen and human rights commissions

\section{P222}

POSITIVE OUTLOOK: WORKING WITH HIV, REHABILITATION AND MENTAL HEALTH WITH FRONT-LINE ASO STAFF AND REHABILITATION PROFESSIONALS

Porch, Wendy; Lewis, Jordan; Dolan, Le-Ann

Toronto, ON

BACKGROUND: Depression has long been recognized as a primary health concern among people living with or vulnerable to HIV (WHO 2008). In Canada and internationally, depression is an issue that affects people living with HIV/AIDS (PHAs) in all geographical locations (BC Prevalence Study 2004, Williams 2005 and WHO 2008). This is often a difficult and challenging issue for front-line workers who, in most cases, are not provided with training on how to deal with this potentially debilitating condition (Trap Doors, 2008). Front-line HIV workers and rehabilitation clinicians, in turn, may experience frustration at their lack of ability to assist in addressing their client's mental health issues.

METHODS: The Positive Outlook Project addressed these issues through building upon previously developed curriculum by the Ontario HIV Treatment Network and the Ontario AIDS Network and providing targeted training to front-line ASO staff and rehabilitation clinicians on working with clients experiencing episodic illnesses and showing signs of depression.

A needs assessment was undertaken to determine local population needs in Canadian cities. Ten workshops were offered across Canada. Each workshop involved a local co-facilitator who was also familiar with local resources and community needs. All workshop participants were engaged in pre/post workshop evaluations. Workshop participants were also interviewed several months after the workshops to discuss any longer term impact the training may have had on their work practices.

RESULTS: Final evaluation results will be available in April 2012. Preliminary feedback from workshop participants suggests that this training increases participant capacity for recognizing signs of depression in clients and increases their confidence in assisting clients. It also increases participant familiarity with a range of intervention modalities which can be used in interacting with clients experiencing mental health issues. Participants also report increased awareness of local mental health resources to assist their clients.

CONCLUSIONS: Preliminary participant feedback from indicates that the training participants have received through the workshop has increased their capacity to recognize and to interact with clients who may be experiencing depression and/or other mental health issues.

\section{P223}

WHAT'S THE BUZZ ABOUT BUZZ?

Robinson, Linda

Windsor, ON

OBJECTIVE: BUZZ is a workshop designed to enhance communication between patients and Health Care Providers (HCPs) and results to date will be described.

BACKGROUND: A study of HIV patients interviewed about their treatment, identified several barriers to communication with their health care teams. Another study called BEAHIV, demonstrated low levels of agreement between patients and HCPs surrounding bothersome symptoms of ARV treatment. With a grant from Janssen, a workshop was designed by a faculty of HIV clinicians from across Canada, in conjunction with a behavioural specialist. The workshop covered several topics: identifying barriers to communication for HIV patients, learning about one's own preferred communication style, how to determine a patient's communication style, and ways to uncover issues associated with the patient's treatment, such as ARV associated side effects. To date, 15 workshops have been conducted across the country. 
RESULTS: At the end of the workshop, $85 \%$ of participants said they would start or continue to identify barriers to communication. At the start of each workshop, several before/after questions were asked. Only $45 \%$ of HCPs felt comfortable in adapting their communication towards patients with different behavioural styles prior to participating in the workshop. This increased to $84 \%$ after the workshop. Prior to the workshop, $68 \%$ of HCPs said they currently engage patients in proactive discussions surrounding ARV associated side effects, increasing to $82 \%$ post workshop. Prior to the workshop, $60 \%$ proactively engaged patients on the impact of ARV side effects on quality of life, which increased to $80 \%$ post workshop. Several weeks after each workshop, a follow up with participants was conducted and it was found that many implemented changes in their practices as a result of what they learned.

CONCLUSION: A workshop designed to enhance communication may have a beneficial effect on reducing communication barriers and enhancing HIV patient care.

\section{P224}

\section{ARE DENTAL COSTS GIVING YOU A TOOTHACHE? PLACING ORAL HEALTH ON THE POLICY AGENDA}

\section{Sangster, Mike; Rosenes, Ron; Stephenson, Leah M} Toronto, ON

ISSUES:

1. PHAs' oral healthcare access challenges relating to their susceptibility to many oral health problems beyond those found in the general population

2. Prohibitive costs and limited public coverage of oral healthcare creating access barriers for many PHAs

3. Stigma and discrimination that still exists amongst dental care professionals who deny PHAs care despite Canadian Dental Association guidelines.

DESCRIPTION: CTAC's oral healthcare "Tools for Access" workshop and draft position paper have been developed to offer recommendations for the future of oral healthcare access for Canada's PHAs. The longterm goal, with the input of all stakeholders, is to affect positive change in accessing oral healthcare, whereby all PHAs in Canada have access to affordable, equitable, and effective oral care, especially those living on low or fixed incomes and with compromised immune systems.

LESSONS LEARNED:

1. Oral health is integral to the overall physical and mental wellbeing of all Canadians, especially those living with HIV and other chronic illnesses.

2. The high cost of dental procedures discourages people on low and/or fixed incomes from accessing proper oral care and treatment.

3. Stigma and discrimination still exists amongst dental care professionals towards their $\mathrm{HIV}^{+}$patients.

4. Education about oral care and HIV is needed, for PHAs and dental care professionals.

\section{RECOMMENDATIONS:}

1. ASOs need their staff to be trained to understand the importance of oral healthcare and good oral hygiene for their clients, and to educate PHAs on the importance of oral health.

2. Canadian and Provincial Dental Associations and ASOs should work together to ensure equitable and affordable access to treatment.

3. Ensure that PHAs living on low and/or fixed incomes have access to the full range of dental services and treatments.

\section{P225}

GETTING TO WORK: PROMOTING AND ASSESSING KNOWLEDGE UPTAKE OF TOOLS TO PROMOTE LABOUR FORCE PARTICIPATION FOR PEOPLE LIVING WITH HIV IN

\section{CANADA}

Worthington, Catherine ${ }^{1}$; Mangion, Martine ${ }^{2}$; Popiel, Melissa ${ }^{2}$; Salbach, Nancy²; O'Brien, Kelly ${ }^{3}$; Rueda, Sergio²;

Solomon, Patricia ${ }^{3}$; Oliver, Brent ${ }^{4}$; Mollison, Ashley ${ }^{1}$

${ }^{1}$ Victoria, BC; ${ }^{2}$ Toronto; ${ }^{3}$ Hamilton, $\mathrm{ON} ;{ }^{4}$ Calgary, AB

OBJECTIVES: As part of a previous study, members of this research team produced a conceptual framework called The HIV and Employment Framework (The Framework) that was summarized in a booklet and a series of fact sheets to highlight labour force participation issues for people living with HIV (PHAs) in Canada. The purpose of this knowledge translation study was to promote and evaluate knowledge uptake of these tools among human resources professionals, vocational rehabilitation workers and AIDS service organization staff.

METHODS: The research team presented The Framework in three webinars and invited participants to continue to engage with the knowledge tools by receiving three email updates in a three-month period. Case-based examples were used in each email to prompt participants to explore ways in which The Framework could be applied to their work. After the final email update, participants were invited to attend a focus group to provide feedback on the tools and discuss their use in practice. An advisory committee that included PHAs, vocational rehabilitation professionals, employers and service providers guided this research process.

RESULTS: Of the 49 members of the target audiences who attended a webinar, 9 agreed to receive the email updates and participate in the focus group. We produced a set of learning tools that were successful in prompting participants to use The Framework in practice. A webinar presentation, three case-based email updates and a 10-page Q\&A document were produced to accompany The Framework booklet and fact sheets. Participants in the focus group $(n=4)$ reported that they liked and used the tools, gained new knowledge, changed the way they approached client interactions and, in some cases, shifted organizational practices to enhance labour force participation for PHAs and for clients with other chronic conditions.

DISCUSSION: This electronically-based knowledge translation approach was accessible for participants and helped to raise awareness and influence practices on labour force participation issues for PHAs.

HIV Vulnerabilities

Vulnérabilités menant au VIH

\section{P226}

\section{FACTEURS DE PROTECTION ET DE VULNÉRABILITÉ AU VIH CHEZ LES JEUNES AYANT SUBI DES ABUS À CARACTÈRE SEXUEL ET ÉMOTIONNEL DURANT L'ENFANCE : ANALYSE DE L'IMPACT DES DÉTERMINANTS SOCIAUX SUR LA SANTÉ SEXUELLE DES JEUNES CANADIENS}

Babin, Caroline

Ottawa, ON

Certains jeunes sont sujets à un risque d'exposition au VIH plus élevé dû à une exposition accrue à certains facteurs de risque. Ces jeunes constituent un des groupes prioritaires identifiés par l'Agence de la santé publique du Canada. Un rapport de la littérature canadienne répertoriant des données probantes sur les facteurs de risque et de protection au VIH, selon un cadre d'analyse des déterminants sociaux, a pour objectif de faire l'état de la situation de ce groupe cible.

Ce rapport est développé en consultation avec un groupe national d'experts provenant de différents horizons soit, recherche, organisations communautaires, analystes de politiques et représentants du groupe cible. La littérature et les projets inclus les publications entre 2004 et 2011 sur la démographie, l'épidémiologie, les facteurs sociaux, la recherche et les initiatives.

Certains groupes de jeunes sont exposés à des facteurs de risque multiples dû aux contextes de marginalisation et de désaffiliation sociale auxquels ils sont affrontés. La compréhension de l'influence des déterminants sociaux sur la santé sexuelle des jeunes est nécessaire à la contextualisation des problématiques sociales et individuelles. Une enfance saine a été rapportée par la littérature comme étant un élément déterminant dans le développement et l'adoption des facteurs de risque et de protection. Labus sexuel et émotionnel ainsi que la négligence ont des impacts néfastes sur l'adoption de comportements sexuels et de consommation sécuritaires.

De plus, les jeunes appartenant aux groupes identifiés comme étant les plus à risque au VIH (jeunes de la rue, jeunes autochtones, jeunes des minorités sexuelles, etc.) rapportent des taux plus élevés d'abus et de négligence. L'estime de soi semble être un facteur de protection rapporté par 
la littérature. La multiplicité des traumas et l'interaction des déterminants influencent les capacités de protection des jeunes quant au VIH. L'élargissement des activités de prévention au VIH à la thématique des abus/négligence permettrait d'explorer de nouvelles avenues de protection pour les jeunes. La collaboration nouvelle de différents acteurs pourrait amener vers d'autres pistes de solution jusqu'ici peu explorées.

\section{P228}

EXAMINING HIV VULNERABILITY AND RESILIENCE

AMONG GAY, BISEXUAL, TWO-SPIRIT AND OTHER MEN WHO HAVE SEX WITH MEN THROUGH A DETERMINANTS OF HEALTH LENS

Boodram, Chris; Hai-Tadesse, Maham; Lary, Tanya

Ottawa, ON

ISSUE: Gay, bisexual, two-spirit and other men who have sex with men (MSM) continue to be the population most affected by HIV in Canada. The Public Health Agency of Canada (PHAC) has developed a Population-Specific HIV/AIDS Status Report summarizing available Canadian evidence on the complex interactions between the determinants of health and the specific vulnerabilties and resiliencies to HIV/AIDS experienced by gay and other MSM.

METHODS: This report was developed in consultation with a national working group composed of community representatives, researchers, and policy and program experts. Academic and grey literature searches were conducted to identify relevant Canadian literature published from 2002 2011, addressing the determinants of health in relation to HIV/AIDS among gay and other MSM in Canada.

LESSONS LEARNED: The relationship between specific risk behaviours, such as inconsistent condom use, unprotected anal intercourse, sex with multiple partners, and HIV vulnerability is well-documented in the literature. Evidence also highlights the impact of risk factors, such as mental health issues, drug use and stressful life events and circumstances, on decision-making regarding safer sex. Additionally, emerging evidence signals the considerable effect of homophobia and heterosexism on determinants of health such as early childhood development, social support networks, income and social status, and access to health services for this population. Multiple sources of marginalization faced by ethnoracial minority gay men and other MSM, as well as two-spirit, gay and other Aboriginal MSM, also affect HIV vulnerability. Social support networks and the history of gay men's activism, including organizing around HIV and AIDS, are important sources of resilience for many members of this population.

RECOMMENDATIONS: Communities, governments, public health practitioners, non-governmental organizations, and researchers are encouraged to use the findings of this report to inform future policy, programming and research to address HIV/AIDS among gay and other MSM.

\section{P229 \\ HIV PREVENTION RESOURCES FOR STREET-INVOLVED YOUTH IN CANADA AND COLOMBIA}

Jalloh, Chelsea ${ }^{1}$; Wylie, John ${ }^{1}$; Rojas, Carlos ${ }^{2}$; Ormond, Margaret ${ }^{1}$; Zuluaga Toro, Marcela ${ }^{2}$; McMillan, Barbara ${ }^{1}$;

Zamora Suaza, Oliver²; Gómez Muñoz, Queeling²;

García Sierra, Adriana ${ }^{2}$; Casey, Catherine ${ }^{1}$

${ }^{1}$ Winnipeg, MB; ${ }^{2}$ Medellín, Colombia

Street-involved youth comprise a population that is overrepresented, yet often underserved, in the field of HIV prevention. Current literature suggests that the vulnerability of this population is exacerbated by factors such as limited literacy, poor access to services, chronic transience, and higher rates of substance use, unprotected sexual behaviour, and sexual exploitation. Traditional prevention approaches are often unable to access, or to have a discernable effect on, this population.

The objective of the initiatives reported here was to create HIV prevention materials, driven by the input of street-involved youth themselves, and to evaluate the success of these materials among the target populations in Winnipeg, Canada and Medellín, Colombia. Prior to resource creation, focus groups with street-involved youth were conducted in each site to discuss existing HIV knowledge, additional health-related topics of interest, and preferences in regards to resource design. With this information, site-specific resources were developed and distributed via community based organizations and directly by project staff who frequented locations where street-involved youth were present. Subsequent evaluation involved mixed method interviews with youth in addition to focus groups in Winnipeg. The evaluation addressed retention of specific information contained in the resource, in addition to individual's perceptions of the content, readability, design, and value of the material. In both sites, the respective resources were positively received by streetinvolved youth who indicated that the topics, design, and language of the resources were reflective of their experiences. Inclusion of artwork grounded in street-culture and narrative excerpts from other street-involved youth were particularly well received. However, specific knowledge uptake was sporadic; some questions were answered incorrectly by between $50 \%-70 \%$ of participants. Further inquiry is necessary to determine what factors, such as reading level, inclusion of graphics, and volume of information, can be modified to improve resource comprehension and information retention among street-involved youth.

Injection Drug Users and Harm Reduction

Utilisateurs de drogue injectable et réduction des méfaits

\section{P230}

\section{SOIVENT USE AND HIV IN WINNIPEG, MANITOBA}

Bell, Courtney P; Ormond, Margaret; Wylie, John;

Mignone, Javier; Fowke, Keith $\mathbf{R}$

Winnipeg, MB

Solvent use is a poorly understood and understudied substance use issue that occurs worldwide. Solvents, a type of inhalant, include common chemicals such as gasoline and lacquer thinner, which have psychoactive effects when inhaled. Manitoba appears to have a population of solvent users that are demographically unique from solvent-using populations described in the limited, existing literature. Individuals that work closely with this population in an outreach/support capacity have observed HIV present within the solvent-using population and clinicians have provided anecdotal evidence that suggests solvent use damages gastrointestinal and respiratory epithelium. HIV disease involves a disruption of the integrity of mucosal barriers as well as a state of chronic immune activation. Destruction of upper respiratory and GI mucosa may have important consequences for solvent users in terms of HIV susceptibility and disease progression. This research aims to investigate both social and biological aspects related to solvent use that may be relevant for HIV through an interdisciplinary approach adopting methodologies from community based research and basic science. The first phase of this research involved partnering with Sunshine House, a community-based organization in Winnipeg, whose client base includes solvent users. Three focus groups and ten qualitative individual interviews with solvent users were carried out as well as 8 interviews with key informants. The purpose of these consultations was to investigate characteristics of the population regarding solvent use and to inquire about the willingness to participate in research. Common themes identified include the use of solvents both recreationally and as a coping mechanism, the importance of relationships, the willingness to participate in research, the obstacles experienced related to solvent use, and the need for a treatment option targeted towards the populations specific needs. The next phase of this research involves obtaining biological samples and exploring the impact of solvent use on the immune system.

\section{P231}

ACCESS, USE AND AVAILABILITY OF SERVICES: RESULTS FROM A NATIONAL ENHANCED HIV SURVEILLANCE SYSTEM AMONG PEOPLE WHO INJECT DRUGS

Bennett, Rachel; Tarasuk, Jill; George, Diana B;

Ogunnaike-Cooke, Susanna; Archibald, Chris P; I-Track Site

Principal Investigators, The

Ottawa, ON

BACKGROUND: I-Track (enhanced surveillance of risk behaviours among people who inject drugs in Canada) conducted Phase 3 of data collection from 2010 to 2012 in 5 sites across Canada. 
METHODS: Information regarding demographics, drug-use, injecting and sexual risk behaviours, and HIV and hepatitis $\mathrm{C}$ testing patterns were collected in face-to-face interviews. A biological sample was collected for HIV antibody testing. Selected descriptive statistics assessed access to, use and availability of health and social services.

RESULTS: A total of 997 participants (as of November 2011) were interviewed; $61 \%$ were male, the average age was 39 years and $47 \%$ selfreported as Aboriginal. Overall HIV seropositivity was 6\%. Among those who had ever used a needle exchange program (NEP) 95\% had used a NEP in the previous 6 months. Use of other health and social services was low (7\% to $57 \%$ ). Ninety-five percent of HIV seropositive participants were under the care of a doctor and $77 \%$ of these were currently taking prescribed HIV medications.

NEP, drug substitution programs, counselling services and referrals were available in all sites. All but one site had vehicle-based mobile NEP services. All sites offered HIV testing and the majority of participants reported having ever been tested for HIV (93\%). The most commonly reported HIV testing location was a doctor's office (34\%). Most participants reported obtaining new clean needles from fixed-site NEPs (69\%) and the majority reported easy access to new clean needles (83\%).

CONCLUSIONS: Use and availability of NEPs was high; however low rates of access to medical, mental health and other social services warrant further investigation as they may indicate specific challenges/barriers at survey sites which impede access/use of these important health and social service supports by this at-risk population.

\section{P232}

\section{TOWARDS A SUPERVISED INJECTION SERVICE IN} MONTRÉAL: WHO? WHAT? HOW?

Goyer, Marie-Eve; Carole, Morissette; Annie, Larouche; Natalia, Gutierrez

Montréal, QC

BACKGROUND: In 2011, as part of its sexually transmitted and bloodborne infections prevention program, Montréal's public health department conducted a feasibility study on the implementation of supervised injection service (SIS). As part of this study, prior experience with injection supervision as well as organizational, ethical and professional issues were documented toward introducing this new practice in Montreal.

METHOD: Three methods were used: 1) a review of Canadian and international expertise through consultation of SIS stakeholders and different documentation sources; 2) focus groups with 25 caregivers from the public and community-based sectors working with IDUs; 3) consultations with professional orders, legal departments of public institutions and emergency services. The process relied on the framework of social innovation.

RESULTS: Montréal caregivers already have much experience with pre- and post-injection interventions. For direct observation of injection, tolerance practices exist in several organizations with varying degrees of formality. Training, experienced caregivers, multidisciplinary teams and service corridors (eg, emergency services) are needed for successful SIS implementation. Together, direction from the public healthcare network and a nursing presence will ascertain the ethic and legal viability of Montréal's SIS.

CONCLUSION: The addition of SIS will complete the continuum of care for IDUs and offer an essential tool to reduce drug overdose mortality and the burden on emergency services. To reach the most vulnerable IDUs, the organization of SIS will need to remain of low-threshold accessibility. Therefore, future SIS must promote peer involvement and be located in community-based organisations. Overall, support from relevant professional orders and Montréal's hands-on experience render SIS implementation feasible. However, the creation of a professional network of trained caregivers and the establishment of institutional collaborations must be set up beforehand.

\section{P233}

DEALING AND HIV RISK: EXPANDING THE FOCUS OF HIV PREVENTION INTERVENTIONS

Kolla, Gillian ${ }^{1}$; Strike, Carol' ${ }^{\text {; Balian, Raffi }}{ }^{\text {; }}$ Altenberg, Jason ${ }^{1}$; Penn, Rebecca ${ }^{1}$; Silver, Rey ${ }^{1}$; Roy, Élise $^{2}$; Millson, Peggy ${ }^{1}$;

Guimond, Tim ${ }^{1}$; Hunt, Neill'; Rotondi, Nooshin ${ }^{1}$

${ }^{1}$ Toronto, ON; ${ }^{2}$ Longueuil, QC; ${ }^{3}$ Faversham, United Kingdom

BACKGROUND: HIV rates in Canada among people who inject drugs have plateaued. Refocusing HIV prevention interventions on groups that have been traditionally excluded from the public health response to HIV, such as people who deal drugs, may provide new avenues for intervention. While often perceived as predatory, the distinction between dealers and drug users is not so clear cut. We examine two areas of HIV risk to identify novel areas to focus HIV prevention.

METHODS: As part of a longitudinal study on the effectiveness of an intervention to decrease initiation to injection drug use, baseline questionnaire data on participants $(n=98)$ were collected from people who injected drugs (previous 30 days) using respondent driven sampling. "Dealers" were identified by asking, "In the past 6 months, have you worked as someone who sold drugs, including small time dealing to friends?" Data were analysed using descriptive statistics and logistic regression.

RESULTS: $69.4 \%$ of participants were men; mean age was 44.6 (range 22-71). $60.4 \%$ of participants reported having engaged in drug dealing. Women were significantly more likely to report drug dealing than men (80.0\% vs. 51.5\%; OR=3.8, 95\% CI 1.4-10.4). Participants reporting dealing were no more likely to report having initiated another person when compared to those who did not deal (OR=1.1, 95\% CI 0.5-2.7). However, they were more likely to report that another person had used a needle after them $(\mathrm{OR}=1.3,95 \% \mathrm{CI} 1.1-1.4)$ or used a spoon/cooker after them $(\mathrm{OR}=3.5,95 \% \mathrm{CI} 1.1-11.5)$ than those who did not report dealing.

CONCLUSION: Seeing the boundary between dealers and users as fluid may offer HIV prevention programs a new opportunity for intervention. Careful considerations of how to engage people who deal drugs in HIV prevention programming may provide a pathway to increase access to sterile injecting equipment and reduce HIV risk.

\section{P234}

SAFE WORKS ACCESS PROJECT (SWAP) IN RURAL AREAS CONTRIBUTING TO LOWER HIV RATES BY PROVIDING SAFE INJECTION EQUIPMENT AND SUPPLIES, AS WELL AS RELATED EDUCATION

Kennedy, Janice ${ }^{1,2}$; Lundrigan, Philip A ${ }^{1}$; Crouse, J ${ }^{1}$ Kelly, Debbie ${ }^{1}$ 'St John's; ${ }^{2}$ Corner Brook, NL

BACKGROUND: The province of Newfoundland and Labrador has the highest median age coupled with the highest rate of unemployment in Canada. It consists of an island portion and a mainland portion. The sparse population is spread over 700 communities.

METHODS: To reach rural communities outside of the St. John's Metro area, the AIDS Committee of Newfoundland and Labrador (ACNL) established an office and hired a SWAP coordinator in Corner Brook, on the west coast of Newfoundland. The SWAP program services clients in the Humber Valley, Bay of Islands and Stephenville. To provide SWAP services, the following are utilized:

- Mobile Van

- Coordination with SWAP office and staff at ACNL in St. John's

- Volunteers pair up with the SWAP coordinator to provide anonymous mobile services

- Natural Helpers to do outreach in Stephenville and other rural communities

- Promotion of program to organizations that work with at-risk clients RESULTS: The SWAP program has been able to establish itself on the west coast of Newfoundland and provide safer injection equipment and supplies for intravenous drug users. The first 6 months of SWAP second year, needles in/out increased. Provision of education creates a more informed user. The program also provides referrals to other supports (eg, methadone/in-patient treatment program). Statistics: 1st year: 12,640 total needles out (1st 6 months 4,765, 2nd-7,875)

2nd year: 1 st 6 months: 13,770 needles out with a return rate of 16,350. These return rates are due in large part to natural helpers who help overcome stigma of drug use. Natural helpers are what make our program so 
successful and what small communities can use to help encourage SWAP use.

CONCLUSIONS: SWAP contributes to reduction in HIV and other infections and provides clients with a connection to non-judgmental support. There is a need for the continuation and expansion of existing services. Research on the benefits and outcomes of the provision of SWAP services in rural areas is required.

\section{P235}

\section{MICRO-ENVIRONMENTAL BARRIERS AND GENDERED} EXPERIENCES OF DIFFERENTIAL VULNERABILITY CONTINUE TO DRIVE SYRINGE-SHARING IN A SETTING OF UNIVERSAL HEALTHCARE, READILY AVAILABLE SYRINGE EXCHANGE SERVICES, AND A SAFE INJECTION FACILITY

Michelow, Warren ${ }^{1}$; Cescon, Angela ${ }^{1}$; Rutherford, Alexander ${ }^{1}$; Small, Will'; Parashar, Surita ${ }^{1}$; O'Brien, Nadia ${ }^{2}$; Pai, Jay'; Montaner, Julio'; ${ }^{1}$ Vasarhelyi, Krisztina ${ }^{1}$

${ }^{1}$ Vancouver, BC; ${ }^{2}$ Montreal, QC

BACKGROUND: Vancouver's Downtown Eastside (DTES) neighbourhood is known for a large and concentrated population of injection drug users (IDUs), low per-capita income, poor housing, and high HIV prevalence. HIV incidence among IDUs in the area has declined since the outbreak of the mid-1990s. While many quantitative studies have identified correlates of persistent syringe-sharing, fewer have qualitatively explored syringe-sharing contexts in this setting of universal healthcare, readily available syringe exchange, and an established supervised injection facility (SIF).

METHODS: In August 2010, two focus group discussions (FGDs) were held, separately for men and women. Peer-recruited participants were DTES residents who self-identified as current or former IDUs. Facilitators elicited data through four serially presented vignettes developed from a review of literature on syringe-sharing in the DTES. The discussions initially related to the syringe-sharing vignette presented and became participant-driven thereafter. Trained note-takers manually recorded discussions. Immediately after the FGDs the study team debriefed, discussed the transcripts of each FGD separately, then identified emergent themes, commonalities and differences between groups. Later, two reviewers further analyzed transcripts for consistency and agreement of themes.

RESULTS: Eleven men and nine women participated, and two overarching themes emerged. First, experiences differed greatly between male and female participants; men described having agency and a community of mutual support, whereas women expressed more isolation and vulnerability. The second theme highlighted the micro-risk environment, where syringe-sharing was driven primarily by social-structural factors that restricted individual ability to enact risk reduction measures. The key determinant of syringe-sharing identified was urgently needing to relieve withdrawal symptoms, an experience mediated by factors including: incarceration; programmatic barriers to using the SIF (line-ups, limited hours of operation, prohibition on assisted injecting); and, when injecting outside the SIF, rushing due to law enforcement presence. CONCLUSIONS: Despite a near optimal environment for risk reduction, syringe-sharing continues under particular circumstances in this setting. Differential vulnerability is mediated by gender and micro-environmental barriers to safer injecting, which continue to drive risk for HIV transmission and undermine risk-reduction efforts.

\section{P237}

\section{'WARTS AND ALL': OPPORTUNITIES AND CHALLENGES OF USING ARTS-BASED METHODS TO FACILITATE A DIALOGUE ABOUT HARM REDUCTION}

Switzer, Sarah; Guta, Adrian; de Prinse, Karen;

Chan Carusone, Soo; Strike, Carol

\section{Toronto, ON}

BACKGROUND: Arts-based methods (ABMs) are increasingly promoted as an effective strategy to engage people living with HIV in research and program evaluations. Many of these projects have been initiated in community-based settings for the purpose of investigating processes external to the host organization. This presentation discusses how ABMs were integrated and adapted for use in a hospital setting to investigate the impact of the organization's harm reduction policy on inpatient and community clients.

METHODS: This presentation draws on ethnographic data collected as part of a capacity building grant to explore the use of ABMs as a community engagement and data collection strategy. Analysis was undertaken of field notes from community-consultations, individual and group data collection sessions, team meetings, and reflexive memos, to offer a detailed account of using ABMs with people living with HIV in a hospital setting.

RESULTS: We critically explore the use of ABMs, and opportunities and challenges that emerged during the conceptualisation and data collection phases. Specifically, we report on the process of collaboratively deciding on which $\mathrm{ABM}$ approaches to pilot and how they would be conducted. Photography and mixed-media emerged as the most popular approaches, garnering interest from the outset. However, the use of these approaches presented a number of unexpected logistical challenges for both participants and research team members. The project required substantial time commitments due to the constraints of a hospital setting, the needs of participants managing advanced HIV, and the sensitive nature of the research topic. We report on strategies used to attend to context and participant's personal, emotional, and practical needs.

CONCLUSIONS: Overall, ABMs offer an effective community-engagement strategy but may require considerable modification for use in a hospital setting with people living with advanced HIV. Research teams are advised to understand the entire process as a data collection opportunity.

\section{P238}

HORMONE, SILICONE AND DRUG INJECTION: ASSESSMENT OF HIV-RELATED INJECTION RISK AMONG TRANSGENDER PEOPLE IN ONTARIO, CANADA

Travers, Robb ${ }^{1}$; Bauer, Greta ${ }^{2}$; Coleman, Todd A ${ }^{2}$; Scanlon, Kyle ${ }^{3}$ 'Waterloo; ${ }^{2}$ London; ${ }^{3}$ Toronto, ON

BACKGROUND: Existing studies of transgender, transsexual or transitioned (trans) people have evaluated injection-related risk behaviours in primarily US urban samples. High prevalences of needle sharing have been observed, for hormone use as well as for illicit drugs.

METHODS: The Trans PULSE Project conducted a respondent-driven sampling survey from 2009-2010 to recruit trans Ontarians age 16 and over $(n=433)$. Descriptive statistics were weighted based on probability of recruitment to represent population estimates, and 95\% confidence intervals were estimated using a modified bootstrapping approach that mimics recruitment chains.

RESULTS: Of trans people, an estimated 36.4\% of female-to-male (FTM) spectrum and 6.0\% of male-to-female (MTF) spectrum people currently inject hormones. Primary sources for obtaining needles for hormone injection included physicians, pharmacies and needle exchanges/ programmes. Low prevalences of needle sharing were observed, despite high frequencies of injection for hormones. Three participants had ever injected silicone and two reported having used a needle that had been used by another person for hormones or silicone (ever) or for street drugs (in the past year only).

CONCLUSIONS: Results suggest that trans people in Ontario engage in the injection of hormones but in largely safe ways. While risk exists, it is occurring in the context of the availability of universal healthcare in Canada, harm reduction interventions that include needle exchange programmes, and consequently, widespread differences in needle access between the two countries. These explanations may be implicated in the very low levels of injection risk behaviours seen in comparison to US studies. Given the large number of trans people who are injecting, there is still a need for trans-appropriate HIV education materials, including those that address risks through injecting equipment for drugs, hormones, and silicone, as well as for continuation of needle access strategies that include needles gauged for hormone use as well as for drug use. 


\section{P239}

\section{WORKING WITH PEER RESEARCHERS IN A NORTHERN COMMUNITY-BASED PROJECT TO REACH HIDDEN USERS OF ILLICIT DRUGS}

Wrath, Kathy; Peters, Heather; Durocher, Holly;

Burmeister, Charlene

Quesnel, BC

The purpose of the Opening Doors to Harm Reduction (ODHR) community-based research project is to engage with hidden populations of people who use illicit drugs and who seldom access mainstream health and social services. Quesnel is a small community in northern British Columba and like many rural communities in Canada, people who use illicit drugs are more hidden than in large urban centres. Rural communities also have fewer resources and this can leave people who use illicit drugs vulnerable to HIV risks inherent in their drug use activities. For those who are already HIV positive, remaining hidden from mainstream health care due to continued drug use also poses risks for themselves, their peers and their partners.

The project utilized a unique approach to accessing extremely hidden populations by relying on Peer Researchers and a Peer Leader. People from the drug using community of Quesnel were recruited and trained in harm reduction and ethical research. The Peer Researchers were able to use their network of peers to connect the research team with people who would otherwise never have been located or who may not have had enough trust to participate.

An overview of the peer researcher recruitment process, training, as well as the benefits and challenges associated with working with peer researchers will be discussed. In the ODHR project, the peer researchers were used not only to recruit participants but also to collect data, share harm reduction information with participants, and refer participants to local services. The success of the peer approach also suggests that peers could be useful in other areas of health care and social services. Working with peers can provide nurses and other care providers with a method of reaching people who are vulnerable to HIV to engage in prevention, harm reduction, testing and treatment.

\section{P240}

\section{CHALLENGES AND SUCCESSES IN LOCATING HIDDEN POPULATIONS OF ILLICIT DRUG USERS IN A SMALL, NORTHERN AND RURAL COMMUNITY}

Wrath, Kathy; Durocher, Holly; Peters, Heather;

Burmeister, Charlene

Quesnel, BC

In small, northern and rural communities in Canada people who use illicit drugs are more hidden than in large urban centers. The concealed nature of this population, combined with the reality of fewer services in small towns, means that people who use illicit drugs have less access to health care and social services which are important in the prevention and treatment of HIV. The purpose of the Opening Doors to Harm Reduction (ODHR) community-based research project is to access hidden populations of people who use illicit drugs with the long range goal of preventing the spread of HIV by improving health and social service access for this population.

Locating members of extremely hidden and stigmatized populations can be difficult, particularly in small, rural communities. The poster presentation provides an overview of the project along with a description of the various recruitment strategies that were used. Strategies included the use of peer researchers, who are people with illicit drug use experience in the community. Peer researchers are valuable, with peers reaching hidden populations that are not accessing available health care and other services. As part of the project, the peers were given a photo-voice project to depict drug use in a small community; a selection of photos is included on the poster. Other recruitment strategies included community-wide mail-outs on the project, use of both hard copy and on-line surveys, traditional advertising on radio and in newspapers, as well as the use of social media (Facebook). The poster describes which strategies were successful, which were not, and recommendations for reaching hidden populations. Although the goal of the project was to recruit hidden populations for research, the successful strategies would also be of benefit in reaching hidden clients for the purpose of service provision and HIV prevention.
Intervention Here and Abroad Intervention ici et ailleurs

P242

LA SATISFACTION DES CLIENTS DES ONG DU VIH/SIDA AU BÉNIN : DIMENSIONS ET FACTEURS EXPLICATIFS À TRAVERS UNE ANALYSE EN COMPOSANTES PRINCIPALES ET UNE ANALYSE DE RÉGRESSION MULTI NIVEAU

Agonnoude, Maurice T; Champagne, François; Leduc, Nicole Montreal, QC

PROBLÉMATIQUE : Les ONG de lutte contre l'épidémie de l'infection à VIH/Sida au Bénin peuvent utiliser l'évaluation comme un outil d'amélioration de la qualité de leurs interventions. Un des aspects de cette évaluation peut être l'estimation de l'effet de ces interventions sur la satisfaction des clients que ces ONG servent. Ce travail est entrepris pour analyser les dimensions et les facteurs explicatifs de la satisfaction de leurs clients. L'objectif général est d'identifier les dimensions et les facteurs organisationnels, contextuels ou individuels explicatifs de la satisfaction des clients des ONG de lutte contre l'épidémie de l'infection à VIH/Sida au Bénin.

MÉTHODE : Il s'agit d'une étude transversale concernant 2413 clients de 31 ONG intervenant dans la prévention dépistage et dans la prise en charge des personnes vivant avec le VIH. Une analyse en composante principale (ACP) et une analyse de régression linéaire multi niveau ont été effectuées.

RÉSULTATS : L'ACP nous a permis d'identifier quatre dimensions de la satisfaction pertinentes à notre contexte et l'analyse de régression les facteurs les influençant. Les implications pour la pratique ont été tirées.

\section{P243}

LESSONS LEARNED FROM A CANADA-AFRICA COLLABORATION: BUILDING COMMUNITY CAPACITY ON NEW PREVENTION TECHNOLOGIES

Buhler, Shayna $^{1}$; Abraham, Lesley${ }^{1}$; Durueke, Florita ${ }^{2}$; LeBlanc, Marc-Andre ${ }^{3}$; Patten, San ${ }^{3}$; Phiri, Bright ${ }^{4}$;

Sesane, Malebo ${ }^{4}$; Stein, Nicci ${ }^{1}$; Ukpong, Morenike ${ }^{2}$

${ }^{1}$ Ottawa, ON; ${ }^{2}$ Lagos, Nigeria; ${ }^{3}$ Halifax, NS; ${ }^{4}$ Johannesburg, South Africa

BACKGROUND: "North-South collaboration" and "South-South collaboration" are key phrases often used in the research world. However, these concepts and the practicality of their implementation is not well defined within the community of civil society organizations (CSOs) working in the new HIV prevention technologies (NPT) field, and successful models for these partnerships have not yet been established.

METHODS: A collaboration was initiated between the Interagency Coalition on AIDS and Development (Canada), New HIV Vaccine and Microbicide Advocacy Society (Nigeria) and the Southern African AIDS Trust (South Africa) to develop and deliver a series of skillsbuilding workshops on interpreting and communicating information around NPTs. At project wrap-up, the partners jointly developed a "lessons learned" toolkit designed to assist other organizations working with international partners to develop standardized training resources, while tailoring for local community education and engagement on HIV/AIDS. The toolkit was developed based on information gathered via an in-person partner meeting as well as a review of meeting minutes and materials developed over the course of the project.

RESULTS: Topics included in the toolkit include discussions on: the partnership process used to develop and deliver the workshops; the standardization of the workshops across different countries and cultures; the importance of building capacity within all partner organizations; successes in leveraging the project to undertake other initiatives; and other lessons learned that are applicable to organizations considering engaging in similar partnerships.The toolkit has been widely disseminated.

CONCLUSIONS: Long-term collaboration amongst CSOs working in the field of biomedical HIV prevention research is feasible and necessary. The success and sustainability of such partnerships beyond the lifetime of the immediate project is best facilitated when projects are jointly designed and flexible enough to adapt to the different needs of partners. 
The toolkit developed through this project can provide a roadmap for other organizations undertaking similar work.

\section{P244 \\ FAISABILITÉ D'UN SYSTÈME DE SURVEILLANCE DE DEUXIÈME GÉNÉRATION DU VIH AUPRÈS DES PERSONNES ORIGINAIRES DE PAYS OÙ LE VIH EST ENDÉMIQUE}

Butoyi, Jérémie $^{1}$; Hot, Aurélie ${ }^{1}$; Bernier, Mélina ${ }^{1}$; Adrien, Alix ${ }^{1}$;

Ogunnaike-Cooke, Susanna ${ }^{2}$

${ }^{1}$ Montreal, QC; ${ }^{2}$ Ottawa, ON

INTRODUCTION : L'Agence de la santé publique du Canada (ASPC), les spécialistes de santé publique, les chercheurs et les organismes communautaires collaborent pour mettre en œuvre des systèmes de surveillance de deuxième génération du VIH, baptisés « Track ». Ces systèmes intègrent de façon systématique la surveillance biologique et la surveillance des comportements à risque, ce qui répond aux recommandations de l'ONUSIDA. Le système de surveillance «E-Track » s'intéresse aux personnes originaires de pays où le VIH est endémique. À Montréal, une première étude de surveillance de seconde génération a déjà été menée par la Direction de santé publique (DSP) chez les Québécois d'origine haïtienne en 2007-2008.

OBJECTIF : Depuis 2009, la Coalition des organismes communautaires québécois de lutte contre le sida, l'ASPC et la DSP ont établi un partenariat afin de déterminer la faisabilité d'un système "E-Track » auprès des communautés montréalaises originaires des pays où le VIH est endémique d'Afrique subsaharienne et des Caraïbes anglophones.

MÉTHODE : Conformément aux directives de l'ONUSIDA (2000), la méthode adoptée comprend la formation d'un comité consultatif représentatif, la réalisation d'un portrait sociodémographique des communautés ciblées, un processus de consultation communautaire et l'organisation d'un atelier sur la faisabilité et l'acceptabilité du système de surveillance de deuxième génération (SDG). Une liste des lieux où rejoindre ces populations et le profil des organisations et groupes communautaires qui les servent au quotidien ont été développés.

RÉSULTATS : Les données quantitatives et qualitatives collectées permettent de confirmer la faisabilité du projet SDG auprès des communautés ciblées. Les résultats de la consultation communautaire soulignent le potentiel de prévention et de sensibilisation autour de la mise en place d'un tel système ainsi que les stratégies à privilégier comme l'importance de combattre la stigmatisation et la nécessité d'adapter le processus pour rejoindre et mobiliser des communautés hétérogènes.

\section{P245}

\section{ADAPTING CANADIAN DIASPORA HEALTH PROMOTION} INTERVENTION TO ADDRESS HIV STIGMA IN CHINA

Li, Alan'ㄹ Ren, Jinma²; Zhu, Xiao-Zhen²; Xue, Yi-le²; Kang, Laiyi²; Calzavara, Liviana $^{1}$

${ }^{1}$ Toronto, ON; ${ }^{2}$ Shanghai, China

BACKGROUND: As an urban centre with 23 million people, Shanghai's HIV cases have more than doubled since 2006. Its diverse PLWHA populations face many unique challenges in their HIV testing, disclosure and access to health care and support. An international research partnership was developed to involve affected stakeholders to assess the needs and identify strategies for improvement.

METHOD: A qualitative research with individual interviews and focus groups was carried out with 50 PLWHAs and 46 health service providers and caregivers to explore challenges and strategies for change. Data were coded and analyzed using N-Vivo software. Findings and recommendations presented to project advisory group made up of diverse stakeholders for validation and feedback.

RESULTS: The study identified the compounding impact of societal and health care sector HIV stigma and discrimination on PLWHAs resulting in delayed testing, non-disclosure, and barriers in accessing treatment and support as a critical need requiring urgent intervention.

Based on experiences of a training program "Can We Talk: empowering PHA health management communication skills" that has been successfully implemented amongst diaspora communities in Canada by the Ethno-racial Treatment Support Network (ETSN), the team adapted it to develop a new training titled: "Let's talk: A workshop to improve communication between PHAs and providers", with two educational modules one for PHAs and one for health care providers. The modules were piloted with 14 PHA peer leaders and 16 health care providers not currently involved in HIV care.

$80 \%$ PHAs and $90 \%$ of health care providers participants rated the workshop as highly effective in increasing their awareness of stigma and challenges facing PHAs, and increasing their skills in communicating with each other on disclosure and health care access issues.

IMPLICATIONS: The successfully piloted intervention is currently being considered for scale up for broader audience and to be integrated as part of core health care providers' training on HIV/AIDS.

\section{P246}

THE LIMITATIONS OF VOLUNTARY MEDICAL MALE CIRCUMCISION AND THE IMPORTANCE OF SUSTAINED CONDOM USE: A KENYAN NEWSPAPER ANALYSIS

Muzyka, Charlene N; Thompson, Laura; Driedger, S Michelle; Bombak, Andrea; Lorway, Robert

Winnipeg, MB

BACKGROUND: Since the completion of three clinical trials indicating that voluntary medical male circumcision (VMMC) is an effective method to reduce men's chances of acquiring HIV, use of the procedure has been advocated in Kenya. Media messages about VMMC shape popular understandings of the benefits and limitations of male circumcision. The objectives of this study were to (1) investigate promotion messages in a popular online newspaper to determine how the limitations of male circumcision are represented, and whether condom use is still being promoted; and (2) gain insight into popular understandings of the limitations of this new procedure through newspaper reader comments.

METHODS: A content analysis was conducted on 34 online media articles published by the Daily Nation during a two year period between January 1, 2008 and December 31, 2010. Information about condom promotion, partial immunity, limitations and complications of the procedure, as well as emergent themes, were analysed.

RESULTS: Few articles, other than Op/Ed articles, communicated the limits of VMMC. Results demonstrated an irregular and occasionally misleading presentation of these topics and a perceived lack of objective information about the risks and limitations of VMMC.

CONCLUSIONS: There is a need for governmental and non-governmental public health organizations to engage with the media to improve risk messaging about the limitation of voluntary medical male circumcision in Kenya.

\section{P247}

SPORTS AS THE MEANS FOR HIV PROGRAMMING: THINKING CRITICALLY

Njelesani, Janet; Cameron, Deb; Gibson, Barbara;

Nixon, Stephanie; Polatajko, Helene

Toronto, ON

BACKGROUND: Operating under the rubric "sport-for-development", the past fifteen years has witnessed a proliferation of non-governmental organizations mobilizing sports as a vehicle for HIV education and prevention in resource-poor settings. An example includes the organization Kicking AIDS Out, which has introduced soccer as a means to build awareness about HIV/AIDS in Zambia. Despite sporting activities having been widely adopted in HIV programming, there has been little research examining the benefits and limitations of this approach. The objective of this study was to use a critical approach to examine staff and youth participants' perspectives on the value, benefits and risks of using sporting activities in HIV programming. A critical social science perspective was adopted to investigate why the sporting activities were selected, whose interests they serve, which power relations are at play, and what assumptions underpin their ongoing acceptance.

METHODS: Case studies were conducted with five sport-for-development organizations in Lusaka, Zambia. Data collection included participant observation and key informant interviews with 29 staff and youth in the sports programme activities. The qualitative data analysis approach by Miles and Huberman (1994) guided analysis.

RESULTS: Three key themes were identified: 1) A dominant ideology of "sport is for boys to play" emerged, which defines boys in opposition 
to girls and encourages spectatorship over participation in HIV programming for girls. 2) Programs are seen as a success when boys reach the goal of playing professional football. This goal appears to be privileged over any other program aim including increased HIV awareness. 3) Programming choices are primarily based on staff preferences and familiarity with sports with minimal/less consideration of youths' or community's needs. CONCLUSION: The use of gendered sporting activities may have numerous positive effects; however, in conjunction with an hierarchical, authoritative approach to HIV programming, it risks perpetuating the disempowering behaviors and attitudes that these programs are attempting to overcome.

MSM

HARSAH

\section{P248}

\section{MALE CALL CANADA STUDY DEMONSTRATES IN AN ERA OF SOCIAL MEDIA THAT PRINT-BASED PERSONAL CLASSIFIED ADVERTISEMENTS REMAIN AN EFFECTIVE MEANS TO RECRUIT GAY, BISEXUAL AND OTHER MSM FOR RESEARCH}

Allman, Dan ${ }^{1}$; Myers, Ted ${ }^{1}$; Alexander, Stephen ${ }^{2}$; Remis, Robert S1; Blais, Martin ${ }^{3}$; Maxwell, John1; Calzavara, Liviana';

Marchand, Rick ${ }^{4}$; Adam, Barry $\mathrm{D}^{5}$; Marlatt, Faria ${ }^{1}$

${ }^{1}$ Toronto; ${ }^{2}$ Ottawa, ON; ${ }^{3}$ Montréal, QC; ${ }^{4}$ Vancouver, BC; ${ }^{5}$ Windsor, ON BACKGROUND: The aim of this paper is to describe the unexpected success of print-based personal classified advertisements (PBPCAs) in an era of social media.

METHODS: Male Call Canada (MCC), a bilingual, nation-wide, tollfree telephone survey, gathered self-report information via 60-90 minute interviews from gay, bisexual and other MSM 16 years-of-age or older on knowledge and attitudes related to HIV and health and wellbeing. The MCC study method was modelled after the 1996 Bisex Survey. For this early analysis, preliminary unweighted data were analyzed with SPSS. RESULTS: MCC spanned the winter months of 2011-2012. In the 11 weeks preceding December 30, 852 calls were received. $41.8 \%$ from Ontario; $17.6 \%$ Quebec; $15.7 \%$ the Prairies; $13.4 \%$ the Maritimes; $10.7 \%$ $\mathrm{BC}$; and $0.82 \%$ from the Territories and Nunavut. Of the resultant 593 interviews, PBPCAs were most frequently cited as the source of recruitment, despite substantial resources targeted to social or traditional display media (See Table).

\section{Male Call Canada Respondents Indicating Recruitment}

Source to Dec 30, 2011 ( $N=593)$

\begin{tabular}{|c|c|c|}
\hline Recruitment source & $\begin{array}{c}\text { \# of } \\
\text { respondents }\end{array}$ & $\begin{array}{c}\% \text { of } \\
\text { respondents }\end{array}$ \\
\hline Print-based personal classified advertisement & 262 & 44.2 \\
\hline Facebook $^{\mathrm{TM}}$ & 140 & 23.6 \\
\hline Word of mouth & 55 & 9.3 \\
\hline Display ad or article in gay publication & 40 & 6.7 \\
\hline Squirt ${ }^{\mathrm{TM}}$ & 40 & 6.7 \\
\hline Craigslist ${ }^{\mathrm{TM}}$ & 24 & 4.0 \\
\hline Email from a listserv & 14 & 2.4 \\
\hline Poster or postcard & 10 & 1.7 \\
\hline Other & 8 & 1.3 \\
\hline Total & 593 & 100 \\
\hline
\end{tabular}

CONCLUSION: In preparing for the study, the research team was informed by recent literature suggesting that social media should be a primary focus, as it would deliver the most effective recruitment of this population. However, surprisingly, as demonstrated with the Bisex Survey fifteen years earlier, PBPCAs provided the most geographicallydiverse and arguably the most cost effective mechanism of recruitment relative to volume and quality of responses received. These results have important implications for other forms of HIV research and prevention that seek to recruit from the diaspora of gay, bisexual and other MSM in Canada.

\section{P249}

ADAPTING HIV PREVENTION INTERVENTIONS FOR LATINO GAY, BISEXUAL AND OTHER MSM (GBMSM) IN CANADA, "MANO EN MANO": PROGRAM IMPLEMENTATION, THEORIES, METHODS AND PRACTICES.

Betancourt, Gerardo $^{1}$; Serrano, Angel ${ }^{1,2}$; Adam, Barry $^{2}$

${ }^{1}$ Toronto; ${ }^{2}$ Windsor, ON

PLAIN LANGUAGE SUMMARY: Interventions in the HIV field promote condom use (behavioural changes), provide HIV information (social-cognitive), and develop resilience and self-efficacy skills at the same time that they promote critical awareness (empowerment). For 3 years "Mano en Mano" (MenM) developed theme interventions for Latino (GBMSM). Constant formative and summative evaluation from participants and co-facilitators feedback, allowed the identification of the intervention's core elements: reduce social isolation, develop individual resilience and critical awareness, provide HIV/STI information, and enhance self-efficacy.

DESCRIPTION: HIV interventions present theoretical and methodological challenges related to the measurement of the intended effects, uncontrolled sources of variability and statistical analysis. "MenM" adopted a core elements format that promoted a constant innovating theme orientation, paying attention to community emerging themes, participants' feedback and allowing multi-method documentation of the program implementation: narratives, surveys, pre-post interviews, knowledge transfer/dissemination activities, focus groups, adopting of new technologies such as internet, pod-cast radio, among others.

RESULTS: "MenM" interventions delivered $(n=15)$. Multiple theme approaches: Newcomers $(n=6)$, Leaders $(n=1)$, Masters $(n=1)$, PHAs $(n=2)$, Drags/Trans ( $n=1)$, Radio pod cast $(n=2)$, Art $(n=1)$, Outreach Peer Educators $(n=1)$. More than 10 abstracts, posters and oral presentations in different conferences (CAHR, OHTN, ACT Research Day), one article published in a peer reviewed academic journal. Graduated $(n=80)$ participants, created $(n=50)$ volunteer and peer education opportunities.

LESSONS LEARNT AND RECOMMENDATIONS:

- There are waiting lists for future participants: former graduates new immigrants and Latino GBMSM new generations that are starting their sexual lives and who have no cultural or social support within their own communities.

- Development over three years to build the capacity building, theoretical, methodological approach and identify the intervention's core elements.

- "MenM" allows community participants to graduate and become cofacilitators, peer sex educators and community leaders.

- The CIHR Centre for Reach (OHTN) supports further evaluation for this intervention.

- There is national (other provinces) and international interest (Queen's University in Colombia) to further develop the core elements of this intervention in Latino populations.

- Innovating forms for funding and sustainability for interventions like "MenM" are needed.

\section{P250}

SAFER SEX DECISION-MAKING AMONG LATINO MSM IN TORONTO: FINDINGS FROM THE "CUENTAME" STUDY Adam, Barry ${ }^{1}$; Betancourt, Gerardo ${ }^{2}$; Rangel, Cristian ${ }^{2}$; Serrano, Angel ${ }^{1,2}$

${ }^{1}$ Windsor; ${ }^{2}$ Toronto, ON

BACKGROUND: This research focuses in understanding the social relations and contextual conditions involved in the sexual practices and vulnerabilities (immigration, language barriers, homophobia, lack of access to services, romantic scripts, etc.) related to HIV and condom use for Latino gay, bisexual and other men who have sex with men in the GTA.

METHODS: In-depth interviews with men who have sex with Latino men (25) were conducted in 2010.

RESULTS: Respondents are well aware of condom use as a form for HIV prevention. The sample is well-educated, perhaps representative of Canadian immigration selection procedures. These men seem to have a high awareness about issues of sexual health and tend to practice safe sex in 
casual relationships. At the same time, those men in relationships tend to be monogamously oriented and due to the nature of their long term partnerships report inconsistent use of condoms. For all the men in our sample, quality of life, that is political and economic stability and life opportunities, seems to be the major driving force behind their immigration process. In addition, sexual orientation issues, such as feeling safe about their sexual orientation and receiving proper health care for those who are HIV positive seem to be influential factors. Most participants now express considerable comfort with their sexuality.

CONCLUSION: The analysis shows that expectations related to trust and romance seem to be relevant factors for safe sex adoption, which are highly valued aspects of sexual intimacy for our participants at different points in their sexual lives. For instance, those who report having dropped the use of condoms have done so after discussion and even HIV testing with their sexual partners.

\section{P251}

EFFECTS OF COUNSELING ON SEXUAL RISK REDUCTION AMONG PARTICIPANTS AT SPOT, A COMMUNITY-BASED RAPID HIV TESTING INTERVENTION FOR MSM IN MONTREAL

Otis, Joanne; Veillette, Ludivine; Blais, Martin; Fadel, Riyas;

Wainberg, Mark; Rousseau, Robert

Montréal, QC

BACKGROUND: SPOT is an ongoing intervention-research project offering free, anonymous, rapid HIV testing to MSM in Montreal. OBJECTIVE: Explore the potential effects of counseling offered at SPOT, based on provincial guidelines, for sexual risk reduction and other psychosocial factors.

METHOD: Between July 2009 and May 2011, 1184 MSM enrolled for a first visit (TO) at SPOT. Of these, 360 returned for a follow-up visit 3 months later (T1). Data were gathered through structured interviews and self-administered questionnaires at T1 and TO. The outcome indicators that were measured included: having had unprotected anal sex with a HIV-positive partner or partner of unknown status (UAS), the importance given to safer sex practices, and perceived barriers with regards to these practices. Each participant's measures at T1 were contrasted with those at TO using the McNemar or the paired t-test.

RESULTS: Although this result is not significant, the proportion of UAS in the last three months has tended to decrease in participants between T0 and T1 (35.1\% vs. 28.9\%, p = 0.06). Participants' perception of the importance of safe practices increased significantly (M: 9.19 vs. $9.38, \mathrm{p}=0.04)$. Perceived barriers to these practices, whether related to sexual sensation-seeking behaviour ( $\mathrm{M}: 2.16$ vs. $1.91, \mathrm{p}=0.0001)$, communicational difficulties $(\mathrm{M}: 2.01 \mathrm{c} .1 .87, \mathrm{p}=0.04)$ or personal problems (M: 2.17 c. $1.99, \mathrm{p}=0.003$ ), seemed less important.

CONCLUSION: Despite significant limitations in the current design (no control group, for example), results so far suggest that the counseling offered at SPOT has beneficial effects for risk reduction and other factors in participants' psychosocial profile. In the future, the potential to add value through counseling based on motivational interviewing will be tested.

\section{P252}

THE OK CHARTER: A MODEL OF VOLUNTARY COMMITMENT BY SAUNAS, PUBLIC AND COMMUNITY ORGANIZATIONS TO WORK TOWARD PREVENTING HIV/ AIDS AND OTHER STBBIS IN MEN WHO HAVE SEX WITH MEN (MSM)

Lacombe, Elysabeth; Rousseau, Robert; Arsenault, Alain;

Boulanger, Jean; Gosselin, Olivier; Labesse, Daniel

Montreal, QC

BACKGROUND: In Montréal, men who have sex with men (MSM) are one of the most vulnerable groups to HIV/AIDS and to other sexually transmitted and blood-borne infections (STBBIs). It is imperative to step up prevention efforts within the gay and bisexual communities, especially in sex on premises venues (saunas, sex clubs, peep shows). The CSSS Jeanne-Mance, RÉZO, the Direction de santé publique de l'Agence de la santé et des services sociaux de Montréal and private businesses worked together to develop the OK Charter. It aims to increase access to protection materials and information, and intensify HIV/STBBI prevention and sexual health promotion activities among MSM who frequent these venues. Our presentation reports on the Charter development process and on the first 9 months of its implementation in four saunas.

METHOD: The OK Charter was developed based on 1) collaboration with gay saunas fostered since 2002 so they can offer their clients preventive interventions; 2) a feasibility study undertaken with owners of these establishments; 3) a review of similar projects carried out in Europe and Australia; and 4) involvement of the saunas in all stages of project design and implementation, through their inclusion on a steering committee.

RESULTS: Launched in summer 2011, the OK Charter defines the commitments and responsibilities of signatory partners, as well as methods for following-up on their commitment. A number of tools were developed, including a companion guide, an implementation grid, a tracking grid, promotional tools and condom dispensers. A communications plan was worked out to promote the OK Charter.

CONCLUSION: A partnership comprised of public, private and community networks makes it possible to better mobilize and involve businesses, and to guide prevention and health interventions for sex on premises venues that target a population vulnerable to HIV and other STBBIs.

New Information Technologies

Nouvelles technologies de l'information

\section{P254}

GOING VIRAL: KEEPING HIV/AIDS ON THE RADAR

Kelly, Debbie; Lundrigan, Philip A; Burt, Kimberly

St John's, NL

BACKGROUND: HIV infection rates in Canada are not decreasing.

Most Canadians consider themselves to be at very low risk; yet $26 \%$ of those infected do not know their status.

Youth feelings of susceptibility to HIV have decreased since 1989.

Stigma and discrimination prevent people from getting tested.

Society requires education regarding HIV to reduce stigma, increase testing rates, reduce transmission, and improve quality of life.

METHODS: This CIHR-funded Café Scientifique facilitated public engagement among a panel of HIV healthcare providers, research experts, and PHAs, to discuss HIV prevalence, transmission, treatment and stigma in NL.

Target audience included youth, parents, educators, and persons infected and affected by HIV.

The free event was hosted by Memorial University, the AIDS Committee of Newfoundland and Labrador, and Eastern Health on World AIDS Day.

Promotion:

- Advertised on local radio stations and a community newspaper

- A video was posted on You Tube, Facebook and Twitter

- 130 posters placed in public locations

- Press release issued day prior to event

RESULTS:

- There were 49 attendees; $79 \%$ completed evaluation forms.

- Audience engaged in lively discussion with panellists, and feedback was very positive.

- Discussion included access to rapid HIV testing, effectiveness of HIV education, how to engage young people specifically, and stigma.

- Event generated extensive media attention: 3 radio and 4 television interviews, and one newspaper article.

Post-event successes:

- Community Health received two new requests for HIV testing following a radio interview regarding the event.

- Interested community stakeholders/healthcare professionals are now pursuing rapid HIV testing in NL.

CONCLUSIONS: Café Scientifique is an effective way to engage the public and inspire collaboration to enhance HIV education and access to HIV testing. Further events/activities are needed to promote HIV testing and enhance HIV education 


\section{P255}

\section{GETTING IT ONLINE SOCIAL MEDIA STRATEGIES IN HIV EDUCATION}

\section{Madsen, Janet; $\underline{\text { Seatter, Erin }}$}

Vancouver, BC

BACKGROUND: Twitter and Facebook have become standard social media tools for maintaining personal connections, following news, and developing communities of interest. They are also increasingly used by various health care professionals. With the amount of online health information, consumers must select where and how to access information. Developing a solid online presence is not only about having a website, but also being a reliable, credible source for information and referral.

Vancouver's Positive Women's Network (PWN) has been using social media to provide HIV prevention education, support service referral and research dissemination since 2009. Why go social with HIV and STI prevention information? Because that's where women are $-84 \%$ of women look for health information online, versus $72 \%$ of men. Women are also more likely to do follow-up research online (http://www.marketingcharts.com/interactive/nine-of-10-women-online-seek-health-infovia-web-1656/). Social networking provides entry to a site and also acts as referral to other resources.

DESCRIPTION: PWN is one of the only women-specific HIV organizations in Canada. PWN launched into social media with two brands: Positive Women's Network website (www.pwn.bc.ca), Facebook and Twitter streams providing post diagnosis HIV education, as well as You Should Know, providing sexual health and HIV prevention education to women over 40 via a stand-alone website (www.youshouldknow.ca), Facebook and Twitter. We were an early adopter of social media in Canada's AIDS services community and are often consulted on creative content and technical development.

LESSONS TO SHARE: Social networking is about connecting with individuals, organizations, and developing an ongoing commitment to community building. Social $=$ personal.

Discussion will include best practices, challenges, and the development of real-life relationships based on online connections. Social networking has tremendous opportunities to enhance community education and support, a boon in Canada where many people live outside of urban centres.

\section{P256}

THE DEVELOPMENT OF A TAILORING PROCESS TO AN ON-LINE CONDOM-HIM WEB-BASED INTERVENTION.

Miranda, Joyal'; Côté, Joséé2, Godin, Gaston 3; Blais, Martin²; Otis, Joanne'2; Guéhéneuc, Yann-Gaël2; Fadel, Ghayas²;

Barton, Luisa ${ }^{1}$; Fowler, Shawn ${ }^{1}$

${ }^{1}$ Toronto, ON; ${ }^{2}$ Montréal; ${ }^{3}$ Laval, QC

BACKGROUND: The internet has become one of the avenues individuals will use to meet sexual partners. As a result, studies have begun to use the internet as a medium to deliver HIV prevention interventions in order to reduce risky sexual behaviors of individuals who do use the web to meet sexual partners.

GOAL/OBJECTIVE: A web-based tailored intervention, "CondomHIM" was developed for HIV+ Men having Sex with Men to increase condom use with their partners that are either $\mathrm{HIV}^{-}$or unknown status. METHODS: Intervention Mapping (IM) was used in the development process of the "Condom-HIM" web-based tailored intervention.

RESULTS: A three-stage approach to intervention tailoring was developed. The first stage was based on the total sum score of participating's baseline assessment of their overall intention and self-efficacy in condom use, resulting in four possible profiles. The second stage of tailoring was based on individual's responses to each item on the questionnaires, resulting in a sub-profile. Rationale is that individual's profiles on total scores should mimic those of the sub-profiles on individual items. The third stage of the tailoring is the validation of profiling. Participants are given a tailored validation message and asked to validate whether or not they have been profiled correctly. Based on the validation response of the participant, participants are given either a tailored web-intervention message and activity or a tailored reinforcement web-intervention message.
CONCLUSION: The use of a three staged approach for tailoring individual's messages combines all three tailoring strategies of personalization, feedback and content matching as suggested within the literature. Compared to generic information, providing tailored messages is seen as more effective since they are more likely to be read, remembered and viewed as personally relevant, thereby engaging individuals, building their self-efficacy and intention to use condoms as well as fostering behavior change.

\section{P257}

HIV-MEDIC ONLINE: EVALUATION OF A WEB-BASED INTERVENTION TO BETTER SUPPORT PEOPLE LIVING WITH HIV (PLHIV) FOR TAKING THEIR ANTIRETROVIRAL THERAPY

Côté, Josée ${ }^{1}$; Rouleau, Geneviève ${ }^{1}$; Guéhéneuc, Yann-Gaël'; Ramirez-Garcìa, Pilar ${ }^{1}$; Godin, Gaston ${ }^{2}$; Otis, Joanne ${ }^{1}$; Fadel, Riyas ${ }^{1}$; Tremblay, Cécile ${ }^{3}$

${ }^{1}$ Montréal; ${ }^{2}$ Quebec; ${ }^{3}$ Sainte-Anne-de-Bellevue, QC

INTRODUCTION: HIV-MEDIC online is a randomized controlled trial available in French and English on a secure Web site, which aims to assess the efficacy of virtual support interventions in the management of antiretroviral therapy to PLHIV.

OBJECTIVE: The objectives of this communication are: 1) to present HIV-MEDIC online, 2) to show the experience of the site's first users, and 3) to reveal the challenges encountered in a study done entirely through the Web.

METHOD: Usability tests to potential users, ie, to PLHIV on antiretroviral therapy, were a prerequisite to launching the study online. Observation and structured interviews were undertaken to determine the usability (ease of use, efficiency, errors, and user satisfaction) of the user interface and the content of the entire process of the randomized controlled trial online.

RESULTS: Usability tests among the users allowed the improvement of the virtual research process. HIV-MEDIC online was developed thanks to the pooling of diversified expertise: a clinical team, community workers, researchers, web design, multimedia team, and computer programmers. Evaluation of an intervention through a randomized controlled trial online presents particular challenges especially in terms of recruitment, ethics, data collection, long-term follow-up, and data security.

CONCLUSION: Accessibility to Web-based interventions provides the possibility for capability and empowerment. From their home or any other place chosen by the individuals accessing the Internet, PLHIV can benefit from this new intervention modality and from the results of this research.

Stigma and Environment

Environnement et stigmas

\section{P259}

\section{STIGMA, HIV AND HEALTH: A QUALITATIVE SYNTHESIS}

Chambers, Lori $\mathrm{A}^{1}$; Rueda, Sergio ${ }^{1}$; Baker, Nico $\mathrm{D}^{1}$;

Wilson, Michael $\mathbf{G}^{1,2}$; Deutsch, Rachel' ${ }^{1}$; Raeifar, Elmira ${ }^{3}$;

Rourke, Sean B ${ }^{1}$

${ }^{1}$ Toronto; ${ }^{2}$ Hamilton, ON; ${ }^{3}$ New York, NY, USA

BACKGROUND: HIV-related stigma acts as a profound stressor in the lives of people with HIV, negatively affecting care, treatment, and support. A growing body of research evidence documents the relationship between HIV-related stigma and health. Research findings suggest that enacted, felt, anticipated, and structural stigma is a pervasive experience for HIV-positive people. Additionally, stigma has a profound impact on the mental and physical health of people with HIV, and in how they adhere to treatment, and access and utilize care.

PURPOSE: To conduct a synthesis of qualitative evidence to explore the intersections of stigma and health for people with HIV.

METHODS: We conducted a systematic search of six electronic databases for journal articles published from January 1996 to February 2009. We also contacted key researchers, and reviewed reference lists of included studies. We included studies with qualitative findings on stigma from the perspective of people with HIV that presented data on physical and 
mental health outcomes, including intermediate health-related factors (e.g., adherence, access to/utilization of care, social supports).

RESULTS: Our search strategy yielded 8,621 references, from which we included 55 studies reporting qualitative findings. For people with HIV, stigma is experienced across multiple social contexts. These experiences impact health, particularly mental health, adherence, access to and utilization of care, and discriminatory practices of health care workers. Our synthesis of qualitative findings from the included studies suggest that other forms of societal marginalization such as gender, ethnoracial identity, sexual identity, and drug use could intensify experiences of stigma. CONCLUSIONS: Our synthesis of qualitative research evidence suggests that stigma profoundly shapes the health of people living with HIV. The intersections of other marginalized identities shape how stigma is enacted, felt and internalized. Social supports, HIV-related knowledge and education, and advocacy can buffer the impacts of stigma and reduce stigmatizing actions and behaviours.

\section{P260}

PEOPLE LIVING WITH HIV/AIDS IN CANADA: A DETERMINANTS OF HEALTH PERSPECTIVE PRELIMINARY FINDINGS OF THE POPULATION-SPECIFIC HIV/AIDS STATUS REPORT

\section{Lary, Tanya}

Ottawa, ON

DESCRIPTION: The Public Health Agency of Canada is developing a Status Report on People Living with HIV/AIDS in Canada (to be released in 2012), as part of a series on key populations in Canada affected by HIV/AIDS. The report summarizes Canadian evidence (peer-reviewed and grey literature) from 2005 to 2011 looking at PHA in Canada. It includes epidemiological data, a description of key issues affecting PHA, an examination of the determinants of health that affect PHA quality of life, and an overview of current research and response activities.

METHODS: This report was developed in consultation with a national working group composed of PHA, community representatives, researchers, and policy and program experts. Academic and grey literature searches were conducted to identify relevant Canadian literature addressing the determinants of health and PHA published from 2005-2011.

LESSONS LEARNED: The relationship between determinants of health and HIV/AIDS is complex, layered and multi-directional. The determinants of health influence the quality of life, treatment adherence, treatment outcomes and mortality of PHA. The experience of living with HIV also influences determinants such as income, employment status and social support networks. Key themes identified include the persistence of stigma and discrimination; the diversity of PHA, and HIV as a life cycle issue. Social support, community engagement, faith and spirituality are key sources of resilience.

RECOMMENDATIONS: Communities, governments, public health practitioners, non-governmental organizations, and researchers are encouraged to use the findings of this report to inform future policy, programming and research to address the needs and realities of PHA. Further research is needed to look at how determinants of health such as income, culture, and gender affect the quality of life of PHA.

\section{P261}

SANS DOMICILE FIXE COMME PASSAGE OBLIGÉ VERS UN LIEU DE VIE STABLE : EXPÉRIENCES D'INTERVENTION DU CENTRE SIDA SECOURS

Legaré, Hélène

Montréal, QC

INTRODUCTION : Certaines personnes vivant avec le VIH/sida (PVVIH) sont parmi les plus vulnérables de notre société : elles peuvent présenter des problèmes de toxicomanie ou de santé mentale compromettant sérieusement la stabilité nécessaire à l'obtention et au maintien d'un lieu de vie stable. Le Centre sida secours accueille, pour de courts séjours, des PVVIH qui n'arrivent pas à être hébergées dans les ressources d'hébergement conventionnelles et ce, pour différentes raisons (désorganisation majeure, problèmes de toxicomanie, atteintes neurologiques, immigrants sans papier, personnes qui sortent de milieu carcéral, etc.). Il n'y a ni limite de nombres de séjours, ni délai minimum entre les diff/ érents séjours.
LEÇONS APPRISES : L'expérience nous porte à croire que les SDF sont dans cette situation parce qu'ils sont incapables de s'investir davantage dans les lieux d'habitation qu'ils occupent au moment où ils les occupent. Vivant dans une situation d'instabilité qui perdure, il leur est difficile de supporter trop de stabilité. Alors, quand dans leur cycle, ils reviennent chez nous, nous avons appris à les accueillir sans trop demander. Notre approche consiste à les laisser venir, à les laisser parler et à travailler avec ce qu'ils veulent bien nous offrir.

RECOMMANDATIONS : Notre expérience nous amène à penser que le statut de «sans domicile fixe » est parfois un passage obligé et qu'il ne faut pas nécessairement tout faire pour le briser. Cette constatation confirme le fait qu'il n'y a pas une solution unique au problème de l'hébergement des personnes sans domicile fixe. Il faut être en mesure d'offrir une variété de type d'hébergement (ou même de suivi dans la rue) pour aider notre clientèle à sortir (à son rythme) de la spirale dans laquelle elle tourne souvent depuis de longs mois, voire de nombreuses années.

\section{P262}

THIS MIGHT BE A STUPID QUESTION BUT...? CREATING SAFE SPACES AND DISPELLING MYTHS: TRAINING WORKERS OUTSIDE OF THE HIV WORLD ON WOMEN AND HIV

\section{Medjuck, Melissa; Summers, Marcie; Grant, Sangam}

Vancouver, BC

ISSUE: Women are one of the fastest growing populations with HIV in Canada. The complex issues women living with HIV (WLHIV) contend with can result in experiences of unique HIV discrimination, which strengthen and reproduce existing inequalities of class, race, gender and sexuality. Negative social responses to WLHIV remain pervasive, and interventions against HIV-related discrimination are integral components of a comprehensive approach to HIV treatment and prevention. One intervention is to train community members on HIV knowledge that will help them engage in practices that uphold WLHIV's human rights.

DESCRIPTION: Positive Women's Network (PWN) provides education, support and resources for WLHIV (called "members") in British Columbia (BC). Since PWN's inception in 1991, members have consistently reported experiencing HIV-related discrimination from service providers not directly involved in the HIV field. Since 2006, PWN has delivered free HIV trainings throughout BC to over 1300 participants, targeting service providers who work with WLHIV, including transition house workers, immigration workers and students in social work and criminology.

LESSONS LEARNED: Service providers not directly involved in the HIV field report having no to limited HIV knowledge. Trainings must start with basic information about HIV and its transmission. Trainings should have a holistic lens and address issues linked to the vulnerabilities that put women at risk for HIV, including poverty, violence, addiction, racism and transphobia. Some challenges to the training program include lack of funding to travel to rural areas, resistance from service providers to recognizing HIV affects their clients, service providers' discomfort with their lack of HIV knowledge, and potential participants having heavy workloads and no time to attend trainings.

NEXT STEPS: Our results indicate that interactive trainings on HIV and WLHIV, facilitated in a non-academic style that does not focus on the medical model of care, significantly improve participants' knowledge.

\section{P264}

ISOLATION OF OLDER ADULTS LIVING WITH HIV: FACTORS VARYING ACCORDING TO SOCIAL NETWORKS

Wallach, Isabelle; Martel, Michel; Ducandas, Xuân;

Trottier, Benoit; Thomas, Réjean

Montréal, QC

BACKGROUND: Research conducted in the United States have shown that people living with HIV 50 years old and over $\left(\mathrm{PLHIV50}{ }^{+}\right.$) tend to deal with isolation for different reasons among which the stigma of HIV and the weakening of their social networks including aging PLHIV. No research has been yet conducted on this issue in Canada. The objective of this communication is to investigate whether PLHIV50+ in Quebec 


\section{Abstracts/Abrégés}

are confronted with isolation and, if it is the case, to thoroughly analyse the factors leading to isolation.

METHODS: As part of a qualitative approach, this research is based on semi-structured interviews conducted with PLHIV $50^{+}$, recruited through the medical clinic l'Actuel (Montreal). Data analysis, performed using the software QDAMiner, based on the method of grounded theory.

RESULTS: The participants actually live experiences of social isolation. However, the analysis shows that the reasons for this isolation depend on some social networks examined. The main reasons for isolation experiments on siblings and parents are HIV stigma, homophobia and the death of parents. However, the withering of relationships with children seems to be caused by the stigma of the way of living and HIV, the loss of former child care or geographical distance. The isolation on the friendly level is based more on a rejection linked to HIV, the internalized stigma of HIV, the side effects of antiretroviral therapies, the death of friends, the cessation of professional activities and financial difficulties. Some differences can emerge from the different sub-groups included.

CONCLUSION: With PLHIV50+ facing experiences of isolation, patterns may vary depending on the relational contexts that must be considered for an appropriate response to the multiple needs of this population. 




\section{J001}

\section{ONCOLYTIC VIRUSES AS A POTENTIAL APPROACH TO ELIMINATE THE HIV RESERVOIR}

Costiniuk, Cecilia T; Cote, Sandra; Carrasco-Medina, Lorna;

Angel, Jonathan B

Ottawa, ON

BACKGROUND: In cancer research, oncolytic viruses (OVs) are actively being studied for their therapeutic potential due to their ability to selectively replicate in, and kill, malignant cells. Similar to cancer cells, HIV-infected cells differ from HIV-uninfected cells in that they have alterations in interferon signaling pathways, the apparent reason for the selectivity of certain OVs. Therefore, use of an OV such as recombinant Maraba virus (MG1) may be a potential approach to eliminate latently infected cells that constitute the HIV reservoir.

OBJECTIVE: To determine whether MG1 will have a greater propensity to target and kill HIV-infected cells compared to non-HIV-infected cells.

METHODS: U1 cells, which are chronically-infected monocytic cells harbouring 2 copies of integrated proviral HIV DNA per cell, were infected with green fluorescent protein (GFP)-encoding MG1 at multiplicities of infection (MOIs) ranging from 0.0000001 to 0.01 . Control cells included U937 cells, the uninfected parent cell line. Flow cytometry was used to quantify active cell infection by MG1, as detected by GFP signal and 7AAD staining was used to examine cell death.

RESULTS: MG1 readily infected both U1 and U937 cell lines. MG1 infected a greater proportion of U1 than U937 cells at MOIs 0.0000001 through 0.0001 , with the extent of infection plateauing at an MOI of 0.0001 in both cell lines. Results from preliminary studies suggest that MG1 induced a greater degree of killing of U1 cells compared to that of HIVuninfected U937 cells at all MOIs tested.

CONCLUSION: Initial investigations suggest that MG1 infects and kills latently HIV-infected cells to a greater degree than the HIV-uninfected parent cells. This appears to be a promising model to facilitate further studies of MG1 as a potential therapy for the eradication of latently HIV-infected cells.

We gratefully acknowledge Dr. David Stojdl for the provision of MG1 virus.

\section{J002 \\ CELL-FREE BIOCHEMICAL CHARACTERISATION OF HIV-1 CRF_A/G INTEGRASE PROTEIN}

Quashie, Peter K; Mesplede, Thibault; Han, Ying-Shan;

Osman, Nathan; Wainberg, Mark A

Montreal, QC

Various subtypes of HIV-1 are responsible for HIV-1 epidemics in different parts of the world with subtypes $\mathrm{C}$ being the most prevalent. Circulating recombinant forms (CRFs) of subtype A such as CRF_A/E and CRF_A/G account for the second and third most abundant epidemics, followed closely by subtype B, which accounts mainly for infections in North America and parts of Europe. Most HIV research and drug discovery has been focussed on subtype-B yet the drugs have for the most part been shown to be effective against other non-B subtypes. One of the latest classes of HIV drugs, integrase inhibitors (INIs), have followed this trend. Recent studies have however shown that resistance mutations against the only approved INI, Raltegravir (RAL), identified in subtype $\mathrm{B}$, do not results in similar levels of resistance in some non-B subtypes. These subtype-specific differences have profound implications for therapy, especially because non-B subtypes are now spreading in western countries. The G118R mutation which was identified as a minor resistance mutation to the experimental INI MK-2048, and which causes minimal RAL resistance in subtype $B$ has been reported to cause major resistance of CRF_A/G virus to RAL. This raises the possibility that A/G virus integrase has profound functional differences with B type enzyme especially in the context of resistance. In order to better characterize A/G integrase, we cloned, expressed and purified the recombinant $A / G$ integrase protein. We extensively characterised the strand transfer and three prime processing activities as well as DNA binding properties compared to recombinant subtype B and C integrase enzymes, which had already been biochemically characterised by our group. These results as well as modelling of subtype A/G integrase structure will be presented.

\section{J004}

A STRAIN-SPECIFIC APPROACH TO HIV-1 VACCINE
DESIGN: A STUDY OF THE SASKATCHEWAN EPIDEMIC
AND BOTSWANA ACUTE INFECTION COHORT

Scott, Jamie K'; Coffin, Niki²; Brooks, James ${ }^{3}$; Novitsky, Vladimir'; Musonda, Rosemary ${ }^{4}$; Gulzar, Naveed ${ }^{1}$; Grewal, Prabvir ${ }^{1}$; Levett, Paul' ; Essex, Myron ${ }^{4}$; Zaharatos, Gerasimos ${ }^{5}$; Wainberg, Mark ${ }^{5}$

${ }^{1}$ Burnaby, BC; ${ }^{2}$ Regina, SK; ${ }^{3}$ Ottawa, ON; ${ }^{4}$ Boston, MA, USA; ${ }^{5}$ Montreal, QC

A major impediment to HIV-1 vaccine design is the virus's extensive genetic variability. During the course of infection, epitopes involved in the neutralizing antibody response shift from strain-specific sites on the envelope protein (Env) to those involved in heterologous neutralization. Only rarely are neutralizing antibodies made against sites common to most or all viruses, and then only 1 year or more after initial infection. We propose that if the genetic variability of circulating HIV strains within a population is limited enough, an effective strain-specific vaccine could be achieved. The HIV-1 epidemic in Saskatchewan is considered one of the worst in the western hemisphere, with an incidence of $~ 19 / 100,000$ ( 200 new infections per year). Our phylogenetic analysis of HIV-1 polymerase (pol) collected between 2004 and 2010 shows distinct clustering, indicating a restricted level of genetic diversity, reflecting the limited diversity of transmitted virus within communities in Saskatchewan. Botswana has the second highest HIV-1 prevalence in the world, estimated at $\sim 25 \%$ in adults. The phylogentic relationships among viruses isolated from newly infected individuals in the town of Mochudi, Botswana, reflect limited Env gene (env) diversity. We hypothesize that, within both the Saskatchewan and Mochudi populations: (i) sera that neutralize autologous (self) virus will also neutralize heterologous (non-self) virus if both viruses are closely related, (ii) the relatedness of viruses in each population is close enough to detect cross-neutralizing activity among HIV+ individuals, and (iii) cross neutralizing activity will follow viral relatedness which in turn will follow social networks; thus knowledge of social networks may predict HIV relatedness. We suggest experiments to test these ideas, and how the results of such studies could be applied to discovery of HIV vaccines in populations where its diversity is limited.

\section{J005}

RECOMBINATION IN CYNOMOLGUS MACAQUES MAFA-B AND MAFA-A ALLELES PRODUCING MHC CLASS I ALLELIC DIVERSITY

Orysiuk, Dallas $^{1}$; Lawrence, Jesse ${ }^{1}$; Prashar, Tarun ${ }^{1}$;

Spangelo, Lisa ${ }^{1}$; Pilon, Richard ${ }^{2}$; Fournier, Jocelyn ${ }^{2}$; Rud, Erling ${ }^{2}$;

Sandstrom, Paul1,2; Plummer, Francis $\mathrm{A}^{\mathbf{1}}$; Luo, $\mathrm{Ma}^{1}$

${ }^{1}$ Winnipeg, MB; ${ }^{2}$ Ottawa, ON

Cynomolgus macaques (Macaca fascicularis, Mafa) are a nonhuman pri mate model commonly used for research in transplantation and infec tious diseases such as SARS, tuberculosis and HIV. Their use in such immune-related studies requires research into their major histocompat ibility complex (MHC). The MHC class I - A and - B genes of cynomolgus macaques are highly polymorphic. These genes encode proteins that present peptides to $\mathrm{CD} 8^{+} \mathrm{T}$ cells to initiate an adaptive immune response. Recombination events are one way the diversity of these alleles can be increased. Such events have been well characterized in humans, but have not been as well characterized in macaques. In order to identify and examine recombinations that create new alleles, it is important to analyze intron sequences. Intron sequences have been shown to be important in understanding the evolutionary mechanisms involved in the 
generation of MHC alleles and loci. Thus far there have been relatively few intron sequences reported for MHC class I alleles in macaques, and this has hampered the understanding of $\mathrm{MHC}$ organization and evolution in macaques. In this study we present evidence of a gene conversion event generating the Mafa-B*099 allele lineage by the combination of Mafa-B*054 and Mafa-B*095 allele lineages. A potential recombination between the Mafa-A3*13 and Mafa-A4:14 lineages was also observed, but it is less clear due to the lack of intron 2 sequence. This report stresses the role that recombination can play in MHC class I diversity in cynomologus macaques, and the importance of introns in identifying and analyzing such events.

\section{J006 \\ INVESTIGATION OF IMMUNE QUIESCENCE: ASSESSING THE ROLE OF T REGULATORY CELLS IN HIV EXPOSED SERO-NEGATIVE (HESN) SUBJECTS}

Abdullahi, Abdirahman ${ }^{1}$; Card, Catherine ${ }^{1}$; Ball, Blake ${ }^{1,2}$; Kimani, Joshua ${ }^{1,2}$; Fowke, Keith ${ }^{1,2}$

${ }^{1}$ Winnipeg, MB; ${ }^{2}$ Nairobi, Kenya

BACKGROUND: Recent research of a cohort of HIV exposed seronegative (HESN) female sex workers has led to the characterization of a phenotype of reduced $\mathrm{T}$ cell immune activation, which we call Immune Quiescence (IQ). The research showed HESN women showed lower levels of cytokine production, lower gene expression in $\mathrm{CD}^{+} \mathrm{T}$ cells and lower levels of activated T cells compared to HIV susceptible women - an overall reduced baseline state. Also, it was shown that these HESN women have elevated levels of $\mathrm{CD}^{+}{ }^{+} \mathrm{CD} 25^{+} \mathrm{FOXP} 3^{+}$regulatory $\mathrm{T}$ cells (Tregs), which may be contributing to the reduced activation state. The proposed project seeks to clarify the role of Tregs in Immune Quiescence in HESN subjects. We hypothesize that in HESN women, Tregs are actively limiting immune activation through their suppression mechanisms.

METHODS: Treg suppression will be assessed by Treg depletion experiments. PBMCs will be depleted of $\mathrm{CD} 25^{+}$Tregs and stimulated with CD3/28 beads and HIV peptides. Treg depleted and mock depleted PBMCs will be stained with fluorescently labeled antibodies and analyzed by flow cytometry for proliferation capacity, cytokine expression and activation markers. We expect higher proliferation, cytokine and activation marker expression in Treg depleted PBMCs of HESN compared to mock depleted, thus suggesting that Tregs are reducing the activation state in HESN women. Preliminary results will be presented.

DISCUSSION: Tregs can be playing a role in reducing immune activation in HESN subjects, thus resulting in an environment of lower HIV replication capacity since HIV replicates more efficiently in activated cells. This model might provide support for the induction of Tregs in the mucosal barrier and therapeutic strategies like microbicides, which reduce immune activation at the mucosal barrier, potentially lowering the chance of initial HIV infection and propagation. This model might also help inform HIV vaccine design.

\section{J007 \\ AN ULTRA-SENSITIVE SEQUENCE-BASED HLA CLASS I TYPING METHOD USING DNA RECOVERED FROM FROZEN PLASMA}

Cotton, Laura A ${ }^{1}$; Rahman, Manal $A^{1}$; Penney, Kali ${ }^{1}$;

Brumme, Zabrina ${ }^{1,2}$

${ }^{1}$ Burnaby, BC; ${ }^{2}$ Vancouver, BC

BACKGROUND: HLA class I typing is an indispensable tool in HIV research, however, the requirement for high-quality, high-concentration template DNA can be limiting. Here we describe a rapid, reliable, highresolution sequence-based HLA class I typing method that uses DNA obtained from frozen plasma.

METHODS: We used a panel comprised of matched frozen PBMC $(\sim 3$ million) and plasma (500 ul) samples from 25 individuals collected between $2001-2007$ and stored at $-80^{\circ} \mathrm{C}$. Total nucleic acids were extracted using commercially-available kits and eluted in $55 \mathrm{ul}$. Extracts were assigned ID codes to ensure that HLA typing was blinded. Nested PCR using universal, locus-specific primers were used to amplify 1000 basepair regions spanning exons 2 and 3 of HLA-A, B and C in independent reactions using $\sim 686 \mathrm{ng}$ (PBMC-derived) and $\sim 18 \mathrm{ng}$ (plasma-derived) total nucleic acids as starting material. Amplicons were bidirectionally sequenced on an automated DNA sequencer using six sequence-specific primers per locus. Chromatograms were analyzed using Sequencher ${ }^{\circledR}$ v4.9 and allele interpretation was performed using free web-based software (http://www.ncbi.nlm.nih.gov/gv/mhc/sbt.cgi?cmd=main).

RESULTS: Median [IQR] nucleic acid concentrations of PBMC and plasma-derived extracts were $85.7 \mathrm{ng} / \mathrm{ul}$ and $2.2 \mathrm{ng} / \mathrm{ul}$ respectively. HLA$\mathrm{A}, \mathrm{B}$ and $\mathrm{C}$ amplification rates were $100 \%$ for $\mathrm{PBMC}$ and plasma-derived DNA. Sequence chromatograms were of uniformly high quality with clearly distinguishable mixed bases regardless of DNA source. Concordance between PBMC and plasma derived DNA was 100\% at the HLA allele group level and $100 \%$ at the protein level. Concordance at the nucleotide level was 100\%. The test panel captured 12 (16), 16 (22) and 11 (14) unique HLA-A, B and C alleles at the allele group (and protein) levels, respectively.

CONCLUSION: This method provides comparable HLA typing specificity to conventional sequence-based approaches that require far higher template DNA concentrations. It could, therefore, be usefully applied in studies where cell samples are unavailable or DNA quantities are limiting.

Clinical Sciences

Sciences cliniques

\section{J008}

ETRAVIRINE WITH 2 NRTIS: AN EFFECTIVE SWITCH OPTION FOR ARV SIMPLIFICATION AND/OR SIDE EFFECT MANAGEMENT

Smith, Graham H; Brunetta, Jason; Sandler, Ina; Crouzat, Fred; Chang, Benny; Fletcher, David; Kovacs, Colin;

Hedgecock, Malcolm; Merkley, Barry; David, Tilley; Loutfy, Mona Toronto, ON

BACKGROUND: Etravirine (ETV) has been approved for use in treatment-experienced patients based on results of the Duet clinical trials. Less experience exists with ETV in earlier stages of treatment. ETV has a favorable genetic barrier, lipid profile, and little associated CNS toxicity. These characteristics make ETV attractive as a switch strategy for simplification and/or management of side effects.

METHODS: A retrospective chart review was conducted at a large urban HIV clinic. All patients who were switched to ETV plus 2 nucleosides and whose viral load (VL) was $<200$ copies $/ \mathrm{ml}$ at the time of switch were included. Maintenance of viral suppression, CD4 and lipid changes at 24 weeks and reason for switch to ETV are reported.

RESULTS: 72 (68 male) patients were identified. Mean age was $43 \pm 10$ and mean duration of HIV infection was $12.5 \pm 7.6$ years. Switches were from efaviren $z=30$, atazanavir $=18$, lopinavir $=16$, other $=8$. Duration of prior regimen was long; median 155 weeks. CNS and GI intolerance were the most common reasons for switches.

At the time of analysis, 53 patients had reached week 24. Four patients had discontinued ETV prior to week 24, $1 \mathrm{LTF} / \mathrm{U}, 14 \mathrm{had}<24$ weeks F/U. 92\% (66/72) maintained VL suppression (ITT-LOCF); 1 patient had a VL of 68 , other failures were 5 patients who stopped/LTF/U prior to week 24 . On treatment CD4 and lipid changes are shown below.

\begin{tabular}{lccc}
\hline Labs & Baseline & $\begin{array}{c}\text { Change from } \mathrm{BL} \\
\text { to WK 24 (OTA) }\end{array}$ & p value \\
\hline CD4 (cells/mm $\left.{ }^{3}\right)$ & $627 \pm 280$ & $+40 \pm 136$ & 0.130 \\
CHOLESTEROL $(\mathrm{mmol} / \mathrm{L})$ & $4.66 \pm 1.18$ & $-0.54 \pm 0.85$ & 0.01 \\
HDL $(\mathrm{mmol} / \mathrm{L})$ & $1.19 \pm 0.35$ & $-0.04 \pm 0.21$ & 0.50 \\
LDL $(\mathrm{mmol} / \mathrm{L})$ & $2.64 \pm 0.89$ & $-0.35 \pm 0.79$ & 0.04 \\
TRIGS $(\mathrm{mmol} / \mathrm{L})$ & $2.02 \pm 2.10$ & $-0.64 \pm 1.84$ & 0.04 \\
\hline
\end{tabular}

CONCLUSION: Switch to ETV plus 2 nucleosides maintained viral suppression, improved lipid profiles and improved side effect profile in this selected group of patients. 
J009

METABOLIC SYNDROME IN PEOPLE LIVING WITH HIV (PHA) AND RECEIVING COMBINED ANTIRETROVIRAL THERAPY (CART) IN COLOMBIA

Mueses, Héctor $\mathrm{F}^{1}$; Galindo, Jaime ${ }^{1}$; Galindo, Ximena ${ }^{1}$;

Martinez, Jorge $\mathbf{L}^{2}$; Alvarado, Beatriz $\mathrm{E}^{2}$

${ }^{1}$ Cali, Colombia; ${ }^{2}$ Kingston, ON

INTRODUCTION: Little information exist on CVD in PHAs from middle-income countries such Colombia. The Canada-Colombia collaboration in HIV research assessed the frequency of and factors associated to MS in PHAs.

METHODS: The assessment of MS was performed in a convenient sample of 102 PHAs on cART followed by the Corporacion de Lucha Contra el SIDA. A structured survey and standard protocols were applied in the participants. MS was defined according to the joint statement of the National Cholesterol Education Program Adult Treatment Panel III (NCEP ATP III) and the International Diabetes Federation (IDF). Multivariate logistic regressions were performed to identify main correlates of MS in PHAs.

RESULTS: The mean age of the participants was $42 \pm 10.5$ (SD). Most patients $(79 \%)$ were on a combination of two NRTI plus one NNRTI (Zidovudine/Lamivudine + Efavirenz [62\%]). Eighty two percent of the patients had a viral load $<40$ copies, $77 \%$ had a CD 4 cell count over 350 , $36 \%$ had more than three years on treatment. About $35 \%$ of patients met the criteria for MS, $30.4 \%$ had two of the criteria and $17 \%$ had one criterion. The most frequent abnormalities were: low HDL $(<40 \mathrm{~g} / \mathrm{dL}$ in men, $<50 \mathrm{mg} / \mathrm{dL}$ in women, $68 \%$ ), high triglycerides ( $>150 \mathrm{mg} / \mathrm{dl}, 60 \%)$, and increased waist circumference ( $>80 \mathrm{~cm}$ in women, $>90 \mathrm{~cm}$ in men, $60 \%$ ). MS was associated with BMI greater than $26(\mathrm{OR}=6.42)$ and longer treatment duration, $>5$ years $(\mathrm{OR}=4.61)$. Factors such as type of antiretroviral drug, age, sex, socioeconomic status, initial clinical stage and smoking, were not related to MS.

CONCLUSIONS: These findings suggest that successful HIV treatment in middle-income country populations will likely be accompanied with a high prevalence of MS as a chronic comorbidity. Thus, early interventions to reduce the occurrence of MS in such populations are needed as part of life-long care of PHAs in Colombia.

\section{J010}

\section{A SURVEY ON THE SEVERITY AND MANAGEMENT OF MENOPAUSAL SYMPTOMS IN MIDDLE-AGED HIV- INFECTED WOMEN}

\section{Hughes, Christine A; Yuksel, Nese; Foisy, Michelle}

\section{Edmonton, $\mathrm{AB}$}

OBJECTIVES: Research regarding normal aging processes, especially menopause in HIV-infected women is limited. Current guidelines do not address management of menopausal symptoms in this population. The purpose of this study was to determine the frequency and severity of menopause symptoms in middle-aged HIV-infected women and how symptoms were managed.

METHODS: This was a cross-sectional survey design. Consecutive women ages 40-60 years were recruited from the Northern Alberta HIV program outpatient clinics. Women enrolled completed a written survey including demographics, menstrual cycle, past medical and surgical history, treatment of menopausal symptoms, and sources of information. Symptoms of menopause were rated using the Menopause Rating Scale (MRS), an 11 item validated scale with scores on each item ranging from 0 (no symptoms) to 4 points (severe symptoms).

RESULTS: Of the 46 women approached, 31 completed the survey. The mean age was 48.5 years; $36 \%$ were hepatitis C co-infected and $19 \%$ had a history of depression. The majority (81\%) were on antiretroviral therapy. Twenty (65\%) women no longer had menstrual periods (mean age of last period 44 years). The mean overall MRS score was $16.16 \pm 8.82$; psychological symptoms were found to have the highest scores (mean $6.45 \pm$ 3.11; highest possible score16), whereas urogenital symptoms scored the lowest (mean $3.43 \pm 3.30$; highest possible score 12). Hot flushes were reported by $22(71 \%)$ women, with $7(23 \%)$ reporting daily symptoms. None of the women were currently on hormone therapy. Eight women reported receiving information about menopause from an external source; most commonly family/friends, pharmacists and doctors.
CONCLUSION: Data from the literature as well as our study suggest an earlier onset of menopause in HIV-infected women. Psychological symptoms appeared to have the greatest severity, however vasomotor and urogenital symptoms were also reported. HIV-infected women may be less likely to attribute symptoms to menopause, and thus increased education in this patient population may be needed. Further research is required to determine optimal treatment.

\section{J011}

\section{ÉVALUATION DE L'HYPERBILIRUBINÉMIE À LONG TERME EN RELATION AVEC L'EXPOSITION À L'ATAZANAVIR CHEZ LES PATIENTS INFECTÉS PAR LE VIH DE LA COHORTE DE LA CLINIQUE MÉDICALE QUARTIER LATIN, MONTRÉAL, CANADA}

Laprise, Claudie; Baril, Jean-Guy; Dufresne, Serge; Côté, Pierre; Trottier, Helen

Montréal, QC

CONTEXTE : Bien que l'on dispose de données significatives sur l'hyperbilirubinémie associée à la prise d'atazanavir, la compréhension de son impact, particulièrement à long terme, reste limitée.

Objectif de l'étude : Évaluer l'ampleur et l'évolution de l'hyperbilirubinémie sur une longue période chez les patients VIH positifs sous atazanavir.

MÉTHODES : Dans le cadre d'une étude de cohorte prospective, l'ampleur de l'hyperbilirubinémie, mesurée à l'aide de la bilirubine sérique totale, a été analysée à partir de courbes Kaplan-Meier, de tests de t et d'un modèle de régression de Cox.

RÉSULTATS OBTENUS : La cohorte compte 2352 patients VIH positifs recrutés au diagnostic, depuis 1996, et suivis généralement tous les 3 à 6 mois. De ce nombre, 62 \% (1431) étaient toujours actifs en 2011. L'âge moyen au recrutement était de 39 ans (écart-type=9), et 92,4\% des patients (2173) sont des hommes. La durée moyenne de suivi est de 6,1 ans (343,2 semaines, écart type=254,7), et 29,7\% (493/1659) des patients prennent ou ont pris atazanavir en cours de suivi, comparativement à ceux qui ont pris d'autres antirétroviraux. Le segment de suivi des patients sous atazanavir a été comparé à celui des patients sous autres thérapies antirétrovirales, soit du 13 août 2000 au 8 septembre 2011. Les courbes de Kaplan Meier ont permis de constater une augmentation de l'incidence cumulative de l'hyperbilirubinémie chez les patients exposés à l'atazanavir par rapport aux patients non exposés. Une analyse du devenir des patients avec hyperbilirubinémie a été effectuée pour établir la proportion de ceux-ci évoluant vers des grades plus élevés et celle évoluant vers des grades moins sévères.

CONCLUSION : La compréhension de l'évolution de l'hyperbilirubinémie sur une longue période permettra aux cliniciens d'améliorer la prise en charge de leurs patients VIH positifs qui en sont atteints.

\section{J012}

\section{A CASE CONTROL STUDY OF SUB-OPTIMAL IMMUNE RECONSTITUTION DESPITE ART INDUCED VIRAL SUPPRESSION AMONG HIV INFECTED INDIVIDUALS IN MANITOBA}

Keynan, Yoav; Darraj, Majid; Chan, Shanna; Becker, Marissa;

Kasper, Ken

Winnipeg, MB

HIV-1 infection is characterized by loss of CD4 T cells, leading to immunodeficiency. Initiation of highly active antiretroviral therapy (HAART) results in increased CD4 counts. Both viral and host factors determine CD4 cell responses with approximately $15-30 \%$ of individuals having suboptimal increase of $\mathrm{CD}^{+} \mathrm{T}$ cell count, most commonly due to lack of compliance to HAART. A smaller fraction of patients will have sub-optimal CD4 increase despite suppression of HIV replication. We sought to characterize the factors associated with decreased immunological response among Manitoba's HIV patient population.

MATERIALS AND METHODS: We conducted a retrospective casecontrol study. Case definition for suboptimal responders was $\mathrm{CD}^{+} \mathrm{T}$ cell increase from baseline of less than $100 \mathrm{CD}^{+} \mathrm{T}$ cells $/ \mathrm{mm}^{3}$ after 48 weeks of potent HAART (defined by viral load $<50$ copies $/ \mathrm{ml}$ ) or lack of increase to above $200 \mathrm{CD}^{+} \mathrm{T}$ cells $/ \mathrm{mm}^{3}$. 
RESULTS: Of $>550$ patients followed by the HSC HIV clinic, 29 individuals met our inclusion criteria. Twenty one were men and average age was 44.96. None of these individuals were co-infected with HCV or HBV and co-morbidities were uncommon. Self reported ethnicity was 9 Aboriginal, 8 African, 11 Caucasian and 1 other. Nadir CD4 ranged from $1-118$ cells $/ \mathrm{mm}^{3}$, with average of 37.9 cells $/ \mathrm{mm}^{3}$. We compared the HAART regimen, presence of OI, co-morbidities, substance use and HLA among cases and controls.

CONCLUSIONS: Several factors have been variably correlated with sub-optimal reconstitution of CD4 count. A low CD4 nadir is a factor previously shown to correlate with poor return of CD4 count and is seen in our cohort. Additional factors associated with failure to reconstitute CD4 numbers will be discussed. Early case identification and linking to health care are important in decreasing the number of individuals with sub-optimal immune response despite of viral suppression.

\section{J019}

\section{ROAD TO WELLNESS}

Benson, Elizabeth I

\section{Maple Ridge, BC}

I recognized that is was critical to collect data respectfully, analyze it in context and use it solely for purpose that benfit the Aboriginal people living with HIV/AIDS from which the data comes. Data files are recorded and stored in secure filing cabinet after support session. Names, Identities, and no identifying information is included in any of the contents on "Road to Wellness" poster. The themes that emerged during Support sessions with $\mathrm{BC}$ Aboriginal people from urban Aboriginal community. Two themes that were prominent in Support session with individuals was the importance of maintaining a wellness plan. Secondly, the presence of Aboriginal staff to talk to about maintaining good health. They stressed the importance of actively participating in Culturally appropriate events that serve Aboriginal people. There were several comments that cultural activities is doing the things that are necessary good health-emotionally, and physically. Engaging them in the evaluation process was viewed with cautious optimism: while it was viewed as positive for Aboriginal people living with HIVAIDS. I am happy with the observations and continued support of Healing Our Spirit in this findings.

\section{J020}

THE CANADIAN TREATMENT ACTION COUNCIL (CTAC): STRENGTHENING THE HIV CO-INFECTION COMMUNITY VIA KNOWLEDGE, TRANSFER, EXCHANGE AND CAPACITY BUILDING

Price, Colleen E; Sutton, Paul; Stephenson, Leah; Binder, Louise D Toronto, ON

ISSUES: In Canada, there are approximately 13,000 people living with HIV and hepatitis $\mathrm{C}$, and many are also co-infected with hepatitis $\mathrm{B}$. In response to deficits in HIV co-infection research, CTAC and partners held the 1st Canadian HIV/HBV/HCV Co-infection Research Summit 2010, engaging multiple stakeholders, including service providers, researchers and community members, about their HIV co-infection related research questions - prioritizing research needs and identifying gaps in HIV co-infection research and service delivery. The outcome was "The Roadmap of HIV Co-infection Research" report (see http://www.ctac.ca/ en/issues/hivhcv-co-infection).

DESCRIPTION: "Getting the Word Out \& Strengthening Community: Knowledge Transfer Exchange and Capacity Building Strategy for HIV/Hepatitis co-infected community, frontline and other relevant stakeholders" was written by Colleen Price with assistance from Louise Binder.

This project focuses on the findings of the Summit, "The Roadmap of HIV Co-infection Research" and creating interactive workshops and webinars that are developed, facilitated and evaluated by co-infected individuals. The objective is to evaluate co-infection research from the Summit and ongoing research to identify research needs and gaps to inform treatment, care, support and policy for people living co-infected.
Communications, leadership and research skills building will be fostered for co-infected members in this project, strengthening community members and community.

METHODS: The KTE and Capacity Building Project Officer was hired, a multidisciplinary Steering Committee was recruited, and all are working in tandem with Project Lead and the CTAC Co-infection Working Group to create materials that will reach out to co-infected people, including those living in rural and remote communities. Co-infected individuals, working group members and the multi-stakeholder Steering Committee have a detailed work plan which identifies roles, responsibilities and activities for 2011-2013.

NEXT STEPS: Interactive workshops will be presented in Toronto, Winnipeg and Montreal.

15 webinars will be created, learning modules posted online.

Workshops will guide treatment, care, support, research and policy for co-infected people.

On-going evaluation.

\section{J021}

\section{'THAT'S ORIGINAL!' HOW AND WHY WE NEED TO THINK ABOUT PRODUCING ORIGINAL KNOWLEDGE IN HIV/AIDS RESEARCH.}

\section{Namaste, Viviane}

Montréal, QC

This presentation seeks to offer a critical perspective on the current state of HIV research in Canada. What kinds of topics are considered important in the field? What constitutes new knowledge? Does research in the field tell us what we already know? What are examples of research which is truly new and innovative? What are the problems in doing original research? Does the infrastructure of research currently in place encourage originality?

This presentation will offer critical reflections on such questions. It is especially designed to help young scholars and graduate students think critically about the topic they define, the theoretical framework they adopt, and the methods they employ. The presentation will offer some useful starting points to think about creating research that tells us more than what is obvious, or more than what we already know.

Given the nature of the topic, in particular a stated objective to have a collective discussion on this question, this work is suited to an oral presentation only.

A successful response to the challenges of the HIV pandemic requires original knowledge. And the production of doctoral level work demands a student make an original contribution. This presentation seeks to start a collective discussion about how and why originality is necessary in the research we do on HIV.

\section{J023}

\section{HOUSING AND HIV IN QUEBEC (CANADA): BUILDING AN INNOVATIVE COMMUNITY-BASED RESEARCH PARTNERSHIP}

Laperrière, Hélène ${ }^{1}$; Zuniga, Ricardo ${ }^{2}$; Blanchard, Michèle ${ }^{2}$; Légaré, Hélène $^{2}$; Richer, Thérèse ${ }^{3}$; Hot, Aurélie $^{2}$

${ }^{1}$ Ottawa, ON; ${ }^{2}$ Montréal; ${ }^{3}$ Québec, QC

INTRODUCTION: No study explores the impact of housing and AIDS in Quebec. This project raises a strategy of partnership between HIV/ AIDS community-based organizations (CBOs) and researchers actively engaged in the fight against AIDS, as well as in the supportive housing of the poorest people living with HIV/AIDS (PLWHA), such as sex workers, drug users, persons affected by mental health problems, homeless youth and women, immigrants. Ten CBOs members of the provincial Coalition of community groups (COCQ-AIDS) have define a project on the issue. How will a collective reflexive practice with community-based organizations and researchers help to innovate a field of research about housing and AIDS in Quebec that values the multiples voices of engaged community actors?

METHODOLOGY: The project has begun with an experimental catalyst project with two of the Coalition members. The objectives are (a) to collect their expertise accumulated on supportive housing with the poorest sectors and (b) to document the community practices through the viewpoints of all the actors involved. We experiment with a form 
of the community-based research with the direct involvement of the participants. We engage PLWHA as peer research assistants from the poorest and most marginalized populations linked to the organizations. They choose the popular education methods with which they feel most comfortable (posters as peer productions, storytelling, photographs and illustrations).

CONCLUSIONS: The ethnological approach increases the understanding of the living milieu where they are inserted. One defining trait of Quebec governance is the participation of civil society organizations in the fabric of public health and social policies. Volunteering in Quebec is historically based on a model of collective responsibility that is quite different from the individual philanthropy prevalent in English Canada. The HIV/AIDS supportive housing in Quebec established some community-based evidences about housing as a response to the social determinants of health for the PLWHA from the poorest sectors.

\section{J024}

\section{LE VIH/SIDA ET LE DROIT SOCIAL EN MILIEU} COMMUNAUTAIRE DES PEUPLES AUTOCHTONES

\section{Bahakoula Mabidi, Louis}

\section{Brazzaville, République du Congo}

CONTEXTE : Le VIH/sida est considéré de nos jours comme une pandémie meurtrière. Les pays en voie de développement sont durement affectés par les ravages de cette tragédie et constitue désormais une grave menace au développement durable. Cette pandémie continue à être immense défi au regard de son impact socio économique négatif sur la population et le développement de tous les pays, surtout ceux en développement dont le Congo Brazzaville.

\section{OBJECTIFS :}

- promouvoir une approche multisectorielle de lutte contre le VIH/ sida et les Infections Sexuellement Transmissibles (IST) dans les communautés minoritaires des peuples autochtones;

- contribuer et accroitre l'accès à l'éducation et aux soins de santés primaires des peuples autochtones;

- renforcer les activités et prévenir les risques du VIH en luttant contre les infections opportunistes;

- contribuer aux interventions de sensibilisation et d'information;

- appuyer et réaliser l'ensemble d'initiatives portant sur les projets de développement socio sanitaire et éducatif en milieu communautaire des peuples autochtones;

METHODOLOGIE : La promotion de la santé à pour objectif majeur d'amener les individus, des groupes et des communautés des peuples autochtones à adopter des comportements sains, par la diffusion de pratiques positives de la santé. Pour connaitre les vulnérabilités potentielles du VIH/sida des populations minoritaires autochtones en milieu communautaire exige beaucoup d'enquête sur les connaissances, attitudes, pratiques et études d'observation qu'il faille consacrer le temps pour y parvenir à dessein.

RESULATS ESCOMPTES : L'impact recherché est l'amélioration du niveau de vie et l'augmentation du bien être des peuples autochtones dans leur environnement et milieu communautaire.

CONCLUSION : L'impact du VIH/sida au secteur des communautés de peuples autochtones, notamment des femmes rurales et les jeunes qui font de l'agriculture de subsistance, manquent des multiples outils d'information et de prévention. Pour planifier et atténuer le VIH/sida et les IST aux peuples autochtones, il faut mettre l'accent sur l'éducation sanitaire pour éviter les souffrances humaines indicibles des peuples autochtones et réduire la gravité de l'infection au VIH/sida dans leur milieu communautaire.

\section{J027}

\section{HIV/AIDS IN LEBANESE PRISONS: A CALL FOR ACTION}

Ammar, Diala $F$

Beirut, Lebanon

Many countries have implemented strategies to alleviate the problem of HIV/AIDS within the prison system. These strategies are not only important in limiting prevention and transmission of HIV/AIDS, but also contribute in optimizing quality of life within the prison system (Polonsky, Kerr \& Harris, 1994). Most Middle Eastern governments have failed to provide appropriate measures to promote and protect the well being of HIV/AIDS infected prisoners (Jenkins, 2004). The case of Lebanon is not an exception. The purpose of this paper was to establish functional administrative management reforms for Roumieh prisons (the country's largest male top-security prison) based on international prison standards as well as cultural considerations. This paper argues Peers in prison, guards and stake holders need the training and education in recognizing hierarchy of services, monitoring procedures, human rights and educational opportunities. The main intent of this particular intervention program is to create a positive prison environment encouraging safety and favorable conditions among infected inmates with constructive ramification towards management of HIV/AIDS in the community.

\section{J028}

AIDS AND STIGMA, CASE STUDY FOR KATIKAMU SUBCOUNTY, UGANDA

Nambi, Sarah

Wobulenzi, Uganda

BACKGROUND: Ever since the first cases were detected in Uganda in 1980s, people with AIDS (PWAs) have been the targets of stigma. AIDS stigma represents a set of shared values, attitudes, and beliefs that can be conceptualized at both cultural and individual levels. At the cultural level, AIDS stigma is manifested in laws, policies, popular discourse, and the social conditions of persons with HIV (PWHIVs) and those at risk for infection. At the individual level, it takes the form of behaviors, thoughts, and feelings that express prejudices against persons infected with HIV

OBJECTIVES: This study/abstract briefly describes current knowledge about AIDS stigma Uganda, in particular case study of Katikamu subcounty. Because of space limitations, it is not intended to provide a thorough literature review but instead highlights some major findings about AIDS stigma and cites representative studies.

METHODS: A cross-sectional study with qualitative and quantitative methods of data collection was conducted among HIV-positive patients aged 15-34 years. A total of 231 respondents were assessed using self reports and questionnaires. Data was collected using a semi structured questionnaire, was entered in Epidural and analyzed with STATA 8 key informant interviews and focus group discussions were held for the qualitative data.

RESULTS: Five in six $(84 \%, \mathrm{n}=194)$ of the respondents were stigmatized. On multivariate analysis, the factors independently associated with AIDS stigma in Katikamu Sub County at individual level were 95\%, and at the cultural level were $80 \%$

CONCLUSION: AIDS is a global pandemic, and persons with HIV (PWHIVs) are stigmatized throughout the world to varying degrees. AIDS stigma around the world is expressed through social ostracism and personal rejection of PWHIVs, discrimination against them.

AIDS epidemic has many parallels to older epidemics of cholera and plague. What differentiates AIDS from the older epidemics is that we now have the collective insight to recognize the impact of stigma on individual lives and public health, as well as the technology to scientifically study stigma and seek to reduce it. 


\section{AUTHOR INDEX/INDEX DES AUTEURS}

\section{A}

Abbas Z ..................P137,P140

Abdullahi A......................J006

Abel K ......................... O005

Abelsohn K . . . . . . . . . . . . O077,P195

Abraham L. . . . . . . . . . . . . . . . . . . . . . . . . O103,P243

Ackad N. . . . . . . . . . . . . . . . . . . O 010

Adam B . . . . . . . . . . . . . P218,P249,P250

Adam BD .................. P248

Adrien A . . . . . . . O013,0024,0099,O107,

.P159,P179,P191,P244

Aggarwal B. . . . . . . . . . . . . . . . . . P070

Agonnoude MT ................. P242

Agudelo JF . . . . . . . . . . . . . . . P104,P107

Ahluwalia A. .................. P211

Ahmad S . . . . . . . . . . . . . O048,O101

Ahmed A . . . . . . . . . . . . . . . . . . . . . P014

Ahmed R . . . . . . . . . . . . . . . . . . P098

Aidala A. . . . . . . . . . . . . . . . . . O105

Aidarus N. . . . . . . . . . . . . . . . . P047

Aiken C...................... P030

Aitken D . . . . . . . . . . . . . . O0 038

Ajamian L . . . . . . . . . . . . . . O004,0005

Akouamba BS . . . . . . . . . . . . . . . P012

Alary M . . . . . . . . . . . . . . . P029,P121

Albert A. . . . . . . . . . . . . . . . . . . P087

Albert AY. . . . . . . . . . . . . . . . . . O091

Albert AY......................... O092

Alenezi O. . . . . . . . . . . . . . . . . . . . O064

Alexander S.................... P248

Al-Ghazawi F. . . . . . . . . . . . . . . . . O O085

Al-Ghazawi FM . . . . . . . . . . P035,P036

Alimenti A. . . . . .0016,0040,0089,P090,P095

Allam O.................... P014

Allan D ...................... P211

Allen V ...................... O0 070

Allman D . . . . . . . . O013,0024,O099,P142, $\mathrm{P} 159, \mathrm{P} 191, \mathrm{P} 248$

Alonso M. . . . . . . . . . . . . . . . . 0057

Altenberg J................... P233

Alvarado BE. . . . . . . . . . . . . . . . . . J009

Ambagala AP. . . . . . . . . . . . . . . . . . P004

Ameli M. . . . . . . . . . . . . . . . P002

Ammar DF....................J027

Ancuta P . . . . . . . . . . . . O028,0083,P037

Andrews J. . . . . . . . . . . . . P116

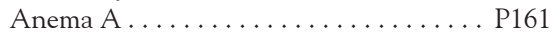

Angel J . . . . . . . . . . . . . . . . . 0038

Angel JB. . . . . . . . . . . . . . . . . P032,P042,J001

Anipindi V. . . . . . . . . . . . . . . . . . O 0087

Annie L . . . . . . . . . . . . . . . P232

Antoniou T . ........ O036,P156,P157,P212

Ao Z .................. O002,P010

Apuzzo L. . . . . . . . . . . . . . . . . . . . O0007,P062

Archibald C............ O035,O101,P114

Archibald CP ....... O017,O046,P126,P136,

$\mathrm{P} 137, \mathrm{P} 140, \mathrm{P} 148, \mathrm{P} 149, \mathrm{P} 231$

Ariane A . . . . . . . . . . . . . . . . . O091

Arsenault A .................... P252

Asad S . . . . . . . . . . . . . . . . P002

Ashkar AA. . . . . . . . . . . . . . . . . . . . 0087

Ashraf T.................... O086

Atkinson M . . . . . . . . . . . . . . . . . . P094

Auger A . . . . . . . . . . . . . . . . 0060

Ayi K .....................P017,P018

Aykroyd G . .................... P125

Azar I . . . . . . . . . . . . . . . . . P0 P041

B

Babin C ................... P226

Bach P ....................... P067
Bacon J. . . . . . . . . . . . . . . . . . . . P204

Bahakoula Mabidi L. . . . . . . . . . . . . . J024

Baidoobonso S . . . . . . . . . . . . . . . P119

Baker ND . . . . . . . . . . . . . . . . . P259

Balachandran A................. P052

Balfour L. . . . . . . . . . . O038,O095,P053,P055

Balian R . . . . . . . . . . . . . . . . P233

Ball B . . . . . . . . . . . . . . . . . . O032,P010,P020,J006

Ball TB............... O057,P022,P028

Balram B. . . . . .............. O014

Baltzer Turje R.................. P109

Bamvita J-M. . . . . . . . . . . . O072, P145

Bangura H . . . . . . . . . . . . . . . P148,P149

Bannister R . . . . . . . . . . . . . . . . . . P009

Baraki B . . . . . . . . . . . . . . O011, 0082

Barbeau B. . . . . . . . . . . . . . . . . O006

Baril J-G. . . . . . . . . P060,0008,0009,0033,

P096,P100,P150,J011

Barnes M . . . . . . . . . . . . . . . . . . P003

Barnes S . . . . . . . . . . . . . . . . P158

Barrett L. . . . . . . . . . . . . . . . . . . . . O029

Barrios L. . . . . . . . . . . . . . . . . P108

Barrios R. . . . . . . . . . . . . . . . . P109

Barry C. . . . . . . . . . . . . . . . . . . . 0057

Barry F . . . . . . . . . . . . . . O021,P177

Barton L..................... P256

Bath M.................... 0015

Bauer G . . . . . . . . . . . . P119,P120,P238

Bauer GR . . . . . . . . . . . . . . . . P125

Baxter L . . . . . . . . . . . . . . . . . . P210

Bayoumi AM . . . . . . . . . . . . . O070,P209

Beaudry S. . . . . . . . . . . . . . . O107

Beaulieu M. . . . . . . . . . . O075,O107,P179

Beaupré-Bériau M . . . . . . . . . . . . . . O075

Becker M . . . . . . . . . . . . O018,P135,J012

Bégin M-A................... O072

Belanger M-C. . . . . . . . . . . . . . . 008

Bell CP................... P230

Bendayan R . . . . . . . . . . . . . O058,0086

Benedict R . . . . . . . . . . . . . . . . . P055

Benko E ..................... O011

Bennett R. . . . . . . . . . . . O046,P126,P231

Benson El . . . . . . . . . . . . . . . . . . J019

Berg KB . . . . . . . . . . . . . . . . . . O091

Bernard NF. . . . . . . . . O025,O026,P023,P033

Bernier A . . . . . . . . . . . . . . . . . . . P037

Bernier M. ............ P110,P192,P244

Berthoumieux S . . . . . . . . . . . . P056

Berthoux L . . . . . . . . . . . . . P024,P030,P043

Best Plummer W . . . . . . . . . . . . . P055

Betancourt G . . . . . . . . . . P213,P249,P250

Bhargava M .................. O014

Bielawny T. . . . . . . . . . . . . . . . . O057

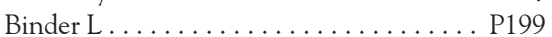

Binder L . . . . . . . . . . . . . . . . . . P208

Binder LD . . . . . . . . . . . . . . . . . . . . J020

Bingham B .................... O106

Biondi MJ. . . . . . . . . . . . . . . O060

Birungi J . . . . . . . . . . . . . . . . . . . . O079

Bisignano A . . . . . . . . . . . . . . . . . P219

Bitnun A . . .0016,0040,0089,0093,P068,P084

BLACCH Study Team, The .......... P119

Blair AH. . . . . . . . . . . . . . . O043, O100,P139

Blair M. . . . . . . . . P211,O049,O050,0051,

Blanchard JF. . . . . . . . . . . . O048,O 101

Blanchard M ................... J023

Blanchette C . ................ P121

Blitz S. . . . . . . . . . . . . . . . O093,P070

Blouin K.................. P121
Bois C. .................... O 014

Boissonault M ................. P064

Boissonnault M . . . . . . . . . . . . . P058

Bolton S. . . . . . . . . . . . . . . . O015

Bombak A ..................... P246

Boodram C................... P228

Borg P. . . . . . . . . . . . P057,P058,P059

Boucher F. . . . . . . . . . . . . . . . . O 0089

Boucher M . . . . . . . . . . . . . . . . O090,P012

Boucher R ................. P184

Boulanger J. . . . . . . . . . . . P252

Boulassel M-R . . . P014,O028,O083,P015,P026

Boulassel R. . . . . . . . . . . . . . . . P040

Bourassa C . . . . . . . . . . . . . P137,P140

Bourbonnais A................. 0022

Bourgeois S. . . . . . . . . . . . . . . . P195

Boven K.................. P058

Bowdish DM ................. O087

Bowen C. . . . . . . . . . . . . . . . . P116

Boyd S . . . . . . . . . . . . . . . . P169

Bozack A ..................... O105

Bozinoff N . . . . . . . . . . . . . . . . P144

Branch DR.................... P045

Bredeek UF . . . . . . . . . . . . . . . . P060

Brennan DJ ................ O115,P214

Brennenstuhl S . . . . . . . . . . . . P214

Brenner BG . . . . . . . . . . O033,P106,P122

Brian WJ . . . . . . . . . . . . . . . . O066

Bridger N . . . . . . . . . . . . . . . . O 018

Brinson C. . . . . . . . . . . . . . . . . . P057

Brisebois C.................. 0077

Brockman M . . . . . . . . . O011,0081,0082

Broeckaert L. . . . . . . . . . . . . . . . O O103

Broliden K . . . . . . . . . . . . . . . . . . О032

Brooks J . . . . . . . . . . . P114,P148,P149,J004

Brophy J . . . . . . . . . . . . . O O . . . . O0,0089, P090

Brophy JC. . . . . . . . . . . . . . . O040,P068,P095

Brouillette M-J........... O037,P015,P077

Brudno M. . . . . . . . . . . . . . . . . . . P004

Brumme C . . . . . . . . . . . . . . . . O081

Brumme CJ................... O034

Brumme Z. . . . . . . . . .0011,0081,0082,J007

Bruneau J . . . O025,О026,O042,О072,P033,P145

Brunet L . . . . . . . . . . . . . . . . . . O063

Brunetta J. . . . . . . . . . . . . . . P058,P094,J008

Buccitelli A . . . . . . . . . . . . . . . . P110

Buccitelli AA............... . . . . . . P192

Buhler S . . . . . . . . . . . . . . O103,P243

Bukassa Kazadi G. . . . . . . . . . . . . . . . P103

Bukassa Kazadi G. . . . . . . . . . . . . . P116

Bullard J . . . . . . . . . . . . . . . . O018,P149

Bulman DE. . . . . . . . . . . . . . . . P193

Bunting C. . . . . . . . . . . . . . . . . . . O099

Burchell A . . . . . . . . . . . . . . . . P152

Burchell AN. . . . . . . . . . . . . . . . . . . . O0970,0077,P069

Burdge D . . . . . . . . . . . . . . . . O039

Burgener A. .................. 0032

Burmeister C . . . . . . . . . . . . . . P239,P240

Burt K.................. O029,P254

Butoyi J..................... P244

Byles M. . . . . . . . . . . . . P211

\section{$\mathrm{C}$}



Caddy S . . . . . . . . . . . . . . . . . . . . 0093

Calzavara L. . . . . . . . . O013,0024,0099,P129, P142,P159,P191,P245

Cameron B, . . ............... O095

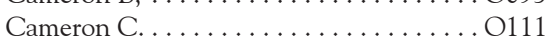

Cameron D. . . . . . . . . . . . . . . . . . P247

Cameron R......................... P194 


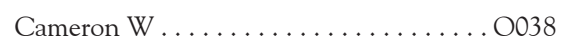

Cameron WD.................. P055

Campbell A .................... P113

Campbell C .................. P211

Campo R ................... P060

Canadian Co-Infection Cohort

Study (CTN 222), for the . . . . . . . . . P074

Canadian Observational Cohort

Collaboration, CANOC . . . . O036,0069,P069

Canadian Pediatric and Perinatal HIV/

AIDS Research Group (CPARG), The ... . O016

Canadian Pediatric Perinatal AIDS

Research Group, The .............. O089

Capina R . . ................. O057

Caprara D. . . . . . . . . . . . . . . . P085

Card C . . . . .................J006

Carl G ..................... P166

Carlson J. . . . . . . . . . .......... О081

Carole M ................... P232

Carrasco-Medina L . . . . . . . . . . . . . . . J001

Carrier M. .................... P076

Carrington M.................. 0081

Carter AJ . . . . . . . . . . . . . . . . . P195

Caruso J . . . . . . . . . . . . . . . . . . P167

Carusone SC . . . ............... P083

Carusone SC, ................ P215

Carvalhal A . . . . . . . . . . . O021,P094,P177

Casey C . . . . . . . . . . . . . . . P229

Cattaneo JS ................... P215

Calzavara L. . . . . . . . . . . . . . . P248

Cedar Project Partnership, for the .. O043,O100,

P124,P130,P139

Center RJ . . . . . . . . . . . . . . . . P023

Ceranto A . . . . . . . . . . . . . . O 115

Cescon A . . O069,P069,P123,P162,P195,P235

Chaban B.................. 0092

Chagnon-Choquet J. . . . . . . . . . . . . . P016

Challacombe LA . . . . . . . . . . . . . . P163

Chambers LA. . . . . . . . . . . . . O105,P259

Champagne F................ P242

Chan AC ................... P078

Chan Carusone S. . . . . . . . . . P203,P237

Chan D. . . . . . . . . . . . . . O034,P108

Chan JK . . . . . . . . . . . . . . . . . . P004

Chan K. . . . . . . . . . . . . . . . . . O069,P066,P069

Chan S. . . . . . . . . . . . . . . . . J J012

Chang B................. P061,J008

Charest H. . . . . . . . . . . . . . О033

Charest L . . . . . . . . . . . . . . . . P064

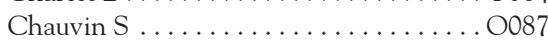

Chavoshi N ................... P124

Chaworth-Musters T . . . . . . . . . . . . O091

Chegwidden W ................. P210

Chelico L . . . . . . . . . . . . . . . O030,P021

Chen Y...................... O079

Cheng A. . . . . . . . . . . P057,P059,P061

Chettiar J . . . . . . . . . . . O045,O117,P111

Chicoine Brathwaite Y . . . . . . O055,P206

Chiu I. . . . . . . . . . . . . . . . . . . . . . P098

Chomont N . . . . . . . . . . . . . . . P040

Chown SA ................... P168

Christian CW .......... O043,O100,P139

Chuck SK. . . . . . . . . . . . . . . . . P058

Chui C . . . . . . . . . . . . . . . . . . O 081

Chung Tun Wong KY ........... P176

CIHR Emerging Team in HIV

Therapy and Aging, The ....... . P090,0039

Clarke C. ....................... P109

Clayton K. . . . . . . . . . . . . . . . . . P047

Clayton KL. . . . . . . . . . . . . . . . . P001

Cleret-Buhot A . . . . . . . . . . . . O083
Clerzius $G$................... P026

Cobbler M-A .................. P196

Cochrane A . . . . . . . . . . . . . . 0003,0084

Cochrane AW .............. O062,P052

Coffin N......................... J004

Cohen C. . . . . . . . . . . . . . P057,P058,P059

Cohen EA .................... O027



Coleman TA ...............P125,P238

Collins E. . . . . . . . . . . O070,P070,P094

Comité consultatif sur les attitudes

envers les PVVIH ............... O107

Compton M . . . . . . . . . . . . . . O O015,P169

Conway B. . . . . . . . O007, $0010,0063,0064$,

............. 0065,0066,0068,P062

Cook D. . . . . . . . . . . ....... 0073

Cooper C . . . . . . . . O010,0036,0038,0063,

......... O065,0066,0067,0068,0069,

$\ldots \ldots \ldots \ldots \ldots \ldots \ldots$
Cooper R . . . . . . . . . . . O009,P097,P098

Corace K . . . . . . . . . . . . . O038,P055

Corroy F. . . . . . . . . . . . . . . P219

Costiniuk CT ....................J001

Côté F. . . . . . . . . . . . . . . O075,0078

Cote H . . . . . . . . . . . . . . . . . P095,P008

Cote HC. . . . . . . . . . . . . . . . O039,P090,P087

Côté J . . . . . . . . . . . . . . . . . . . . O022,O078,P256,P257

Côté P . . . . . . ........... O068,P096,J011

Cote $S \ldots . \ldots \ldots \ldots \ldots \ldots \ldots \ldots$. . . . J001

Cote SC . . . . . . . . . . . . . . . . . P038

Cotton LA . . . . . . . . . . . . . . . . . . . . . J007

Coutlee F . . . . . . . . . . . . . . . . . . . . O093

Couture M.................... O022

Cox D..................... P164

Cox J . . . . . . . O . 063,0065,0067,0068,

...............P074,P110,P122,P192

Crawley AM. . . . . . . . . . . . . . P032

Crossie CL . . . . . . . . . . . . . . . . . . P039

Crouse J . . . . . . . . . . . . . . . . P234

Crouzat F . . . . . . . . . . . . . . . . J008

CTN 236 Study Group, The ......... O093

Czarnecki C . . . . . . . . . . . . . . . . . O057

\section{D}

Da Fonseca S . . . . . . . . . . . . . . . P040 Daher A . . . . . . . . . . . . . P007,P013,P026

Dahl C . . . . . . . . . . . . . . . . . O090

Dandekar S. . . . . . . . . . . . . . . . O058

Daniel M .................... O042

Daniels S . . . . . . . . . . . . . . . P007,P013

Daniels SM. . . . . . . . . . . . . . . P041

Daniuk C.................... 0057

Darraj M. . . . . . . . . . . . . . . . . . J012

Dassa C. ...................... O107

David T ...................... J008

Davidson M . . . . . . . . . . . . . . . . P199

de Pokomandy A . . . . . . . . . . . . . . P195

de Prinse K. . . . . . . . . O O113,P083,P203,P237

De Souza LR. . . . . . . . . . . . . . . . . P102

Deans GD. . . . . . . . . . . . . . . P146

Deering KN . . . . . . . . . . O080,O109,P111

DeGrado W ................... O061

deJesus E. . . . . . . . . . P057,P059,P060,P061

Demetrakopoulos AS. . . . . . . . P216,P217

Deutsch R. . . . . . . . . . . . . . . . P259

Dewey CE. .................... O044

Diallo AB.................. $\mathrm{P012}$

Diaz Llamas R . . . . . . . . . . . . . . P192

Dickson N . . . . . . . . . . . . . . . . O044

Diekema C.................... 0092

Dimeo M .................... P078

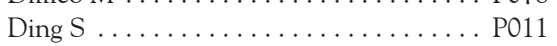

Dion H. . . . . . . . . . . . . . . . . . P063,P064

Dionne M.................... О075

Dolan L-A . . . . . . . . . . . . . . . . . P222

Donald J . . . . . . . . . . . . . . . . 0061

Donatelli J . . . . . . . . . . . . . . . P220

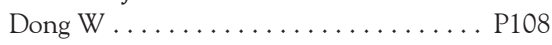

Dore GJ .................. P146

Dow B. .................. P116

Driedger SM. . . . . . . . . . . . . . P246

Drouin M-C................... О075

Dube A. . . . . . . . . . . . P153,P202

Ducandas X . . ............. O114,P264

Duff A ..................... P199

Duff P................. O106,O 117

Duff PK.................... O0 79

Dufresne S . . . . . . . . . . . P096,P150,J011

Duncan F . . . . . . . . . . . . . . . . . . O064

Durand M. . . . . . . . . . . . P076,P150

Durocher H . . . . . . . . . . . . . . . P239,P240

Durueke F. . . . . . . . . . . . . . . P243

Dykeman M . . . . . . . . . . . . . P193

Dzamba M ................... P004

\section{$\mathrm{E}$}

El Helou P . . . . . . . . . . . . . . . . . . . . O009

El-Far M. . . . . . . . . . . . . . . . . P040

El-Helou P . . . . . . . . . . . . . . . . . . . O0007,P062

Elston D . . . . . . . . . . . . . O021,P177

Embree J................... P010

Emlet CA...................................... 214

Emmanuel F. . . . . . . . . . . . . O101,0048

Engler K . . . . . . . . . . . . . . . . . O 012

Essex M .................... J004

\section{$\mathrm{F}$}

Fadel G. . . . . . . . . . . . O051,0052,P256

Fadel R................ O049,P251,P257

Falkenhagen A................. P002

Faller E. . . . . . . . . . . . . . . P035,P036

Faller EM . . . . . . . . . . . . . . . . 0085

Falutz J . . . . . . . . . . . . . . . . . P049

Farley JD . . . . . . . . . . . . . . P0 P

Faye R. .................... P140

Fellows L . . . . . . . . . . . . . . . O037,P077

Feng C . . . . . . . . . . . . . . . O O $045, \mathrm{O} 117$

Feng CX . . . . . . . . . O080,0109,P111

Feng $\mathrm{Y} \ldots \ldots \ldots \ldots \ldots \ldots \ldots \ldots \ldots \ldots$

Fergusson D . . . . . . . . . . . . . . 0095

Fergusson P................. O0 19

Ferlatte O............. O054,P112,P168

Fernandes JR . . . . . . . . . . . . . . P042

Fernet M. . . . . . . . . . . . . . O O $078, P 181$

Ferreira VH .............. O084, 0087

Fielden $S \ldots \ldots \ldots \ldots \ldots \ldots \ldots \ldots$. . . . . . . . . . . . . . . . O 19

Finch L...................... O0 037

Finney CA ............... P017,P018

Finzi A . . . . . . . . . . . . . . . . 0001

Fischer B. . . . . . . . . . . . . . . . . P146

Fisher M................... P204

Fletcher D . . . . . . . . . . . . . . . . J008

Fletcher S. . . . . . . . . . . . . . P072

Flicker S. . . . . . . . . . . . . . . . P190

Flores-Soto RA ................ . P043

Foisy M. . . . . . . . . . . . . . . . O009,P099,J010

Foisy MM . . . . . . . . . . . . . . . . . . . P097,P098

Fontaine $\mathrm{J} \ldots \ldots \ldots \ldots \ldots \ldots \ldots \ldots \ldots$. $\ldots \ldots 16$

Forbes J. . . . . . . . . . . . . . . . . О040

Forbes JC . . . . . . . . . . . . . . O091,P090

Forrest JI. . . . . . . . . . O102,P123,P162,P198

Fournier J . . . . . . . . . . . . P004,J005

Fowke K............ O029,P010,J006

Fowke KR. . . . . . . . . . . . . . P019,P230 
Fowler $S \ldots \ldots \ldots \ldots \ldots \ldots \ldots \ldots$ P256

Fraser C . . . . . . . . . . . . . O007,P062

French MA.................. O076

Fujiwara T . . . . . . . . . . . . . P005

\section{G}

Gadawski I . . . . . . . . . . . . . . . . . 0039

Gagnon D. . . . . . . . . . . . . . P121

Gagnon F...................... O 112

Gagnon M . . . . . . . . . . . . O020,P170

Gagnon V. . . . . . . . . . . . . . . . . . . . O098

Gahagan J. . . P113,P153,P199,P200,P201,P202

Gajayanaka N . . . . . . . . . . . . . P003

Galindo J . . . . . . . . . . . . .P104,P107,J009

Galindo P. . . . . . . . . . . . . . . . . . . P104

Galindo X. . . . . . . . . . . . . . . . . . . . J009

Gallagher L . . . . . . . . . . . . . . . . . O064

Gallant J....................... P059

Gallant M...................... О029

Gallant S . . . . . . . . . . . . . . P063,P064

Garber $\mathrm{G} \ldots \ldots \ldots \ldots \ldots \ldots \ldots . \ldots . \ldots 38$

García Sierra A . . . . . . . . . . . . . P229

Gardner S. . . . . . . . . . . . . O . . . . . . . . P10,0077,P152

Gartner K..................... P103,P128

Gaspar MA................. P171

Gathe $J \ldots \ldots \ldots \ldots \ldots \ldots \ldots$. . . . . . . . . 61

Gatignol A...........P007,P013,P026,P041

Gauthier J. . . . . . . . . . . . . . . . . P080,P100

George C . . . . ............... O115

George DB . . . . . . . . . . . . O046,P126,P231

Gheorghe M................ 0017

Gibson B . . . . . . . . . . . . . . . . P247

Giguere P. . . . . . . . . . . . . . O O 038, P079

Gil DM................... P091,P092

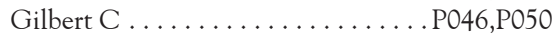

Gilbert L. . . . . . . . . . . . . . . . . . . P075

Gilbert M . . O071,O073,O104,P112,P127,P148

Gill J.................... 0065,0067

Gill JM ...................... P155

Gill MJ . . . . . . . . . . . . . . . 0056,P094

Gillis J ...................... O036

Gilmore N . . . . . . . . . . . . . . . . O012

Girard G. . . . . . . . . . . . . . . . . O053

Glazier RH. . . . . . . . . . P156,P157,P212

Godin G. .................P256,P257

Gogolishvili D . . . . . . . . . . . . O105,P094

Golubkov O.................... О033

Gómez Muñoz Q . . . . . . . . . . . . . . . P229

Gosselin A . . . . . . . . . . . . . O028,0083,P037

Gosselin O . . . . . . . . . . . . . . . P252

Gotte M . . . . . . . . . . . . . . . . . . . . . . 0060

Gough K..................... P078

Gough KA . . . . . . . . . . . . . . . . . P081

Goulet J-P . . . . . . . . . . . . . . . O083,,P037

Goyer M-E . . . . . . . . . . . . . P232,O112

Grant M..................... O029

Grant MD . . . . . . . . . . . . . . P023

Grant S. . . . . . . . . . . . . . . P262

Grebely J . . . . . . . . . . . . . . . P146

Green T . . . . . . . . . . . . . . . . . . P087

Greene BD .................. P176

Greene S . . . . . . . . O021,O077,P177,P195

Green-Walker L. . . . . . . . . . . . . . P131

Grewal P.......................J004

Gu C. . . . . . . . . . . . . . . . . O001

Guéhéneuc Y-G . . . . . . . .......P256,P257

Guillemi S . . . . . . . . . .P065,P066,P091,P092

Guimond T . ................. P233

Gulzar N. . . . . . . . . . . . . . . . . . 0061,J004

Gustafson D . . . . .............. P193

Gustafson P . . . . . . . . . . . . . . . . . O104

Gustafson R............. 0071,0104
Guta A . . . . . . . . . . . O020,O113,P203,P237

Gutierrez N . . . . . . . . . . . . . O098, O112

Guyer B . . . . . . . . . . . . . . . . P058,P060

\section{$\mathrm{H}$}

Haaland M . . . P052

Haddad E. . $\mathrm{O} 083, \mathrm{P} 037$

Hagen B . . . . . . . . . . . . . . . . . . P186 Haig T . . . . . . . . . .049,0050,0051,0052

Haight J . . . . . . . . . . . . . . . . P184

Hai-Tadesse M. . . . . . . . . . . . . . . P P228

Hall J . . . . . . . . . . . . . . . . . . . . P099

Halverson J. . . . . . O017,O0035,P114,P148,P149



Han Y....................... P005

Han Y-S ..................... J002

Hanass-Hancock J . . . . . . . . . . . . O O111

Hapanowicz M. . . . . . . . . . . . . . . . P110,P192

Hardy I. . . . . . . . . . . . . . . . . . . О0 033

Hare K . . . . . . . . . . . . . . . P153,P202

Harrigan PR . . . . . . . . . . . . . . . . P108

Harrigan R . . . . . . . . . . . . O034,P160

Harrigan RP . . . . . . . . . . . . . . . O0 035

Harris G . . . . . . . . . . . . . . . P153,P202

Harris M. . . . . . . . .0009,P065,P066,P091,P092

Hart T . . . . . . . . . . . . . . . . . . P218

Hart TA . . . . . . . . . . O115,P129,P214

Hasselrot K. . . . . . . . . . . . . . . O032

Haubrich R. . . . . . . . . . . . . . . . . O081

Heckerman D. . . . . . . . . . . . . . O 0081

Hedgecock M......................J008

Hennink M . . . . . . . . . . . . . P137,P140

Henrick BM.................. O031

Henry K . . . . . . . . . . . . . . . . . . . . . P061

Higgins NM . . . . . . . . . . . . . . . P080,P100

Hill J........................ O092

Hill-Mann A . . . . . . . . . . . . . . . . P201

Hiltz ER ..................... P172

HIV and Rehabilitation Policy

Research Team, The ............ P201

Hoe D. . . . . . . . . . . . . . . . . . . P218

Hogg RS. . . . . . O071,P138,P165,O034,O036, O069,O102,O104,O108,O118,

$\ldots \ldots \ldots \ldots \ldots \ldots \ldots \ldots \ldots \ldots \ldots$ P161,P162,P198

Hopkins S. . . . . . . . . . . . . . P143

Hopman W . . . . . . . . . . . . . P147

Hot A. . . . . . . . . . . . . . . . . P244,J023

Houston $\mathrm{S} \ldots \ldots \ldots \ldots \ldots \ldots \ldots$. . . . . P099

Hsiung G-YR . . . . . . . . . . . . . . P091,P092

Huchet E . . . . . . . . . . . . . . . . . P0 P0

Hudson P . . . . . . . . . . . . . . . . . . P202

Hudson PL . . . . . . . . . . . . . . . . . . . P153

Huff $\mathrm{H} \ldots \ldots \ldots \ldots \ldots \ldots . \ldots . \ldots . \ldots . \ldots 95$

Hughes A . . . . . . . . . . . . . . . . . . . . . . O044

Hughes C. . . . . . . . . . . . . . . . P097,P099

Hughes CA ..................J010

Hui $\mathrm{C} \ldots \ldots \ldots \ldots \ldots \ldots \ldots \ldots \ldots . . . \ldots 219$

Hukezalie K . . . . . . . . . . . . . . . . P008

Hull M . . . . O O067,P065,P091,P092,P123,P162

Humphreys S . . . . . . . . . . . . . . P165

Hunt K. . . . . . . . . . . . . . . . . O074,P128

Hunt N.................. P233

Husbands WC . ................. P215

\section{I}

Ibanescu R-I . . . . . . . . . . . . . . . . . . O033

Ibanez-Carrasco F. . . . . . . . . . . . . . P204,P214

Illing $\mathrm{V} \ldots \ldots \ldots \ldots \ldots \ldots \ldots \ldots \ldots . \ldots \ldots$



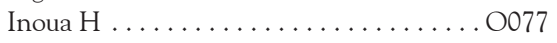

Ion A ................. O021,P177
Iqbal S . . . . . . . . . . . . . . . . . P102

I-Track Site Principal Investigators, The . O046, P126,P231

\section{$\mathrm{J}$}

Jacob A. . . . . . . . . . . . . . . . . P166

Jain S . . . . . . . . . . . . . . . O059

Jalloh C . . . . . . . . . . . . . . . . . P229

Jamal R. . . . . . . . . . . . . . . . . . . . P109

James L......................... . . . . .

Jandourek A. . . . . . . . . . . . . . . . . P057

Jasinghe VJ. . . . . . . . . . . . . . P003

Jayappa K...................... O002

Jenabian M-A .................. P015

Ji H. . . . . . . . . . . . . . . . . P114

Jiang $\mathrm{W} \ldots \ldots \ldots \ldots \ldots \ldots \ldots \ldots . \ldots \ldots 60 . \ldots \ldots$

Joanne $\mathrm{O} \ldots \ldots \ldots \ldots \ldots \ldots \ldots \ldots . \ldots . \ldots . \ldots \ldots 7$

Joffe AM. . . . . . . . . . . . . . . . . . . . P098

Jollimore J . . . . . ............ P112

Jolly AM. . . . . . . . . . . . . . . . . P151

Joncas L . . . . . . . . . . . . . . . . . 0075

Jones B . . . . . . . . . . . . . . . . . . . . . P047

Jongbloed K . . . . . . . . . . . . . . . P130,P139

Joseph L . . . . . . . . . . . . . . . . . . . . . О014

Joshi S . . . . . . . . . . . . . . . . P002

Jung $\mathrm{D} \ldots \ldots \ldots \ldots \ldots \ldots \ldots \ldots$ P045

\section{$\mathrm{K}$}

Kafka J .................... 0087

Kafka JK . . . . . . . . . . . . . . . . . . O084,P044

Kaida A . . . . . . . . . . .......... P195

Kain K . . . . . . . . . . . . . . P017,P018

Kakal J ...................... P036

Kakkar F. . . . . . . . . . . . . . . . O090,P086

Kang L . . . . . . . . . . . . . . . . . P142,P245

Kanters S . . . . . . . . . . . . . P123,P162

Kantors S . . . . . . . . . . . . . . . . . O071

Kapiriri L . . . . . . . . . . . . . . . . P178

Kappes JC. . . . . . . . . . . . . . . . . . . . . O059

Karanja S . . . . . . . . . . . . . P115,P118

Karapita S. .................P214,P215

Karatzios C................... P068

Kariri A . . . . . . . . . ........ P118

Kasper K. . . O O007,O018,P058,P062,P135,J012

Kaul R . .O011,O070,P017,P018,P044,P048,P131

Kaushic C. . . . . . . . . . . O084,O . . . . . . . . . P087,P044,P048

Kearney B. . . . . . . . . . . . . . . . . . . . . P059,P061

Keleher B ..................... O038

Kell AJ . . . . . . . . . . . . . . . . . . . P0 P003

Kelly C.........O013,O024,O099,P159,P191

Kelly D . . . . . . . . . . . . . . . . . . . P234,P254

Kelly EM .................... . P073

Kelly TL........................ P095

Kema IP . . . . . . . . . . . . . . . . . P015

Kennedy J. . . . . . . . . . . . . . . . . . P234

Kennedy L . . . . . . . . . . . . . . . . . P177

Kent SJ..................... P023

Kerr T. . . . . . . . . . . . . . O041,P160

Kerrison C . . . . . . . . . . . . . . . . . . . . P084 . . . . P131

Kesler MA . . . . . . . . . . . . . . . . . P131

Keynan Y . . . . . . . . . . . . O018,P135,J012

Khan I ................... P116

Kim CJ....................... P048

Kim J .................... P148

Kim J ...................... P149

Kim P ................... P127

Kimani J . . . . . . . . . . . . . . . . . . . O057,P019,P022,J006

Kimani M............. O057,P019,P022

King $\mathrm{K} \ldots \ldots \ldots \ldots \ldots \ldots \ldots \ldots \ldots \ldots \ldots$

$\mathrm{Kis} \mathrm{O} \ldots \ldots \ldots \ldots \ldots \ldots \ldots \ldots$

Kiwanuka N.................. О047

Klaric K-A ....................... O061 
Klein M ...................... P144

Klein MB . . . . O0 036,0063,О065,0066,О067, O068,0069,P069,P074,P080,P150

Knapp D.................... О081

Ko K.................... О056

Kobinger G. . . . . . . . . . . . . . . . O057

Kok S.................... P132

Kolla G...................... P233

Konrad S . . . . . . . . . . . O074,P103,P128

Konsmo EM . . . . . . . . . . . . . . O O110,P190

Koski L....................... $\bigcirc 037$

Kottorp A.................... P200

Kovacs C . . . . . . . . . . . . . . . . . . . . . P044,P070,J008

Kozak RA..................... О060

Krajden M .................... O073

Krajden M ......... O093,P127,P146,P149

Kramer VG. . . . . . . . . . . . . . . . . . . . . P105

Kravcik S . . . . . . . . . . . . . . . . P079

Krentz HB . . . . . . . . . . . . . . . . O056

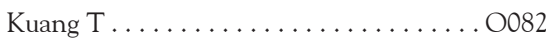

Kukreti V...................... 0096

Kvaratskhelia M................ O060

Kwag M .................. O073,P112

Kwaramba G . . . . . . . . O021,O077,P177

Kyle R. ....................... P144

\section{$\mathrm{L}$}

La D ...................... O057

La Fleur C . . . . . . . . . . . . . . . . . P055

La Porte C . . . . . . . . . . . . . . . . . . . . P010,0011,0038,0095

Labbé A-C . . . . . . . . . . . . . . . . . . P029

Labbé L. . . . . . . . . . . . . . . . . . P100

Labesse D ................ O112,P252

Labra $\mathrm{O} \ldots \ldots \ldots \ldots \ldots \ldots \ldots \ldots . \ldots \ldots$

Lacap P. . . . . . . . . . . . . . . . . . . 0057

Lachowsky NJ . . . . . . . . . . . . . . . . O044

Lacombe E . . . . . . . . . . . P252,P110,P192

Lai C. ................... P146

Lainé S. . . . . . . . . . . . . . . . . . . . . P013

Lajoie J . . . . . . . . . . . . . . . P019,P029

Lamarre V . . . . . . . . . O090,P068,P086,P090

Lambert A . . . . . . . . . . . . . . . . . . . . . P046

Lambert G . . . . . . . . . . P110,P122,P192

Lamoureux N . . . . . . . . . . . . . . . . . . . . . . . . . . . . . . . .

Landry S. . . . . . . . . . . . . . . . . O006

Laperrière H. . . . . . . . . . . . . . . . . . . . J023

Laplante F . . . . . . . . . . . . . . . . P061

Lapointe N . . . . . . . . . O016,0089,0090,P012, .P086,P090,P100,P181

Laprise C . . . . . . . . . . . . . . . P096,J011

Laroche $\mathrm{M} \ldots \ldots \ldots \ldots \ldots \ldots \ldots \ldots \ldots \ldots . . . . . \ldots 096$

Larocque E . . . . . . . . . . . . . . . . O006

Larouche A . . . . . . . . . . . . . 0098,0112

Lary T.................. P228,P260

Lavigne C. . . . . . . . . . . . . . . . . . . P003

Lavoie J . . . . . . . . . . . . . . . . . . . . P054 . . . . . P063,P064

Lavoie S . . . . . . . . . . . . . . . P063,P064

Lawless J . . . . . . . . . . . . . . . . . . . . . 0038

Lawrence J . . . . . . . . . . . . . . . . . . J005

Lax-Vanek J . . . . . . . . . . . . . . . . . . . P176

Le A ............... O011,0081,0082

Leahy R . . . . . . . . . . . . . . . . . P218

Leaver C. . . . . . . . . . . . . . . P216,P217

LeBlanc M-A . . . . . . . . . . . . . . . O103,P243

LeBlanc R. . . . . . . . . . . . . . . . . P023

Lebouche B ................... O012

Lebouché B .................... P015

Lebounga Vouma JI . . . . . . . . . . . . . P179

Leclerc P. . . . . . . . . . . O098,O112,P121,P133

Leduc N . . . . . . . . . . . . . . . . . . P242

Lee C . . . . . . . . . . . . . . . . . . . . 0038



Legaré H. . . . . . . . . . . . . . . . . . P261

Légaré H. . . . . . . . . . . . . . . . . . J023

Legault D . . . . . . . . . . . . . . . . . . . P064

Legault M. .................. 0033

Leifso K . . . . . . . . . . . . . . . . . . . P095

Lemieux B . . . . . . . . . . . . . . . . . O096

Leobon A . . . . . . . . . . . . . . O055,P206

Lepik C. . . . . . . . . . . . . . . . . . . O061

Lepik K. . . . . . . . . . . . . . . P123,P162

Lepist I . . . . . . . . . . . . . . . . . . . . . P009

Lesnikova D . . . . . . . . . . . . . . . . . . P164

Lessard R . . . . . . . . . . . . . . . . . . . O112

Lester RT . . . . . . . . . . . . . . . P088,P118

Levesque M . . . . ................ P007

Levett P . . . . . . . . . . . . . . . . . . . . . . J004

Lévy JJ . . . . . . . . . . . . . . O012,O078,P181

Lewellyn D. . . . . . . . . . . . . . . . . P201

Lewis J . . . . . . . . . . . . . . . P222

Li A ............. P117,P219,P245

LI T . . . . . . . . . . . . . . . . O097

Li Y........................ P114

Liang B .................... P020

Liddell M . . . . . . . . . . . . . . . . . . . P199

Light L . . . . . . . . . . . . . . . . . . . . . O077

Lim HJ . . . . . . . . . . . . . . O074,P103

Lim H-J . . . . . . . . . . . . . . P128

Lima V . . . . . . . . . . . . . . . . . . P165

Lima VD. . . . . . . . . . P065,P066,P154,P160

Lindsay $J \ldots \ldots \ldots \ldots \ldots \ldots \ldots \ldots$. P211

Lingwood CA. . . . . . . . . . . . . . . O062

Lioznov D. . . . . . . . . . . . . . . . . P164

Listgarten J . . . . . . . . . . . . . . . . . O081

Liu C . . . . . . . . . . . . . . . . . . . . . P059

Liu J . . . . . . . . . . . . . . . . O017,P001,P131

Liu LR ................... P020

Liu TT . . . . . . . . . . . . . . . P067

Llamas R. . . . . . . . . . . . . . . . . . P110

Lloyd K. . . . . . . . . . . . . . . . . P137,P140

Lo C . . . . . . . . . . . . . . . . . . . P047

Logie C. . . . . . . . . . . . . . . . . . O023

Logue K . . . . . . . . . . . . . . . . . . . . . P060

Longpré D . . . . . . . . . . . . . . P063,P064

Lord E. . . . . . . . . . . . . . . . . . . . P012

Lorway R . . . . . . . . . . . . . . . P246

Louch D . . . . . . . . . . . . . . . . . . . . . P084

Louie M . . . . . . . . . . . . . . . . . O100

Loutfy M. . . . . . O007,0023,0077,0093,P017, P018,P062,P129,P131,P156,P157, P158,P212,P218,J008

Loutfy MR . . O021,O036,O069,P069,P177,P195

Love E . . . . . . . . . . . . . . . . O038

Love RP ..................... P021

Lu S ...................... 0061

Lundrigan PA. . . . . . . . . . . . . P234,P254

Luo M. . . . . . . . . . . . . O057,P020,J005

Luyombya H.................. P219

\section{$\mathbf{M}$}

Maan EJ . . . . . . . . . . . . . O039,0091,P088 MacDonald KS. . . . . . . . . . . . . . . . . P0 P04

MacDougall G . . . . . . . . . . . . . . . P084

MacGillivray J . . . . . . . . . . . . . . . P085

Machouf N. . .0036,O069,P063,P064,P069,P150

MacKay R. . . . . . . . . . . . . . P218

MacPherson P . . . . . . . . . O038,0085,P055

MacPherson PA ........... P035,P036,P051

Madeleine D................. P056

Madsen J. . . . . . . . . . . . . . . . . . . . P255

Maginley JJ. . . . . . . . . . . . . . . . . P088

Mahal D.................... 0092

Mahood G . . . . . . . . . . . . . . . . . P220

Maitlan D. . . . . . . . . . . . . . P219
Malik N ................... O048, O101

Mallal S ..................... O081

Mallick R...................... O095

Man P...................... P066

Mancount Study Team, The ......... O071

Mangion M ................... P225

Manning E................... P170

Manno M.................... P152

Mansour S . . . . ............... P076

Mao Y....................... O001

Marchand R............ O054,P112,P248

Marcotte S..................... O096

Margolese S . . . . . . . . . . . . . . . . . O O O 077, P195

Margolick J.................. O007,P062

Markle T ................... 0082

Marlatt F . . . . . . . . . . . . . P248

Marsh AK. . . . . . . . . . . . . . . . . P P004

Martel M ................ O114,P264

Martin B. .................... P167

Martin E. . . . . . . . . . . . . . . . O081, 0082

Martin N . . . . . . . . . . . . . . . . . . P181

Martinez JL. . . . . . . . . . . . . . . . . . . P104,J009

Martinez-Cajas J. . . . . . . . . . . . . P147,P107

Marzouk R.................... P014

Masching R ............... P137,P140

Massé B ...................... O022

Massie L... . . . . . . . . . . . . . . . . . O078

Masson J-Y................... О006

Mathews M ................. P193

Maurais E. . . . . . . . . . . P074,P110,P192

Maurice J . . . . . . . . . . . . . . . . . . P153

Maxwell J.................P218,P248

Mayo N ................ O037,P077

Mazen H. . . . . . . . . . . . . . . . . 0066

Mazzulli T................. O011,0070

Mbuagbaw LC . . . . . . . . . . . . . . . P115

McCallum M ................... P105

McCarthy SD . . . . . . . . . . . . . . P0 P045

McClarty LM . . . . . . . . . . . . . . . . P135

McCombe JA . . . . . . . . . . . . . . . . P094

McCorrister S. . . . . . . . . . . . . . . P028

McDougall P . . . . . . . . . . . . . . . . P109

McEwen O....................... P194

McFadyen A.................... О050

McGee F. . . . . . . . . . . . . . . . . . O0 070

McGiffin C. .................. P211

McGovern RA................. O034

McGuire M ........... O071,O104,P136

McKinney M . . . . . . . . . . . . . . . . P169

McKye J ...................... P110

McKye JA. . . . . . . . . . . . . . . . . . P192

McMillan B . . . . . . . . . . . . . . . P229

Medford R . . . . . . . . . . . . . . . P147

Medjuck M................. P262

Meili R................. P082,P186

Melendez-Pena CE. ............ O066,P041

Melnychuk L . . . . . . . . . . . . . . . . . . O004

Mensah MN................... О078

Merindol N . . . . . . . . . . . . . . . . P012

Merkley B...................J008

Merks H. . . . . . . . . . . . . . . . P114

Merritt B ................. P200

Mesnard J-M .................... O006

Mesplede T. ...........P005,P027,J002

Meyers A .................... P022

Mfunyi CM ................... P046

Mian F .................. 0087

Michelow W. . O071,O102,O104,P138,P198,P235

Mignone J. . . . . . . . . . . . . . P230

Milan D ....................P123,P162

Miller C................. P130 
Miller P .................... 0056

Milligan RS . . . . . . . . . . . . . . P054

Milloy M-J . . . . . . . . . . . . . . . . P141

Mills A................... P057,P059

Mills E ...................... O095

Millson P ................ O070,P233

Millson PM ..................... P143

Min JE ...................... P141

Miranda J ..................P207,P256

Miron M-J ................... O088

Mishra S.................... 0048

Miura T ................... 0082

Mo T . . . ............. O081,P108

Mogk K................... O032

Moisi D. . . . . . . . . . O033,P105,P106

Mollison A.................... P225

Momar N . . . . . ................ O066

Mondal P . . . . . . . . . . . . . . . P128

Mondal PK..................... O074

Mondou I . . . . . . . . . . . . . . . . . . . . O O112

Money D . . . . . . . . . . . O016,0089,,0092

Money DM. . . . . . . O039,0091,0093,P087,

$\mathrm{P0} 88, \mathrm{P} 090$

Montaner J. . . O041,O045,O068,O106,O117, P065,P066,P091,P092,P160,P235

Montaner JS . . . . . . . O034,O036,O069,O108, P069,P111,P123,P141,P154,

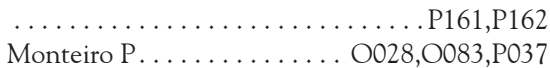

Monteith K . . . . . . . . . . . . . P110,P192

Montero M. . . . . . . . . . . . . . . . . . . O061

Moodie E ................ O067,P122

Moodie EE . . . . . . . . . . . O063,P074,P080

Moore D. . . . . . . O071,O102,P123,P162,P198

Moore DM .................... O079

Moravan V.................... O070

Morfaw FIL. . . . . . . . . . . . . . . . . P115

Morin G. . . . . . . . . . . . . . . . . P181

Morissette C. . . . . . . O098,O112,P121,P133

Mossman K. . . . . . . . . . . . . . . . . . . 0087

Motard J . . . . . . . . . . . . . . . . . . P007

Motorina A . . . . . . . . . . . . . . . . P012

Mouland A. . . . . . . . . . . . . . . O004,P026

Mouland AJ . . . . . . . . . . O005, O088, P039

Mtambo A . . . . . . . . . . . . . . . P065,P066

M-Track Study Team, The . . . . . . . . P136

Mueller K. . . . . . . . . . . . . . . . . . O084,,0087

Mueses H . . . . . . . . . . . . . . . . . P107

Mueses HF . . . . . . . . . . . . . . . . . . P104,J009

Mujib S. . . . . . . . . . . . . . . . . . P047

Muldoon KA ................ O079,0109

Murphy D..................... P063

Murray B . . . . . . . . . . . . . . . . . P009

Murray J ..................... P218

Murray M.................. О039

Murray MC . . . . . . . . . . . . . . P087,P088

Murzin K ................... P215

Musonda R........................J004

Muyinda H. . . . . . . . . . . . . . . . . . . O047

Muzyka CN ................... P246

Mwimanzi P.................. O082

Myers T . . . . . . . . . . P129,P142,P248

$\mathrm{N}$

Nahas Chebli G................. O022

Nahmias S . . . . . . . . . . . . . . . P136

Namaste V . . . . . . . . . . . . . . . . . . . J021

Nambi S.....................J028

Natalia G . . . . . . . . . . . . . . . P232

Nazli A. . . . . . . . . . . . O084,O. . . . . . . . P087,P044,P048

Ndumbi P. . . . . . . . . . . . . . P049,P075

Ndungu J . . . . . . . . . . . . . . . P211
Ndyanabo E ................... P211

Nesbitt A ..................... O045

Newman A. . . . . . . . . . . . . . . . . P147

Newman PA.................. O115

Newman R.................... P125

Ngolobe MH . . . . . . . . . . . . . . . . . O079

Nguyen T..................... P071



Nikolaenko S................... P164

Ning Z . . . . . . . . . . . . . . . . . P142

Nixon S . . . . . . . . . . . . . O111,P247

Njelesani J . . . . . . . . . . . . . . . . . P247

Noël L . . . . . . . . . . . . . . . . . . . P121

Nosyk B . . . . . . . . . . . . . . O080, P154,P160

Novitsky V. . . . . . . . . . . . . . . . . . . . . J004

Nurbhai M.................... P137

\section{$\mathrm{O}$}

O'Brien K................... P225

O'Brien KK . . . . . . . . . . . . . . . . P209,P210

O'Brien N. . . . . . . . . . . . . . . . . P195,P235

Ochsenbauer C . . . . . . . . . . . . . . . . O059

Ogilvie GS . . . . . . . . . . . . . . . P088

Ogunnaike-Cooke S . . . . . O O . . . . P126,P136,

......................P231,P244

Ogunnaike-Cooke SO . . . . . . . . . P137,P140

Ohnona F. . . . . . . . . . . . . . . . . P122

OHTN Cohort Study Research

Team, The ................ 0077,0070

Oliveira M . . . . . . . . . . . . P005,P105,P106

Oliver B . . . . . . . . . . . . . . . . . . P225

Omange WR .................... P022

Ongolo-Zogo P.................. P115

Ormond M. . . . . . . . . . . . . . . . . P229,P230

Orysiuk D. . . . . . . . . . . . . . . . . . J005

Osborne BJ . . . . . . . . . . . . . . O011,P044

Osman N . . . . . . . . . . . . . . . . . . . P005, J002

Ostrowski M. . . . . . . . . . O062,P001,P047

Otis J . . . . . . . O . O . . . . . O

............... O078,P122,P181,P206,

.

Oulanova O ................... P055

Owino M ................... P117

\section{$\mathrm{P}$}

Pacheco B. . . . . . . . . . . . . . . . . . . O001 Pai J . . . . . . . . . . O102,P138,P198,P235 Palladini L . . . . . . . . . . . . . . . . . . . . . . P1937, P077

Palmer AK . . . . . . . . . . . . . . . . . . P195

Pan SW ................. O100,P139

Pankovich J . . . . . . . . . . . . . . . . . . . P164

Pant Pai N . . . . . . . . . . . . O014,P144,P155

Papp E ....................... P006

Paquette D . . . . . . . . . . . . O104,P137,P140

Parashar S. . . . . . . . O108,O118,P141,P235

Parent R................ O0075,P121

Parmar P. . . . . . . . . . . . . . . . . P035,P036

Parsons M. . . . . . . . . . . . . . . . . . P033

Parsons MS. .................... P023

Patel M. . . . . . . . . . . . . . . . . . . P015

Patel SH. . . . . . . . . . . . . . . . . . O0 047

Patocs AE. . . . . . . . . . . . . . . . . P180

Patten S . . . . . . . . . . . . . . . O103,P243

Patterson TL . . . . . . . . . . . . . . P161

Pawlica P . . . . . . . . . . . . . . . . P024,P030

Payne M. . . . . . . . . . . . . . . . . . P109

Pearce ME . . . . . . . . O043,O100,P130,P139

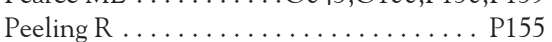

Peissel N. . . . . . . . . . . . . . . . . . . O103

Peltier D. . . . . . . . . . . . . O O116,P184,P187

Peng J . . . . . . . . . . . . . . . . . O002,P010

Penn R . . . . . . . . . . . . . . . . . . . . P233
Penney K . . . . . . . . . . . . . . . . . . . . J007

Pereyra F. ...................... O08

Perreault J-P. . . . . . . . . . . . . . . . . P007

Perreault Y . . . . . . . . . . . P216,P217

Persad I. . . . . . . . . . . . . . . . . O115

Peters H . . . . . . . . . . . . . . O057,P239,P240

Petitclerc C . . . . . . . . . . . . . . . . O008

Pham QT .................... P030

Pham TN .................... O027

Phiri B . . . . . . . . . . . . . . . . P243

Pick N . . . . . . . O039,0063,0065,0068,

. O092,0094,P074,P087,P088

Pierre Pitman L . . . . . . . . . . . . . . P125

Pillet S . . . . . . . . . . . . . . . . . . . . O057

Pilon R. . . . . . . . . . . . . . . . O057,P004,J005

Pindera C................ O018,P135

Pinto JC . . . . . . . . . . . . . . . . . O057

Piontkowsky D.................. P060

Pipe A ...................... O038

Plourde MB . . . . . . . . . . . . . P030,P043

Plummer A. . . . . . . . . . . . . . P059,P061

Plummer F . . . . . . . . O032,P010,P019,P028

Plummer FA. . . . . . . . . . O057,P020,P022,J005

Poitras M . . . . . . . . . . . . . . P137,P140

Polatajko H . . . . . . . . . . . . . . . P247

Poon K. . . . . . . . . . . . . . . . . . . . P219

Popiel M. .................... P225

Popovic V.................. P060,P061

Porch W.................... P222

Potter B . . . . . . . . . . . . . . . P076

Potter M. . .0063,0065,0067,0068,P074,P080

Potvin L . . . . . . . . . . . . . . . . . . O 107

Poudrier J . . . . . . . . . . . . . P016,P025,P029

Powell L . . . . . . . . . . . . . . . . . . P125

Power C . . . . . . . . . . . . . . . . . . P031

Prasad R................... P158

Prashar T .....................J005

Prego C. . . . . . . . . . . . . . . . . . . O057

Prentice T . . ............. O116,P187

Price CE.....................J020

Primeau M-A . . . . . . . . . . . . . . 0052

Proulx-Boucher K . . . . . . . . . . . . . P181

Pugh D ................... P125

Puskas C....................... P141



Quashie PK ................ P005,J002

Quirk E. .................... P059

\section{$\mathrm{R}$}

Raboud J. . . . . . . . . . . . . . . . . . O070,O093,P070,P152

Raboud JM . . . . . . . . . . . . O036,O069,P069

Rachlis A . . . . . . . . . . . . . . . . . . . P2597,P058,P061,P062,P094

Raeifar E. . . . . . . . . . . . . . . . . . . P259

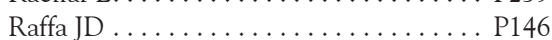

Raghunauth J................. P055

Rahman MA ....................J007

Ramanathan S . . . . . . . . . . . . . P P057

Ramdahin S . . . . . . . . . . . . . . . . . P010

Ramirez-Garcì P. . . . . . . . . . O022,P257

Ramotar K . . . . . . . . . . . . . . . . . P055

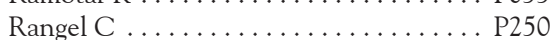

Rank C................0035,P148,P149

Rans S . . . . . . . . . . . . . . . . P176

Ransy DG. . . . . . . . . . . . . . . . P012

Rashbaum B.................. P057

Ray A...................... P009

Read S ...................... O040

Read SE . . . . . . . . . . . . . . . . . P002,P084

Rebecca GJ.................... P088

Reid B. . . . . . . . . . . . . . . . . O038 
Reinhard RJ . . . . . . . . . . . . . . . P208

Rekart M ................... O073

Remis RS . . . . . . . O017,O035,P131,P248

Ren J.................... P245

Riahi M .................... P076

Richardson CG ................. P124

Richer T......................J023

Riddler S . . . . . . . . . . . . . . . . . O081

Rini J . . . . . . . . . . . . . . . . P001

Rivas C. . . . . . . . . . . . . . . . . . . . P093

Robert W . . . . . . . . . . . . O071,O104

Robinson G .................. P210

Robinson L. . . . . . . . . . . . . . . . . P223

Robinson S. . . . . . . . . . . . . . . . . . O O077

Roger M . . . . . . . .0033,P016,P029,P122,P150

Rogers T.................... O103

Rojas C. . . . . . . . . . . . . . . P229

Rollet K . . . . . . O063,0065,0067,0068,P074

Rollet KC . . . . . . . . . . . . . . . . P150

Rosenes R. . . . . . . . . . . O095,P094,P164,P224

Rosenthal KL.................... O059

Ross E....................... P201

Roth EA ................. O102,P198

Rotondi N . . . . . . . . . . . . . . . P233

Rouleau D . . . . . . . . . O068,0096,P023,P074

Rouleau G . . . . . . . . . . . . . O022,P257

Rourke S. . . . . . . . . . . . . . . P204,P214

Rourke SB ........ O036,О069,0070,O105,

.P069,P094,P152,P259

Rousseau R. . . . . . . O049,0050,0051,O052, $\mathrm{P} 122, \mathrm{P} 251, \mathrm{P} 252$

Routy J-P . . . . . . O012,O026,O027,O028, O033, $0083,0096, \mathrm{P} 014, \mathrm{P} 015$

$\ldots \ldots \ldots \ldots \ldots \ldots \ldots \ldots \ldots \ldots \ldots \ldots \ldots \ldots \ldots$
Roy $\mathrm{A} \ldots \ldots \ldots \ldots \ldots$

Roy E .................... P133

Roy É ................ O042,P121,P233

Rozenbaum W ................. O012

Rud E . . . . . . . . . . . . . . . . . . J005

Rueda S . . . . . . O O105,P094,P214,P225,P259

Rumman A. . . . . . . . . . . . P067,P107

Rushowick B .................. P116

Rutherford A . . . . . . . . . . P132,P235

\section{$\mathrm{S}$}

Sabbah A .................... P027

Saeed S. . . . . . . . . . . . . . . . . O066

Sahar S. . . . . . . . . . . . . . . O065,0067

Sainsbury J . . . . . . . . . . . . . . P020

Sakac D . . . . . . . . . . . . . . . . . P045

Salbach N. . . . . . . . . . . . . . . . P225

Salim M...................... O048

Salit I . . . . . . . . . . . . . . . . . . . P070

Salters K................. O108, O 118

Salters KA . . . . . . . . . . . . . . . P141,P161

Salway Hottes T. . . . . . . . . . . . O O54,P127

Samarani S. . . . . . . . . . . . . . . . P014

Samji H . . . . . . . . O069,P069,P123,P162,P165

Sampalis J. . . . . . . . . . . . . . . . . O010

Samson J. . . . . . . . . . . . . . . . P012,P181

Samson L . . O016,O040,0089,P068,P090,P095

Samuels C . . . . . . . . . . . . . . P216,P217

Sanchez M . . . . . . . . . . . . . . . . P195

Sandler I. . . . . . . . . . . . . . . . . . . J008

Sandre D . . . . . . . . . . . . . . . O0 038

Sandstrom P. . . . . . . . . . . O057,P004,P114,

P148,P149,J005

Sangster M. . . . . . . . . . . . . . . . P224

Sankaran S. . . . . . . . . . . . . . . . . . O058

Sant $\mathrm{N} \ldots \ldots \ldots \ldots \ldots \ldots \ldots \ldots \ldots . \ldots \ldots$

Satkunarajah M ................. P001

Sattha B.................... 0039



Sax P . . . . . . . . . . . . . . . . . P059

Saxton P..................... O0 44

Scanlon K. . . . . . . . . . . . . . . P120,P238

Scarborough R ..............P007,P041

Schechter MT . . . . . . O043,O047,O100,P124,

P130,P139

Scott JK . . . . . . . . . . . . . . . . O0661,J004

Seatter E. .................... P255

Sekaly R-P . . . . . . . . . . . . O083,P037,P040

Seki J . . . . . . . . . . . . . . . . . . . . 0096

Serghides L. . . . . . . . . . . P006,P017,P018

Serhir B . . ................ P121

Serrano A. ................... P249,P250

Sesane M ..................... P243

Sewankambo NK . . . . . . . . . . . . . . O047

Shafran S. . . . . . . . . . . . . . . . . . P099

Shah RR. . . . . . . . . . . . . . . . . P085

Shah VB.................... P030

Shahin R ................. P143

Shaipanich T . . . . . . . . . . . . . P066

Shannon K. . . . . . . O045,0079,0080,0106,

$. \mathrm{O} 109, \mathrm{O} 117, \mathrm{P} 111, \mathrm{P} 160$

Sharma J. . . . . . . . . . . . . . . O014,P155

Sharma T . . . . . . . . . . . . . . . . P083

Shaw E.................... P007,P026

Shaw S..................... P135

Shaw SY................. O101,P151

Sheehan N. . . . . . . . . . . . . . . . . 0096,P150

Sheehan NL. . . . . . . . . . . . . . . . P100

Sheth P. . . . . . . . . . . . . P017,P018,P044

Sheth PM.................... O011

Shivkumar S. . . . . . . . . . . . P144,P155

Shorkey A ................ P176

Shoveller J ...................... O117

Shoveller JS . . . . . . . . . . . . . . . . . . . . O109

Shubert V..................... O105

Shum W..................... P071

Siegel S. .................... P178

Siemieniuk RA ............... О056

Silver $R \ldots \ldots \ldots \ldots \ldots \ldots \ldots \ldots \ldots . . . \ldots 233$

Simo A..................... O106

Simpson D . . . . . . . . . . . . . . . . . P109

Simwaba P .................. O 111

Sin D ....................... P066

Sinck L. . . . . . . . . . . . . . . P013,P041

Singer J. . . . . . . . . . . . . . . . . . . . . . . O096

Singhal N. . . . . . . . . . . . . . . . . . O095

Singhroy DN ............... P005,P027

Skinner S . . O074,P082,P103,P116,P128,P186

Slater K . . . . . . . . . . . . . . . . . . P003

Smaill F . . . . . . . . . . . . . . . . . . O . . . . . .

Small W.................... P235

Smieja M .................... O040

Smith G. . . . . . . . . . . . . . . O009,P060

Smith GH ..................J008

Smith LA . . . . . . . . . . . . . . . . . . . . P116

Smith S . . . . . . . . . . O021,O077,P177

Sodroski J. . . . . . . . . . . . . . . . . . O001

Sohi GK. . . . . . . . . . . . . . . . P008

Solomon P . . . . . . . O111,P209,P210,P225

Somers J . . . . . . . . . . . . . . . P161

Song R ..................... O025

Soudeyns H . . . . . . . . O090,P012,P086,P090

Spaans J . . . . . . . . . . . . . . . . . . . 0095

Spangelo L . . . . . . . . . . . . . . . . . . . . J J005

Spira B .................. P106,0012

Spittal PM . . O O043,O047,O100,P124,P130,P139

St Pierre C . . . . . . . . . . . . . . . . P046

Stals S. . . . . . . . . . . . . . . . . . P003

Stapleton S. . . . . . . . . . . . . . . . O029
Steele (nee Taleski) SJ. . . . . . . . . . . . . P142

Stein C...................... P113

Stein DR . . . . . . . . . . . . . . . P028

Stein N. . . . . . . . . . . . . . . . . P243

Steinberg M............. O073,P112

Stephenson L . . . . . . . . . . . . . . P208,J020

Stephenson LM . . . . . . . . . . . . . . . P184,P224

Stevenson B. . . . . . . . . . . . . O O102,P198

Stewart K . . . . . . . . . . . . . O009,P082,P186

Strathdee SA . . . . . . . . . . . . . O080,P111

Stratton N . . . . . . . . . . . . . . . P218

Strike C . . . . ...............

$\ldots \ldots \ldots \ldots \ldots \ldots \ldots$ P212,P233,P237

Su RC ..................... P022

Subra C ................. P050

Sugden $\mathrm{S} \ldots \ldots \ldots \ldots \ldots \ldots \ldots \ldots$. . . . . . . . . . 35

Sugden SM. . . . . . . . . . . . . . . . P051

Sullivan A . . . . . . . . . . . . . . . . . . O 017

Sullivan AB .................. O035

Summerlee AJ .................. O044

Summers M ................... P262

Sutton P.....................J020

Swantee C .................. O017,0035

Swenson LC. . . . . . . . . . . . . . . P108

Switzer S. . .

.O113,P203,P237

\section{$\mathrm{T}$}

Tallon BJ ................... 0026

Tan D . . . . . . . . . . . . . O009,P117,P158

Tan $\mathrm{DH} \ldots \ldots \ldots \ldots \ldots \ldots \ldots \ldots \ldots$ P078



Tan X ...................... O026

Tang D . . . . . . . . . . . . . . O057

Tarasuk J. . . . . . . . . . . . . . . . . . . . . . O46,P126,P137,P140,P231

Tasca G. . . . . . . . . . . . . . . . 0038

Tasca GA . . . . . . . . . . . . . . . P055

Taylor J J. . . . . . . . . . . . . . . . . . . P108

Teegee M................ О043,P139

Tellier P-P ..................... O0 014

Tessier P . . . . . . . . . . . . . . . . P050

Thabane $\mathrm{L} \ldots \ldots \ldots \ldots \ldots \ldots \ldots \ldots$. . . . . . . . . . . .

Thabane L ................... P118

Tharao W.......... O077,P131,P178,P195

Tharao WE. . . . . . . . . . . . . . . O023

Thériault J . . . . . . . . . . . . . . P181

Therrien R . . . . . . . . . . . . . . . . . P100

Thibodeau V . . . . . . . . . . . . . . . . . P029

Thierry AR. . . . . . . . . . . . . . . . . . P0 P03

Thomas R.......... O114,P063,P064,P150

Thomas R. . . . . . . . . . . . . . . . . P264

Thomas V................... P130,P139

Thompson L. . . . . . . . . . . . . . O018,P246

Thompson LH . . . . . . . . . . . . . . . . O048,O101

Thorne A . . . . . . . . . . . . O . 0039,0040,P090

Thumath M..................... P169

Thumath MA.................. O015

Tinmouth J..................... P070

Tomas K .................... 0017

Tong $\mathrm{L} \ldots \ldots \ldots \ldots \ldots \ldots \ldots \ldots \ldots \ldots$. . . . . . . . . . . . . . . . . . . .

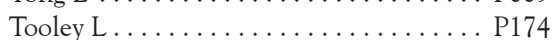

Torresilla C. . . . . . . . . . . . . . . . O006

Tossonian H. . . . . . . . O007,0010,0064,P062

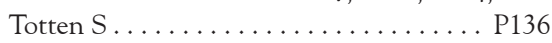

Toy J ..................... P065

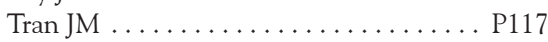

Travers R . . . . . . . . . . . . P120,P238

Tremblay C . . O O022,P015,P023,P076,P150,P257

Tremblay CL . . . . . . . . . . . . P056

Tremblay M ...................... O084

Trentham B ................ P210

Trottier B . . . . . . . O114,P063,P064,P150,P264

Trottier G............... P181 
Trottier H. . . . . . . . . . . . . . . . . . P096,J011

Trottier S . . . . . . . . . . . . . . . . . O093

Truong A . . . . . . . . . . . . . . . . P071

Trussler T . . . . . . . O054,O071,O104,P112

Tsai S . . . . . . . . . . . . . . . . . O061

Tsarevsky I . . . . . . . . . . . . . . . . . P100

Tseng A ..................... O096

Tsoukas C............ O036,0069,P069

Tsoukas CM . . . . . . . . . . . O026,P049,P075

Tucker M . . . . . . . . . . . . . . . . P153

Tucker R. . . . . . . . . . . . . . . P202

Tuff J. . . . . . . . . . . . . . . . . O057

Tupper P................... P132

Tynan A-M ................. P210

Tyndall M . . .O013,O024,O063,O068,P159,P191

Tyndall MW...................... O099

Tyndall MW. . . . . . . . . . . . . . . . . . . . P146

\section{$\mathrm{U}$}

Ueno $\mathrm{T} \ldots \ldots \ldots \ldots \ldots \ldots \ldots \ldots . \ldots . \ldots . \ldots 2$

Ukpong M . . . . . . . . . . . . . . . P243

Underwood MR ................. P005

Urquia M . . . . . . . . . . . . . . . . . . . P085 V

Vaillancourt É ................... P133

Valiente-Echeverria FA . . . . . . . . . . . . . O004

van Borek N. . . . . . . . . . . . . . . . P08 8

Van Caeseele P. ............... O018,P148

van der Kop ML. . . . . . . . . . . . . . . P088,P118

van Schalkwyk JE . . . . . . . . . . O O091,P087

Vasarhelyi K.................. P132,P235

Vassal A . . . . . . . . . . . . . . . . . . . . P150

Veillette L . . . . . . . . . . . . . . . O049,P251

Veillette M...................... P030

Veillette-Bourbeau L . . . . . . O O050,0051,О052

Vézina S . . . . . . . . . . . . . . P063,P064

Vicol L.......................... O092

Vincent H . . . . . . . . . . . . . . . . . . . P184

Vyboh K. . . $.0004,0005$

\section{W}

Wachihi C ................... O057

Wacleche VS ................. О028

Wagner EC. . . . . . . . . . . . . . . . . 0091,0092

Wainberg M...... О033,О049,0050,О051,

...............P106,P122,P251,J004

Wainberg MA ..O052,P005,P023,P027,P105,J002

Walker B .................... O082

Walker S. . . . . . . . . . . P153,P201,P202
Wallach I . . . . . . . . . . . . . O O114,P264 Walmsley S. . . O058,0063,0065,О067,0068, ............ O093,0095,P058,P061,P074 Walsh JG . . . . . . . . . . . . . . . P031 Walters M. .................... O024 Wang B. . . . . . . . . . . . O002,0079,P057,P060 Wang L.......................... O001

Wang S. . . . . . . . . . . . . . . . O061

Wang X . . . . . . . . . . . . O002,P010

Ward B . . . . . . . . . . . . . . . . . . P068

Ward NJ . . . . . . . . . . . . . . . . . . P041

Wasmuth J . . . . . . . . . . . . . . P017,P018

Waters ST .................... P124

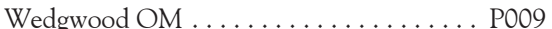

Wei X........................ P061

WelTel BC Study Team . . . . . . . . . . . . . P088

Wentzell J. . . . . . . . . . . . . . . . . . . . . . P079

Werb D..................... O041

Werb R......................... O009

Wertheimer S.................... P199

Westmacott G . . . . . . . . . . . O032,P028

White K . . . . . . . . . . . . . . . . . P059,P061

White S . . . . . . . . . . . . . O O13,P191

White SJ. . . . . . . . . . . . . O024,P143,P159

Willer DO ..................... P004

Wilson CL . . . . . . . . . . . . . . . P183,P190

Wilson MG ............. O105,P176,P259

Wilton J ....................... O103

Wingate K . . . . . . . . . . . . . . . . . . P087

Wobeser W. . . . . . . . . . . . . . . P147,P164

Wobeser WL . . . . . . . . . . . . . . . . . P067

Wohl D. . . . . . . . . . . . . . . . . . P059

Woit C ....................... P032

Wong AY . . . . . . . . . . . . . . . . O096

Wong G . . . . . . . . . . . . . . . . . O057

Wong J . . . . . . . . . . . . . . . . . . . . . . P0 P08

Wong JP ....................... P219

Wong L. . . . . . . . . . . . . . . . . . . . . . P P080

Wong RW................ O062,P052

Wood E. . . . . . . . . . . . . . . . . O041,P160

Woodman K........................ O056

Woods C. . .................... P108

Woods CK . . . . . . . . . . . . . . . . . . O034

Worthington C . . . . O013,0024,O099,P159,

.................. P191,P204,P225

Wrath K................P239,P240

Wu J . . . . . . . . . . . . . . . . P210

Wu K ....................... 0035

Wu S ..................... 061
Wylie J ..................P229,P230

Wylie JL..................... P151

\section{$\mathrm{X}$}



Xu H. . . . . . . . . . . . . . . . . . . . P021,P105

Xue $Y-1 \ldots \ldots \ldots \ldots \ldots \ldots \ldots \ldots$. P245

Xue-Zhong M................... P045

Y

Yale $K \ldots \ldots \ldots \ldots \ldots \ldots \ldots \ldots$ P057

Yam KK ...................... P068

Yamamoto A . . . . . . . ........ P091,P092

Yao X ..................... O002,P010

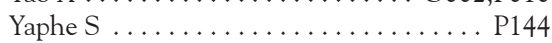

Yatsishin S . . . . . . . . . . . . . . . . . P164

Yee J ........................ P190

Yee JJ ..................... O110

Yi TJ ........................ O011

Yip B................... P154,P165

Yoong D . . . . . . . . . . . . . . . . . . P078

Yoong DM . . . . . . . . . . . . . . P081

Young J . . . . . . . . . . . . . . . . . . . . . . О068

Youth TA ................... O110

Yuan X-Y . . . . . . . . . . . . . . . . . . 0057

Yudin $\mathrm{MH} \ldots \ldots \ldots \ldots \ldots \ldots . . . . . .2085, \mathrm{P} 102$

Yuksel N......................J010

$\mathrm{Z}$

Zack E.................... P210

Zagorski B. .................... P157

Zaharatos G . .................... J004

Zahariadis G. ............... P148,P149

Zamora Suaza O . . . . . . . . . . . . . . . P229

Zanet DL ................ О039,P090

Zang G.................. O042,O072

Zanoni P.................... P033

Zhang H. . . . . . . . . . . . O043,O100,P124,P139

Zhang W .......... O108,P065,P161,P162

Zhao XY .................... P152

Zheng Y ...................... O002

Zhong L . . . . . . . . . . . . . . P058

Zhu X-Z ..................... P245

Zipursky AR.................... P094

Zolopa A ..................... P059

Zuluaga Toro M ................. P229

Zuniga R......................J023

Zylak C................ P137,P140, 


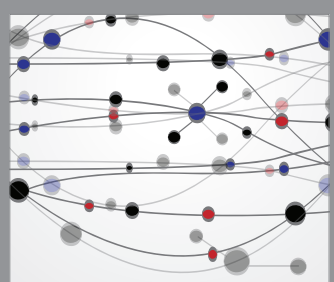

The Scientific World Journal
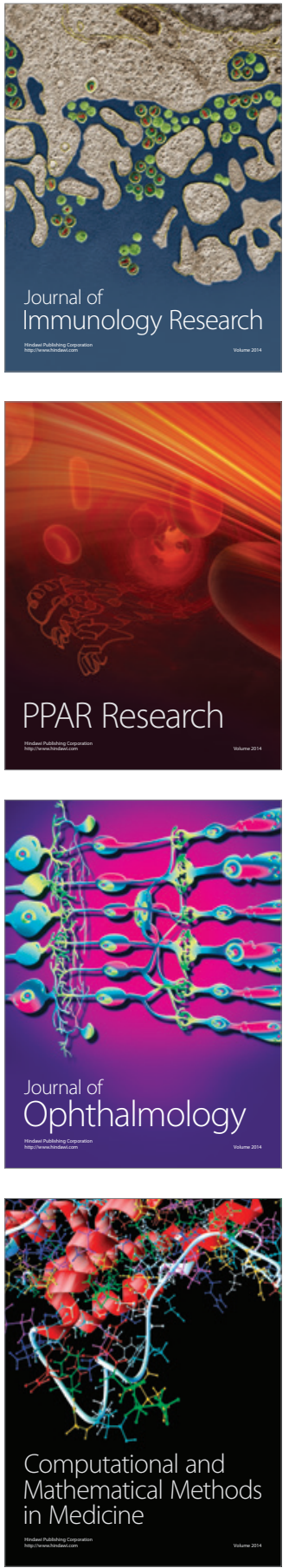

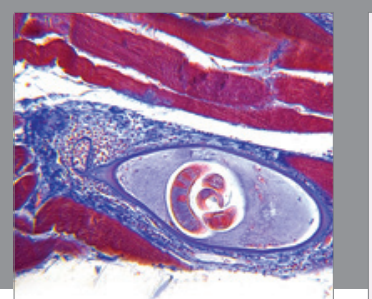

Gastroenterology Research and Practice

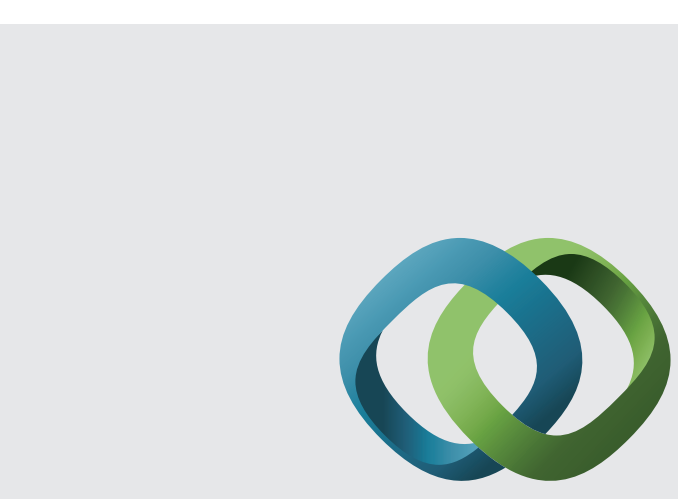

\section{Hindawi}

Submit your manuscripts at

http://www.hindawi.com



Disease Markers
